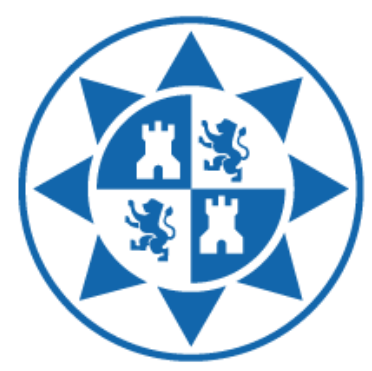

Universidad Politécnica de Cartagena Departamento de Estructuras y Construcción

\title{
Diseño óptimo robusto de estructuras utilizando meta-modelos estocásticos
}

Jesús Martínez Frutos

2014 



\title{
Universidad
}

Politécnica

de Cartagena

DT-16

\section{CONFORMIDAD DE SOLICITUD DEAUTORIZACIÓN DE DEPÓSITO DE TESIS DOCTORAL POR EL/LA DIRECTOR/A DE LA TESIS}

D. Pascual Martí Montrull, Director de la Tesis doctoral "Diseño óptimo robusto de estructuras utilizando meta-modelos estocásticos”.

\section{INFORMA:}

Que la referida Tesis Doctoral, ha sido realizada por D. Jesús Martínez Frutos, dentro del programa de doctorado Análisis y Diseño Avanzado de Estructuras, dando mi conformidad para que sea presentada ante la Comisión de Doctorado para ser autorizado su depósito.

La rama de conocimiento en la que esta tesis ha sido desarrollada es:

\author{
Ciencias \\ Ciencias Sociales y Jurídicas \\ $X$ Ingeniería y Arquitectura
}

En Cartagena, a 15 de Enero de 2014

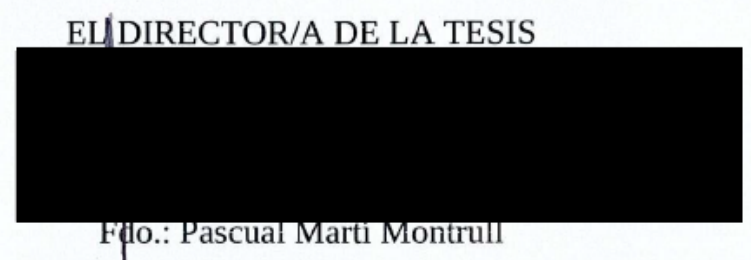

\section{COMISIÓN DE DOCTORADO}





\section{CONFORMIDAD DE DEPÓSITO DE TESIS DOCTORAL POR LA COMISIÓN ACADÉMICA DEL PROGRAMA}

D. Pascual Martí Montrull, Presidente de la Comisión Académica del Programa Análisis y Diseño Avanzado de Estructuras.

\section{INFORMA:}

Que la Tesis Doctoral titulada, "Diseño óptimo robusto de estructuras utilizando metamodelos estocásticos", ha sido realizada, dentro del mencionado programa de doctorado, por D.Jesús Martínez Frutos , bajo la dirección y supervisión del Dr. Pascual Martí Montrull.

En reunión de la Comisión Académica de fecha 15 de enero de 2014 , visto que en la misma se acreditan los indicios de calidad correspondientes y la autorización del Director de la misma, se acordó dar la conformidad, con la finalidad de que sea autorizado su depósito por la Comisión de Doctorado.

La Rama de conocimiento por la que esta tesis ha sido desarrollada es:

nCiencias

口Ciencias Sociales y Jurídicas

Xngeniería y Arquitectura

En Cartagena, a 15 de enero de 2014

P.S. EL MIEMBRO DE LA COMISIÓN ACADÉMICA DEL PROGRAMA

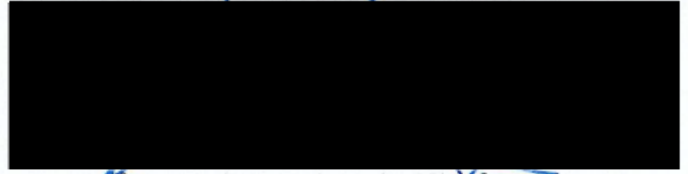

Fado: Mariano Victoria Nidolas

\section{COMISIÓN DE DOCTORADO}



A mis padres y hermana.

Por todo el tiempo que les robé pensando en mí... 



\section{Agradecimientos}

Me gustaría que estas líneas sirvieran para expresar mi más profundo y sincero agradecimiento a todas aquellas personas que con su ayuda han colaborado en la realización del presente trabajo. En especial al director de esta investigación el Dr. Pascual Martí por darme la oportunidad de realizar esta tesis, y a la Universidad Politécnica de Cartagena por la formación y apoyo económico recibido durante este periodo.

Quisiera hacer extensiva mi gratitud a mis compañeros del Departamento de Estructuras y Construcción. A todos los que directa e indirectamente ayudaron a la realización de esta tesis. En especial a David, por su orientación, su infinita paciencia y sus ánimos durante todo este tiempo.

A todos mis amigos y compañeros, en especial a Raquel por su apoyo constante, sus consejos y su comprensión durante este tiempo.

A mi hermana María Dolores, por su cariño y aliento en los momentos más difíciles.

Un especial agradecimiento merece la comprensión y apoyo recibido por mis padres. Que estas líneas sirvan como testimonio de mi amor y eterno agradecimiento por mi existencia. Por sacrificar gran parte de vuestra vida en mi educación y bienestar. Porque nunca podré devolver el tiempo que os he robado pensando en mí.

A todos ellos, muchas gracias. 



\section{Resumen}

Uno de los principales retos del diseño óptimo robusto es su aplicación a problemas con modelos de simulación de elevado coste computacional. En estos casos, el problema de diseño óptimo robusto puede resultar una tarea inabordable como consecuencia de: (1) el elevado coste computacional del modelo de simulación, (2) el número de evaluaciones requeridas por el proceso de propagación de incertidumbre y (3) el anidamiento del proceso de propagación de incertidumbre y de optimización multi-objetivo. Este problema ha motivado gran parte de los trabajos realizados en los últimos años en este campo. Actualmente, técnicas como la reducción de modelos, los meta-modelos o las superficies de respuesta, son ampliamente utilizadas para reducir el coste computacional de los modelos de simulación de alta fidelidad utilizados en el proceso de diseño. A pesar de su creciente aplicación en el campo de la optimización determinista, el desarrollo de meta-modelos estocásticos capaces de representar la respuesta aleatoria en el espacio de diseño, es un campo de reciente exploración.

En esta tesis, el problema de diseño óptimo robusto se aborda bajo un enfoque probabilista, en el que la respuesta aleatoria de la estructura se caracteriza por su distribución de densidad de probabilidad. Bajo este enfoque probabilista, la resolución del problema de diseño óptimo robusto incluye dos procesos anidados: (1) la propagación de incertidumbre y (2) la optimización multi-objetivo. El primero consiste en obtener la respuesta aleatoria de la estructura como consecuencia de la incertidumbre introducida por las variables y/o parámetros aleatorios del problema. El segundo consiste en la aplicación de técnicas de optimización multi-objetivo para obtener un conjunto de soluciones de compromiso entre la robustez y la respuesta estructural.

Esta tesis propone una solución eficiente del problema de diseño óptimo robusto de estructuras. La eficiencia de la metodología se obtiene minimizando el número de evaluaciones del modelo de simulación. Para ello se propone una estrategia adaptativa basada en un meta-modelo estocástico de la respuesta aleatoria de la estructura. Este meta-modelo emula la respuesta aleatoria de la estructura en el espacio de diseño y proporciona predicciones, acerca de la estadística del problema, con un bajo coste computacional. El meta-modelo 
estocástico propuesto se basa en: (1) la utilización de métodos de reducción dimensional para la estimación eficiente de los momentos estadísticos de la estructura, (2) la utilización de modelos de predicción Kriging para la aproximación de los momentos estadísticos en el espacio de diseño y (3) la reconstrucción de la distribución de probabilidad de la respuesta a partir de los momentos estadísticos, utilizando una distribución de Pearson. La incertidumbre epistémica introducida por la aproximación se incorpora en el proceso de optimización multi-objetivo (MO) mediante una estrategia adaptativa basada en el indicador de hipervolumen del frente de soluciones no dominadas que permite una resolución eficiente del problema multi-objetivo.

Las principales aportaciones del trabajo se presentan en tres capítulos. El Capítulo 4 presenta el algoritmo para la resolución eficiente del problema de optimización multi-objetivo, que subyace en el problema de diseño óptimo robusto. El Capítulo 5 presenta el algoritmo de reducción dimensional, dedicado a la estimación de los momentos estadísticos de la respuesta estructural. El Capítulo 6 presenta el meta-modelo estocástico propuesto para la resolución del problema de diseño óptimo robusto. Las contribuciones que se presentan en cada capítulo se validan numéricamente mediante la resolución de benchmarks existentes en la literatura, así como problemas estructurales que incluyen: no linealidades geométricas, del material y de contacto; incertidumbres en cargas, material y geometría; e incertidumbres modeladas con diferentes distribuciones de probabilidad y campos aleatorios. Los problemas estructurales resueltos abarcan tanto estructuras de nudos articulados como modelos continuos.

Palabras clave: Diseño óptimo robusto de estructuras - Modelos Kriging - Procesos Gaussianos • Optimización multi-objetivo • Algoritmos de análisis Bayesiano • Optimización bajo incertidumbre • Meta-modelos estocásticos • Métodos de reducción dimensional 


\section{Abstract}

One of the main challenges in the field of Robust Design Optimization (RDO) is its application to real world problems, especially when the simulation model involves computationally-expensive black-box functions (e.g. a finite element model). In these cases, the robust optimization problem is a highly demanding computational task due to (1) the computationally-expensive simulation model, (2) the high number of calls to the simulation model, and (3) the optimization problem, which involves two nested processes: the uncertainty propagation process and the multi-objective optimization process. The former aims to obtain the response of the structure given an uncertainty modelled by some stochastic variables of the problem. The latter instanciates an multi-objective optimization algorithm to obtain a trade-off between the robustness of the structural design and the structural performance. Techniques such as meta-models or surrogate models have been widely used to address this computational problem. However, the development of stochastic meta-models able to represent the random response with respect to random inputs is still an open issue.

This work presents an efficient procedure to address the RDO problem minimizing the number of evaluations of the simulation model and using an adaptive strategy that replaces the response of the problem by a stochastic meta-model. The stochastic meta-model is based on: (1) Dimension Reduction Methods for obtaining the statistical moments of the response; (2) Kriging models for approximating the statistical moments of the response within the design domain; and (3) the Pearson family of distributions for reconstructing the probability density distribution of the original response. The multi-objective optimization problem is solved using an adaptive strategy based on the information provided by the stochastic meta-model. A special interest has been given to reduce and eventually to eliminate the epistemic uncertainty introduced by the use of this meta-model instead of the original model. This epistemic uncertainty is quantified and incorporated in the multi-objective optimization by the expected improvement in the hypervolume of the front of non-dominated solutions. The contributions presented in this thesis are validated numerically by solving both classical benchmarks from the literature and structural problems. The structural problems include: truss structures and continuous models; geometric, material and contact nonlinearities; 
multiple sources of uncertainties (loads, material and geometry); and several probability distributions and random fields. The results show that the proposed algorithm requires a fewer number of evaluations of the simulation model with respect to widely used methods in this field of study.

Keywords: Robust Design Optimization of structures • Kriging Models - Gaussian Processes - Multi-objective Optimization • Meta-model Assisted Optimization - Bayesian Analysis - Optimization Under Uncertainty $\bullet$ Stochastic Meta-models $\bullet$ Dimension Reduction Methods 


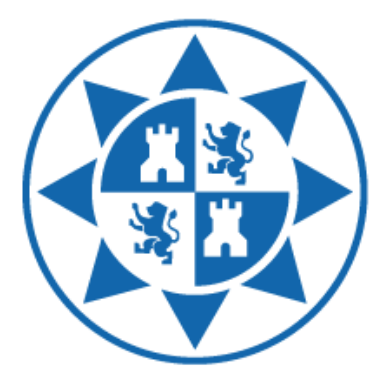

Universidad Politécnica de Cartagena

Departamento de Estructuras y Construcción

\title{
Diseño óptimo robusto de estructuras utilizando meta-modelos estocásticos
}

Tesis doctoral

\author{
Realizada por: \\ Jesús Martínez Frutos \\ Dirigida por: \\ Dr. Pascual Martí Montrull
}

Cartagena, 29 de marzo de 2014 



\section{Índice general}

1. Introducción 1

1.1. Contexto del problema . . . . . . . . . . . . . . . . 3

1.2. Declaración del problema $\ldots \ldots \ldots \ldots \ldots$

1.3. Objetivos de la tesis . . . . . . . . . . . . . . . 9

1.3.1. Objetivo principal. Desarrollo de un meta-modelo estocástico adaptativo para la resolución del problema de DOR ...................... 9

1.3.2. Objetivo parcial I. Actualización de los meta-modelos en las regiones alrededor de la región óptima $\ldots \ldots$. . . . . . 9

1.3.3. Objetivo parcial II. Propagación de incertidumbre con modelos no-intrusivos de alto coste computacional . . . . 9

1.3.4. Objetivo parcial III. Implementación de una herramienta para el diseño claro, flexible y encapsulado de algoritmos de optimización bajo incertidumbre asistida por meta-modelos 10

1.4. Principales contribuciones . . . . . . . . . . . . . . . . 11

1.5. Organización de la tesis . . . . . . . . . . . . . . 11

1.6. Lista de publicaciones . . . . . . . . . . . . . . . 13

1.6.1. Revistas . . . . . . . . . . . . . . . . 13

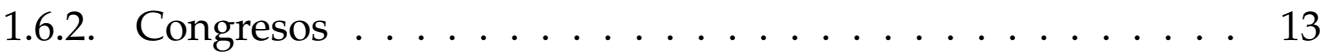

2. Revisión del problema de diseño óptimo robusto de estructuras 15

2.1. Introducción . . . . . . . . . . . . . . . . . 17

2.2. Formulación del problema de optimización bajo incertidumbre . . 17

2.2.1. Función objetivo . . . . . . . . . . . . . . . . . . . 19

2.2.2. Restricciones de igualdad . . . . . . . . . . . . . . . 19

2.2.3. Restricciones de desigualdad . . . . . . . . . . . . 20

2.2.4. Discusión . . . . . . . . . . . . . . . . . . 21

2.3. Criterios de robustez para la resolución del problema de DOR . . . 22

2.3.1. Criterios no probabilistas . . . . . . . . . . . . 23

2.3.2. Criterios probabilistas . . . . . . . . . . . . 26

2.4. Formulación multi-objetivo del problema DOR . . . . . . . . 28

2.4.1. Implementación numérica . . . . . . . . . . . . . . . . . 32

2.5. Revisión del problema de DOR y perspectivas . . . . . . . . . . 32

2.6. Ejemplos . . . . . . . . . . . . . . . . 34

2.6.1. Estructura articulada de 38 barras sometida a carga de compresión . . . . . . . . . . . . . . . 34 
2.7. Discusión y conclusiones . . . . . . . . . . . . . . . . 37

3. Modelos de predicción basados en procesos Gaussianos de regresión 39

3.1. Introducción . . . . . . . . . . . . . . . . . 41

3.1.1. Formulación del problema . . . . . . . . . . . . . . . . 41

3.1.2. Revisión del estado del arte y perspectivas . . . . . . . . . . . . 44

3.1.2.1. Superficies de respuesta polinómicas . . . . . . . . 45

3.1.2.2. Funciones de base radial . . . . . . . . . . . . 46

3.2. Definiciones previas . . . . . . . . . . . . . . . . 47

3.2.1. Espacio de probabilidad . . . . . . . . . . . . . . 4 47

3.2.2. Interpretación frecuentista de la probabilidad . . . . . . . . 48

3.2.3. Probabilidad condicionada. Teorema de Bayes . . . . . . . . 48

3.2.4. Variable aleatoria . . . . . . . . . . . . . . . . . . 49

3.2.5. Vector aleatorio . . . . . . . . . . . . . . . . . . . 50

3.3. Procesos Gaussianos . . . . . . . . . . . . . . . . 51

3.3.1. Definición .................. 51

3.3.2. Discretización del proceso estocástico . . . . . . . . . . 53

3.3.3. Ejemplos de funciones de correlación estacionarias . . . . . 55

3.3.3.1. Función de correlación lineal . . . . . . . . . . . 55

3.3.3.2. Función de correlación exponencial . . . . . . . . . 55

3.3.3.3. Función de correlación exponencial cuadrática o Gaussiana .................. . . 55

3.3.3.4. Función de correlación exponencial-Gaussiana . . 55

3.3.3.5. Función de correlación esférica . . . . . . . . 56

3.3.3.6. Función de correlación cúbica . . . . . . . . . . . . 56

3.3.3.7. Función de correlación spline . . . . . . . . . . . . 56

3.4. Predicción con procesos Gaussianos de regresión . . . . . . . . . . . 58

3.4.1. Modelo de regresión por mínimos cuadrados generalizada . 58

3.4.2. Modelo de regresión basado en Procesos Gaussianos . . . . 60

3.4.3. Predicción lineal insesgada óptima (Best Linear Unbiased

Prediction ): Universal Kriging . . . . . . . . . . . . . . . 61

3.4.3.1. Propiedades de la predicción lineal insesgada óptima . . . . . . . . . . . . . . . . . . 64

3.4.4. Predicción lineal insesgada empírica óptima (Empirical Best

Linear Unbiased Predictor) . . . . . . . . . . . . . . . . . 66

3.4.4.1. Estimación de los hiperparámetros mediante el método de máxima verosimilitud . . . . . . . . . . 66

3.4.5. Metodología propuesta . . . . . . . . . . . . 68

3.4.5.1. Etapa 1: Generación de observaciones . . . . . . 68

3.4.5.2. Etapa 2: Estimación de hiperparámetros y entrenamiento del meta-modelo . . . . . . . . . 68

3.4.5.3. Etapa 3: Validación . . . . . . . . . . . . 69

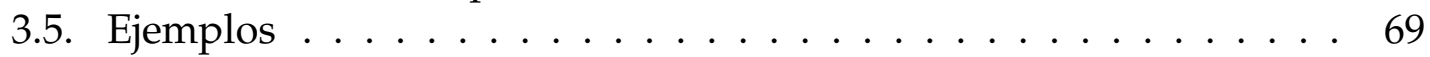

3.5.1. Modelización mediante campos aleatorios de la incertidumbre espacial del espesor de una lámina cilíndrica 
3.5.2. Influencia de la función de correlación en la predicción mediante procesos Gaussianos . . . . . . . . . . . . 72

3.5.3. Clasificación de modelos de predicción mediante errores de validación cruzada . . . . . . . . . . . . . . . . . . . . . . . . . . 86

3.6. Discusión y conclusiones . . . . . . . . . . . . . . . . 80

4. Optimización Multi-objetivo con modelos de predicción Bayesiana 83

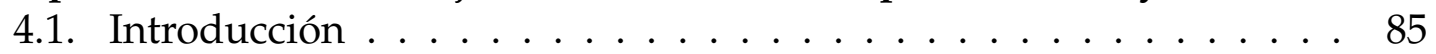

4.1.1. Formulación del problema . . . . . . . . . . . . . . 86

4.1.2. Revisión del estado del arte y perspectivas . . . . . . . . 87

4.2. Definiciones previas ... . . . . . . . . . . . . 93

4.2.1. Formulación del problema multi-objetivo . . . . . . . . . 94

4.3. Criterios heurísticos para la optimización Bayesiana mono-objetivo 95

4.3.1. Probabilidad de mejora . . . . . . . . . . . . . . . . . 95

4.3.2. Mejora esperada . . . . . . . . . . . . . . . . . . . 97

4.3.3. Algoritmo EGO (Efficient Global Optimization) (Jones et al., 1998) . . . . . . . . . . . . . . . . 997

4.4. Metodología propuesta . . . . . . . . . . . . . . . . . . 99

4.4.1. Mejora esperada en el hipervolumen dominado . . . . . . 102

4.4.2. Tratamiento de las restricciones de desigualdad . . . . . . . 104

4.4.3. Búsqueda de puntos en el espacio de diseño factible . . . . . 105

4.4.4. Consideraciones acerca del algoritmo . . . . . . . . . 107

4.4.4.1. Resolución de los subproblemas de optimización . 107

4.4.4.2. Tratamiento de evaluaciones fallidas del modelo de simulación . . . . . . . . . . . . . . . 112

4.4.4.3. Restricciones de bajo coste computacional . . . . . 113

4.4.4.4. Explotación del meta-modelo . . . . . . . . . . . 113

4.5. Ejemplos . . . . . . . . . . . . . . . . . . . . . 113

4.6. Discusión y conclusiones . . . . . . . . . . . . . . . . . 127

5. Propagación de incertidumbre con métodos de reducción dimensional y modelos Kriging

5.1. Introducción . . . . . . . . . . . . . . . . . . . . 131

5.1.1. Formulación del problema . . . . . . . . . . . . . . . . 131

5.1.2. Revisión del estado del arte y perspectivas . . . . . . . . 134

5.1.2.1. Discusión . . . . . . . . . . . . . . . . . . 138

5.2. Propagación de incertidumbre con métodos de reducción

dimensional . . . . . . . . . . . . . . . . . . . . . . . 139

5.2.1. Aproximación univariable . . . . . . . . . . . . . . . . . 139

5.2.2. Aproximación bivariable . . . . . . . . . . . . . . 140

5.2.3. Aproximación multivariable . . . . . . . . . . . . . 141

5.2.4. Momentos estadísticos de la respuesta . . . . . . . . . . . 142

5.2.5. Integración de las integrales de dimensión reducida . . . . . 143

5.3. Metodología propuesta . . . . . . . . . . . . . . . . . . . 145

5.3.1. Motivación . . . . . . . . . . . . . . . . . . . . 145

5.3.2. Implementación del algoritmo . . . . . . . . . . . 146 
5.4. Ejemplos . . . . . . . . . . . . . . . . . . . 152

5.4.1. Ejemplo TCPI-1 . . . . . . . . . . . . . . . . . 152

5.4.2. Ejemplo TCPI-2 . . . . . . . . . . . . . . . . 157

5.4.3. Ejemplo TCPI-3 . . . . . . . . . . . . . . . . . . . . . 161

5.4.4. Ejemplo TCPI-4 . . . . . . . . . . . . . . . . . . . . 164

5.4.4.1. Descripción del modelo . . . . . . . . . . . . . . 164

5.4.4.2. Análisis estocástico . . . . . . . . . . . . . 168

5.4.5. Ejemplo TCPI-5 . . . . . . . . . . . . . . . . . . 171

5.4.5.1. Introducción . . . . . . . . . . . . . 171

5.5. Discusión y conclusiones . . . . . . . . . . . . . . . . 176

6. Diseño óptimo robusto utilizando meta-modelos estocásticos 179

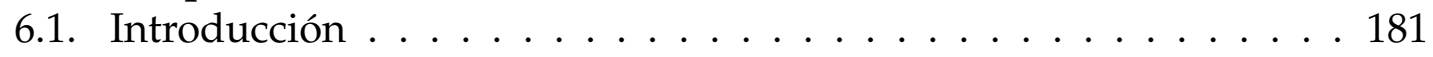

6.1.1. Formulación del problema . . . . . . . . . . . . . . . . . 181

6.1.2. Revisión del estado del arte y perspectivas . . . . . . . . 183

6.1.2.1. Formulaciones del problema de optimización bajo incertidumbre con meta-modelos . . . . . . . . 183

6.1.2.2. Diseño óptimo robusto con meta-modelos . . . . . 184

6.1.2.3. Discusión y perspectivas . . . . . . . . . . . . 188

6.2. Meta-modelo estocástico utilizando modelos Kriging y métodos de reducción dimensional . . . . . . . . . . . . . . . . . . . 190

6.3. Aplicación al problema multi-objetivo de diseño óptimo robusto . . 193

6.4. Ejemplos . . . . . . . . . . . . . . . . . . . . . . . . 194

6.4.1. Aplicación al diseño óptimo robusto de problemas multi-objetivo con restricciones de fiabilidad . . . . . . . . 194

6.4.1.1. Introducción . . . . . . . . . . . . . . . . 194

6.4.1.2. Configuración de los experimentos . . . . . . . 195

6.4.1.3. Validación . . . . . . . . . . . . . . . . 197

6.4.2. Problema TCMO1 . . . . . . . . . . . . . . . . . . . . . 198

6.4.3. Problema TCMO2 a TCMO4 . . . . . . . . . . . . . . . . . 204

6.4.4. Problema TCMO5 . . . . . . . . . . . . . . . . . . . . . 211

6.4.5. Aplicación al diseño óptimo robusto de estructuras de nudos articulados . . . . . . . . . . . . . . . . . . . 211

6.4.5.1. Problema TCSO1. Diseño óptimo robusto de una estructura de 4 barras con incertidumbre en material 211

6.4.5.2. Problema TCSO2. Diseño óptimo robusto de una torre de 25 barras . . . . . . . . . . . . . . . . 214

6.4.5.3. Problema TCSO3: Diseño óptimo robusto de un domo de 120 barras . . . . . . . . . . . . . . 220

6.4.6. Aplicación al diseño óptimo robusto de láminas imperfectas frente a pandeo . . . . . . . . . . . . . . . . . . . . 224

6.4.6.1. Diseño óptimo robusto de una lámina cilíndrica sin rigidizadores. Scordelis-Lo shell roof . . . . . . . 224

6.4.6.2. Modelo de elementos finitos . . . . . . . . . . . 224

6.4.6.3. Modelización de las variaciones aleatorias en el espacio . . . . . . . . . . . . . 225 
6.4.6.4. Modelado de la imperfección . . . . . . . . . 226

6.4.6.5. Formulación del problema de DOR . . . . . . . . . 227

6.4.6.6. Metamodelos estocásticos . . . . . . . . . . . 227

6.5. Discusión y conclusiones . . . . . . . . . . . . . . . . . . 229

7. Conclusiones 235

7.1. Resumen y principales contribuciones . . . . . . . . . . . . . 237

7.2. Trabajos futuros . . . . . . . . . . . . . . . 239

$\begin{array}{ll}\text { Referencias } & 243\end{array}$ 



\section{Índice de figuras}

1.1. Evolución en el uso de los recursos computacionales (adaptado de Simpson et al. 2008). . . . . . . . . . . . . . . . . . . . . . . 4

1.2. Contecto del trabajo de la tesis . . . . . . . . . . . 6

1.3. Declaración del problema. . . . . . . . . . . . . . . . 8

1.4. ooSBOUU toobox . . . . . . . . . . . . . . . . . . . 10

2.1. Función de Branin. $x$ es una variable de diseño determinista y $z$ un parámetro de incertidumbre. . . . . . . . . . . 23

2.2. Interpolación en el dominio de diseño del criterio de robustez basado en el peor caso (ecuación (2.17)) . . . . . . . . . . . 25

2.3. (a) Valor óptimo $f^{*}(z)$ en el dominio estocástico; (b) Interpolación en el dominio de diseño del criterio de robustez basado en la máxima desviación frente a la solución óptima (ecuación (2.19)) . . . . . . . . 26

2.4. Interpolación en el dominio de diseño del criterio de robustez basado en el cuantil de la distribución (ecuación (2.20)) . . . . . . . 27

2.5. Interpolación en el dominio de diseño del criterio de robustez basado en los momentos estadísticos de la respuesta (ecuaciones $(2.21)$ y $(2.22))$. . . . . . . . . . . . . . . . . 29

2.6. Interpolación en el dominio de diseño del criterio de robustez basado en la diferencia de percentiles (ecuación (2.24)) . . . . . . . . 30

2.7. Solución del problema MO de DOR de la función de Branin. (a) Soluciones óptimas sobre la interpolación de $\mu_{f(x, z)}$; (b) soluciones óptimas sobre la interpolación de $\sigma_{f(x, z)} ;$ (c) Conjunto de soluciones óptimas en el espacio de las funciones objetivo. . . . . . . . . . . . 31

2.8. Diagrama de flujo del problema multi-objetivo de DOR. . . . . . . . 32

2.9. Estructura de 38 barras de nudos articulados. (a) Universo estructural, condiciones de contorno y cargas. (b) DOD. . . . . . . . 35

2.10. Diseños óptimos robustos de la estructura de 38 barras de la Figura 2.9 para una variabilidad del $10 \%$ del módulo de Young y diferentes valores del coeficiente $\alpha$. En rojo barras a tracción, en azul barras a

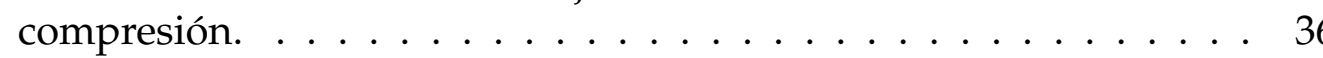

3.1. Diagrama de flujo del problema de predicción con procesos Gaussianos. 
3.2. Etapas del proceso de creación del meta-modelo. (a) Realizaciones del modelo a priori; (b) Etapa 1: generación de observaciones; (c) Etapa 2: estimación de parámetros del modelo y entrenamiento del meta-modelo; (d) Etapa 3: validación del meta-modelo. . . . . . . . .

3.3. Número de publicaciones relacionadas con optimización basada en meta-modelos durante el período 1980-2012 ${ }^{1}$. . . . . . . . . . . . . .

3.4. Meta-modelos de la funcion $\mathscr{M}=(6 x-2)^{2} \sin \left((6 x-2)^{2}\right)$ (Forrester et al., 2008). (a) Superficie polinómica de orden 3; (b) función de base radial multicuadrática. La línea azul continua representa el valor de la predicción. El área gris representa un intervalo de confianza de la predicción del $95 \%$. . . . . . . . . . . . . . . . . . . 47

3.5. funciones de correlación para $0 \leq\left|x_{i}-x_{i}^{\prime}\right| \leq 1 \ldots \ldots$. . . . . . 57

3.6. Realización de un proceso estocástico estacionario con media cero y funciones de correlación exponencial (línea azul) y exponencial cuadrática (línea roja). . . . . . . . . . . . . . . . . . . .

3.7. Interpolación de la función $\mathscr{M}(x)=(6 x-2)^{2} \cdot \sin \left((6 x-2)^{2}\right)$ (Forrester et al., 2008) mediante la PLIO. Función de correlación Gaussiana y regresión constante. . . . . . . . . . . . . . . . .

3.8. Consistencia asintótica de la PLIO para la aproximación unidimensional de la función $\mathscr{M}(x)=(6 x-2)^{2} \cdot \sin \left((6 x-2)^{2}\right)$ (Forrester et al., 2008). (a) error cuadrático medio de la predicción; (b) valor esperado de la predicción. . . . . . . . . . . . . . . . 65

3.9. Ilustración del error de validación cruzada en el cuarto punto del diseño de experimentos para la función $\mathscr{M}=(6 x-2)^{2} \sin \left((6 x-2)^{2}\right) . \quad 71$

3.10. Geometría de la lámina cilíndrica Scordelis-Lo (Scordelis y Lo, 1961). 72

3.11. Realización del campo aleatorio del espesor de la lámina cilíndrica Scordelis-Lo (Scordelis y Lo, 1961).

3.12. Modelos Kriging de la función $\mathscr{M}=(6 x-2)^{2} \sin \left((6 x-2)^{2}\right)$ con diferentes funciones de correlación. Las figuras de la izquierda representan el valor medio de la predicción. Las figuras de la derecha representan la función de verosimilitud reducida $\psi(\theta)$ y el resultado del problema de optimización (3.96). . . . . . . . . . . .

3.12. (Continuación). Modelos Kriging de la función $\mathscr{M}=(6 x-$ $2)^{2} \sin \left((6 x-2)^{2}\right)$ con diferentes funciones de correlación. Las figuras de la izquierda representan el valor medio de la predicción. Las figuras de la derecha representan la función de verosimilitud reducida $\psi(\theta)$ y el resultado del problema de optimización (3.96). .

3.13. Modelos Kriging de la función $\mathscr{M}=(6 x-2)^{2} \sin \left((6 x-2)^{2}\right)$ para diferentes valores del parámetro $\theta$ : (a) aproximación de alta calidad con un valor óptimo del parámetro $\theta$; (b) aproximación con baja calidad debido a una selección defectuosa del parámetro $\theta$. . . . 76

3.14. Modelos de predicción de la función de Rastrigin bidimensional (Törn y Zilinskas, 1989). . . . . . . . . . . . . . . . . . . . . . 78

3.15. Clasificación de los modelos Kriging de la función de Rastrigin. (a) y (c) Estimación basada en errores de validación cruzada;(b) y (d) Estimación basada en muestra de validación. . . . . . . . . . . . 
4.1. Ejemplo del frente de Pareto en el espacio de funciones objetivo masa y desplazamiento máximo para una estructura de 13 barras.

4.2. Diagrama de flujo del problema optimización Multi-objetivo asistida por procesos Gaussianos en el contexto de problemas de optimización bajo incertidumbre. . . . . . . . . . . . . . . . 87

4.3. Interpretación gráfica de la probabilidad de mejora. . . . . . . . 96

4.4. Implementación del algoritmo EGO para la minimización de la función $y(x)=-\sin (x)-\exp (x / 100)+10$ en el intervalo $[0,10]$. Las figuras de la izquierda representan la función original (línea roja discontinua) junto con el modelo de predicción Kriging (línea azul continua) para el conjunto de datos disponible. Las figuras de la derecha muestran la mejora esperada y su valor máximo que corresponde al punto candidato para mejorar el meta-modelo. . . . 100

4.5. Ilustración de la mejora esperada en el hipervolumen dominado. (a) hipervolumen dominado para un conjunto de soluciones óptimas; (b) mejora en el hipervolumen dominado producida por la inclusión de un nuevo punto $\left\{f_{1}\left(\mathbf{x}_{c}\right), f_{2}\left(\mathbf{x}_{c}\right)\right\}$ en el frente; (c) valor esperado de mejora en el hipervolumen dominado por la inclusión de un nuevo punto $\left\{\mu_{\hat{f}_{1}}\left(\mathbf{x}_{c}\right), \mu_{\hat{f}_{2}}\left(\mathbf{x}_{c}\right)\right\} \ldots \ldots \ldots \ldots . \ldots \ldots$

4.6. Ilustración del criterio $\mathscr{C}_{1}$ basado en una muestra inicial de seis puntos (circulos negros), junto con el límite de la restricción verdadera (línea gris). También se muestran los puntos de actualización en iteraciones anteriores (cuadrados blancos), y el candidato para la actualización en la iteración actual (cuadrado verde). . . . . . . . . . . . . . . . . . . . . 106

4.7. Problema Gomez \#3. Función objetivo y restricción (a-b); modelos Kriging iniciales de la función objetivo y restricción (c-d); región factible del modelo real y de la aproximación inicial (e-f). . . . . . . 108

4.8. Evolución del criterio de búsqueda en el ejemplo Gomez \#3. La muestra inicial se presenta como círculos negros. Los cuadrados blancos representan los puntos añadidos hasta la iteración. Los cuadrados verdes representan el candidato que maximiza el criterio en cada iteración. . . . . . . . . . . . . . . . . . . . . . . . . . 109

4.9. Evolución del criterio de búsqueda en el problema MOP2. La muestra inicial se presenta como círculos negros. Los cuadrados blancos representan los puntos añadidos hasta la iteración. Los cuadrados verdes representan el candidato que maximiza el criterio en cada iteración. . . . . . . . . . . . . . . . . . . . . . 118

4.10. Evolución del criterio de búsqueda en el problema TNK. La muestra inicial se presenta como círculos negros. Los cuadrados blancos representan los puntos añadidos hasta la iteración. Los cuadrados verdes representan el candidato que maximiza el criterio en cada iteración. . . . . . . . . . . . . . . . . . . . . . . 120 
4.11. Evolución del criterio de búsqueda en el problema DEB. La muestra inicial se presenta como círculos negros. Los cuadrados blancos representan los puntos añadidos hasta la iteración. Los cuadrados verdes representan el candidato que maximiza el criterio en cada iteración. . . . . . . . . . . . . . . . . . . . 122

4.12. Evolución del criterio de búsqueda en el problema SRN. La muestra inicial se presenta como círculos negros. Los cuadrados blancos representan los puntos añadidos hasta la iteración. Los cuadrados verdes representan el candidato que maximiza el criterio en cada iteración. . . . . . . . . . . . . . . . . . . . 124

4.13. Frentes de Pareto de los problemas ZDT1, ZDT2, Y ZDT3. . . . . . 126

5.1. Contexto del análisis estocástico de estructuras. . . . . . . . . . . . 132

5.2. Colocación de puntos en un espacio normalizado estándar tridimensional $\left(S=1\right.$ y $\left.N_{1}=4\right) \ldots \ldots \ldots 148$

5.3. Colocación de puntos en un espacio normalizado estándar bidimensional $\left(S=2, N_{1}=4, N_{2}=4,8\right) \ldots \ldots$. . . . . . 150

5.4. Colocación de puntos en un espacio normalizado estándar bidimensional. (a) puntos del método BIDRM . (b) $\left(S=2, N_{1}=4\right.$,

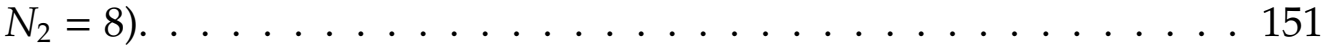

5.5. Problem TCPI-1. Localización de los experimentos $\left(S=1 ; N_{1}=4\right) .154$

5.6. Problema TCPI-1. (a) $\hat{Y}\left(X_{1}\right)$, (b) $\hat{Y}\left(X_{2}\right)$, (c) $\hat{Y}\left(X_{3}\right),(d)-\log \left(P_{R E S S}\right.$ RMS $)$ de $\hat{Y}\left(X_{1}\right),(e)-\log \left(P_{R E S S}\right.$ RMS $)$ de $\hat{Y}\left(X_{2}\right)$, (f) - log $\left(\right.$ PRESS $\left._{\text {RMS }}\right)$ de $\hat{Y}\left(X_{3}\right) .155$

5.7. Problem TCPI-1. Funciones de regresión y correlación resultantes tras la realización de un total de 1000 experimentos. (a) $\mathrm{Y}\left(\mathrm{X}_{1}\right),(\mathrm{b})$ $Y\left(X_{2}\right),(c) Y\left(X_{3}\right) \ldots \ldots \ldots \ldots . \ldots \ldots \ldots$

5.8. Problem TCPI-1. Función de densidad de probabilidad acumulada de 5.34. . . . . . . . . . . . . . . . . . . 158

5.9. Fortiniś clutch: esquema del ensamblado. . . . . . . . . . . . . . 158

5.10. Problem TCPI-2. Localización de los experimentos para una descomposición bidimensional con $N_{1}=6$ y $N_{2}=8$. . . . . . . 160

5.11. Problem TCPI-3. Estructura de 23 barras. . . . . . . . . . . . . . . 162

5.12. Problem TCPI-3. función de densidad de probabilidad de la función de estado límite. . . . . . . . . . . . . . . . . . . . . 163

5.13. Problem TCPI-4. Geometría, discretización y deformación del modelo de elementos finitos para el ensayo T1 (Coelho, 2004). . . . 166

5.14. Problem TCPI-4. Curva tensión-deformación real en escala logarítmica del acero. . . . . . . . . . . . . . . . . . . 167

5.15. Problem TCPI-4. (a) Curva carga-desplazamiento; (b) deformación principal máxima; (c) detalle de la deformación principal máxima en el ala del perfil. . . . . . . . . . . . . . . . . . . . . . 169

5.16. Problem TCPI-4. Función de densidad de probabilidad de la carga última del T-Stub. (a) Resultados para una reducción unidimensional, (b) resultados para una reducción bidimesional. $\quad 170$

5.17. Problema Scordelis-Lo shell roof (I). Geometría de la lámina cilíndrica Scordelis-Lo Scordelis y Lo (1961). 
5.18. Problema Scordelis-Lo shell roof (I). Realizaciones de los campos aleatorios con diferentes factores de correlación (l): (a)-(b) $l=$ $350 \mathrm{~mm}$; (b)-(c) $l=3500 \mathrm{~mm}$; (d)-(e) $l=5000 \mathrm{~mm} . \ldots 174$

5.19. Problema Scordelis-Lo shell roof (I). Función de densidad de probabilidad del desplazamiento vertical $U_{z}$. . . . . . . . . . 176

6.1. Diagrama de flujo del problema de optimización bajo incertidumbre. (a) Enfoque anidado convencional; (b) contexto del problema. . . . . . . . . . . . . . . . .

6.2. Formulaciones del problema de optimización bajo incertidumbre con meta-modelos. (a) Anidada; (b) anidada/meta-modelo; (c) meta-modelo/anidada; (d) meta-modelo/anidada/meta-modelo. . . 185

6.3. Meta-modelos de los dos primeros momentos estadísticos y cuantiles de la función 6.8 . . . . . . . . . . . . . . . . . . . . . 193

6.4. Problema TCMO1. Meta-modelos de los momentos estadísticos. . 201

6.5. Problema TCMO1. Meta-modelos de los cuantiles 0.75 y 0.95 de las restricciones. . . . . . . . . . . . . . . . 202

6.6. Problema TCMO1. Conjunto de soluciones óptimas de Pareto en el espacio de las variables de diseño (superior) y en el espacio de las funciones objetivo (inferior). . . . . . . . . . . . . . 203

6.7. Problemas TCMO2 a TCMO4. Evolución del criterio de búsqueda. 205

6.8. Problemas TCMO2 y TCMO3. Conjunto de soluciones óptimas de Pareto en el espacio de las variables de diseño (superior) y en el espacio de las funciones objetivo (inferior) obtenidas para el caso TCMO2 (a-c) y para el caso TCMO3 (d-f). . . . . . . . . . . . . . 206

6.9. Problema TCMO4. Conjunto de soluciones óptimas de Pareto en el espacio de las funciones objetivo. . . . . . . . . . . . . . . . 207

6.10. Problema TCMO2. Evolución del criterio de búsqueda. La muestra inicial se presenta como círculos negros. Los cuadrados blancos representan los puntos añadidos hasta la iteración. Los cuadrados verdes representan el candidato que maximiza el criterio en cada iteración. . . . . . . . . . . . . . . . . . . 208

6.11. Problema TCMO3. Evolución del criterio de búsqueda. La muestra inicial se presenta como círculos negros. Los cuadrados blancos representan los puntos añadidos hasta la iteración. Los cuadrados verdes representan el candidato que maximiza el criterio en cada iteración. . . . . . . . . . . . . . . . . . . . 209

6.12. Problema TCMO4. Evolución del criterio de búsqueda. La muestra inicial se presenta como círculos negros. Los cuadrados blancos representan los puntos añadidos hasta la iteración. Los cuadrados verdes representan el candidato que maximiza el criterio en cada iteración. . . . . . . . . . . . . . . . . . . 210

6.13. Problema TCMO5. Frentes de Pareto obtenidos tras aplicar el algoritmo NSGA-II sobre los meta-modelos actualizados (b) y sin

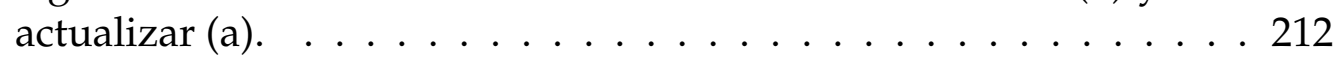

6.14. Problema TCSO1: geometría de la estructura. . . . . . . . . . . . 213 
6.15. Problema TCS01. Evolución del criterio de búsqueda. La muestra inicial se presenta como círculos negros. Los cuadrados blancos representan los puntos añadidos hasta la iteración. Los cuadrados verdes representan el candidato que maximiza el criterio en cada iteración. . . . . . . . . . . . . . . . . . . . . 215

6.16. Problema TCS01. Soluciones óptimas de máxima "performance" (Diseño A) y máxima robustez (Diseño B). . . . . . . . . . . . . . 216

6.17. Problema TCS01. (a-b) conjunto de soluciones óptimas de Pareto sobre los modelos Kriging de las funciones objetivo tras 50 iteraciones del algoritmo de actualización. (c) frente de Pareto tras 50 iteración de la estrategia adaptativa (64 puntos en total); (d) frente de Pareto utilizando meta-modelos con una muestra inicial de 64 puntos. . . . . . . . . . . . . . . . . . . 216

6.18. Problema TCSO2. Estructura de 25 barras. . . . . . . . . . . . . . . . 218

6.19. Problema TCS02. Frentes de Pareto: (a) Solución obtenida con NSGA-II y simulación MonteCarlo; (b) solución obtenida con NSGA-II y EKDRM; (c) Solución obtenida con el algoritmo EHV-EMO. . . . . . . . . . . . . . . . . . . . . . . 219

6.20. Problema TCS02. (a) diseño con mínima $\mu_{c}$; (b) diseño con mínima

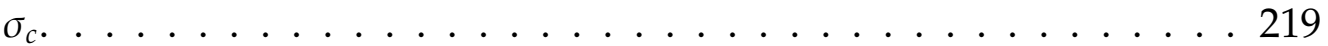

6.21. Problema TCS03. Domo de 120 barras de nudos articulados. . . . . 220

6.22. Problema TCS03. Frentes de Pareto: (a) solución obtenida con NSGA-II y simulación MonteCarlo; (b) solución obtenida con NSGA-II y EKDRM ( $\left.\mathrm{S}=1, \mathrm{~N}_{1}=1\right)$; (c) solución obtenida con EHV-EMO. (d) Evolución del criterio de búsqueda CEI. . . . . . . . 223

6.23. Geometría de la lámina cilíndrica Scordelis-Lo (Scordelis y Lo, 1961).224

6.24. Análisis de no lineal de la lámina Scordelis-Lo shell roof con la configuración perfecta. (a) Deformada para la carga crítica (10x); (b) curva factor de carga crítica - desplazamiento. . . . . . . . . . 225

6.25. Problema Scordelis-Lo shell roof (II). Realizaciones de los campos aleatorios de E, $F_{y}, \mathrm{y} h . \ldots 226$

6.26. Problema Scordelis-Lo shell roof (II). Tres primeros modos de pandeo (a)-(c), y forma de la imperfección geométrica para una realización

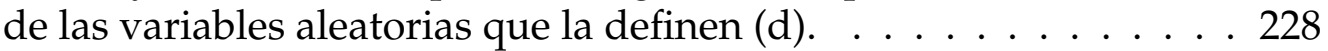

6.27. Problema Scordelis-Lo shell roof (II). Evolución del criterio de búsqueda. La muestra inicial se presenta como círculos negros. Los cuadrados blancos representan los puntos añadidos hasta la iteración. Los cuadrados verdes representan el candidato que maximiza el criterio en cada iteración. . . . . . . . . . . . . . . . . 230

6.28. Problema Scordelis-Lo shell roof (II). (a) Frente de Pareto, (b) evolución del criterio de búsqueda CEI. $\hat{\mu}_{v o l}$ en $m^{3}$. . . . . . . . . . . 232

6.29. Problema Scordelis-Lo shell roof (II). Soluciones óptimas de Pareto (puntos en color rojo). En color gris se representa el dominio de diseño no factible.

6.30. Problema Scordelis-Lo shell roof (II). (a) Diseño con mínima $\mu_{v o l}$. (b) Diseño con mínima $\operatorname{COV}_{\mathrm{P}_{\text {crit }}} \ldots \ldots$. . . . . . . . . . . . . 233 


\section{Índice de tablas}

4.1. Principales métodos de resolución del problema de optimización multi-objetivo. . . . . . . . . . . . . . . . 88

4.2. Resumen de los algoritmo de optimización MO basados en análisis Bayesiano. . . . . . . . . . . . . . . . . . 92

4.3. Problemas test utilizados en esta tesis. . . . . . . . . . . . . . . 114

4.4. Configuración de los parámetros del algoritmo EHV-EMO. . . . . . 114

4.5. Configuración de los parámetros del algoritmo NSGA-II. . . . . . . 115

4.6. Resumen de los resultados numéricos (I) . . . . . . . . . . . 117

4.7. Resumen de los resultados numéricos (II). . . . . . . . . . . 117

5.1. Ejemplos numéricos. . . . . . . . . . . . . . . . . . . . 153

5.2. Problem TCPI-1. Momentos estadísticos obtenidos con EKDRM y simulación MonteCarlo. . . . . . . . . . . . . . . . . . . . . . . 154

5.3. Problem TCPI-1. Probabilidades de $Y$ para pequeños valores de la distribución de probabilidad acumulada. . . . . . . . . . . . 157

5.4. Problem TCPI-2. Distribución de las variables aleatorias. . . . . . . 159

5.5. Problem TCPI-2. Momentos estadísticos de la respuesta obtenidos mediante una descomposición unidimensional con $N_{1}=2, N_{1}=4$, y $N_{1}=6$ respectivamente. . . . . . . . . . . . . . . . . . 159

5.6. Problem TCPI-2. Momentos estadísticos de la respuesta obtenidos mediante una descomposición bidimensional, polinomios del caos y cuadratura. . . . . . . . . . . . . . . . . . . . . . . 161

5.7. Problem TCPI-3. Distribución de probabilidad de las variables aleatorias. . . . . . . . . . . . . . . . . . . . 161

5.8. Problem TCPI-3. momentos estadísticos de la función de estado límite obtenidos mediante descomposiciones unidimensionales y bidimensional. . . . . . . . . . . . . . . . . . . . . 162

5.9. Problem TCPI-4. Distribución de probabilidad de las variables aleatorias. . . . . . . . . . . . . . . . . . . . . 168

5.10. Problem TCPI-4. Momentos estadísticos de la función de la resistencia última obtenidos mediante descomposición unidimensionales y bidimensional. . . . . . . . . . . . . . . 170

5.11. Problema Scordelis-Lo shell roof (I). Parámetros de los campos aleatorios lognormales de $E$ y $h . \ldots$. . . . . . . . . . . . . . 173

5.12. Problema Scordelis-Lo shell roof (I). Momentos estadísticos del desplazamiento según el eje $z$ del punto central de la lámina. . . . . 175 
5.13. Problema Scordelis-Lo shell roof (I). Momentos estadísticos de la tensión de Von Mises máxima de la lámina. . . . . . . . . . . . . . . 175

6.1. Formulación de los problemas TCMO\#. . . . . . . . . . . . . . . . . 195

6.2. Distribución de probabilidad de los problemas TCMO\#. . . . . . . . 196

6.3. Parámetros utilizados para los casos TCMO\#. . . . . . . . . . . . 197

6.4. Problemas TCMO\#. Resumen de los resultados numéricos para $P_{S}=0.75 \ldots \ldots \ldots \ldots \ldots$

6.5. Problemas TCMO\#. Resumen de los resultados numéricos para

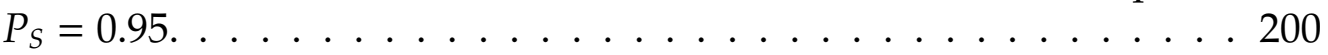

6.6. Problema TCMO1. Número de evaluaciones total. . . . . . . . . . . 202

6.7. Problema TCSO\#. Parámetros del algoritmo EHV-EMO . . . . . . . 212

6.8. Problema TCSO2. Coordenadas de los nudos de la estructura de 25 barras. . . . . . . . . . . . . . . 217

6.9. Problema TCSO2. Distribución de las variables aleatorias. . . . . . . 217

6.10. Problema TCS02. Resumen de los resultados numéricos. . . . . . . 218

6.11. Problema Scordelis-Lo (II). Parámetros de los campos aleatorios de

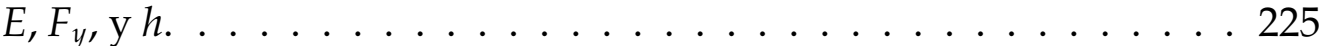

6.12. Problema Scordelis-Lo shell roof (II). Resumen de los resultados numéricos. . . . . . . . . . . . . . . . . . . . . . . 229 


\section{Lista de algoritmos}

1. Ajuste y selección del modelo de predicción Kriging. . . . . . . . . 70

2. Algoritmo EGO (Jones et al., 1998) . . . . . . . . . . . . . . . . . . . . 98

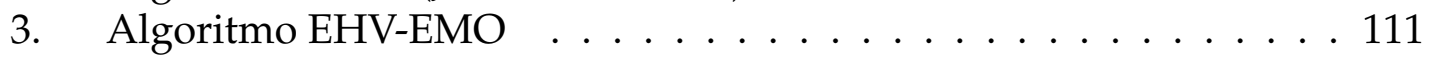

4. Algoritmo EKDRM . . . . . . . . . . . . . . . . . . . . 147

5. Meta-modelo estocástico . . . . . . . . . . . . . . . . . . . . . . . 192 



\section{Lista de abreviaturas}

CR Criterio de robustez.

BIDRM BIvariate Dimension-Reduction Method.

CMA-ES Covariance Matrix Adaptation Evolution Strategy.

DEMM Deterministic Equivalent Modeling Method.

DIRect Dividing Rectangles.

DOD Diseño Óptimo Determinista.

DOF Diseño Óptimo basado en Fiabilidad.

DOR Diseño Óptimo Robusto.

EGO Efficient Global Optimization.

EHV - EMO Expected HyperVolume Efficient Multi-objective Optimization.

EHVI Expected HyperVolume Improvement.

EI Expected Improvement.

EKDRM Extended Kriging based Dimension Reduction Method.

FDP Función de Densidad de Probabilidad.

FORM First Order Reliability Method.

GD Generational distance.

GEI Generalize Expected Improvement.

HDMR High-Dimensional Model Representation.

HLRI Hasofer-Lind Reliability Index.

HR Hypervolume Ratio.

HV Hypervolume.

HVI Hypervolume Improvement. 


\begin{tabular}{|c|c|}
\hline $\begin{array}{l}\text { KDRM } \\
K G-M O G A\end{array}$ & $\begin{array}{l}\text { Kriging-based Dimension Reduction Method. } \\
\text { Kriging-with-DOE MOGA. }\end{array}$ \\
\hline MARS & Multi-Adaptive Regression Splines. \\
\hline$M C$ & MonteCarlo. \\
\hline$M L E$ & Maximum Likelihood Estimation. \\
\hline$M M A$ & Method of Moving Asymptotes. \\
\hline MO & Multi-Objetivo. \\
\hline MOEAs & MultiObjective Evolutionary Algorithms. \\
\hline MPFE & Maximum Pareto Front Error. \\
\hline$M P P$ & Most Probable Point. \\
\hline$M R D$ & Métodos de Reducción Dimensional. \\
\hline$M R U$ & Método de Reducción Unidimensional. \\
\hline NIPC & Nonintrusive Polynomial Chaos method. \\
\hline$N S G A-I I$ & Nondominated Sorting Genetic Algorithm II. \\
\hline$N S G A-I I-A N N$ & $\begin{array}{l}\text { Nondominated Sorting Genetic Algorithm II \& } \\
\text { Artificial Neural Network. }\end{array}$ \\
\hline ooSBOUU & $\begin{array}{l}\text { object oriented toolbox for Surrogate-Based } \\
\text { Optimization Under Uncertainty Toolbox. }\end{array}$ \\
\hline PI & Propagación de Incertidumbre. \\
\hline PLIEO & $\begin{array}{l}\text { Predicción Lineal Insesgada Empírica } \\
\text { Óptima. }\end{array}$ \\
\hline PLIO & Predicción Lineal Insesgada Óptima. \\
\hline PoI & Probability of Improvement. \\
\hline PRESS & PREdiction Sum of Squares. \\
\hline PSP & Pareto Set Pursuing. \\
\hline RMSE & Root Mean Square Error. \\
\hline RRDO & Reliability-based Robust Design Optimization. \\
\hline$S C$ & Stochastic Collocation method. \\
\hline$S M S-E M O A 2$ & $\begin{array}{l}\text { S-Metric Selection Evolutionary MultiObjective } \\
\text { Algorithm. }\end{array}$ \\
\hline SORM & Second Order Reliability Method. \\
\hline SPEA2 & Strength Pareto Evolutionary Algorithm 2. \\
\hline$S R S M$ & Stochastic Response Surface Method. \\
\hline TSPA & chebycheff Saddle Point Approximation. \\
\hline
\end{tabular}


VRDO Vulnerability-based

Robust Design

Optimization. 


\section{Lista de símbolos}

$\Omega \quad$ espacio muestral.

$\Phi(\cdot)$ función de densidad de probabilidad acumulada estándar.

$\beta_{j} \quad$ índice de fiabilidad de la restricción j-ésima.

C matriz de covarianza.

F matriz de regresión.

K matriz de rigidez.

$\mathbf{R}$ matriz de correlación.

Y vector de observaciones.

Z vector aleatorio Gaussiano.

$\Lambda$ matriz diagonal con las desviaciones estándar de cada componente de la variable $\mathrm{X}$.

a vector de pesos de la predicción lineal insesgada óptima.

f vector de cargas.

u vector de desplazamientos.

$\mathbf{x}$ vector de variables de entrada del modelo de simulación.

y vector de respuestas del modelo de simulación.

$\Phi \quad$ matriz de Vandermonde.

$\Psi \quad$ matriz de Gram.

$\psi \quad$ función de verosimilitud reducida.

$\psi_{e} \quad$ vector de distancias euclídea a los centros de la base funcional (función de base radial).

$\boldsymbol{\theta}$ vector de hyperparámetros del meta-modelo.

$f \quad$ funciones de regresión.

$\boldsymbol{\beta} \quad$ vector de pesos del modelo de regresión.

$\lambda \quad$ vector de multiplicadores de Lagrange.

$\delta_{X} \quad$ coeficiente de desviación de $X$.

$\emptyset \quad$ conjunto vacío. 
$\gamma_{j} \quad$ factor de seguridad de la restricción j-ésima.

$\hat{Y}_{-i} \quad$ predicción de validación cruzada del punto de diseño $i$.

$\hat{\mathscr{M}} \quad$ modelo de predicción o meta-modelo.

$n_{g} \quad$ número de restricciones de desigualdad.

$n_{h} \quad$ número de restricciones de igualdad.

$\kappa_{X} \quad$ coeficiente de asimetría de $X$.

$\lambda \quad$ autovalores solución de la ecuación integral de Fredholm de segundo orden.

$\mathbb{P} \quad$ operador probabilidad.

$\mathbb{R} \quad$ espacio de números reales.

N espacio de números naturales.

$\mathscr{C}_{1} \quad$ criterio de búsqueda de candidatos factibles (algoritmo EHV-EMO).

$\mathscr{C}_{2}$ criterio de búsqueda de candidatos a formar parte del frente de Pareto (algoritmo EHV-EMO).

$\mathscr{D}$ conjunto de observaciones del modelo de simulación.

$\mathscr{D}_{f, j} \quad$ dominio de fallo de la restricción j-ésima.

$\mathscr{D}_{\text {fact }}$ dominio factible.

$\mathscr{F} \quad$ colección de todos los sucesos aleatorios de un espacio muestral.

$\mathscr{G} \mathscr{P} \quad$ proceso estocástico Gaussiano.

$\mathscr{M} \quad$ modelo de simulación.

$\mathscr{N}$ distribución de probabilidad normal.

$\mathscr{P}$ medida de probabilidad o función de probabilidad.

$\mathscr{R} \quad$ función de correlación.

$\mathscr{X}$ conjunto de muestras del diseño de experimentos.

$\mathscr{Y}$ conjunto de respuestas del diseño de experimentos.

$\mu_{X} \quad$ media de $X$.

$\mu_{\hat{Y}_{0}} \quad$ valor medio de la predicción lineal insesgada óptima.

$\phi(\cdot) \quad$ función de densidad de probabilidad estándar.

$\rho_{X, Y} \quad$ coeficiente de correlación de X e Y.

$\mathrm{Crit}_{\max }$ criterio de parada (algoritmo EHV-EMO).

$\mathrm{It}_{\max }$ número máximo de iteraciones (algoritmo EHV-EMO). 
NumFeas $_{\min }$ número mínimo de diseños factibles (algoritmo EHV-EMO).

$\mathrm{Tol}_{\mathrm{g}} \quad$ tolerancia de las restricciones de desigualdad (algoritmo EHV-EMO).

$\mathbb{E}[\cdot] \quad$ operador esperanza matemática.

$\sigma_{X} \quad$ desviación estándar de $X$.

$\sigma_{\hat{Y}_{0}}^{2} \quad$ varianza de la predicción lineal insesgada óptima.

$\varphi \quad$ autovectores solución de la ecuación integral de Fredholm de segundo orden.

$\xi \quad$ variables aleatoria Gaussiana estándar.

$\mathbf{c}^{(i)} \quad$ i-ésimo origen de la base funcional (función de base radial).

$P_{\mathrm{F}, j} \quad$ probabilidad de fallo de la restricción j-ésima.

$P_{\mathrm{S}, j} \quad$ probabilidad de que el diseño sea factible de la restricción j-ésima.

$\mathrm{EI}(\mathbf{x}) \quad$ función de mejora esperada.

$\mathrm{I}(\mathbf{x}) \quad$ función de mejora.

$\operatorname{PoI}(\mathbf{x}) \quad$ función de probabilidad de mejora.

$g_{j}(\mathbf{x}) \quad$ restricción de desigualdad j-ésima.

$h_{k}(\mathbf{x}) \quad$ restricción de igualdad k-ésima.

$\| \bullet \quad$ norma euclídea.

$N_{\text {evals }} \quad$ número de evaluaciones del modelo de simulación.

$C[X, Y] \quad$ covarianza de $\mathrm{X}$ e $\mathrm{Y}$.

$\operatorname{COV}_{X} \quad$ coeficiente de variación de $X$.

d dimensión del vector de respuestas del modelo de simulación.

$f(\mathbf{x}) \quad$ función objetivo.

$F_{X}(x) \quad$ función de probabilidad acumulada.

$f_{X}(x) \quad$ función de densidad de probabilidad.

M orden de la expansión de Karhunen-Loève.

$m$ dimensión del diseño de experimentos.

n dimensión del vector de respuestas del modelo de simulación. 
$N_{i} \quad$ número de puntos en la dimensión i (algoritmo EKDRM).

$p \quad$ orden del modelo de regresión.

Q fracción de variabilidad.

$R^{2} \quad$ coeficiente de determinación.

S orden de la descomposición (algoritmo EKDRM).

$\operatorname{Var}[X] \quad$ varianza de $X$.

$w_{j, k} \quad$ peso del punto de integración k-ésimo para la variable aleatoria j-ésima.

$x_{i}^{\text {inf }}$ límite inferior de la variable de diseño i-ésima.

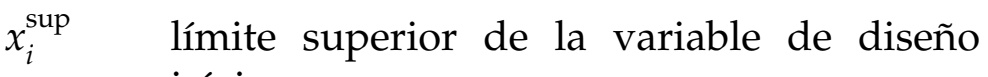
i-ésima.

$Y(\mathbf{x}, \omega)$ proceso estocástico. 
"The ethics of our profession today does not allow any design for a structure without optimization."

-Feynman (1988)

\section{Introducción}

\section{Índice}

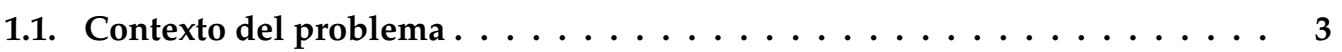

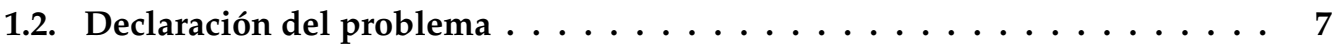

1.3. Objetivos de la tesis $\ldots \ldots \ldots \ldots \ldots \ldots \ldots \ldots \ldots$

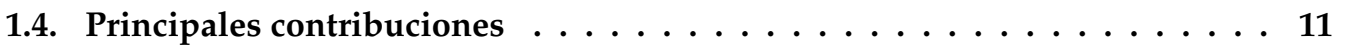

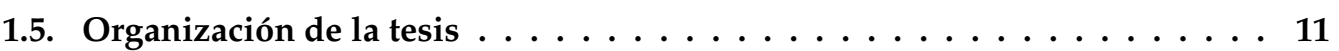

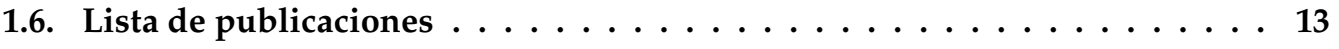





\subsection{Contexto del problema}

El propósito de esta sección es presentar el contexto en el que se enmarca esta tesis. En los capítulos 3 a 5 se presenta en profundidad cada uno de los diferentes campos que rodean al problema de Diseño Óptimo Robusto (DOR), junto con una revisión del estado del arte, las limitaciones y posibles líneas de investigación.

Las diferentes ramas de la ciencia y la ingeniería tienen en común la necesidad de explicar y reproducir el comportamiento de diferentes fenómenos físicos. En este sentido, la realización de pruebas experimentales resulta de gran utilidad para la reproducción de dichos fenómenos, permitiendo un mejor conocimiento de los mismos. Sin embargo, existen problemas en los que no es posible o no resulta práctico la realización de ensayos experimentales (ej. propagación de sismos, diseño de perfiles aerodinámicos, etc.). Por esta razón, la simulación numérica se ha convertido en uno de los pilares de la ingeniería moderna, ya que permite la validación de diferentes soluciones y teorías sin necesidad de experimentación física.

Durante las dos últimas décadas, la ley de Moore (Moore, 1975) muestra un incremento exponencial de la potencia computacional. Dicho crecimiento permite la integración de modelos de simulación numérica junto con algoritmos de optimización, lo que ha supuesto un profundo impacto en el proceso de diseño. Las técnicas de diseño basadas en la experiencia, o en procedimientos de "prueba-error", han dado paso a metodologías sistemáticas que permiten obtener diseños óptimos con ayuda de ordenadores. Un ejemplo de ello se encuentra en el campo de la ingeniería aeroespacial y aeronáutica, donde las técnicas de optimización estructural se utilizan habitualmente en el proceso de diseño, reduciendo el peso de las estructuras y cumpliendo requerimientos tales como rigidez, resistencia, vibraciones o cargas críticas (Adeli y Cheng, 1994; Gu y Kang, 1997; Y.X. Gu et al., 1998). A pesar de los avances alcanzados en velocidad de procesamiento, memoria y almacenamiento, el coste computacional sigue siendo una limitación en el campo de la optimización. Autores como Venkataraman y Haftka (2004) asocian esta limitación a que los recursos computacionales han sido utilizados para aumentar la complejidad del problema. La Figura 1.1 muestra la evolución en el uso de los recursos computacionales en los campos de la simulación, análisis, optimización y técnicas de meta-modelos. En la actualidad, las técnicas de simulación se enfrentan principalmente a tres limitaciones: (1) el coste computacional, (2) los problemas de alta dimensionalidad y (3) la fidelidad de los modelos de simulación (Shan y Wang, 2010a,b,c). El compromiso entre modelos de alta fidelidad y bajo coste computacional supone un reto en el campo de la optimización y en particular en el campo del DOR.

Habitualmente, el modelo matemático utilizado en la simulación incluye ecuaciones en derivadas parciales que emulan el comportamiento del fenómeno físico en estudio. Estos modelos matemáticos con frecuencia requieren de métodos numéricos de alto coste computacional para su resolución (ej. método 


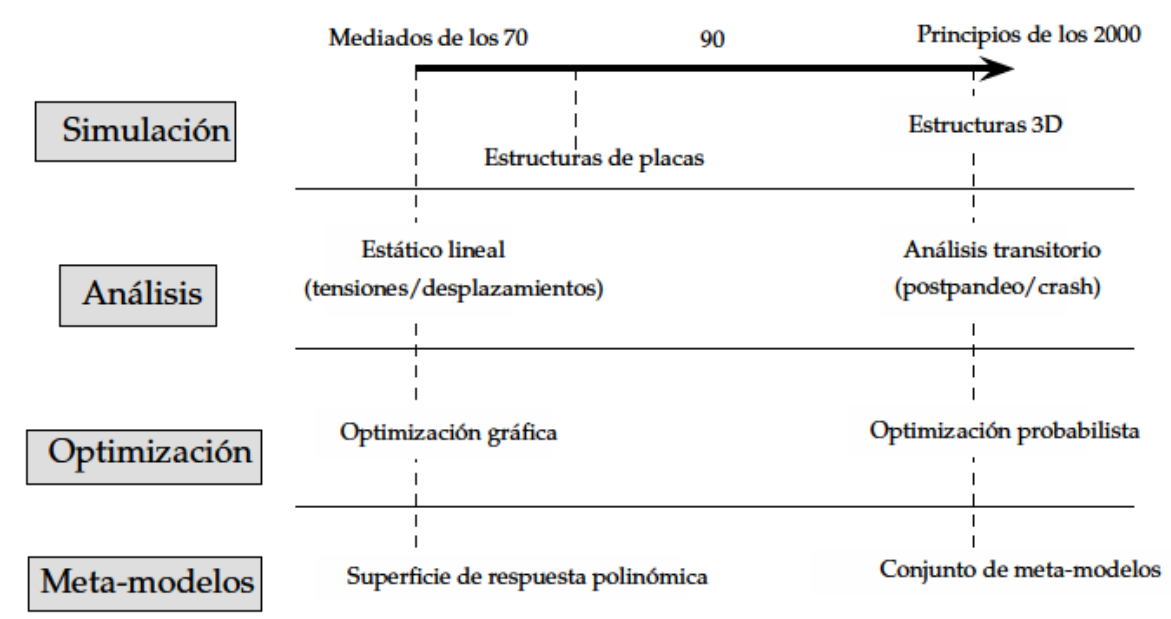

Figura 1.1: Evolución en el uso de los recursos computacionales (adaptado de Simpson et al. 2008).

de elementos finitos, dinámica de fluidos computacional, etc.). En estos casos, el coste computacional para una sola evaluación puede ser de horas o incluso días. En el campo de la industria del automóvil, un único análisis frente a impacto ("crash test") puede consumir de 36 a 160 horas (Gu, 2001). La necesidad de reproducir fenómenos físicos cada vez más complejos con una mayor precisión, ha provocado que aumente el interés por desarrollar nuevas técnicas de simulación con modelos de alto coste computacional (Shan y Wang, 2010a,b,c). Una de las líneas que ha recibido mayor atención es la utilización de modelos de aproximación. Estos modelos, conocidos habitualmente como meta-modelos (proporcionan un modelo del modelo de simulación (Kleijnen, 1986)), actúan como sustitutos del modelo de simulación proporcionando evaluaciones con un bajo coste computacional. Las investigaciones realizadas en los últimos años muestran a los meta-modelos como una técnica de gran utilidad en tareas como la exploración del espacio de diseño, la formulación del problema, o actuando como soporte de la optimización frente a diferentes necesidades de la industria (optimización multi-objetivo, optimización global u optimización bajo incertidumbre) (Wang y Shan, 2007).

"Computation-intensive design problems are becoming increasingly common in manufacturing industries. The computation burden is often caused by expensive analysis and simulation processes in order to reach a comparable level of accuracy as physical testing data. To address such a challenge, approximation or meta-modeling techniques are often used".

Wang y Shan (2007)

A pesar de la gran evolución que han experimentado los métodos de simulación, aun existen discrepancias que alejan al modelo de simulación de la realidad. La principal fuente de discrepancia surge como consecuencia de la idealización de las condiciones que rodean al sistema en estudio. En el campo de la ingeniería estructural, el efecto de las variaciones en las condiciones de carga, propiedades de los materiales, geometría o condiciones de contorno, suelen ser 
modelizadas a partir de valores deterministas. No se considera de forma explícita la influencia de fuentes de incertidumbre que son inherentes al problema. La creciente necesidad de predecir con mayor fidelidad el comportamiento real de la estructura, ha impulsado en las últimas dos décadas el desarrollo de métodos de análisis estocástico capaces de determinar la influencia de la incertidumbre de los parámetros de entrada en las respuestas. Los métodos de análisis estocástico han propiciado el desarrollo de los métodos de optimización bajo incertidumbre. Los métodos de optimización determinista proporcionan diseños que son óptimos en teoría y bajo condiciones cercanas a las nominales. Lejos de las condiciones nominales, el comportamiento puede degradarse o incluso dar lugar a diseños no factibles.

"Determistic optimization is actually just the opposite of robustness".

Egorov et al. (2002)

La combinación de técnicas de optimización, simulación numérica y propagación de incertidumbre da lugar a métodos de optimización bajo incertidumbre ("Optimization under uncertainty") (Schuëller y Jensen, 2008) capaces de proporcionar diseños óptimos más robustos y fiables. Los métodos de optimización bajo incertidumbre se clasifican principalmente en dos familias: los métodos de Diseño Óptimo basado en Fiabilidad (DOF) (Frangopol y Maute, 2004) y los métodos de DOR (Beyer y Sendhoff, 2007). Mientras que los primeros se centran en buscar diseños seguros frente a eventos extremos, los segundos tratan de determinar diseños insensibles a la variabilidad de los factores que intervienen en la respuesta estructural. Ambas formulaciones buscan objetivos distintos y pueden considerarse complementarias. En este sentido, recientes trabajos como los de Lagaros et al. (2007) proponen una formulación híbrida del problema bajo el nombre Reliability-based Robust Design Optimization (RRDO). En comparación con el problema de diseño óptimo determinista, el problema de DOR resulta más exigente como consecuencia de su inherente carácter multi-objetivo y de su integración con métodos de simulación estocásticos.

En la actualidad, la integración del DOR y de los métodos de simulación estocásticos tiene por delante un gran número de retos que están recibiendo una gran atención por parte de los investigadores. Entre ellos está el tratamiento de modelos de alta dimensionalidad y de modelos no-intrusivos de alto coste computacional. Esta tesis se contextualiza en el campo de DOR de modelos no intrusivos de elevado coste computacional. 
Thesis problem domain

Uncertainty quantification for computationally-expensive black-box type problems

Sparse grid (Smolyak) collocation methods

o Stochastic Decomposition Methods

- Dimension reduction methods
- Model approximation techniques

- Coarse-to -fine model based techniques

○ Non-gradient optimization algorithms

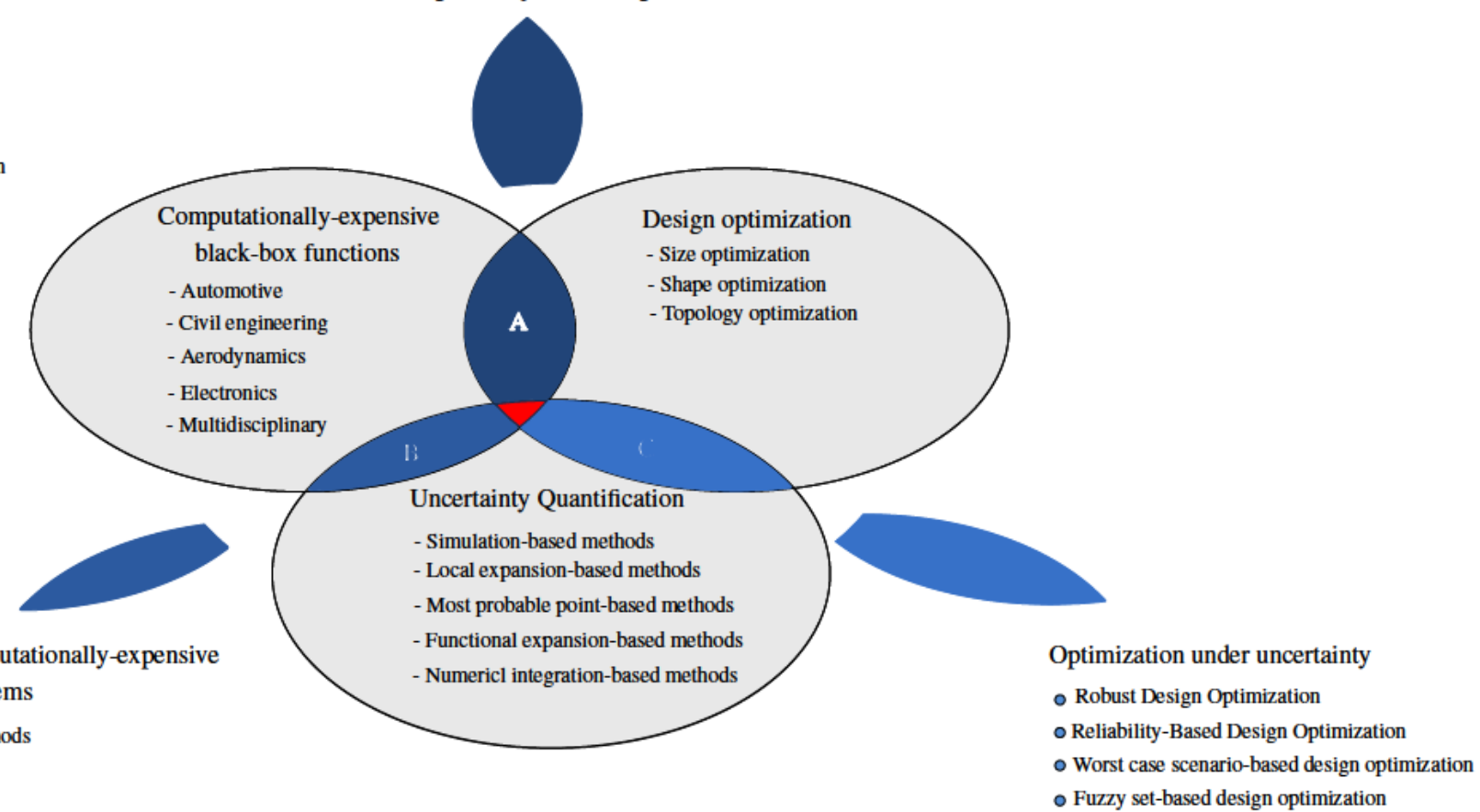

Figura 1.2: Contecto del trabajo de la tesis 
La Figura 1.2 muestra un diagrama de Venn que representa el contexto en el que se encuadra el trabajo realizado en esta tesis. El problema que se aborda en esta tesis se encuentra en la intersección común de tres campos: optimización estructural, propagación de la incertidumbre y simulación con modelos no-intrusivos de alto coste computacional. Las intersecciones de estos campos da lugar a tres subcampos que están en relación directa con la presente tesis: (a) optimización de modelos no-intrusivos de alto coste computacional (capítulos 3 y 4), (b) cuantificación de incertidumbre con modelos no-intrusivos de alto coste computacional (capítulo 5) y (c) optimización probabilista (capítulo $6)$.

"The integration of optimization methodologies with computational analyses/simulations faces multiple challenges. The most eminent challenges arise from high-dimensionality of problems, computationally-expensive analysis/simulation, and unknown function properties (i.e., black-box functions)".

Shan y Wang (2010a)

\subsection{Declaración del problema}

En el problema de DOR es necesario establecer un índice de robustez que caracterice la sensibilidad del diseño frente a variaciones en los parámetros estructurales. Actualmente existen métodos que abordan el problema de DOR bajo un enfoque probabilista. Estos métodos consideran los momentos estadísticos (media y desviación estándar) de la respuesta estructural como un índice de robustez del diseño. Estas medidas estadísticas pueden ser obtenidas mediante diversas técnicas de cuantificación de incertidumbre disponibles en la actualidad.

Cuando se aplica a problemas "reales" de ingeniería, el proceso de propagación de incertidumbre puede resultar una tarea de elevado coste computacional por dos razones. En primer lugar, la utilización de software "in house" o comercial obliga en muchos casos a utilizar métodos de propagación de incertidumbre no-intrusivos. Estos métodos por lo general requieren de un elevado número de evaluaciones del modelo de simulación. En segundo lugar, los problemas "reales" de ingeniería suelen involucrar complejos modelos de simulación de alto coste computacional. En estos caso, la utilización del proceso de propagación de incertidumbre durante la optimización puede dar lugar a un problema de inabordable. El propósito principal de este trabajo es establecer una metodología que permita la resolución del problema de DOR con un coste computacional razonable.

El problema del alto coste computacional y la aplicación del DOR a la resolución de casos "reales", ha motivado la mayoría de los trabajos en este campo realizados en los últimos años. Actualmente, técnicas como la reducción de modelos, los meta-modelos o las superficies de respuesta son ampliamente 
utilizadas para reducir el coste computacional de modelos de simulación de alta fidelidad durante el proceso de diseño. A pesar de su creciente aplicación en el campo de optimización determinista, el desarrollo de meta-modelos estocásticos capaces de representar la respuesta aleatoria en el espacio de diseño es un campo de reciente exploración (Coelho et al., 2011), siendo escasos los trabajos existentes en esta línea. La utilización de meta-modelos estocásticos para la resolución del problema de diseño óptimo robusto plantea nuevas oportunidades para su aplicación eficiente a casos reales así como nuevos retos (Coelho et al., 2011). En el presente trabajo se propone una estrategia de modelización jerárquica basada en la utilización de técnicas de reducción de dimensión y modelos Kriging para la construcción del meta-modelo estocástico. La metodología propuesta combina tres ideas: (1) la descomposición del espacio de diseño y del espacio aleatorio, (2) un procedimiento adaptativo basado en los modelos Kriging para obtener el conjunto de diseños óptimos con el menor número de evaluaciones del código de simulación, y (3) La utilización de técnicas de reducción de dimensión para aproximar los momentos estadísticos de la respuesta en el dominio aleatorio.

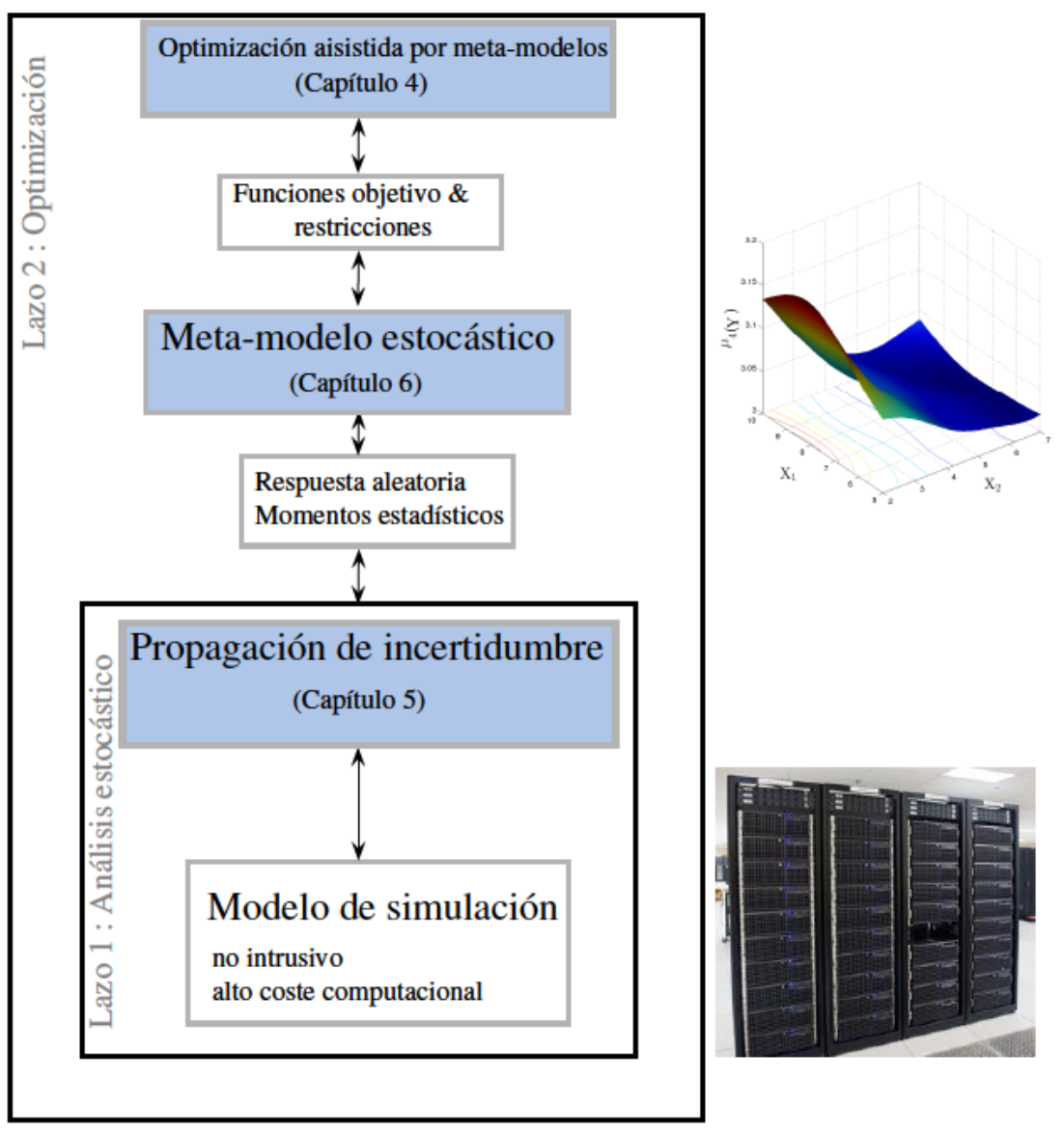

Figura 1.3: Declaración del problema. 


\subsection{Objetivos de la tesis}

\subsubsection{Objetivo principal. Desarrollo de un meta-modelo estocástico adaptativo para la resolución del problema de DOR}

En esta tesis se trata la resolución eficiente del problema de DOR cuando se trabaja con modelos de simulación (ej. modelos de elementos finitos) de alto coste computacional. Este problema se aborda mediante la utilización de meta-modelos estocásticos que aproximan la respuesta probabilista de la estructura en el espacio de diseño.

El objetivo principal de la tesis es el desarrollo y validación de un nuevo meta-modelo estocástico para la resolución eficiente del problema de DOR con modelos de simulación de alto coste computacional. La consecución del objetivo principal propuesto involucra los siguientes dos objetivos parciales: (1) propagación de incertidumbre con modelos no-intrusivos de alto coste computacional y (2) actualización de los meta-modelos en las regiones alrededor de la región óptima.

\subsubsection{Objetivo parcial I. Actualización de los meta-modelos en las regiones alrededor de la región óptima}

La utilización de meta-modelos estocásticos en el campo del DOR plantea nuevas necesidades, entre las que se encuentra la actualización de los meta-modelos cuando la región de interés no está delimitada por un único punto de diseño (optimización mono-objetivo) sino por una región más amplia alrededor de los diseños óptimos (Coelho et al., 2011). Por lo tanto, uno de los objetivos que se plantea en el trabajo desarrollado en esta tesis es una metodología para la actualización del meta-modelo estocástico basada en la teoría del aprendizaje estadístico (Hurtado, 2004).

\subsubsection{Objetivo parcial II. Propagación de incertidumbre con modelos no-intrusivos de alto coste computacional}

Los modelos de simulación de alto coste computacional son habitualmente complejos modelos de elementos finitos que requieren para su resolución de software comercial. Esto implica ciertas limitaciones, como la de no poder modificar el código de simulación. Por ello, los meta-modelos estocásticos deberán estar basados en métodos eficientes de propagación de incertidumbre que no requieran modificaciones del código de simulación (perturbaciones de variables). Así mismo se investigará en métodos áltamente paralelizables. Los meta-modelos estocásticos propuestos en este trabajo se basan en métodos de integración numérica, denominados Métodos de Reducción Dimensional (MRD). 
Los métodos de reducción dimensional han demostrado su eficiencia en la aproximación de momentos estadísticos de bajo orden (Lee y Chen, 2009).

\subsubsection{Objetivo parcial III. Implementación de una herramienta para el diseño claro, flexible $y$ encapsulado de algoritmos de optimización bajo incertidumbre asistida por meta-modelos}

Una de las principales dificultades a la hora de la implementación de algoritmos de optimización bajo incertidumbre asistidos por meta-modelos reside en la gran diversidad de formulaciones del problema (Eldred y Burkardt, 2009). Existen diferentes tipos de meta-modelos, técnicas adaptativas y técnicas de cuantificación de incertidumbre, así como diferentes posibilidades a la hora de la integración de dichas técnicas en el proceso de cuantificación de incertidumbre y de optimización. Sin embargo, el paradigma de la programación orientada a objetos proporciona una herramienta de gran utilidad para la implementación de algoritmos de optimización bajo incertidumbre de una forma fácil y elegante. Así, el tercer objetivo parcial que se plantea en esta tesis es el desarrollo de una toolbox orientada a objetos que aborde los siguientes campos:

- Meta-modelos. Exploración y visualización, diseño de experimentos, ajuste, validación.

- Propagación de incertidumbre. Estimación de momentos estadísticos, probabilidades, análisis de sensibilidad.

- Optimización. Algoritmos de optimización local y global con un único objetivo, con múltiples objetivos y algoritmos asistidos por meta-modelos.

Los principales objetivos de la toolbox son el diseño claro, flexible y encapsulado del código, que permita la investigación en algoritmos de optimización bajo incertidumbre asistidos por meta-modelos.

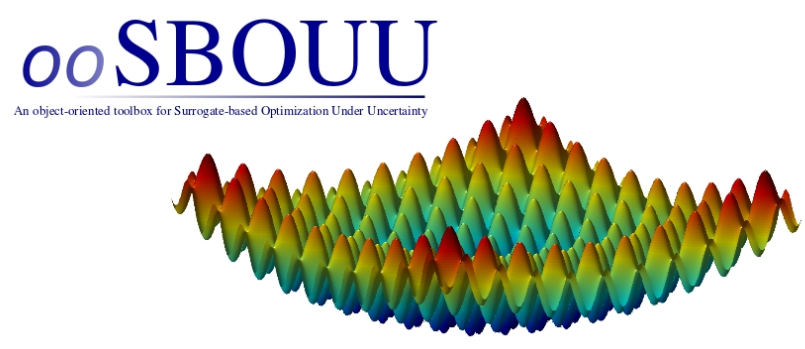

Figura 1.4: ooSBOUU toobox 


\subsection{Principales contribuciones}

Las principales contribuciones se presentan en los Capítulos 4 a 6:

1. Un meta-modelo estocástico de la respuesta aleatoria basado en modelos Kriging y métodos de reducción dimensional.

2. Algoritmo Expected HyperVolume Efficient Multi-objective Optimization (EHV-EMO) dedicado a la resolución del problema de optimización MO con modelos de simulación de alto coste computacional.

3. Algoritmo Extended Kriging based Dimension Reduction Method (EKDRM) dedicado a la resolución eficiente del problema de propagación de incertidumbre con modelos de simulación de alto coste computacional.

4. Implementación de una herramienta para el diseño claro, flexible y encapsulado de algoritmos de optimización bajo incertidumbre asistida por meta-modelos.

\subsection{Organización de la tesis}

Esta tesis se organiza en siete capítulos. El Capítulo 1 está dedicado a presentar la motivación, el contexto y los objetivos del trabajo realizado. El Capítulo 2 presenta formalmente el problema de Diseño Óptimo Robusto en el campo de optimización estructural. Se presenta la formulación general del problema de optimización bajo incertidumbre, las diferentes formulaciones que derivan del mismo, así como el problema multi-objetivo de DOR. Se utiliza un enfoque probabilista donde las incertidumbres están completamente caracterizadas por distribuciones de densidad de probabilidad. Bajo este enfoque, los principales criterios de robustez se ilustran mediante la resolución de ejemplos académicos. Finalmente, se presenta una revisión de los principales trabajos que tratan el problema de DOR con aplicación al diseño estructural. Dicha revisión pone de manifiesto los principales retos existentes actualmente así como posibles líneas de investigación.

El Capítulo 3 está dedicado a la presentación del problema de predicción utilizando procesos Gaussianos de regresión, también conocidos como modelos Kriging. Estas técnicas son ampliamente utilizadas en el campo de machine learning como técnicas de aprendizaje supervisado. En este capítulo se presenta formalmente el problema de predicción así como algunas de las principales contribuciones en este campo. Se presta especial atención a la relación entre los modelos de predicción Gaussianos y otras técnicas convencionales como los modelos de regresión por mínimos cuadrados, funciones de base radial, o máquinas de soporte vectorial. El capítulo finaliza con la resolución de tres 
ejemplos que ilustran problemas de interés desde el punto de vista de esta tesis: (1) el proceso de selección de los hiperparámetros del modelo de predicción, (2) la selección empírica de las funciones de correlación y regresión del modelo de predicción mediante errores de validación cruzada y (3) la representación espacial de la incertidumbre de propiedades estructurales mediante campos aleatorios.

El Capítulo 4 trata el problema de optimización multi-objetivo con algoritmos de análisis Bayesiano. En este capítulo se utiliza la información estadística proporcionada por los procesos Gaussianos (media y varianza de la predicción) para una resolución eficiente de problemas de optimización multi-objetivo (MO) con modelos de simulación de alto coste computacional. Se realiza una revisión de los trabajos existentes en la literatura, en especial los que utilizan algoritmos basados en análisis Bayesiano. Resultado de la revisión realizada se propone un algoritmo basado en la esperanza del hipervolumen de soluciones no-dominadas y en la probabilidad de diseño factible. Este criterio se utiliza para guiar el proceso de selección de nuevos puntos candidatos a formar parte de la muestra de observaciones iniciales. Los nuevos puntos mejoran el meta-modelo en las regiones cercanas al conjunto de soluciones óptimas. El algoritmo propuesto se valida mediante la resolución de un conjunto de benchmarks, clásicos en el campo de optimización multi-objetivo.

El Capítulo 5 trata el problema de propagación de incertidumbre. Esta etapa consiste en obtener la respuesta estocástica del modelo de simulación como consecuencia de la incertidumbre aleatoria de las variables de entrada. Se realiza una revisión de las técnicas existentes en la literatura para la resolución del problema. Se discuten las ventajas y limitaciones de cada una de ellas. Se toma como punto de partida los métodos de reducción dimensional por ser los que mejor se adaptan a las características del problema tratado en esta tesis. Se propone un algoritmo en el que se utilizan modelos Kriging para mejorar la eficiencia del proceso de integración. El algoritmo propuesto se valida numéricamente mediante la resolución tanto de ejemplos analíticos y como de modelos estructurales de alto coste computacional.

El Capítulo 6 plantea la resolución del problema multi-objetivo de DOR mediante un meta-modelo estocástico que aproxima la respuesta aleatoria de la estructura en el dominio de diseño. Se propone un meta-modelo estocástico basado en: (1) métodos de reducción dimensional para la estimación eficiente de los momentos estadísticos, (2) modelos Kriging para aproximar los momentos en el espacio de diseño y (3) reconstrucción de la distribución de probabilidad utilizando distribuciones Pearson. La eficiencia y precisión del meta-modelo estocástico se valida mediante la resolución de: (1) problemas multi-objetivo de DOR con restricciones de probabilidad, (2) problemas de DOR de estructuras de nudos articulados y (3) el DOR de láminas imperfectas frente a pandeo global.

El Capítulo 7 presenta las conclusiones y principales contribuciones de la 
tesis. Así mismo se presentan las principales líneas de investigación y trabajos futuros derivadas del trabajo realizado.

\subsection{Lista de publicaciones}

A continuación se detallan las publicaciones en revistas y congresos derivadas del trabajo realizado en esta tesis.

\subsubsection{Revistas}

- Diseño óptimo robusto utilizando modelos Kriging: aplicación al diseño óptimo robusto de estructuras articuladas

J. Martínez-Frutos, P. Martí.

Revista internacional de métodos numéricos cálculo $\mathrm{y}$ diseño en ingeniería. 2013. Aceptado para publicación. DOI:http://dx.doi.org/10.1016/j.rimni.2013.01.003.

\subsubsection{Congresos}

- Diseño óptimo robusto de estructuras basado en modelos Kriging J. Martínez-Frutos, P. Martí.

Congress on Numerical Methods in Engineering, CMNE2011. Coimbra, 14-17 June 2011.

- Diseño óptimo probabilista de uniones semirrígidas mediante simulación numérica y modelos Kriging

P. Martí, C. Diaz, J. Martínez-Frutos, M.V. Nicolás.

Congress on Numerical Methods in Engineering, CMNE2011. Coimbra, 14-17 June 2011.

- Metamodel-based multi-objective robust design optimization of structures

J. Martínez-Frutos, P. Martí.

Computer Aided Optimum Design in Engineering XII. ISBN: 978-1-84564-598-4 eISBN: 978-1-84564-599-1. Pages: 292. Book Series: WIT Transactions on The Built Environment. Series Volume: 125.

- Diseño óptimo de vigas armadas de acero mediante elementos finitos, metamodelos y algoritmos de optimización

P. Martí, J. Martínez-Frutos y M. Martínez

Congress on Numerical Methods in Engineering. 25 - 28 Junio 2013, Bilbao, España. 

"Deterministic optimization is actually just the opposite of robustness."

\section{2 \\ Revisión del problema de diseño óptimo robusto de estructuras}

\section{Índice}

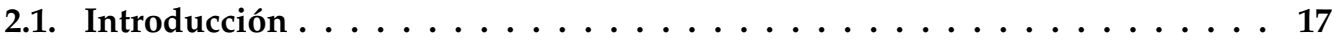

2.2. Formulación del problema de optimización bajo incertidumbre . . . . . 17

2.3. Criterios de robustez para la resolución del problema de DOR . . . . . . 22

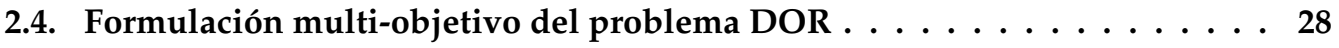

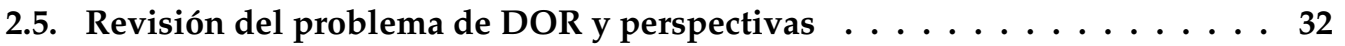

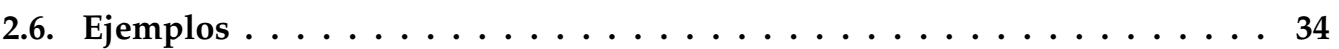

2.7. Discusión y conclusiones $\ldots \ldots \ldots \ldots \ldots \ldots \ldots \ldots \ldots \ldots$ 



\subsection{Introducción}

El problema de optimización estructural se ha tratado tradicionalmente desde un enfoque determinista (Diseño Óptimo Determinista (DOD)) sin considerar de forma explícita el efecto de fuentes de incertidumbre en la respuesta estructural. Las soluciones del problema determinista se encuentran habitualmente en los límites del espacio de diseño, por lo que son especialmente sensibles a variaciones en las variables y/o parámetros que definen la estructura tales como: imperfecciones en el proceso de fabricación, variabilidad en las propiedades del material o condiciones de carga desconocidas. Dichas variaciones pueden provocar una dramática disminución de la calidad del diseño óptimo, o incluso dar lugar a diseños no factibles. Por este motivo resulta imprescindible incorporar la incertidumbre existente en la formulación del problema de optimización.

Frente a los métodos de DOD, los métodos de optimización bajo incertidumbre tienen en cuenta la aleatoriedad de las variables y/o parámetros de diseño para obtener soluciones más robustas y fiables. Dentro de estos métodos, el DOR busca minimizar el efecto de las incertidumbres en la respuesta estructural (Apley et al., 2005; Balling et al., 1986; Chen et al., 2001; Du y Chen, 1999; Gu et al., 2000; Gunawan y Azarm, 2005; Liu et al., 2006; McAllister y Simpson, 2003; Mourelatos y Liang, 2006).

Este capítulo está dedicado a presentar formalmente la formulación del problema de DOR en el campo de la optimización estructural. El capítulo consta de ocho secciones. La sección 2.2 introduce la formulación del problema de optimización con incertidumbre, junto con una discusión de las diferentes formulaciones que derivan de la misma. La sección 2.3 presenta los principales criterios de robustez existentes en la literatura. La sección 2.4 formula el problema multi-objetivo de DOR. La sección 2.5 revisa las principales contribuciones existentes en la literatura que tratan el problema de DOR en el campo de optimización estructural. El capítulo finaliza con un ejemplo de aplicación (sección 2.6) y la presentación de las conclusiones alcanzadas (sección 2.7).

\subsection{Formulación del problema de optimización bajo incertidumbre}

El problema de optimización que considera de forma explícita las incertidumbres asociadas a las variables y/o parámetros de diseño se conoce en la literatura como problema de optimización bajo incertidumbre (Calafiore y Dabbene, 2008) o problema de optimización estocástica (stochastic optimization problem) (Jurecka et al., 2007). Esta última denominación puede dar lugar a confusión con los métodos de optimización estocástica. Estos últimos utilizan técnicas de muestreo estocástico, tales como búsqueda aleatoria o algoritmos heurísticos, para la resolución del problema de optimización determinista. 
Para evitar confusión, en esta tesis se utiliza el término "optimización bajo incertidumbre" para hacer referencia a este tipo de problema.

El problema de DOD se puede formular como sigue:

$$
\text { DOD }\left\{\begin{array}{lll}
\min _{\mathbf{x}} & f(\mathbf{x}) ; & \mathbf{x} \in \mathbb{R}^{\mathrm{n}} \\
\text { s.a. : } & g_{j}(\mathbf{x}) \leq 0 ; & j=1, \ldots, n_{g}, \\
& h_{k}(\mathbf{x})=0 ; & k=1, \ldots, n_{h} \\
& x_{i}^{\text {inf }} \leq x_{i} \leq x_{i}^{\text {sup }} ; & i=1, \ldots, n,
\end{array}\right.
$$

donde $\mathbf{x}$ es el vector de variables de diseño, $f(\mathbf{x})$ es la función objetivo, $n_{g}, n_{h}$ y $n$ son el número de restricciones de desigualdad, igualdad y de variables de diseño respectivamente, $x_{i}^{\text {inf }}$ y $x_{i}^{\text {sup }}$ son los límites inferior y superior de la variable de diseño i-ésima.

El problema de optimización presentado en la ecuación (2.1) puede estar sometido a incertidumbre tanto en las variables de diseño $\mathbf{x}$ ) como en parámetros que afectan a la respuesta estructural. Dicha incertidumbre se puede incorporar explícitamente en la formulación utilizando un enfoque probabilista. Por simplicidad en la formulación se considera que las variables de diseño aleatorias se pueden descomponer en una parte nominal determinista más una contribución aleatoria $\mathbf{x}+\mathbf{Z}$. El vector $\mathbf{Z}$ contiene las incertidumbres asociadas a cada variable aleatoria así como las relacionadas con parámetros aleatorios fuera del alcance del diseñador. Dado un espacio de probabilidad $(\Omega, \mathscr{F}, \mathscr{P}), \mathbf{Z}$ es un vector aleatorio completamente caracterizado por una función de densidad de probabilidad $p_{\mathbf{Z}}(\mathbf{Z})$ definida a partir del valor esperado $\mu_{\mathrm{Z}}$ y la matriz de covarianza $\mathrm{C}$ (capítulo 3 , sección 3.2.5). Introduciendo el vector $\mathbf{Z}$, el problema (2.1) se transforma en el problema de optimización bajo incertidumbre:

$$
\text { OBI }\left\{\begin{array}{lll}
\min _{\mathbf{x}} & f(\mathbf{x}, \mathbf{Z}) ; & \mathbf{x} \in \mathbb{R}^{\mathrm{n}} . \\
\text { s.a. : } & g_{j}(\mathbf{x}, \mathbf{Z}) \leq 0 ; & j=1, \ldots, n_{g} ; \forall \mathbf{z} \in \mathbf{\Omega}, \\
& h_{k}(\mathbf{x}, \mathbf{Z})=0 ; & k=1, \ldots, n_{h} ; \forall \mathbf{z} \in \mathbf{\Omega}, \\
& x_{i}^{\text {inf }} \leq x_{i} \leq x_{i}^{\text {sup }} ; & i=1, \ldots, n,
\end{array}\right.
$$

donde $f(\mathbf{x}, \mathbf{Z}), g_{j}(\mathbf{x}, \mathbf{Z}), \mathbf{y} h_{k}(\mathbf{x}, \mathbf{Z})$ ya no son valores deterministas, sino respuestas aleatorias con funciones de densidad de probabilidad $p_{f}(f), p_{g_{j}}\left(g_{j}\right)$ y $p_{h_{k}}\left(h_{k}\right)$, respectivamente. Dichas funciones de probabilidad se obtienen mediante métodos de propagación de incertidumbre que son descritos más adelante en el capítulo 5 .

El problema (2.2) no se puede resolver directamente mediante algoritmos de optimización convencionales y requiere una transformación previa de las respuestas aleatorias a valores deterministas. Existen diferentes enfoques en la literatura para esta transformación. En lo que resta de sección se presentan las diferentes posibilidades para la transformación de la función objetivo, restricciones de igualdad y restricciones de desigualdad. 


\subsubsection{Función objetivo}

En el problema (2.2), la función objetivo $f(\mathbf{x}, \mathbf{Z})$ es una función aleatoria como consecuencia del vector $\mathbf{Z}$. En este contexto un diseño $\mathbf{x}^{*}$ es óptimo si verifica la relación:

$$
f\left(\mathbf{x}^{*}, \mathbf{Z}\right) \leq \mathrm{f}(\mathbf{x}, \mathbf{Z}), \quad \forall \mathbf{x} \in \mathscr{D}_{\mathrm{fac}} \wedge \forall \mathbf{z} \in \mathbf{\Omega},
$$

es decir, no existe ningún diseño $\mathbf{x}$ en el dominio de diseño factible $\mathscr{D}_{f a c}$ que mejore el valor de la función objetivo en el punto $\mathbf{x}^{*}$ para cualquier realización $\mathbf{z} \in \boldsymbol{\Omega}$ del vector de variables aleatorias. Un punto $\mathbf{x}^{*}$ que satisface la ecuación (2.3) es solución del problema (2.2) para cualquier realización del vector de variables aleatorias. Por este motivo a dicha solución se le denomina en la literatura diseño óptimo robusto.

En la práctica es difícil encontrar un DOR $\mathbf{x}^{*}$ que verifique la relación (2.3) para cualquier realización $\mathbf{z} \in \mathbf{\Omega}$. Por este motivo es necesario buscar un criterio que aproxime de forma adecuada la relación (2.3) y proporcione valores deterministas para evaluar la bondad de cada diseño durante la optimización. Dicho criterio se denomina en la literatura criterio de robustez (CR) y su elección depende de la información disponible acerca de las variables aleatorias y en última instancia del criterio de diseño.

\subsubsection{Restricciones de igualdad}

En el problema de DOD, las restricciones de igualdad se deben satisfacer para la solución óptima. En el caso del problema bajo incertidumbre, debido a la naturaleza probabilista del mismo, no es posible garantizar que las restricciones de igualdad sean estrictamente satisfechas para cualquier realización de las variables aleatorias $\mathbf{z} \in \boldsymbol{\Omega}$. Sin embargo, las restricciones de igualdad que representan la física del problema (ej. ecuaciones de equilibrio) deben satisfacerse estrictamente para garantizar la fiabilidad del diseño final. La forma de tratar este tipo de restricciones en el problema bajo incertidumbre ha dado lugar a numerosos trabajos que se pueden clasificar en tres categorías:

- relajación de la restricción de igualdad (Fares et al., 2002; Messac y Ismail-Yahaya, 2002; Ramakrishnan y Rao, 1991) sustituyéndola por dos restricciones de desigualdad que garanticen el cumplimiento de la restricción original con una tolerancia $\epsilon$ :

$$
-\frac{\epsilon}{2} \leq h_{k}(\mathbf{x}, \mathbf{Z}) \leq+\frac{\epsilon}{2} ; k=1, \ldots, n_{h}
$$

- Satisfacción de la restricción en su valor medio (Eggert, 1991; Putko et al., 1991; Siddall, 1983; Sundaresan et al., 1993): 


$$
\mathbb{E}\left[h_{k}(\mathbf{x}, \mathbf{Z})\right]=0 ; k=1, \ldots, n_{h},
$$

o satisfacción de la restricción para el valor medio de las variables aleatorias:

$$
h_{k}(\mathbf{x}, \mathbb{E}[\mathbf{Z}])=0 ; k=1, \ldots, n_{h} .
$$

- Sustitución de la restricción de igualdad en la función objetivo y en las restricciones de desigualdad (Das, 2000).

Una comparación de las ventajas e inconvenientes de cada uno de los enfoques anteriores queda fuera del alcance del presente trabajo. Dicha comparación, junto con una extensa revisión del problema con restricciones de igualdad, se presenta en el trabajo de Rangavajhala et al. (2007).

\subsubsection{Restricciones de desigualdad}

En comparación con las restricciones de igualdad, el tratamiento de las restricciones de desigualdad en el problema con incertidumbre ha recibido una mayor atención en la literatura (Balling et al., 1986; Otto y Antonsson, 1999; Parkinson et al., 1993; Taguchi, 1996). La forma más directa de transformar la restricción de desigualdad es garantizar que se cumple con un $100 \%$ de probabilidad:

$$
\mathbb{P}\left[g_{j}(\mathbf{x}, \mathbf{Z}) \leq 0\right]=1 \quad \forall \mathbf{z} \in \mathbf{\Omega} ; j=1, \ldots, n_{g} .
$$

Sin embargo, en la mayoría de aplicaciones reales no existe un diseño que garantice este nivel de cumplimiento y se define un valor escalar asociado a la probabilidad de fallo del diseño $\mathbf{x}$ :

$$
P_{\mathrm{F}, j}=\mathbb{P}\left[g_{j}(\mathbf{x}, \mathbf{Z})>0\right] \quad \forall \mathbf{z} \in \mathbf{\Omega} ; j=1, \ldots, n_{g} .
$$

A partir del concepto de probabilidad de fallo, la restricción de desigualdad de la ecuación (2.2) se puede reformular como sigue:

$$
\mathbb{P}\left[g_{j}(\mathbf{x}, \mathbf{Z})>0\right] \leq P_{\max , j} \quad \forall \mathbf{z} \in \boldsymbol{\Omega} ; j=1, \ldots, n_{g},
$$

donde $P_{m a x, j}$, es el valor de la probabilidad de fallo máxima admisible para la restricción j-ésima. El complemento de la probabilidad de fallo representa la probabilidad de que el diseño sea factible y se denomina fiabilidad:

$$
P_{\mathrm{S}, j}=1-P_{\mathrm{F}, j} \quad \forall \mathbf{z} \in \mathbf{\Omega} ; j=1, \ldots, n_{g} .
$$


Este concepto de fiabilidad es el que da nombre a una de las ramas en el campo de la optimización bajo incertidumbre: el diseño óptimo fiable (DOF).

La probabilidad de fallo viene dada por la integral:

$$
P_{\mathrm{F}, j}=\mathbb{P}\left[g_{j}(\mathbf{x}, \mathbf{Z})>0\right]=\int_{\mathscr{D}_{f, j}} p_{\mathbf{Z}}(\mathbf{Z}) d \mathbf{z} \quad j=1, \ldots, n_{g},
$$

donde $\mathscr{D}_{f, j}=\left\{\mathbf{x} \in \mathbb{R}^{\mathrm{n}} \mid g_{j}(\mathbf{x})>0 \wedge \mathrm{x}_{i}^{\text {inf }} \leq \mathrm{x}_{i} \leq \mathrm{x}_{i}^{\text {sup }}\right\}$ es el dominio de fallo. La principal dificultad para la evaluación de la probabilidad de fallo definida en (2.11) reside en: (1) el dominio de integración es implícito y (2) la dimensión de la integral es igual a la dimensión del vector $\mathbf{Z}$. Salvo en algunos casos especiales, la integral (2.11) no puede ser resuelta analíticamente por lo que es necesario utilizar métodos como el First Order Reliability Method (FORM) (Hasofer y Lind, 1974) y el Second Order Reliability Method (SORM) (Breitung, 1984) para su resolución.

\subsubsection{Discusión}

Considerando las transformaciones anteriores, la formulación general del problema de optimización bajo incertidumbre queda como sigue:

$$
\text { OBI }\left\{\begin{array}{lll}
\min _{\mathbf{x}} & \mathrm{CR}(\mathbf{x}) ; & \mathbf{x} \in \mathbb{R}^{\mathrm{n}} \\
\text { s.a. : } & \mathbb{P}\left[g_{j}(\mathbf{x}, \mathbf{Z})>0\right] \leq P_{\text {max }, j} ; & \forall \mathbf{z} \in \mathbf{\Omega} ; j=1, \ldots, n_{g}, \\
& \mathbb{E}\left[h_{k}(\mathbf{x}, \mathbf{Z})\right]=0 ; & \forall \mathbf{z} \in \mathbf{\Omega} ; k=1, \ldots, n_{h}, \\
& x_{i}^{\text {inf }} \leq x_{i} \leq x_{i}^{\text {sup }} ; & i=1, \ldots, n,
\end{array}\right.
$$

donde $\mathrm{CR}(\mathbf{x})$ representa el criterio de robustez de la estructura.

En la formulación (2.12), las fuentes de incertidumbre afectan tanto a la función objetivo como a las restricciones. En la literatura se distinguen dos casos particulares dependiendo de las funciones afectadas por las incertidumbres: el problema de DOF y el problema de DOR.

Diseño óptimo fiable. El término diseño óptimo fiable (Enevoldsen y Sorensen, 1994; Gasser y Schüller, 1997; Papadrakakis y Plevris, 2005) hace referencia de forma exclusiva a problemas en los que la función objetivo es determinista y está sujeta a restricciones probabilistas como las presentadas en la sección 2.2.3:

$$
\text { DOF }\left\{\begin{array}{lll}
\min _{\mathbf{x}} & f(\mathbf{x}) ; & \mathbf{x} \in \mathbb{R}^{\mathrm{n}} \\
\text { s.a. : } & \mathbb{P}\left[g_{j}(\mathbf{x}, \mathbf{Z})>0\right] \leq P_{\text {max }, j} ; & \forall \mathbf{z} \in \mathbf{\Omega} ; j=1, \ldots, n_{g}, \\
& \mathbb{E}\left[h_{k}(\mathbf{x}, \mathbf{Z})\right]=0 ; & \forall \mathbf{z} \in \mathbf{\Omega} ; k=1, \ldots, n_{h}, \\
& x_{i}^{\text {inf }} \leq x_{i} \leq x_{i}^{\text {sup }} ; & i=1, \ldots, n .
\end{array}\right.
$$


Diseño óptimo robusto. A diferencia de los métodos de DOF que se centran en buscar soluciones óptimas con un determinado nivel de fiabilidad, el DOR (Doltsinis y Kang, 2004) se centra en la búsqueda de soluciones óptimas menos sensibles frente a variaciones en las variables y/o parámetros de diseño. El problema de DOR se centra principalmente en satisfacer la ecuación (2.3) mediante un Criterio de robustez (CR). En este contexto, no es tan importante garantizar un cierto nivel de fiabilidad en las restricciones, es suficiente garantizar un cierto grado de robustez de las mismas. Trabajos como los de Parkinson et al. (1993) y Messac y Ismail-Yahaya (2002) reformulan las restricciones de desigualdad en función de sus dos primeros momentos estadísticos, desplazándolas de modo que se garantice un nivel suficiente de cumplimiento. La magnitud de dicho desplazamiento está relacionada con la fiabilidad deseada en el diseño óptimo final a través de un factor $\beta_{j}$, que multiplica la desviación estándar de la restricción. La formulación del problema DOR queda como sigue:

$$
\text { DOR }\left\{\begin{array}{lll}
\min _{\mathbf{x}} & \mathrm{CR}(\mathbf{x}) ; & \mathbf{x} \in \mathbb{R}^{\mathrm{n}}, \\
\text { s.a. }: & \mu_{g_{j}(\mathbf{x}, \mathbf{z})}+\beta_{j} \sigma_{g_{j}(\mathbf{x}, \mathbf{z})} \leq 0 ; & j=1, \ldots, n_{g}, \\
& \sigma_{h_{k}(\mathbf{x}, \mathbf{z})} \leq \sigma_{\mathrm{k}}^{+} ; & k=1, \ldots, n_{h}, \\
& x_{i}^{\text {inf }} \leq x_{i} \leq x_{i}^{\text {sup }} ; & i=1, \ldots, n_{x},
\end{array}\right.
$$

donde $\mathbf{x}$ es el vector de variables de diseño, $\mathbf{z}$ es el vector de variables aleatorias, $g_{j}(\mathbf{x}, \mathbf{z})\left(j=1, \ldots, n_{g}\right)$ son las restricciones de desigualdad; y $\sigma_{h_{k}(\mathbf{x}, \mathbf{z})}\left(k=1, \ldots, n_{h}\right)$ representan restricciones sobre la desviación estándar de la respuesta. El parámetro $\beta_{j}>0$ es el índice de fiabilidad para la restricción de desigualdad j-ésima. $\sigma_{k}^{+}$es el límite superior admisible para la desviación estándar de la respuesta estructural.

Desde un punto de vista estructural, el DOF se centra en garantizar la seguridad estructural frente a daños causados directa o indirectamente por eventos extremos. Por otra parte, el DOR se centra en garantizar un grado de robustez de la respuesta estructural durante su vida útil frente a variaciones en las condiciones de diseño.

\subsection{Criterios de robustez para la resolución del problema de DOR}

La solución del problema de DOR depende en gran medida del criterio de robustez adoptado. A diferencia del problema basado en fiabilidad, en el caso del DOR no existe una formulación unificada del mismo. En la literatura existen diversos criterios de robustez, cuya selección es función de la información disponible acerca de la incertidumbre del problema y del criterio de diseño. Se pueden distinguir dos enfoques: el enfoque no probabilista y el enfoque probabilista. 


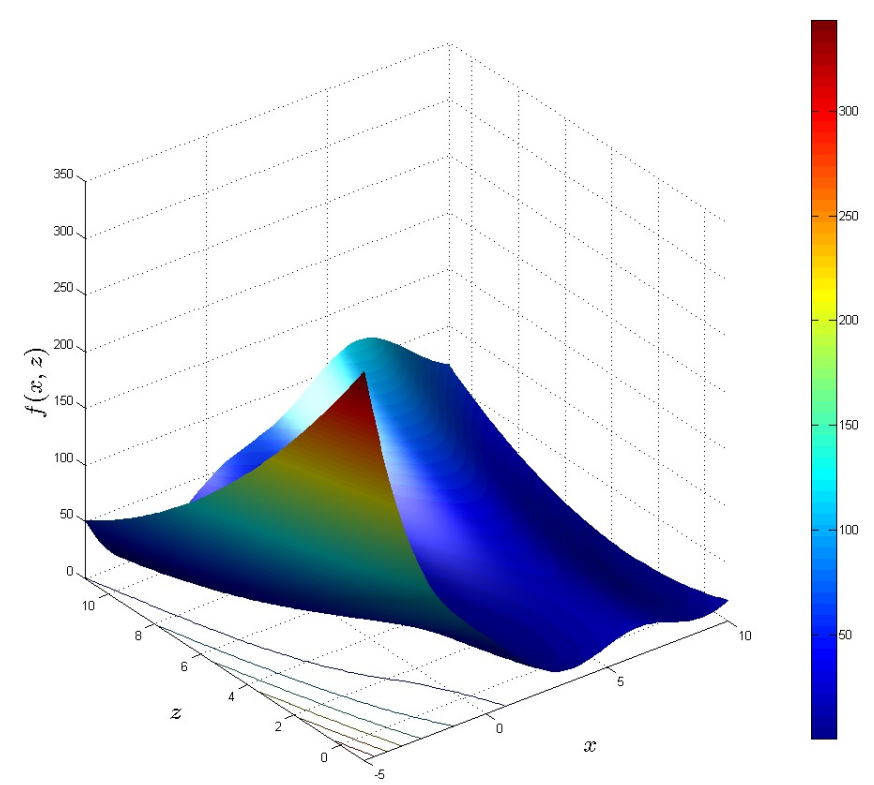

Figura 2.1: Función de Branin. $x$ es una variable de diseño determinista y $z$ un parámetro de incertidumbre.

Ilustración 2.1. Los criterios de robustez presentados a lo largo de esta sección se ilustran mediante la resolución de un problema de DOR. La función objetivo utilizada es la función de Branin (Branin, 1972):

$$
f(x, z)=\left(z-\frac{5.1}{4 \pi^{2}} x^{2}+\frac{5}{\pi} x-6\right)^{2}+10\left(1-\frac{1}{8 \pi}\right) \cos (x)+10
$$

que depende de una variable de diseño determinista $-5 \leq x \leq 10$ y un parámetro $z$ representativo de la incertidumbre del problema (probabilista o no probabilista). La Figura 2.1 muestra la representación gráfica de la función en el espacio de diseño $(x)$ y el espacio estocástico $(z)$.

\subsubsection{Criterios no probabilistas}

Estos criterios están asociados a problemas en los que no se dispone de información suficiente para caracterizar la incertidumbre mediante funciones de densidad de probabilidad. En estos casos,la incertidumbre se modela mediante conjuntos convexos (Ben-Haim y Elishakoff, 1990; Pantelides y Ganzerli, 1998) o conjuntos difusos (Sakawa, 1993). En el contexto del diseño óptimo robusto lo habitual es considerar las incertidumbre dentro de un conjunto acotado y utilizar una estrategia de anti-optimización (McWilliam, 2000) para determinar la combinación menos favorable de las mismas en dicho conjunto. 


\section{Criterio minimax basado en el peor caso}

Este criterio es conocido en la literatura como: minmax approach (Wald, 1950), robust counterpart approach (Ben-Tal y Nemirovski, 1998), worst case analysis (Lombardi y Haftka, 1998), y optimization with anti-optimization (McWilliam, 2000). El criterio de robustez se define como:

$$
\mathrm{CR}(\mathbf{x})=\sup _{\mathbf{z} \in \mathbf{Z}} f(\mathbf{x}, \mathbf{z}),
$$

donde se busca el supremo del conjunto de funciones $f(\mathbf{x}, \mathbf{z})$, es decir el menor valor real que es mayor o igual a cada valor del conjunto $f(\mathbf{x}, \mathbf{z})$. Dado un conjunto acotado $\mathbf{Z}=\left[\mathbf{z}^{\text {inf }}, \mathbf{z}^{\text {sup }}\right]$ representativo de la incertidumbre, el problema de minimización se formula como:

$$
\mathrm{CR}(\mathbf{x})=\operatorname{máx}_{\mathbf{z}}\left\{f(\mathbf{x}, \mathbf{z}) \mid \mathbf{z}^{\text {inf }} \leq \mathbf{z} \leq \mathbf{z}^{\text {sup }}\right\} .
$$

Para cada vector de variables de diseño $\mathbf{x}$, se busca la situación más desfavorable en todo el rango de $\mathbf{z} \in \mathbf{Z}$ mediante la resolución del problema de minimización de (2.17). En el campo de la optimización estructural, autores como Elishakoff et al. (1994) y Lombardi y Haftka (1998) combinan el criterio minimax junto con técnicas de optimización determinista para la resolución del problema de optimización bajo incertidumbre.

Ilustración 2.1 (continuación). Bajo la hipótesis de que no existe información suficiente para la caracterización probabilista del parámetro $z$, se considera un rango de variación de dicho parámetro comprendido entre $z \in[-1,11]$. La Figura 2.2 muestra el criterio de robustez (ec. (2.17)) en el dominio de diseño. Esta representación es una interpolación del criterio a partir de 150 puntos equiespaciados en el dominio de diseño, para los cuales se resuelve el problema de maximización definido en la ecuación (2.17). En este caso el valor mínimo del $\mathrm{CR}(x)$ se alcanza en el punto $x=0.6712$.

\section{Criterio minimax basado en la máxima desviación frente a la solución óptima}

Este criterio se basa en la minimización de la máxima desviación frente a la solución óptima debido a la incertidumbre del problema (Savage, 1951). La máxima desviación frente a la solución óptima se define como la diferencia entre el mejor valor de la función objetivo en el dominio de diseño factible $\left(\mathscr{D}_{f a c}\right)$ dado un valor de $\mathbf{z}$ :

$$
f^{*}(\mathbf{z})=\inf _{\mathbf{x} \in \mathscr{P}_{\text {fac }}} f(\mathbf{x}, \mathbf{z}),
$$




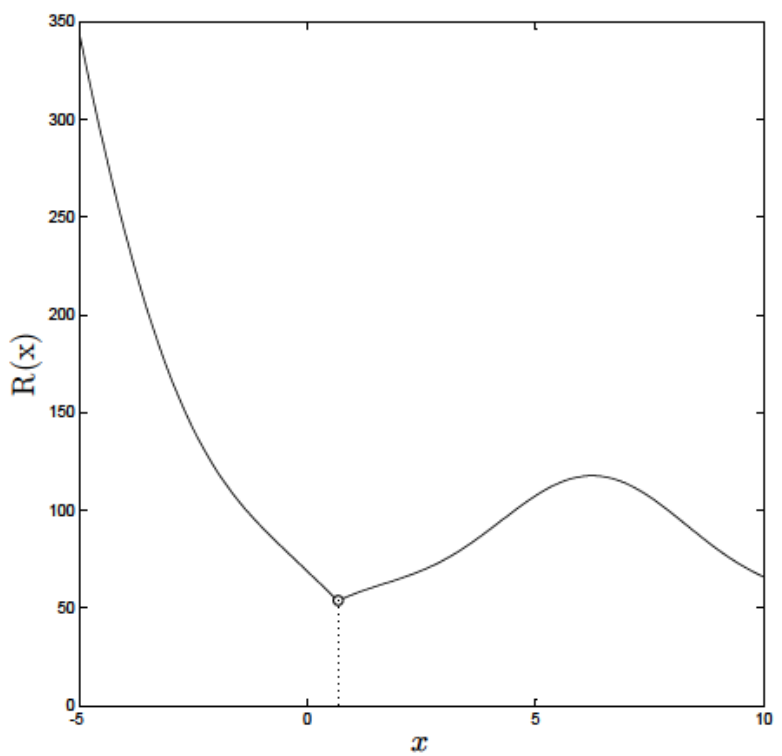

Figura 2.2: Interpolación en el dominio de diseño del criterio de robustez basado en el peor caso (ecuación (2.17)).

y el valor de la función objetivo dado un valor de $\mathbf{x}$ :

$$
\mathrm{CR}(\mathbf{x})=\sup _{\mathbf{z} \in \mathbf{Z}}\left\{f(\mathbf{x}, \mathbf{z})-f^{*}(\mathbf{z})\right\}
$$

En primer lugar se obtiene el mejor valor de la función objetivo para cada valor del vector de incertidumbre $\mathbf{z}$, posteriormente se busca el escenario más desfavorable mediante el problema de maximización.

Ilustración 2.1 (continuación). Se considera el mismo rango de variación del parámetro $z$. En este caso, el criterio de robustez requiere de la búsqueda previa del valor óptimo $f^{*}(z)$. Dicho valor valor se representa en la Figura 2.3a en el dominio estocástico. Conocido $f^{*}(z)$, se puede evaluar la desviación respecto de la solución óptima para cada combinación de $x$ y $z$. El valor máximo de la desviación en cada punto del espacio de diseño se representa en la Figura 2.3b. En este caso el valor mínimo de CR $(x)$ se alcanza en el punto $x=0.9577$.

Los enfoques minimax resultan desde el punto de vista computacional muy costosos puesto que para cada iteración del proceso de optimización es necesario analizar el caso más desfavorable mediante una optimización adicional. Además, tienden a proporcionar resultados muy conservadores. 


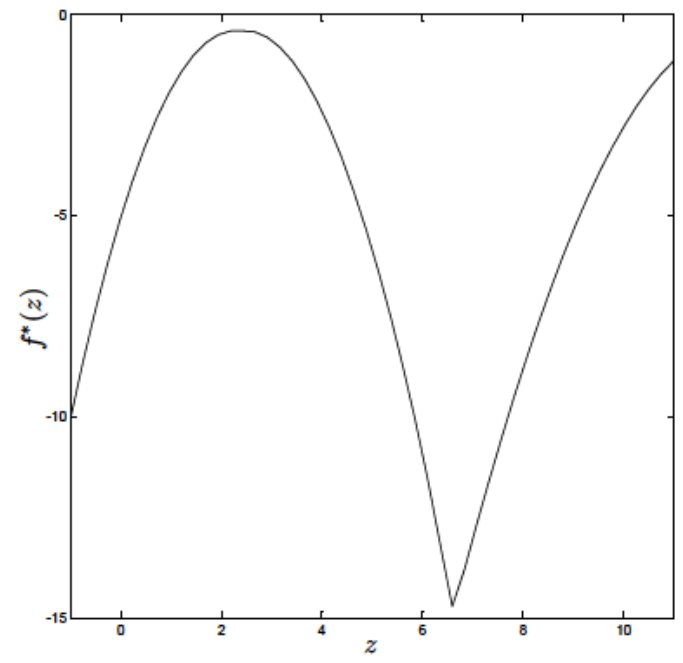

(a)

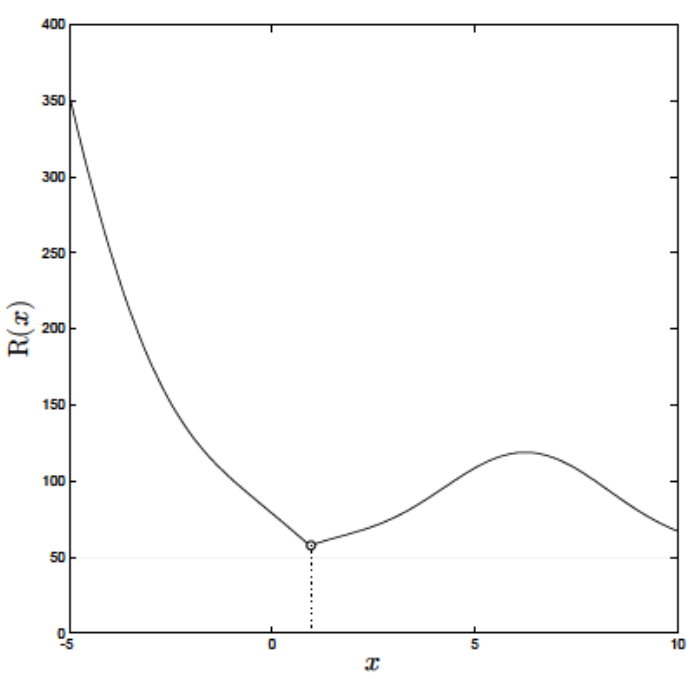

(b)

Figura 2.3: (a) Valor óptimo $f^{*}(z)$ en el dominio estocástico; (b) Interpolación en el dominio de diseño del criterio de robustez basado en la máxima desviación frente a la solución óptima (ecuación (2.19)).

\subsubsection{Criterios probabilistas}

Estos criterios están asociados a problemas en los que la incertidumbre se representa mediante un vector aleatorio $\mathbf{Z}$ caracterizado por una función de densidad de probabilidad $p_{\mathbf{Z}}(\mathbf{Z})$. A partir de dicha distribución y utilizando métodos de propagación de incertidumbre (capítulo 5) se obtiene la función de densidad de probabilidad de la función objetivo aleatoria $p_{f}(f, \mathbf{x})$.

\section{Criterio basado en el cuantil de la distribución}

Siguiendo la línea de los criterios basados en el caso más desfavorable, cuando se dispone de información probabilista se puede establecer un criterio de robustez basado en el cuantil $q$ de la distribución:

$$
\mathrm{CR}(\mathbf{x})=\Phi^{-1}(\mathbf{x}, q),
$$

donde $\Phi(\cdot)^{-1}$ es la inversa de la función de distribución acumulada de la respuesta aleatoria $f(\mathbf{x}, \mathbf{Z})$. En comparación con los criterios minimax, el problema de minimización se sustituye por un problema de propagación de incertidumbre para estimar la distribución de probabilidad de la función objetivo aleatoria.

Ilustración 2.1 (continuación). Para la resolución del problema utilizando criterios probabilistas se asume que el parámetro $z$ es un parámetro aleatorio que sigue una distribución de probabilidad normal $\mathscr{N}(5,2)$. A partir de dicha distribución es posible estimar la distribución de probabilidad de la función $f(x, z)$. En este ejemplo se utiliza una simulación MonteCarlo con 1e4 evaluaciones 


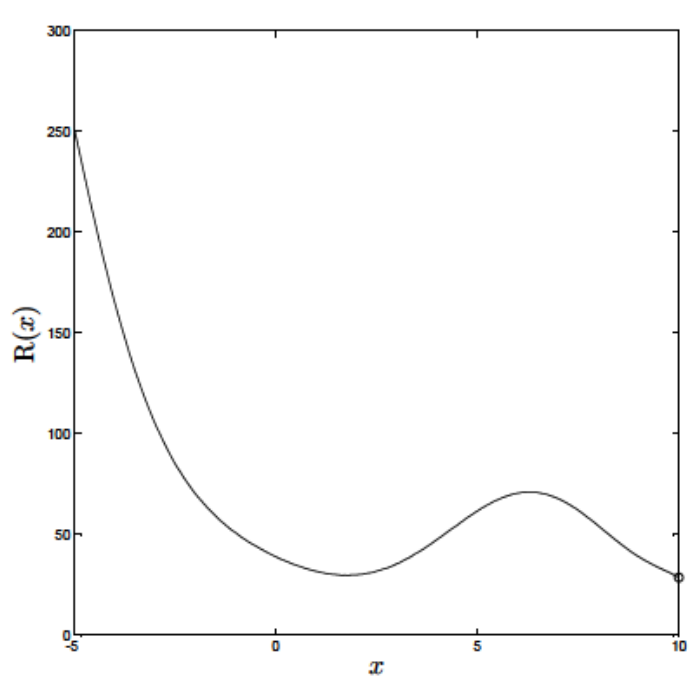

(a) $\mathrm{CR}(\mathbf{x})=\Phi^{-1}(\mathbf{x}, 0.95)$

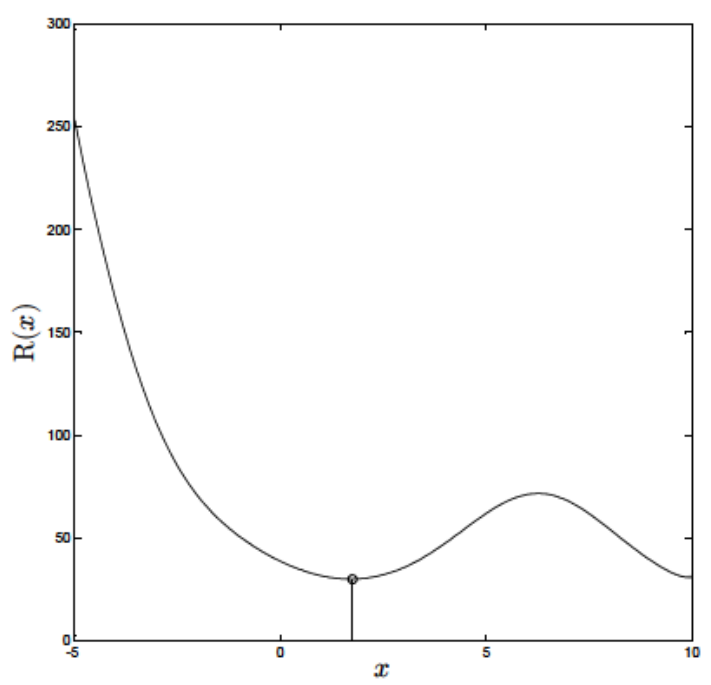

(b) $\mathrm{CR}(\mathbf{x})=\Phi^{-1}(\mathbf{x}, 0.99)$

Figura 2.4: Interpolación en el dominio de diseño del criterio de robustez basado en el cuantil de la distribución (ecuación (2.20)) .

del modelo de simulación. Esta simulación permite estimar el cuantil, la diferencia de percentiles y los momentos estadísticos de $f(x, z)$ que serán utilizados más adelante.

La Figura 2.4 muestra el criterio de robustez en el dominio de diseño basado en el cuantil $95 \%$ (Figura 2.4a) y en el cuantil $99 \%$ (Figura 2.4b). El mínimo valor del criterio se obtiene en puntos distintos: en $x=9.999$ para el cuantil $95 \%$ y en $x=1.746$ para el cuantil $99 \%$.

\section{Criterio basado en los momentos estadísticos}

Entre los criterios que adoptan un enfoque probabilista, uno de los criterios más extendidos en la literatura es la utilización de los dos primeros momentos estadísticos de la respuesta. Utilizando el valor medio de la función $f(\mathbf{x}, \mathbf{Z})$ el criterio de robustez se define como:

$$
\mathrm{CR}(\mathbf{x})=\mathbb{E}[f(\mathbf{x}, \mathbf{Z})]=\mu_{f}=\int_{\Omega} f(\mathbf{x}, \mathbf{Z}) p_{\mathbf{Z}}(\mathbf{Z}) d \mathbf{Z},
$$

donde $\mathbf{x}$ es un vector de variables de diseño deterministas, $\mathbf{z}$ es un vector aleatorio y $\mathbb{E}[\cdot]$ es el operador esperanza matemática. Al igual que los criterios minimax y el criterio de robustez basado en el cuantil, el valor medio de la respuesta no tiene en cuenta la dispersión de la misma. Esta dispersión se considera utilizando la varianza o la desviación estándar de la respuesta como criterio de robustez:

$$
\mathrm{CR}(\mathbf{x})=\mathbb{E}\left[f(\mathbf{x}, \mathbf{Z})-\mu_{f}\right]^{2}=\sigma_{f}^{2}=\int_{\Omega}\left[f(\mathbf{x}, \mathbf{Z})-\mu_{f}\right]^{2} p_{\mathbf{Z}}(\mathbf{Z}) d \mathbf{Z} .
$$


A diferencia de los criterios minimax, los criterios de robustez presentados en las ecuaciones (2.21) y (2.22) no requieren de la resolución de un problema de optimización. Sin embargo, se requiere la resolución de una integral multidimensional en el dominio probabilista. Por lo general, la resolución analítica o mediante integración numérica directa de dicha integral resulta imposible o de gran dificultad en problemas de alta dimensionalidad (ver capítulo 5). Por ello en los últimos años se han desarrollado métodos alternativos que abordan su resolución de forma eficiente y precisa. Uno de los retos del DOR es encontrar un equilibrio entre la precisión y la eficiencia en la estimación de dichos momentos (Du y Chen, 1999).

Ilustración 2.1 (continuación). Las Figuras 2.5a y 2.5b representan los criterios de robustez basados en las ecuaciones (2.21) y (2.22) respectivamente. El mínimo valor del criterio se obtiene en: $x=9.915$ para el problema de minimización de media y en $x=0.695$ para el problema de minimización de varianza. Las Figuras $2.5 \mathrm{c}$ a $2.5 \mathrm{~d}$ muestran la distribución de probabilidad de la respuesta para ambos diseños óptimos. El criterio de mínima media proporciona un diseño con menores valores de $f(x, z)$ a costa de una mayor dispersión. En contraposición, el criterio de mínima varianza garantiza una distribución de probabilidad con menos dispersión a costa de un aumento de la función objetivo $f(x, z)$. Ambos criterios están en conflicto y proporcionan soluciones "extremas", por lo que es necesario buscar soluciones de compromiso que se ajusten adecuadamente al criterio del diseñador.

\section{Criterio basado en la diferencia de percentiles}

Siguiendo la línea del criterio de robustez basado en la varianza, Du y Chen (2004) proponen un criterio basado en la diferencia de percentiles de la función objetivo aleatoria:

$$
\mathrm{CR}(\mathbf{x})=f^{\alpha_{2}}-f^{\alpha_{1}}
$$

donde $f^{\alpha_{1}}$ y $f^{\alpha_{2}}$ son dos valores de la distribución aleatoria $f(\mathbf{x}, \mathbf{Z})$ dados por:

$$
\mathbb{P}\left(f(\mathbf{x}, \mathbf{Z}) \leq f^{\alpha_{i}}\right)=\alpha_{i}, \forall i=1,2,
$$

Ilustración 2.1 (continuación). La Figura 2.6 muestra el criterio de robustez en el dominio de diseño basado en la diferencia entre los percentiles $\alpha_{1}=0.05 \mathrm{y}$ $\alpha_{2}=0.95$. El mínimo valor de la diferencia se obtiene en $x=0.7351$.

\subsection{Formulación multi-objetivo del problema DOR}

La revisión realizada pone de manifiesto la existencia de una gran variedad de criterios de robustez en la literatura. La selección del criterio es función de la 

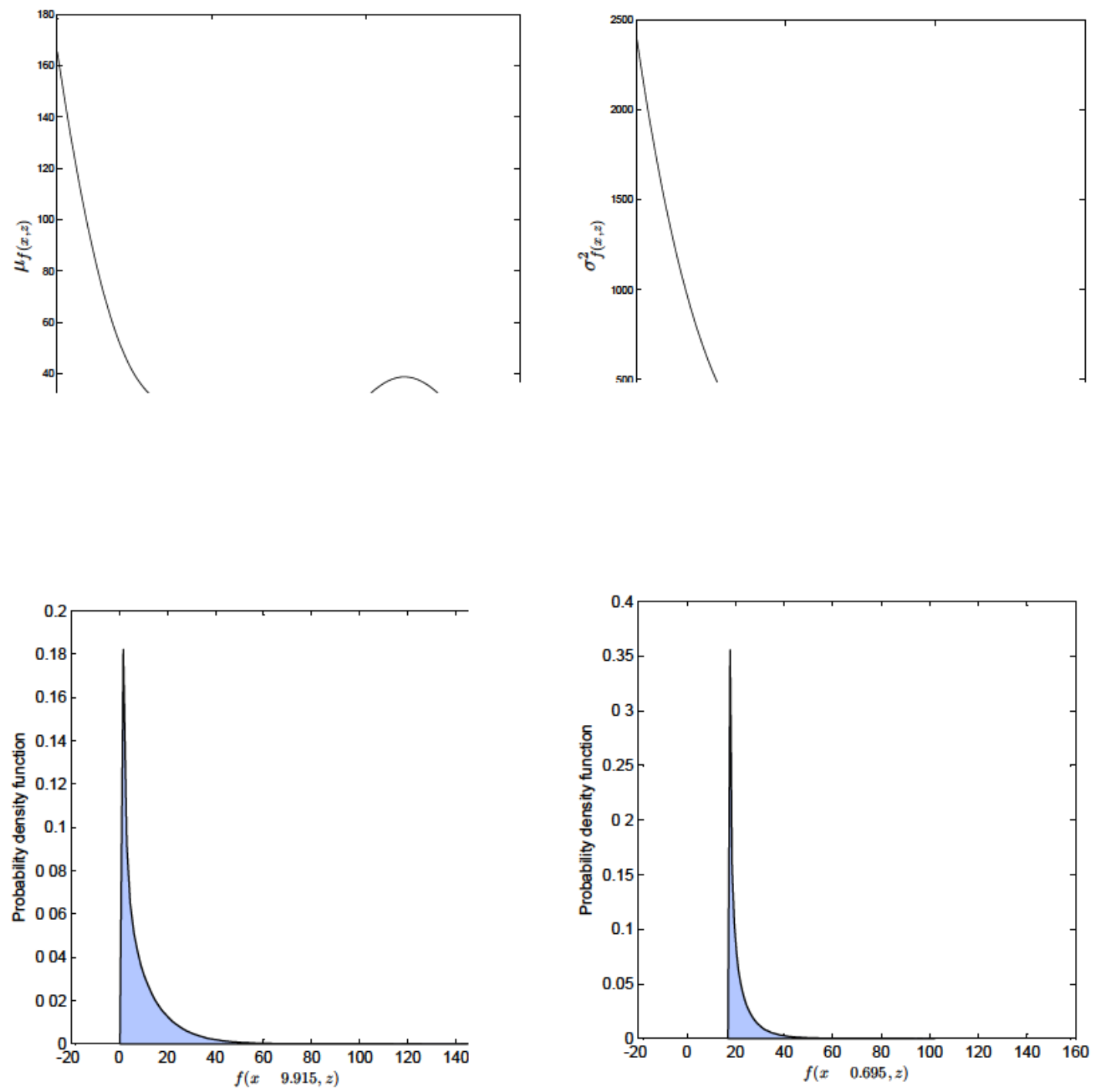

(c) PDF de $f(x=9.915, z)$

(d) PDF de $f(x=0.695, z)$

Figura 2.5: Interpolación en el dominio de diseño del criterio de robustez basado en los momentos estadísticos de la respuesta (ecuaciones (2.21) y (2.22)) .

información disponible acerca de la incertidumbre del problema y del criterio del diseñador. Como consecuencia, no existe una formulación unificada del problema de DOR. El enfoque más extendido es tratar el problema desde un punto de vista probabilista utilizando los dos primeros momentos estadísticos de las respuestas. Estos dos criterios se encuentran habitualmente en conflicto y es necesario encontrar una solución de compromiso entre el diseño de mejor respuesta y el diseño con mínima varianza. El problema se formula como un 


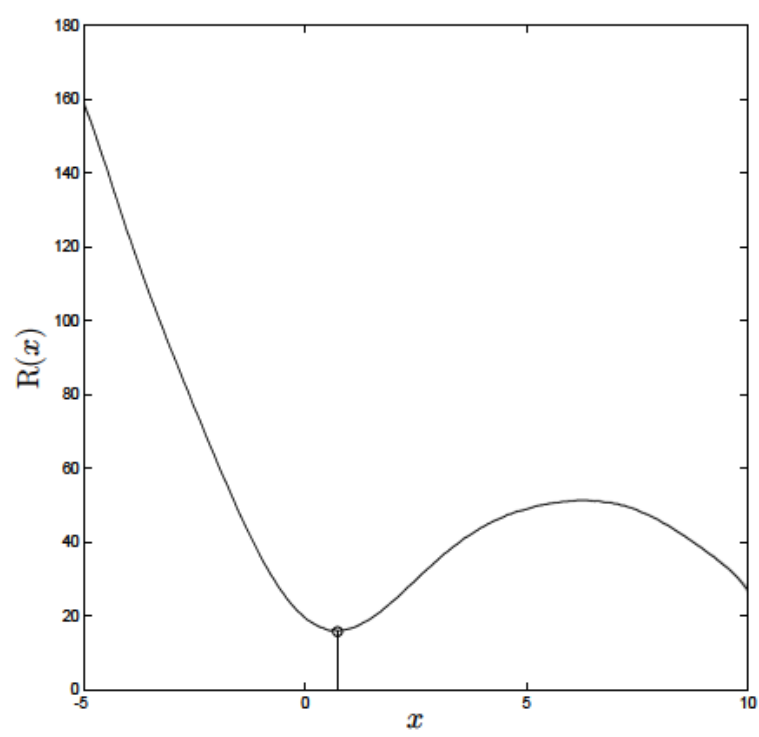

Figura 2.6: Interpolación en el dominio de diseño del criterio de robustez basado en la diferencia de percentiles (ecuación (2.24)).

problema de optimización multi-objetivo (Doltsinis y Kang, 2004):

$$
\begin{aligned}
& \min _{\mathbf{x}}\left\{\mu_{f(\mathbf{x}, \mathbf{z})}, \sigma_{f(\mathbf{x}, \mathbf{z})}\right\} \\
& \text { s.a. : } \quad \mu_{g_{j}(\mathbf{x}, \mathbf{z})}+\beta_{j} \sigma_{g_{j}(\mathbf{x}, \mathbf{z})} \leq 0 ; \quad j=1, \ldots, n_{g}, \\
& \sigma_{h_{k}(\mathbf{x}, \mathbf{z})} \leq \sigma_{k}^{+} ; \quad k=1, \ldots, n_{h}, \\
& x_{i}^{\text {inf }} \leq x_{i} \leq x_{i}^{\text {sup }} ; \quad i=1, \ldots, n_{x}
\end{aligned}
$$

Las primeras $n_{g}$ restricciones son equivalentes a:

$$
\gamma_{j} \leq \beta_{j}, j=1, \ldots, n_{g},
$$

donde $\gamma_{j}=-\mu_{g_{j}(\mathbf{x}, \mathbf{z})} / \sigma_{g_{j}(\mathbf{x}, \mathbf{z})}$ es el factor de seguridad de la restricción j-ésima. La restricción de desigualdad que aparece en la ecuación (2.25) es similar a la del problema de diseño óptimo robusto basado en fiabilidad. Sin embargo, mientras que en el problema de fiabilidad es necesario evaluar la probabilidad de fallo en el punto más probable, en el problema de DOR no es necesario evaluar de forma precisa dicha probabilidad. Grandes valores del coeficiente $\beta_{j}$ demandan una mayor robustez de la respuesta estructural frente a variaciones. En el problema de DOR el índice de fiabilidad representa la robustez del diseño frente a incertidumbres en las restricciones. Esta formulación presenta claras ventajas durante la etapa de toma de decisiones del diseñador, sin embargo el coste computacional asociada suele ser elevado.

Ilustración 2.1 (continuación). El problema de optimización multi-objetivo no presenta restricciones y está compuesto por los criterios de robustez presentados en las ecuaciones (2.21) y (2.22). Las Figuras $2.7 \mathrm{a}$ y $2.7 \mathrm{~b}$ representan gráficamente las dos funciones objetivo. El problema de optimización se resuelve mediante 
la aplicación del algoritmo multi-objetivo NSGA-II (Deb et al., 2002) sobre los momentos estadísticos de la respuesta. La estrategia seguida para la resolución del problema es la presentada en la sección 2.4.1y en la Figura 2.8.

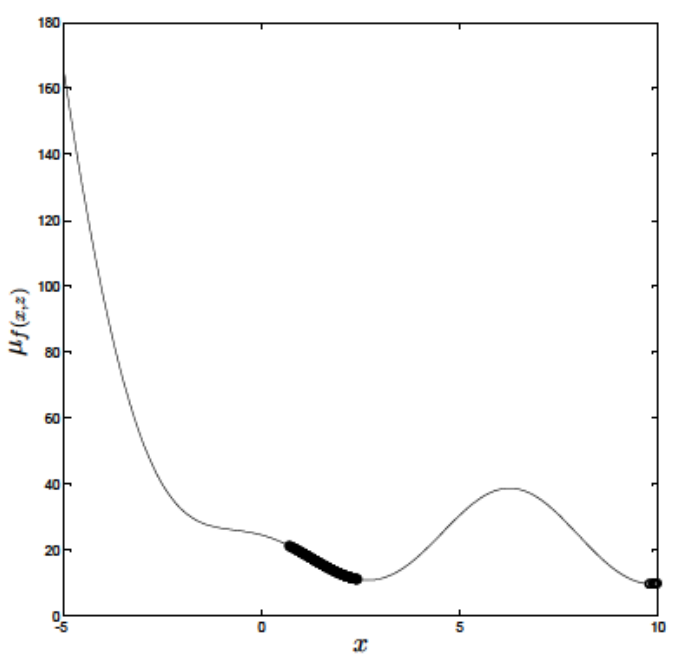

(a)

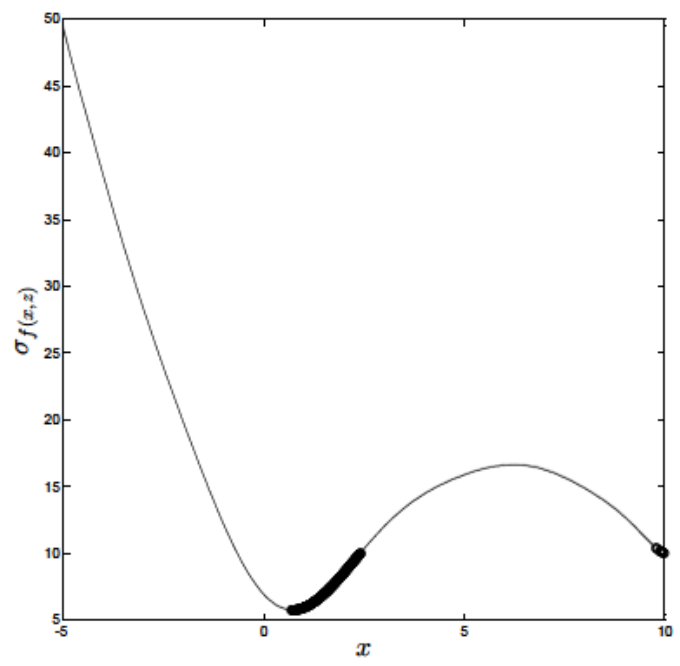

(b)

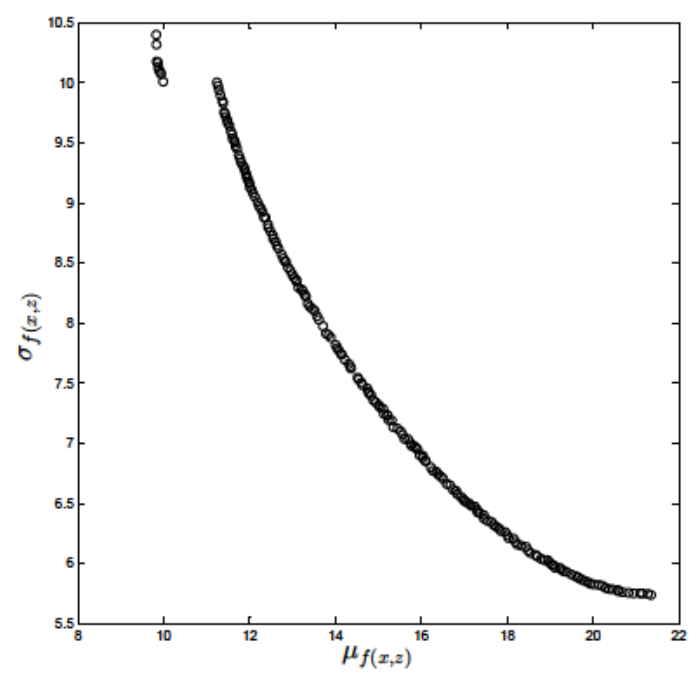

(c)

Figura 2.7: Solución del problema MO de DOR de la función de Branin. (a) Soluciones óptimas sobre la interpolación de $\mu_{f(x, z)}$; (b) soluciones óptimas sobre la interpolación de $\sigma_{f(x, z)} ;$ (c) Conjunto de soluciones óptimas en el espacio de las funciones objetivo.

Se obtienen 100 soluciones óptimas repartidas en dos regiones. Estas soluciones representan un compromiso entre el diseño con mínima media y el diseño con mínima dispersión (Figura 2.7c). 


\subsubsection{Implementación numérica}

La resolución del problema DOR formulado en la ecuación (2.25) consta de dos etapas: (1) la Propagación de Incertidumbre (PI) y (2) la optimización Multi-Objetivo (MO). La primera etapa consiste en la propagación de la incertidumbre de las variables de entrada en el modelo de simulación, con el objetivo de estimar los momentos estadísticos y las probabilidades de la respuesta. La segunda etapa consiste en la utilización de algoritmos de optimización MO dirigidos a la búsqueda de soluciones de compromiso entre el mínimo coste y la máxima robustez.

Las dos etapas son tareas que demandan un elevado número de evaluaciones del modelo de simulación, lo cual se traduce en un alto coste computacional en el caso de modelos de simulación FEM. Adicionalmente, en el proceso de DOR el coste computacional se puede incrementar dramáticamente como consecuencia del anidamiento entre la etapa de PI y de optimización MO (Figura 2.8). Es preciso reducir al máximo el número de evaluaciones del modelo de simulación.

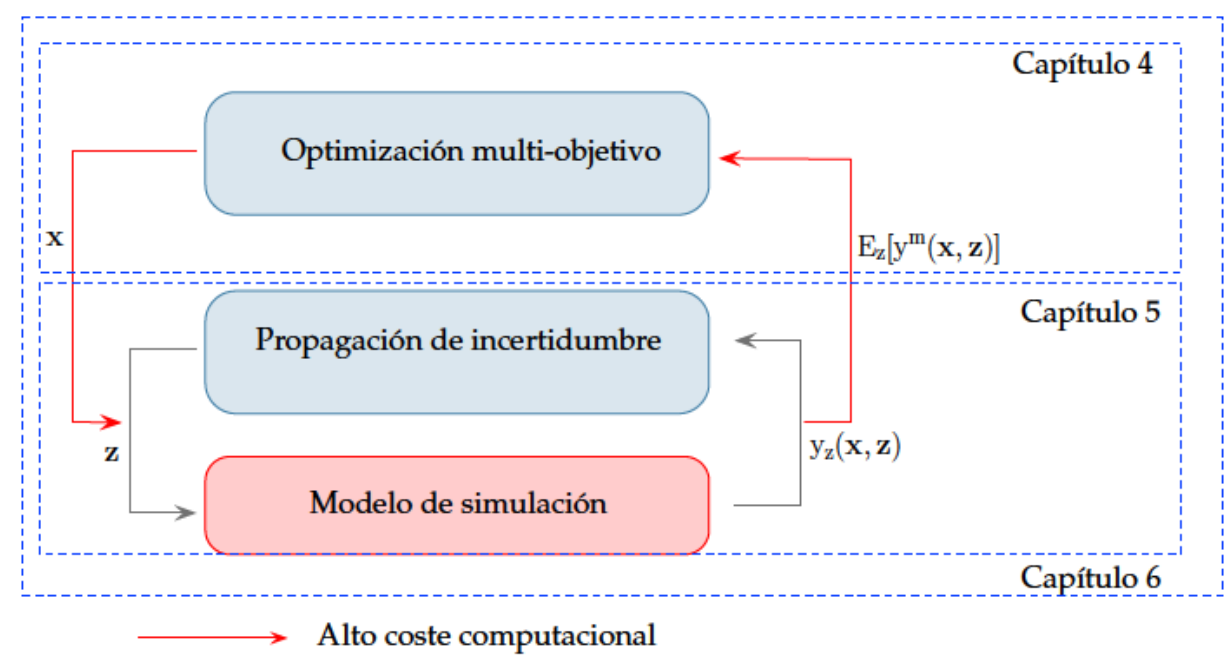

Figura 2.8: Diagrama de flujo del problema multi-objetivo de DOR.

\subsection{Revisión del problema de DOR y perspectivas}

El problema de DOR tiene su origen en el campo de la ingeniería de procesos y desde el mismo ha sido aplicado a muy diversas ramas de la ingeniería. El alcance de esta revisión se limita a los trabajos más significativos en el campo de la optimización estructural. Una revisión en detalle de la evolución de los métodos de DOR en un contexto más general se presenta en trabajos previos como los de Beyer y Sendhoff (2007).

Los primeros intentos para tener en cuenta las incertidumbres en el proceso de diseño están directamente relacionados con los métodos Taguchi de diseño 
robusto (Taguchi, 1996). El método Taguchi considera las variaciones en la respuesta de un sistema a través de un factor de ruido, que el diseñador no puede controlar. El diseño robusto con métodos Taguchi ha sido ampliamente desarrollado en campos de la ingeniería, tales como el diseño de productos industriales o de procesos. Sin embargo, su aplicación en el campo de diseño estructural se limita a unos pocos trabajos, como los de Chi y Bloebaum (1996), que utilizan los métodos Taguchi para resolver problemas estructurales con variables continuas y discretas. Por otra parte, Park et al. (1994) y Lee et al. (1994) tratan la optimización de problemas sin restricciones con variables discretas aplicados a estructuras articuladas. Autores como Montgomery (1991), Nair et al. (1992), Trosset (1996) y Box y Fung (1986) ponen de manifiesto las deficiencias de los métodos Taguchi de diseño robusto como métodos de optimización. Estas deficiencias se resumen en: (1) los métodos Taguchi están basados en técnicas de diseño de experimentos, lo que resulta desfavorable para problemas con modelos de elementos finitos, (2) por lo general la relación entre las variables de entrada y la respuesta estructural presenta un alto grado de no linealidad y (3) los métodos de DOR convencionales tratan a los parámetros de diseño mediante factores de control discretos, por lo que no ofrecen un nivel de flexibilidad suficiente para tratar problemas con parámetros continuos.

Los métodos de DOR aplicados al campo de optimización estructural se dividen en dos categorías: los métodos que utilizan la idea de Taguchi basados en diseño de experimentos y los métodos basados en la minimización de una función objetivo que tiene en cuenta la variabilidad de la respuesta estructural.

Durante la década de los ochenta y principios de los noventa, algunos autores como Balling et al. (1986), Bennett y Lust (1990) o Ramakrishnan y Rao (1991), enfocan el problema de DOR como una etapa de pos-proceso de la optimización determinista. En esta etapa utilizan información de primer orden de las restricciones y de la función objetivo para mejorar la robustez del diseño óptimo determinista. A lo largo de la década de los noventa, los trabajos desarrollados en este campo están basados en su mayoría en la aplicación de técnicas Taguchi. Destacan las aportaciones de autores como Lautenschlager y Eschenauer (1999) que incorporan la incertidumbre asociada con otros parámetros ajenos a las variables de diseño.

El mayor avance en los métodos que tratan el problema de DOR se produce durante la última década. Los trabajos desarrollados en este período se pueden clasificar en dos categorías: (1) los dedicados a estudiar mejoras en la formulación del problema y (2) los dedicados a la aplicación de las técnicas de DOR a la resolución de problemas demandados por la industria.

En cuanto a los trabajos dedicados a estudiar mejoras en la formulación del problema, Lee y Park (2001) formulan el problema de DOR como un problema de optimización con dos objetivos: la media y la desviación estándar de la función objetivo determinista. Sandgren y Cameron (2002) resuelven el problema 
planteado por Lee y Park (2001) utilizando simulación MonteCarlo (MC) junto con un algoritmo híbrido basado en algoritmos genéticos y programación no lineal. Sandgren y Cameron (2002) obtienen diseños óptimos con menor sensibilidad frente a las variaciones, pero con un alto coste computacional derivado del acoplamiento entre el algoritmo genético y la simulación MC. Doltsinis y Kang (2004) resuelven problemas de DOR de estructuras articuladas en régimen estático y dinámico. En los últimos cinco años aparecen nuevas líneas de investigación basadas en formulaciones "mixtas" del problema. La filosofía de estas nuevas formulaciones es conseguir diseños óptimos robustos, permitiendo al diseñador determinar explícitamente la probabilidad de que la respuesta estructural no supere cierto umbral. En esta línea, se sitúan formulaciones como: Reliability-based Robust Design Optimization (RRDO) (Lagaros et al., 2007; Lee, 2008; Lee et al., 2008) y Vulnerability-based Robust Design Optimization (VRDO) (Papadopoulos y Lagaros, 2009), basadas en la introducción de restricciones probabilistas en la formulación del problema de DOR.

En cuanto a la aplicación industrial de los métodos de DOR, destacan trabajos como los de Lee (2010) en los que estudian el problema de DOR de estructuras aeroespaciales frente a imperfecciones geométricas y cargas extremas. Utilizan superficies de respuesta y modelos Kriging para aliviar el problema del alto coste computacional. Por otra parte, Marano et al. (2010) estudian diferentes formulaciones del problema de DOR aplicado a la elección de antivibratorios en estructuras sometidas a vibraciones aleatorias. Lönn et al. (2010) abordan el problema de tubos de aluminio sometidos a un impacto axial considerando las incertidumbres asociadas al proceso de fabricación. Utilizan redes neuronales para reducir el coste computacional. En el campo de la ingeniería civil, se han desarrollado trabajos como los de Lagaros et al. (2007), que aplican técnicas de optimización probabilista junto con redes neuronales para aumentar los niveles de fiabilidad y seguridad de estructuras resistentes a sismos. También Aoues y Chateauneuf (2008) aplican métodos de DOF para obtener estructuras de hormigón armado más económicas y seguras.

\subsection{Ejemplos}

\subsubsection{Estructura articulada de 38 barras sometida a carga de compresión}

En este ejemplo se estudia el problema de DOR de topología de una estructura de nudos articulados sometida a un estado tensional de compresión. El universo estructural inicial está formado por 38 barras de nudos articulados tal y como muestra la Figura 2.9. Este ejemplo ha sido estudiado previamente por otros autores como Asadpoure et al. (2011). Los parámetros F y $L$ tienen un valor determinista de 1 y 8 respectivamente, mientras que el módulo de Young de cada barra se asume aleatorio con una distribución de probabilidad $\mathscr{N}(100,10)$. Dichas 


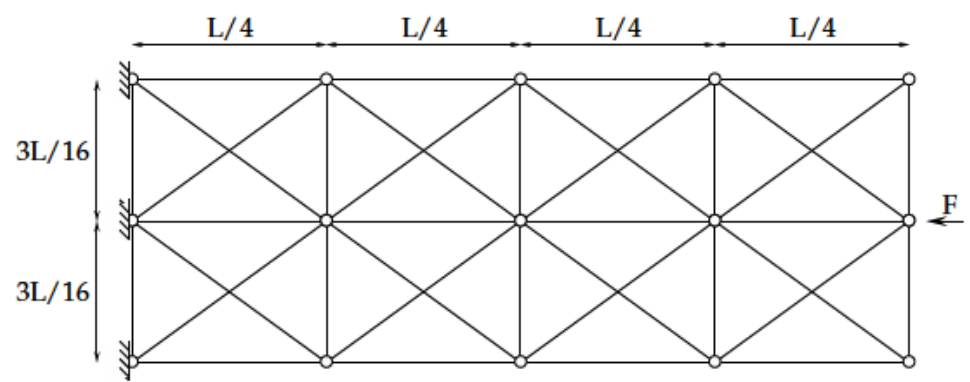

(a) Universo estructural

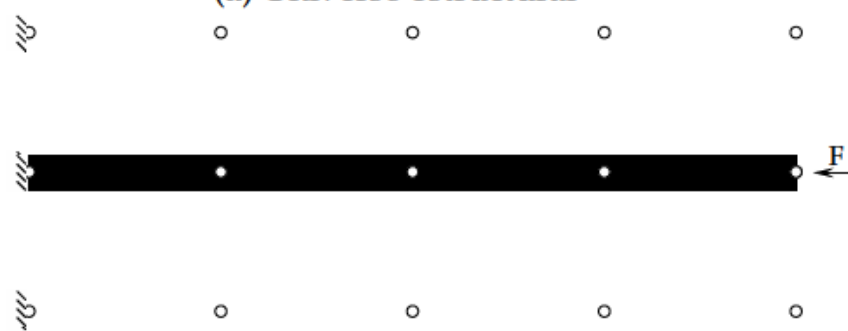

(b) Diseño óptimo determinista

Figura 2.9: Estructura de 38 barras de nudos articulados. (a) Universo estructural, condiciones de contorno y cargas. (b) DOD.

variables aleatorias se consideran independientes entre sí. El problema de DOD consiste en la minimización de la compliance de la estructura bajo la restricción de volumen $\left(V \leq L^{2} / 32\right)$. Para la resolución del problema de DOR se adopta la formulación $\mathrm{MO}$ presentada en la ecuación (2.25). El problema $\mathrm{MO}$ se resuelve mediante su escalarización a un problema con un solo objetivo utilizando una suma ponderada. La formulación del problema queda como sigue:

$$
\begin{array}{ll}
\min _{\mathbf{A}} & \alpha \frac{\mu_{c}}{\mu_{c}^{*}}+(1-\alpha) \frac{\sigma_{c}}{\sigma_{c}^{*}} . \\
\text { s.a. : } & \mathbf{K u}=\mathbf{f}, \\
& \sum_{i=1}^{38} A_{i} \cdot L_{i} \leq \frac{L^{2}}{32}, \quad i=1, \ldots, 38, \\
& 0 \leq A_{i} \leq 1 ; \quad
\end{array}
$$

donde $\alpha \in \mathbb{R}^{+}$es el vector de pesos cuyo valor está comprendido entre 0 y 1 . $\mu_{c}^{*}$ y $\sigma_{c}^{*}$ son los valores obtenidos al resolver el problema de mínima media y mínima desviación estándar respectivamente. En cada iteración del proceso de optimización la media y la desviación estándar se estiman mediante una simulación MC con un total de 1e4 evaluaciones del modelo de simulación. Variando el valor de $\alpha$ se generan diversos subproblemas de optimización cuya solución representa un compromiso entre los diseños de mínima media $(\alpha=1)$ y mínima desviación estándar $(\alpha=0)$. Los subproblemas de optimización se resuelven mediante el algoritmo Method of Moving Asymptotes (MMA) propuesto por Svanberg (1987).

La Figura 2.9b muestra la solución del problema determinista frente a un valor nominal de la carga de $F=100$. En este caso todo el material se sitúa en las barras centrales, que representan el camino más corto desde la carga a los apoyos. 


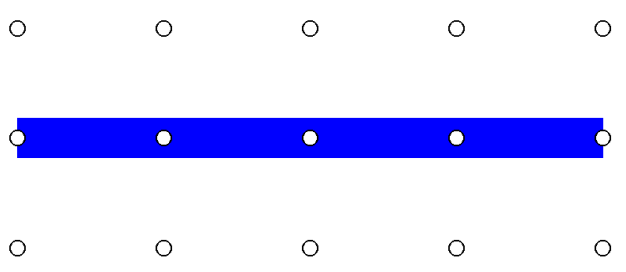

(a) $\alpha=1$

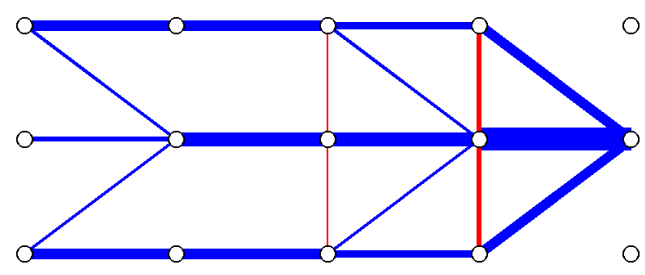

(c) $\alpha=0.6$

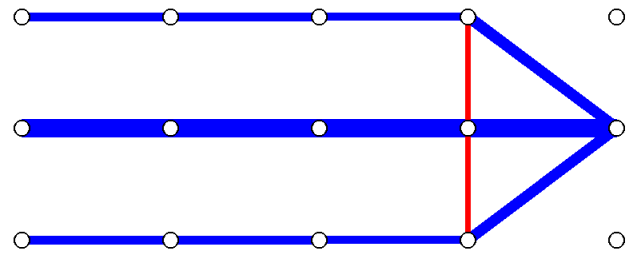

(b) $\alpha=0.767$

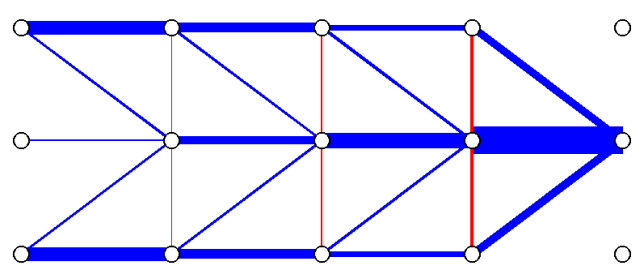

(d) $\alpha=0$

Figura 2.10: Diseños óptimos robustos de la estructura de 38 barras de la Figura 2.9 para una variabilidad del $10 \%$ del módulo de Young y diferentes valores del coeficiente $\alpha$. En rojo barras a tracción, en azul barras a compresión.

Está solución es óptima pero poco robusta al convertirse en una estructura inestable frente a perturbaciones en la geometría o en la carga horizontal.

La Figura 2.10 muestra las estructuras resultantes para diferentes valores del parámetro $\alpha$. A medida que el coeficiente $\alpha$ se aproxima a cero la importancia de la desviación estándar en la función objetivo escalarizada aumenta y como consecuencia aparecen un mayor número de barras en el diseño final. El aumento del número de barras incrementa la estabilidad de la estructura y el grado de robustez de la misma. Este ejemplo pone de manifiesto la importancia de incluir información acerca de la dispersión de la respuesta en el criterio de robustez. La Figura 2.10a muestra la estructura que resulta al utilizar como función objetivo únicamente la media de la compliance $(\alpha=1)$. Esta estructura es idéntica al DOD. Esto se debe a que, asumiendo que todas las variables aleatorias tienen la misma variabilidad y el problema es lineal, el valor esperado de la solución coincide con el valor determinista:

$$
\begin{aligned}
\mathbb{E}\left[\mathbf{u}\left(\mathbf{E}_{\text {Young }}\right)\right] & =\mathbb{E}\left[\left(\mathbf{E}_{\text {Young }} \mathbf{K}_{0}\right)^{-1} \cdot \mathbf{f}\right] \\
& =\mathbb{E}\left[\mathbf{E}_{\text {Young }}^{-1} \cdot \mathbf{K}_{0}^{-1} \cdot \mathbf{f}\right] \\
& =\mathbb{E}\left[\mathbf{E}_{\text {Young }}^{-1}\right] \cdot \mathbf{K}_{0}^{-1} \cdot \mathbf{f} \\
& =\mathbb{E}\left[\mathbf{E}_{\text {Young }}\right]^{-1} \cdot \mathbf{K}_{0}^{-1} \cdot \mathbf{f} .
\end{aligned}
$$

En presencia de incertidumbres en cargas y/o geometría, la matriz de rigidez y sus derivadas presentan una estructura diferente al caso determinista y se producen cambios en la topología considerando únicamente la media en la 
función objetivo.

Respecto al coste computacional, el número total de evaluaciones del modelo de simulación necesarias para la obtención del conjunto de diseños óptimos es igual al producto entre el número de subproblemas de optimización resueltos, el número de evaluaciones durante el proceso de optimización, y el número de evaluaciones realizadas durante la simulación MC. En este ejemplo, el número total de evaluaciones fue de 51e6.

\subsection{Discusión y conclusiones}

Este capítulo trata el problema de optimización estructural cuando se considera explícitamente la incertidumbre de las variables de diseño y/o de parámetros de gran influencia en la respuesta estructural. En particular se han presentado las diferentes formulaciones existentes en el campo de optimización bajo incertidumbre, presentando especial atención al problema de DOR. Para ello se han revisado en profundidad las diferentes formulaciones del problema DOR así como los trabajos más significativos desarrollados hasta la fecha dentro del campo de la optimización estructural. Como resultado de esta revisión se alcanzan las siguientes conclusiones:

Formulación del problema de diseño óptimo robusto. Los resultados del ejemplo de ilustración muestran diseños óptimos robustos distintos en función del criterio de robustez seleccionado. Por lo que la selección de dicho criterio resulta en sí mismo un problema de toma de decisiones. En dicho problema interviene la información disponible acerca de la incertidumbre (probabilista o no probabilista) y el criterio de diseño (mejor coste o mayor robustez). En este sentido, la formulación probabilista MO del problema de DOR resulta de gran utilidad puesto que proporciona diferentes soluciones y permite la combinación de diferentes criterios de robustez. Esta formulación se utiliza como punto de partida en esta tesis.

Junto con las ventajas anteriormente mencionadas, la formulación $\mathrm{MO}$ del problema de DOR también plantea retos como el alto coste computacional necesario para su resolución. Dicho coste computacional procede de dos procesos principalmente: (1) el proceso de PI necesario para la estimación de los momentos estadísticos de la respuesta y (2) el anidamiento con el algoritmo de optimización MO.

Estado actual del problema de DOR en el campo de la optimización estructural. En comparación con otros campos de la ingeniería, el problema de DOR de estructuras ha sido tratado con menos frecuencia en la literatura. A diferencia del problema de DOF, cuya formulación está muy asentada, en el caso del problema 
de DOR no existe una definición matemática unificada acerca del criterio de robustez estructural. Entre los criterios más adoptados se encuentran la relación señal-ruido de Taguchi, la varianza de la respuesta estructural, la diferencia de percentiles (Huang y Du, 2007), o el índice de robustez que mide el riesgo total de la estructura a partir de las consecuencias directas (Baker et al., 2008). Gran parte de los trabajos existentes en la literatura tienen en cuenta únicamente variaciones en las variables de diseño, sin considerar incertidumbre en otros parámetros y en las cargas. Las líneas de investigación actuales estudian enfoques unificados capaces de considerar la incertidumbre tanto en variables como en parámetros, facilitando de este modo su aplicación práctica.

En el campo de la optimización estructural garantizar la fiabilidad del diseño final resulta de vital importancia. Por este motivo existe una tendencia a formulaciones mixtas que permitan al diseñador controlar la robustez estructural así como determinar explícitamente la probabilidad de fallo de la estructura. La aplicación de dichas formulaciones junto con modelos de simulación de alta fidelidad suponen un reto en la actualidad. Por este motivo se plantea la necesidad de investigar en nuevas técnicas numéricas que consigan disminuir el coste computacional del problema y que permitan su aplicación en problemas "reales". En este sentido, autores como Schuëller y Jensen (2008) destacan la importancia de la eficiencia cuando se investiga en nuevos métodos para la resolución de problemas de optimización bajo incertidumbre:

"Efficient procedures to deal with optimization considering uncertainties should become the rule and not the exception in future engineering design".

Schuëller y Jensen (2008)

La necesidad de resolver problemas de aplicación práctica ha consolidado una línea de investigación basada en la utilización de meta-modelos como sustitutos del modelo de simulación de alto coste computacional. La utilización de meta-modelos permite la resolución sistemática y eficiente de problemas de optimización con sofisticados modelos de simulación, proporcionando una gran flexibilidad para la resolución del problema de DOR frente a diferentes tipos de incertidumbre, comportamiento estructural y métricas de robustez estructural. Sin embargo, como se describe en mayor detalle en el Capítulo 6, estos enfoques presentan importantes limitaciones, tales como su ineficiencia en problemas de media/alta dimensionalidad o el tratamiento de la incertidumbre asociada al modelo de aproximación.

Las líneas de investigación actuales tratan de mejorar los métodos existentes potenciando tres aspectos fundamentales: la eficiencia, la precisión y la aplicabilidad. La revisión realizada pone de manifiesto la necesidad de investigar en una metodología que permita la resolución de problemas de aplicación práctica de una forma eficiente y sistemática. 
"Essentially, all models are wrong, but some are useful."

\section{Modelos de predicción basados en procesos Gaussianos de regresión}

\section{Índice}

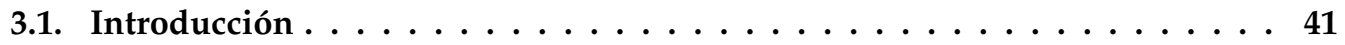

3.2. Definiciones previas $\ldots \ldots \ldots \ldots \ldots \ldots \ldots \ldots \ldots \ldots \ldots \ldots \ldots \ldots$

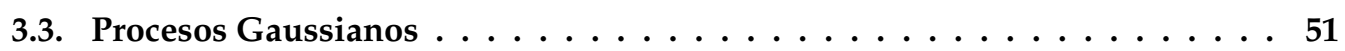

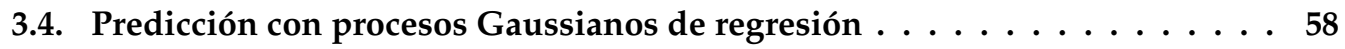

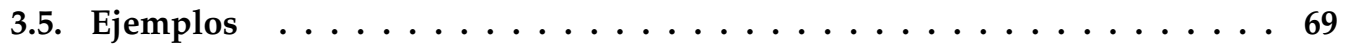

3.6. Discusión y conclusiones $\ldots \ldots \ldots \ldots \ldots$ 



\subsection{Introducción}

En este capítulo se aborda el problema de búsqueda de un modelo de predicción que emule los resultados obtenidos mediante una simulación computacional o experimental. Este problema es común en diversos campos de la ciencia e ingeniería. En función del campo de aplicación el modelo obtenido se conoce en la literatura como: hipótesis (Murphy, 2012) o emulador (Rasmussen y Williams, 2006), superficie de respuesta (Box y Wilson, 1951), modelo sustituto (Fabian et al., 1959) o meta-modelo ("model of model"). Este último término, acuñado por (Kleijnen, 1975), será el utilizado en este trabajo para hacer referencia a este tipo de modelos. Aunque la mayor parte del capítulo puede aplicarse de manera indiferente a la predicción de respuestas obtenidas mediante experimentos físicos o computacionales, el objetivo en este trabajo es su aplicación a la predicción de respuestas obtenidas mediante modelos de alto coste computacional.

En el resto de esta sección se presenta la formulación del problema de predicción junto con una breve revisión del estado del arte que motiva la utilización de modelos de predicción basados en procesos Gaussianos o modelos Kriging. La sección 3.2 presenta algunas definiciones básicas de la teoría de probabilidad para una familiarización con la notación utilizada en el resto del capítulo. La sección 3.3 presenta la formulación de un proceso estocástico Gaussiano. Este modelo se utiliza para la modelización espacial de la incertidumbre de propiedades estructurales (sección 3.3.2), así como para la predicción de modelos de simulación de alto coste computacional (sección 3.4). Los conceptos presentados en este capítulo se ilustran con tres ejemplos en la sección 3.5. La sección 3.6 recoge las conclusiones alcanzadas en el capítulo.

\subsubsection{Formulación del problema}

El problema que se trata en este capítulo consiste en la búsqueda de una función matemática $\hat{\mathscr{M}}$, denominada en adelante meta-modelo, que emule la respuesta obtenida a partir de un modelo $\mathbf{y}=\mathscr{M}(\mathbf{x})$.

$\mathscr{M}$ es una aplicación que asigna a cada vector de variables $\mathbf{x} \in \mathbb{X} \subseteq \mathbb{R}^{n}$ una salida $\mathbf{y} \in \mathbf{Y} \subseteq \mathbb{R}^{d}$ que puede ser el resultado de un experimento físico o computacional. Desde el punto de vista de ingeniería estructural $\mathscr{M}$ puede representar un modelo de elementos finitos mediante el cual se calcula una respuesta $(y)$ en función de la dimensiones geométricas de la estructura $(\mathbf{x})$. En esta tesis se limita el problema a la emulación de una salida escalar de un modelo de simulación computacional $(d=1)$ libre de ruido. La relación estadística entre la entrada y la salida se obtiene a partir de un conjunto de observaciones $\mathscr{D}=\left\{\left(\mathbf{x}^{i}, y^{i}\right), \ldots,\left(\mathbf{x}^{m}, y^{m}\right)\right\}$ y de unas hipótesis a priori acerca de la física del problema. 


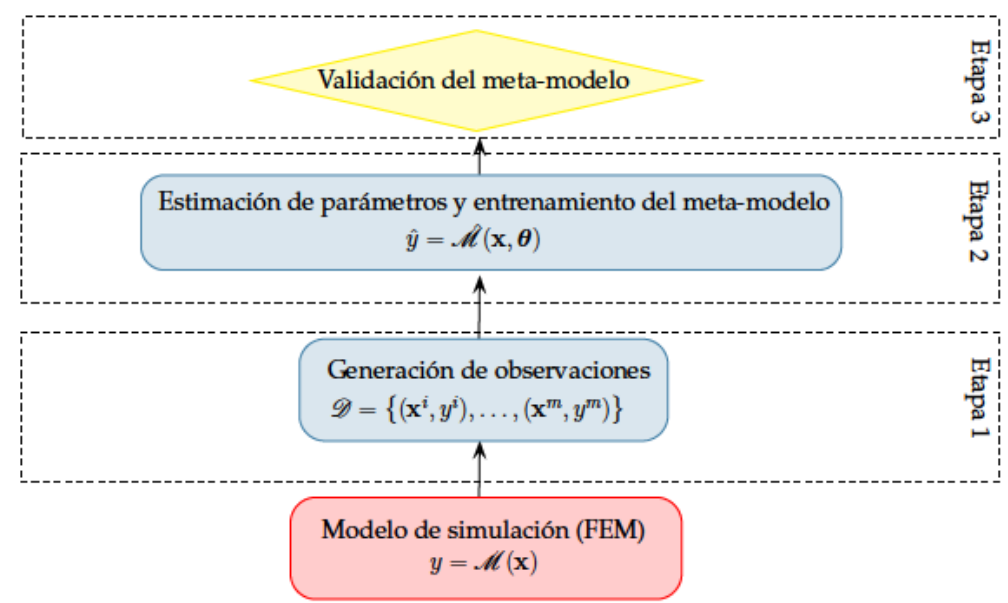

Figura 3.1: Diagrama de flujo del problema de predicción con procesos Gaussianos.

"A metamodel is a relatively small, simple model intended to mimic the behavior of a large complex model, called the object model, that is, to reproduce the object model's input-output relationships".

Davis y Bigelow (2003)

El proceso de creación de un meta-modelo consta de tres etapas (Figura 3.1). Una primera etapa denominada Generación de observaciones consiste en la generación de $\mathscr{D}$ (Figura 3.2b). Estas observaciones se utilizan en el proceso de búsqueda en el espacio de posibles funciones $\mathscr{M}$ que emulen el comportamiento de la función $\mathscr{M}$. Este proceso es conocido como aprendizaje supervisado en el campo del aprendizaje estadístico (Rasmussen y Williams, 2006). Algunos autores como Rasmussen y Williams (2006) adoptan un enfoque Bayesiano mediante el cual asignan una probabilidad a priori a cada posible función $\hat{\mathscr{M}}$, dando una probabilidad elevada a funciones consideradas como más adecuadas para emular $\mathscr{M}$. En la práctica resulta difícil establecer que tipo de funciones son las más adecuadas, puesto que la función $\mathscr{M}$ es a priori desconocida. Por esta razón, el enfoque adoptado en este trabajo consiste en asignar una estructura a priori a $\hat{\mathscr{M}}(\mathbf{x}, \boldsymbol{\theta})$ que es función de un conjunto de parámetros $\boldsymbol{\theta}$ (sección 3.4.2) y utilizar un algoritmo de optimización para buscar el conjunto de parámetros óptimos que mejor ajuste el modelo a las observaciones. El proceso de ajuste se realiza en la etapa 2, denominada estimación de parámetros del modelo y entrenamiento del meta-modelo. La tercera etapa consiste en la validación del meta-modelo obtenido mediante la estimación de indicadores de la precisión global del meta-modelo, tales como el coeficiente de determinación o el error cuadrático medio.

La Figura 3.2 ilustra las tres etapas del proceso de creación del meta-modelo. En un principio se considera que la única información que se conoce acerca de la función $\mathscr{M}$ es que dicha función es continua y diferenciable. Bajo esta información la Figura 3.2a muestra 5 posibles realizaciones de una función (proceso Gaussiano con media cero y correlación exponencial cuadrática) que $a$ priori representa dicha información previa. La Figura 3.2c muestra el valor medio 
y la varianza de la función a posteriori. Esta función se obtiene optimizando los parámetros de correlación utilizando las observaciones de la Figura 3.2b. La Figura $3.2 \mathrm{~d}$ presenta gráficamente la precisión del meta-modelo comparando las predicciones del modelo frente a las observaciones de $\mathscr{M}$.

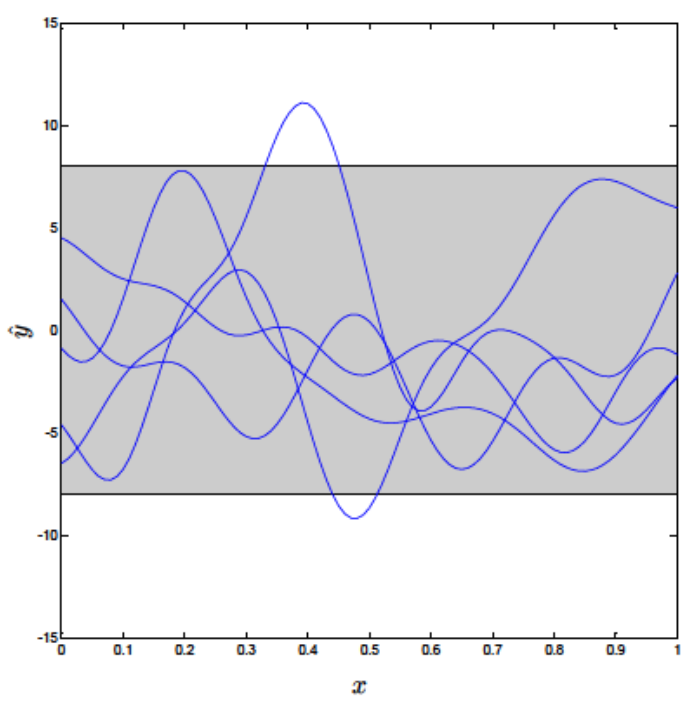

(a)

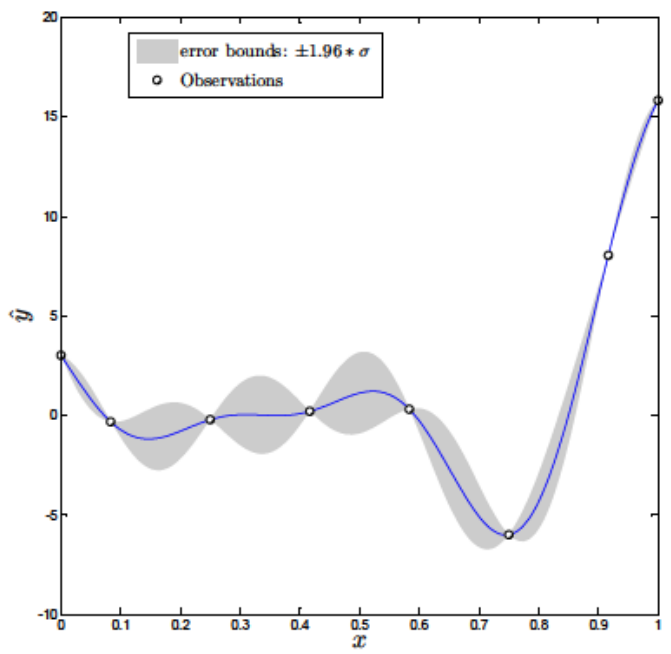

(c)

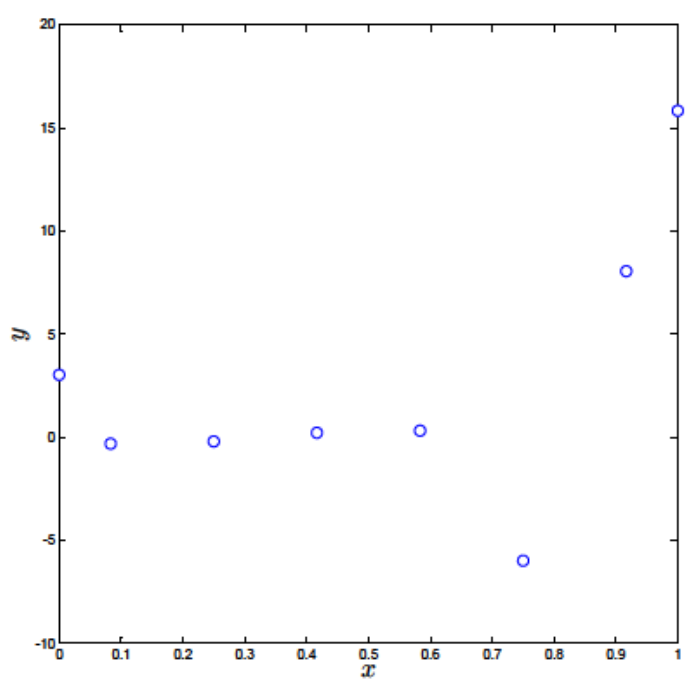

(b)

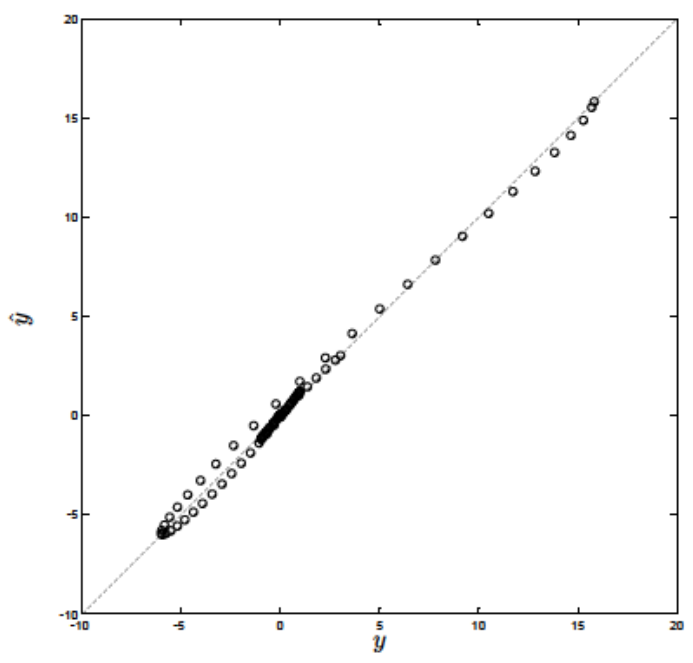

(d)

Figura 3.2: Etapas del proceso de creación del meta-modelo. (a) Realizaciones del modelo a priori; (b) Etapa 1: generación de observaciones; (c) Etapa 2: estimación de parámetros del modelo y entrenamiento del meta-modelo; (d) Etapa 3: validación del meta-modelo. 


\subsubsection{Revisión del estado del arte y perspectivas}

El origen de las técnicas de meta-modelos está íntimamente ligado con el desarrollo de métodos de optimización y su aplicación a la resolución de problemas "reales" de ingeniería. Su origen se remonta a la década de 1980 en el campo de la optimización multidisciplinar. En este campo el acoplamiento entre modelos de elementos finitos y técnicas de optimización pone de manifiesto dos problemas. En primer lugar, la utilización de modelos de elementos finitos dificulta la obtención de una expresión explícita de la función objetivo y de las restricciones. En segundo lugar, el acoplamiento simulación-optimización supone un coste computacional prohibitivo. Esta problemática queda patente en trabajos como los de Sobieszczanski-Sobieski y Haftka (1997):

"The primary challenges in MDO are computational expense and organizational complexity".

Abstract, Sobieszczanski-Sobieski y Haftka (1997)

En la literatura existe un gran número de técnicas de meta-modelos como: las redes neuronales (Haftka et al., 1998; Papadrakakis et al., 1998), los modelos Multi-Adaptive Regression Splines (MARS) (Friedman, 1991) y las máquinas de soporte vectorial (Smola y Schölkopf, 2004) entre otras. La mayoría de publicaciones en el campo de la optimización basada en meta-modelos se concentra en la última década (Figura $3.3^{1}$ ), en gran medida como respuesta a necesidades de la industria como la optimización multi-objetivo, la optimización multidisciplinar o la optimización probabilista. Autores como Wang y Shan (2007), Simpson et al. (2008), Queipo et al. (2005) y Forrester y Keane (2009) aportan una revisión de la evolución desarrollada y la aplicación de estos métodos durante la última década.

En este trabajo se ha decidido la utilización de modelos de predicción basados en procesos Gaussianos, también conocidos como modelo Kriging, por sus características de interpolación, Gaussianidad y consistencia asintótica (sección 3.4.3.1). Más allá de actuar como simples aproximaciones, estos modelos representan el comportamiento del modelo de simulación como una realización de un proceso estocástico Gaussiano proporcionando en cada punto del espacio de diseño información acerca del error cometido por la aproximación. Esta información resulta de gran utilidad para el desarrollo de algoritmos de optimización basados en análisis Bayesiano (Bayesian analysis algorithms) (Mockus et al., 1978; Zilinskas, 1975) que exploran globalmente el espacio de diseño en la búsqueda del óptimo.

Una revisión en detalle de la utilización de técnicas de meta-modelos queda fuera del alcance del presente trabajo y se remite al lector a trabajos como los antes mencionados. En esta sección se presentan los fundamentos de dos de

\footnotetext{
${ }^{1}$ Los datos fueron obtenidos mediante la herramienta Google Scholar, realizando una búsqueda de publicaciones con la frase: "surrogate-based design optimization" OR "surrogate-based optimization" OR "Metamodel assisted optimization"
} 


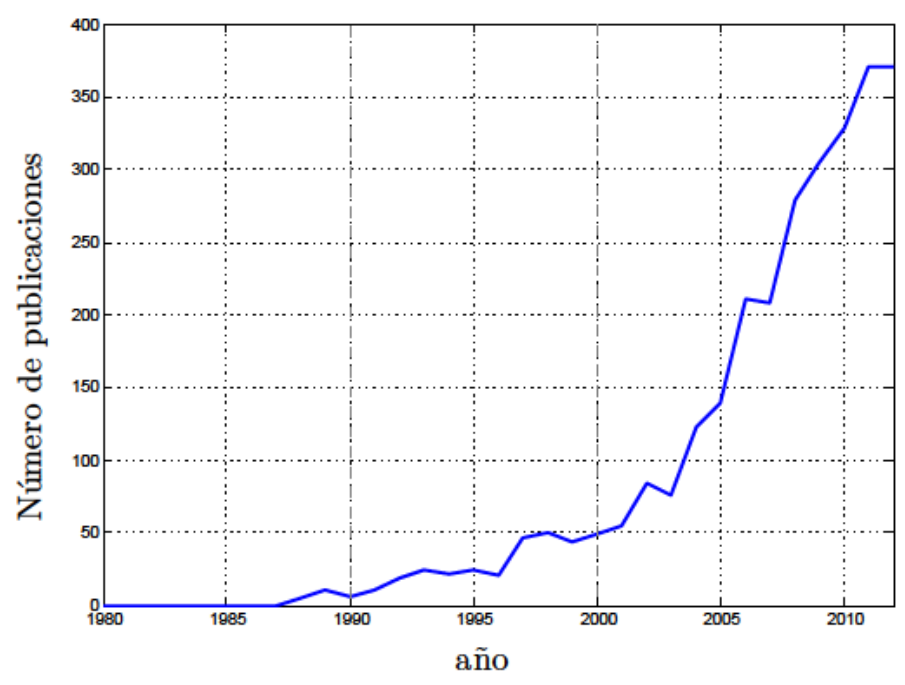

Figura 3.3: Número de publicaciones relacionadas con optimización basada en meta-modelos durante el período 1980-2012 ${ }^{1}$.

estas técnicas: las superficies de respuesta polinómicas (Box y Wilson, 1951) y las funciones de base radial (Dyn et al., 1986; Fang y Horstemeyer, 2006; Hardy, 1971). La primera por su importancia en los orígines de la aplicación de los meta-modelos en el campo de la propagación de incertidumbre. La segunda por su relación con los meta-modelos utilizados en esta tesis, los modelos Kriging.

\subsubsection{Superficies de respuesta polinómicas}

Dado un conjunto de observaciones $\mathscr{D}=\left\{\left(\mathbf{x}^{(1)}, y^{(1)}\right), \ldots,\left(\mathbf{x}^{(m)}, y^{(m)}\right)\right\}$ de una función escalar $y=\mathscr{M}(\mathbf{x})$, una aproximación polinómica de la función $\mathscr{M}$ de orden $p$ se expresa como sigue:

$$
\mathscr{M}(x, p, \boldsymbol{\theta})=\theta_{0}+\theta_{1} x+\theta_{2} x^{2}+\ldots+\theta_{p} x^{p}=\sum_{i=0}^{p} \theta_{i} x^{i},
$$

el vector de parámetros $\boldsymbol{\theta}=\left\{\theta_{0}, \theta_{1}, \ldots, \theta_{p}\right\}^{T}$ es la estimación de máxima verosimilitud obtenida mediante la solución del problema de mínimos cuadrados:

$$
\min _{\boldsymbol{\theta}} \sum_{i=1}^{m}\left[y^{(i)}-\mathbf{\Phi} \boldsymbol{\theta}\right]^{2},
$$

donde $\boldsymbol{\Phi}$ es la matriz de Vandermonde:

$$
\boldsymbol{\Phi}=\left[\begin{array}{ccccc}
1 & x_{1} & x_{1}^{2} & \ldots & x_{1}^{p} \\
1 & x_{2} & x_{2}^{2} & \ldots & x_{2}^{p} \\
\ldots & \ldots & \ldots & \ldots & \ldots \\
1 & x_{m} & x_{m}^{2} & \ldots & x_{m}^{p}
\end{array}\right]
$$


Bajo la hipótesis de un modelo Gaussiano del error, el estimador de máxima verosimilitud de $\boldsymbol{\theta}$ es:

$$
\boldsymbol{\theta}=\left(\boldsymbol{\Phi}^{\mathrm{T}} \boldsymbol{\Phi}\right)^{-1} \boldsymbol{\Phi}^{\mathrm{T}} \mathbf{y}
$$

La ecuación (3.1) se puede entender como una expansión en serie de Taylor truncada en el término $p+1$. El orden $p$ de la expansión se puede determinar mediante diferentes técnicas (Cherkassky y Mulier, 1989). Una de ellas es la validación cruzada que se presentará en la sección 3.4.5.2.

\subsubsection{Funciones de base radial}

Dado un conjunto de observaciones $\mathscr{D}=\left\{\left(\mathbf{x}^{(1)}, y^{(1)}\right), \ldots,\left(\mathbf{x}^{(m)}, y^{(m)}\right)\right\}$ de una función escalar $y=\mathscr{M}(\mathbf{x})$, la función de aproximación de base radial se expresa como sigue:

$$
\hat{\mathscr{M}}(\mathbf{x}, \boldsymbol{\theta})=\sum_{i=1}^{n_{c}} \theta_{i} \psi_{e}\left(\left\|\mathbf{x}-\mathbf{c}^{i}\right\|\right)
$$

donde $\mathbf{c}^{(i)}$ es el i-ésimo origen de la base funcional y $\psi_{e}$ es el vector de dimensión $n_{c}$ que contiene los valores de la distancia euclídea entre el punto para el cual se busca la predicción $\mathbf{x}$ y los centros $\mathbf{c}^{(i)}$ de la base funcional. El número de parámetros es igual a $n_{c}$ en el caso de utilizar funciones fijas tales como: lineal $(r)$, cúbica $\left(r^{3}\right)$, thin plate spline $\left(r^{2} \ln (r)\right)$. Por otra parte, las funciones de tipo paramétrico como: la Gaussiana $\left(e-r^{2} /\left(2 \sigma^{2}\right)\right)$, la multicuadrática $\left(\left(r^{2}+\sigma^{2}\right)^{1 / 2}\right)$ o la multicuadrática inversa $\left(\left(r^{2}+\sigma^{2}\right)^{-1 / 2}\right)$, dotan al modelo de la ecuación (3.5) de una mayor flexibilidad a costa de aumentar la dificultad en el proceso de ajuste.

El vector de parámetros $\theta$ se obtiene de la condición de interpolación:

$$
\sum_{i=1}^{n_{c}} \theta_{i} \psi\left(\left\|\mathbf{x}^{(j)}-\mathbf{c}^{(i)}\right\|\right)=y^{(j)}, j=1, \ldots, m .
$$

La principal ventaja de las funciones de base radial respecto a otros meta-modelos reside en que la ecuación (3.6) es lineal en términos de $\boldsymbol{\theta}$, pero permite emular respuestas con un alto grado de no linealidad. El sistema (3.6) tiene solución única cuando $n_{c}=m$. Seleccionando el origen de las funciones en los puntos de la muestra inicial $\left(\mathbf{c}^{(i)}=\mathbf{x}^{(i)}, i=1, \ldots, m\right)$, el problema (3.6) se simplifica al sistema de ecuaciones matricial:

$$
\Psi \boldsymbol{\theta}=\mathbf{y} \longrightarrow \boldsymbol{\theta}=\Psi^{-1} \mathbf{y},
$$

donde $\Psi$ es la matriz de Gram. La Figura 3.4 muestra la predicción de la función $\mathscr{M}=(6 x-2)^{2} \sin \left((6 x-2)^{2}\right)$ (Forrester et al., 2008) utilizando superficies de respuesta polinómicas y funciones de base radial. 


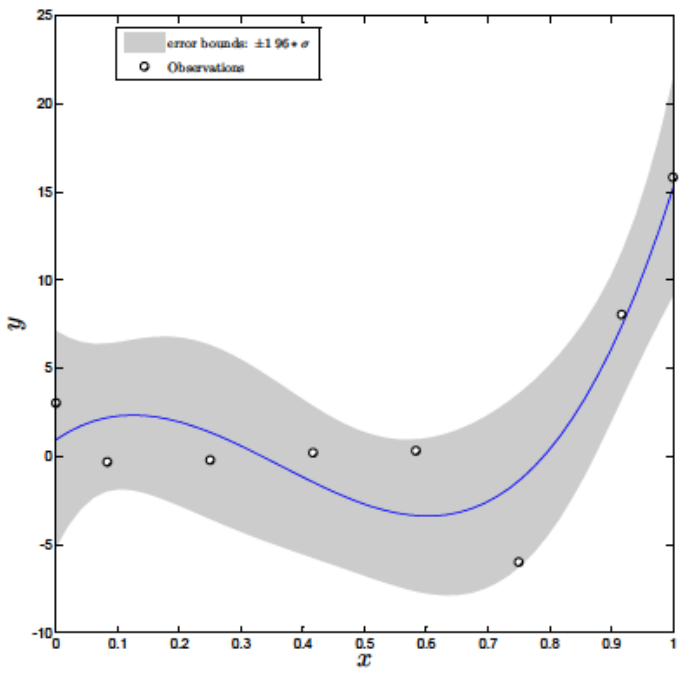

(a)

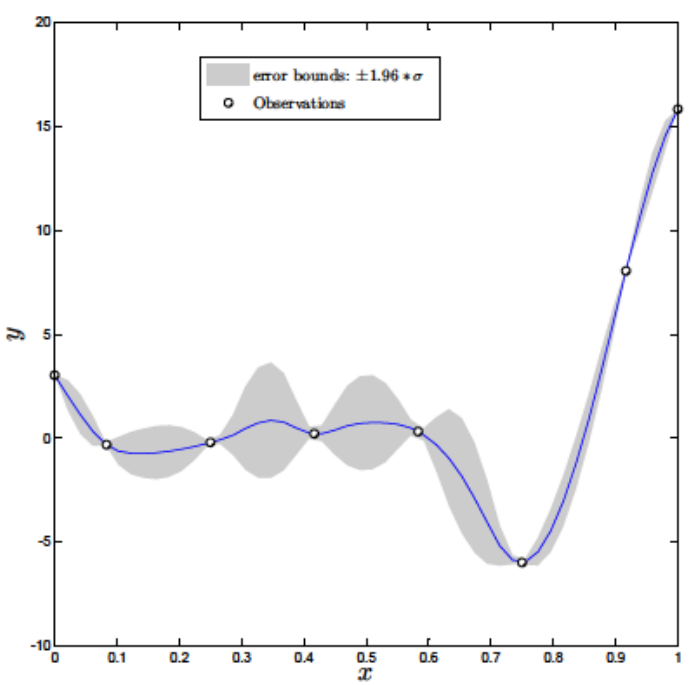

(b)

Figura 3.4: Meta-modelos de la funcion $\mathscr{M}=(6 x-2)^{2} \sin \left((6 x-2)^{2}\right)$ (Forrester et al., 2008). (a) Superficie polinómica de orden 3; (b) función de base radial multicuadrática. La línea azul continua representa el valor de la predicción. El área gris representa un intervalo de confianza de la predicción del $95 \%$.

\subsection{Definiciones previas}

El objetivo de esta sección es presentar los fundamentos matemáticos imprescindibles así como establecer la notación que será utilizada a lo largo de todo este documento. La introducción que se realiza en esta sección está inspirada en las referencias Henk (2004) y Kolmogorov (1950).

\subsubsection{Espacio de probabilidad}

Un espacio de probabilidad o probabilístico sirve para modelizar un experimento aleatorio. Un espacio de probabilidad $(\Omega, \mathscr{F}, \mathscr{P})$ está integrado por tres componentes. En primer lugar, $\Omega$ es el conjunto de los posibles resultados del experimento, denominados sucesos elementales. En segundo lugar, $\mathscr{F}$ es la colección de todos los sucesos aleatorios, que es una sigma álgebra sobre $\Omega$. El $\operatorname{par}(\Omega, \mathscr{F})$ es un espacio medible. Por último $\mathscr{P}$ es lo que se conoce como medida de probabilidad o función de probabilidad, que es una aplicación $\mathbb{P}: \mathscr{F} \longmapsto[0,1]$ que asigna una probabilidad a todo suceso verificando los siguientes axiomas de Kolmogorov:

$$
\begin{aligned}
\mathbb{P}(A) & \geq 0 \quad \forall A \in \mathscr{F}, \\
\mathbb{P}(\Omega) & =1 \\
\mathbb{P}(A \cup B) & =\mathbb{P}(A)+\mathbb{P}(B) \quad \forall A, B \in \mathscr{F}, A \cap B=\emptyset .
\end{aligned}
$$

A partir de estos axiomas se verifica: 


$$
\begin{aligned}
\mathbb{P}(\emptyset) & =0 \\
\mathbb{P}(\bar{A}) & =1-\mathbb{P}(A), \\
\mathbb{P}(A \cup B) & =\mathbb{P}(A)+\mathbb{P}(B)-\mathbb{P}(A \cap B) \quad \forall A, B \in \mathscr{F} .
\end{aligned}
$$

\subsubsection{Interpretación frecuentista de la probabilidad}

El espacio de probabilidad es un modelo matemático que asigna a cada suceso un número real. Existen diferentes teorías que interpretan dicho modelo matemático y le dan un significado real. La interpretación más difundida es la frecuentista. La interpretación frecuentista entiende que la probabilidad de un suceso es el límite de la frecuencia relativa de aparición de dicho suceso cuando el experimento se repite indefinidamente.

Si realizamos un experimento un número de veces $n$, y obtenemos como resultado el evento $A$ un número de veces $n_{A}$, la frecuencia de aparición del suceso $A$ se define como:

$$
\operatorname{Freq}(A)=\frac{n_{A}}{n} .
$$

Cuando el número de realizaciones del experimento $(n)$ tiende a infinito, la frecuencia relativa de obtención del evento $A$ tiende a la probabilidad de $A$ :

$$
\mathbb{P}(A)=\lim _{n \rightarrow \infty} \frac{n_{A}}{n} .
$$

\subsubsection{Probabilidad condicionada. Teorema de Bayes}

La probabilidad de un evento $A$ condicionada a un evento $B$ se define como:

$$
\mathbb{P}(A \mid B)=\frac{\mathbb{P}(A \cap B)}{\mathbb{P}(B)} .
$$

Cuando la probabilidad de $B$ no afecta a la probabilidad de $A$, dichos eventos se denominan independientes, y $\mathbb{P}(A \mid B)=\mathbb{P}(A)$. Dos eventos independientes verifican:

$$
\mathbb{P}(A \cap B)=\mathbb{P}(A) \mathbb{P}(B) .
$$

Teorema 1 (Teorema de Bayes (Bayes, 1763).) Sea $\left\{A_{1}, \ldots, A_{i}, \ldots, A_{n}\right\}$ un conjunto de sucesos mutuamente excluyentes y exhaustivos, tales que la probabilidad de cada uno de ellos es distinta de cero. Sea B un suceso cualquiera del que se conocen las probabilidades condicionadas $\mathbb{P}\left(B \mid A_{i}\right)$. Entonces, la probabilidad $\mathbb{P}\left(A_{i} \mid B\right)$ viene dada por la expresión:

$$
\mathbb{P}\left(A_{i} \mid B\right)=\frac{\mathbb{P}\left(B \mid A_{i}\right) \mathbb{P}\left(A_{i}\right)}{\mathbb{P}(B)},
$$


donde:

- $\mathbb{P}\left(A_{i}\right)$ son las probabilidades a priori.

- $\mathbb{P}\left(B \mid A_{i}\right)$ es la probabilidad de $B$ dado $A_{i}$.

- $\mathbb{P}\left(A_{i} \mid B\right)$ son las probabilidades a posteriori.

El teorema de Bayes es de gran relevancia puesto que vincula la probabilidad de $A$ dado $B$ con la probabilidad de $B$ dado $A$, y es la base de la estadística Bayesiana utilizada a lo largo del capítulo.

\subsubsection{Variable aleatoria}

Sea $(\Omega, \mathscr{F}, \mathscr{P})$ un espacio de probabilidad, una variable aleatoria $X$ es una aplicación $X: \Omega \longmapsto \mathscr{D}_{X}$. Si $\mathscr{D}_{X} \subset \mathbb{R}$ es un conjunto discreto, la variable aleatoria $X$ se denomina variable aleatoria discreta, en cualquier otro caso se denomina continua. Una variable aleatoria está totalmente definida por su función de distribución acumulada (cumulative distribution function $\mathrm{CDF}$ ) denotada por $F_{X}(x)$ :

$$
F_{X}(x)=\mathbb{P}(X \leq x)
$$

En el caso de una variable aleatoria discreta, el dominio de definición está representado por el conjunto $\mathscr{D}_{\mathrm{X}}=\left\{x^{(i)}, i \in \mathbb{N}\right\}$. La función de masas de probabilidad se define como:

$$
p_{i}=\mathbb{P}\left(X=x^{(i)}\right)
$$

Haciendo uso de (3.20), la función de distribución acumulada queda como:

$$
F_{X}(x)=\sum_{i \in \mathbb{N}} p_{i} \mathbf{1}_{\left\{x \geq x^{(i)\}}\right.}(x)
$$

donde $\mathbf{1}_{\left\{x \geq x^{(i)\}}\right.}(x)$ es una función cuyo valor es 1 si $x \geq x^{(i)}$ y 0 en el resto de casos. Para una variable aleatoria continua, la Función de Densidad de Probabilidad (FDP) se define como:

$$
f_{X}(x)=\lim _{h \rightarrow 0, h>0} \mathbb{P}(x \leq X \leq x+h) / h .
$$

El operador esperanza matemática se denota por $\mathbb{E}[\cdot]$. El valor esperado o media de una variable aleatoria $X$ se define como:

$$
\mu_{X}=\mathbb{E}[X]=\int_{\mathscr{D}_{X}} x f_{X}(x) d x
$$


El n-ésimo momento estadístico ordinario y central de una variable aleatoria $X$ se definen respectivamente como:

$$
\begin{aligned}
\mu_{n}^{\prime}=\mathbb{E}\left[X^{n}\right] & =\int_{\mathscr{D}_{X}} x^{n} f_{X}(x) d x, \\
\mu_{n}=\mathbb{E}\left[\left(X-\mu_{X}\right)^{n}\right] & =\int_{\mathscr{D}_{X}}\left(x-\mu_{X}\right)^{n} f_{X}(x) d x .
\end{aligned}
$$

La varianza, desviación estándar y coeficiente de variación de $X$ se definen como:

$$
\begin{aligned}
\operatorname{Var}[X] & =\mathbb{E}\left[\left(X-\mu_{X}\right)^{2}\right], \\
\sigma_{X} & =\sqrt{\operatorname{Var}[X]}, \\
\operatorname{COV}_{X} & =\frac{\sigma_{X}}{\mu_{X}} .
\end{aligned}
$$

Los momentos estadísticos centrados y normalizados de tercer/cuarto orden se denominan coeficiente de desviación/asimetría y se definen como:

$$
\begin{aligned}
& \delta_{X}=\frac{1}{\sigma_{X}{ }^{3}} \mathbb{E}\left[\left(X-\mu_{X}\right)^{3}\right], \\
& \mathcal{K}_{X}=\frac{1}{\sigma_{X}{ }^{4}} \mathbb{E}\left[\left(X-\mu_{X}\right)^{4}\right] .
\end{aligned}
$$

La covarianza entre dos variables aleatorias $X$ e $Y$ es:

$$
C[X, Y]=\mathbb{E}\left[\left(X-\mu_{X}\right)\left(Y-\mu_{Y}\right)\right] .
$$

El coeficiente de correlación de dos variables aleatorias se obtiene normalizando la covarianza de las dos variables entre las respectivas desviaciones estándar:

$$
\rho_{X, Y}=\frac{C[X, Y]}{\sigma_{X} \sigma_{Y}} .
$$

\subsubsection{Vector aleatorio}

Dado un espacio de probabilidad $(\Omega, \mathscr{F}, \mathscr{P})$. Un vector aleatorio $\mathbf{X}$ es una aplicación $\mathbf{X}: \Omega \longmapsto \mathscr{D}_{\mathbf{X}} \subset \mathbb{R}^{q}$, con $q \geq 2$ el tamaño del vector $\mathbf{X}$. $\mathbf{X}$ puede ser considerado como un conjunto de variables aleatorias: $\mathbf{X}=\left\{\mathrm{X}_{1}, \mathrm{X}_{2}, \ldots, \mathrm{X}_{\mathrm{q}}\right\}^{\mathrm{T}}$. La esperanza del vector aleatorio $\mathbf{X}$ se define como el vector del valor esperado de cada una de las variables aleatorias: 


$$
\mu_{\mathrm{X}}=\left(\mu_{X_{1}}, \mu_{X_{2}}, \ldots, \mu_{X_{q}}\right)^{T}
$$

Por otra parte $\mathbf{C}(\mathbf{R})$ es una matriz cuadrada simétrica que representa la matrix de covarianza (correlación) que se define como:

$$
\begin{array}{r}
\mathbf{C}_{i, j}=\operatorname{Cov}\left[X_{i}, X_{j}\right], \\
\mathbf{R}_{i, j}=\rho_{X_{i}, X_{j}} .
\end{array}
$$

La matriz de covarianza y la de correlación satisfacen la relación:

$$
\mathrm{C}=\boldsymbol{\Lambda} \mathbf{R} \Lambda
$$

donde $\Lambda$ es una matriz diagonal con las desviaciones estándar de cada componente de $\mathbf{X}$. El vector aleatorio $\mathbf{X}$ queda definido mediante su FDP conjunta. En el caso particular de vectores aleatorios Gaussianos dicha función viene dada por la expresión:

$$
f_{\mathbf{X}}(\mathbf{x})=(2 \pi)^{-q / 2}|\mathbf{C}|^{-1 / 2} \exp \left[-\frac{1}{2}\left(\mathbf{x}-\mu_{\mathbf{x}}\right)^{T} \cdot \mathbf{C}^{-1} \cdot\left(\mathbf{x}-\mu_{\mathbf{x}}\right)\right]
$$

\subsection{Procesos Gaussianos}

\subsubsection{Definición}

Dado un espacio de probabilidad $(\Omega, \mathscr{F}, \mathscr{P})$ y un parámetro contínuo $\mathbf{x} \in \mathbb{X}$, un proceso estocástico (o aleatorio) se define como una aplicación:

$$
Y(\mathbf{x}): \mid \begin{array}{ccc}
\mathbb{X} \times(\Omega, \mathscr{F}, \mathscr{P}) & \rightarrow & \mathbb{Y}, \\
(\mathbf{x}, \omega) & \rightarrow & y(\mathbf{x}),
\end{array}
$$

donde $y(\mathbf{x}) \equiv Y(\mathbf{x}, \omega)$ es una realización del proceso. Cuando $\mathbb{X}$ representa el tiempo, la aplicación se denomina proceso estocástico (3.39) y cuando es un conjunto abierto de $\mathbb{R}^{1,2,3}$ se denomina campo estocástico o aleatorio. Para un valor dado $\mathbf{x}_{0} \in \mathbb{X}, Y\left(\mathbf{x}_{0}, \omega\right)$ es una variable aleatoria en el espacio de probabilidad $(\Omega, \mathscr{F}, \mathscr{P})$. Por el contrario, para un valor dado $\omega_{0} \in \Omega, Y\left(\mathbf{x}, \omega_{0}\right)$ es una función de $\mathbf{x} \in \mathbb{X}$.

El proceso estocástico Gaussiano es el proceso estocástico más simple y de mayor aplicación práctica puesto que está totalmente caracterizado por sus dos primeros momentos estadísticos: su función media $\mu(\mathbf{x}) \equiv \mathbb{E}[Y(\mathbf{x})]$, y su función de covarianza $C\left(\mathbf{x}, \mathbf{x}^{\prime}\right) \equiv \mathbb{E}\left[(\mathrm{Y}(\mathbf{x})-\mu(\mathbf{x}))\left(\mathrm{Y}\left(\mathbf{x}^{\prime}\right)-\mu\left(\mathbf{x}^{\prime}\right)\right)\right]$. Dado $\mu(\mathbf{x})$ y $C\left(\mathbf{x}, \mathbf{x}^{\prime}\right)$ el proceso estocástico Gaussiano se denota por: 


$$
Y(\mathbf{x}) \sim \mathscr{G} \mathscr{P}\left(\mu(\mathbf{x}), C\left(\mathbf{x}, \mathbf{x}^{\prime}\right)\right), \quad\left(\mathbf{x}, \mathbf{x}^{\prime}\right) \in \mathbb{X} \times \mathbb{X} .
$$

Atendiendo al comportamiento del proceso estocástico al desplazarse sobre el espacio indexado $\mathbb{X}$, se pueden considerar las siguientes hipótesis:

- Proceso estocástico estacionario. Un proceso estocástico se dice estacionario si es invariante a la translación. Como consecuencia su función media es constante y su función de covarianza solo depende de la distancia entre dos valores (x, $\left.\mathbf{x}^{\prime}\right)$ (Purgachev, 1967).

- Proceso estocástico isotrópico. Un proceso estocástico se dice isotrópico si es invariante a la rotación. Como consecuencia un proceso estocástico isotrópico es también estacionario y su función de correlación se expresa como:

$$
\mathscr{R}\left(\mathbf{x}, \mathbf{x}^{\prime}\right)=\mathscr{R}\left(\left\|\mathbf{x}-\mathbf{x}^{\prime}\right\|_{2}\right), \quad \mathbf{x}, \mathbf{x}^{\prime} \in \mathbb{X} \times \mathbb{X},
$$

donde $\|\bullet\|$ denota la norma euclídea en $\mathbb{R}^{n}$. En el contexto del problema de creación de un meta-modelo, esta propiedad no se cumple debido al diferente significado físico de cada componente del vector $\mathbf{x}$, el cual en el caso de un problema estructural puede representar propiedades de diferente orden de magnitud tales como propiedades del material, geometría o cargas. Para solventar este problema se normalizan los datos de entrada mediante el valor medio $\mu_{X_{i}}$ y la desviación estándar $\sigma_{X_{i}}$ de cada componente del vector $\mathbf{x}$. Estos valores se obtienen a partir de la muestra del diseño de experimentos $\mathscr{X}=\left\{x^{i}, i=1, \ldots, m\right\}$. De esta forma el espacio indexado normalizado $\overline{\mathbb{X}}$ se define por:

$$
\left\{\bar{x}_{i}=\frac{x_{i}-\mu_{X_{i}}}{\sigma_{X_{i}}}\right\} \in \overline{\mathbb{X}} .
$$

- Proceso estocástico anisótropo por componentes. Un proceso estocástico se dice anisótropo por componentes si su función de correlación se define a partir del producto de las funciones de correlación unidimensionales:

$$
\mathscr{R}\left(\mathbf{x}, \mathbf{x}^{\prime}\right)=\prod_{i=1}^{n} \mathscr{R}_{i}\left(x_{i}-x_{i}^{\prime}\right), \quad \mathbf{x}, \mathbf{x}^{\prime} \in \mathbb{X} \times \mathbb{X} .
$$

Esta propiedad resulta de interés desde el punto de vista de la meta-modelización puesto que permite considerar en el meta-modelo la posibilidad de que la función que se intenta emular no tenga la misma 
dependencia respecto de todas las variables. Si uno de los componentes de $\mathbf{x}$ tiene una influencia escasa en la respuesta $y$, sería interesante que la función de correlación presentara un rango amplio que tienda a uniformizar la variación de la respuesta en dicho componente. Por otra parte, si la influencia de uno de los componentes de $\mathbf{x}$ es grande, interesaría una función de correlación cuyo rango sea corto que permita capturar fluctuaciones en dicho componente.

\subsubsection{Discretización del proceso estocástico}

Un proceso estocástico es un conjunto infinito de variables aleatorias indexadas en un espacio $\mathbb{X}$, lo que resulta inabordable desde el punto de vista computacional. La discretización del proceso estocástico $Y(\mathbf{x}, \omega)$ consiste en aproximar dicho proceso por un conjunto finito de variables aleatorias $\left\{\chi_{i}, i=1, \ldots, n\right\}$ contenidas en un vector aleatorio $\chi$ :

$$
Y(\mathbf{x}, \omega) \stackrel{\text { discretization }}{\longrightarrow} \hat{Y}(\mathbf{x}, \chi(\omega))
$$

Desde la década de los 80 se han desarrollado un gran número de métodos destinados a la resolución del problema de discretización. Una revisión y comparación de los mismo se presenta en los trabajos de Li y Kiureghian (1993) y Sudret y Kiureghian (2000). Los diferentes métodos se pueden clasificar en tres grupos: métodos de discretización puntual, métodos de discretización media y métodos de expansión en series. Los métodos basados en expansión de series resultan más eficientes en el sentido de que requieren de un menor número de variables para alcanzar una buena aproximación del proceso. Los métodos basados en expansión en series representan el proceso de forma exacta mediante una serie que se descompone en variables aleatorias y funciones deterministas dependientes de $\mathbf{x}$. La aproximación $\hat{Y}(\mathbf{x}, \mathcal{X}(\omega))$ se obtiene mediante el truncamiento de la serie. En este trabajo se adopta la expansión de Karhunen-Loève (KL) (Ghanem y Spanos, 2003; Loeve, 1977) como método de discretización de campos aleatorios utilizados para representar variaciones espaciales de imperfecciones geométricas o de propiedades del material. Por esta razón dicha expansión se describe brevemente a continuación.

Dado un proceso aleatorio $Y(\mathbf{x}, \omega)$ con media $\mu(\mathbf{x})$ y función de covarianza $C\left(\mathbf{x}, \mathbf{x}^{\prime}\right)=\sigma(\mathbf{x}) \sigma\left(\mathbf{x}^{\prime}\right) \mathscr{R}\left(\mathbf{x}, \mathbf{x}^{\prime}\right)$, la expansión de Karhunen-Loève del proceso $Y(\mathbf{x}, \omega)$ se define como:

$$
Y(\mathbf{x}, \omega)=\mu(\mathbf{x})+\sum_{i=1}^{\infty} \sqrt{\lambda_{i}} \xi_{i}(\omega) \varphi_{i}(\mathbf{x}), \mathbf{x} \in \mathbb{X},
$$

donde $\left\{\xi_{i}, i \in \mathbb{N}^{+*}\right\}$ es un conjunto infinito de variables aleatorias de media cero y desviación unitaria no correlacionadas. $\left\{\left(\lambda_{i}, \varphi_{i}\right), i \in \mathbb{N}^{+*}\right\}$ son un conjunto infinito 
de autovalores y autovectores solución de la ecuación integral de Fredholm de segundo orden:

$$
\int_{\mathbb{X}} C\left(\mathbf{x}, \mathbf{x}^{\prime}\right) \varphi_{i}\left(\mathbf{x}^{\prime}\right) d \mathbf{x}^{\prime}=\lambda_{i} \varphi_{i}\left(\mathbf{x}^{\prime}\right), i \in \mathbb{N}^{+*}, \mathbf{x} \in \mathbb{X} .
$$

En (3.46) $C\left(\bullet, \bullet^{\prime}\right)$ representa la función de covarianza acotada, simétrica y definida positiva, y por lo tanto el conjunto de autofunciones $\left\{\varphi_{i}, i \in \mathbb{N}^{+*}\right\}$ forma una base ortogonal completa.

En la práctica la expansión de Karhunen-Loève de la ecuación (3.45) se representa en función de los autovalores de mayor orden. Esto se realiza truncando la expansión en el término de orden $M$ :

$$
\hat{Y}(\mathbf{x}, \omega)=\mu(\mathbf{x})+\sum_{i=1}^{M} \sqrt{\lambda_{i}} \xi_{i}(\omega) \varphi_{i}(\mathbf{x}), \mathbf{x} \in \mathbb{X},
$$

donde los autovalores se ordenan en orden descendente $\lambda_{1}>\lambda_{2}>\ldots>\lambda_{M-1}>$ $\lambda_{M}>0$. Cada autovalor considerado en la expansión contribuye a la varianza total del proceso estocástico, que es la traza de la matriz de covarianza. Los autovalores de mayor valor tienen una contribución mayor a la varianza total del proceso, lo que posibilita truncar la serie en el término $M$ despreciando los autovalores de menor orden de magnitud. La calidad de la aproximación se expresa mediante la fracción de variabilidad:

$$
Q=\frac{\sum_{i=1}^{M} \lambda_{i}}{\operatorname{traza}(\mathbf{C})}: 0 \leq Q \leq 1 .
$$

El número $M$ de variables aleatorias consideradas en la expansión se ajusta para garantizar una calidad suficiente (ej. $Q>0.95$ ). De este modo es posible una reducción considerable de la dimensión del problema y posibilita su utilización en procesos de propagación de incertidumbre junto con modelos de simulación de alto coste computacional. La base formada por las autofunciones $\varphi_{i}(\mathbf{x})$ es óptima en relación a cualquier otra base puesto que minimiza el error cuadrático medio que resulta al truncar la serie en el término de orden $M$.

Cuando el proceso estocástico es Gaussiano se demuestra (Loeve, 1977) que la expansión truncada de Karhunen-Loève es convergente y $\left\{\xi_{i}\right\}$ es un conjunto de variables aleatorias normales independientes. Para procesos estocásticos no Gaussianos, la expansión KL existe pero las variables aleatorias que aparecen en la expansión pueden estar correlacionadas y su distribución es desconocida $a$ priori. En estos casos la FDP se puede identificar a partir de datos experimentales tal y como muestran trabajos como los de Ghanem y Doostan (2006) y Desceliers et al. (2007). 


\subsubsection{Ejemplos de funciones de correlación estacionarias}

Para estudiar el efecto del tipo de función de correlación sobre la realización del proceso estocástico Gaussiano, se presentan en esta sección una selección de las funciones de correlación utilizadas habitualmente. Las características de dichas funciones de correlación se ilustran mediante la generación de una realización de un proceso estocástico Gaussiano con media cero y varianza unitaria.

\subsubsection{Función de correlación lineal}

La función de correlación lineal se define como:

$$
\mathscr{R}\left(\mathbf{x}, \mathbf{x}^{\prime}, \boldsymbol{\theta}\right)=\prod_{i=1}^{n} \max \left(0,1-\theta_{i}\left|x_{i}-x_{i}^{\prime}\right|\right),
$$

donde $\left\{\theta_{i}>0, \forall i=1, \ldots, n\right\}$ son los parámetros de escala.

\subsubsection{Función de correlación exponencial}

La función de correlación exponencial se define como:

$$
\mathscr{R}\left(\mathbf{x}, \mathbf{x}^{\prime}, \boldsymbol{\theta}\right)=\exp \left(-\sum_{i=1}^{n} \theta_{i}\left|x_{i}-x_{i}^{\prime}\right|\right),
$$

donde $\left\{\theta_{i}>0, \forall i=1, \ldots, n\right\}$ son los parámetros de escala.

\subsubsection{Función de correlación exponencial cuadrática o Gaussiana}

La función de correlación exponencial cuadrática o Gaussiana se define como:

$$
\mathscr{R}\left(\mathbf{x}, \mathbf{x}^{\prime}, \boldsymbol{\theta}\right)=\exp \left(-\sum_{i=1}^{n} \theta_{i}\left|x_{i}-x_{i}^{\prime}\right|^{2}\right),
$$

donde $\left\{\theta_{i}>0, \forall i=1, \ldots, n\right\}$ son los parámetros de escala.

\subsubsection{Función de correlación exponencial-Gaussiana}

La función de correlación exponencial Gaussiana se define como:

$$
\mathscr{R}\left(\mathbf{x}, \mathbf{x}^{\prime}, \boldsymbol{\theta}, \theta_{n+1}\right)=\exp \left(-\sum_{i=1}^{n} \theta_{i}\left|x_{i}-x_{i}^{\prime}\right|^{\theta_{n+1}}\right),
$$

donde $\left\{\theta_{i}>0, \forall i=1, \ldots, n\right\}$ son los parámetros de escala, y $0<\theta_{n+1} \leq 2$ el parámetro de forma. El parámetro de forma controla el comportamiento cerca del origen $\left(\left|x_{i}-x_{i}^{\prime}\right|=0\right)$. Un valor de $\theta_{n+1}=2$ equivale a una función de correlación exponencial cuadrática con un comportamiento parabólico cerca del origen. Un valor de $\theta_{n+1}=1$ resulta en una función de correlación lineal con dicho comportamiento cerca del origen. 


\subsubsection{Función de correlación esférica}

La función de correlación esférica se define como:

$$
\begin{aligned}
\mathscr{R}\left(\mathbf{x}, \mathbf{x}^{\prime}, \boldsymbol{\theta}\right) & =\prod_{i=1}^{n} 1-1.5 \eta_{i}+0.5 \eta_{i}^{3}, \\
\eta_{i} & =\min \left\{1, \theta_{i} \mid x_{i}-x_{i}^{\prime}\right\},
\end{aligned}
$$

donde $\left\{\theta_{i}>0, \forall i=1, \ldots, n\right\}$ son los parámetros de escala.

\subsubsection{Función de correlación cúbica}

La función de correlación cúbica se define como:

$$
\begin{aligned}
\mathscr{R}\left(\mathbf{x}, \mathbf{x}^{\prime}, \boldsymbol{\theta}\right) & =\prod_{i=1}^{n} 1-3 \eta_{i}^{2}+2 \eta_{i}^{3}, \\
\eta_{i} & =\min \left\{1, \theta_{i}\left|x_{i}-x_{i}^{\prime}\right|\right\},
\end{aligned}
$$

donde $\left\{\theta_{i}>0, \forall i=1, \ldots, n\right\}$ son los parámetros de escala.

\subsubsection{Función de correlación spline}

La función de correlación spline se define como:

$$
\begin{array}{ll}
\mathscr{R}\left(\mathbf{x}, \mathbf{x}^{\prime}, \boldsymbol{\theta}\right)=\prod_{i=1}^{n} \varsigma\left(\eta_{i}\right), & \\
\varsigma\left(\eta_{i}\right)=\left\{\begin{array}{cl}
1-15 \eta_{i}^{2}+30 \eta_{i}^{3} & \forall 0 \leq \eta_{i} \leq 0.2, \\
1.25\left(1-\eta_{i}\right)^{3} & \forall 0.2<\eta_{i}<1, \\
0 & \forall \eta_{i} \geq 1,
\end{array}\right.
\end{array}
$$

donde $\eta_{i}=\theta_{i}\left|x_{i}-x_{i}^{\prime}\right|$ y $\left\{\theta_{i}>0, \forall i=1, \ldots, n\right\}$ son los parámetros de escala.

La Figura 3.5 representa gráficamente las funciones de correlación mostradas anteriormente para diferentes valores del parámetro de escala. En todos los casos la correlación decrece al aumentar la distancia $\left|x_{i}-x_{i}^{\prime}\right|$. El parámetro $\theta_{i}$ muestra el radio de influencia en el espacio de un punto $x_{i}$. Un valor bajo de $\theta_{i}$ significa que todos los puntos tienen una alta correlación y la respuesta es similar en todos los puntos del espacio. Un valor elevado de $\theta_{i}$ significa que existen diferencias significativas de la respuesta en el espacio. En el caso de problemas multidimensionales los elementos del vector $\boldsymbol{\theta}$ son un indicador de las dimensiones con mayor influencia en la respuesta.

Las funciones de correlación mostradas en la Figura 3.5 se pueden clasificar en dos grupos: aquellas con un comportamiento lineal en el origen (lineal, exponencial, esférica) y aquellas que presentan un comportamiento parabólico cerca del origen (exponencial cuadrática, cúbica, spline). La función exponencial-Gaussiana puede presentar ambos comportamientos dependiendo 

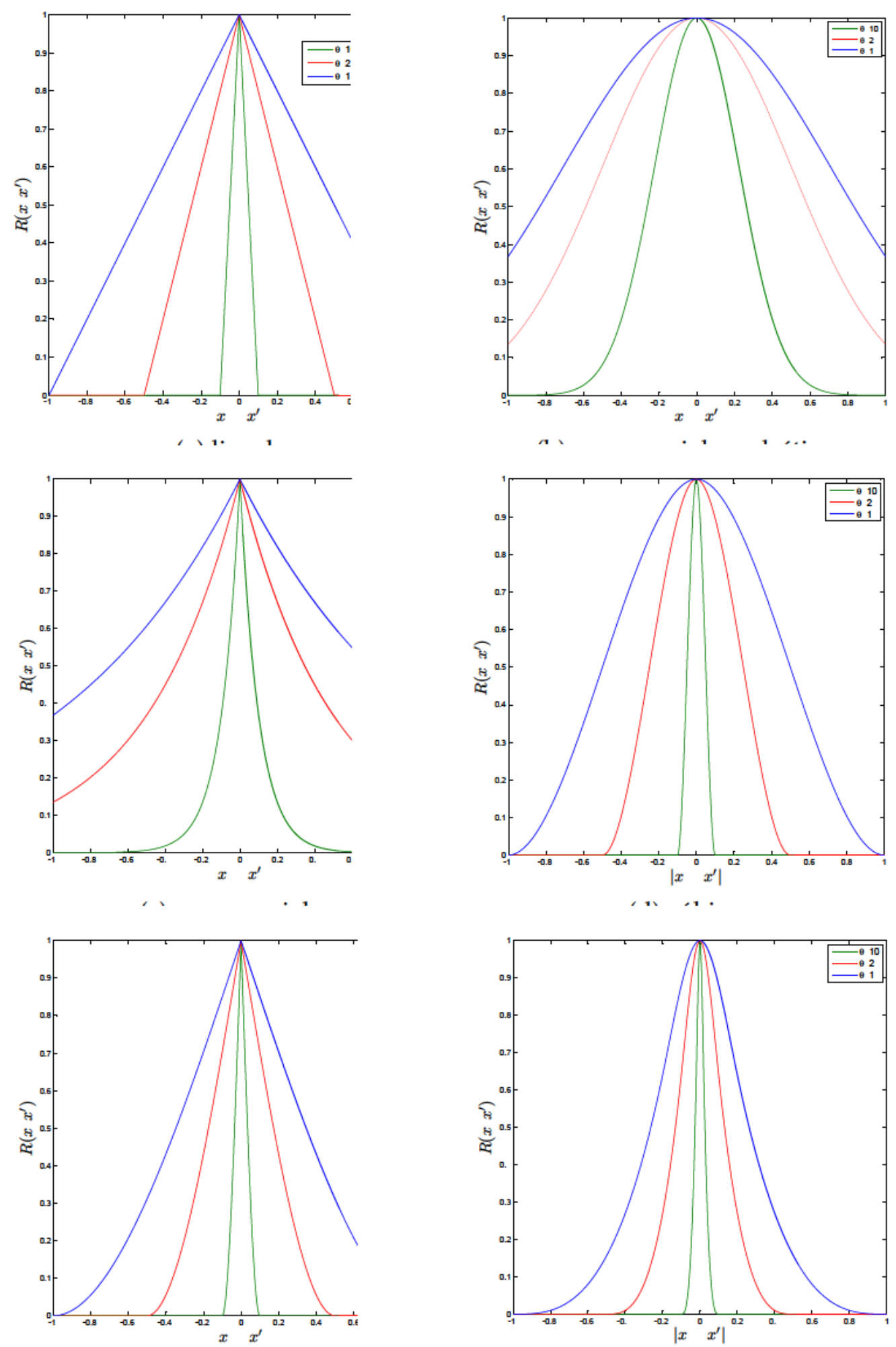

(e) esférica

(f) spline

Figura 3.5: funciones de correlación para $0 \leq\left|x_{i}-x_{i}^{\prime}\right| \leq 1$. 
del parámetro de forma $\theta_{n+1}$. Si $\theta_{n+1}=1$ se obtiene la función exponencial, mientras que si $\theta_{n+1}=2$ se obtiene la exponencial cuadrática. Las funciones de correlación del tipo lineal, exponencial y esférica son de clase $\mathscr{C}^{0}$ (continuas pero no diferenciables). Por el contrario, las funciones de correlación exponencial cuadrática, cúbica y spline son del tipo $\mathscr{C}^{\infty}$ por lo que permiten generar realizaciones de procesos estocásticos infinitamente diferenciables. La Figura 3.6 muestra las diferencias en la diferenciabilidad de dos realizaciones de un proceso estocástico estacionario con media cero, utilizando funciones de correlación exponencial y exponencial cuadrática.

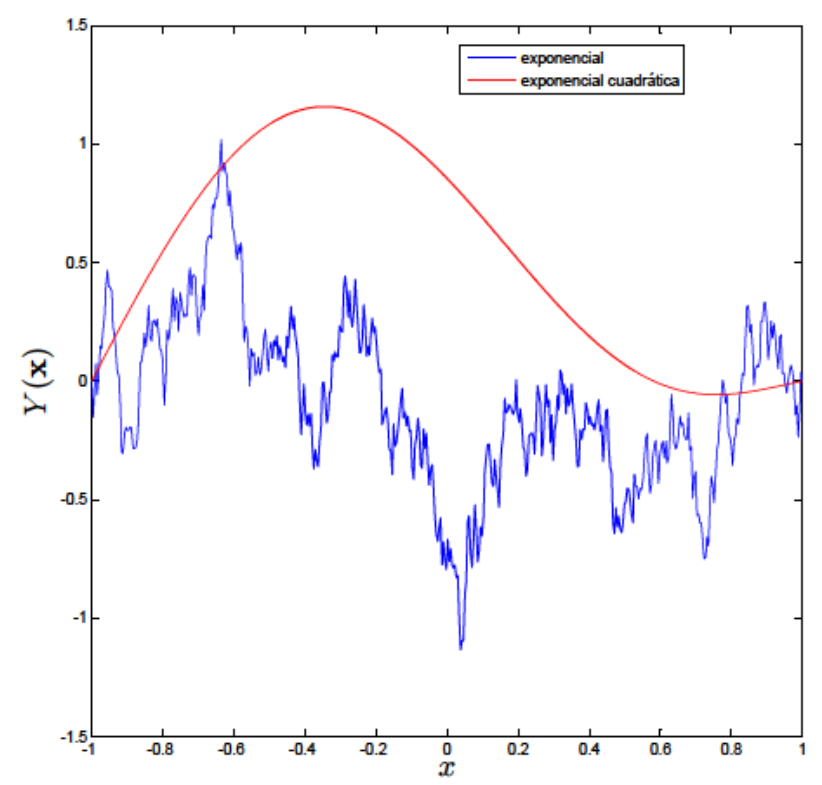

Figura 3.6: Realización de un proceso estocástico estacionario con media cero y funciones de correlación exponencial (línea azul) y exponencial cuadrática (línea roja).

\subsection{Predicción con procesos Gaussianos de regresión}

En esta sección se presenta los fundamentos matemáticos del modelo de predicción utilizado en esta tesis. Estos fundamentos se presentan en cuatro etapas: (1) modelo de regresión por mínimos cuadrados generalizada, (2) modelo de regresión basado en Procesos Gaussianos, (3) modelo de la Predicción Lineal Insesgada Óptima (PLIO) y (4) modelo de Predicción Lineal Insesgada Empírica Optima (PLIEO).

\subsubsection{Modelo de regresión por mínimos cuadrados generalizada}

El modelo de regresión por mínimos cuadrados es uno de los más utilizados en la práctica debido a su simplicidad conceptual y su eficiencia. La resolución del problema de mínimos cuadrados es parte fundamental dentro de problemas más 
amplios como el problema de cuantificación de incertidumbre con meta-modelos, en el que se utilizan superficies de respuesta polinómicas (Box y Wilson, 1951) o meta-modelos estocásticos como polinomios del caos (Blatman y Sudret, 2010; Ghanem y Spanos, 2003; Maitre y Knio, 2010) para emular el modelo de simulación. Así mismo juegan un papel fundamental en la teoría de aprendizaje estadístico (Rasmussen y Williams, 2006). El problema de regresión por mínimos cuadrados generalizada consiste en minimizar la distancia entre la predicción del modelo y las medidas reales, y se formula como sigue:

$$
Y_{i}=\sum_{j=1}^{p} \beta_{j} f_{j}\left(\mathbf{x}^{(i)}\right)+Z_{i}, \quad i=1, \ldots, m
$$

donde $\mathbf{Y}=\left\{Y_{1}, Y_{2}, \ldots, Y_{m}\right\}^{T}$ es un vector de observaciones, $\boldsymbol{\beta}=\left\{\beta_{1}, \beta_{1}, \ldots, \beta_{p}\right\}^{T}$ es un vector de pesos, $f=f_{1}, f_{2}, \ldots, f_{p}$ es un conjunto de funciones de regresión, y $\mathbf{Z}=$ $\left\{Z_{1}, Z_{2}, \ldots, Z_{m}\right\}^{T}$ es un vector aleatorio Gaussiano cuyos momentos estadísticos de primer y segundo orden son:

$$
\begin{array}{r}
\mathbb{E}[\mathbf{Z}]=0, \\
\operatorname{COV}[\mathbf{Z}, \mathbf{Z}]=\sigma^{2} \mathbf{R},
\end{array}
$$

donde $\sigma^{2} \in \mathbb{R}$ es la varianza y $\mathbf{R} \in \mathbb{R}^{\mathrm{m} \times \mathrm{m}}$ es la matriz de correlación que se asume conocida. Si la matriz de correlación es la identidad resulta el problema de mínimos cuadrados ordinario (ordinary least-squares problem OLS), mientras que para un caso más general donde la matriz de correlación sea una matriz simétrica definida positiva se obtiene el problema de mínimos cuadrados generalizado. Debido al modelo de regresión lineal, el vector de observaciones $\mathbf{Y}$ es un vector aleatorio que sigue una distribución normal con media $\mathbf{F} \boldsymbol{\beta}$ y varianza $\sigma^{2} \mathbf{R}$, donde $\mathbf{F}$ es la matriz de regresión cuyos términos se definen como $F_{i j}=f_{j}\left(x^{(i)}\right), i=1, \ldots, m, j=1, \ldots, p$.

El problema de regresión consiste en encontrar el valor óptimo de los parámetros $\boldsymbol{\beta}^{*} \in \mathbb{R}^{p}$ y $\sigma^{* 2} \in \mathbb{R}^{+}$, solución del problema de minimización:

$$
\left\{\boldsymbol{\beta}^{*}, \sigma^{2^{*}}\right\}=\arg \min _{\boldsymbol{\beta}, \sigma^{2}}-\log \mathrm{L}\left(\mathbf{y} \mid \boldsymbol{\beta}, \sigma^{2}\right),
$$

donde $\left\{\boldsymbol{\beta}^{*}, \sigma^{* 2}\right\}$ es la estimación de máxima verosimilitud (maximum likelihood estimation) y $\mathrm{L}\left(\mathbf{y} \mid \boldsymbol{\beta}, \sigma^{2}\right)$ es la función de densidad de probabilidad del vector aleatorio $\mathbf{Y}$, donde :

$$
\mathrm{L}\left(\mathbf{y} \mid \boldsymbol{\beta}, \sigma^{2}\right)=\frac{1}{\left(\left(2 \pi \sigma^{2}\right)^{m}|\mathbf{R}|\right)^{1 / 2}} \exp \left[-\frac{1}{2 \sigma^{2}}(\mathbf{y}-\mathbf{F} \boldsymbol{\beta})^{\mathrm{T}} \mathbf{R}^{-1}(\mathbf{y}-\mathbf{F} \boldsymbol{\beta})\right] .
$$


La solución del problema de optimización sin restricciones definido en la ecuación (3.58) se resuelve mediante la utilización de las condiciones de optimalidad de primer orden:

$$
\left\{\begin{array}{lcl}
\nabla_{\beta^{*}} \mathrm{~L}\left(\mathbf{y} \mid \boldsymbol{\beta}^{*}, \sigma^{2}\right) & =\frac{1}{\sigma^{2}}\left(\mathbf{F}^{\mathrm{T}} \mathbf{R}^{-1} \mathbf{y}-\mathbf{F}^{\mathrm{T}} \mathbf{R}^{-1} \mathbf{F} \boldsymbol{\beta}^{*}\right)=\mathbf{0}, \\
\frac{\partial \mathrm{L}\left(\mathbf{y} \boldsymbol{\beta}, \sigma^{*}\right)}{\partial \sigma^{2^{*}}} & =-\frac{m}{2} \frac{1}{\sigma^{2}}+\frac{1}{2 \sigma^{*}}(\mathbf{y}-\mathbf{F} \boldsymbol{\beta})^{\mathrm{T}} \mathbf{R}^{-1}(\mathbf{y}-\mathbf{F} \boldsymbol{\beta})=0 .
\end{array}\right.
$$

La estimación de mínimos cuadrados generalizada viene dada por la resolución del sistema de ecuaciones (3.60):

$$
\begin{cases}\boldsymbol{\beta}^{*} & =\left(\mathbf{F}^{\mathrm{T}} \mathbf{R}^{-1} \mathbf{F}\right)^{-1}\left(\mathbf{F}^{\mathrm{T}} \mathbf{R}^{-1} \mathbf{y}\right) \\ \sigma^{2^{*}} & =\frac{1}{m}\left(\mathbf{y}-\mathbf{F} \boldsymbol{\beta}^{*}\right)^{\mathrm{T}} \mathbf{R}^{-1}\left(\mathbf{y}-\mathbf{F} \boldsymbol{\beta}^{*}\right) .\end{cases}
$$

La solución del problema ordinario de mínimos cuadrados $\left\{\boldsymbol{\beta}_{\text {ord }}{ }^{*}, \sigma_{\text {ord }}^{2}{ }^{*}\right\}$ es una solución particular de la ecuación (3.61) cuando la matriz de correlación es la identidad:

$$
\left\{\begin{array}{l}
\boldsymbol{\beta}_{\text {ord }}{ }^{*}=\left(\mathbf{F}^{\mathrm{T}} \mathbf{F}\right)^{-1}\left(\mathbf{F}^{\mathrm{T}} \mathbf{y}\right) \\
\sigma_{\text {ord }}^{2}=\frac{1}{m}\left(\mathbf{y}-\mathbf{F} \boldsymbol{\beta}^{*}\right)^{\mathrm{T}}\left(\mathbf{y}-\mathbf{F} \boldsymbol{\beta}_{\text {ord }}{ }^{*}\right) .
\end{array}\right.
$$

\subsubsection{Modelo de regresión basado en Procesos Gaussianos}

La regresión basada en procesos Gaussianos asume que la función $\mathscr{M}$ que relaciona la entrada $\mathbf{x}$ con la salida $y$ del modelo de simulación es una realización de un proceso estocástico Gaussiano condicionado, cuya expresión viene dada por la contribución de dos términos:

$$
Y=\underbrace{\sum_{j=1}^{p} \beta_{j} f_{j}(\mathbf{x})}+\underbrace{Z(\mathbf{x})}_{(2)} .
$$

(1)

El término (1) es un modelo de regresión lineal, combinación de una base funcional $f=f_{1}, f_{2}, \ldots, f_{p}$ y de unos pesos $\beta=\left\{\beta_{1}, \beta_{1}, \ldots, \beta_{p}\right\}^{T}$. El término (2) es un proceso estocástico Gaussiano totalmente caracterizado por una media:

$$
\mathbb{E}[Z(\mathbf{x})]=0, \quad \forall \mathbf{x} \in \mathbb{X}
$$

y una función de covarianza:

$$
\operatorname{Cov}\left[Z(\mathbf{x}), Z\left(\mathbf{x}^{\prime}\right)\right]=\sigma^{2} \mathscr{R}\left(\mathbf{x}, \mathbf{x}^{\prime}, \boldsymbol{\theta}\right),
$$

donde $\sigma^{2}$ denota la varianza del proceso Gaussiano y $\mathscr{R}$ es la función de correlación estacionaria (sección 3.3.3) que en el caso de procesos Gaussianos solo depende de la diferencia $\mathbf{x}-\mathbf{x}^{\prime}$ y de un vector de hiperparámetros $\boldsymbol{\theta}$. El 
problema de regresión utilizando procesos Gaussianos es una extensión del problema regresión por mínimos cuadrados generalizado con la diferencia de que el término (2) de la expresión (3.63) es un proceso estocástico Gaussiano función de $\mathbf{x}$ en lugar de un vector aleatorio Gaussiano.

Gracias a la hipótesis de Gaussianidad del término (2) de la ecuación (3.63) y la linealidad del termino (1) de esa misma ecuación, un vector $\mathbf{Y}=$ $\left\{\mathscr{M}\left(\mathbf{x}^{(1)}\right), \mathscr{M}\left(\mathbf{x}^{(1)}\right), \mathscr{M}\left(\mathbf{x}^{(2)}\right), \ldots, \mathscr{M}\left(\mathbf{x}^{(m)}\right)\right\}^{T}$ de observaciones, es una realización de un vector aleatorio que sigue una distribución normal:

$$
\mathbf{Y} \sim \mathscr{N}_{m}\left(\mathbf{F} \beta, \sigma^{2} \mathbf{R}\right)
$$

donde $\mathbf{F}$ es la matriz de regresión cuyos términos se definen como $F_{i j}=f_{j}\left(\mathbf{x}^{(i)}\right), i=$ $1, \ldots, m, j=1, \ldots, p, \mathrm{y} \mathbf{R}$ es la matriz de correlación cuyos términos se definen como $R_{i j}=\mathscr{R}\left(\mathbf{x}^{(i)}-\mathbf{x}^{(j)}, \boldsymbol{\theta}\right)$.

\subsubsection{Predicción lineal insesgada óptima (Best Linear Unbiased Prediction ): Universal Kriging}

A partir del modelo de regresión con procesos Gaussianos presentado en la sección 3.4.2, en esta sección se obtiene la expresión de la PLIO de una respuesta desconocida $Y_{0}$. Esta predicción es también conocida en la literatura como predicción Kriging o Universal Kriging en honor del ingeniero de minas Daniel G. Krige (Krige, 1951) pionero en el campo de la geoestadística, quien fue el primero en proponer esta metodología. El término Kriging fue acuñado por el ingeniero francés Georges Matheron (Matheron, 1962) quien formalizó dicha técnica basada en la labor investigadora previa de Krige.

Por definición la PLIO $\hat{Y}\left(\mathbf{x}^{(0)}\right)$ de $Y_{0} \equiv Y\left(\mathbf{x}^{(0)}\right)$ tiene las siguientes tres propiedades:

- Linealidad. Puede obtenerse a partir de la combinación lineal de $m$ observaciones $\mathbf{Y}$ mediante el vector de pesos $\mathbf{a}_{0} \equiv \mathbf{a}\left(\mathbf{x}^{(0)}\right) \in \mathbb{R}^{\mathrm{m}}$ :

$$
\hat{Y}\left(\mathbf{x}^{(0)}\right)=\mathbf{a}_{0}^{T} \mathbf{Y}
$$

- Insesgabilidad.

$$
\mathbb{E}\left[\hat{Y}_{0}-Y_{0}\right]=0
$$

- Optimalidad. Es óptima en el sentido de que minimiza el error cuadrático medio:

$$
\hat{Y}_{0}=\arg \min _{Y_{0}^{*}} \mathbb{E}\left[\left(Y_{0}^{*}-Y_{0}\right)^{2}\right]
$$


donde $Y_{0}^{*}$ representa una predicción lineal insesgada.

El problema consiste en encontrar el vector de pesos óptimo $\mathbf{a}_{0}^{*}$ que satisface las tres condiciones anteriores:

$$
\begin{aligned}
& \mathbf{a}_{0}^{*} \quad=\arg \min _{\mathbf{a}_{0}} \mathbb{E}\left[\left(\mathbf{a}_{0}^{T} \boldsymbol{Y}-Y_{0}\right)^{2}\right], \\
& \text { s.a. } \mathbb{E}\left[\mathbf{a}_{0}^{T} \boldsymbol{Y}-Y_{0}\right]=0 \text {. }
\end{aligned}
$$

Considerando el modelo de la ecuación (3.63), la diferencia entre $Y_{0}$ y su predicción lineal $\hat{Y}_{0}$ se reduce a:

$$
\begin{aligned}
\hat{Y}_{0}-Y_{0} & =\mathbf{a}_{0}^{T} \boldsymbol{Y}-Y_{0} \\
& =\mathbf{a}_{0}^{T}(\boldsymbol{F} \boldsymbol{\beta}+\boldsymbol{Z})-\left(\boldsymbol{f}_{0}^{T} \boldsymbol{\beta}+Z_{0}\right) \\
& =\mathbf{a}_{0}^{T} \boldsymbol{Z}-Z_{0}+\left(\mathbf{a}_{0}^{T} \boldsymbol{F}-\boldsymbol{f}_{0}^{T}\right) \boldsymbol{\beta} .
\end{aligned}
$$

Haciendo uso de (3.73) la condición de predicción insesgada queda como sigue:

$$
\begin{aligned}
\mathbb{E}\left[\hat{Y}_{0}-Y_{0}\right]= & \mathbb{E}\left[\mathbf{a}_{0}^{T} \boldsymbol{Z}-Z_{0}+\left(\mathbf{a}_{0}^{T} \boldsymbol{F}-\boldsymbol{f}_{0}^{T}\right) \boldsymbol{\beta}\right] \\
= & \underbrace{\mathbb{E}\left[\mathbf{a}_{0}^{T} \boldsymbol{Z}-Z_{0}\right]}_{=0(3.64)}+\left(\mathbf{a}_{0}^{T} \boldsymbol{F}-\boldsymbol{f}_{0}^{T}\right) \boldsymbol{\beta} \\
\Downarrow & \\
& \left(\mathbf{a}_{0}^{T} \boldsymbol{F}-\boldsymbol{f}_{0}^{T}\right)=0 .
\end{aligned}
$$

Utilizando (3.73) y (3.77) la predicción del error cuadrático medio quedaría como sigue:

$$
\begin{aligned}
& \mathbb{E}\left[\left(\hat{Y}_{0}-Y_{0}\right)^{2}\right]=\mathbb{E}\left[\left(\mathbf{a}_{0}^{T} \boldsymbol{Z}-Z_{0}\right)^{2}\right]
\end{aligned}
$$

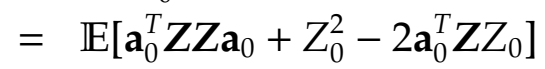

$$
\begin{aligned}
& =\mathbf{a}_{0}^{T} \mathbb{E}[Z Z] \mathbf{a}_{0}+\mathbb{E}\left[Z_{0}^{2}\right]-2 \mathbf{a}_{0}^{T} \mathbb{E}\left[Z Z_{0}\right] \\
& =\mathbf{a}_{0}^{T} \underbrace{\mathbb{E}[\mathbf{Z Z}]}_{\equiv \sigma^{2} \mathbf{R}} \mathbf{a}_{0}+\underbrace{\mathbb{E}\left[Z_{0}^{2}\right]}_{\equiv \sigma^{2}}-2 \mathbf{a}_{0}^{T} \underbrace{\mathbb{E}\left[\mathbf{Z Z} Z_{0}\right]}_{\equiv \sigma^{2} r_{0}} \\
& =\mathbf{a}_{0}^{T} \sigma^{2} \mathbf{R} \mathbf{a}_{0}+\sigma^{2}-2 \mathbf{a}_{0}^{T} \sigma^{2} r_{0} \\
& =\sigma^{2}\left(1+\mathbf{a}_{0}^{T}\left(\mathbf{R} \mathbf{a}_{0}-2 \mathbf{r}_{0}\right)\right) \text {. }
\end{aligned}
$$

El problema de optimización con restricción de igualdad de la ecuación (3.70), tras sustituir (3.77) y (3.83), queda como sigue:

$$
\begin{aligned}
& \mathbf{a}_{0}^{*}=\arg \operatorname{mí}_{\mathbf{a}_{0}} \sigma^{2}\left(1+\mathbf{a}_{0}^{T}\left(\mathbf{R a}_{0}-2 \mathbf{r}_{0}\right)\right), \\
& \text { s.a. } \quad \mathbf{a}_{0}^{T} \boldsymbol{F}-\boldsymbol{f}_{0}^{T}=0 .
\end{aligned}
$$


Esta restricción de igualdad se incorpora a la función objetivo mediante el método aumentado de Lagrange (Belegundu y Chandrupatla, 2011) para construir la función objetivo aumentada:

$$
L\left(\boldsymbol{a}_{0}, \boldsymbol{\lambda}_{0}\right)=\sigma^{2}\left(1+\boldsymbol{a}_{0}^{T}\left(\boldsymbol{R} \boldsymbol{a}_{0}-2 \boldsymbol{r}_{0}\right)\right)+\boldsymbol{\lambda}_{0} \mathbf{a}_{0}^{T} \boldsymbol{F}-\boldsymbol{f}_{0}^{T}
$$

El problema de optimización sin restricciones definido en la ecuación (3.85) se resuelve mediante la utilización de las condiciones de optimalidad de primer orden:

$$
\left\{\begin{array}{l}
\nabla_{\boldsymbol{a}_{0}} L=2 \sigma^{2}\left(\boldsymbol{R} \boldsymbol{a}_{0}-\boldsymbol{r}_{0}\right)+\boldsymbol{F} \overline{\boldsymbol{\lambda}}_{0}=0 \\
\nabla_{\lambda_{0}} L=\boldsymbol{F}^{T} \boldsymbol{a}_{0}-\boldsymbol{f}_{0}=0
\end{array}\right.
$$

cuyas incógnitas son $\bar{\lambda}_{0}=\frac{\lambda_{0}}{2 \sigma^{2}}$ y $\boldsymbol{a}_{0}$. Multiplicando la primera ecuación del sistema (3.86) por $-\boldsymbol{F}^{T} \boldsymbol{R}^{-1}$ y sumando a la segunda obtenemos una ecuación lineal cuya solución es:

$$
\overline{\boldsymbol{\lambda}}_{0}^{*}=\left(\boldsymbol{F}^{T} \boldsymbol{R}^{-1} \boldsymbol{F}\right)^{-1}\left(\boldsymbol{F}^{T} \boldsymbol{R}^{-1} \boldsymbol{r}_{0}-\boldsymbol{f}_{0}\right) .
$$

Sustituyendo (3.87) en (3.86) obtenemos la solución óptima del vector de pesos:

$$
\boldsymbol{a}_{0}^{*}=\boldsymbol{R}^{-1}\left(\boldsymbol{r}_{0}-\boldsymbol{F}\left(\boldsymbol{F}^{T} \boldsymbol{R}^{-1} \boldsymbol{F}\right)^{-1}\left(\boldsymbol{F}^{T} \boldsymbol{R}^{-1} \boldsymbol{r}_{0}-\boldsymbol{f}_{0}\right)\right) .
$$

Haciendo uso de (3.88) es posible obtener los momentos estadísticos de la predicción $\hat{Y}_{0}$. Sustituyendo el valor óptimo del vector de pesos $\boldsymbol{a}_{0}$ (ec. (3.88)) en la ecuación (3.67) se obtiene la expresión de la media de $\hat{Y}_{0}$ :

$$
\begin{aligned}
\mu_{\hat{Y}_{0}} & =\boldsymbol{a}_{0}^{* T} \boldsymbol{Y} \\
& =\left(\boldsymbol{r}_{0}-\boldsymbol{F}\left(\boldsymbol{F}^{T} \boldsymbol{R}^{-1} \boldsymbol{F}\right)^{-1}\left(\boldsymbol{F}^{T} \boldsymbol{R}^{-1} \boldsymbol{r}_{0}-\boldsymbol{f}_{0}\right)\right)^{T} \boldsymbol{R}^{-1} \mathbf{y} \\
& =\boldsymbol{r}_{0} \boldsymbol{R}^{-1} \boldsymbol{Y}-\left[\left(\boldsymbol{F}^{T} \boldsymbol{R}^{-1} \boldsymbol{F}\right)^{-1} \boldsymbol{F}^{T} \boldsymbol{R}^{-1} \boldsymbol{r}_{0}+\left(\boldsymbol{F}^{T} \boldsymbol{R}^{-1} \boldsymbol{F}\right)^{-1} \boldsymbol{f}_{0}\right]^{T} \boldsymbol{F}^{T} \boldsymbol{R}^{-1} \boldsymbol{Y} \\
& =\boldsymbol{f}_{0}{ }^{T} \underbrace{\left(\boldsymbol{F}^{T} \boldsymbol{R}^{-1} \boldsymbol{F}\right)^{-1} \boldsymbol{F}^{T} \boldsymbol{R}^{-1} \boldsymbol{Y}}_{\hat{\boldsymbol{\beta}}}+\boldsymbol{r}_{0}^{T} \boldsymbol{R}^{-1}(\boldsymbol{Y}-\boldsymbol{F} \underbrace{\left.\left(\boldsymbol{F}^{T} \boldsymbol{R}^{-1} \boldsymbol{F}\right)^{-1} \boldsymbol{F}^{T} \boldsymbol{R}^{-1} \boldsymbol{Y}\right)}_{\hat{\boldsymbol{\beta}}} \\
& =\boldsymbol{f}_{0}^{T} \hat{\boldsymbol{\beta}}+\boldsymbol{r}_{0}^{T} \boldsymbol{R}^{-1}(\boldsymbol{Y}-\boldsymbol{F} \hat{\boldsymbol{\beta}}) .
\end{aligned}
$$

Así mismo, la expresión de la varianza se obtiene mediante la sustitución de (3.88) en (3.83):

$$
\begin{aligned}
\sigma_{\hat{\Upsilon}_{0}}^{2} & =\sigma^{2}\left[1+\boldsymbol{a}_{0}^{* T}\left(\boldsymbol{R} \boldsymbol{a}_{0}^{*}-2 \boldsymbol{r}_{0}^{*}\right)\right] \\
& =\sigma^{2}\left[1+\left(\boldsymbol{r}_{0}-\boldsymbol{F}\left(\boldsymbol{F}^{T} \boldsymbol{R}^{-1} \boldsymbol{F}\right)^{-1} \boldsymbol{u}_{0}\right)^{T} \boldsymbol{R}^{-1}\left(\left(\boldsymbol{r}_{0}-\boldsymbol{F}\left(\boldsymbol{F}^{T} \boldsymbol{R}^{-1} \boldsymbol{F}\right)^{-1} \boldsymbol{u}_{0}\right)-2 \boldsymbol{r}_{0}\right)\right] \\
& =\sigma^{2}\left[1-\left(\boldsymbol{r}_{0}-\boldsymbol{F}\left(\boldsymbol{F}^{T} \boldsymbol{R}^{-1} \boldsymbol{F}\right)^{-1} \boldsymbol{u}_{0}\right)^{T} \boldsymbol{R}^{-1}\left(\boldsymbol{r}_{0}+\boldsymbol{F}\left(\boldsymbol{F}^{T} \boldsymbol{R}^{-1} \boldsymbol{F}\right)^{-1} \boldsymbol{u}_{0}\right)\right] \\
& =\sigma^{2}\left[1-\left(\boldsymbol{r}_{0}^{T} \boldsymbol{R}^{-1} \boldsymbol{r}_{0}-\left(\boldsymbol{F}\left(\boldsymbol{F}^{T} \boldsymbol{R}^{-1} \boldsymbol{F}\right)^{-1} \boldsymbol{u}_{0}\right)^{T} \boldsymbol{R}^{-1} \boldsymbol{F}\left(\boldsymbol{F}^{T} \boldsymbol{R}^{-1} \boldsymbol{F}\right)^{-1} \boldsymbol{u}_{0}\right)\right] \\
& =\sigma^{2}[1-\boldsymbol{r}_{0}^{T} \boldsymbol{R}^{-1} \boldsymbol{r}_{0}+\boldsymbol{u}_{0}^{T} \underbrace{\left(\boldsymbol{F}^{T} \boldsymbol{R}^{-1} \boldsymbol{F}\right)^{-1}\left(\boldsymbol{F}^{T} \boldsymbol{R}^{-1} \boldsymbol{F}\right)}_{I}\left(\boldsymbol{F}^{T} \boldsymbol{R}^{-1} \boldsymbol{F}\right)^{-1} \boldsymbol{u}_{0}] \\
& =\sigma^{2}\left[1-\boldsymbol{r}_{0}^{T} \boldsymbol{R}^{-1} \boldsymbol{r}_{0}+\boldsymbol{u}_{0}^{T}\left(\boldsymbol{F}^{T} \boldsymbol{R}^{-1} \boldsymbol{F}\right)^{-1} \boldsymbol{u}_{0}\right]
\end{aligned}
$$


donde por simplicidad se ha introducido $\boldsymbol{u}_{0} \equiv \boldsymbol{F}^{T} \boldsymbol{R}^{-1} \boldsymbol{r}_{0}-f_{0}$.

Dada la ecuación (3.63), en la literatura se presentan tres variantes del modelo de PLIO:

- Simple Kriging: función de regresión constante $f(\mathbf{x})=1$ y coeficiente $\beta$ conocido a priori.

- Ordinary Kriging: función de regresión constante $f(\mathbf{x})=1$ y coeficiente $\beta$ estimado a partir de las observaciones $(\hat{\beta})$.

- Universal Kriging: función de regresión dependiente de $\mathbf{x}$ y vector de coeficientes $\beta$ estimado a partir de las observaciones $(\hat{\beta})$.

\subsubsection{Propiedades de la predicción lineal insesgada óptima}

Los modelos Kriging reúnen tres propiedades que los diferencian frente a otros meta-modelos y que los convierte en una herramienta de enorme utilidad dentro del marco definido en este trabajo, estas son: la interpolación, la Gaussianidad, y la consistencia asintótica.

- Interpolación. La predicción lineal insesgada óptima es una interpolación de las observaciones del diseño inicial $\mathscr{D}=\left\{x_{i}, y_{i}\right\} \forall i=1, \ldots, m$ tal y como muestra la Figura 3.7. Sea $x_{i} \in \mathscr{D}$ un valor del conjunto de diseños inicial, $\mathbf{r}\left(x_{i}\right)$ es la i-ésima columna de la matriz de correlación $\mathbf{R}$, y el producto $\mathbf{R}^{-1} \mathbf{r}\left(x_{i}\right)=$ $\mathbf{e}_{i}$ la i-ésima columna de la matriz unidad. Utilizando estas relaciones el valor esperado de la PLIO es:

$$
\begin{aligned}
\mu_{\hat{Y}_{i}} & =f\left(\boldsymbol{x}_{i}\right)^{T} \boldsymbol{\beta}^{*}+r\left(x_{i}\right)^{T} \boldsymbol{R}^{-1}\left(\boldsymbol{Y}-\boldsymbol{F} \boldsymbol{\beta}^{*}\right) \\
& =f\left(\boldsymbol{x}_{i}\right)^{T} \boldsymbol{\beta}^{*}+\boldsymbol{e}_{i}\left(\boldsymbol{Y}-\boldsymbol{F} \boldsymbol{\beta}^{*}\right) \\
& \left.=f\left(\boldsymbol{x}_{i}\right)^{T} \boldsymbol{\beta}^{*}+y_{i}-\boldsymbol{F}_{i,} \boldsymbol{\beta}^{*}\right)=y_{i} .
\end{aligned}
$$

- Asintóticamente consistente. La PLIO o Universal Kriging es asintóticamente consistente cuando la función de correlación es regular, es decir $\mathscr{R}(\boldsymbol{x}, \boldsymbol{x}, \boldsymbol{\theta})=1$. Esta propiedad se traduce en que a medida que crece el tamaño de la muestra las estimaciones que proporciona el modelo Kriging en punto $x_{i}$ se aproximan cada vez más al valor de la observación de la función real en dicho punto. En el caso del modelo Kriging, al ser este un estimador insesgado, para garantizar su consistencia es suficiente garantizar que cuando el tamaño de la muestra tiende a infinito (es decir, se hace muy grande), la varianza del estimador se aproxime a cero (Figura 3.8).

- Gaussianidad. Al ser una combinación lineal de observaciones Gaussianas la predicción Kriging resulta también una variable aleatoria Gaussiana con media $\mu_{\hat{y}}(x)$ (3.89) y varianza $\sigma_{\hat{y}}^{2}(x)$ (3.90). Esta propiedad junto con las anteriores son la base de los algoritmos basados en análisis Bayesiano, que utilizan información acerca de la incertidumbre epistémica en cada punto del espacio para la búsqueda eficiente de óptimos globales. 


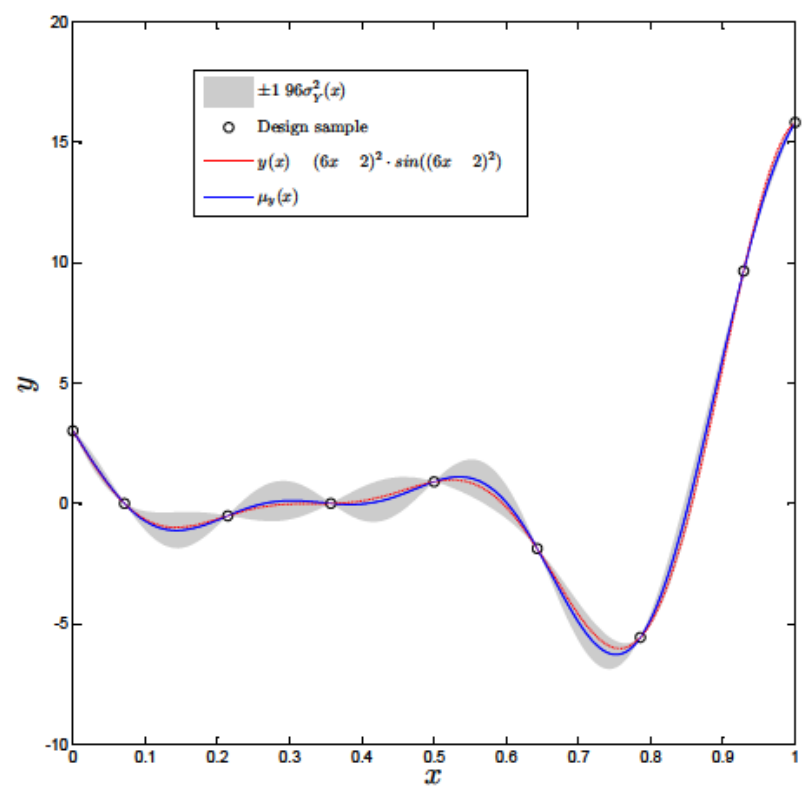

Figura 3.7: Interpolación de la función $\mathscr{M}(x)=(6 x-2)^{2} \cdot \sin \left((6 x-2)^{2}\right)$ (Forrester et al., 2008) mediante la PLIO. Función de correlación Gaussiana y regresión constante.

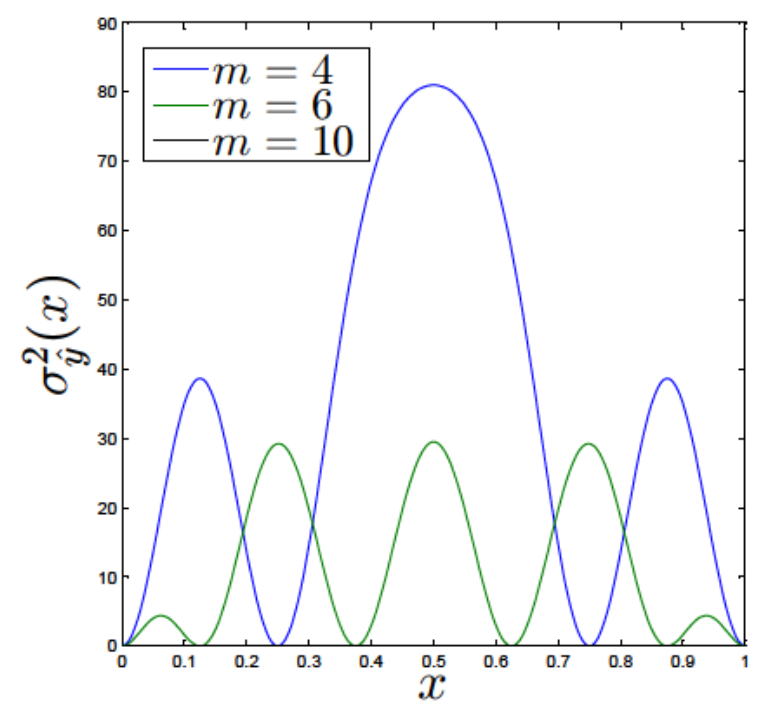

(a)

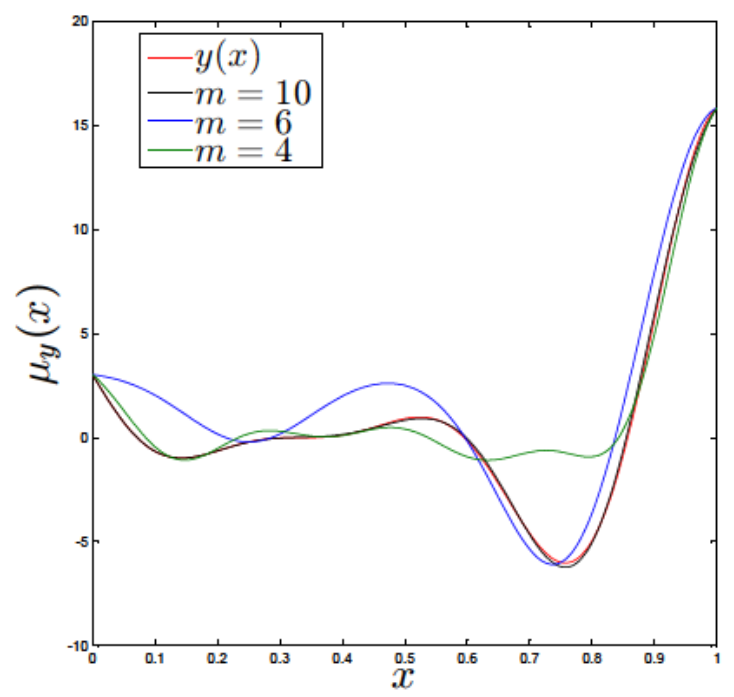

(b)

Figura 3.8: Consistencia asintótica de la PLIO para la aproximación unidimensional de la función $\mathscr{M}(x)=(6 x-2)^{2} \cdot \sin \left((6 x-2)^{2}\right)$ (Forrester et al., 2008). (a) error cuadrático medio de la predicción; (b) valor esperado de la predicción. 


\subsubsection{Predicción lineal insesgada empírica óptima (Empirical Best Linear Unbiased Predictor)}

En el desarrollo anterior se asume una función de correlación conocida, bien sea por información acerca de la física del problema o mediante procedimientos iterativos de prueba y error (Clarke et al., 2003; Yesilyurt et al., 1996). Sin embargo, en el contexto del diseño de experimentos por ordenador el tipo de función de correlación así como los parámetros que la definen son raramente conocidos y es preciso realizar una estimación. El problema de determinación de la función de correlación es un problema de optimización en el espacio de todas las posibles funciones. En este problema un enfoque adecuado es la utilización de un algoritmo de optimización guiado por una métrica representativa de la calidad del modelo (ej. validación cruzada, distribución posterior de los hiperparámetros $P(\theta \mid \mathscr{D})$ utilizando estimación Bayesiana, o estimación de máxima verosimilitud). Una revisión en detalle de estas diferentes técnicas se recoge en la referencia (Santner y Notz, 2003). En esta tesis se propone la utilización de los errores de validación cruzada para determinar las funciones de regresión y de correlación, junto con la estimación de máxima verosimilitud para la estimación de los hiperparámetros de la función de correlación. La predicción resultante se denomina PLIEO.

\subsubsection{Estimación de los hiperparámetros mediante el método de máxima verosimilitud}

El método de máxima verosimilitud (Maximum Likelihood Estimation (MLE)) para la estimación de los parámetros $\left\{\boldsymbol{\beta}, \sigma^{2}, \theta\right\}$ que aparecen en el modelo de predicción óptima lineal insesgada consiste en la maximización del logaritmo de la función de probabilidad:

$$
\left\{\boldsymbol{\beta}^{*}, \sigma^{2^{*}}, \theta^{*}\right\}=\underset{\boldsymbol{\beta}, \sigma^{2}, \theta}{\arg \min }-\log \mathrm{L}\left(\mathbf{y} \mid \boldsymbol{\beta}, \sigma^{2}, \theta\right),
$$

donde $\left\{\boldsymbol{\beta}^{*}, \sigma^{2 *}, \theta^{*}\right\}$ es la estimación de máxima verosimilitud (maximum likelihood estimation) y $\mathrm{L}\left(\mathbf{y} \mid \boldsymbol{\beta}, \sigma^{2}, \theta\right)$ es la función de densidad de probabilidad del vector aleatorio (y) que viene dada por la expresión:

$$
\mathrm{L}\left(\boldsymbol{y} \mid \boldsymbol{\beta}, \sigma^{2}, \theta\right)=\frac{1}{\left(\left(2 \pi \sigma^{2}\right)^{m}|\boldsymbol{R}(\theta)|\right)^{1 / 2}} \exp \left[-\frac{1}{2 \sigma^{2}}(\boldsymbol{y}-\boldsymbol{F} \boldsymbol{\beta})^{\mathrm{T}} \boldsymbol{R}(\theta)^{-1}(\boldsymbol{y}-\boldsymbol{F} \boldsymbol{\beta})\right] .
$$

La estimación de máxima verosimilitud de los parámetros $\left\{\boldsymbol{\beta}, \sigma^{2}\right\}$ es la obtenida en la sección 3.4.1 para el problema de estimación generalizada por mínimos cuadrados, con la única diferencia de que en este caso ambos parámetros son función del vector de hiperparámetros $\boldsymbol{\theta}$ :

$$
\begin{cases}\boldsymbol{\beta}^{*}(\boldsymbol{\theta}) & =\left(\mathbf{F}^{\mathrm{T}} \mathbf{R}(\boldsymbol{\theta})^{-1} \mathbf{F}\right)^{-1}\left(\mathbf{F}^{\mathrm{T}} \mathbf{R}^{-1} \mathbf{y}\right) \\ \sigma^{2^{*}}(\boldsymbol{\theta}) & =\frac{1}{m}\left(\mathbf{y}-\mathbf{F} \boldsymbol{\beta}^{*}\right)^{\mathrm{T}} \mathbf{R}(\boldsymbol{\theta})^{-1}\left(\mathbf{y}-\mathbf{F} \boldsymbol{\beta}^{*}\right)\end{cases}
$$


No existe una solución analítica de la solución óptima del vector de hiperarámetros $\boldsymbol{\theta}$ y requiere de métodos numéricos para su resolución. Para simplificar el problema de optimización, las expresiones de $\boldsymbol{\beta}^{*}(\boldsymbol{\theta})$ y $\sigma^{2^{*}}(\boldsymbol{\theta})$ se sustituyen en la ecuación (3.93) y se toman logaritmos para mejorar los problemas asociados a regiones potencialmente llanas o cercanas a cero. De este modo la expresión del estimador MLE quedaría como sigue:

$$
\begin{aligned}
-\log \left(\mathrm{L}\left(\boldsymbol{y} \mid \boldsymbol{\beta}, \sigma^{2}, \theta\right)\right) & =\frac{m}{2}+\frac{m}{2} \log (2 \pi)+\frac{m}{2} \log \left(\sigma^{2^{*}}(\boldsymbol{\theta})\right)+\frac{1}{2}(\log (|\boldsymbol{R}(\theta)|)) \\
& =\frac{m}{2} \log (\psi(\theta))+\frac{m}{2}(\log (2 \pi)+1)
\end{aligned}
$$

donde se ha introducido la función $\psi(\theta)=\sigma^{2^{*}}(\boldsymbol{\theta})|\boldsymbol{R}(\theta)|^{1 / m}$, conocida como función de verosimilitud reducida (Jones, 2001). De acuerdo con Sacks et al. (1989) el problema de minimización de la función (3.95) es equivalente a la minimización de la función de verosimilitud reducida:

$$
\begin{aligned}
& \boldsymbol{\theta}^{*}=\underset{\theta}{\arg \min _{\boldsymbol{\theta}}} \boldsymbol{\psi}(\theta), \\
& \text { s.a }: \boldsymbol{\theta}>0 .
\end{aligned}
$$

A pesar de la reducción del problema de optimización, éste sigue siendo el punto de mayor coste computacional en el proceso de obtención del modelo de predicción. Además, tal y como muestran trabajos como los de Martin (2009), el proceso de optimización puede presentar dificultades numéricas, tales como un mal condicionamiento de la matriz de correlación, múltiples óptimos locales y grandes regiones cercanas al óptimo .

El problema de optimización global definido en (3.96) no tiene solución analítica y se resuelve mediante la utilización de técnicas de optimización. En la literatura existen trabajos que hacen uso de la continuidad y diferenciabilidad de la función de verosimilitud reducida durante la optimización. Park y Baek (2001) desarrollan una expresión analítica del gradiente de la función (3.96) para su utilización en un método de optimización quasi-Newton. Zhang y Leithead (2005) van un paso más allá y desarrollan una expresión analítica del Hessiano de (3.96), empleándolo junto con un método de optimización Trust-Region. Staum (2009) utiliza un algoritmo de programación matemática no lineal implementado en la función fmincon de MATLAB. Toal y Keane (2008a) y Toal y Keane (2008b) utilizan un algoritmo genético seguido por dynamic hill climber para la resolución del problema de optimización. Más recientemente, Roustant et al. (2010) utilizan el algoritmo genético basado en gradiente propuesto por Sekhon y Mebane (2011). En esta línea, Toal et al. (2011) proponen la utilización de un algoritmo híbrido basado en enjambre de partículas, con el objetivo de facilitar una exploración global del espacio de diseño y una rápida convergencia al óptimo mediante la utilización de información de primer orden. 


\subsubsection{Metodología propuesta}

El Algoritmo 1 muestra el proceso de obtención del modelo de predicción Kriging utilizado en esta tesis. El algoritmo propuesto consta de las tres etapas descritas a continuación.

\subsubsection{Etapa 1: Generación de observaciones}

En esta etapa se obtiene un conjunto de muestras $\left(\mathscr{X} \in \mathbb{X}^{m}\right)$ en el espacio de variables de entrada $(\mathbb{X})$ utilizando técnicas de diseño de experimentos. Dichas muestras se analizan con el modelo de simulación $(\mathscr{M})$ generando el conjunto de respuestas: $\mathscr{Y}=\mathscr{M}(\mathscr{X})=\left\{\mathscr{M}\left(\mathbf{x}^{(1)}\right), \mathscr{M}\left(\mathbf{x}^{(2)}\right), \ldots, \mathscr{M}\left(\mathbf{x}^{(m)}\right)\right\}$. Las muestras y las respuestas forman el conjunto de observaciones $(\mathscr{D})$ que se utiliza para la estimación de los hiperparámetros del meta-modelo en la etapa 2.

Se genera un conjunto de $\mathrm{K}$ modelos $\left(\hat{\mathscr{M}}_{i}\left(\boldsymbol{\theta}, \boldsymbol{\beta}, \sigma^{2}\right), \quad \forall i=1, \ldots, K\right)$ que difieren en su estructura a priori: función de correlación y modelo de regresión. La selección del modelo que más se adecua a las observaciones obtenidas, se realiza en la etapa 2 mediante técnicas de validación cruzada.

\subsubsection{Etapa 2: Estimación de hiperparámetros y entrenamiento del meta-modelo}

En esta etapa se obtienen las expresiones de los modelos a posteriori condicionados a las observaciones obtenidas: $\hat{\mathscr{M}}_{i}\left(\boldsymbol{\theta}, \boldsymbol{\beta}, \sigma^{2} \mid \mathscr{X}, \mathscr{Y}\right) \quad \forall i=1, \ldots, K$. Para ello se obtiene el conjunto de hyperparámetros $\left(\boldsymbol{\theta}, \boldsymbol{\beta}, \sigma^{2}\right)$ para cada uno los $\mathrm{K}$ modelos definidos en la etapa 1.

El valor de $\beta$ y $\sigma^{2}$ se obtiene de forma explícita a partir de la ecuación (3.94). Sin embargo, la obtención del valor de $\boldsymbol{\theta}$ requiere de la resolución del problema de optimización definido en la ecuación (3.96). Dicho problema se resuelve utilizando un algoritmo híbrido basado en un algoritmo genético seguido de un algoritmo de programación cuadrática secuencial.

Una vez obtenidos los $\mathrm{K}$ modelos a posteriori se selecciona el que mejor se adecua a la características del problema utilizando técnicas de validación cruzada.

Clasificación utilizando validación cruzada. Las funciones de regresión y de correlación se seleccionan empíricamente a través de un indicador de la calidad global de la aproximación obtenido a partir de los errores de validación cruzada. La técnica de validación cruzada es ampliamente utilizada en el campo de los meta-modelos. Muestra de ello son las aportaciones de autores como Viana et al. (2009), Jin et al. (2001a) y Meckesheimer et al. (2002), que utilizan los errores de validación cruzada para clasificar los meta-modelos por su calidad, así como seleccionar el mejor meta-modelo de entre un conjunto. 
La técnica de validación cruzada consiste en la división del diseño de experimentos inicial $\mathscr{D}=\left\{\left(\mathbf{x}^{(i)}, Y^{(i)}\right), i=1, \ldots, m\right\}$ en un número $S$ de subconjuntos mutuamente excluyentes $\left\{\mathscr{D}_{S}, s=1, \ldots, S\right\}$. Dichos subconjuntos son utilizados para obtener un número $S$ de predicciones de validación cruzada $\left\{\hat{Y}_{-s}, S=1, \ldots, S\right\}$. Cada una de estas predicciones se obtiene ajustando el modelo Kriging utilizando todos los subconjuntos a excepción del subconjunto s-ésimo. La Figura 3.9 ilustra el error de validación cruzada en el cuarto punto de una muestra inicial de 8 puntos para la función $\mathscr{M}=(6 x-2)^{2} \sin \left((6 x-2)^{2}\right)$. Haciendo uso de las predicciones de validación cruzada, los errores de validación cruzada conocidos como el PREdiction Sum of Squares (PRESS) (Meckesheimer et al., 2002) se obtienen como sigue:

$$
e_{s}=Y_{s}-\hat{Y}_{-s} .
$$

A partir del PRESS se estiman indicadores de la calidad global del meta-modelo tales como el Root Mean Square Error (RMSE) o el coeficiente de determinación $\left(R^{2}\right)$ :

$$
\begin{aligned}
\text { PRESS }_{\text {RMSE }} & =\sqrt{\frac{\sum_{i=1}^{m}\left(Y_{i}-\hat{Y}_{-i}\right)^{2}}{m},} \\
\text { PRESS }_{R^{2}} & =1-\frac{\sum_{i=1}^{m}\left(Y_{i}-\hat{Y}_{-i}\right)^{2}}{\sum_{i=1}^{m}\left(Y_{i}-\bar{Y}\right)^{2}},
\end{aligned}
$$

donde $\hat{Y}_{-i}$ es la predicción de validación cruzada del punto de diseño $i$.

La utilización de errores de validación cruzada no requiere de evaluaciones adicionales del modelo de simulación, por lo que el coste computacional únicamente se ve incrementado en la medida en que nuevos problemas de optimización necesitan ser resueltos. Este coste computacional es directamente proporcional con el número de subconjuntos $(K)$, pero es sensiblemente inferior en comparación con la resolución del modelo de simulación. Por otra parte, un aspecto destacable es que dicha metodología es totalmente paralelizable, lo que permite reducir en gran medida el coste computacional de los procesos de optimización subyacentes mediante su resolución en paralelo.

\subsubsection{Etapa 3: Validación}

La precisión global del meta-modelo se estima utilizando el coeficiente $\mathrm{R}^{2}$ obtenido mediante errores de validación cruzada (3.99).

\subsection{Ejemplos}

En esta sección se presentan tres ejemplos que ilustran: (1) la modelización de incertidumbre espacial de propiedades de estructuras continuas (sección 3.5.1), 


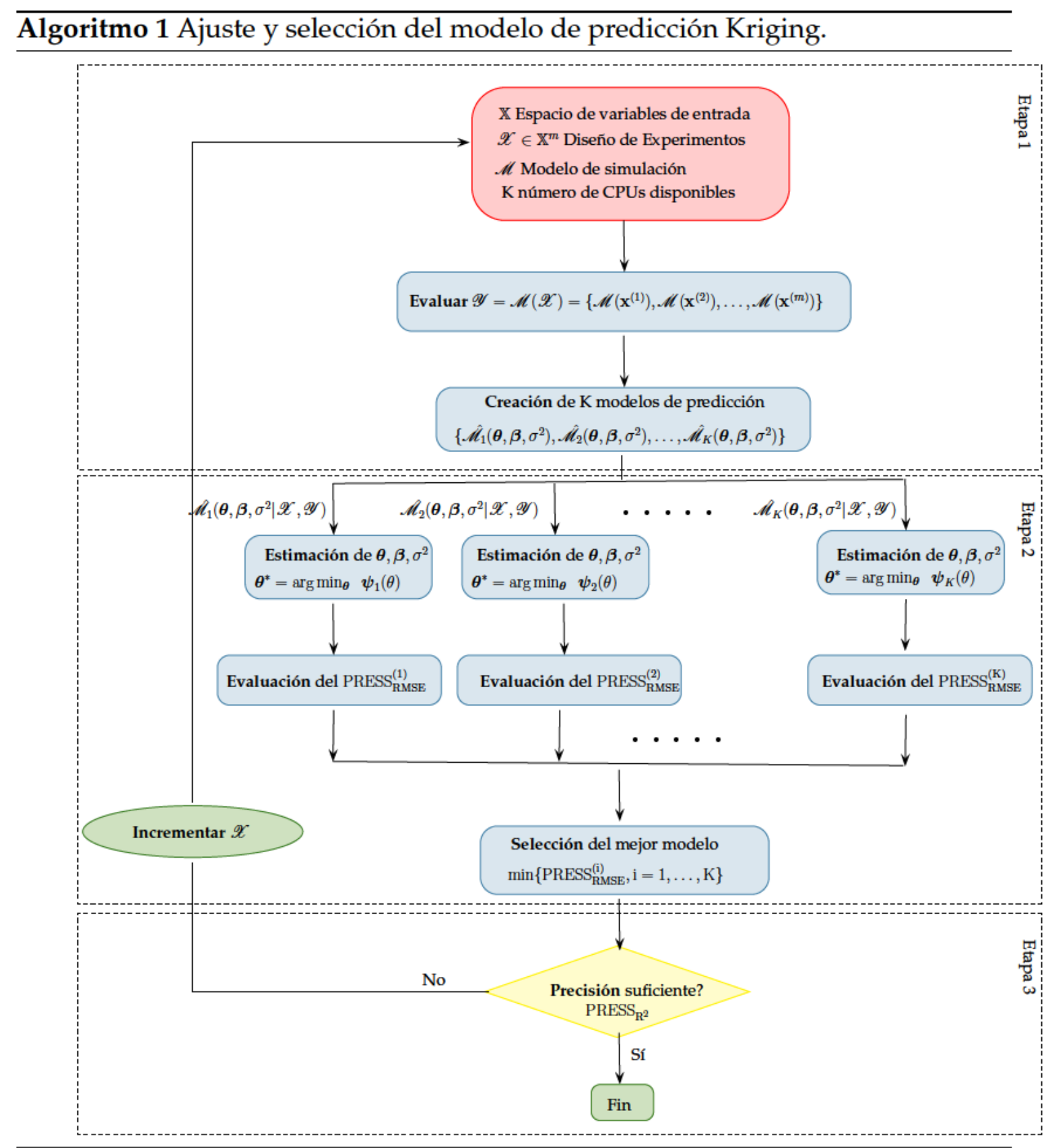




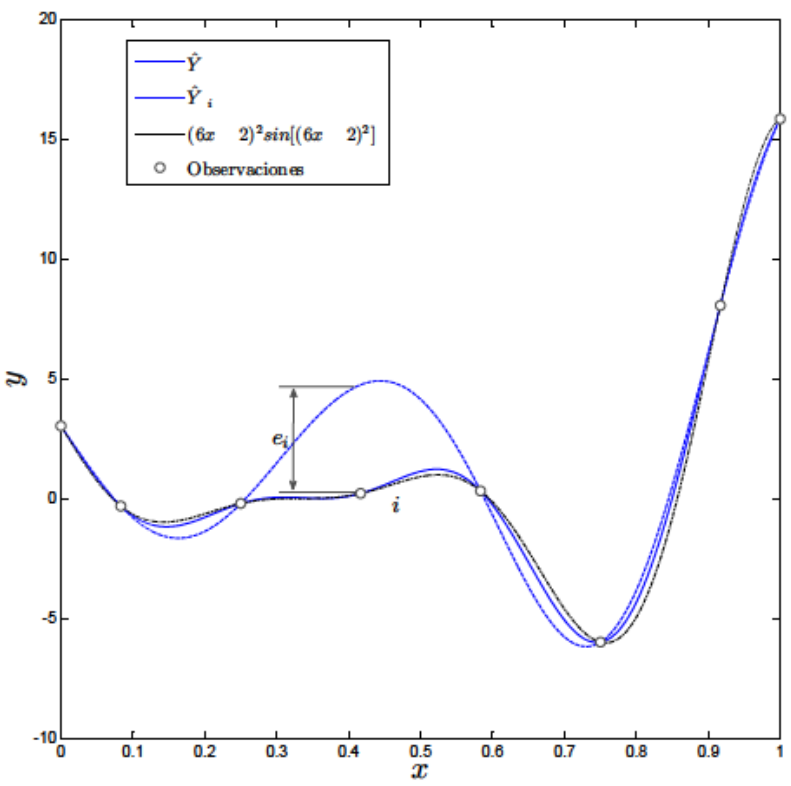

Figura 3.9: Ilustración del error de validación cruzada en el cuarto punto del diseño de experimentos para la función $\mathscr{M}=(6 x-2)^{2} \sin \left((6 x-2)^{2}\right)$.

(2) la influencia de la función de correlación en la predicción mediante procesos Gaussianos (sección 3.5.2), y (3) la utilización de errores de validación cruzada para la clasificación y selección de las funciones de regresión y correlación (sección 3.5.3).

\subsubsection{Modelización mediante campos aleatorios de la incertidumbre espacial del espesor de una lámina cilíndrica}

Este ejemplo ilustra el proceso de modelización de la incertidumbre espacial de propiedades estructurales tales como el espesor, propiedades del material, imperfecciones geométricas, etc. Se considera una lámina cilíndrica (Figura 3.10) cuyo espesor $(h)$ está sometido a variaciones en el espacio. Estas variaciones se modelizan mediante un campo aleatorio de tipo lognormal con un valor medio de $\mu_{h}=76 \mathrm{~mm}$ y una covarianza $\operatorname{Cov}_{h}=0.05$. El campo aleatorio se obtiene a partir de la transformación (3.100):

$$
F(x, \alpha)=\exp \left(\lambda_{h}+\xi_{h} U_{h}(x, \alpha)\right)
$$

donde $U_{h}$ es un campo Gaussiano estandar, y $\lambda_{h}=4.3295$ y $\xi_{h}=0.05$ son los parámetros de localización y escala respectivamente. La función de correlación utilizada es la exponencial cuadrática y la función de covarianza es:

$$
C\left(x-x^{\prime}, \alpha-\alpha^{\prime}\right)=\exp \left[-\frac{\left(x-x^{\prime}\right)^{2}}{l^{2}}-\frac{\left(R \alpha-R \alpha^{\prime}\right)^{2}}{l^{2}}\right],
$$




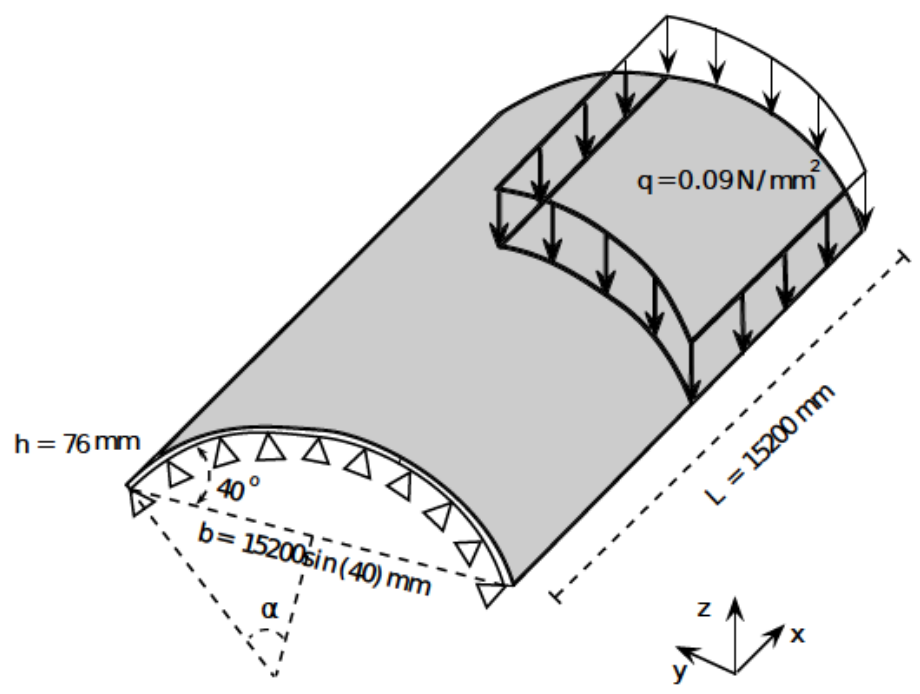

Figura 3.10: Geometría de la lámina cilíndrica Scordelis-Lo (Scordelis y Lo, 1961).

donde $l$ es la longitud de correlación con un valor $l=5000 \mathrm{~mm}$ para ambas direcciones del espacio. El campo aleatorio se discretiza mediante la expansión de Karhunen-Loéve (3.47) sobre la malla de elementos finitos. Se utiliza el centroide de los elementos lámina, cuyas coordenadas cilíndricas están contenidas en el vector $\mathscr{X}=\left\{x_{i}, \alpha_{i}, i=1, \ldots, N_{e}\right\}$. Esta discretización transforma el problema continuo en un conjunto finito de variables aleatorias $\left\{Y_{i}, i=1, \ldots, N_{e}\right\}$ que siguen una distribución normal con media y covarianza:

$$
\begin{aligned}
\mu_{i} & =\mu\left(x_{i}, \alpha_{i}\right), i=1, \ldots, N_{e} \\
C_{i j} & =C\left(x_{i}-x_{j}, \alpha_{i}-\alpha_{j}\right), i, j=1, \ldots, N_{e} .
\end{aligned}
$$

La expansión de Karhunen-Loéve se trunca en el término $M$, es decir se consideran los autovalores cuya contribución es mayor a la varianza total del proceso. En este ejemplo se adopta un valor de $M=19$ que captura el $95 \%$ de la variabilidad total del proceso, tal y como muestra la Figura 3.11e. Las Figuras 3.11a a 3.11d muestran la contribución de los primeros cuatro autovalores al campo aleatorio final (Figura 3.11f).

\subsubsection{Influencia de la función de correlación en la predicción mediante procesos Gaussianos}

Este ejemplo presenta una comparación entre los diferentes modelos Kriging ajustados a partir de un mismo conjunto de observaciones para la función $\mathscr{M}=(6 x-2)^{2} \sin \left((6 x-2)^{2}\right)$ (Forrester et al., 2008). El objetivo es ilustrar el efecto de la función de correlación en el problema de optimización subyacente, así como las principales propiedades (interpolación, consistencia, y Gaussianidad) del predictor PLIEO. Se considera el mismo modelo de regresión constante $(f(x)=1)$ 


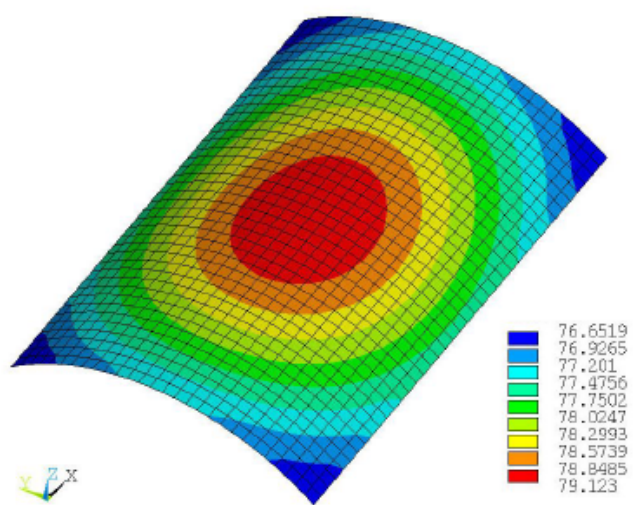

(a) Contribución $\lambda_{1}=18.6720$

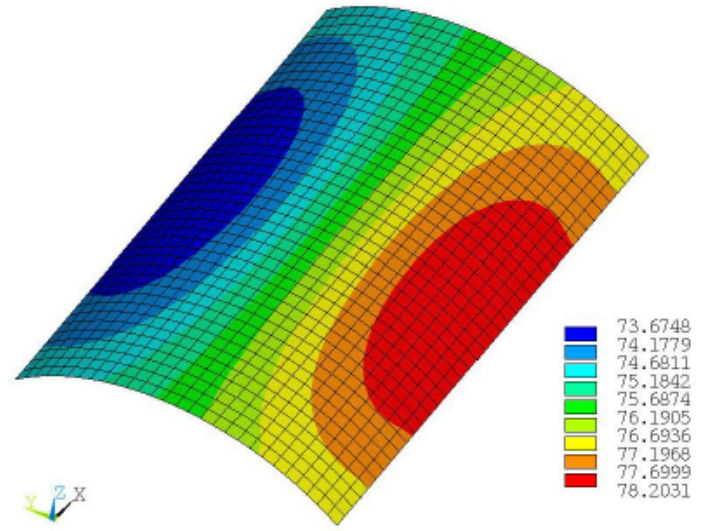

(c) Contribución $\lambda_{3}=12.3989$

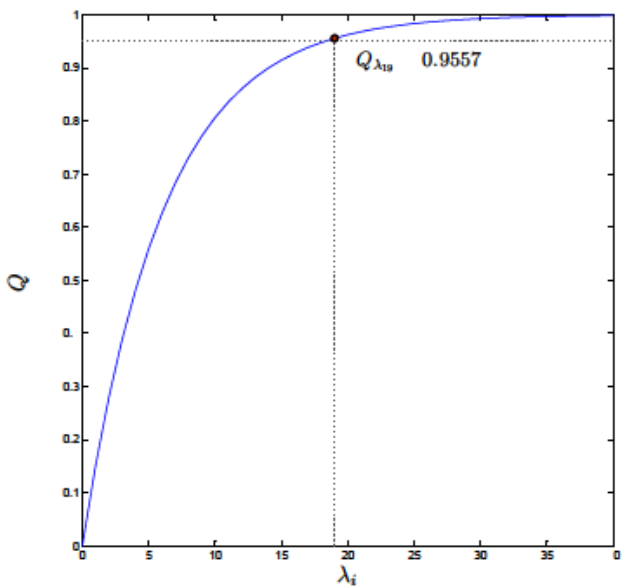

(e) Fracción de variabilidad

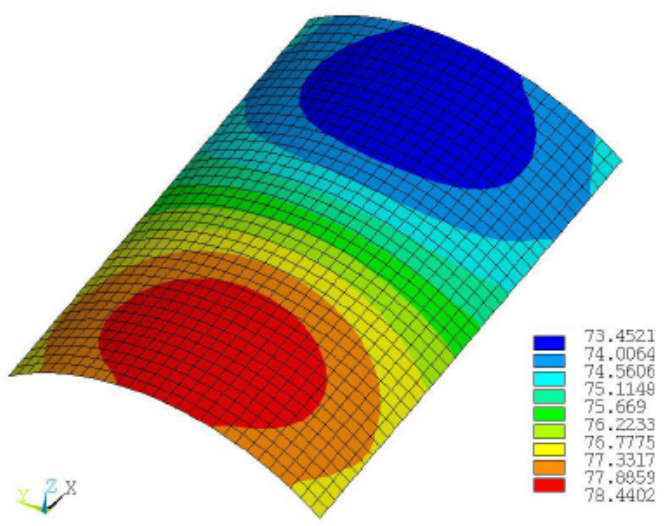

(b) Contribución $\lambda_{2}=14.6610$

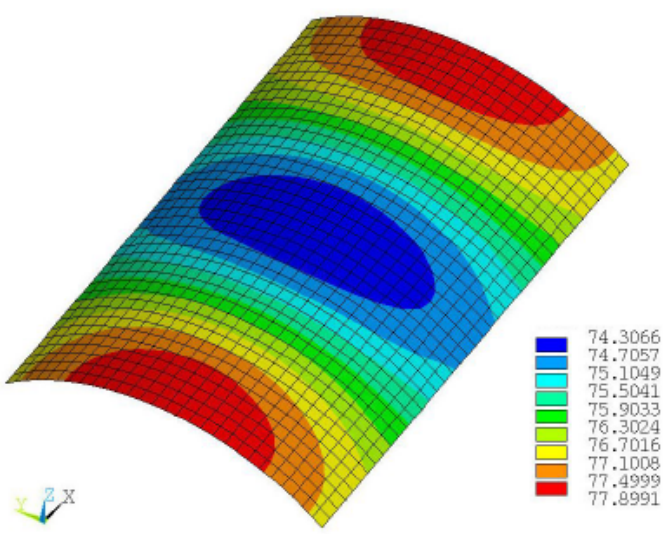

(d) Contribución $\lambda_{4}=9.8468$

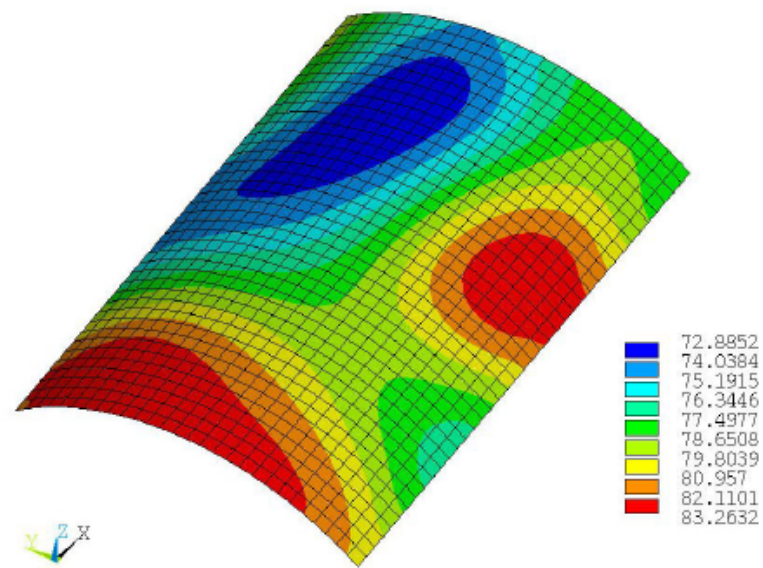

(f) Realización del campo aleatorio $(M=19)$

Figura 3.11: Realización del campo aleatorio del espesor de la lámina cilíndrica Scordelis-Lo (Scordelis y Lo, 1961). 

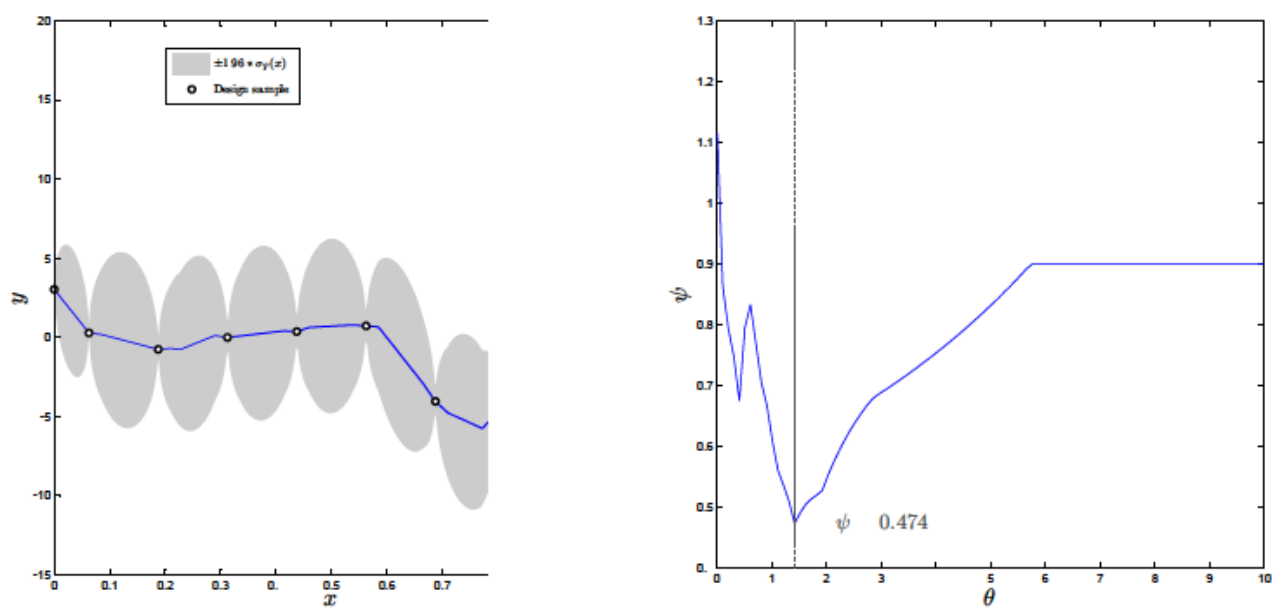

(a) Función de correlación lineal (3.49)
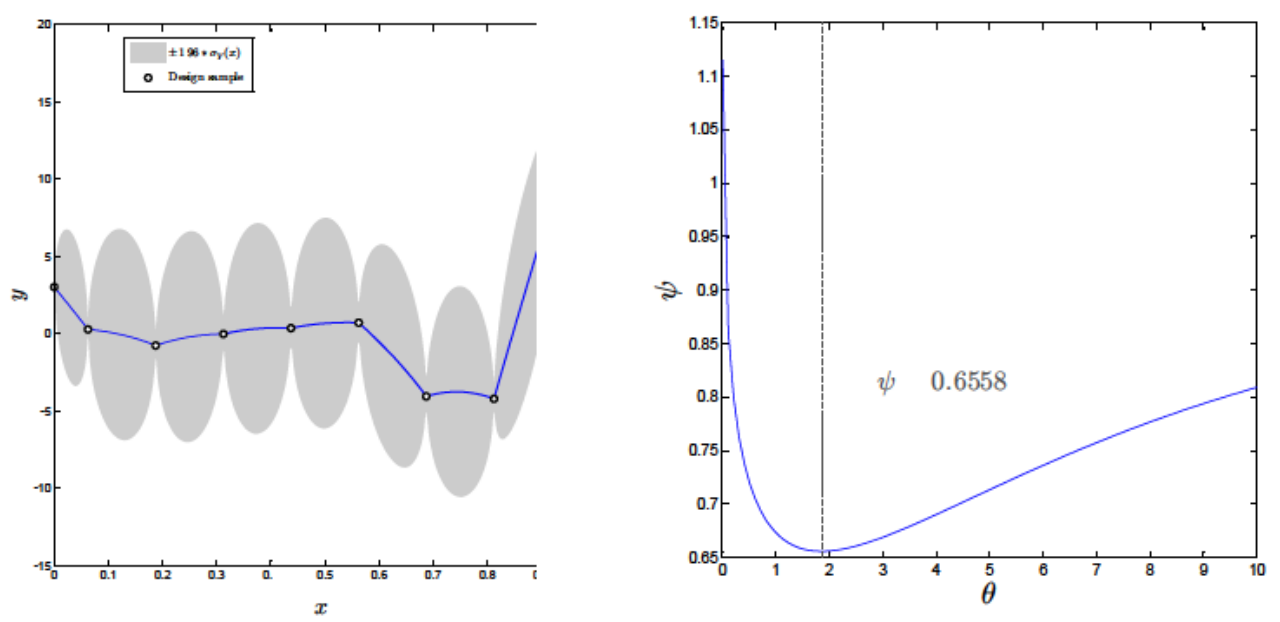

(b) Función de correlación exponencial (3.50)
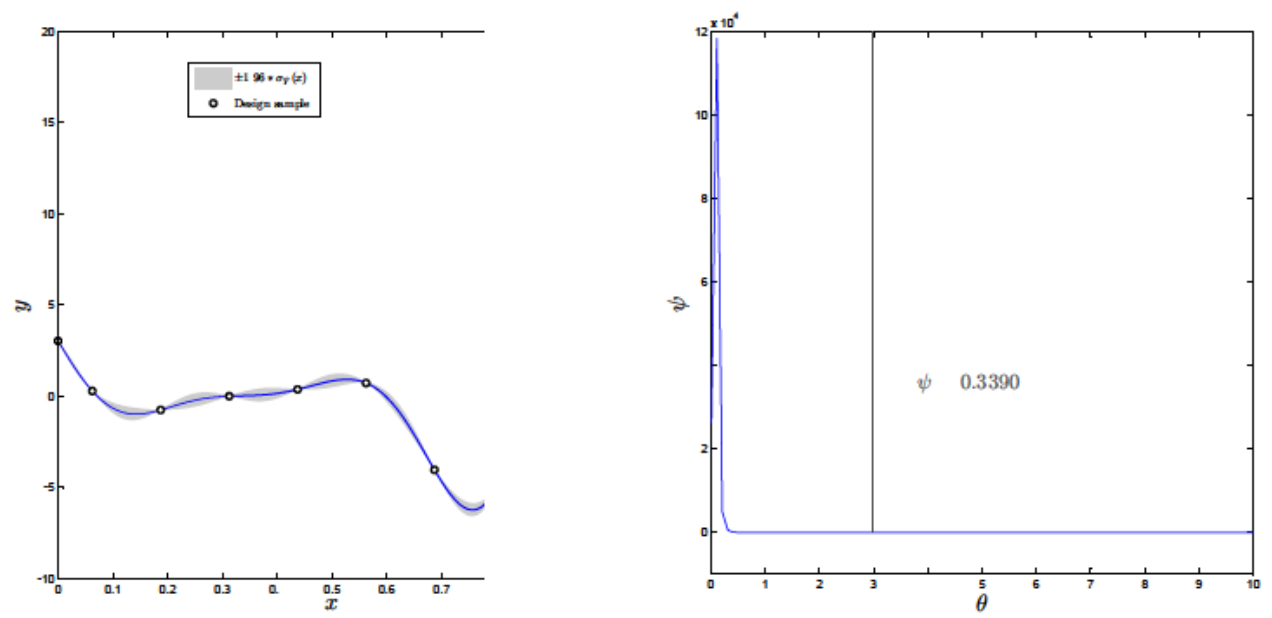

(c) Función de correlación exponencial cuadrática (3.51)

Figura 3.12: Modelos Kriging de la función $\mathscr{M}=(6 x-2)^{2} \sin \left((6 x-2)^{2}\right)$ con diferentes funciones de correlación. Las figuras de la izquierda representan el valor medio de la predicción. Las figuras de la derecha representan la función de verosimilitud reducida $\psi(\theta)$ y el resultado del problema de optimización (3.96). 

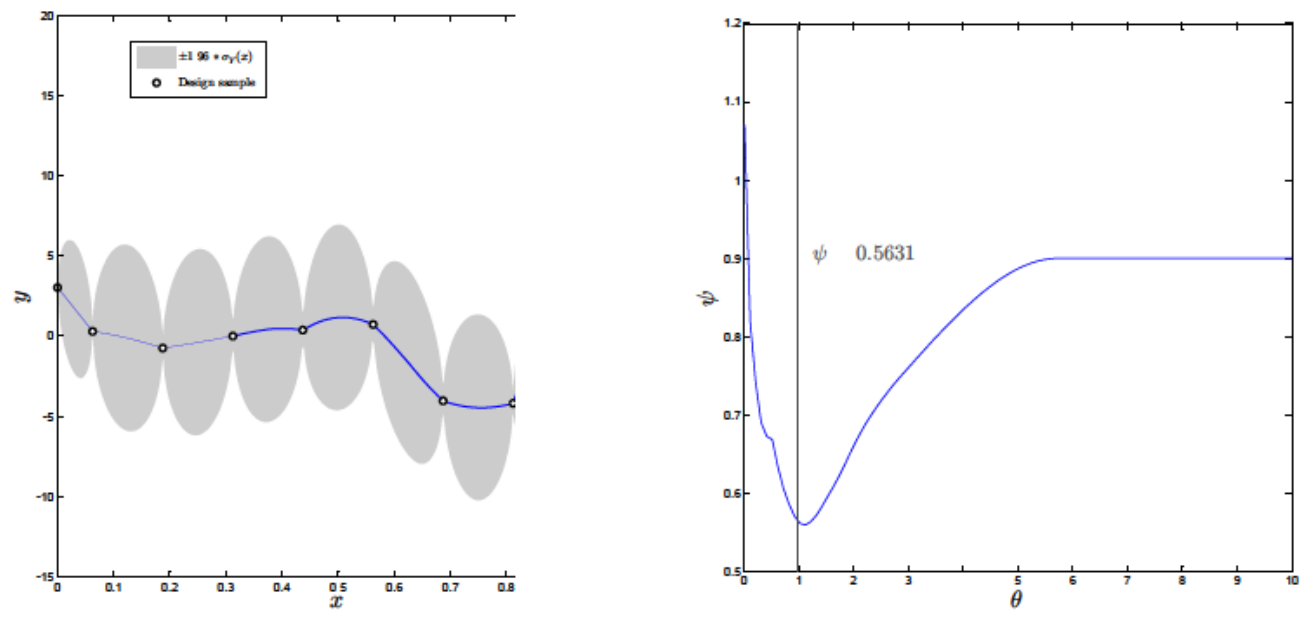

(d) Función de correlación esférica (3.53)
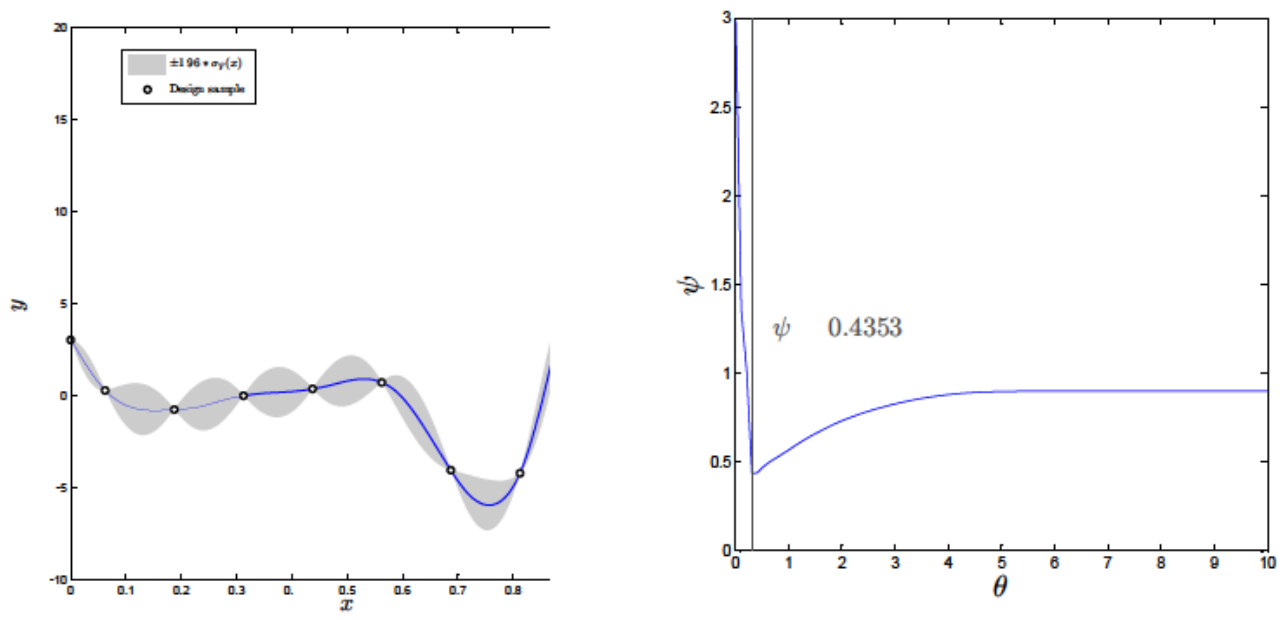

(e) Función de correlación spline (3.55)

Figura 3.12: (Continuación). Modelos Kriging de la función $\mathscr{M}=(6 x-2)^{2} \sin \left((6 x-2)^{2}\right)$ con diferentes funciones de correlación. Las figuras de la izquierda representan el valor medio de la predicción. Las figuras de la derecha representan la función de verosimilitud reducida $\psi(\theta)$ y el resultado del problema de optimización (3.96). 
para todos los modelos Kriging. La Figura 3.12 muestra el valor medio de la predicción junto con la función de verosimilitud reducida en función de la longitud de correlación.

Una primera conclusión que se obtiene a la vista de la Figura 3.12, es que el problema de optimización aumenta en dificultad a medida que la función de correlación es más suave, a excepción de la función de correlación lineal (Figura 3.12a) que presenta varios óptimos locales. La función de correlación cuadrática (Figura 3.12c) es claramente el peor caso por presentar una gran región llana alrededor del óptimo global.

La Figura 3.13 muestra el efecto de una selección defectuosa del parámetro $\theta$ para una función de correlación spline. Esta figura pone de manifiesto la importancia de una resolución precisa del problema de optimización definido en la ecuación (3.96). Los resultados deben ser revisados en detalle, puesto que una solución no óptima del problema puede empeorar dramáticamente el ajuste tal y como muestra la Figura 3.13b.

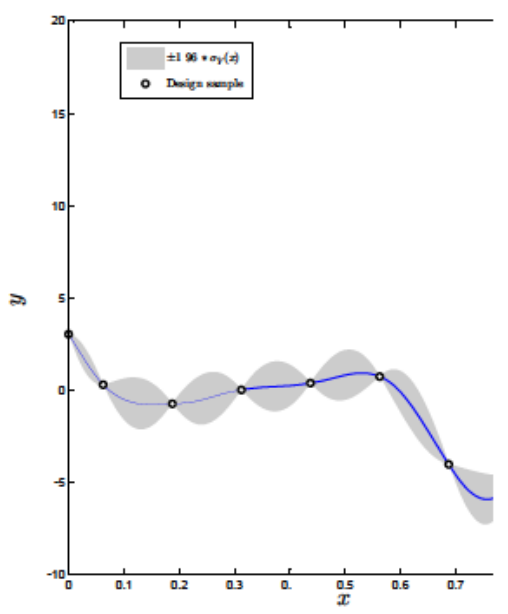

(a) $\theta=0.4353$

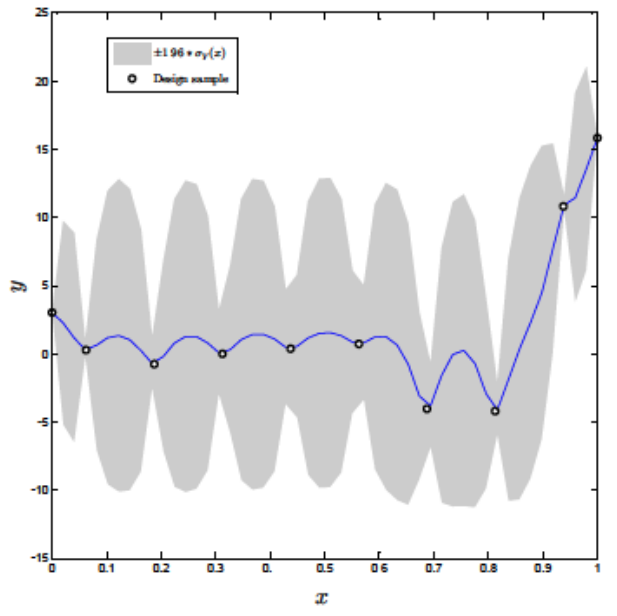

(b) $\theta=0.01$

Figura 3.13: Modelos Kriging de la función $\mathscr{M}=(6 x-2)^{2} \sin \left((6 x-2)^{2}\right)$ para diferentes valores del parámetro $\theta$ : (a) aproximación de alta calidad con un valor óptimo del parámetro $\theta$; (b) aproximación con baja calidad debido a una selección defectuosa del parámetro $\theta$.

\subsubsection{Clasificación de modelos de predicción mediante errores de validación cruzada}

Este tercer ejemplo ilustra la metodología presentada en la sección 3.4.5.2 para la selección de la función de correlación y de regresión del modelo a partir de los errores de validación cruzada. Se utiliza como función test la función de Rastrigin 
bidimensional utilizada en la literatura como benchmark para el estudio de la convergencia de algoritmos de optimización global (Törn y Zilinskas, 1989):

$$
y(\mathbf{x})=10 d+\sum_{i=1}^{d}\left(x_{i}^{2}-10 \cos \left(2 \pi x_{i}\right)\right), x_{i} \in[-5.12,5.12] ; d=2 .
$$

Con este ejemplo se pretende ilustrar la validez de la utilización de los errores de validación cruzada con el objetivo de: (1) seleccionar el mejor meta-modelo de entre un conjunto y (2) estimar el coeficiente de determinación sin utilizar evaluaciones adicionales del modelo de simulación. Todos los modelos Kriging se ajustan a partir de una muestra de 100 puntos generada mediante un Hipercubo Latino obtenido con la maximización de la mínima distancia entre puntos. El conjunto de modelos de prueba está formado por las posibles combinaciones de: regresión constante, lineal, cuadrática y cúbica, y correlación Gaussiana (3.51), exponencial (3.50), exponencial-Gaussiana (3.52), spline (3.55), cúbica (3.54) y esférica (3.53) . La Figura 3.14 presenta el valor medio y la varianza de la predicción para los casos: regresión lineal+correlación Gaussiana y regresión lineal+correlación cúbica. Esta última es la que resulta de la minimización de los errores de validación cruzada.

En el problema de optimización (3.96) se consideran unos límites de los hiperparámetros $1 e-5 \leq \theta_{1,2} \leq 20$. La Figura 3.15 muestra la clasificación del modelos en función del PRESS $R^{2}$ (Figura 3.15a) y del PRESS RMSE $_{\text {( Figura }}$ 3.15c). Para analizar la precisión de la estimación de los indicadores $R^{2}$ y RMSE utilizando validación cruzada, se considera como referencia el valor obtenido de los mismos utilizando una nueva muestra de validación. Esta muestra está formada por $1 e+06$ puntos repartidos de manera uniforme en el dominio de diseño. Los resultados obtenidos con la muestra de validación se muestran en la Figura $3.15 \mathrm{~b}$ para el caso del $\mathrm{R}^{2}$ y en la Figura $3.15 \mathrm{~d}$ para el RMSE. Si comparamos los resultados de referencia con los obtenidos mediante validación cruzada se concluye que: (1) en todos los casos el mejor modelo es el correspondiente a una función de regresión lineal y una función de correlación cúbica, (2) cuando la muestra inicial es suficiente los errores de validación cruzada permiten estimar con una precisión aceptable estimadores del error en el ajuste global del modelo, tales como el coeficiente de determinación $R^{2}$ o el RMSE y (3) la estimación del RMSE mediante errores de validación cruzada permite clasificar un conjunto de meta-modelos de acuerdo con su precisión y seleccionar el de mejor ajuste.

A la vista de la clasificación de la Figura 3.15c la combinación de regresión lineal junto con correlación cúbica resulta la más adecuada para aproximar la función (3.104). El valor óptimo del vector de hiperparámetros es $\boldsymbol{\theta}^{*}=\{0.0219,0.0216\}$ y la función de máxima verosimilitud reducida en dicho punto es $\psi\left(\boldsymbol{\theta}^{*}\right)=1.3160$. 


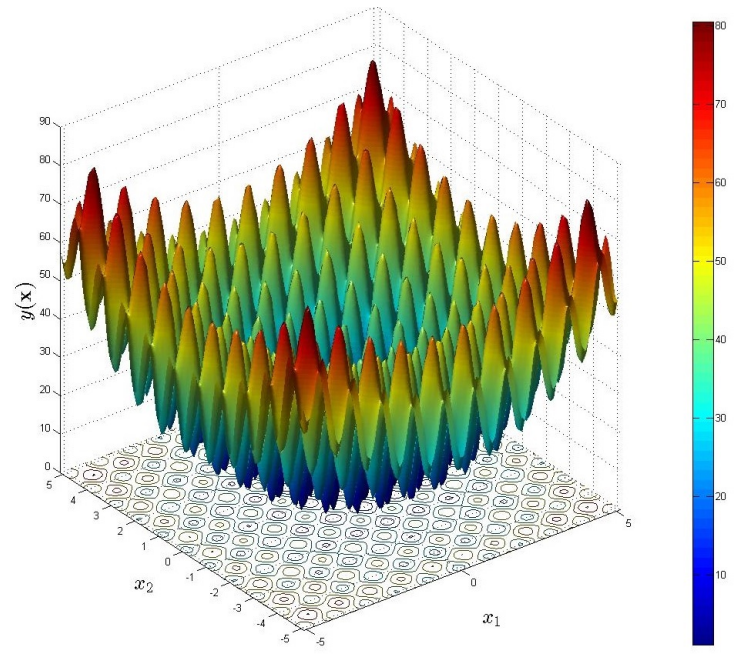

(a) función de Rastrigin para $d=2$

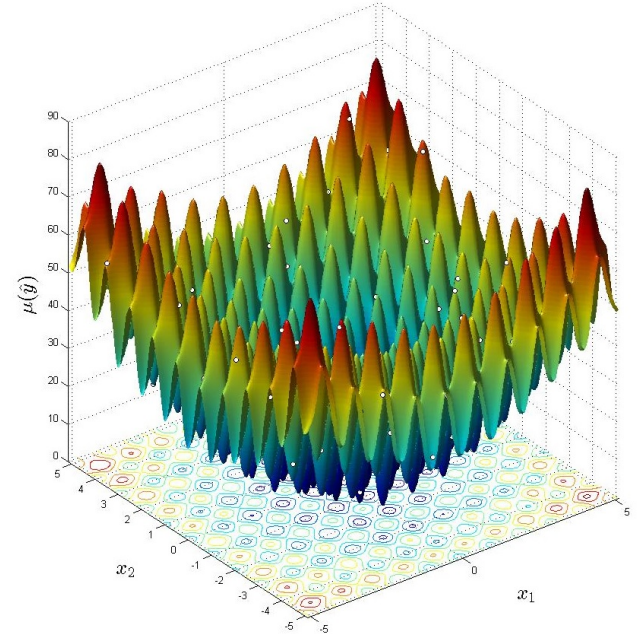

(b) correlación cúbica; $\mu_{\hat{y}}$

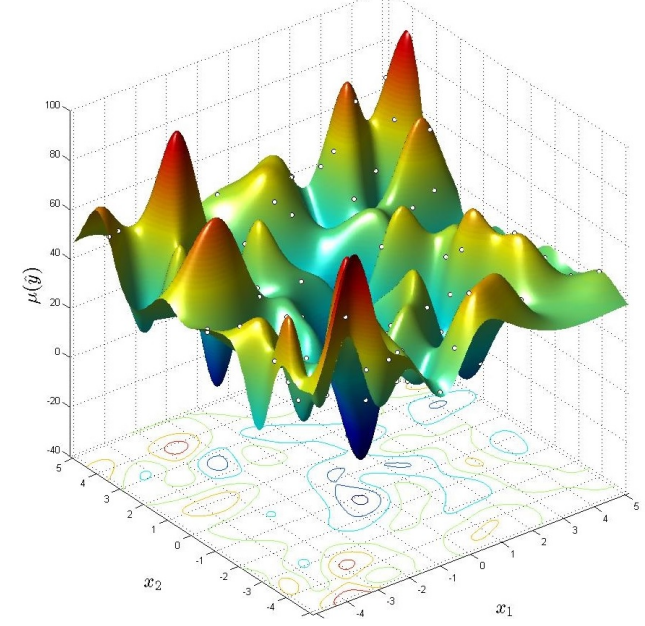

(d) correlación Gaussiana; $\mu_{\hat{y}}$

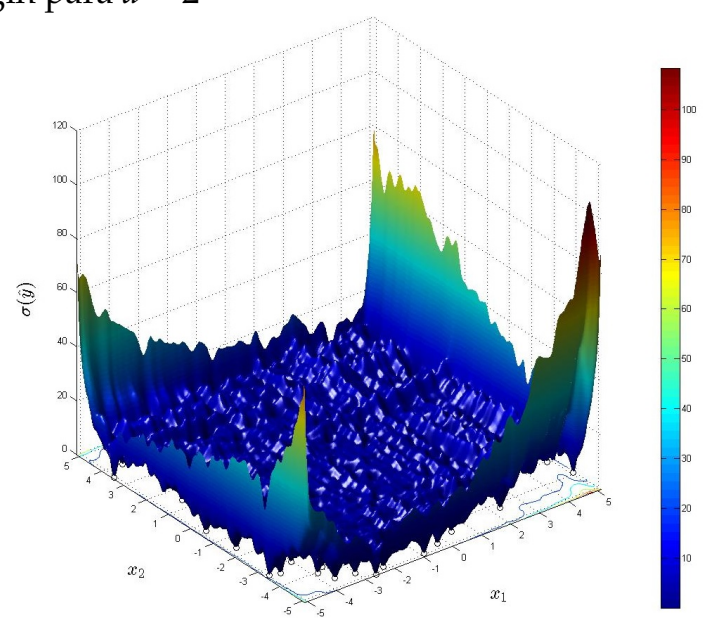

(c) correlación cúbica; $\sigma_{\hat{y}}^{2}$

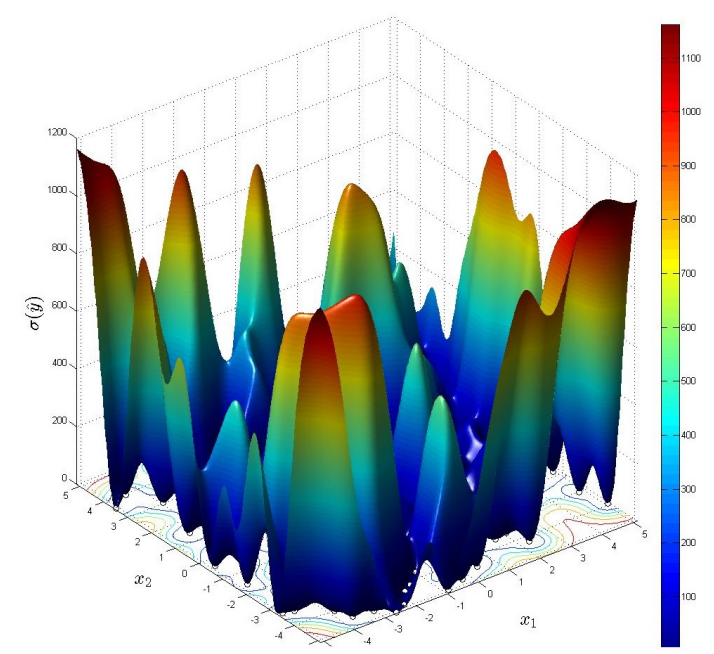

(e) correlación Gaussiana; $\sigma_{\hat{y}}^{2}$

Figura 3.14: Modelos de predicción de la función de Rastrigin bidimensional (Törn y Zilinskas, 1989). 

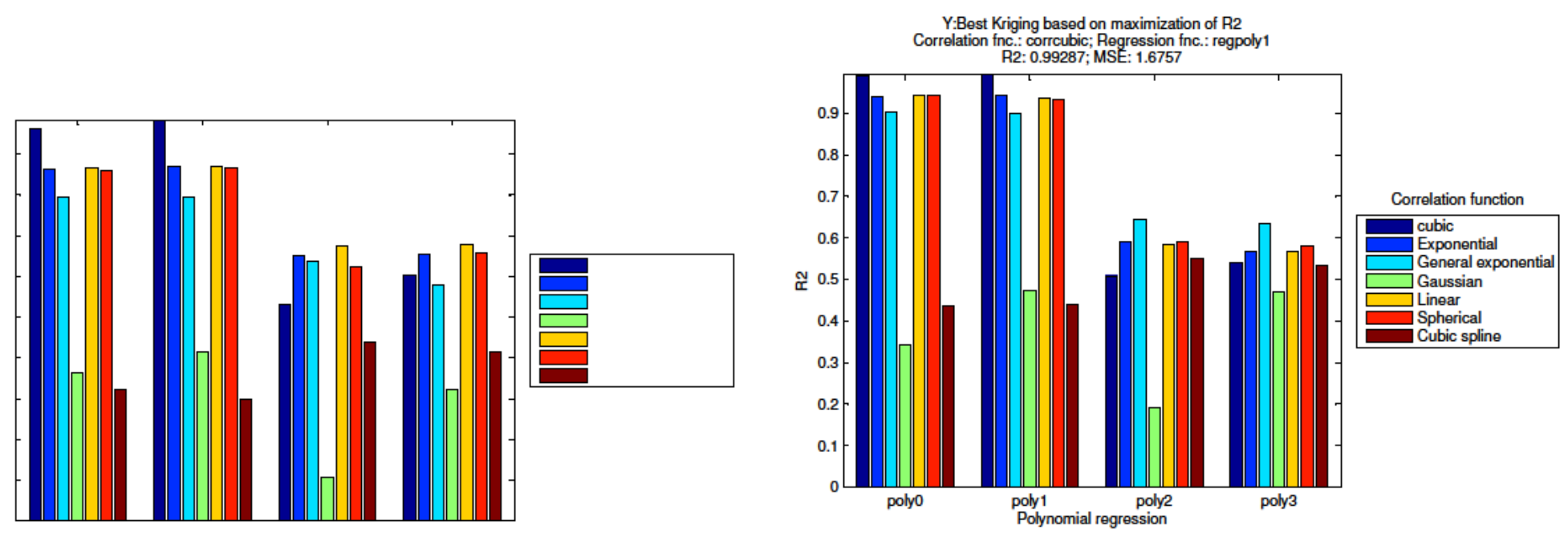

ช้

(b) $R^{2}$
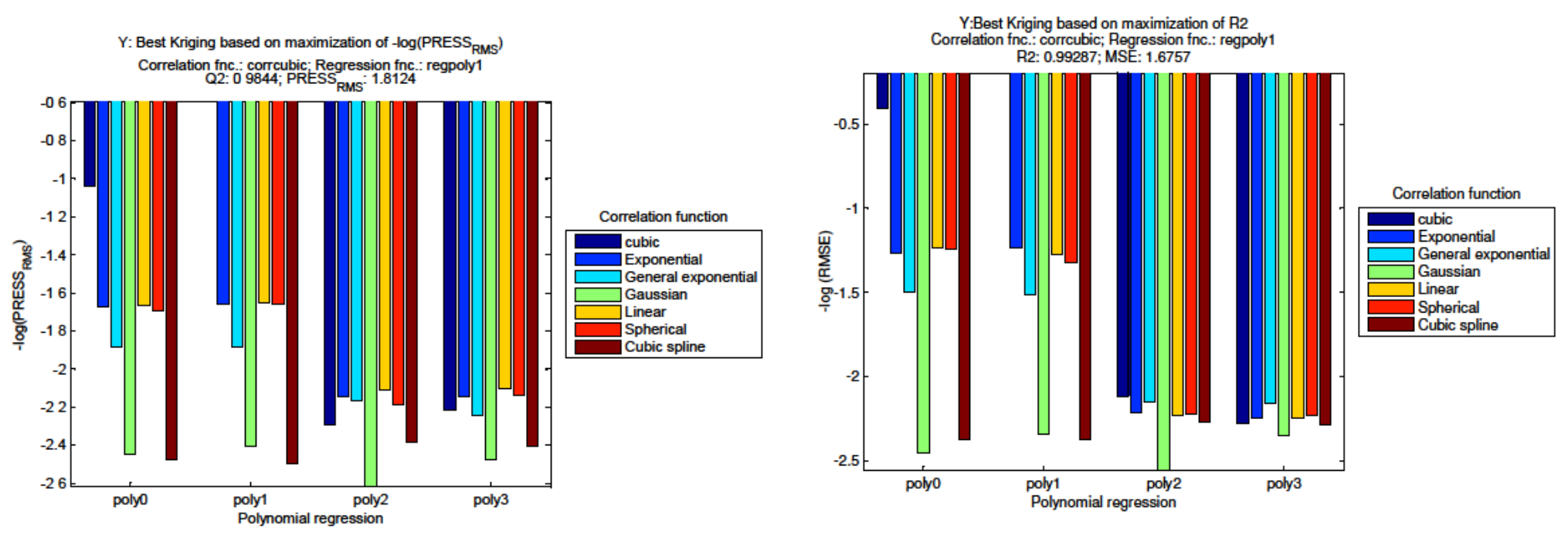

(d) RMSE

Figura 3.15: Clasificación de los modelos Kriging de la función de Rastrigin. (a) y (c) Estimación basada en errores de validación cruzada;(b) y (d) Estimación basada en muestra de validación. 
Analizando las características del modelo seleccionado (regresión lineal y correlación cúbica) se obtienen las siguientes conclusiones: (1) la función de correlación cúbica presenta un comportamiento parabólico cerca del origen, adecuado para aproximar funciones continuas y diferenciables como en este caso y (2) el valor de los hiperparámetros es muy similar en ambas direcciones, lo cual se ajusta al comportamiento isotrópico de la función de Rastrigin $2 \mathrm{~d}$ (3.104). Por lo tanto el modelo obtenido verifica la isotropía, continuidad y diferenciabilidad de la función original.

\subsection{Discusión y conclusiones}

En este capítulo se ha abordado el problema de la búsqueda de un modelo de predicción (meta-modelo) que emule la respuesta obtenida mediante un modelo de simulación de alto coste computacional. Se ha realizado un revisión del estado del arte del problema. Fruto de esta revisión se seleccionan los modelos Kriging, ya que se ajustan de forma adecuada a las características del problema que se aborda en esta tesis. Los modelos Kriging proporcionan una gran flexibilidad a la hora de aproximar respuestas de distinta naturaleza. En este sentido se propone un algoritmo para la selección del modelo de correlación y de regresión que mejor se ajuste a la naturaleza del problema. Fruto del trabajo realizado se alcanzan las conclusiones:

\section{Revisión del estado del arte.}

1. Los modelos Kriging, en concreto su variante más genérica el Universal Kriging, son una herramienta de gran utilidad en el contexto del problema de cuantificación y optimización bajo incertidumbre. Entre las principales ventajas destacan sus características de interpolación, su flexibilidad (permiten utilizar diferentes modelos de regresión y diferentes funciones de correlación), y la predicción probabilista (Gaussiana). Esta última permite obtener intervalos de confianza y probabilidades en las regiones de interés. Dichos intervalos de confianza estiman la incertidumbre asociada al meta-modelo debida a la falta de información en la muestra inicial (incertidumbre epistémica). Dicha incertidumbre puede ser reducida mediante la introducción de nuevos puntos en la muestra utilizada para el ajuste, haciendo uso de la consistencia asintótica del modelo. Este hecho posibilita la utilización de técnicas adaptativas de muestreo dirigidas a la optimización global, clasificación probabilista o cuantificación de incertidumbre. Por sus características de interpolación, Gaussianidad, y consistencia asintótica, los modelos Kriging son seleccionados como meta-modelo de partida en este trabajo.

2. Bajo ciertas hipótesis, el modelo Kriging en sus diferentes variantes equivale formalmente a otros modelos de predicción. El modelo Kriging en su forma más genérica corresponde a un modelo de regresión ordinario cuando la correlación es nula. La variante Simple Kriging equivale 
formalmente a funciones de base radial seleccionando los centros en los puntos de la muestra (Emmerich, 2005). Para ciertas funciones de correlación, el modelo Kriging proporciona predicciones equivalentes a modelos MARS (Multivariate adaptive regression splines) y/o thin-plate splines (Koehler y Owen, 1996).

Metodología propuesta para el ajuste del meta-modelo. Analizando el proceso de ajuste del modelo Kriging se obtienen las siguientes conclusiones:

1. Desde el punto de vista computacional, los modelos Kriging pierden la comparación frente a otros meta-modelos como funciones de base radial o especialmente superficies de respuesta polinómicas. La construcción del modelo Kriging requiere de la resolución de un problema de optimización multimodal para la obtención del vector de hiperparámetros $\boldsymbol{\theta}$. El vector óptimo de hiperparámetros requiere una factorización de orden $O\left(n^{3}\right)$ para cada evaluación del algoritmo de optimización, donde $n$ es el número de puntos en la muestra inicial. Por lo tanto, el coste de la optimización se incrementa dramáticamente al incrementar el tamaño de la muestra. El coste de evaluar la función (3.96) puede incrementarse rápidamente para problemas de alta dimensionalidad dado que el tamaño de la muestra inicial está íntimamente ligado con la dimensión del problema $d$. Así mismo el número de hiperparámetros es, dependiendo de la función de correlación, del orden de $2 d$, por lo que la complejidad del problema de optimización aumenta al incrementar la dimensionalidad del problema. Por esta razón habitualmente se restringe su aplicación a problemas con un número de variables de entrada $d \leq 50$.

2. Es imprescindible una selección apropiada de los hiperparámetros para obtener una representación adecuada de la función en el espacio de diseño. La selección precisa de estos parámetros mediante el estimador de máxima verosimilitud requiere de la utilización de algoritmos de optimización global. La optimización de la función de máxima verosimilitud es un problema altamente multi-modal y por lo tanto no puede resolverse de manera fiable mediante la utilización de técnicas de optimización basadas en gradiente.

3. La precisión de los modelos Kriging es muy sensible al mal condicionamiento de la matriz de correlación, circunstancia que ocurre cuando los puntos de la muestra inicial están muy juntos.

4. La estimación del error cuadrático medio a partir de errores de validación cruzada resulta una herramienta eficaz para la clasificación y selección de las funciones de correlación y regresión. Así mismo, permite reducir en gran medida el coste computacional de los procesos de optimización subyacentes mediante su resolución en paralelo. 

"The only thing that is certain is that nothing is certain."

— Gaius Plinius Secundus (AD 23 - AD 79)

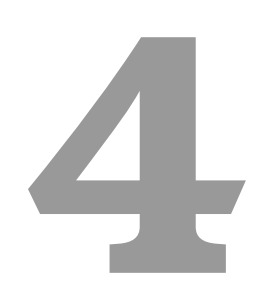

\section{Optimización Multi-objetivo con modelos de predicción Bayesiana}

\section{Índice}

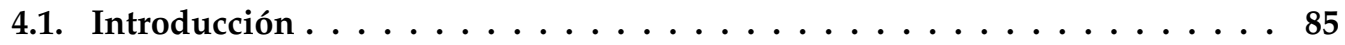

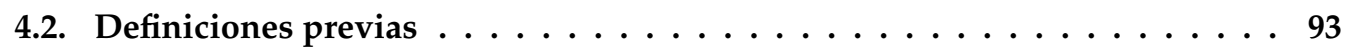

4.3. Criterios heurísticos para la optimización Bayesiana mono-objetivo . . . . 95

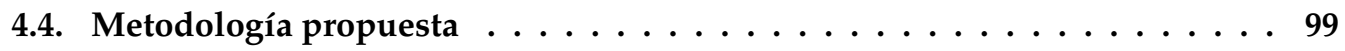

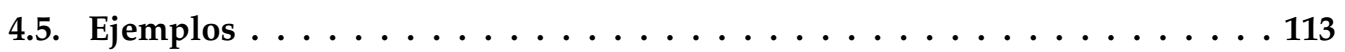

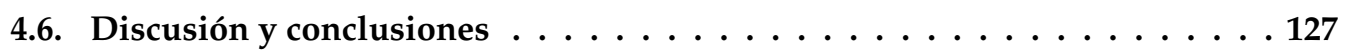





\subsection{Introducción}

Las modelos de predicción basados en procesos Gaussianos permiten emular la respuesta de modelos de simulación deterministas de alto coste computacional, aportando información acerca de la incertidumbre en la predicción. Estos modelos pueden actuar bien como aproximaciones globales en la totalidad del espacio de diseño, o bien integrarse en diferentes etapas de procesos de optimización. En este último caso, conocido en la literatura como diseño óptimo basado en modelos sustitutos (surrogate-based design optimization), los meta-modelos se crean durante el proceso de optimización para aproximar de forma precisa el modelo de simulación en ciertas regiones del espacio de diseño (ej. regiones cercanas al óptimo).

Este capítulo se centra en la utilización de la información estadística proporcionada por los procesos Gaussianos (media y varianza de la predicción) para una resolución eficiente de problemas de optimización $\mathrm{MO}$ con modelos de alto coste computacional. Con el término resolución eficiente se hace referencia a la resolución del problema de optimización utilizando el mínimo número de evaluaciones del modelo de simulación. Los métodos que abordan este problema son habitualmente referidos en la literatura como algoritmos de optimización basados en análisis Bayesiano (Bayesian analysis algorithms) (Mockus et al., 1978; Zilinskas, 1975) o métodos de aprendizaje activo (active learning) (Zuluaga et al., 2013). En este capítulo se aborda el problema de optimización multi-objetivo subyacente en la formulación del problema de diseño óptimo robusto (sección 2.4). En este caso, el acoplamiento entre los procesos de propagación de incertidumbre y de optimización puede incrementar el coste computacional drásticamente. Para aliviar dicho problema, en esta tesis se plantea la utilización de modelos de predicción Kriging para aproximar la estadística del problema en el espacio de diseño. La información estadística proporcionada por el modelo de predicción permite estimar la esperanza de mejora del frente de soluciones no-dominadas del problema $\mathrm{MO}$ en cada punto del espacio de diseño. Dicho criterio puede ser utilizado para guiar el proceso de selección de nuevos puntos candidatos a formar parte de la muestra de observaciones inicial, mejorando el meta-modelo en las regiones cercanas al conjunto de soluciones óptimas.

El resto de la sección presenta el problema de optimización multi-objetivo con procesos Gaussianos y una revisión del estado del arte del mismo. La sección 4.2 contiene algunas definiciones básicas del problema de optimización multi-objetivo necesarias para la familiarización con la notación utilizada en el resto del capítulo. La sección 4.3 revisa algunos criterios heurísticos, utilizados en el problema de optimización con un único objetivo, directamente relacionados con el criterio multi-objetivo propuesto. La sección 4.4 propone un procedimiento para la resolución eficiente del problema MO basada en la maximización de la mejora esperada del hipervolumen dominado respecto del frente de Pareto aproximado. El procedimiento propuesto se aplica a la resolución de múltiples benchmarks (ver 4.5) adaptados de la literatura y se compara con conocidos 


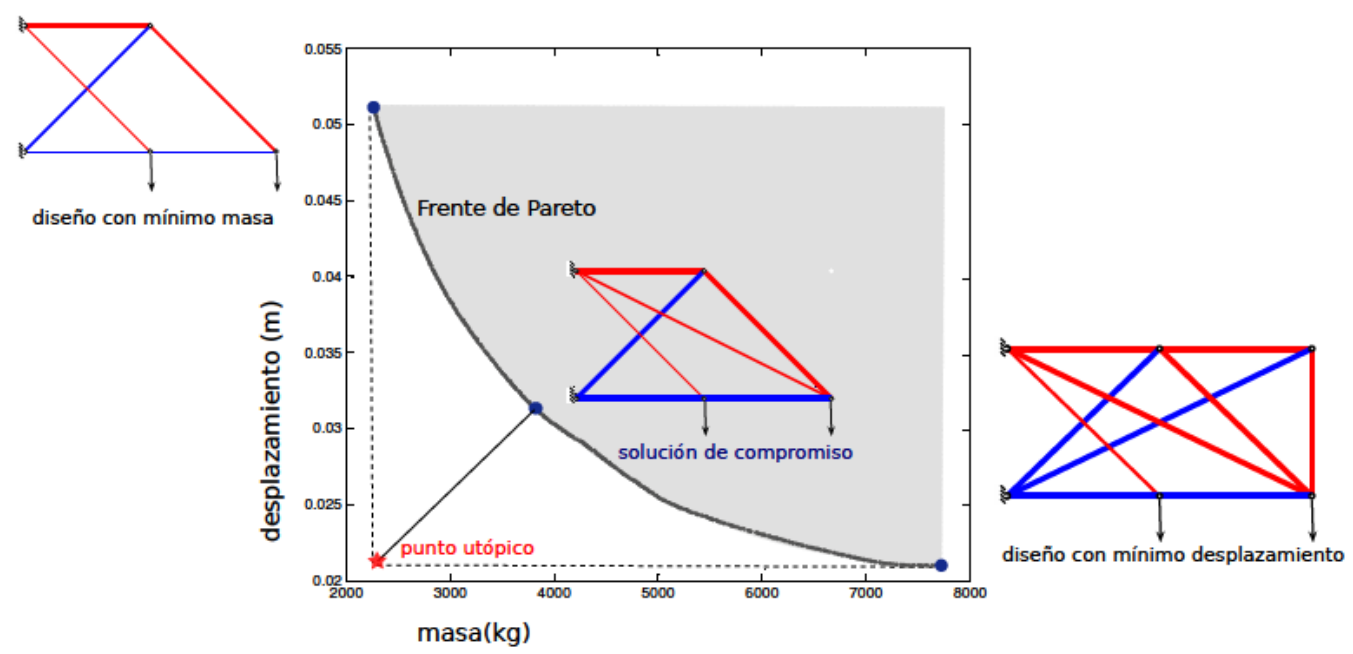

Figura 4.1: Ejemplo del frente de Pareto en el espacio de funciones objetivo masa y desplazamiento máximo para una estructura de 13 barras.

algoritmos de optimización MO como el NSGA-II (Deb et al., 2002). Finalmente, la sección 4.6 presenta las conclusiones del capítulo.

\subsubsection{Formulación del problema}

En muchos problemas de ingeniería es necesario encontrar un equilibrio entre varios objetivos. Por ejemplo, en el campo de la ingeniería estructural es habitual buscar soluciones estructurales con poco peso, bajo coste, alta fiabilidad frente a eventos extremos o baja sensibilidad frente a variaciones en las condiciones de diseño. Habitualmente las soluciones óptimas de estos objetivos divergen y no existe un único diseño que resulte óptimo para todos ellos. Por este motivo es necesaria la utilización de un criterio de optimalidad que permita obtener un conjunto de diseños que representen el mejor compromiso entre los diversos objetivos.

El criterio de optimalidad más ampliamente utilizado en la literatura es el de óptimo de Pareto. Formalmente, un conjunto de soluciones óptimas de Pareto son aquellas para las cuales no es posible mejorar alguna de las funciones objetivo sin empeorar al menos otra. Por este motivo, el conjunto de soluciones óptimas de Pareto también se denomina soluciones no-dominadas, dado que no existe ningún otro diseño que mejore simultáneamente el valor de todas las funciones objetivo. La Figura 4.1 ilustra este concepto para el problema clásico de minimización de masa y desplazamiento de una estructura de 13 barras. El lugar geométrico del conjunto de soluciones en el espacio de las funciones objetivo se denomina frente de Pareto. Todos los puntos que quedan por encima y a la derecha de dicho frente son soluciones dominadas por los puntos del frente. 


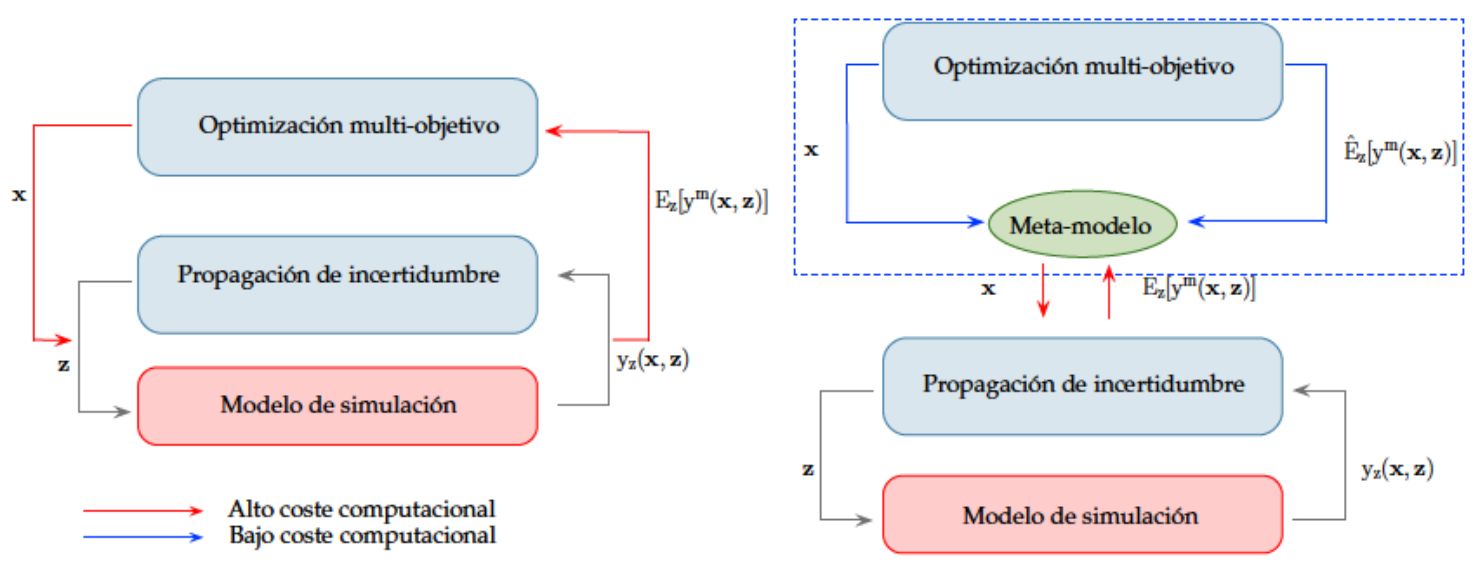

Figura 4.2: Diagrama de flujo del problema optimización Multi-objetivo asistida por procesos Gaussianos en el contexto de problemas de optimización bajo incertidumbre.

El problema que se aborda en este capítulo consiste en estimar el frente de soluciones óptimas de Pareto con un bajo coste computacional, es decir minimizando el número de evaluaciones del modelo de simulación tanto como sea posible.

El interés del problema propuesto se justifica a partir de las dificultades reconocidas en la literatura para la resolución del problema MO (ver sección 4.1.2). En primer lugar, a diferencia del problema con un único objetivo, la solución del problema MO está formada por un conjunto de diseños óptimos. El orden de magnitud del coste computacional del proceso de optimización es mayor, bien por un masivo número de evaluaciones (ej. algoritmos evolucionarios multi-objetivo) o bien por la necesidad de resolver varios subproblemas de optimización (ej. métodos de escalarización). Adicionalmente, el coste computacional se puede incrementar drásticamente cuando se utilizan modelos de simulación de alta fidelidad, o cuando son necesarios métodos de propagación de incertidumbre anidados en el proceso de optimización (ver Figura 4.2). Por motivos prácticos, para este tipo de aplicaciones el número de evaluaciones del modelo de simulación o del proceso de propagación de incertidumbre es limitado.

\subsubsection{Revisión del estado del arte y perspectivas}

La revisión del estado del arte se realiza de mayor a menor generalidad comenzado por: (1) métodos de resolución del problema $\mathrm{MO}$, (2) métodos de resolución del problema $\mathrm{MO}$ basados en meta-modelos y (3) algoritmos de optimización MO basados en análisis Bayesiano.

Métodos de resolución del problema MO. En la actualidad existen una gran variedad de métodos que tratan el problema de optimización MO. Algunos de estos métodos se muestran en la Tabla 4.1, donde se clasifican en tres categorías atendiendo a la forma en la que combinan las etapas de búsqueda de soluciones 
óptimas y toma de decisiones. Los métodos a priori (toma de decisión previa a la búsqueda) requieren información acerca de la toma de decisiones al comienzo del proceso de optimización, ponderando o al menos clasificando cada función objetivo. Por otra parte, los métodos progresivos incluyen la etapa de toma de decisiones durante la búsqueda de candidatos. El número de métodos englobados en esta categoría es bajo, debido a que dichos métodos requieren de la intervención del usuario durante la optimización. Finalmente, en los métodos a posteriori la toma de decisión se realiza al finalizar el proceso de optimización, una vez el conjunto de soluciones óptimas de Pareto es completamente conocido. Una revisión en detalle de cada uno de estos métodos queda fuera del alcance de este trabajo, y se puede encontrar en publicaciones como las de Collette y Siarry (2003) y Marler y Arora (2004).

\begin{tabular}{ccc}
\hline Métodos a priori & Métodos a posteriori & Métodos progesivos \\
\hline \hline Weighted global criterion method & Physical programming & Surrogate Worth Tradeoff \\
Weighted sum method & Normal boundary intersection method & Fradel method \\
Lexicographic method & Normal constraint method & Step method \\
Weighted min-max method & Multi-objective evolutionary algorithms & Jahn method \\
Exponential weighted criterion & & Geoffrion method \\
Weighted product method & & Simplex method \\
Goal programing methods & & \\
Bounded objective function method & & \\
Physical programming & & \\
\hline
\end{tabular}

Tabla 4.1: Principales métodos de resolución del problema de optimización multi-objetivo.

Entre todos los métodos recogidos en la Tabla 4.1, los métodos a priori y los métodos a posteriori son los que han recibido una mayor atención para la obtención de soluciones óptimas de Pareto. Los primeros representan la forma más directa y simple de abordar el problema. Transforman el problema multi-objetivo en un problema con un único objetivo mediante una función de escalarización. La modificación recursiva de la función de escalarización permite la obtención de diferentes puntos del frente de Pareto. Sin embargo, no está claro a priori la función de escalarización a utilizar (ej. los pesos en el caso de una suma ponderada), ni tampoco como debe ser modificada dicha función para obtener un conjunto de soluciones óptimas uniformemente distribuidas en el frente. Esta deficiencia se hace especialmente patente en métodos de escalarización basados en una suma líneal ponderada (Weighted sum method), en los que no es posible encontrar puntos óptimos en regiones no convexas del frente (Boyd y Vandenberghe, 2004). A pesar de que este problema puede ser resuelto mediante la utilización de funciones de escalarización no líneales (ej. función de escalarización de Tchebycheff), la necesidad de resolver varios problemas de optimización descarta este tipo de métodos en esta tesis. 
Por otra parte, entre los métodos a posteriori existen métodos como los algoritmos evolucionarios multi-objetivo (MultiObjective Evolutionary Algorithms (MOEAs)) (Coello et al., 2002) que intentan evitar las limitaciones de los métodos de escalarización. Estos algoritmos no necesitan definir de forma explícita una función de escalarización a priori para combinar las funciones objetivo. Dentro de este grupo, algoritmos como el Strength Pareto Evolutionary Algorithm 2 (SPEA2) propuesto por Zitzler et al. (2002), el S-Metric Selection Evolutionary MultiObjective Algorithm (SMS-EMOA2) propuesto por Beume et al. (2007), y principalmente el Nondominated Sorting Genetic Algorithm II (NSGA-II) propuesto por Deb et al. (2002) han ganado una gran popularidad en los últimos años.

Métodos de resolución del problema MO basados en meta-modelos. Los métodos basados en algoritmos evolucionarios resuelven gran parte de las limitaciones existentes en los métodos de escalarización y permiten obtener una aproximación del frente de Pareto uniforme en un solo proceso de optimización. Sin embargo, requieren un gran número de evaluaciones de las funciones objetivo. Esto resulta inabordable o al menos poco práctico en problemas con modelos de simulación de alto coste computacional. Por este motivo, existen trabajos en la literatura que buscan minimizar dicho número de evaluaciones integrando meta-modelos en diferentes etapas de la optimización. En su mayoría, estos trabajos buscan la integración de meta-modelos junto con algoritmos evolucionarios multi-objetivo (Zhang et al., 2010). Gaspar-Cunha y Vieira (2004) utilizan redes neuronales para aproximar localmente la función objetivo y generar nuevos puntos de búsqueda de forma eficiente. Wang y Shan (2005) presentan el algoritmo Pareto Set Pursuing (PSP) en el que utilizan superficie de respuesta polinómicas junto con una estrategia de diseño de experimentos adaptativo para aproximar el frente de Pareto. Voutchkov y Keane (2006) utilizan modelos Kriging como sustitutos del modelo de simulación junto con el algoritmo NSGA-II. En su trabajo, Voutchkov y Keane construyen un meta-modelo para cada función objetivo a partir de una muestra de puntos inicial. El algoritmo NSGA-II es utilizado sobre los meta-modelos para la búsqueda del frente de soluciones óptimas de Pareto. Los diseños obtenidos son utilizados como puntos candidatos para la actualización y mejora de los meta-modelos. Siguiendo esta línea otros autores como Martínez-Frutos y Martí (2011) proponen una estrategia jerárquica de meta-modelos junto con el algoritmo NSGA-II para la resolución del problema de diseño óptimo robusto multi-objetivo. Utilizan modelos Kriging para emular los momentos estadísticos de la respuesta estructural. Al igual que Voutchkov y Keane, Martínez-Frutos y Martí utilizan parte de los puntos perteneciente al frente de Pareto para refinar el meta-modelo. Li et al. (2008) y Li et al. (2009) proponen una mejora respecto de los algoritmos genéticos multi-objetivo denominada Kriging-with-DOE MOGA (KG-MOGA), en la que se utilizan modelos Kriging para aproximar la función de aptitud (fitness) de cada individuo, junto con técnicas de diseño de experimentos adaptativas para asistir el proceso de reproducción. Nain y Deb (2005) presentan una estrategia basada en el algoritmo NSGA-II y redes neuronales (Nondominated Sorting Genetic Algorithm 
II \& Artificial Neural Network (NSGA-II-ANN)). El algoritmo NSGA-II-ANN utiliza el modelo de simulación durante $n$ generaciones del algoritmo NSGA-II y un modelo de redes neuronales durante las $m-n$ generaciones siguientes. Cada $m$ generaciones se ajusta un nuevo meta-modelo a partir de las muestras obtenidas del modelo de simulación. Emmerich (2005) propone un algoritmo basado en el SMS-EMOA2 y asistido por meta-modelos. Utilizan el hipervolumen como criterio de selección junto con procesos Gaussianos. Una revisión en mayor detalle se puede encontrar en los trabajos de citetKnowles2008 y Zhou et al. (2011).

Algoritmos de optimización MO basados en análisis Bayesiano. Los trabajos presentados anteriormente utilizan parte de soluciones no-dominadas para la actualización del meta-modelo. Esta forma de proceder proporciona en ocasiones soluciones satisfactorias convergiendo al frente de Pareto real (Gaspar-Cunha y Vieira, 2004; Martínez-Frutos y Martí, 2011; Voutchkov y Keane, 2006). Sin embargo, al igual que ocurre en el problema con un único objetivo, dicho enfoque únicamente se basa en las soluciones óptimas para la actualización del meta-modelo (explotación) y no considera la incertidumbre epistémica del mismo (exploración). Para obtener unos resultados satisfactorios es necesario incorporar dicha incertidumbre durante la optimización, de otro modo es probable que el algoritmo de optimización converja hacia falsos óptimos (Ulmer et al., 2003). Este problema ha sido ampliamente tratado en la literatura mediante la utilización de algoritmos de optimización basados en análisis Bayesiano (Mockus et al., 1978; Zilinskas, 1975). En estos algoritmos, la función objetivo se emula mediante un proceso estocástico (generalmente un proceso Gaussiano o modelo Kriging). El proceso estocástico permite realizar hipótesis a priori sobre las características de la función a aproximar (ej. selección de funciones de correlación y regresión) y guiar la selección de nuevos puntos (infill points) en el espacio de diseño mediante la utilización de criterios heurísticos (infill sampling criterion) basados en la predicción de incertidumbre del modelo. En el campo de optimización con un único objetivo, el algoritmo Efficient Global Optimization (EGO) propuesto por Jones et al. (1998) es uno de los que gozan de una mayor popularidad. El algoritmo EGO utiliza el criterio de mejora esperada generalizada (Generalize Expected Improvement (GEI)) para seleccionar puntos en regiones del espacio con alta incertidumbre (exploración) o en regiones donde es altamente probable mejorar la función objetivo (explotación). A partir del trabajo original de Jones et al. (1998), son numerosas las aportaciones en este campo. Dichas aportaciones abordan problemas como el tratamiento de las restricciones de desigualdad (Sasena et al., 2000), la convergencia de diferentes criterios heurísticos (Sasena et al., 2002) o la búsqueda simultánea de varios puntos candidatos (Sóbester et al., 2004; Viana y Haftka, 2010). Una excelente revisión del estado del arte en este campo se encuentra en Forrester et al. (2008) y Forrester y Keane (2009).

A pesar del gran desarrollo mostrado en el campo de optimización con un único objetivo, las técnicas de optimización basadas en análisis Bayesiano no están tan extendidas desde el punto de vista multi-objetivo. Los trabajos 
desarrollados en esta línea son de reciente aparición y se pueden clasificar en dos categorías. En primer lugar, existen enfoques que transforman el problema multi-objetivo en un problema con un único objetivo y posteriormente utilizan algoritmos de optimización Bayesiana sobre el problema escalarizado. Este es el caso del algoritmo ParEGO propuesto por Knowles (2006). El algoritmo ParEGO es una extensión del EGO Jones (2001) en la que se utilizan modelos Kriging y el criterio de mejora esperada para optimizar una suma ponderada de las funciones objetivo. Zhang et al. (2010) proponen el método MOEA/D-EGO. Descomponen el problema multi-objetivo en un conjunto de subproblemas para los cuales se construye un modelo de predicción basado en procesos Gaussianos. Los nuevos candidatos se seleccionan a partir del criterio de mejora esperada. Martínez-Frutos y Martí (2013) adaptan el algoritmo EGO junto con un método de escalarización aumentado de Tchebycheff para la resolución del problema de diseño óptimo robusto multi-objetivo. Hawe y Sykulski (2008) revisan las posibilidades de la utilización de métodos de escalarización junto con modelos Kriging para la resolución del problema de optimización multi-objetivo. El principal defecto de los enfoques basados en la escalarización del problema reside en que el criterio heurístico utilizado para la búsqueda de candidatos funciona para dicha función de escalarización, pero su convergencia no está garantizada para el problema MO.

Frente a los enfoques anteriores, existe otra línea de investigación cuyo objetivo es desarrollar criterios heurísticos multi-objetivo. A diferencia del problema de optimización con un único objetivo, en el caso multi-objetivo no es evidente el criterio heurístico a utilizar para evaluar la calidad de la predicción. Los criterios heurísticos multi-objetivo existentes en la literatura se clasifican en tres grupos:

- La mejora esperada con respecto a un valor deseado de las funciones objetivo. Jeong et al. (2006) proponen una extensión del algoritmo EGO para problemas multi-objetivo denominada EGOMOP, que evalúa la mejora esperada de cada función objetivo y maximiza múltiples criterios. Bautista (2009) propone el algoritmo Emax en el que se utilizan procesos Gaussianos que guían la búsqueda de soluciones óptimas a través de la máxima mejora esperada del frente de Pareto.

- La probabilidad de mejora respecto del conjunto de soluciones no-dominadas. Keane (2006) presenta versiones multi-objetivo de los criterios de probabilidad de mejora y de mejora esperada para problemas con dos funciones objetivo. Hawke y Sykulski (2008) proponen el concepto de nivel de mejora (Level of Improvement LoI), en el que se tiene en cuenta la cantidad de puntos del frente de Pareto que son dominados por un nuevo punto. En base a esta idea, Couckuyt et al. (2012) definen varios criterios estadísticos entre los que se encuentra la probabilidad de mejora con un mínimo nivel de mejora de tres (probabilidad de que un nuevo punto domine al menos tres puntos del actual frente de Pareto). 


\begin{tabular}{|c|c|c|c|c|}
\hline Autor & Algoritmo & Criterio & Escalarización & Restricciones \\
\hline 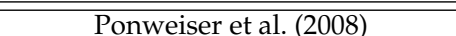 & SMS-EGO & 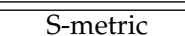 & $\overline{\mathrm{N} \text { No }}$ & $\overline{\mathrm{No}}$ \\
\hline Obayashi (2006) & Multi-EGO & $\mathrm{EI}^{(2)}$ & No & No \\
\hline Henkenjohann y Kunert (2007) & $(-)$ & $\mathrm{EIDI}^{(7)}$ & Sí & No \\
\hline Couckuyt et al. (2012) & EMO & $\mathrm{PoI}^{(1)}+\mathrm{LoI}^{(3)}$ & No & No \\
\hline Keane (2006) y Forrester et al. (2008) & $(-)$ & $\mathrm{PoI}^{(1)} / \mathrm{EI}^{(2)}$ & Sí & No \\
\hline Bautista (2009) & EMAX & EPI $^{(6)}$ & No & No \\
\hline Jeong et al. (2006) & EGOMOP & $\mathrm{EI}^{(2)}$ & Sí & No \\
\hline Knowles (2006) & ParEGO & $\mathrm{EI}^{(2)}$ & Sí & No \\
\hline Zhang y Leithead (2005) & MOEA-EGO & $E I^{(2)}$ & Sí & No \\
\hline Zhang et al. (2010) & MOEA/D-EGO & $E I^{(2)}$ & Sí & No \\
\hline Hawe y Sykulski (2008) & GEI+WMM ${ }^{(5)}$ & $\mathrm{GEI}^{(4)}$ & Sí & No \\
\hline
\end{tabular}

Tabla 4.2: Resumen de los algoritmo de optimización MO basados en análisis Bayesiano.

- La mejora esperada en el hipervolumen con respecto al conjunto de soluciones no-dominadas (Emmerich et al., 2008). Se basan en la utilización del denominado hipervolumen (Zitzler et al., 2007) para evaluar el volumen encerrado por el frente de Pareto real y su predicción. A diferencia de los criterios anteriores que se centran en la mejora de cada función objetivo, el criterio basado en la mejora esperada del hipervolumen busca la mejora del conjunto de soluciones no-dominadas en el espacio de las funciones objetivo. Ponweiser et al. (2008) proponen el algoritmo SMS-EGO, en el que combinan el método EGO con una métrica (S-metric) basada en el hipervolumen. Utilizan el algoritmo Covariance Matrix Adaptation Evolution Strategy (CMA-ES) para maximizar el criterio de búsqueda.

En la Tabla 4.2 se recoge un resumen de los principales algoritmos de optimización MO basados en análisis Bayesiano. Analizando las características de los algoritmos presentados, se pueden extraer las siguientes conclusiones y posibles líneas de mejora:

- La mayoría de los algoritmos abordan el problema MO mediante su escalarización a un problema con un único objetivo, utilizando criterios como la mejora esperada o la probabilidad de mejora para la actualización de los meta-modelos.

- Los algoritmos consideran únicamente restricciones en los límites de las variables de diseño. No consideran restricciones de alto coste computacional aproximadas por meta-modelos.

- Los algoritmos que abordan el problema utilizando criterios heurísticos multi-objetivo son escasos. No existe en la actualidad un trabajo que estudie y compare de forma exhaustiva el funcionamiento de los diferentes criterios, y es necesaria una mayor investigación para establecer conclusiones definitivas acerca de las bondades y de los defectos de los 
diferentes criterios. Sin embargo, los trabajos iniciales de Ponweiser et al. (2008) y Couckuyt et al. (2012) muestran que el criterio basado en la mejora esperada del hipervolumen mantiene un equilibrio entre la eficiencia y la uniformidad del frente de soluciones no-dominadas en comparación con el criterio de mejora esperada de las funciones objetivo. En esta tesis se sigue la línea propuesta por Ponweiser et al. (2008) y se estudia la utilización de un criterio basado en el indicador del hipervolumen dominado.

- La evaluación del hipervolumen dominado requiere una primera aproximación del frente de Pareto, es decir la existencia de soluciones factibles entre la muestra inicial utilizada para el ajuste del meta-modelo. Este problema no ha recibido especial atención en la literatura debido a que los trabajos que abordan el problema $\mathrm{MO}$ solo consideran restricciones en los límites de las variables de diseño. En este caso todos los puntos de la muestra inicial son factibles y es posible aproximar el frente de soluciones no dominadas sin especial dificultad. Sin embargo, cuando existen restricciones aproximadas por meta-modelos, el número de diseños factibles puede ser inexistente o insuficiente para generar una primera aproximación del frente. El tratamiento de la incertidumbre de los meta-modelos de las restricciones en el contexto $\mathrm{MO}$, así como la búsqueda eficiente de puntos factibles en el espacio de diseño, son áreas en las que los actuales algoritmos de optimización MO pueden ser mejorados y/o extendidos.

En este capítulo se propone una metodología para la resolución eficiente del problema de optimización $\mathrm{MO}$ en el contexto del diseño óptimo robusto. En comparación con los algoritmos existentes en la literatura, la principal contribución de la metodología propuesta es la inclusión de restricciones de alto coste computacional en el problema. En concreto se tratan dos problemas: (1) como incluir la incertidumbre de los meta-modelos de las restricciones en el criterio de búsqueda y (2) como buscar puntos de diseño factible de forma eficiente cuando el espacio factible es incierto.

\subsection{Definiciones previas}

En esta sección se presenta la formulación del problema de optimización MO junto con algunas definiciones relacionadas con la solución. Las definiciones presentadas son adaptadas de las publicaciones de Arora (1989) y Ehrgott (1997) por lo que una descripción en mayor detalle puede encontrarse en dichas referencias. 


\subsubsection{Formulación del problema multi-objetivo}

El problema de optimización multi-objetivo se formula como sigue:

$$
\begin{array}{cl}
\min _{\mathbf{x}} \mathbf{f}(\mathbf{x})= & \left\{f_{1}(\mathbf{x}), f_{2}(\mathbf{x}), \ldots, f_{n_{f}}(\mathbf{x})\right\} ; \\
\text { s. a. : } \quad g_{j}(\mathbf{x}) \leq 0 ; \quad j \in \mathbb{R}^{\mathrm{n}} . & \quad j=1, \ldots, n_{g}, \\
& h_{k}(\mathbf{x}) \leq 0 ; \quad k=1, \ldots, n_{h}, \\
& x_{i}^{\text {inf }} \leq x_{i} \leq x_{i}^{\text {sup }} ; i=1, \ldots, n,
\end{array}
$$

donde: $\mathbf{x}$ es el vector de variables de diseño; $f(\mathbf{x})$ es la función objetivo; $g_{j}(\mathbf{x})$ es la restricción de desigualdad j-ésima; $h_{k}(\mathbf{x})$ es la restricción de igualdad $k$-ésima; $n_{g}(\mathbf{x})$ es el número de restricciones de desigualdad; $n_{h}(\mathbf{x})$ es el número de restricciones de igualdad; $x_{i}^{\text {inf }}$ es el límite inferior de la variable de diseño i-ésima; $x_{i}^{\text {sup }}$ es el límite suprior de la variable de diseño i-ésima; y $n$ es el número de variables de diseño.

Definición (optimalidad de Pareto). Un vector de diseño $\mathbf{x}^{*} \in \mathscr{D}_{\text {fact }}$ es un óptimo de Pareto si y solo si no existe otro $\mathbf{x} \in \mathscr{D}_{\text {fact }}$ tal que:

$$
f_{i}(\mathbf{x}) \leq f_{i}\left(\mathbf{x}^{*}\right) \forall i=1, \ldots, n_{f},
$$

con $f_{i}(\mathbf{x})<f_{i}\left(\mathbf{x}^{*}\right)$ para al menos una función objetivo i. $\mathscr{D}_{\text {fact }}$ es el espacio de diseño factible.

Definición (dominancia de Pareto fuerte). Un vector $\mathbf{u}$ se dice que domina estrictamente a $\mathbf{v}(\mathbf{u}<<\mathbf{v})$ si y solo $s i \forall i \in\left\{1, \ldots, n_{f}\right\}, u_{i}<v_{i}$.

Definición (dominancia de Pareto). Un vector $\mathbf{u}$ se dice que domina a $\mathbf{v}(\mathbf{u}<\mathbf{v})$ si y solo si $\forall i \in\left\{1, \ldots, n_{f}\right\}, u_{i} \leq v_{i}$ y para al menos un $i \in\left\{1, \ldots, n_{f}\right\}: u_{i}<v_{i}$.

Definición (dominancia de Pareto débil). Un vector u se dice que domina débilmente a $\mathbf{v}(\mathbf{u} \leq \mathbf{v})$ si y solo si $\forall i \in\left\{1, \ldots, n_{f}\right\}, u_{i} \leq v_{i}$.

Definición (incompatibilidad). Dos vectores $\mathbf{u}$ y $\mathbf{v}$ son incompatibles si $\mathbf{u} \not \mathbf{v} \mathrm{y}$ $\mathbf{v} \npreceq \mathbf{u}$.

Definición (punto utópico). Un punto $\mathbf{f}^{\circ}$ en el espacio definido por las funciones objetivo se denomina punto utópico si $\mathbf{f}^{\circ}=\min \left[f_{i}(\mathbf{x})\right] \forall \mathbf{x} \in \mathscr{D}_{\text {fact }} \wedge \forall i=1, \ldots, n_{f}$. $\mathrm{Al}$ punto utópico también se le conoce como punto ideal.

Definición (conjunto de óptimos de Pareto). Al conjunto de todas las soluciones no-dominadas se le denomina: conjunto de óptimos de Pareto $\mathbf{P}^{*}$ :

$$
\mathbf{P}^{*}=\left\{\mathbf{x}^{*} \in \mathscr{D}_{\text {fact }} \mid \nexists \mathbf{x} \in \mathscr{D}_{\text {fact }} \text { tal que } \mathbf{x} \leq \mathbf{x}^{*}\right\} .
$$


Definición (frente de Pareto). La representación gráfica del conjunto de óptimos de pareto $\mathbf{P}^{*}$ en el espacio definido por las funciones objetivo se denomina frente de Pareto (FP).

Definición (solución de compromiso). La mejor solución en el conjunto de óptimos de Pareto es la más cercana al punto utópico. Dicha solución se denomina solución de compromiso. Habitualmente el criterio considerado para determinar la proximidad de la solución al punto utópico es la distancia Euclídea $D(\mathbf{x})$ entre ambos puntos en el espacio de las funciones objetivo:

$$
D(\mathbf{x})=\left\|\mathbf{f}(\mathbf{x})-\mathbf{f}^{o}\right\|=\left\{\sum_{i=1}^{k}\left[f_{i}(\mathbf{x})-f_{i}^{o}\right]\right\}^{1 / 2} .
$$

\subsection{Criterios heurísticos para la optimización Bayesiana mono-objetivo}

La utilización de técnicas de análisis Bayesiano en optimización comienza a ganar en popularidad a raíz de los trabajos de Jones et al. (1998) y Mockus et al. (1978) a comienzos de la década de los 90. El objetivo es la resolución de problemas de optimización global con funciones objetivo que involucran modelos de simulación de alto coste computacional.

Los métodos de optimización basados en análisis Bayesiano asumen que la función objetivo se aproxima por un proceso Gaussiano:

$$
\hat{f}(\mathbf{x}) \sim \mathscr{N}\left(\mu_{\hat{f}}(\mathbf{x}), \sigma_{\hat{f}}^{2}(\mathbf{x})\right) .
$$

A diferencia de los algoritmos de optimización convencionales, los algoritmos basados en análisis Bayesiano utilizan la información proporcionada por el modelo de predicción Gaussiano para buscar puntos que mejoren el modelo de predicción en su búsqueda del óptimo. Dicha búsqueda se realiza a partir de criterios heurísticos tales como la probabilidad de mejora (Probability of Improvement (PoI)) o la mejora esperada (Expected Improvement (EI)).

\subsubsection{Probabilidad de mejora}

Dado un conjunto de observaciones inicial $\mathscr{D}=\left\{\left(\mathbf{x}^{(i)}, f^{(i)}\right) \forall i=1, \ldots, m\right\}$ y un modelo de predicción inicial $\hat{f}(\mathbf{x}) \sim \mathscr{N}\left(\mu_{\hat{f}}(\mathbf{x}), \sigma_{\hat{f}}^{2}(\mathbf{x})\right)$, Jones et al. (1998) definen la función de mejora como:

$$
\mathrm{I}(\mathbf{x}) \equiv\left\{\begin{array}{cl}
f_{\min }-\mu_{\hat{f}}(\mathbf{x}) & \text { si } \mu_{\hat{f}}(\mathbf{x}) \leq f_{\text {min }} \\
0 & \text { si } \mu_{\hat{f}}(\mathbf{x})>f_{\text {min }}
\end{array}\right.
$$




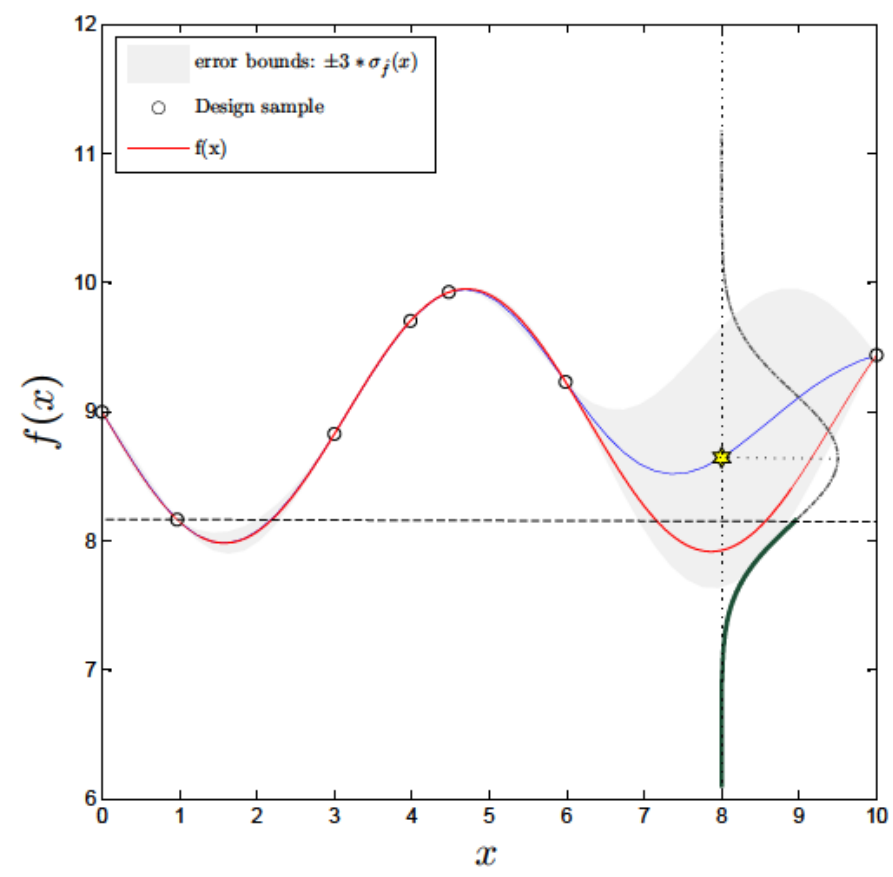

Figura 4.3: Interpretación gráfica de la probabilidad de mejora.

donde $f_{\min }=\min \left\{f^{(i)}=\mathscr{M}\left(\mathbf{x}^{(i)}\right), i=1, \ldots, m\right\}$ es el mínimo valor de las observaciones del modelo de simulación $(\mathscr{M})$ existente en el conjunto inicial $\mathscr{D}$. Dado que el modelo de predicción es Gaussiano, la función I(x) es también Gaussiana y truncada para valores positivos. Utilizando la función de densidad de probabilidad, la probabilidad de mejora se puede evaluar como:

$$
\operatorname{PoI}(\mathbf{x})=\mathbb{P}[\mathrm{I}(\mathbf{x})]=\frac{1}{\sigma_{f}(\mathbf{x}) \sqrt{2 \pi}} \int_{-\infty}^{0} \exp \frac{-\left(\mathrm{I}(\mathbf{x})-\mu_{f}(\mathbf{x})^{2}\right.}{2 \sigma_{\hat{f}}^{2}(\mathbf{x})} \mathrm{dI}(\mathbf{x}) .
$$

La ecuación (4.7) se puede analizar gráficamente en la Figura 4.3. En dicha figura la línea roja representa la función $y(x)=-\sin (x)-\exp (x / 100)+10$ en el intervalo $[0,10]$. La línea azul representa el valor medio de la predicción de la función $y(x)$ en dicho intervalo. Esta predicción se obtiene a partir de 7 observaciones (círculos blancos). La distribución de probabilidad vertical mostrada en la Figura 4.3 corresponde a una distribución normal con media $\mu_{\hat{y}}(x=8)$ y desviación estándar $\sigma_{\hat{y}}(x=8)$. En línea discontinua se representa el valor mínimo de las observaciones de la función $y(x)$ existente en el conjunto de 7 observaciones. La zona de la distribución bajo la línea discontinua representa la probabilidad de mejora en el punto $x=8$ respecto del mejor valor en el conjunto $\mathscr{D}$. 


\subsubsection{Mejora esperada}

En lugar de buscar regiones con cierta probabilidad de mejora, Jones et al. (1998) proponen cuantificar la cantidad de mejora esperada mediante la esperanza de la función I(x). La función de mejora esperada se define como:

$$
\mathrm{EI}(\mathbf{x})=\mathbb{E}[\mathrm{I}(\mathbf{x})]=\int_{-\infty}^{f_{\min }}\left(f_{\min }-f\right) \phi\left(\frac{f-\mu_{\hat{f}}(\mathbf{x})}{\sigma_{\hat{f}}^{2}(\mathbf{x})}\right) d f,
$$

y tras una integración por partes se obtiene la expresión:

$$
\mathrm{EI}(\mathbf{x})=\mathrm{E}[\mathrm{I}(\mathbf{x})] \equiv\left\{\begin{array}{cl}
\left(f_{\min }-\mu_{\hat{f}}(\mathbf{x})\right) \Phi\left(\frac{f_{\min }-\mu_{\hat{f}}(\mathbf{x})}{\sigma_{f}^{2}(\mathbf{x})}\right)+\sigma_{\hat{f}}^{2}(\mathbf{x}) \phi\left(\frac{f_{\min }-\mu_{f}(\mathbf{x})}{\sigma_{f}(\mathbf{x})}\right) & \text { si } \sigma_{\hat{f}}^{2}(\mathbf{x})>0, \\
0 & \text { si } \sigma_{\hat{f}}^{2}(\mathbf{x})=0,
\end{array}\right.
$$

donde $\Phi(\cdot)$ y $\phi(\cdot)$ son la función de distribución acumulada y la función de densidad de probabilidad respectivamente. La ecuación (4.9) se interpreta gráficamente en la Figura 4.3. En este caso, el valor de la mejora esperada viene dado por el área encerrada por la distribución Gaussiana y la línea discontinua.

\subsubsection{Algoritmo EGO (Efficient Global Optimization) (Jones et al., 1998)}

El algoritmo EGO propuesto por Jones et al. (1998) se basa en la búsqueda de nuevos puntos de actualización del meta-modelo mediante la maximización de la función de mejora esperada:

$$
\mathbf{x}^{*}=\arg \operatorname{máx}_{\mathbf{x} \in \mathbb{R}^{\mathrm{n}}} \operatorname{EI}(\mathbf{x}) .
$$

Tal y como se detalla en el Algoritmo 2, se requiere un espacio de variables de entrada, un diseño de experimentos inicial y un modelo de simulación que permita obtener nuevas observaciones. El algoritmo comienza con la evaluación del diseño de experimentos inicial con el modelo de simulación $\mathscr{M}$.

El conjunto de observaciones del modelo de simulación se emplea para ajustar el modelo Kriging mediante el estimador de máxima verosimilitud. El candidato a formar parte del diseño de experimentos en la siguiente iteración $($ It +1$)$ se obtiene maximizando la función de mejora esperada a partir de la información estadística proporcionada por el modelo de predicción. El modelo Kriging permite la evaluación vectorizada con un bajo coste computacional, por lo que se pueden utilizar técnicas de búsqueda exhaustiva para la resolución de este subproblema de optimización. En concreto, Jones et al. (1998) proponen el algoritmo Dividing Rectangles (DIRect) basado en la división del espacio de diseño n-dimensional en un conjunto de hiperrectangulos, los cuales se subdividen 


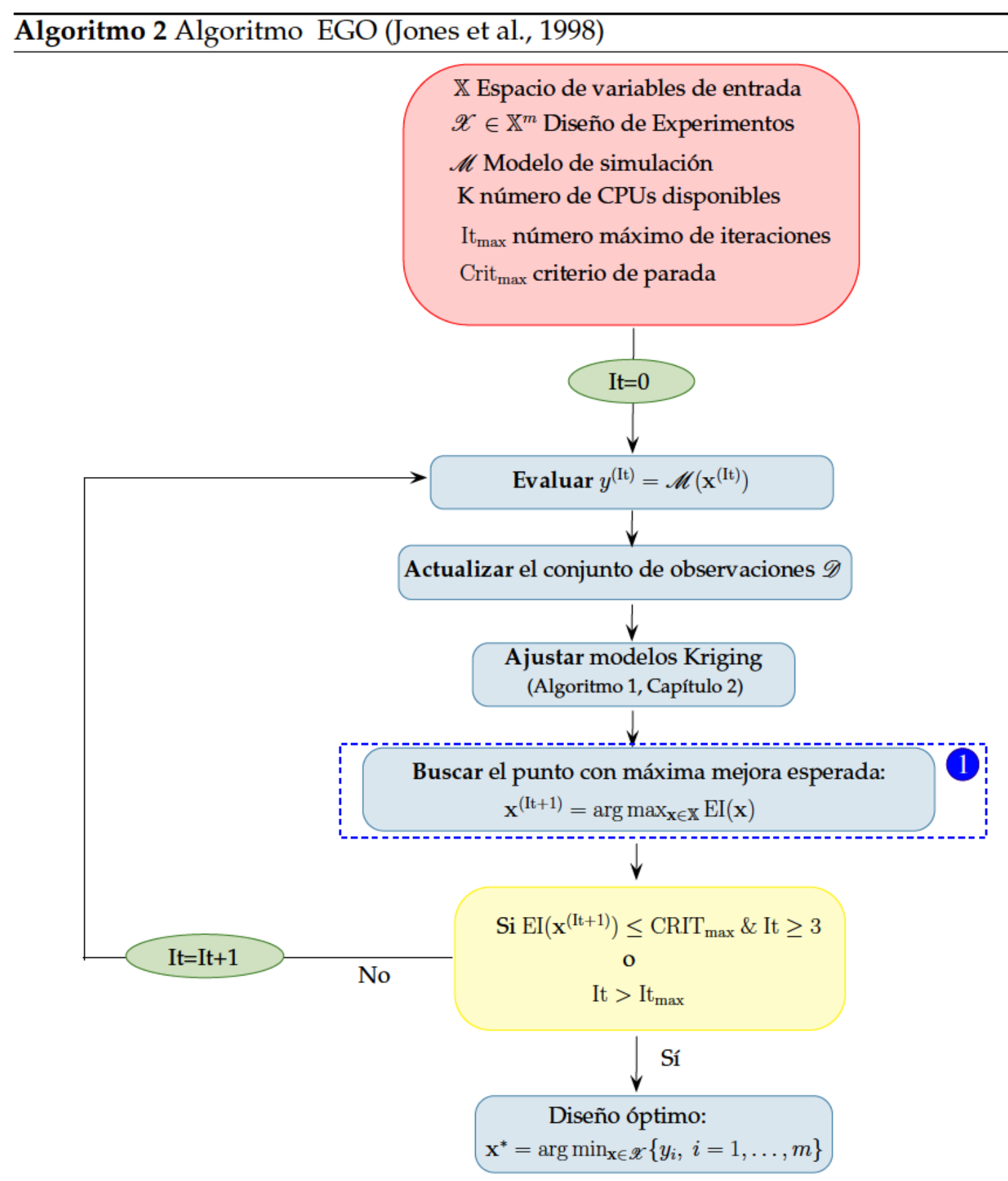

(1) Subproblema de optimización 
secuencialmente hasta alcanzar el diseño óptimo. El algoritmo EGO continúa hasta alcanzar un número máximo de iteraciones $\left(\mathrm{It}_{\max }\right)$ o verificar un cierto criterio de parada ( Crit $\left._{\max }\right)$.

La convergencia del algoritmo depende en gran medida de la capacidad del modelo de predicción de capturar el comportamiento de la respuesta del modelo de simulación. A partir del trabajo original de Jones et al. (1998), se han propuesto modificaciones encaminadas a mejorar sus propiedades de convergencia. Destaca la aportación de Schonlau (1997) que propone la modificación del criterio de búsqueda mediante la utilización de un criterio de mejora esperada generalizada GEI. Con este nuevo criterio incluyen un parámetro adicional para controlar explícitamente la exploración de zonas del espacio con alta incertidumbre en la predicción.

Ilustración 4.1. La Figura 4.4 muestra una ejecución del algoritmo EGO para la minimización de la función $y(x)=-\sin (x)-\exp (x / 100)+10$ en el intervalo [0,10]. La función unidimensional presenta dos mínimos locales: $f_{1}^{*}(x=1.5809)=7.9841$ y $f_{2}^{*}(x=7.8648)=7.9182$. El algoritmo finaliza tras 6 iteraciones cumpliendo con el criterio de parada $C$ rit $_{\max }=1 e-3$. En la iteración número 6 se alcanza un valor óptimo de $y(x=7.8635)=7.9182$, evitando el mínimo local situado en $x=1.5809$.

\subsection{Metodología propuesta}

A diferencia del problema con un único objetivo, en el caso multi-objetivo no está claro cual es el mejor criterio de búsqueda. En este trabajo se propone el algoritmo EHV-EMO, inspirado en el concepto de mejora esperada del hipervolumen dominado (Emmerich et al., 2008). El criterio propuesto se aplica a la resolución del problema de optimización $\mathrm{MO}$ con restricciones de alto coste computacional. La estrategia propuesta consiste en dos etapas que se describen a continuación.

- Etapa 1: aproximación inicial del frente de Pareto. La búsqueda de nuevas soluciones no dominadas se basa en la maximización de la mejora esperada del hipervolumen dominado. Dicho criterio requiere de la evaluación del hipervolumen respecto de un conjunto de soluciones no-dominadas, por lo que es imprescindible una primera aproximación del frente para iniciar el algoritmo. Sin embargo, en presencia de restricciones, el número de diseños factibles puede ser inexistente o insuficiente para generar una primera aproximación del frente. Por esta razón, la primera etapa consiste en la búsqueda eficiente de puntos factibles en el espacio de diseño utilizando la información estadística proporcionada por los modelos de predicción de las restricciones. 


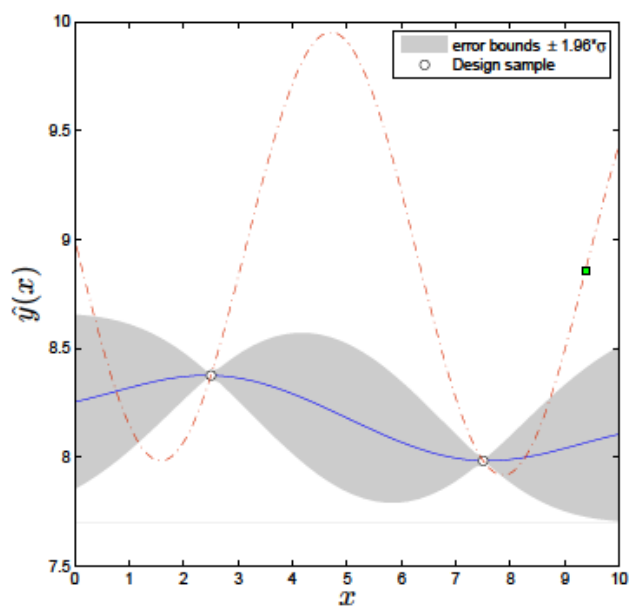

(a) Modelo Kriging (Iter=1)

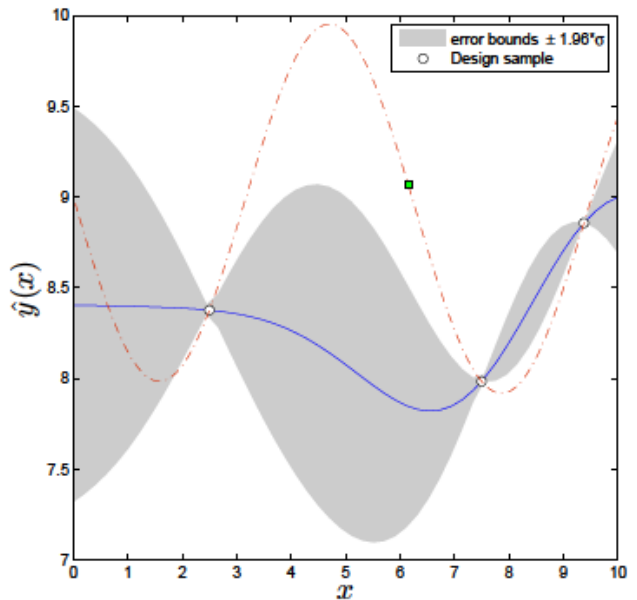

(c) Modelo Kriging (Iter=2)

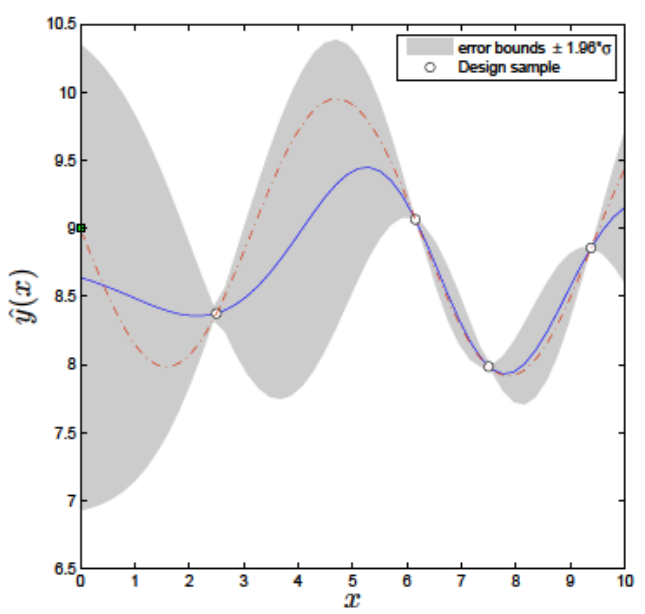

(e) Modelo Kriging (Iter=3)

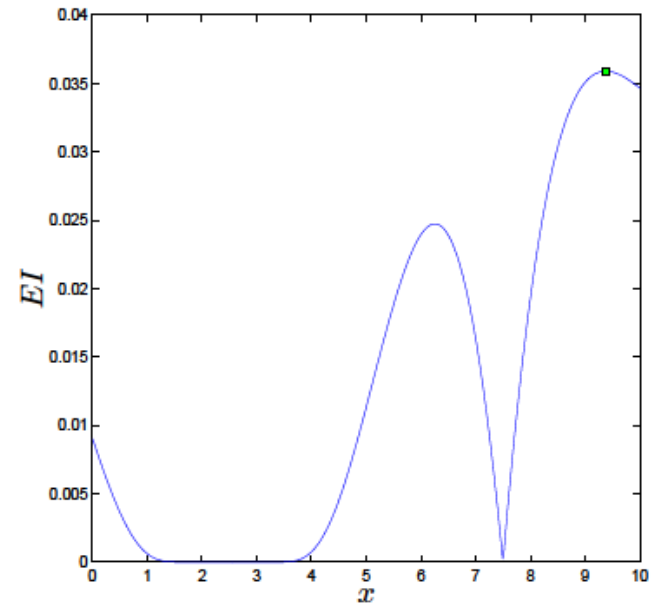

(b) EI (Iter=1)

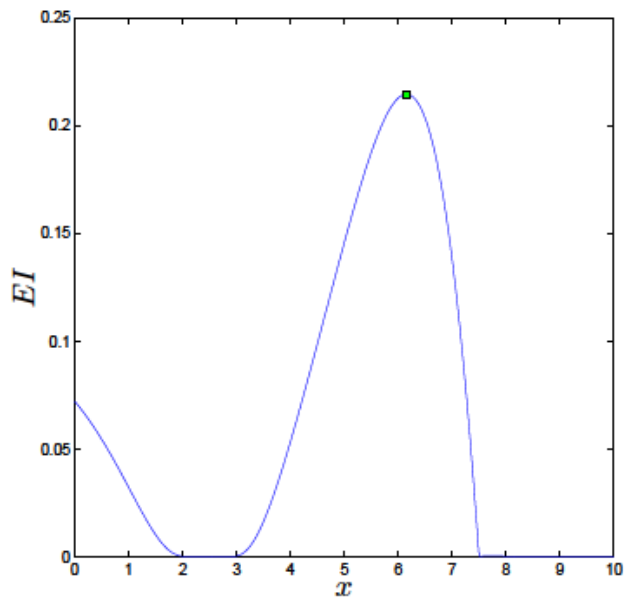

(d) EI (Iter=2)

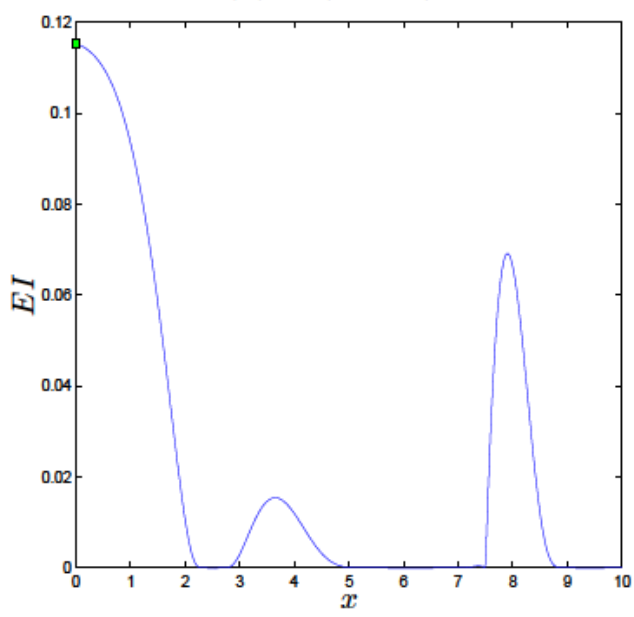

(f) EI (Iter=3)

Figura 4.4: Implementación del algoritmo EGO para la minimización de la función $y(x)=$ $-\sin (x)-\exp (x / 100)+10$ en el intervalo [0,10]. Las figuras de la izquierda representan la función original (línea roja discontinua) junto con el modelo de predicción Kriging (línea azul continua) para el conjunto de datos disponible. Las figuras de la derecha muestran la mejora esperada y su valor máximo que corresponde al punto candidato para mejorar el meta-modelo. 


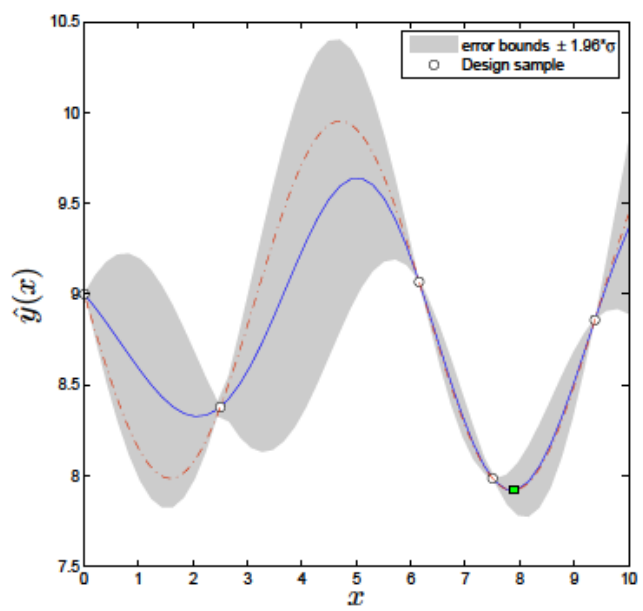

(g) Modelo Kriging (Iter $=4$ )

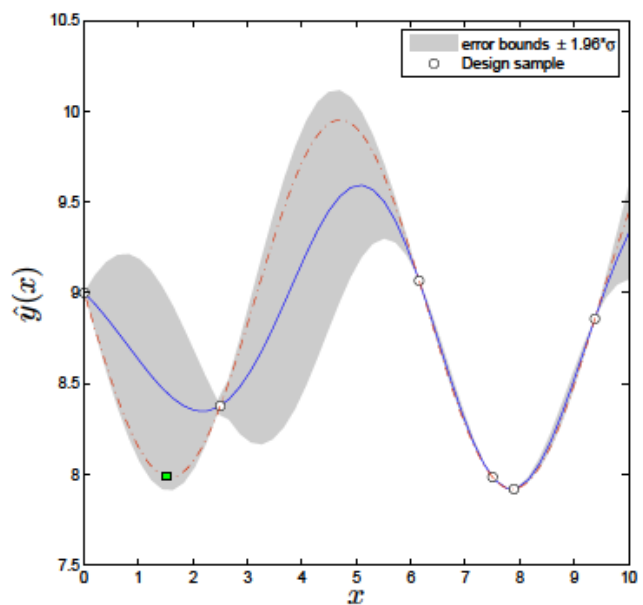

(i) Modelo Kriging (Iter=5)

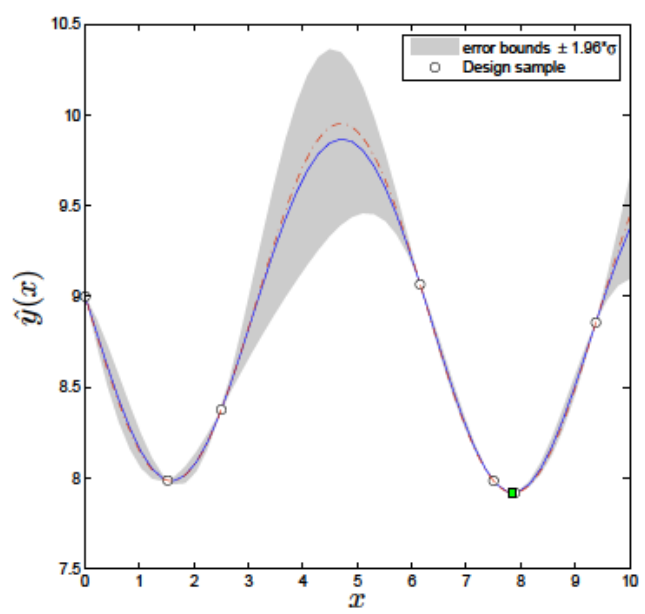

(k) Modelo Kriging (Iter=6)

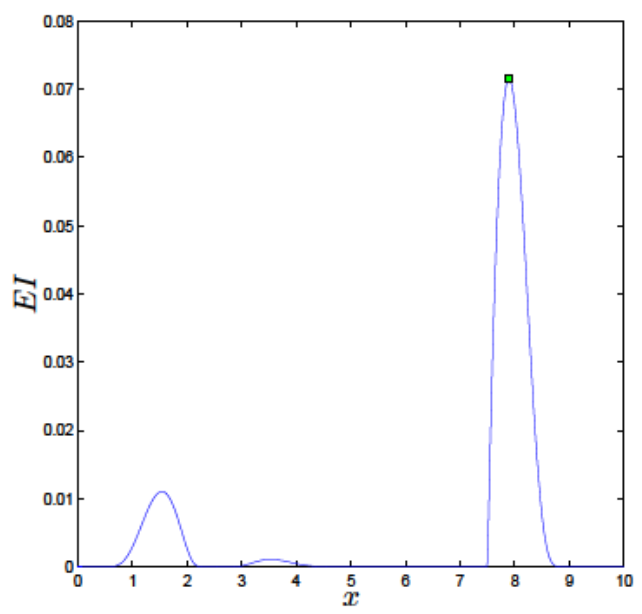

(h) EI (Iter=4)

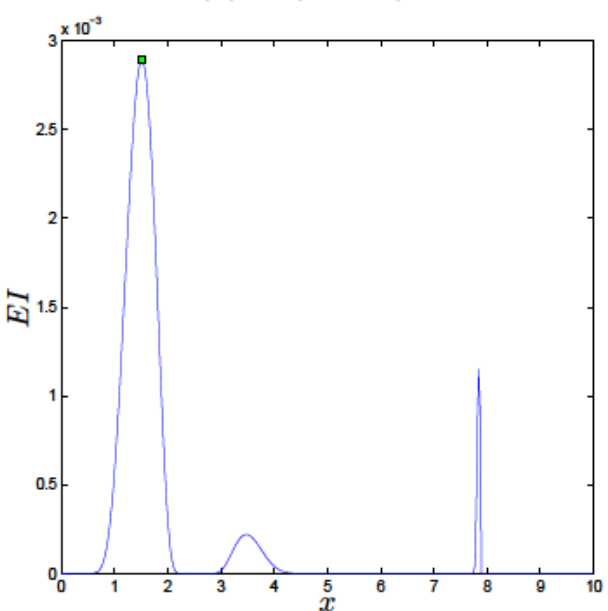

(j) EI (Iter=5)

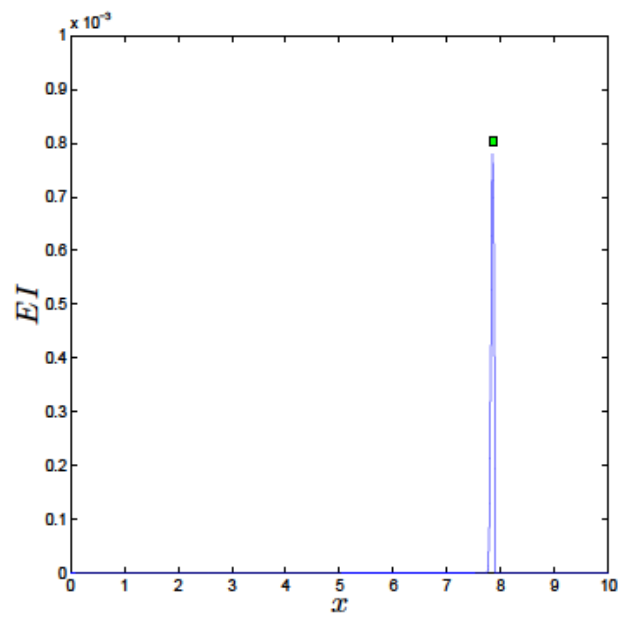

(1) EI (Iter=6)

Figura 4.4: (Continuación). Implementación del algoritmo EGO para la minimización de la función $y(x)=-\sin (x)-\exp (x / 100)+10$ en el intervalo [0,10]. Las figuras de la izquierda representan la función original (línea roja discontinua) junto con el modelo de predicción Kriging (línea azul continua) para el conjunto de datos disponible. Las figuras de la derecha muestran la mejora esperada y su valor máximo que corresponde al punto candidato para mejorar el meta-modelo. 
- Etapa 2: búsqueda de nuevas soluciones factibles no-dominadas. La búsqueda se realiza mediante la maximización de la mejora esperada del hipervolumen dominado respecto del frente de Pareto aproximado. Las restricciones de desigualdad se incluyen en el proceso de búsqueda mediante la modificación del criterio heurístico utilizando el concepto de probabilidad de solución factible.

\subsubsection{Mejora esperada en el hipervolumen dominado}

El criterio heurístico utilizado es la mejora esperada en el hipervolumen dominado (Expected HyperVolume Improvement (EHVI)). Dicho criterio está basado en el indicador de Hypervolume (HV), también conocido como S-metric (Zitzler y Thiele, 1998). El HV se utiliza habitualmente en la literatura para evaluar la calidad de las soluciones no-dominadas del problema MO. El HV se define a partir de la medida de Lebesgue de un conjunto como:

$$
\operatorname{HV}\left(P, \mathbf{f}^{r e f}\right)=\text { Lebesgue }\left(\left\{\mathbf{f} \in \mathbb{R}^{n_{f}} \mid P \leq \mathbf{f} \leq \mathbf{f}^{r e f}\right\}\right) .
$$

Gráficamente el indicador de hipervolumen dominado es la región acotada inferiormente por el frente de soluciones no-dominadas $(P)$ y superiormente por un punto de referencia $\left(\mathbf{f}^{r e f}\right)$ (Figura $4.5 \mathrm{a}$ ). En este trabajo, dicho punto de referencia corresponde al máximo valor de las funciones objetivo en la muestra inicial.

Haciendo un paralelismo con el problema con un único objetivo, la mejora obtenida en el $\mathrm{HV}$ al incluir un punto candidato $\mathbf{x}_{c}$ en el conjunto de soluciones no-dominadas $P=\left\{\mathbf{f}^{(1)}, \ldots, \mathbf{f}^{\left(n_{P}\right)}\right\}$ se define como:

$$
\operatorname{HVI}\left(P, \mathbf{f}\left(\mathbf{x}_{c}\right), \mathbf{f}^{r e f}\right)=\operatorname{HV}\left(P \cup \mathbf{f}\left(\mathbf{x}_{c}\right), \mathbf{f}^{r e f}\right)-\operatorname{HV}\left(P, \mathbf{f}^{r e f}\right) .
$$

El Hypervolume Improvement (HVI) está acotado superiormente por el conjunto de soluciones no-dominadas tal y como muestra la Figura 4.5b. Cuando la respuesta $\mathscr{M}\left(\mathbf{x}_{c}\right)$ es conocida, el HVI es un valor determinista. Sin embargo, en el contexto de los algoritmos de optimización Bayesiana, esta respuesta se desconoce y únicamente se dispone de una predicción en forma de distribución Gaussiana multivariable $\mathscr{N}\left(\mu_{\hat{\mathbf{f}}}\left(\mathbf{x}_{c}\right), \sigma_{\hat{\mathbf{f}}}\left(\mathbf{x}_{c}\right)\right)$. En este caso, el interés reside en conocer cuál es el valor esperado de mejora en el hipervolumen (EHVI) al incluir a $\mathbf{f}\left(\mathbf{x}_{c}\right)$ en el conjunto de soluciones no-dominadas (Figura 4.5c). Utilizando la función de densidad de probabilidad, la EHVI se define como:

$$
\operatorname{EHVI}(\mathbf{x})=\int_{\hat{\mathbf{f}} \in \mathbb{R}^{n_{f}}} \operatorname{HVI}\left(P, \hat{\mathbf{f}}, \hat{\mathbf{f}}^{r e f}\right) \cdot \phi_{\mathbf{x}}(\hat{\mathbf{f}}) d \hat{\mathbf{f}} .
$$

La integral (4.13) se puede resolver utilizando métodos de integración como MonteCarlo. Sin embargo, la utilización de dicha metodología puede dar lugar a resultados poco precisos. En este trabajo se utiliza el algoritmo propuesto por Emmerich et al. (2008) para calcular de forma directa la mejora esperada en el hipervolumen sin necesidad de integración numérica. Este algoritmo consiste en: 


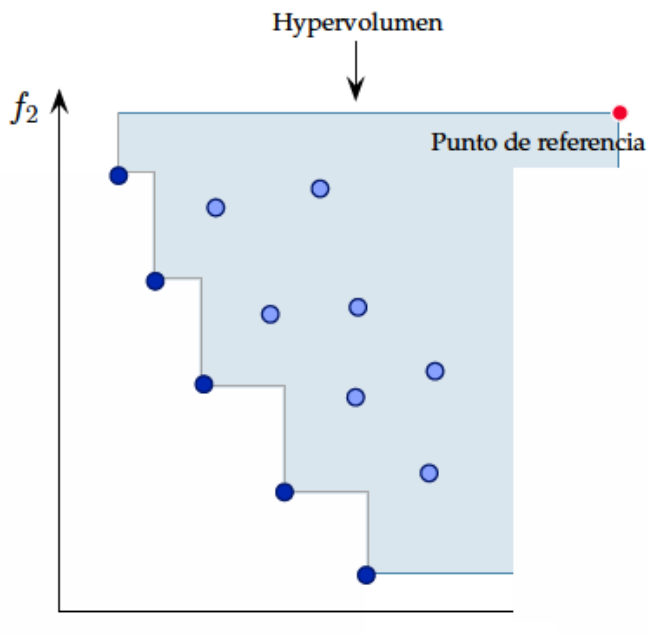

(a)

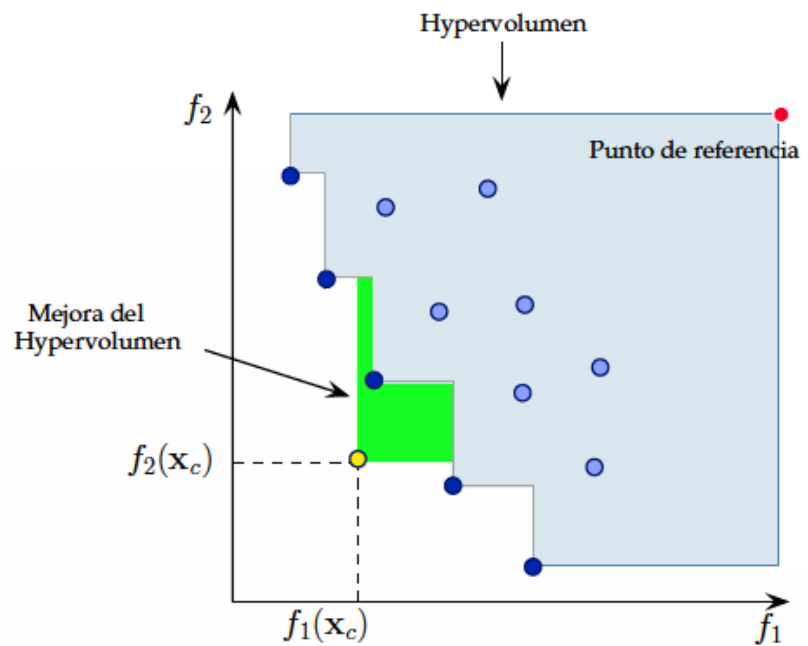

(b)

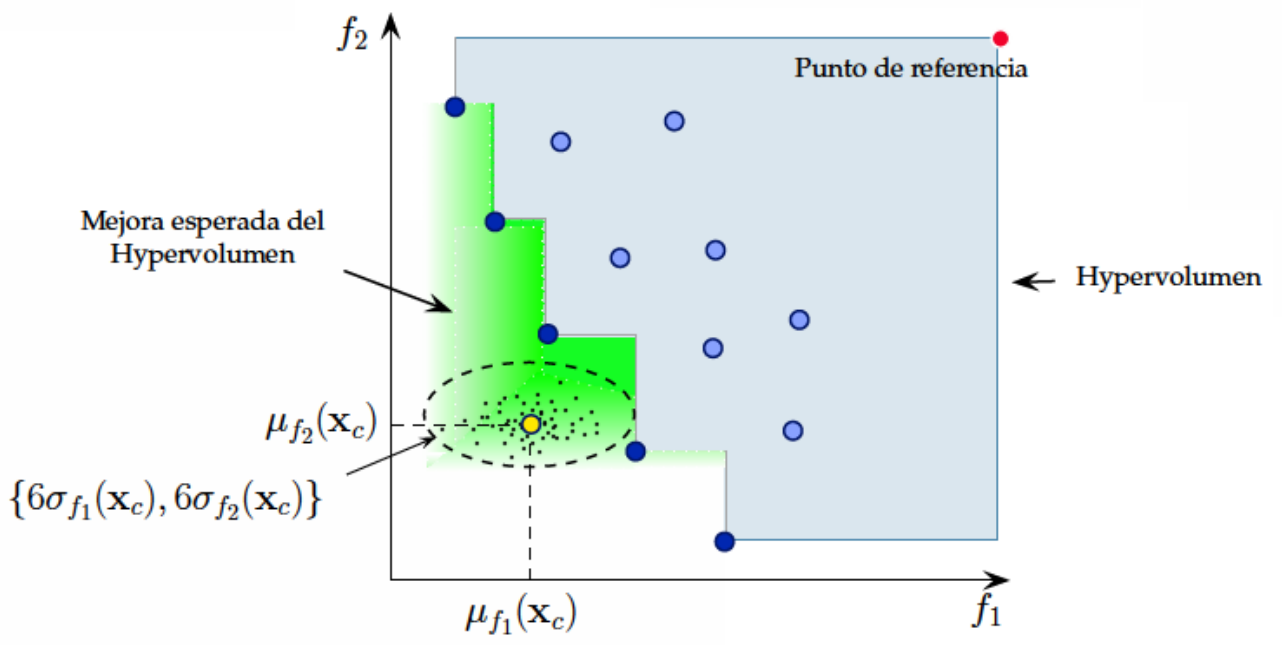

(c)

Figura 4.5: Ilustración de la mejora esperada en el hipervolumen dominado. (a) hipervolumen dominado para un conjunto de soluciones óptimas; (b) mejora en el hipervolumen dominado producida por la inclusión de un nuevo punto $\left\{f_{1}\left(\mathbf{x}_{c}\right), f_{2}\left(\mathbf{x}_{c}\right)\right\}$ en el frente; (c) valor esperado de mejora en el hipervolumen dominado por la inclusión de un nuevo punto $\left\{\mu_{\hat{f}_{1}}\left(\mathbf{x}_{c}\right), \mu_{\hat{f}_{2}}\left(\mathbf{x}_{c}\right)\right\}$ 
(1) dividir el espacio de integración en un grid de celdas, (2) detectar el conjunto de celdas activas, es decir celdas cuyos límites inferiores dominan el punto de referencia y son dominados por al menos un punto del frente de Pareto, y (3) resolver la integral (4.13) sobre cada celda activa del grid. Una descripción en mayor detalle del procedimiento se puede encontrar en el trabajo de Emmerich et al. (2008).

\subsubsection{Tratamiento de las restricciones de desigualdad}

Tal y como muestra la Tabla 4.2, la mayoría de los algoritmos de análisis Bayesiano que tratan el problema de optimización MO han sido desarrollados en problemas que consideran únicamente restricciones en los límites de las variables de diseño. En este trabajo se aborda la resolución del problema de optimización $\mathrm{MO}$ en el contexto del diseño óptimo robusto. En este contexto el problema presenta restricciones de desigualdad que involucran procesos de propagación de incertidumbre para la estimación de probabilidades o momentos estadísticos de la respuesta. Por este motivo, dichas restricciones son aproximadas mediante modelos de predicción Kriging cuya incertidumbre epistémica debe ser considerada en el proceso de optimización.

En el campo de la optimización con un único objetivo existen diferentes enfoques que tratan el problema de las restricciones. Estos enfoques se pueden clasificar en dos categorías:

1. Métodos que transforman el problema con restricciones en un problema sin restricciones utilizando una penalización (Sasena et al., 2000), la probabilidad de factibilidad (Schonlau, 1997) o la esperanza de factibilidad (Audet et al., 2000).

2. Métodos que abordan directamente el problema considerando las restricciones estadísticamente (Sasena et al., 2001).

En este trabajo se investiga la eliminación explícita de la restricción en el problema MO mediante la utilización de la probabilidad de factibilidad (Schonlau, 1997). Se propone transformar el problema de optimización en un problema sin restricciones multiplicando la función objetivo (EHVI (4.13)) por la probabilidad de que el diseño sea factible. De esta forma el criterio de búsqueda $\mathscr{C}_{1}$ queda como sigue:

$$
\mathscr{C}_{1}=\operatorname{EHVI}(\mathbf{x}) \cdot \prod_{j=1}^{n_{g}} \mathbb{P}\left(\hat{g}_{j}(\mathbf{x}) \leq 0\right)=\operatorname{EHVI}(\mathbf{x}) \cdot \prod_{j=1}^{n_{g}} \Phi\left(\frac{\operatorname{Tol}_{\mathrm{g}}-\mu_{\hat{g_{j}}}(\mathbf{x})}{\sigma_{\hat{g_{j}}}(\mathbf{x})}\right),
$$

donde $\Phi(\cdot)$ es la distribución de probabilidad acumulada, $\mu_{\hat{g_{j}}}(\mathbf{x})$ y $\sigma_{\hat{g}_{j}}(\mathbf{x})$ es la media y la desviación estándar de la predicción de la restricción j-ésima 
respectivamente, $\mathrm{y} \mathrm{Tol}_{\mathrm{g}}=0$ es la tolerancia de la restricción.

Ilustración 4.2. El procedimiento propuesto se ilustra en la Figura 4.6 correspondiente a tres iteraciones del problema (Deb et al., 2002):

$$
\operatorname{DEB}\left\{\begin{array}{llc}
f_{1}(\mathbf{x}) & = & x_{1}, \\
f_{2}(\mathbf{x}) & = & \left(1+x_{2}\right) / x_{1}, \\
g_{1}(\mathbf{x}) & = & x_{2}+9 x_{1} \geq 6, \\
g_{2}(\mathbf{x}) & = & -x_{2}+9 x_{1} \geq 1, \\
0.1 \leq x_{1} \leq 1.0,0 \leq x_{1} \leq 5 . &
\end{array}\right.
$$

En esta figura se muestran los contornos del criterio $\mathscr{C}_{1}$ en el espacio de diseño. La línea gris representa el límite de las restricciones originales evaluadas con la expresión analítica. Inicialmente (Iteración 0) las restricciones se aproximan con modelos Kriging a partir de una muestra de seis puntos (círculos negros). La incertidumbre inicial de dichos modelos provoca la existencia de regiones de potencial mejora del criterio $\mathscr{C}_{1}$ fuera del espacio de diseño factible original (Figura 4.6b). En las iteraciones posteriores se muestra como la utilización de la probabilidad de factibilidad combina la exploración de zonas de alta incertidumbre fuera del espacio factible original (Iteración 13) con la búsqueda de potenciales soluciones no dominadas (Iteración 15). A medida que el algoritmo evoluciona, se reduce la incertidumbre epistémica de los modelos Kriging y como consecuencia los contornos de $\mathscr{C}_{1}$ se ajustan a la restricción original (Figura 4.6f).

\subsubsection{Búsqueda de puntos en el espacio de diseño factible}

La evaluación de la mejora esperada en el hipervolumen requiere de una primera aproximación del frente de soluciones no-dominadas $\mathscr{P}$. Para ello se utiliza el conjunto de observaciones iniciales de las funciones objetivo y de las restricciones $\mathscr{D}=\{\mathscr{X}, \mathscr{Y}=\mathscr{M}(\mathbf{x} \in \mathscr{X})\}$. El conjunto de soluciones no-dominadas se obtienen utilizando el criterio de dominancia de Pareto (ver sección 4.2) sobre el subconjunto $\mathscr{D}_{\text {fact }} \subset \mathscr{D}$ de soluciones factibles:

$$
\mathscr{P}=\left\{\mathbf{x}^{*} \in \mathscr{D}_{\text {fact }} \subset \mathscr{D} \mid \nexists \mathbf{x} \in \mathscr{D}_{\text {fact }} \text { tal que } \mathbf{x} \leq \mathbf{x}^{*}\right\}
$$

Como se mencionó con anterioridad, el número de observaciones existentes en el diseño de experimentos inicial es limitado y habitualmente el número de diseños factibles es inexistente o insuficiente. Por esta razón, es necesaria una primera etapa consistente en una búsqueda eficiente de puntos factibles en el espacio de diseño.

La estrategia propuesta consiste en la búsqueda en el espacio de diseño de 


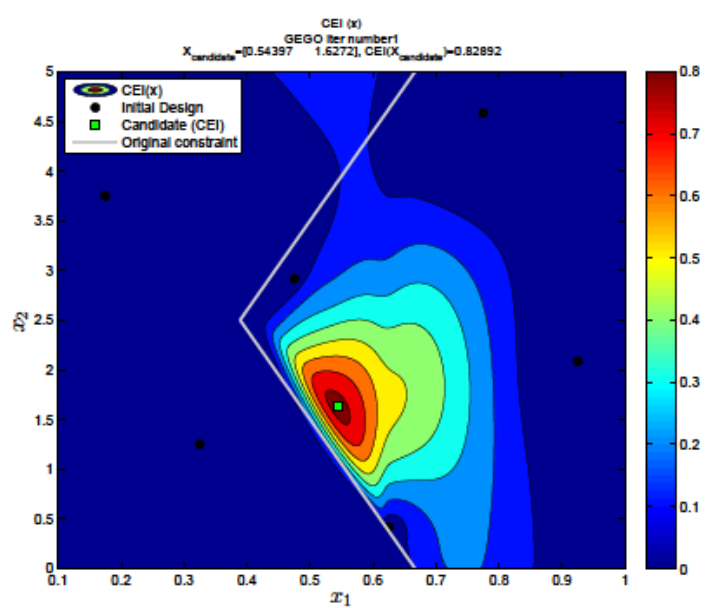

(a) $\mathscr{C}_{1}$ (Iter=1)

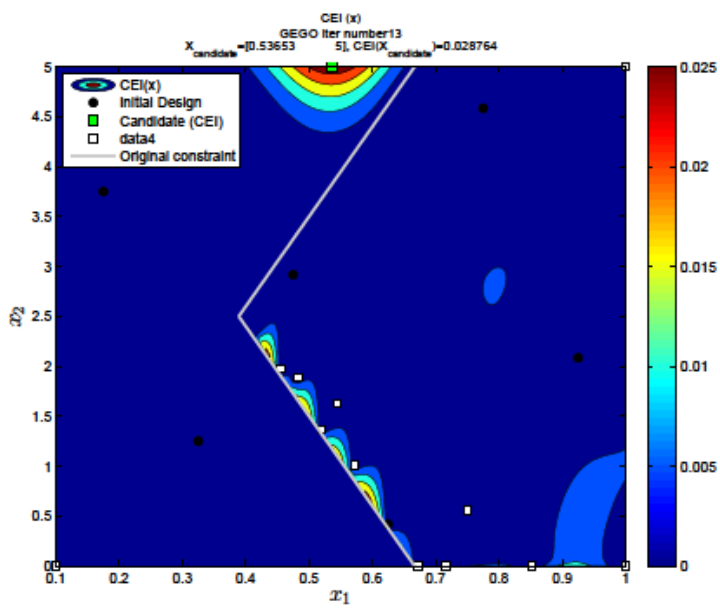

(c) $\mathscr{C}_{1}$ (Iter=13)

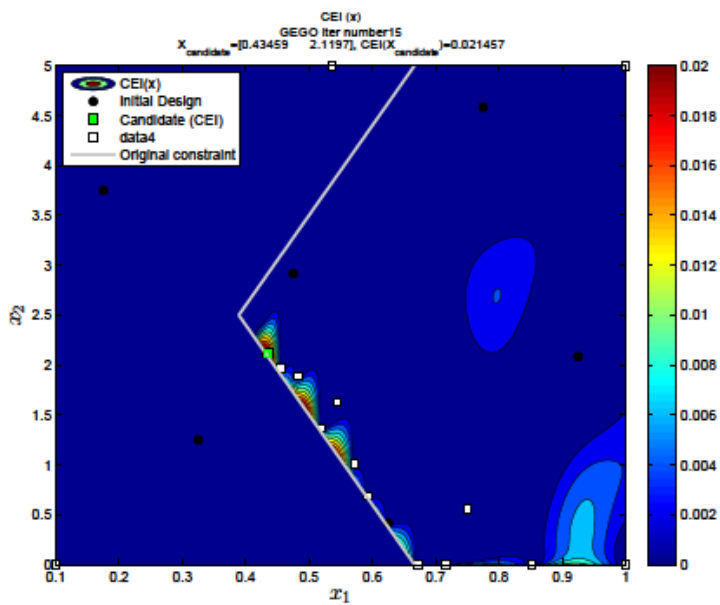

(e) $\mathscr{C}_{1}($ Iter=15)

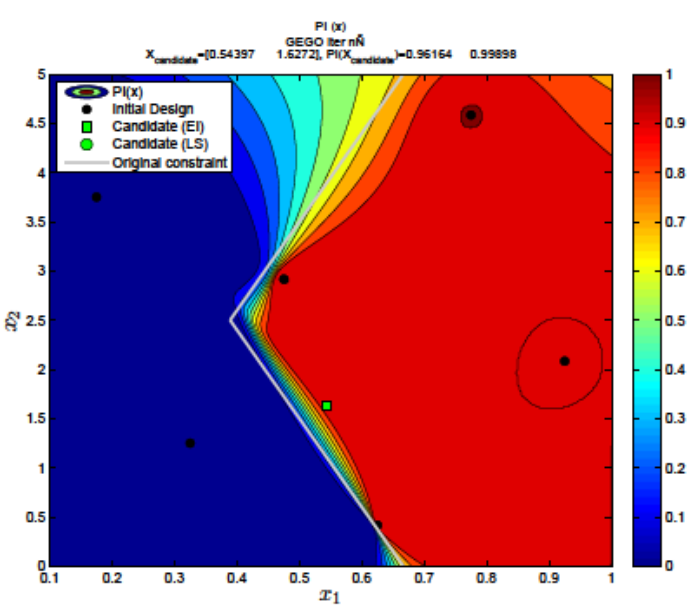

(b) $\mathbb{P}\left(\hat{g}_{j}(\mathbf{x}) \leq 0\right)($ Iter $=1)$

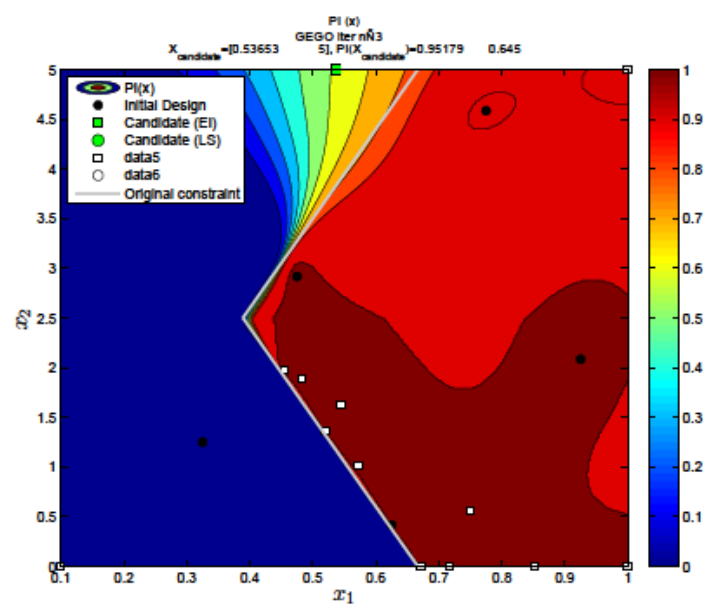

(d) $\mathbb{P}\left(\hat{g}_{j}(\mathbf{x}) \leq 0\right)($ Iter $=13)$

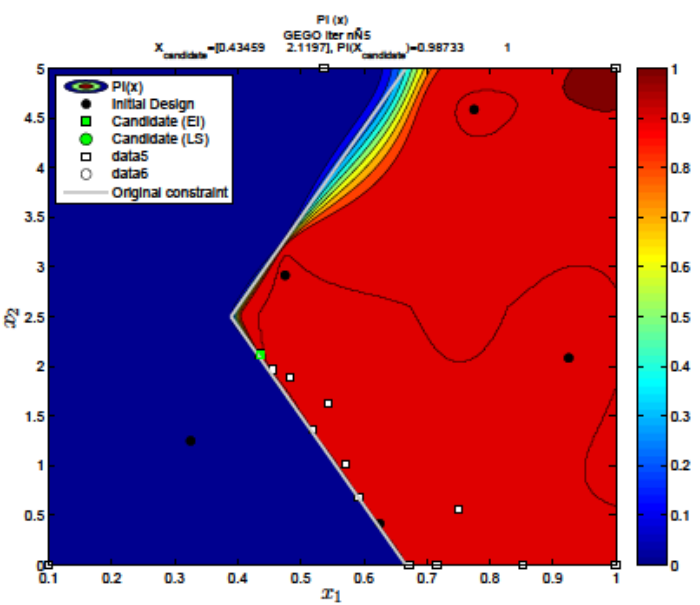

(f) $\mathbb{P}\left(\hat{g}_{j}(\mathbf{x}) \leq 0\right)($ Iter $=15)$

Figura 4.6: Ilustración del criterio $\mathscr{C}_{1}$ basado en una muestra inicial de seis puntos (circulos negros), junto con el límite de la restricción verdadera (línea gris). También se muestran los puntos de actualización en iteraciones anteriores (cuadrados blancos), y el candidato para la actualización en la iteración actual (cuadrado verde). 
puntos que maximicen el criterio:

$$
\mathscr{C}_{2}=\prod_{i=1}^{n_{g}} \sigma_{\hat{g}_{j}}(\mathbf{x}) \cdot \mathbb{P}\left(\hat{g}_{j}(\mathbf{x}) \leq 0\right)=\prod_{i=1}^{n_{g}} \sigma_{\hat{g}_{j}}(\mathbf{x}) \cdot \Phi\left(\frac{\operatorname{Tol}_{\mathrm{g}}-\mu_{\hat{g}_{j}}(\mathbf{x})}{\sigma_{\hat{g}_{j}}(\mathbf{x})}\right),
$$

donde $\mathbb{P}\left(\hat{g}_{j}(\mathbf{x}) \leq 0\right)$ es la probabilidad de que el diseño sea factible y $\sigma_{\hat{g}_{j}}(\mathbf{x})$ es la desviación estándar de la predicción, indicador de la incertidumbre del modelo. Multiplicar la desviación por la probabilidad tiene el efecto reducir el criterio $\mathscr{C}_{2}$ cuando la probabilidad de que el diseño sea factible es baja o cuando la incertidumbre del modelo es baja.

Ilustración 4.3. La estrategia utilizada para la búsqueda de puntos en el espacio factible se ilustra mediante la resolución del problema Gomez\#3 (Gomez y Levy., 1982):

$$
\begin{cases}\min _{\mathbf{x}} f(\mathbf{x}) & = \\ \text { s. } a: g(\mathbf{x}) & \left(4-2.1 x_{1}^{2}+\frac{x_{1}^{4}}{3}\right) x_{1}^{2}+x_{1} x_{2}+\left(-4+4 x_{2}^{2}\right) x_{2}^{2}, \\ -1 \leq x_{i} \leq 1, i=1,2 . & -\sin \left(4 \pi x_{1}\right)+2 \sin ^{2}\left(2 \pi x_{2}\right) \leq 0,\end{cases}
$$

Las Figuras 4.7a a 4.7b muestran la representación gráfica de las funciones originales $f(\mathbf{x})$ y $g(\mathbf{x})$. El alto carácter multimodal de la función de restricción divide el espacio de diseño en 20 regiones factibles desconectadas entre sí (Figura 4.7e). Lo que dificulta su resolución eficiente mediante métodos convencionales basados en gradiente por la necesidad de múltiples reinicializaciones.

Ambas funciones se aproximan con dos modelos Kriging (Figuras 4.7c a 4.7d) a partir de una muestra inicial de 22 puntos. Dicha muestra inicial proporciona una excelente aproximación de la función objetivo. Sin embargo no ocurre lo mismo con la restricción, que resulta difícil de aproximar debido a su alto comportamiento multimodal. Como consecuencia la aproximación inicial del espacio factible (Figura 4.7f) es muy pobre.

La Figura 4.8 muestra la evolución del problema a mediada que avanza el número de iteraciones. En la iteración 135, 19 de las 20 regiones son detectadas. En la iteración 150 todas las regiones han sido detectadas, alcanzando un número total de 172 puntos de los que 109 son factibles.

\subsubsection{Consideraciones acerca del algoritmo}

\subsubsection{Resolución de los subproblemas de optimización}

La estrategia propuesta se muestra en el Algoritmo 3. Existen dos subproblemas de optimización que corresponden a la maximización de $\mathscr{C}_{1}$ y $\mathscr{C}_{2}$. Para su resolución en este trabajo se utiliza el algoritmo de optimización 


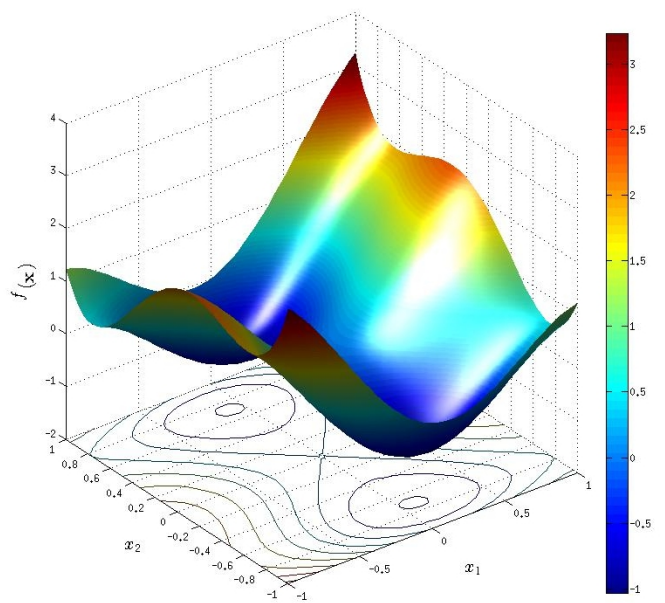

(a) $f(\mathbf{x})$

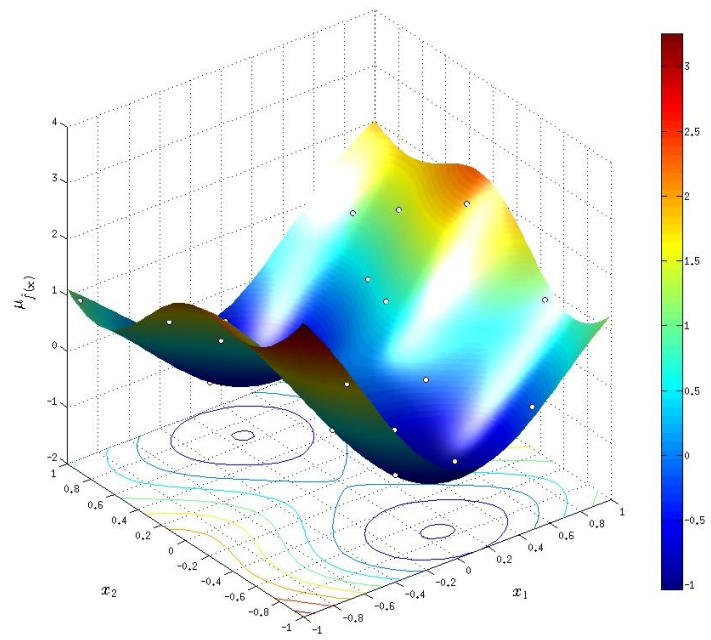

(c) $\hat{f}(\mathbf{x})$

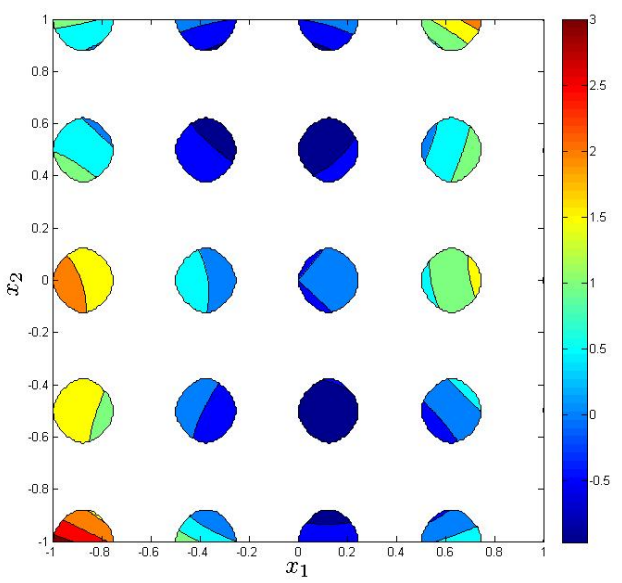

(e) $f(\mathbf{x})$ región factible

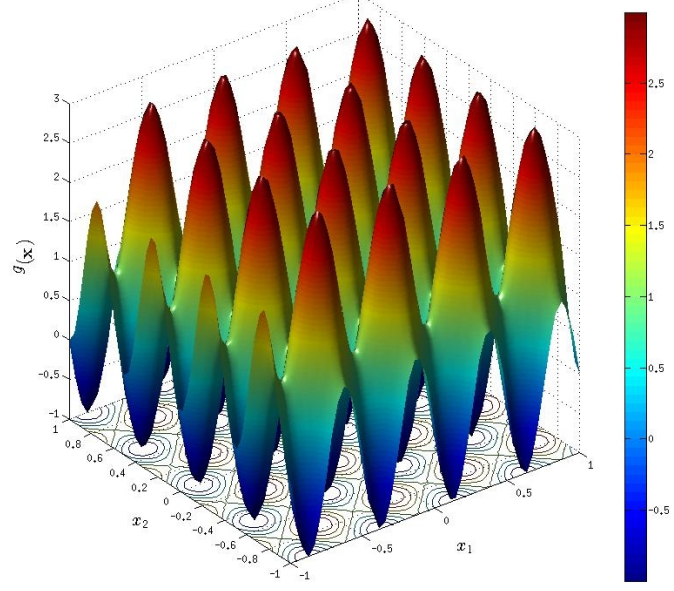

(b) $g(\mathbf{x})$

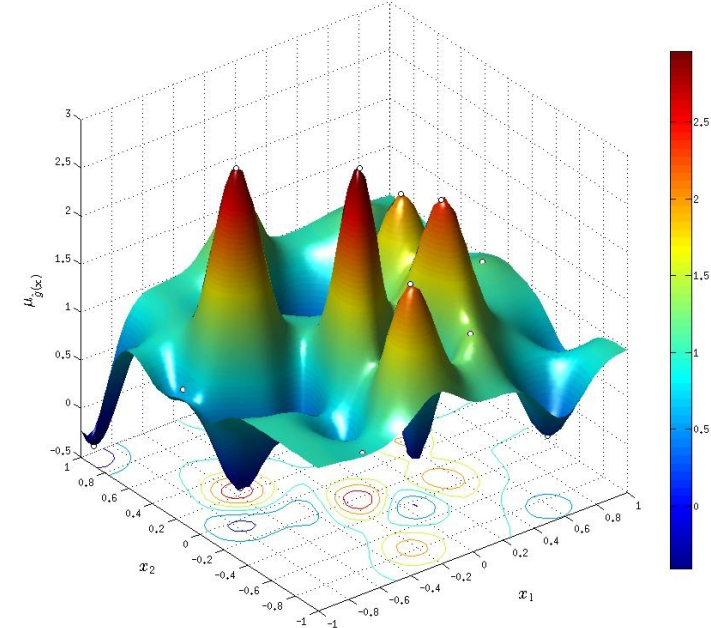

(d) $\hat{g}(\mathbf{x})$

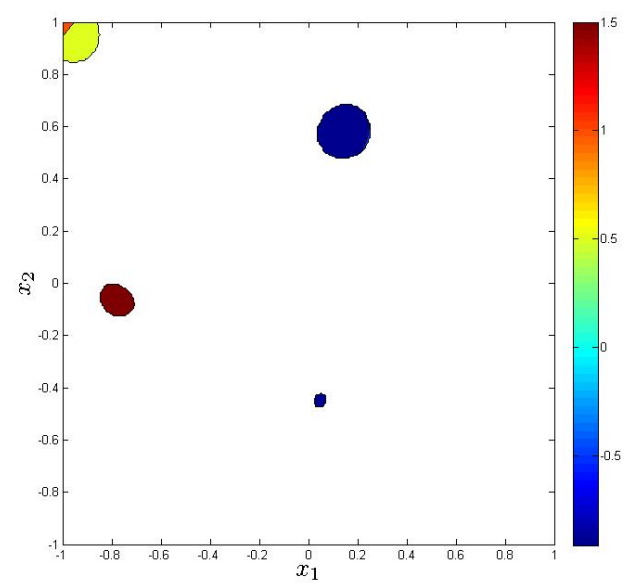

(f) $\hat{f}(\mathbf{x})$ región factible

Figura 4.7: Problema Gomez \#3. Función objetivo y restricción (a-b); modelos Kriging iniciales de la función objetivo y restricción (c-d); región factible del modelo real y de la aproximación inicial (e-f). 


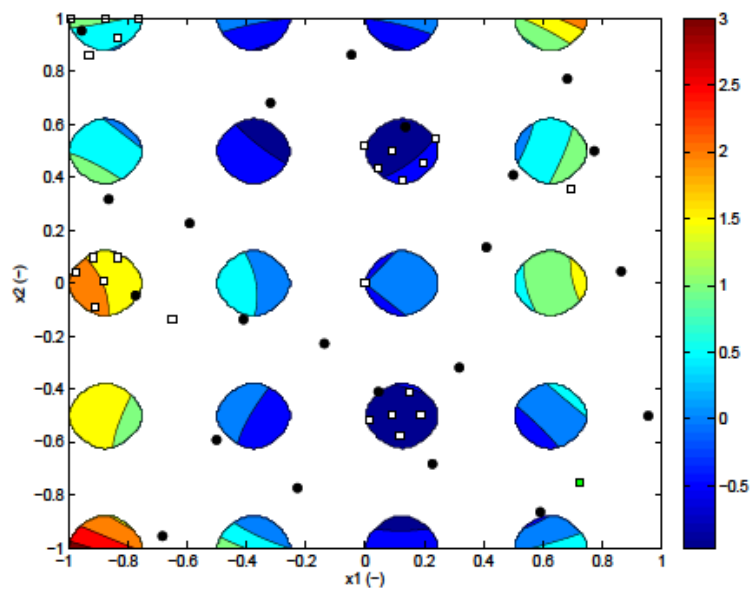

(a) Iter $=25$

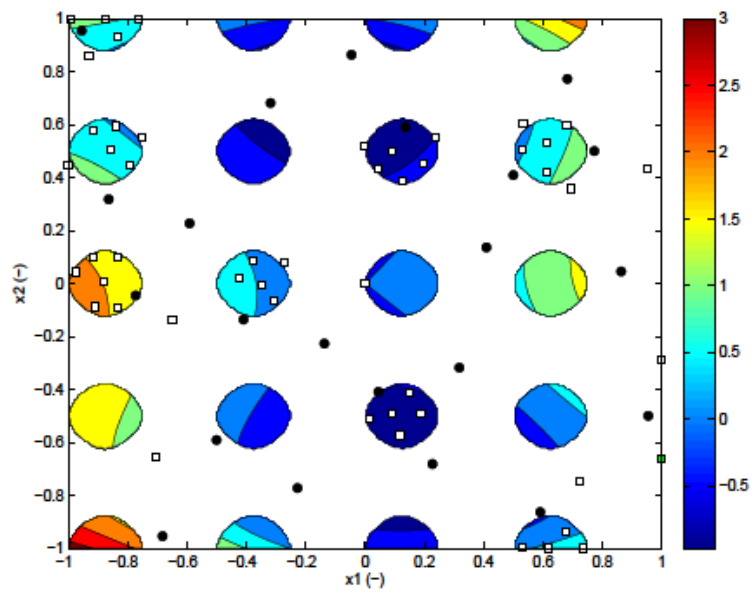

(c) Iter $=50$

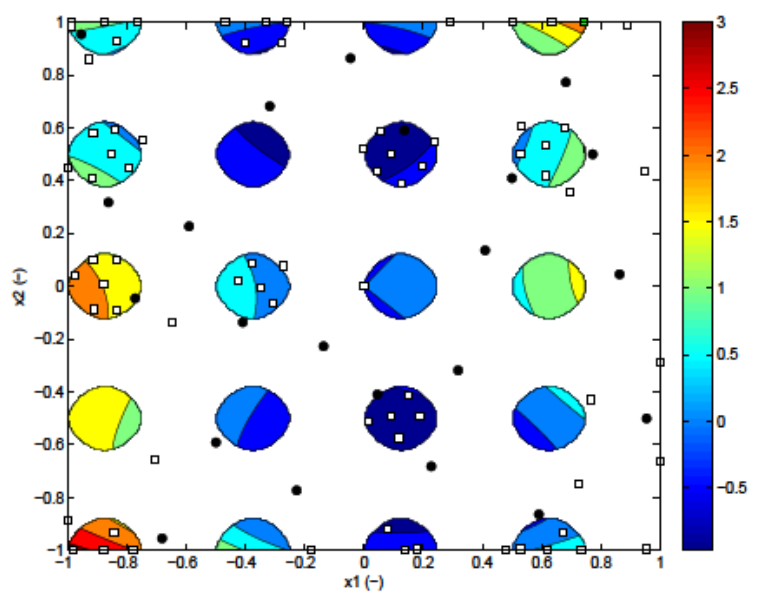

(e) Iter $=75$

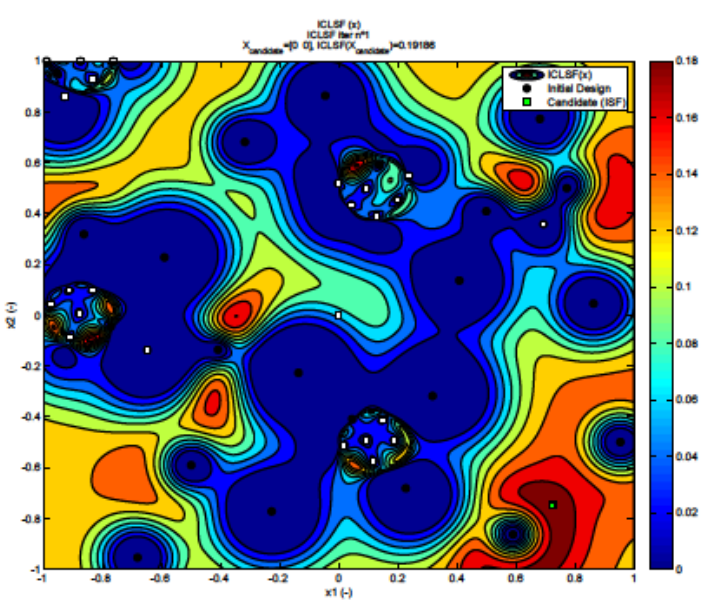

(b) Iter $=25$

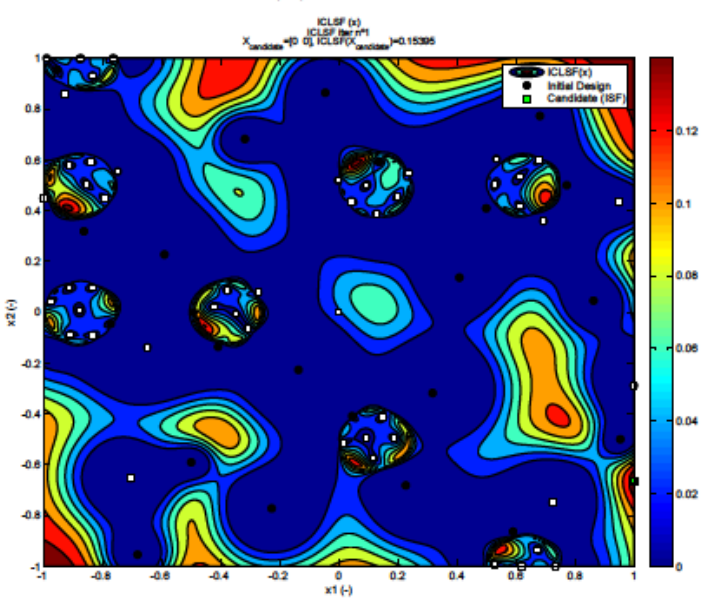

(d) Iter $=50$

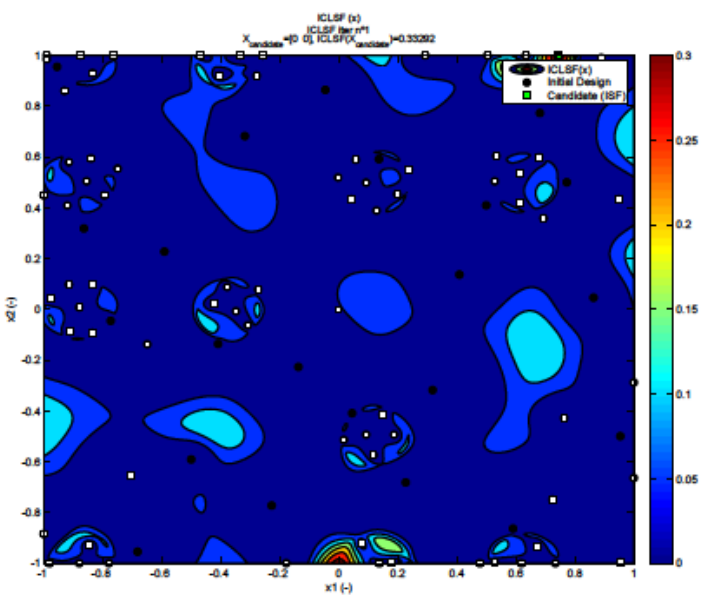

(f) Iter $=75$

Figura 4.8: Evolución del criterio de búsqueda en el ejemplo Gomez \#3. La muestra inicial se presenta como círculos negros. Los cuadrados blancos representan los puntos añadidos hasta la iteración. Los cuadrados verdes representan el candidato que maximiza el criterio en cada iteración. 


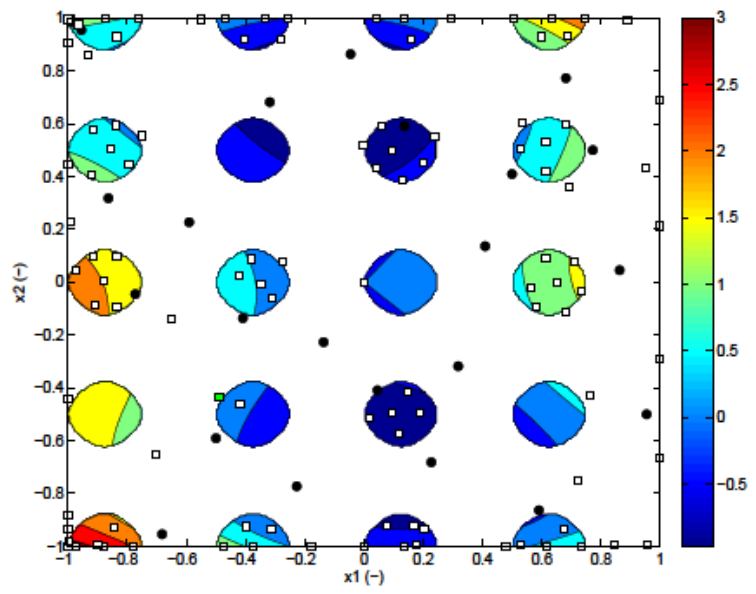

(g) Iter $=110$

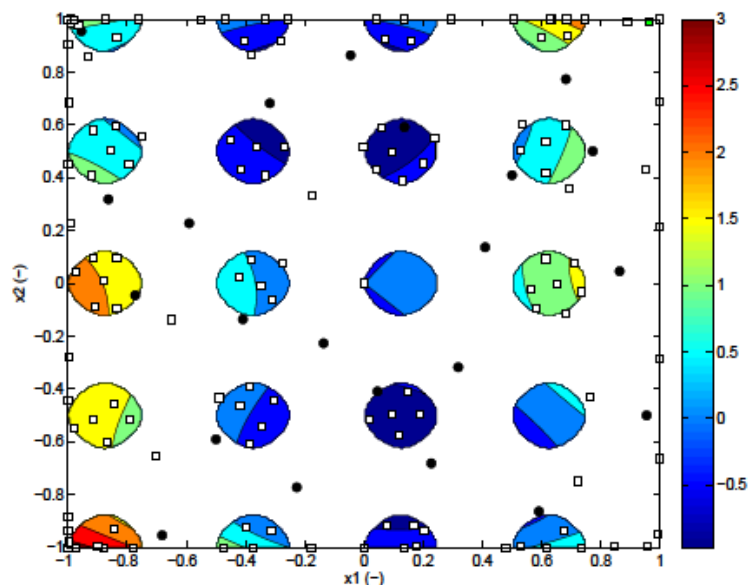

(i) Iter $=135$

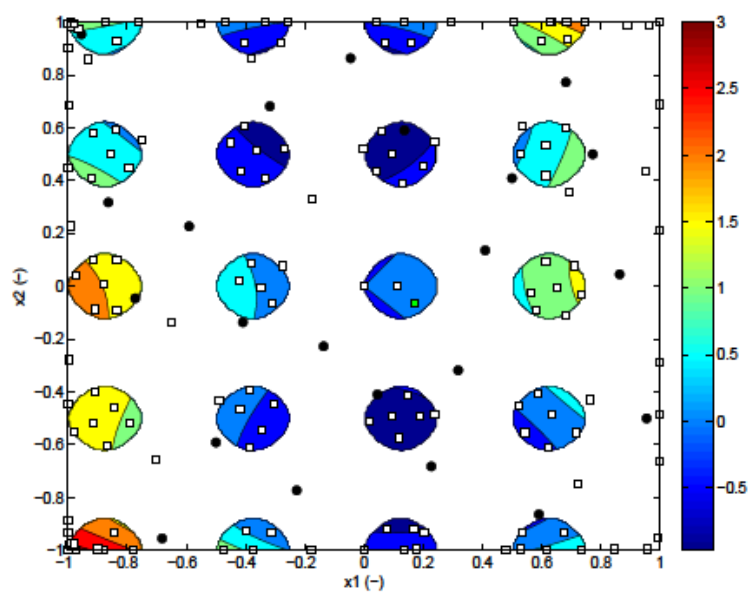

(k) Iter $=150$

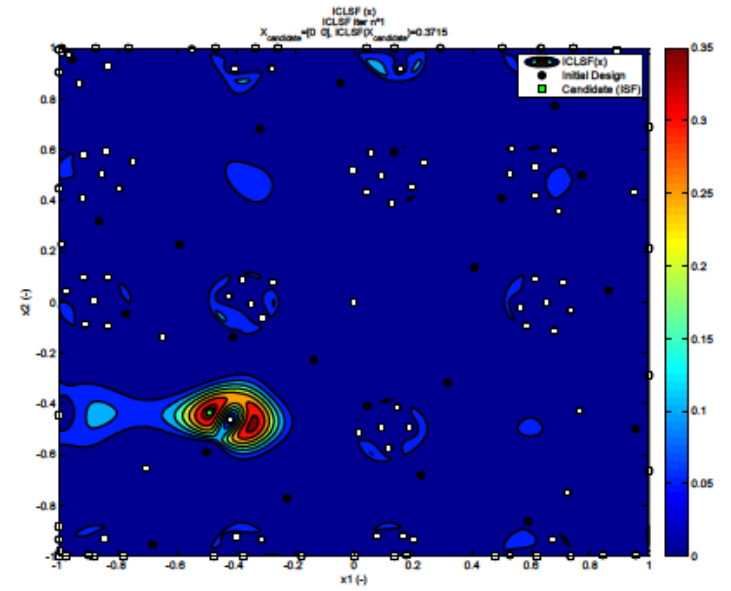

(h) Iter $=110$

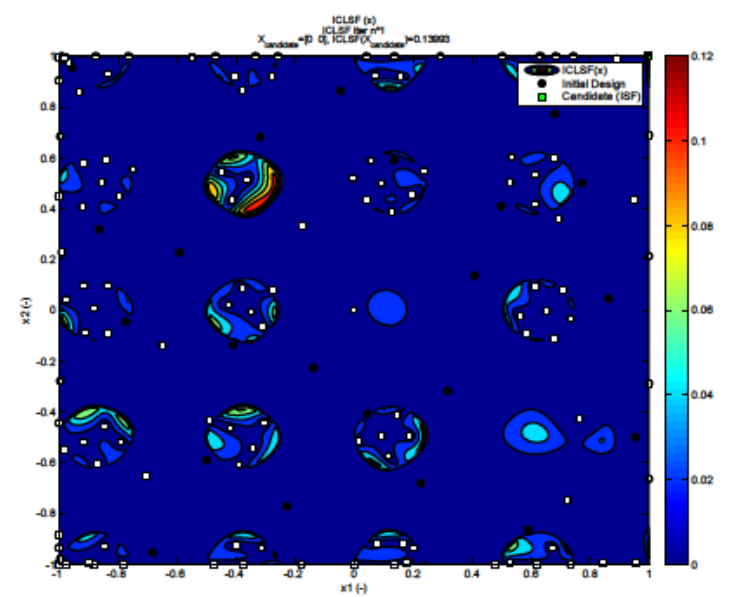

(j) Iter $=135$

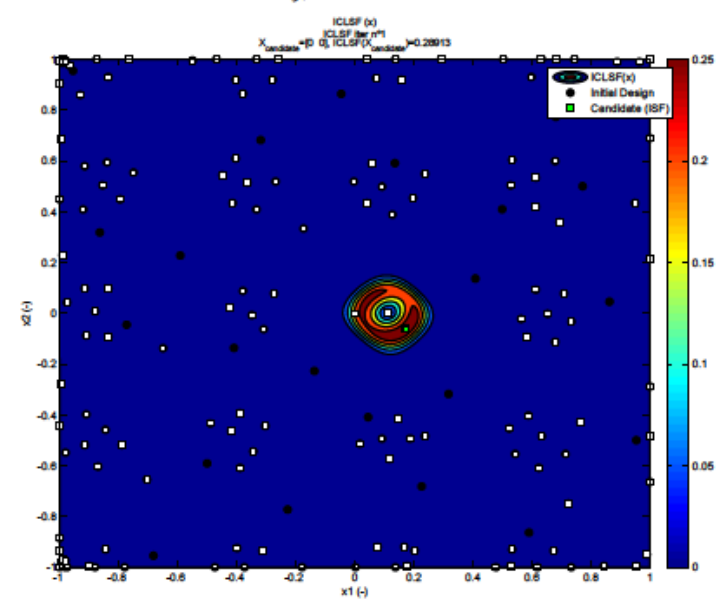

(1) Iter $=150$

Figura 4.8: (Continuación). Evolución del criterio de búsqueda en el ejemplo Gomez \#3. La muestra inicial se presenta como círculos negros. Los cuadrados blancos representan los puntos añadidos hasta la iteración. Los cuadrados verdes representan el candidato que maximiza el criterio en cada iteración. 


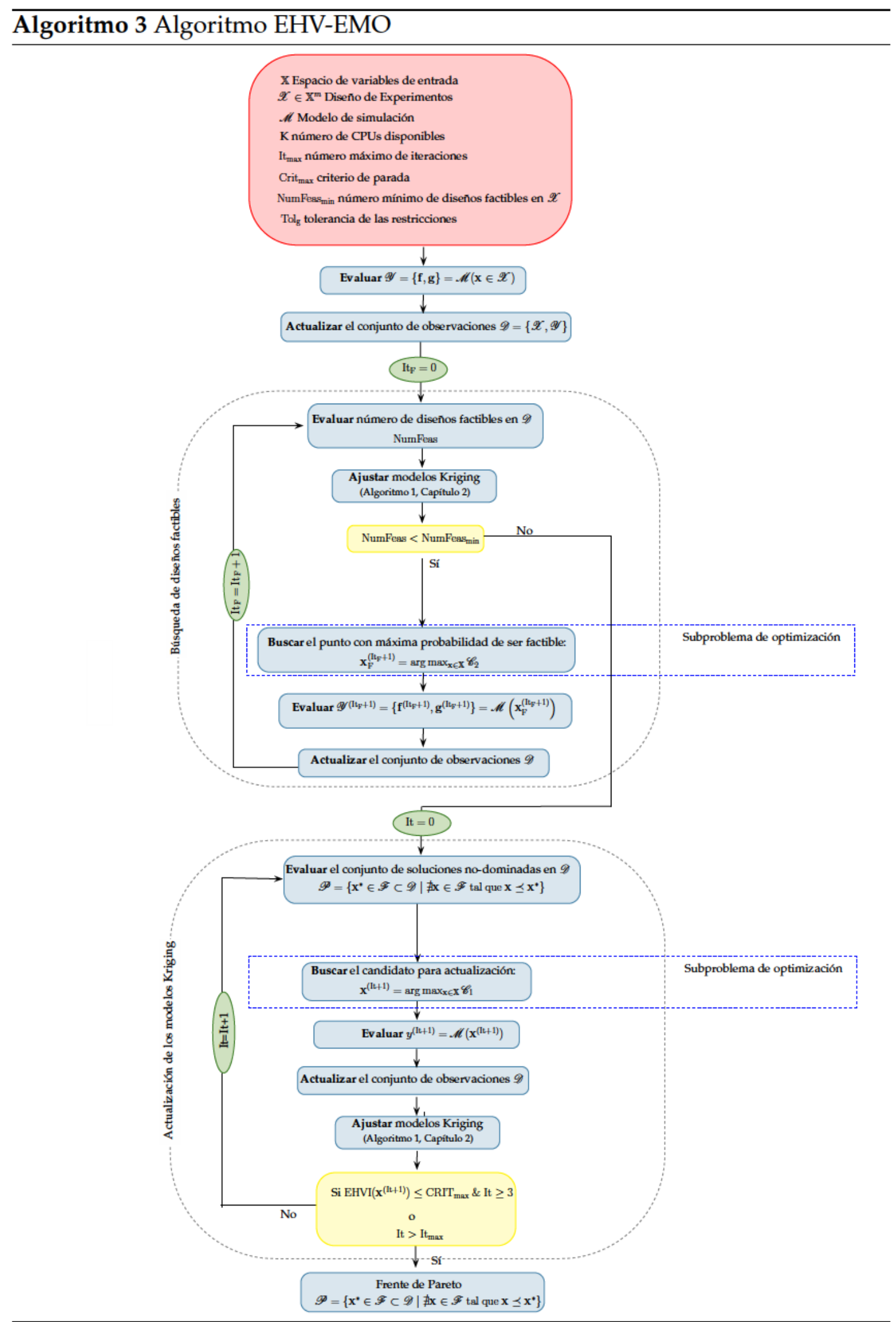


global DIRect utilizado previamente en este campo por Jones et al. (1998). El algoritmo DIRect fue introducido originalmente por Perttunen et al. (1993) como una modificación del problema de optimización de Lipschitz. El algoritmo está enfocado a la resolución de problemas de optimización global con restricciones en las variables de diseño y funciones objetivo pertenecientes a $\mathbb{R}$.

El algoritmo DIRect no requiere de información acerca de los gradientes de la función objetivo. Utiliza información de orden cero de las iteraciones previas para decidir el lugar donde muestrear en la siguiente iteración. Por esta razón, este algoritmo es muy apropiado en problemas "reales" que involucran modelos de simulación no-intrusivos (black box). Carter et al. (2002) presentan ejemplos donde utilizan el algoritmo DIRect para la resolución de este tipo de problemas. De acuerdo con Perttunen et al. (1993), el algoritmo converge hacia el óptimo global de la función objetivo. A cambio, puede requerir un elevado número de evaluaciones de la misma. Este problema no limita su utilización en este trabajo puesto que se aplica a la minimización de los criterios $\mathscr{C}_{1}$ y $\mathscr{C}_{2}$ evaluados utilizando los modelos Kriging de la función objetivo y restricciones.

En comparación con otros algoritmos de búsqueda, el algoritmo DIRect se muestra muy competitivo debido al equilibrio entre la búsqueda local y global y al bajo número de parámetros que es preciso ajustar para su ejecución.En este trabajo, se utiliza la implementación del algoritmo en MATLAB (Finkel, 2003) incluida en la toolbox object oriented toolbox for Surrogate-Based Optimization Under Uncertainty Toolbox (ooSBOUU). Los parámetros utilizados para su ejecución son:

- Factor de Jones: 0.0001.

- Máximo número de evaluaciones de la función objetivo : 1000.

- Máximo número de iteraciones: 1000.

- Máximo número de divisiones de rectángulos: 1000.

\subsubsection{Tratamiento de evaluaciones fallidas del modelo de simulación}

Las soluciones de los subproblemas de optimización son evaluadas con el modelo de simulación $\mathscr{M}$ y los resultados se utilizan para la actualización de los modelos Kriging de funciones objetivo y restricciones. Si el modelo de simulación devuelve soluciones fallidas (ej. falta de convergencia, o la combinación de parámetros genera geometrías imposibles) los modelos de predicción no se pueden actualizar y el algoritmo queda bloqueado. Para evitar dicho bloqueo, la respuesta fallida del modelo de simulación se sustituye por el límite superior de la predicción utilizando los modelos Kriging existentes $\left(\mu_{\hat{\mathbf{f}}}\left(\mathbf{x}^{*}\right)+\sigma_{\hat{\mathbf{f}}}\left(\mathbf{x}^{*}\right)\right)$. Estudios previos en el campo de optimización con un único objetivo (Forrester et al., 2008) muestran que la utilización de $\mu_{\hat{\mathbf{f}}}\left(\mathbf{x}^{*}\right)+\sigma_{\hat{\mathbf{f}}}\left(\mathbf{x}^{*}\right)$ como nuevas observaciones permite dirigir la optimización hacia la región de diseño factible. Este nuevo valor reduce la mejora esperada a cero en este punto, por lo que no puede ser seleccionado nuevamente en el futuro. 


\subsubsection{Restricciones de bajo coste computacional}

La estrategia propuesta para resolver el problema con restricciones se basa en la probabilidad de que el diseño sea factible. Atendiendo al tiempo consumido para su evaluación, las restricciones se pueden clasificar en restricciones de alto y de bajo coste computacional. Las restricciones de alto coste computacional son aproximadas por modelos de predicción Kriging. En base a la información estadística proporcionada por dichos modelos se estima la probabilidad de que un diseño sea factible. En el caso de restricciones de bajo coste computacional dicha probabilidad toma valores de 0 (diseño no factible) y de 1 (diseño factible) dado que su evaluación es determinista y no presenta incertidumbre epistémica.

\subsubsection{Explotación del meta-modelo}

Los meta-modelos obtenidos tras la finalización del algoritmo de optimización tienen una precisión elevada en la región cercana al frente de soluciones no-dominadas. Por este motivo pueden ser reutilizados junto con un algoritmo de optimización MO convencional (ej. NSGA-II) para incrementar el número de soluciones no-dominadas sin necesidad de evaluaciones adicionales del modelo de simulación.

\subsection{Ejemplos}

Introducción. El algoritmo EHV-EMO se valida numéricamente mediante la resolución de un conjunto de ejemplos habitualmente utilizados como test en el campo de la optimización multi-objetivo. Los ejemplos son adaptados de los trabajos de Deb et al. (2002) (seis problemas) y Veldhuizen (1999) (un problema). Estas funciones fueron utilizadas con anterioridad por autores como Knowles (2006), Couckuyt et al. (2012) o Zuluaga et al. (2013), para la validación de algoritmos de optimización Multi-objetivo basados en análisis Bayesiano.

Los siete ejemplos seleccionados abarcan: problemas con y sin restricciones de desigualdad, funciones de dos a seis variables de diseño, funciones donde el frente de Pareto sigue complicadas curvas en el espacio de diseño, funciones con frentes de Pareto convexos y cóncavos y frentes de Pareto desconectados. Los problemas que se estudian tratan una gran variedad de dificultades. Sin embargo, el alcance de la validación realizada es limitado: no se tratan problemas de alta dimensionalidad, y el espacio objetivo se limita a dos funciones. Este caso es el habitualmente encontrado en el problema de diseño óptimo robusto (performance vs robutness).

Un resumen de los siete benchmarks seleccionados se presenta en la Tabla 4.3. Dicha tabla muestra la dimensión, función objetivo y restricciones del problema. Una descripción en mayor detalle de cada uno de los problemas se puede encontrar en las referencias originales de Deb et al. (2002) y Veldhuizen (1999). 


\begin{tabular}{|c|c|c|c|c|}
\hline Problema & $\mathrm{n}$ & $\begin{array}{l}\text { Límites de } \\
\text { variables }\end{array}$ & $\begin{array}{l}\text { Funciones } \\
\text { objetivo }\end{array}$ & Restricciones \\
\hline $\mathrm{MOP}^{(a)}$ & 2 & $-2 \leq x_{i} \leq 2, i=1,2$ & $f_{1}(\mathbf{x})=1-\exp \left\{-\sum_{i=1}^{2}\left(x_{i}-\frac{1}{\sqrt{2}}\right)^{2}\right\}$ & $(-)$ \\
\hline $\mathrm{TNK}^{(b)}$ & 2 & $0 \leq x_{i} \leq \pi, i=1,2$ & $\begin{array}{l}f_{1}(\mathbf{x})=x_{1} \\
f_{2}(\mathbf{x})=x_{2}\end{array}$ & $\begin{array}{l}g_{1}(\mathbf{x})=-x_{1}^{2}-x_{2}^{2}+1+ \\
0.1 \cos \left(16 \arctan \left(x_{1} / x_{2}\right)\right) \leq 0 \\
g_{2}(\mathbf{x})=\left(x_{1}-0.5\right)^{2}+\left(x_{2}-0.5\right)^{2}- \\
0.5 \leq 0\end{array}$ \\
\hline $\mathrm{DEB}^{(b)}$ & 2 & $0.1 \leq x_{1} \leq 1.0,0 \leq x_{1} \leq 5$ & $\begin{array}{l}f_{1}(\mathbf{x})=x_{1} \\
f_{2}(\mathbf{x})=\left(1+x_{2}\right) / x_{1}\end{array}$ & $\begin{array}{l}g_{1}(\mathbf{x})=x_{2}+9 x_{1} \geq 6 \\
g_{2}(\mathbf{x})=-x_{2}+9 x_{1} \geq 1\end{array}$ \\
\hline $\operatorname{SRN}^{(b)}$ & 2 & $-20 \leq x_{i} \leq 20, i=1,2$ & $\begin{array}{l}f_{1}(\mathbf{x})=\left(x_{1}-2\right)^{2}+\left(x_{2}-2\right)^{2}+2 \\
f_{2}(\mathbf{x})=9 x_{1}-\left(x_{2}-2\right)^{2}\end{array}$ & $\begin{array}{l}g_{1}(\mathbf{x})=x_{1}^{2}+x_{2}^{2} \leq 225 \\
g_{2}(\mathbf{x})=x_{1}-3 x_{2} \geq-10\end{array}$ \\
\hline $\mathrm{ZDT1}^{(b)}$ & 4 & $\leq x_{i} \leq 1, i=1,2, \ldots, n$ & $\begin{array}{l}f_{1}(\mathbf{x})=x_{1} \\
f_{2}(\mathbf{x})=g(\mathbf{x})\left[1-\sqrt{x_{1} / g(\mathbf{x})}\right]\end{array}$ & $g(\mathbf{x})=1+9\left(\sum_{i=2}^{n}\right) /(n-1)$ \\
\hline $\mathrm{ZDT}^{(b)}$ & 4 & $0 \leq x_{i} \leq 1, i=1,2, \ldots, n$ & $\begin{array}{l}f_{1}(\mathbf{x})=x_{1} \\
f_{2}(\mathbf{x})=g(\mathbf{x})\left[1-\left(x_{1} / g(\mathbf{x})\right)^{2}\right]\end{array}$ & $g(\mathbf{x})=1+9\left(\sum_{i=2}\right) /(n-1)$ \\
\hline ZDT3 ${ }^{(b)}$ & 6 & $0 \leq x_{i} \leq 1, i=1,2, \ldots, n$ & $\begin{array}{l}f_{1}(\mathbf{x})=x_{1} \\
f_{2}(\mathbf{x})=g(\mathbf{x})\left[1-\sqrt{x_{1} / g(\mathbf{x})}-\frac{x_{1}}{g(\mathbf{x})} \sin \left(10 \pi x_{1}\right)\right]\end{array}$ & $g(\mathbf{x})=1+9\left(\sum_{i=2}^{n}\right) /(n-1)$ \\
\hline
\end{tabular}

Tabla 4.3: Problemas test utilizados en esta tesis.

\begin{tabular}{lclllll}
\hline Problema & $\begin{array}{c}m \\
\left(\mathscr{X} \in \mathbb{X}^{m}\right)\end{array}$ & NumFeas $_{\min }$ & $\mathrm{It}_{\max }$ & Crit $_{\max }$ & Tolg $_{\mathrm{g}}$ & $\mathrm{K}$ \\
\hline \hline MOP2 & 10 & - & 200 & $1 \mathrm{e}-4$ & $1 \mathrm{e}-6$ & 80 \\
TNK & 10 & 10 & 200 & $1 \mathrm{e}-4$ & $1 \mathrm{e}-6$ & 80 \\
DEB & 6 & 10 & 200 & $1 \mathrm{e}-4$ & $1 \mathrm{e}-6$ & 80 \\
SRN & 10 & 5 & 200 & $1 \mathrm{e}-4$ & $1 \mathrm{e}-6$ & 80 \\
ZDT1 & 40 & 20 & 200 & $1 \mathrm{e}-4$ & $1 \mathrm{e}-6$ & 80 \\
ZDT2 & 40 & 20 & 200 & $1 \mathrm{e}-4$ & $1 \mathrm{e}-6$ & 80 \\
ZDT3 & 60 & 30 & 200 & $1 \mathrm{e}-4$ & $1 \mathrm{e}-6$ & 80 \\
\hline
\end{tabular}

Tabla 4.4: Configuración de los parámetros del algoritmo EHV-EMO.

Configuración de la experimentación numérica. La Tabla 4.4 recoge la configuración de los parámetros del algoritmo EHV-EMO para cada uno de los benchmarks estudiados. En todos los casos, las funciones objetivo y las restricciones se aproximan por modelos Kriging a partir de un diseño de experimentos generado mediante un Hypercubo Latino cuasi-óptimo (minimizando la máxima distancia entre puntos (Dam et al., 2007)). Los modelos Kriging se ajustan utilizando una función de correlación exponencial-Gaussiana y una función de regresión constante.

El conjunto de diseños óptimos de Pareto obtenido se compara con el frente de Pareto generado con el algoritmo evolucionario NSGA-II. Los parámetros de este último se muestran en la Tabla 4.5. 


\begin{tabular}{ll}
\hline Parámetro & Valor \\
\hline \hline Población & 200 \\
Máximo número de geneneraciones & 200 \\
Probabilidad de cruce & 0.9 \\
Valor real de la probabilidad de mutación & $1 / d^{(a)}$ \\
Valor real del parámetro SBX & 10 \\
Valor real del parámetro de mutación & 50 \\
\hline & \\
(a) dimensión del problema
\end{tabular}

Tabla 4.5: Configuración de los parámetros del algoritmo NSGA-II.

Indicadores del rendimiento del algoritmo. Para evaluar como de cerca está la solución obtenida de la solución de referencia (NSGA-II), se utilizan tres índices (Coello et al., 2002; Zitzler et al., 2003) representativos de las diferencias entre los resultados de referencia y los obtenidos mediante el algoritmo EHV-EMO. Los indicadores utilizados son:

- Generational distance (GD). Esta métrica ha sido utilizada en otros estudios como los de Veldhuizen y Lamont (1998. Air Force Inst. Technol.) y representa como de lejos está el frente de Pareto conocido del frente de referencia. Se define como:

$$
\mathrm{GD}=\frac{\left(\sum_{i=1}^{n} d_{i}^{p}\right)^{1 / p}}{n},
$$

donde $n$ es el número de soluciones óptimas de Pareto en el frente conocido; $p=2$ y $d_{i}$ es la distancia Euclídea (en el espacio de funciones objetivo) entre cada vector de diseño óptimo y el miembro más cercano del frente de referencia. Un resultado de cero indica que ambos frentes son iguales, mientras que un valor distinto de cero indica una desviación del frente de Pareto obtenido respecto de la referencia.

- Maximum Pareto Front Error (MPFE) (Veldhuizen, 1999). Cuantifica el peor escenario en términos de la mayor distancia en el espacio de las funciones objetivo entre cualquier individuo en el frente de Pareto aproximado y el más cercano en el frente de referencia. Se define como:

$$
\mathrm{MPFE}=\operatorname{máx}_{i} d_{i} .
$$

- Hypervolume Ratio (HR)(Veldhuizen, 1999; Zitzler et al., 2003). Calcula el hipervolumen del espacio objetivo encerrado por el frente de Pareto aproximado y un punto de referencia. Esta métrica requiere de la definición de un punto de referencia (Veldhuizen, 1999). De acuerdo con (Coelho y Bouillard, 2011) el punto de referencia es seleccionado como el vector de 
peores valores de cada función objetivo (considerando los dos frentes de Pareto). Se define como:

$$
\mathrm{HR}=\frac{\operatorname{HV}\left(P_{\text {known }}, \mathbf{f}^{\text {ref }}\right)}{\operatorname{HV}\left(P_{\text {true }}, \mathbf{f}^{\text {ref }}\right)},
$$

donde $P_{\text {known }}$ es el frente de Pareto obtenido y $P_{\text {true }}$ es el frente de Pareto real o de referencia. En esta tesis $P_{\text {known }}$ es el frente de Pareto obtenido mediante el algoritmo EHV-EMO (en adelante $P_{E H V-E M O}$ ) y $P_{\text {true }}$ es el frente de Pareto de referencia obtenido mediante el algoritmo NSGA-II (en adelante $\left.P_{N S G A-I I}\right)$.

Para tener en cuenta diferentes ordenes de magnitud de las funciones objetivo, se lleva a cabo la siguiente adimensionalización:

$$
f_{k}^{s c}=\frac{f_{k}}{\tilde{f}_{k}}
$$

donde:

$$
\begin{array}{r}
\tilde{f}_{k}=\operatorname{máx}\left\{\operatorname{máx}_{i}\left(f_{k}^{\mathrm{EHV}-\mathrm{EMO}, \mathrm{i}}\right), \operatorname{máx}_{j}\left(f_{k}^{\mathrm{NSGA}-\mathrm{II}, \mathrm{j}}\right)\right\}- \\
\operatorname{mín}\left\{\operatorname{mín}_{i}\left(f_{k}^{\mathrm{EHV}-\mathrm{EMO}, \mathrm{i}}\right), \operatorname{mín}_{j}\left(f_{k}^{\mathrm{NSGA}-\mathrm{II}, \mathrm{j}}\right)\right\} .
\end{array}
$$

Dada la aleatoriedad del algoritmo evolucionario, los diseños óptimos pertenecientes al frente de Pareto de referencia pueden variar de una ejecución a otra. Por esta razón, para cada caso se han realizado 10 ejecuciones obteniendo la media y desviación estándar de los distintos indicadores. En el caso del problema MOP2, el frente de Pareto de referencia se aproxima mediante una muestra de 200 puntos en la solución analítica del problema:

$$
P_{X}=\left\{x: x_{1}=x_{2} \forall-\frac{1}{\sqrt{2}} \leq x_{1} \leq \frac{1}{\sqrt{2}},-\frac{1}{\sqrt{2}} \leq x_{2} \leq \frac{1}{\sqrt{2}}\right\} .
$$

Resultados. Los resultados obtenidos en cada uno de los benchmarks se presentan gráficamente en las Figuras 4.9 a 4.13 y numéricamente en las tablas 4.6 y 4.7 .

Las Figuras 4.9 a 4.12 muestran la evolución del criterio de búsqueda $\mathscr{C}_{1}$ junto con el frente de soluciones óptimas de Pareto resultante en cada una de las iteraciones. Dicha representación se limita a los problemas con un número de variables de diseño de dos dada la dificultad para su representación en un espacio de mayor dimensión. Para los problemas con número de variables 
de diseño mayor se representa el frente de soluciones de Pareto final (Figura 4.13). A la vista de los resultados obtenidos, el algoritmo EHV-EMO evoluciona satisfactoriamente hacia el frente de Pareto de referencia. Destaca la relación entre el número de puntos cercanos al frente de Pareto y el número total.

Coste computacional. La Tabla 4.6 muestra un resumen del número de evaluaciones del modelo de simulación en cada etapa del algoritmo EHV-EMO. En la Tabla 4.6 se recoge: el número de evaluaciones del modelo de simulación en el diseño de experimentos $(m)$, el número de iteraciones realizadas en la búsqueda de diseños factibles $\left(\mathrm{It}_{\mathrm{F}}\right)$, el número de iteraciones realizadas en la búsqueda del frente de diseños óptimos (It), el número total de evaluaciones del modelo de simulación $\left(N_{\text {evals }}\right)$ y el número de soluciones óptimas de Pareto $\left(N_{\mathscr{P}}\right)$. Se observa un mayor número de evaluaciones para los problemas de mayor dimensionalidad (ZDT1 a ZDT3) y para problemas con zonas desconectadas en el frente de Pareto (TNK).

La Tabla 4.7 recoge el valor medio de los indicadores de calidad de la solución tras 10 repeticiones de los experimentos numéricos. En todos los casos el valor medio del ratio de hipervolumen está cercano a 1 con valores comprendidos entre 0.9681 y 0.9996 . Estos valores indican una buena precisión en la aproximación discreta obtenida del frente de Pareto.

\begin{tabular}{lcllll}
\hline Problema & $m$ & It $_{\mathrm{F}}$ & It & $N_{\text {evals }}$ & $N_{\mathscr{P}}$ \\
\hline \hline MOP2 & 10 & - & 44 & 54 & 42 \\
TNK & 10 & 15 & 78 & 103 & 27 \\
DEB & 6 & 9 & 29 & 44 & 26 \\
SRN & 10 & 7 & 27 & 44 & 28 \\
ZDT1 & 40 & 32 & 98 & 170 & 23 \\
ZDT2 & 40 & 22 & 86 & 148 & 31 \\
ZDT3 & 60 & 52 & 139 & 251 & 27 \\
\hline
\end{tabular}

Tabla 4.6: Resumen de los resultados numéricos (I).

\begin{tabular}{llllll}
\hline Problema & $\mu_{\mathrm{HV} V_{\text {EHV-EMO }}}$ & $\mu_{\mathrm{HV}_{\text {ref }}}$ & $\mu_{\mathrm{HR}}$ & $\mu_{\mathrm{GD}}$ & $\mu_{\mathrm{MPFE}}$ \\
\hline \hline MOP2 & 0.3063 & 0.3164 & 0.9681 & 0.002 & 0.1667 \\
TNK & 0.2847 & 0.2929 & 0.9720 & 0.001 & 0.017 \\
DEB & 0.4113 & 0.4166 & 0.9873 & 0.0012 & 0.0147 \\
SRN & 0.9898 & 0.9937 & 0.9961 & $9.6886 \mathrm{e}-4$ & 0.0097 \\
ZDT1 & 0.6372 & 0.6611 & 0.9638 & 0.0015 & 0.0164 \\
ZDT2 & 0.4931 & 0.4933 & 0.9996 & $6.3587 \mathrm{e}-4$ & 0.0154 \\
ZDT3 & 0.7528 & 0.7608 & 0.9894 & $8.5611 \mathrm{e}-4$ & 0.0090 \\
\hline
\end{tabular}

Tabla 4.7: Resumen de los resultados numéricos (II). 


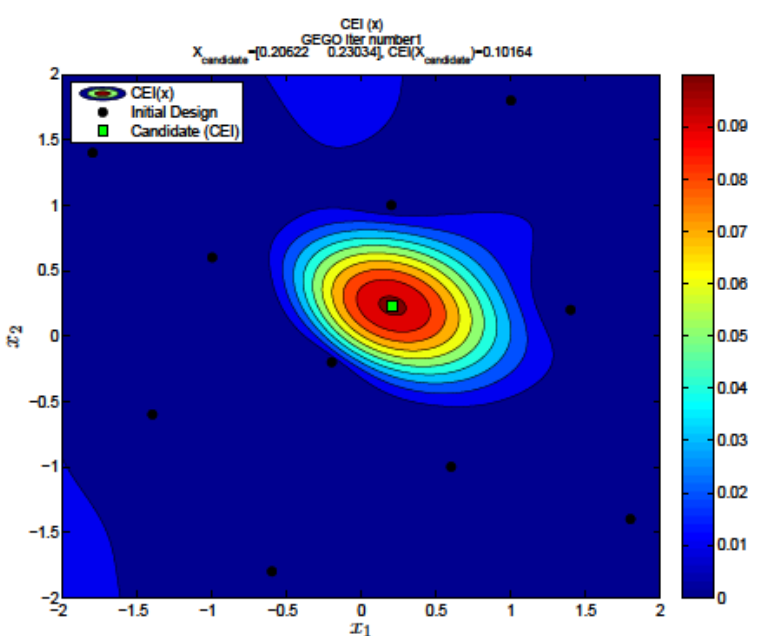

(a) Iter $=1$

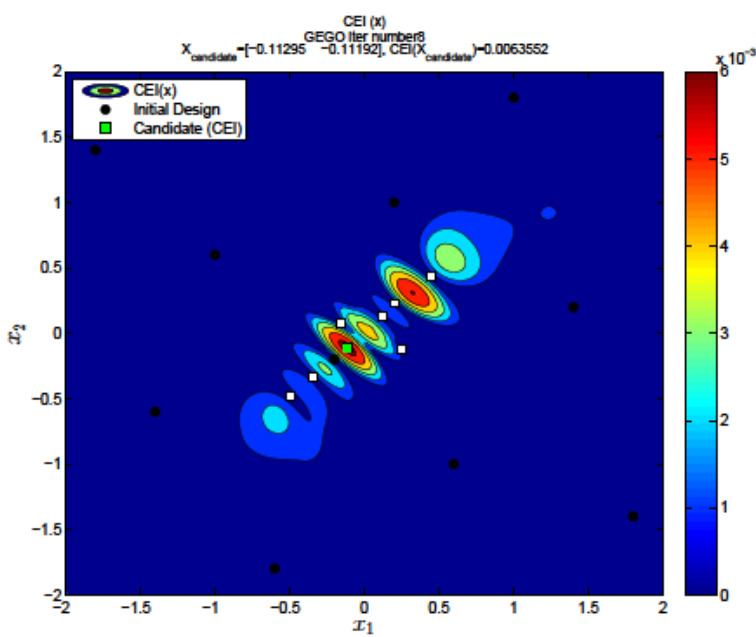

(c) Iter $=8$

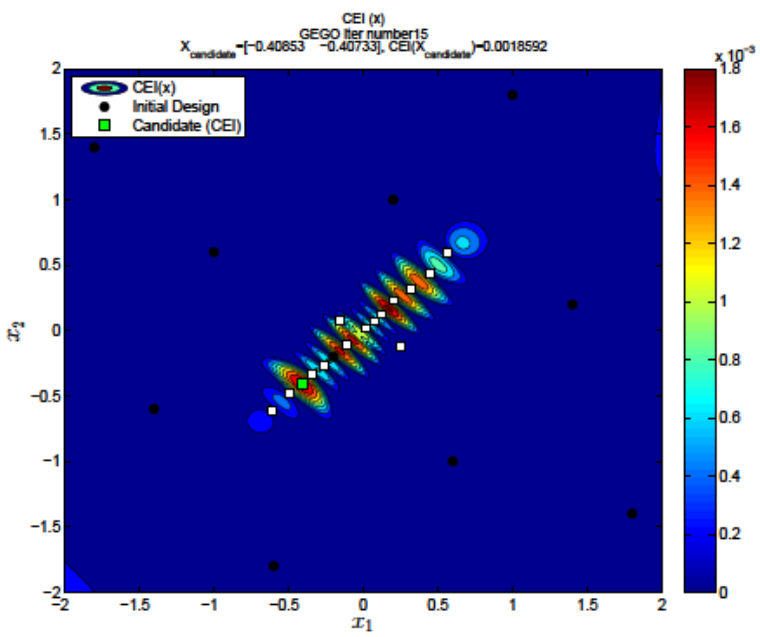

(e) Iter $=15$

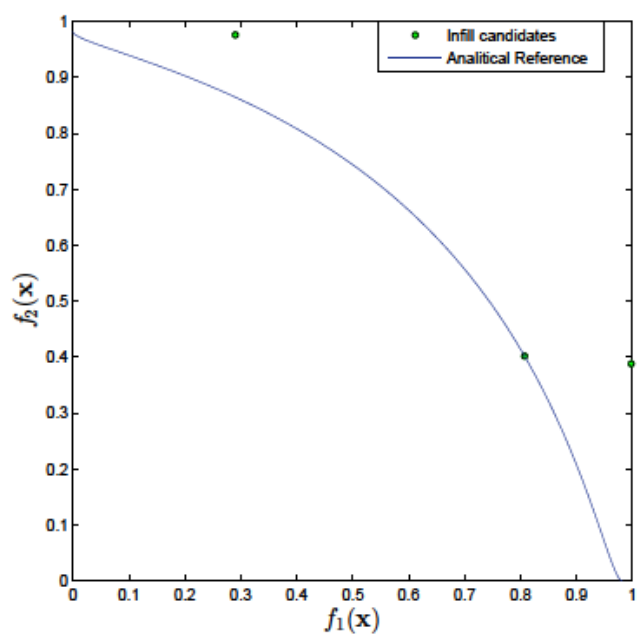

(b) Iter $=1$

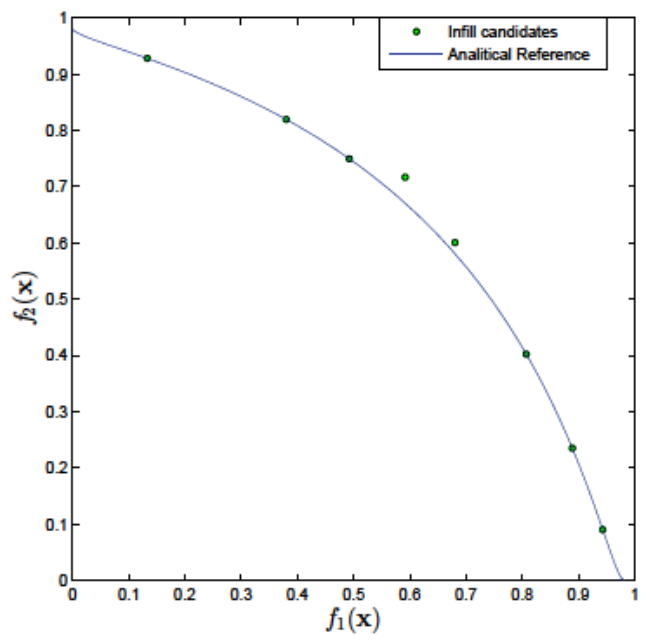

(d) Iter $=8$

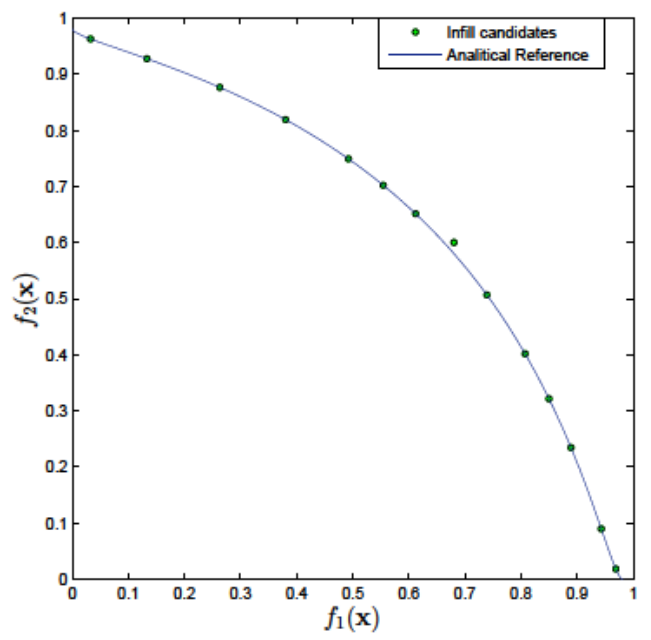

(f) Iter $=15$

Figura 4.9: Evolución del criterio de búsqueda en el problema MOP2. La muestra inicial se presenta como círculos negros. Los cuadrados blancos representan los puntos añadidos hasta la iteración. Los cuadrados verdes representan el candidato que maximiza el criterio en cada iteración. 


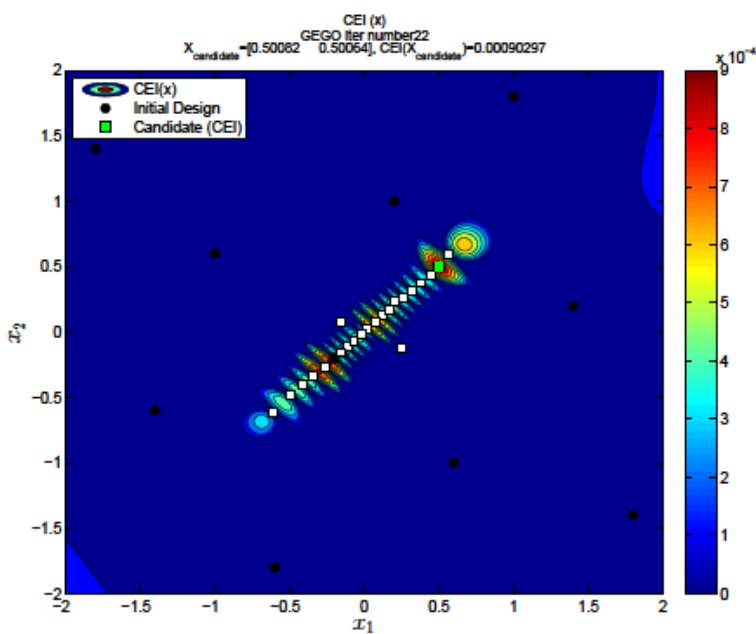

(g) Iter $=22$

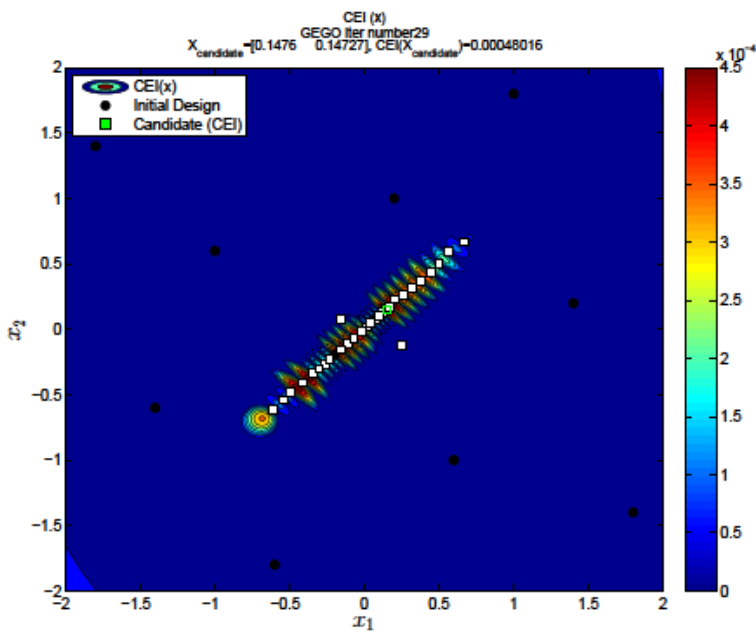

(i) Iter $=29$

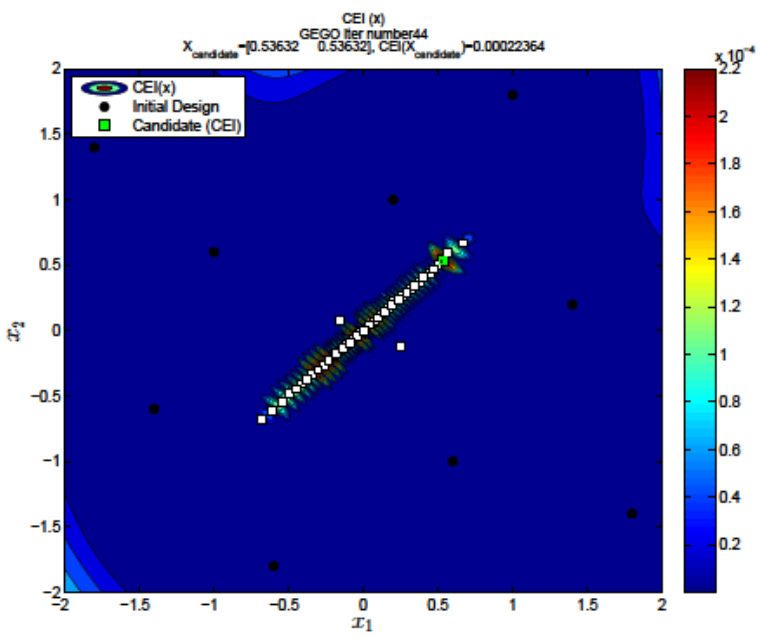

(k) Iter $=44$

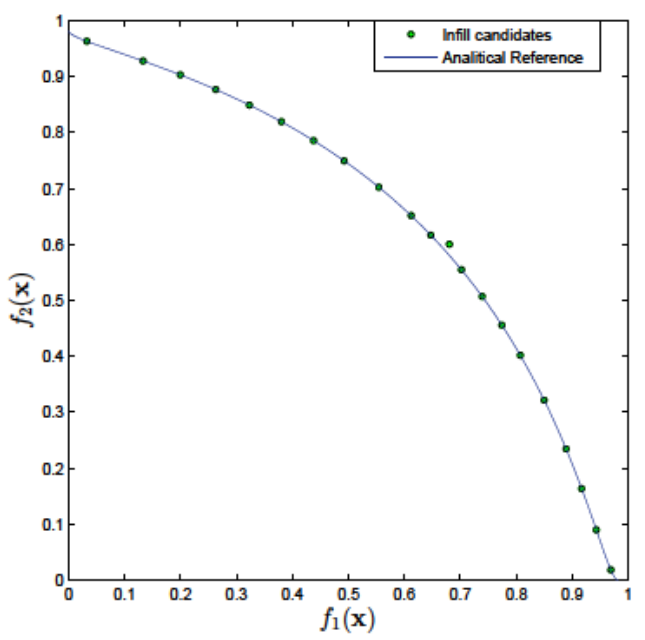

(h) Iter $=22$

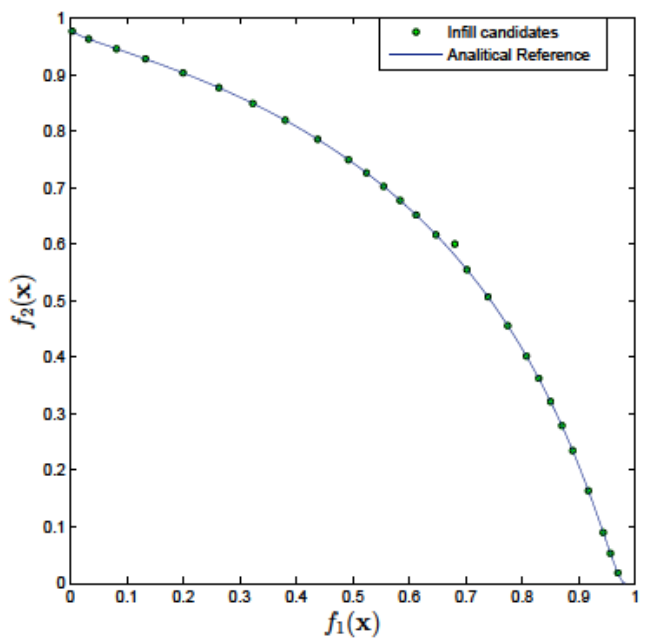

(j) Iter $=29$

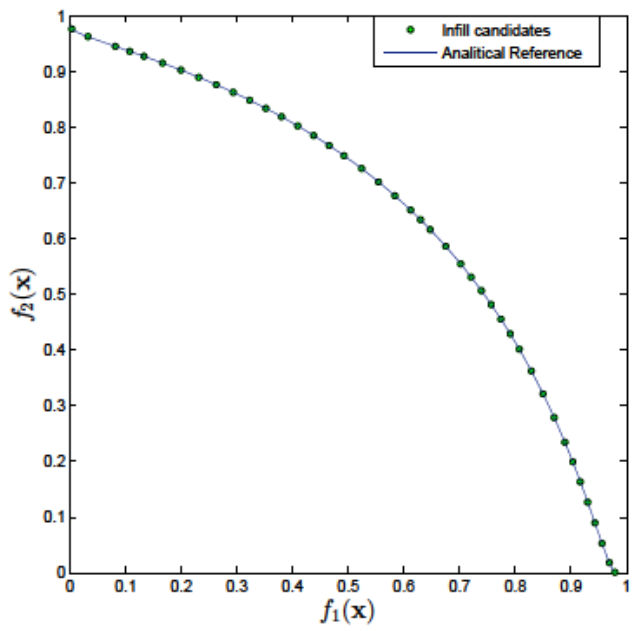

(l) Iter $=44$

Figura 4.9: (Continuación). Evolución del criterio de búsqueda en el problema MOP2. La muestra inicial se presenta como círculos negros. Los cuadrados blancos representan los puntos añadidos hasta la iteración. Los cuadrados verdes representan el candidato que maximiza el criterio en cada iteración. 


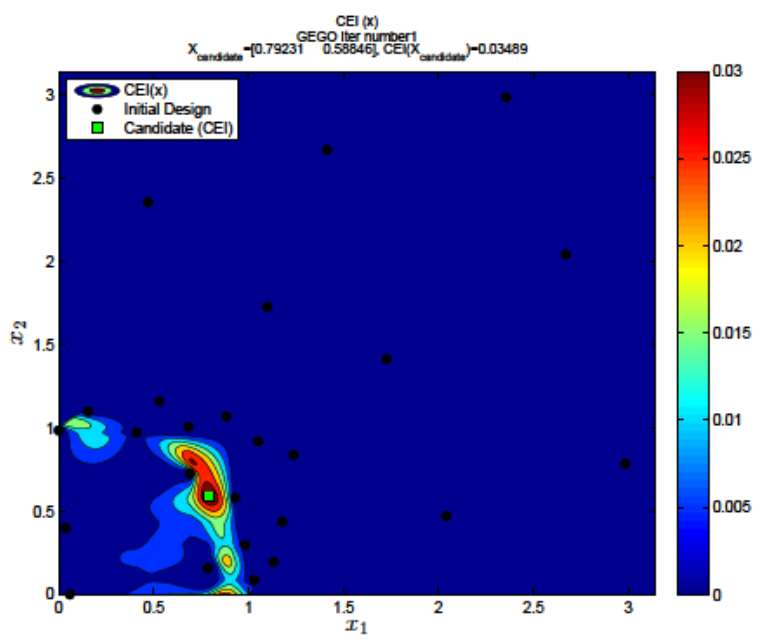

(a) Iter $=1$

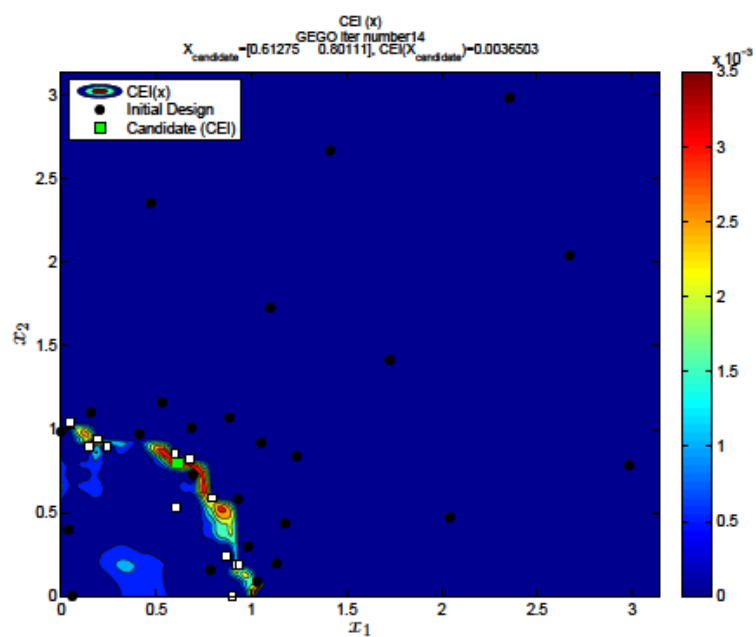

(c) Iter $=14$

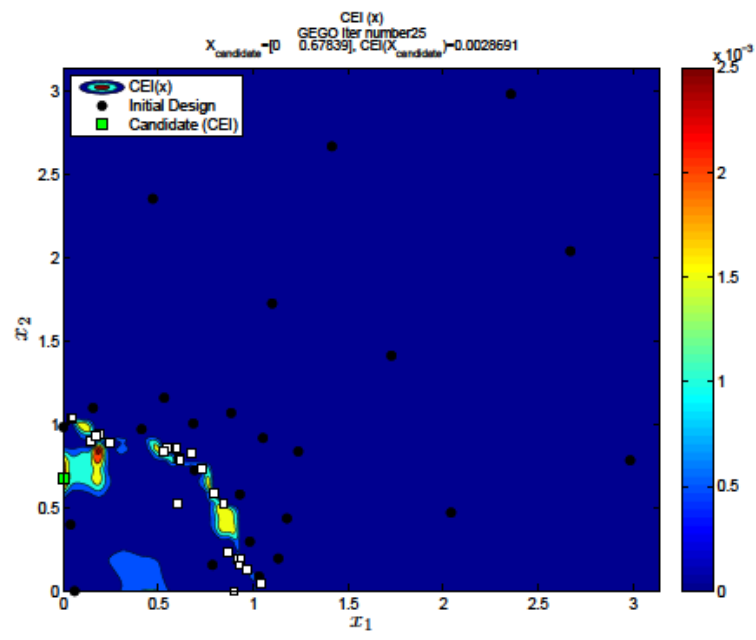

(e) Iter $=25$

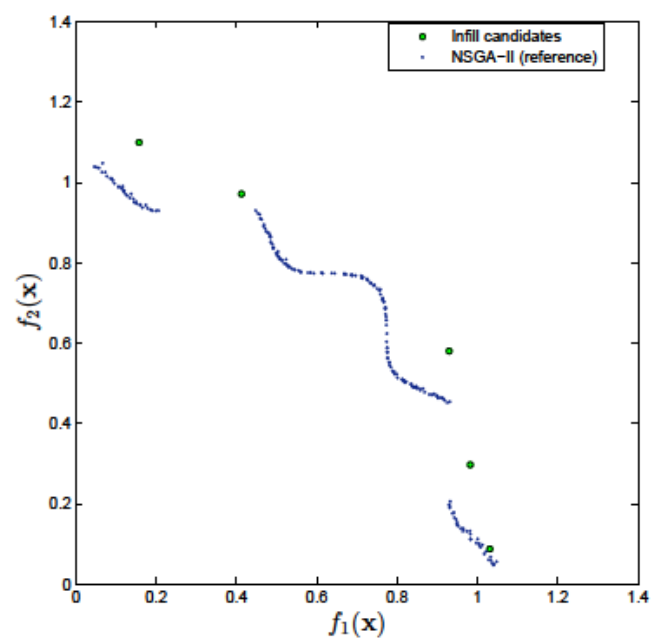

(b) Iter=1

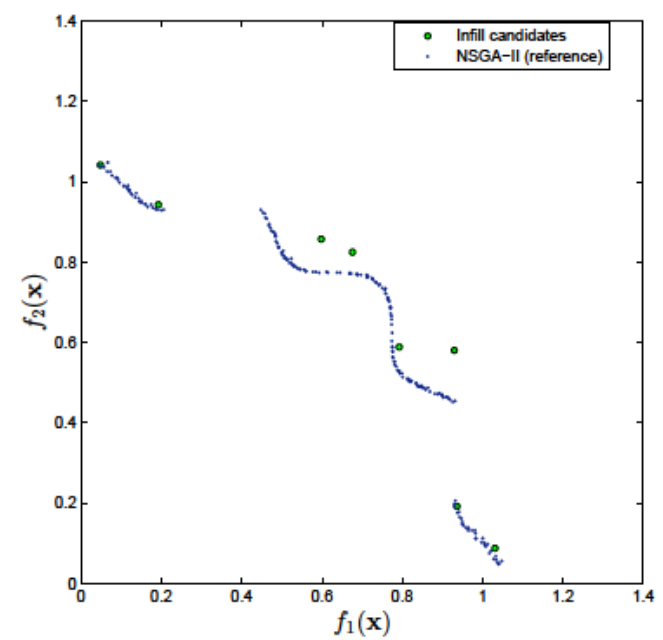

(d) Iter $=14$

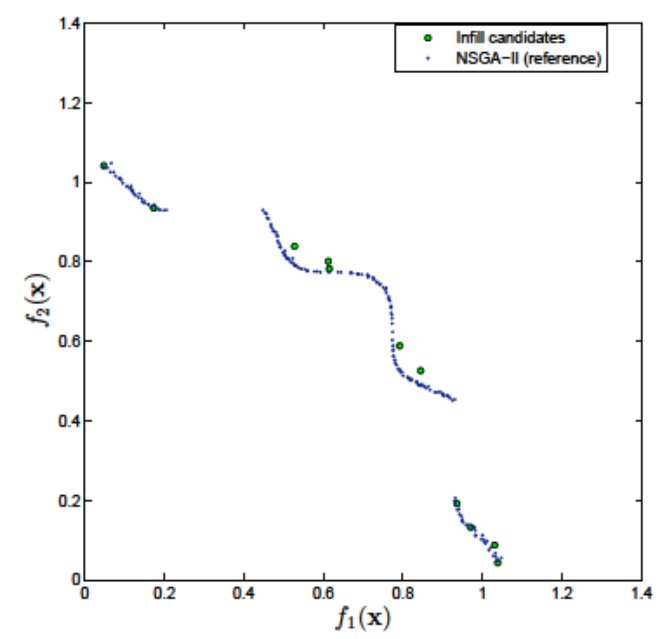

(f) Iter $=25$

Figura 4.10: Evolución del criterio de búsqueda en el problema TNK. La muestra inicial se presenta como círculos negros. Los cuadrados blancos representan los puntos añadidos hasta la iteración. Los cuadrados verdes representan el candidato que maximiza el criterio en cada iteración. 


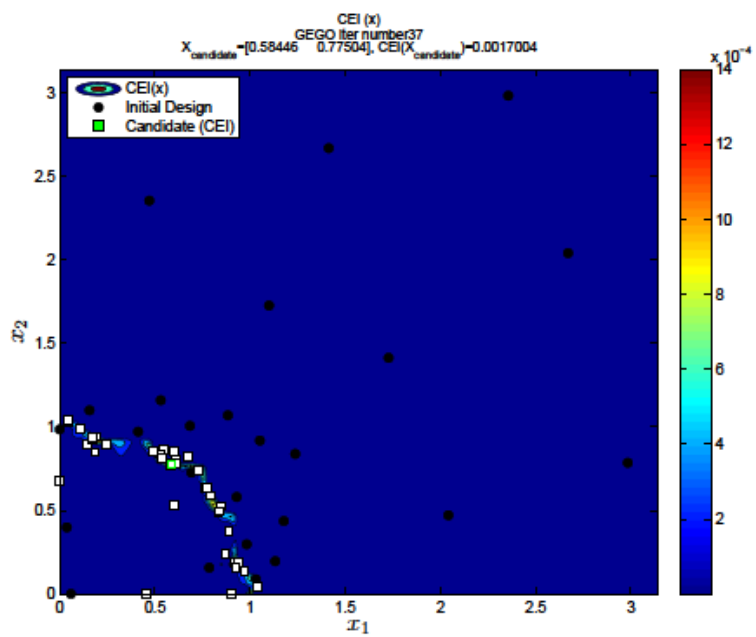

(g) Iter $=37$

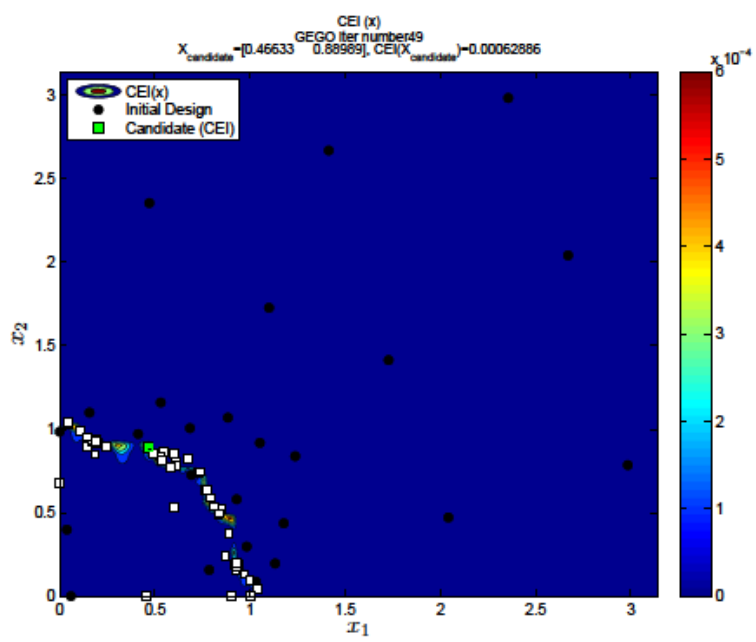

(i) Iter $=49$

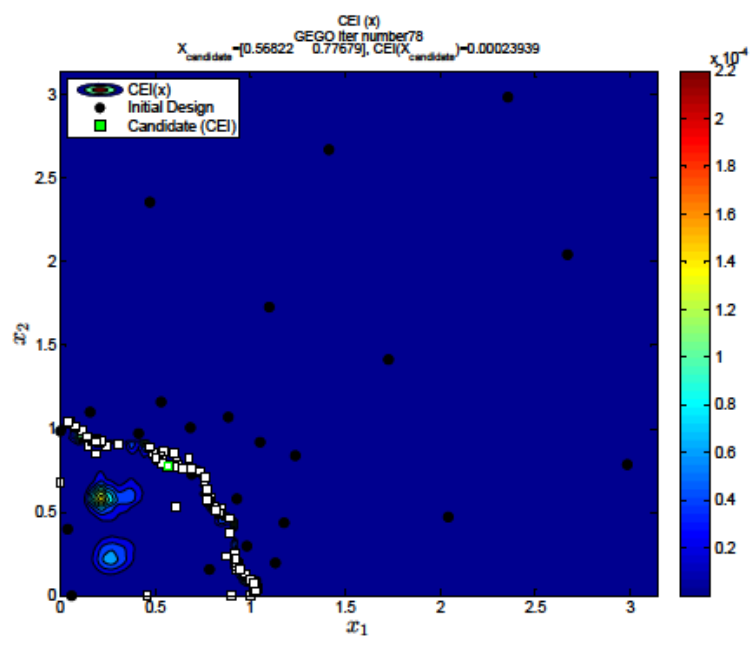

(k) Iter $=78$

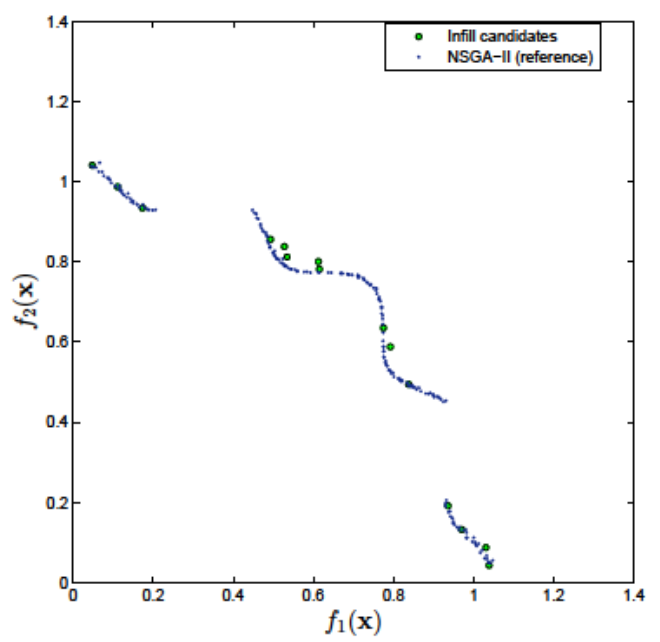

(h) Iter $=37$

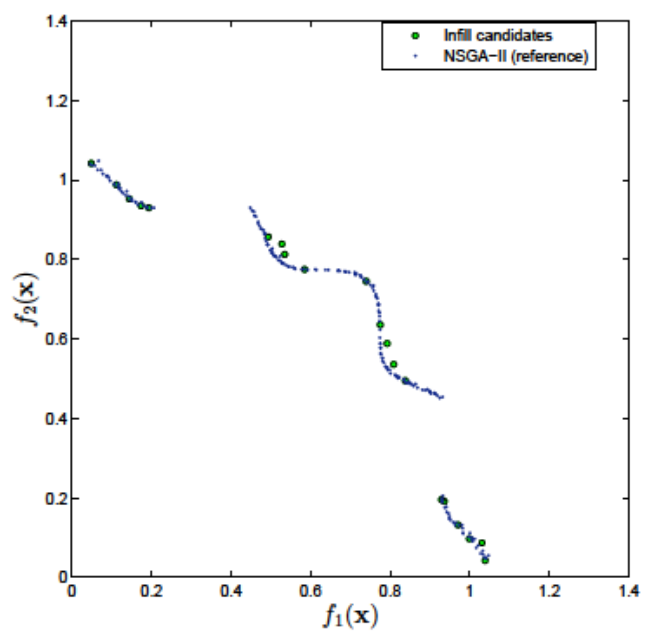

(j) Iter $=49$

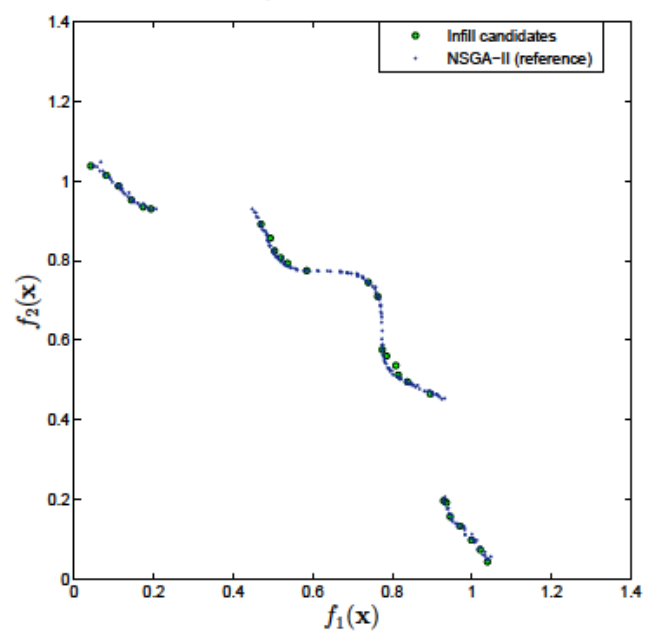

(1) Iter $=78$

Figura 4.10: (Continuación). Evolución del criterio de búsqueda en el problema TNK. La muestra inicial se presenta como círculos negros. Los cuadrados blancos representan los puntos añadidos hasta la iteración. Los cuadrados verdes representan el candidato que maximiza el criterio en cada iteración. 


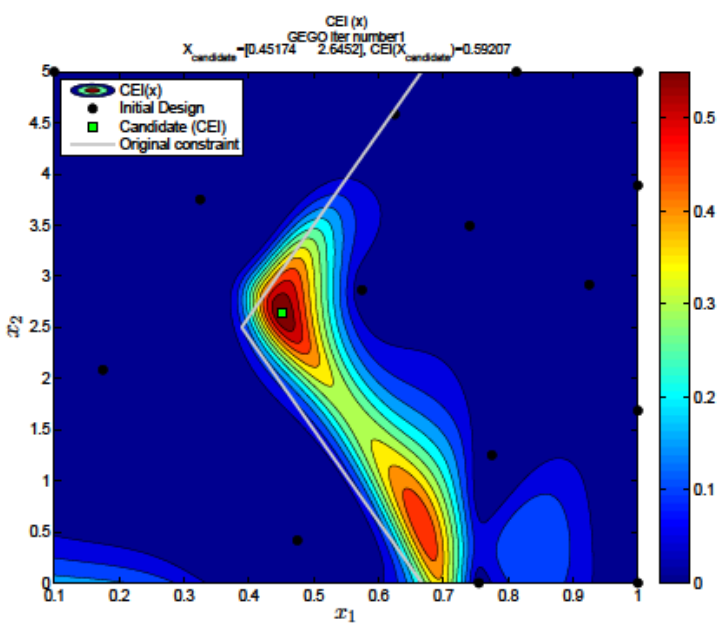

(a) Iter $=1$

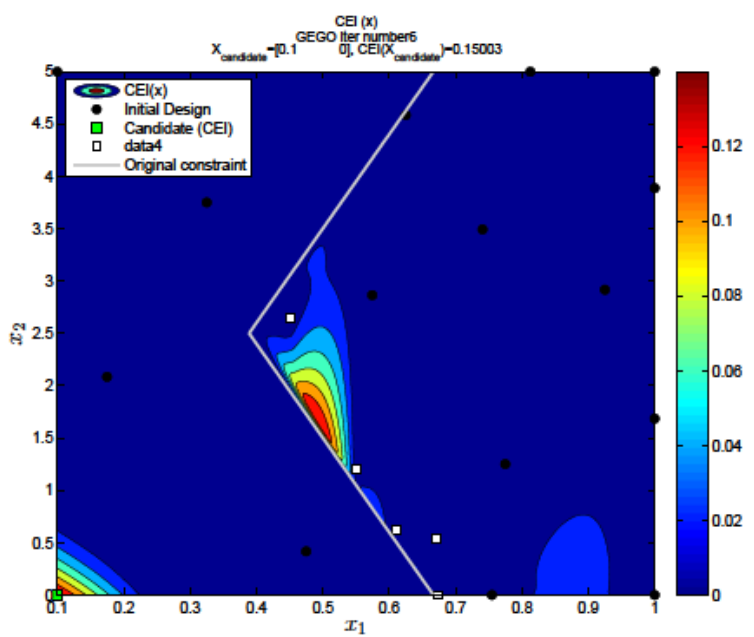

(c) Iter $=6$

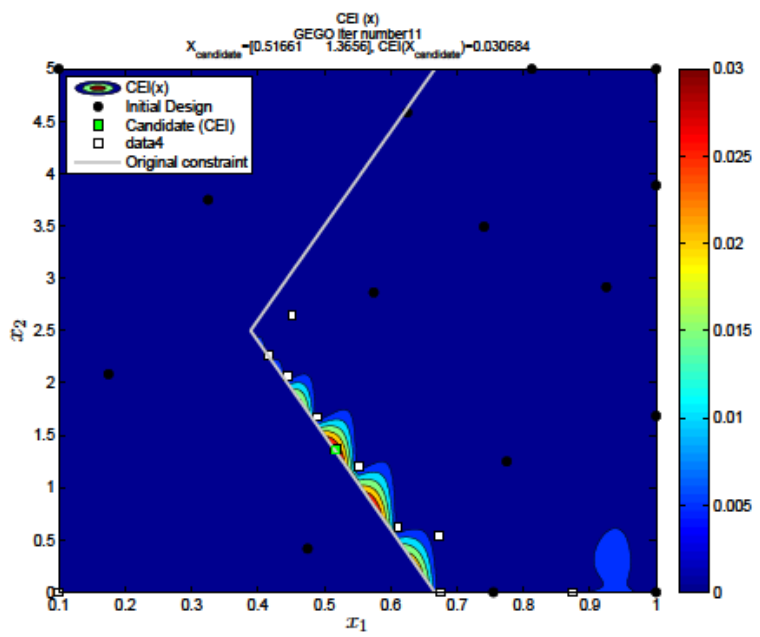

(e) Iter $=11$

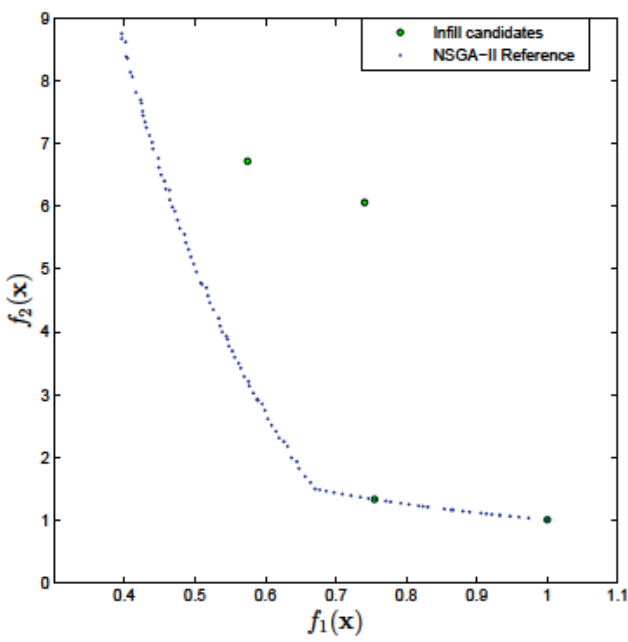

(b) Iter $=1$

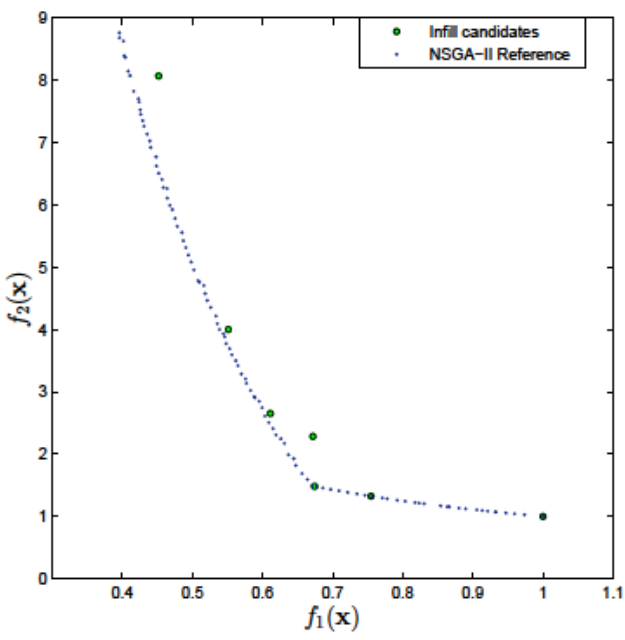

(d) Iter $=6$

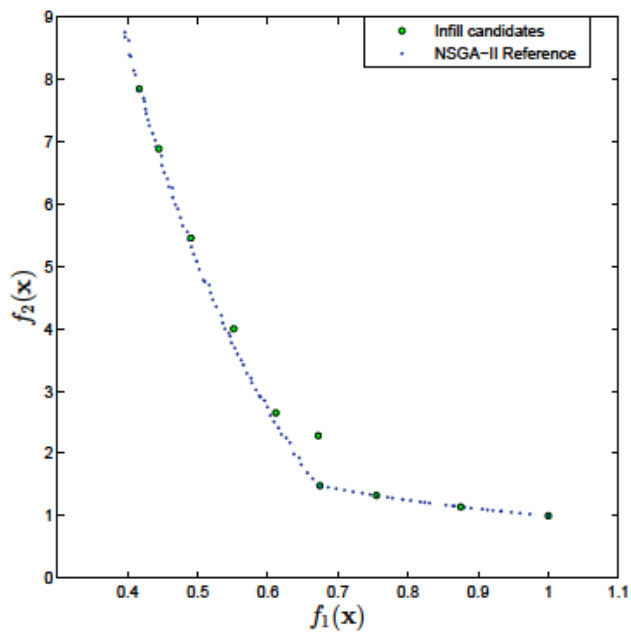

(f) Iter $=11$

Figura 4.11: Evolución del criterio de búsqueda en el problema DEB. La muestra inicial se presenta como círculos negros. Los cuadrados blancos representan los puntos añadidos hasta la iteración. Los cuadrados verdes representan el candidato que maximiza el criterio en cada iteración. 


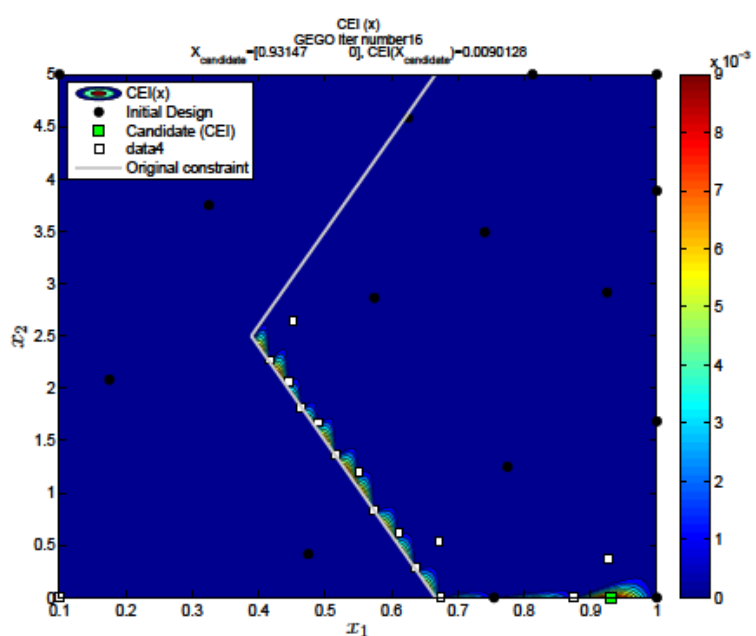

(g) Iter $=16$

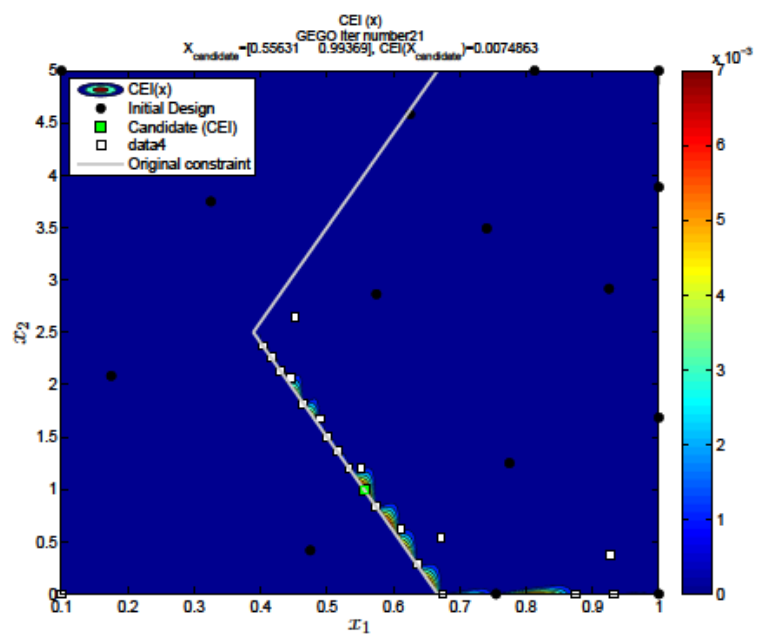

(i) Iter $=21$

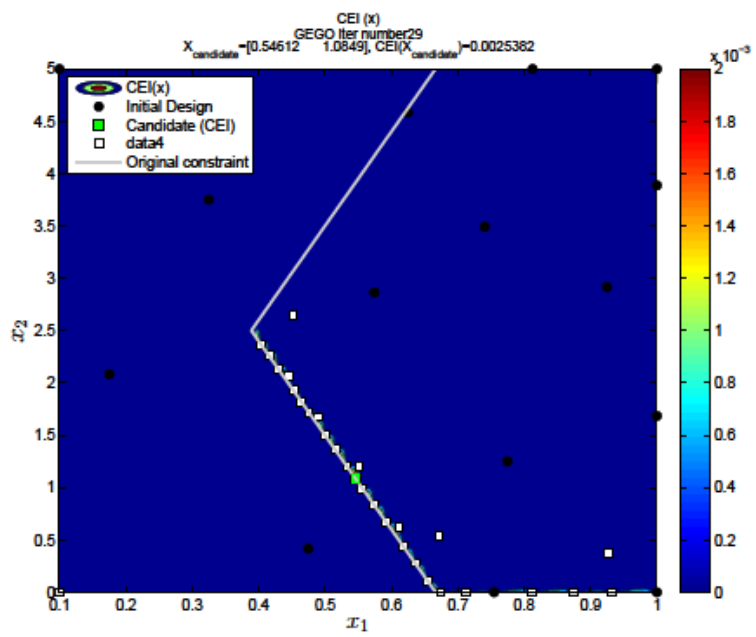

(k) Iter $=29$

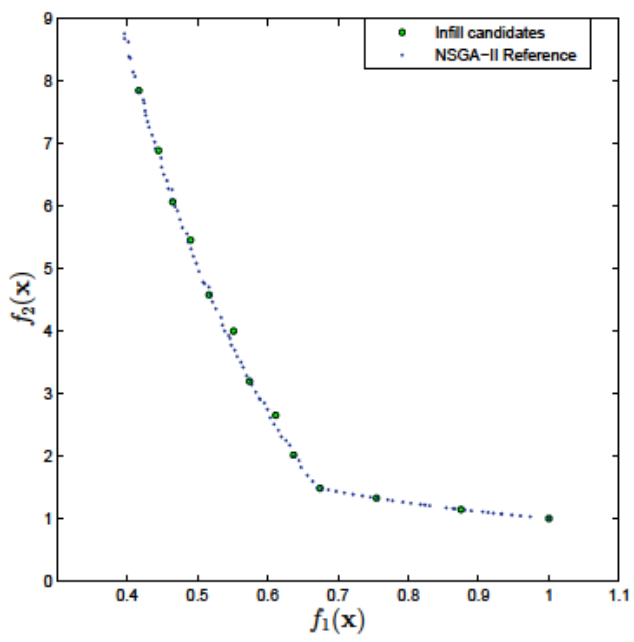

(h) Iter $=16$

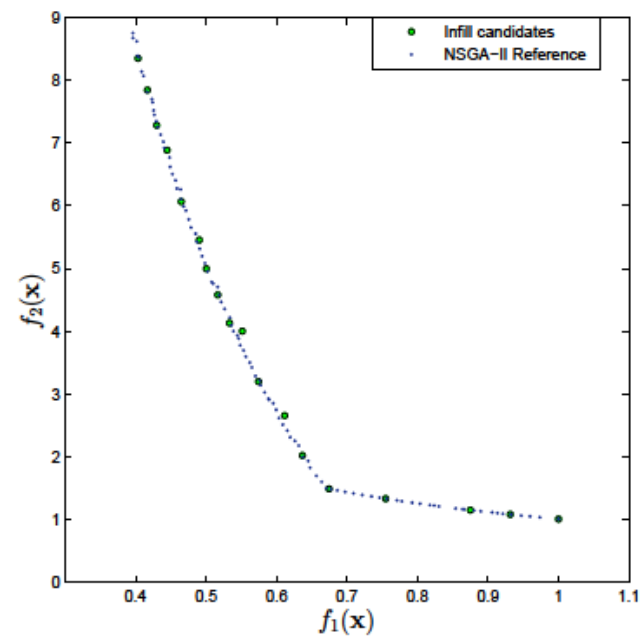

(j) Iter $=21$

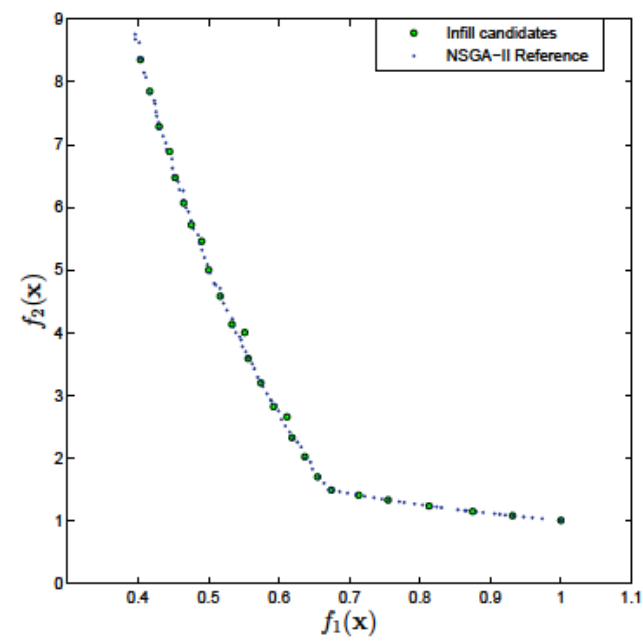

(1) Iter $=29$

Figura 4.11: (Continuación). Evolución del criterio de búsqueda en el problema DEB. La muestra inicial se presenta como círculos negros. Los cuadrados blancos representan los puntos añadidos hasta la iteración. Los cuadrados verdes representan el candidato que maximiza el criterio en cada iteración. 


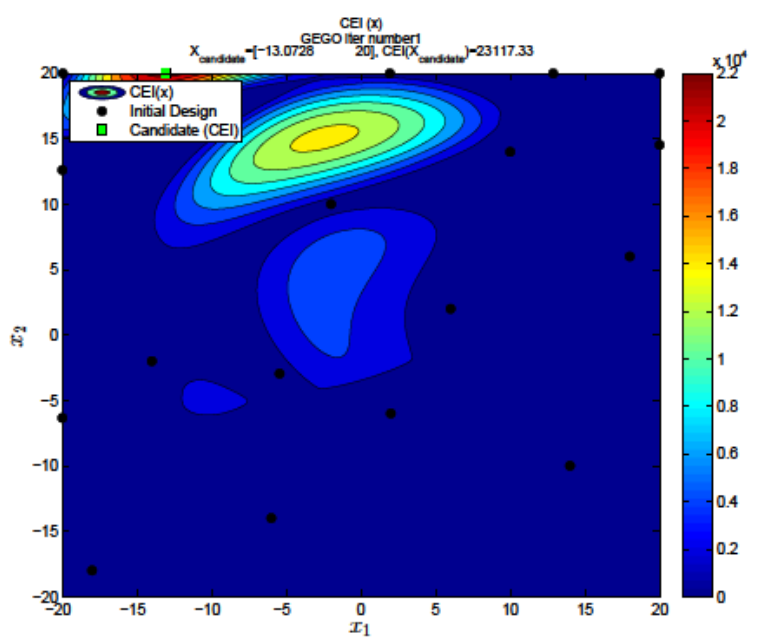

(a) Iter $=1$

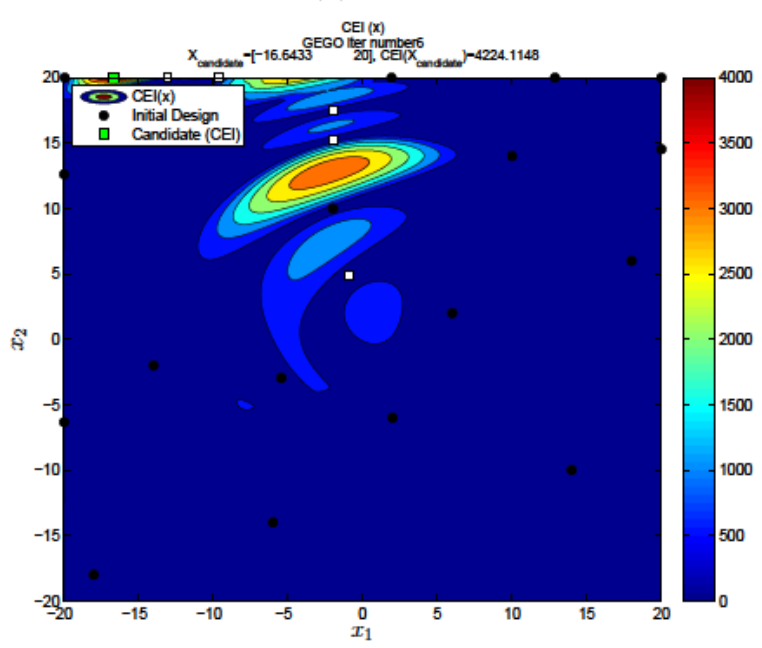

(c) Iter $=6$

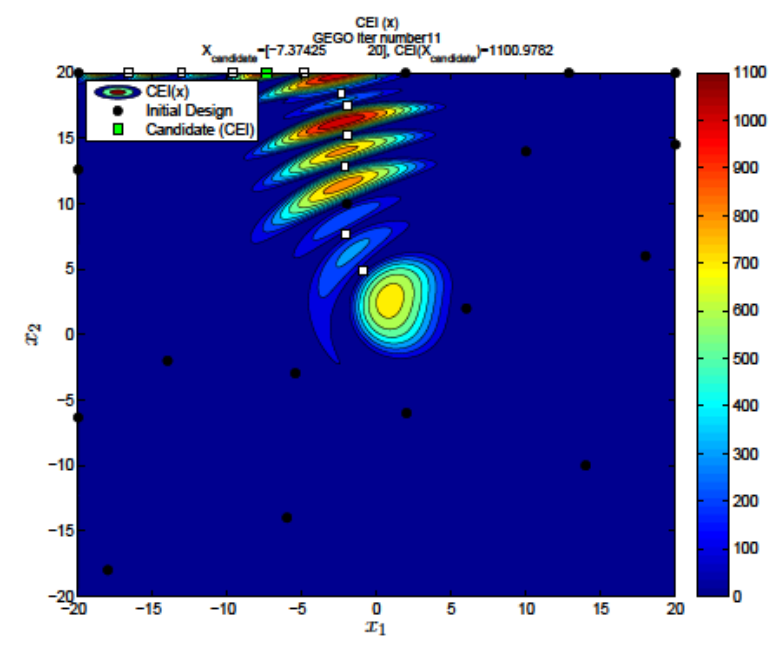

(e) Iter $=11$

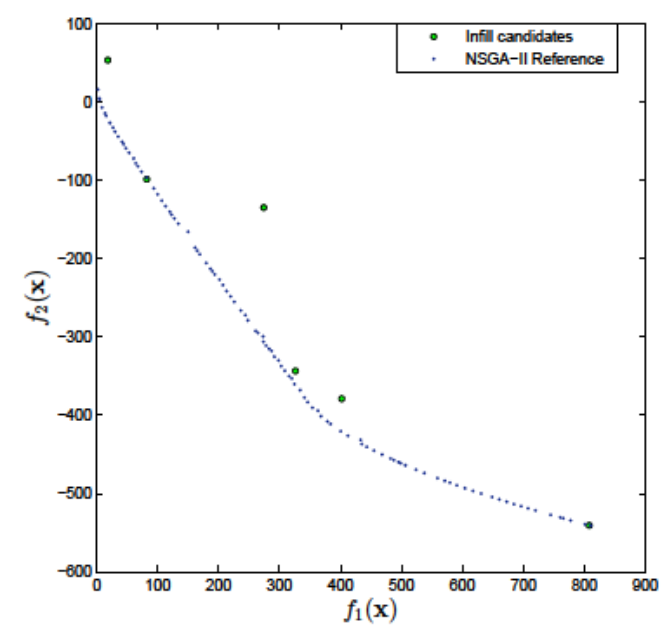

(b) Iter=1

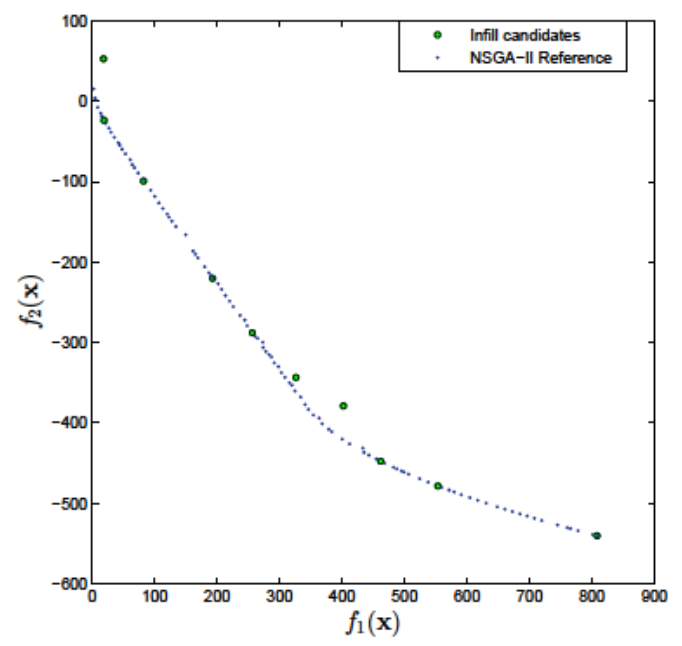

(d) Iter $=6$

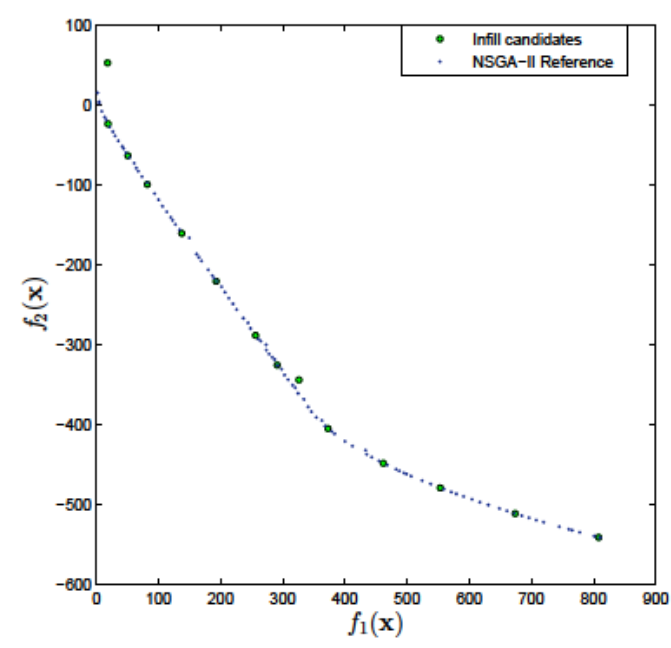

(f) Iter=11

Figura 4.12: Evolución del criterio de búsqueda en el problema SRN. La muestra inicial se presenta como círculos negros. Los cuadrados blancos representan los puntos añadidos hasta la iteración. Los cuadrados verdes representan el candidato que maximiza el criterio en cada iteración. 


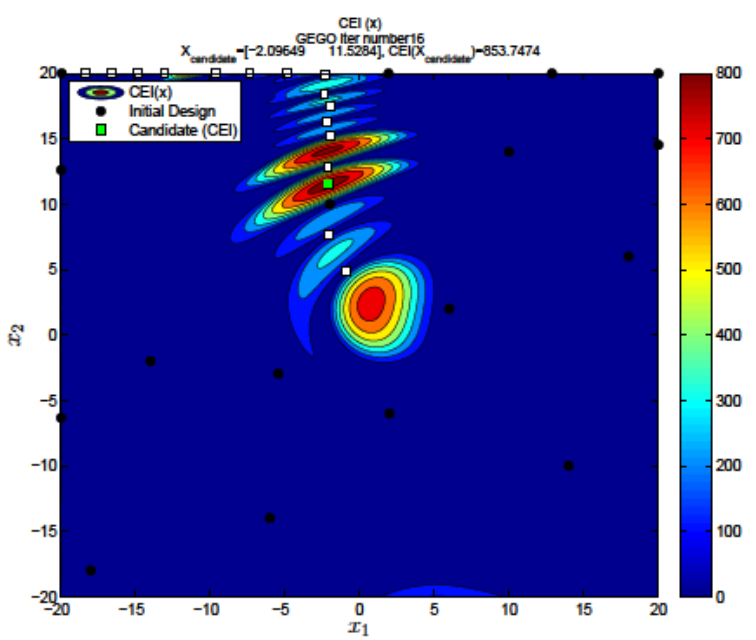

(g) Iter $=16$

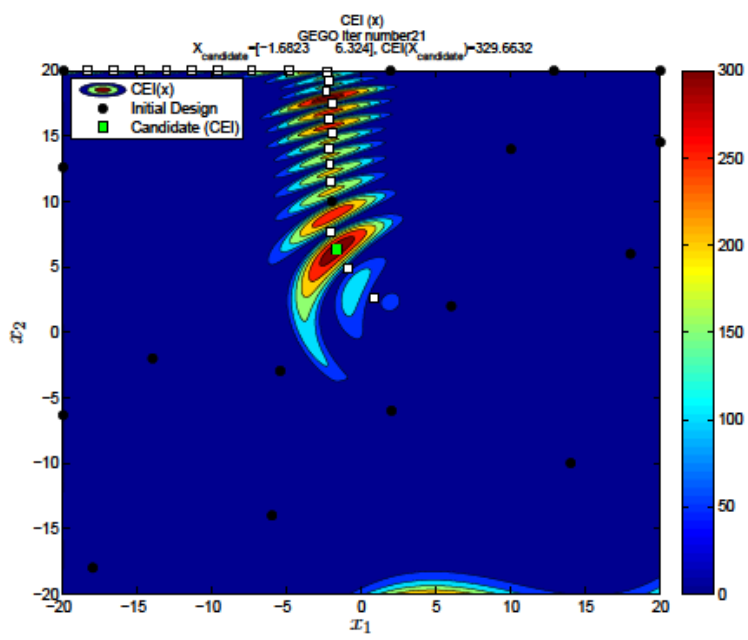

(i) Iter $=21$

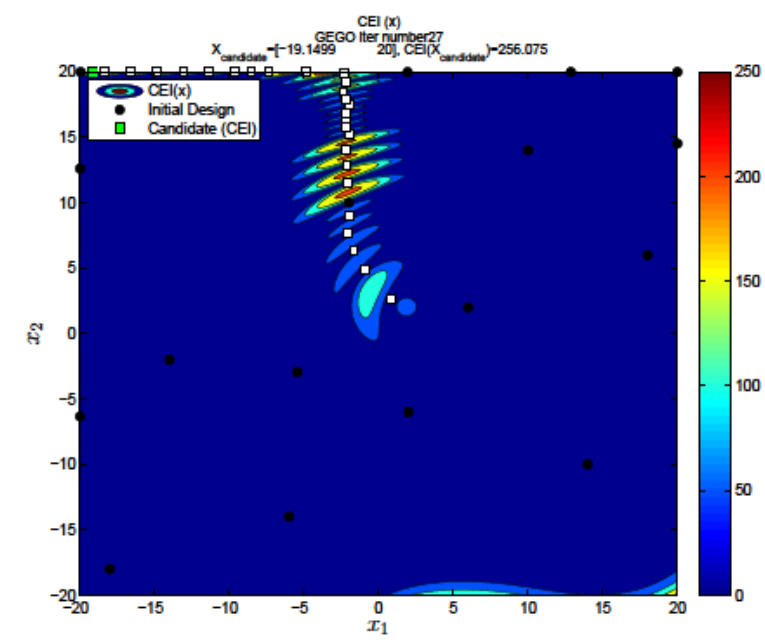

(k) Iter $=27$

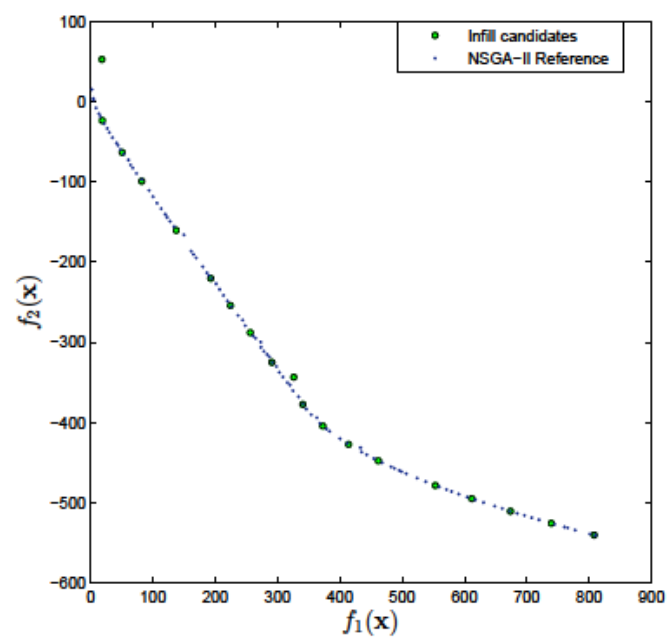

(h) Iter $=16$

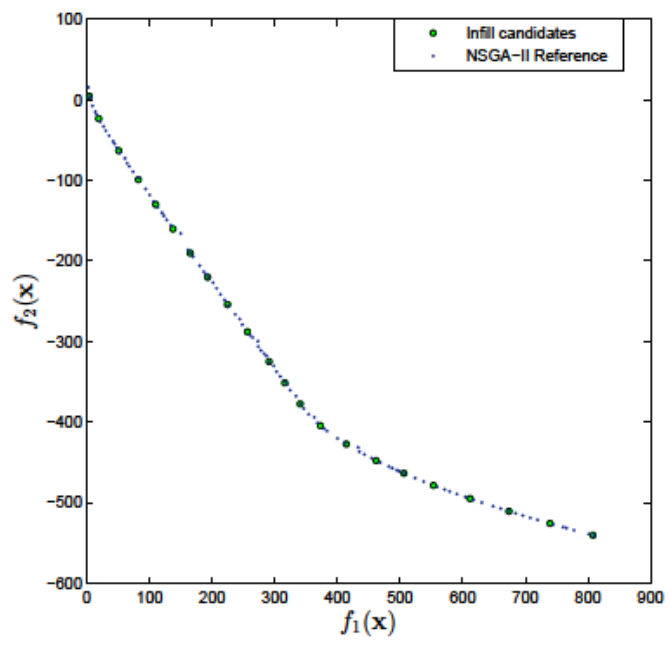

(j) Iter $=21$

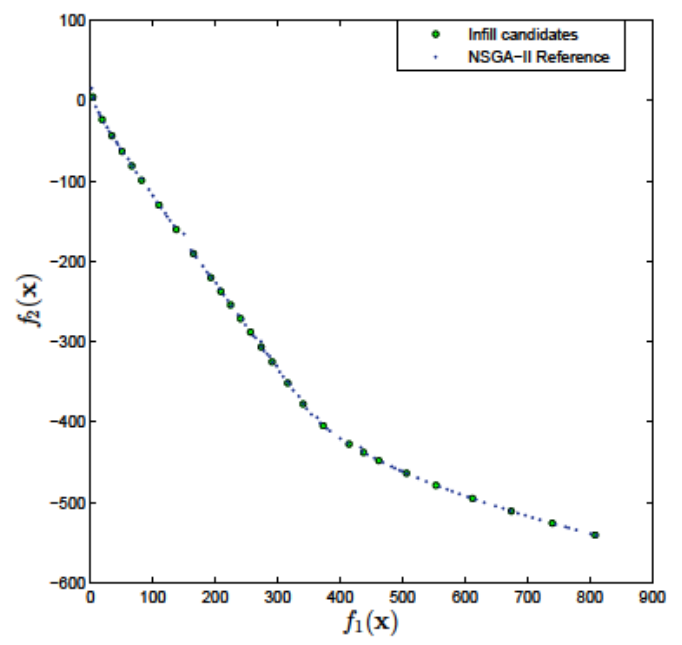

(l) Iter $=27$

Figura 4.12: (Continuación). Evolución del criterio de búsqueda en el problema SRN. La muestra inicial se presenta como círculos negros. Los cuadrados blancos representan los puntos añadidos hasta la iteración. Los cuadrados verdes representan el candidato que maximiza el criterio en cada iteración. 


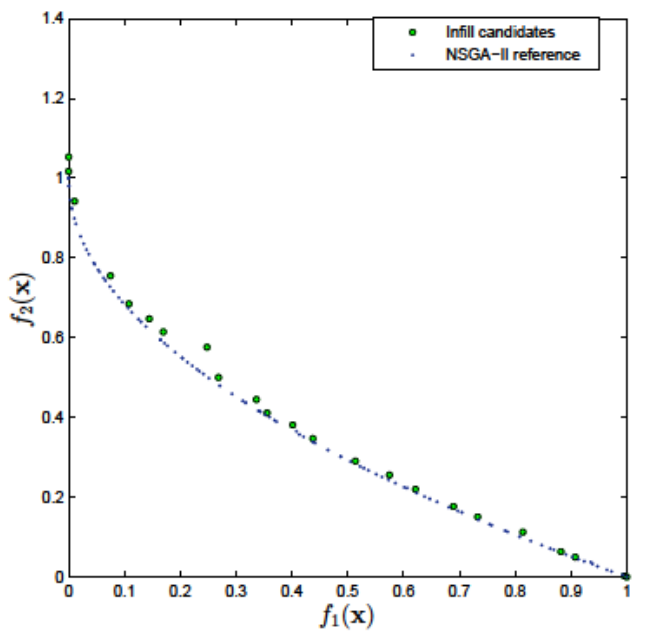

(a)

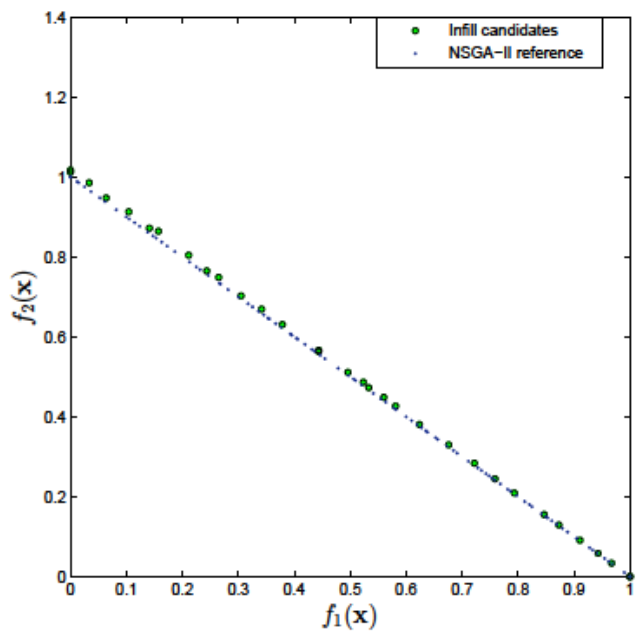

(b)

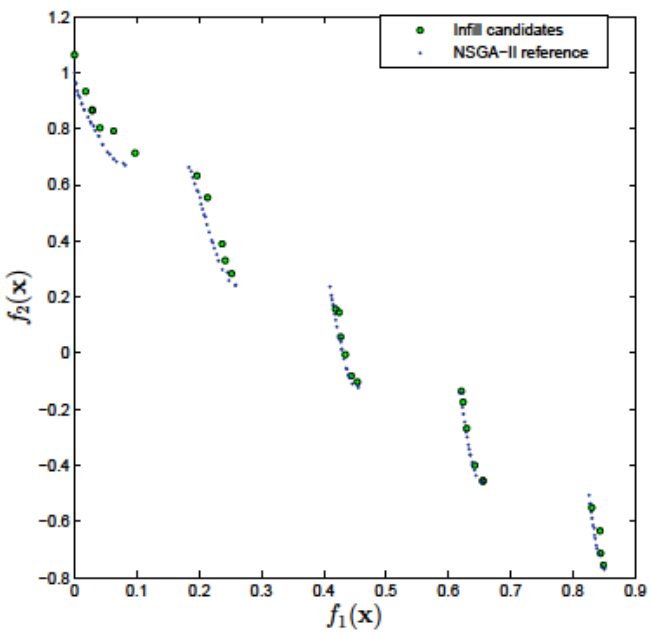

(c)

Figura 4.13: Frentes de Pareto de los problemas ZDT1, ZDT2, Y ZDT3. 


\subsection{Discusión y conclusiones}

En este capítulo se ha tratado el problema de la resolución eficiente de problemas de optimización multi-objetivo con modelos de simulación de alto coste computacional. Se ha realizado una revisión de la literatura del problema de optimización multi-objetivo con meta-modelos, presentando especial atención a los diferentes enfoques que resuelven el problema utilizando procesos Gaussianos y análisis Bayesiano. Se ha propuesto el algoritmo EHV-EMO, que utiliza la información estadística proporcionada por los modelos Kriging para estimar la mejora esperada en el hipervolumen del frente de soluciones dominadas. Este criterio se utiliza como criterio de búsqueda de nuevos puntos candidatos a formar parte de la muestra inicial. La precisión y eficiencia del algoritmo propuesto se estudia con la resolución de un conjunto de benchmarks utilizados en el campo de la optimización multi-objetivo. Como resultado del trabajo realizado se alcanzan las siguientes conclusiones:

\section{Revisión del estado del arte.}

1. Si lo comparamos con el problema con un único objetivo, el problema de optimización MO requiere un coste computacional mayor, bien por un masivo número de evaluaciones (ej. algoritmos evolucionarios multi-objetivo) o bien por la necesidad de resolver varias optimizaciones con un único objetivo (ej. métodos de escalarización). Este problema se agudiza cuando se utilizan modelos de simulación de alto coste computacional para la evaluación de las funciones objetivo, o cuando son necesarios métodos de propagación de incertidumbre anidados en el proceso de optimización (optimización bajo incertidumbre). En estos casos, se utilizan técnicas de meta-modelos para la resolución eficiente del problema.

2. La utilización de parte de las soluciones no-dominadas para la actualización del meta-modelo proporciona en ocasiones soluciones satisfactorias convergiendo al frente de Pareto real. Sin embargo, al igual que ocurre en el problema con un único objetivo, dicho enfoque únicamente se basa en las soluciones óptimas para la actualización del meta-modelo (explotación) y no considera la incertidumbre epistémica del mismo (exploración).

3. Los trabajos existentes en la literatura que tratan el problema de optimización multi-objetivo con algoritmos Bayesianos están basados en su mayoría en la escalarización previa del problema MO. La utilización de criterios heurísticos sobre el problema escalarizado no garantiza la convergencia a la solución del problema $\mathrm{MO}$ original.

4. Entre los enfoques que utilizan criterios heurísticos multi-objetivo, los métodos basados en la mejora esperada del hipervolumen proporcionan soluciones no-dominadas precisas y uniformemente distribuidas en el espacio de las funciones objetivo. Por esta razón, es el criterio a partir del 
cual se desarrolla el algoritmo propuesto en este capítulo.

\section{Validación numérica.}

1. El criterio $\mathscr{C}_{2}$ utilizado para la búsqueda de puntos en el espacio de diseño factible se ha mostrado eficiente en la resolución de problemas con espacios factibles complejos desconectados entre sí.

2. A partir de los resultados obtenidos en la experimentación numérica, se puede afirmar que: la utilización del valor esperado del hipervolumen como criterio de búsqueda permite aproximar el frente de soluciones no-dominadas de forma precisa y uniforme en el espacio objetivo.

3. Los ejemplos estudiados ponen de manifiesto que el algoritmo propuesto es eficiente para la aproximación del frente de soluciones óptimas de Pareto en problemas multi-objetivo con restricciones de alto coste computacional. La relación entre el número de soluciones óptimas encontradas y el número de evaluaciones es superior en comparación con algoritmos convencionales, tales como el NSGA-II.

4. El tiempo de ejecución del algoritmo se consume principalmente en la resolución de los subproblemas de optimización. De los dos subproblemas, el mayor coste computacional se debe a la maximización de $\mathscr{C}_{1}$, puesto que involucra la evaluación del valor esperado del hipervolumen. El problema de maximización de $\mathscr{C}_{2}$ permite la evaluación vectorizada del criterio de búsqueda, por lo que el coste computacional es considerablemente inferior.

5. Aunque en este trabajo se han utilizado modelos Kriging (procesos Gaussianos) (Matheron, 1962) para aproximar las funciones objetivo y las restricciones, el algoritmo EHV-EMO permite la utilización de otros meta-modelos tales como funciones de base radial (Fang y Horstemeyer, 2006), máquinas de soporte vectorial (Smola y Schölkopf, 2004), etc.

6. La precisión y convergencia del algoritmo propuesto depende en gran medida de la habilidad del modelo Kriging de capturar la respuesta del simulador por medio de las funciones de regresión y de correlación. 
—Philip II, king of Macedon (382-336 BC)

\section{Propagación de incertidumbre con métodos de reducción dimensional y modelos Kriging}

\section{Índice}

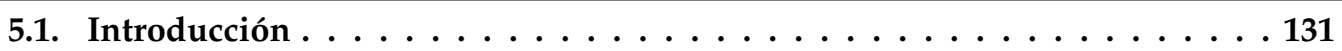

5.2. Propagación de incertidumbre con métodos de reducción dimensional . . 139

5.3. Metodología propuesta . . . . . . . . . . . . . . . . . . 145

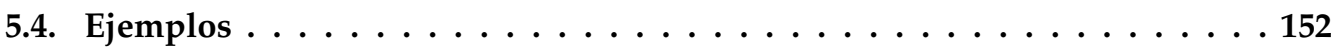

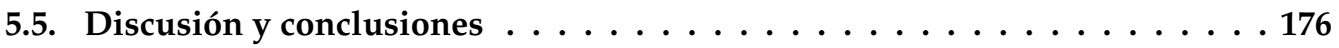





\subsection{Introducción}

La existencia de fuentes de incertidumbre resulta inevitable cuando se abordan problemas de ingeniería. En el campo de la ingeniería estructural, la incertidumbre puede aparecer en cuatro etapas durante la vida de una estructura: en el diseño, en la fabricación, en la vida útil y durante el envejecimiento de la misma. Atendiendo a su origen, se distinguen dos tipos de incertidumbre (Ayyub y McCuen, 2003): la incertidumbre aleatoria y la incertidumbre epistémica. La primera se debe a la variabilidad intrínseca de la repuesta estructural y únicamente se puede reducir a través de modificaciones del diseño. La segunda está asociada a la falta de conocimiento y por lo tanto se puede reducir con un aumento del mismo.

El análisis estocástico de estructuras investiga el comportamiento estocástico de la estructura debido a fluctuaciones en los parámetros que influyen en la respuesta de la misma. Este capítulo se centra en una de las etapas que forman parte del análisis estocástico de estructuras, la Propagación de Incertidumbre (PI). Esta etapa consiste en obtener la respuesta estocástica del modelo de simulación como consecuencia de la incertidumbre aleatoria de las variables de entrada. La mayor parte de métodos de propagación de incertidumbre obtienen la respuesta estocástica evaluando repetidamente el modelo de simulación. El principal problema reside en el elevado número de evaluaciones requerido para una estimación precisa de la respuesta aleatoria, lo que resulta incompatible con la utilización de modelos de simulación de alto coste computacional (ej. modelos FEM). Este capítulo tiene por objeto la resolución eficiente del problema de propagación de incertidumbre en el contexto del diseño óptimo robusto. Se propone un algoritmo basado en el método de reducción dimensional, que utiliza modelos de predicción Kriging para asistir el proceso de integración numérica.

El resto de esta sección presenta la formulación del problema de PI, junto con una revisión de los métodos existentes en la literatura. La sección 5.2 presenta las bases de los métodos de reducción dimensional para el análisis estocástico de estructuras. La sección 5.3 propone una extensión de estos métodos que incluye modelos de predicción Kriging para aliviar algunas de las carencias recogidas en la literatura. La sección 5.4 presenta la validación numérica de la metodología propuesta, mediante la resolución de ejemplos analíticos y de modelos estructurales de alto coste computacional. Finalmente, la sección 5.5 presenta una discusión del método propuesto junto con las conclusiones del capítulo.

\subsubsection{Formulación del problema}

El problema de análisis estocástico de estructuras consta de tres etapas (Figura 5.1): (1) el modelo de simulación, (2) la cuantificación de la incertidumbre en las variables de entrada y (3) la propagación de la incertidumbre de las variables de entrada. Una vez obtenida la respuesta aleatoria en términos de distribución 
de probabilidad, esta distribución puede ser utilizada para evaluar los valores probabilistas de las respuestas frente al fallo (fiabilidad) o la sensibilidad del diseño frente a perturbaciones (robustez). El objetivo final es el que determina el método de propagación de incertidumbre más adecuado.

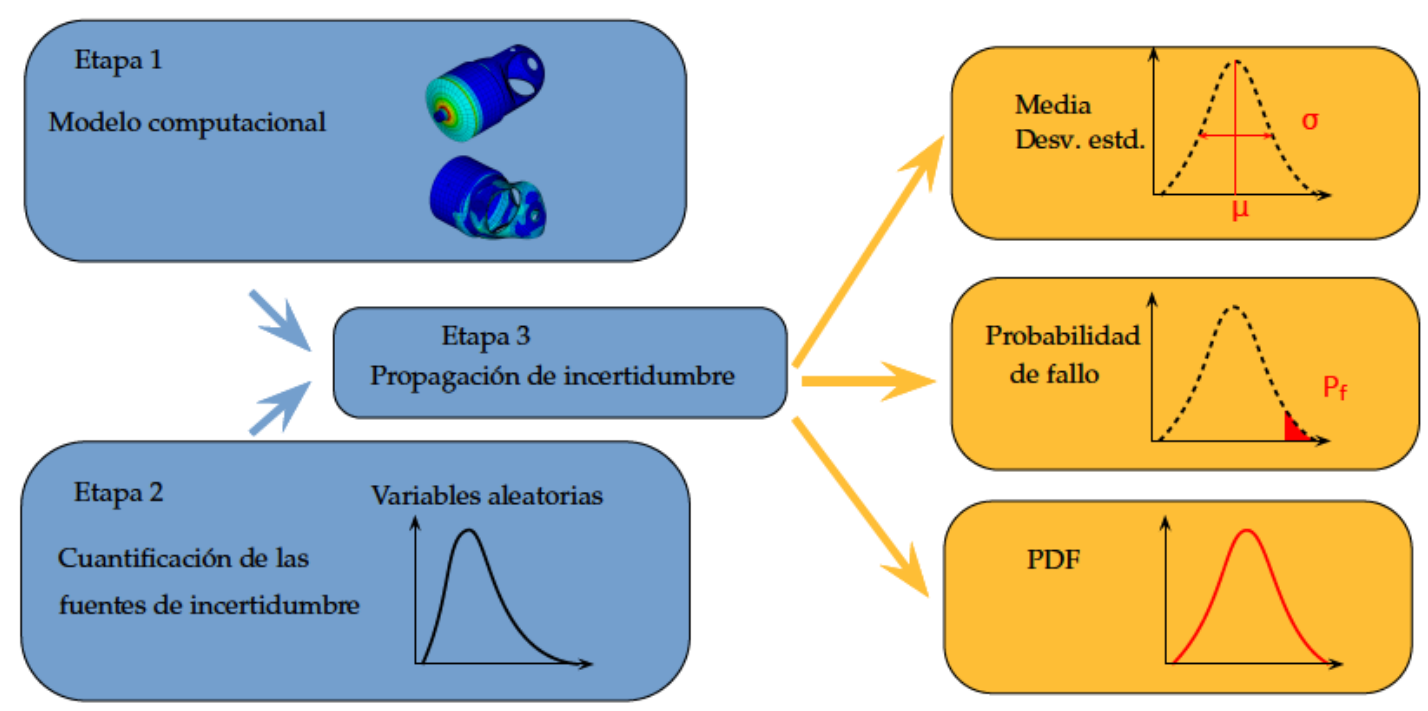

Figura 5.1: Contexto del análisis estocástico de estructuras.

Etapa 1: Modelo de simulación. Esta etapa consiste en la modelización de la respuesta estructural a partir de un conjunto de ecuaciones diferenciales. Estas ecuaciones se discretizan y el sistema resultante se resuelve para obtener las respuestas estructurales. El modelo de simulación se puede representar matemáticamente como:

$$
\mathbf{y}=\mathscr{M}(\mathbf{x})
$$

donde $\mathbf{x} \in \mathbb{X} \subseteq \mathbb{R}^{n}$ es un vector que contiene $n$ parámetros de entrada del modelo (ej. geometría, propiedades del material o cargas); e y $\in Y \subseteq \mathbb{R}^{d}$ es un vector que contiene $d$ respuestas de la estructura (ej. desplazamientos, tensiones o deformaciones). El modelo $\mathscr{M}$ se considera un modelo determinista en el sentido que un mismo vector de entrada proporciona la misma salida.

En la práctica, el modelo de simulación es un modelo no intrusivo (black-box) debido a la utilización de software "in house" o comercial. Esta característica se considera una hipótesis de partida en este trabajo para la resolución del problema de propagación de incertidumbre. En consecuencia, se asume la hipótesis de que el modelo de simulación solo puede ser analizado en un conjunto finito de puntos. 
Etapa 2: Cuantificación de la incertidumbre (modelo probabilista). Las incertidumbres que actúan en el modelo se modelan mediante un vector aleatorio $\mathbf{Z}$ con una distribución de probabilidad $p_{\mathbf{Z}}(\mathbf{z})$. Queda fuera del alcance de este trabajo describir en detalle como se obtiene la distribución de las variables aleatorias en función de la información disponible. Sin embargo, existen ciertas guías recogidas en la literatura Sudret (2012):

- Si existe un conjunto de medidas reales, el enfoque clásico es utilizar métodos de inferencia estadística, tales como el principio de máxima verosimilitud para estimar la distribución de probabilidad (Gaussiana, lognormal, Gumble, Weibull, etc.) que mejor se ajuste a los datos.

- Si no existe información disponible, la decisión se basa en la experiencia. En ingeniería civil, se encuentran disponibles códigos como el JCSS probabilistic model que aportan información acerca de la modelización de cargas y propiedades de materiales (ver http://www.jcss.byg.dtu.dk/).

- Si existen medidas reales, pero su cantidad es limitada, se aconseja utilizar técnicas Bayesianas. Se identifica una distribución a priori basada en la experiencia y se obteniene una distribución a posteriori utilizando la información disponible.

Etapa 3: Propagación de incertidumbre El proceso de propagación de incertidumbre tiene por objeto obtener la respuesta estocástica del modelo de simulación como consecuencia de la incertidumbre aleatoria de las variables de entrada. Existen diferentes técnicas en la literatura encaminadas a tal fin. El método más apropiado depende de la información disponible (derivadas o no), de la precisión que se desea obtener, del coste computacional y de si el objetivo final es estimar un momento estadístico de bajo orden (análisis de robustez), una probabilidad (análisis de fiabilidad) o la totalidad de la función de densidad de probabilidad (Figura 5.1).

En el problema de diseño óptimo robusto se busca establecer un criterio de robustez que caracterice la sensibilidad del diseño frente a variaciones en los parámetros estructurales (capítulo 2). Bajo un enfoque probabilista, dicho criterio se formula en función de los dos primeros momentos estadísticos de la respuesta estructural (media y desviación estándar). La evaluación del momento estadístico de orden $m$ de una función $y(\mathbf{x})$ se obtiene con la resolución de la integral múltiple:

$$
\mathbb{E}\left[y^{m}(\mathbf{x})\right]=\int_{\mathbf{\Omega}} y^{m}(\mathbf{x}) \cdot f_{\mathbf{X}}(\mathbf{x}) \cdot d \mathbf{x},
$$

donde $\mathbf{x}$ es un vector aleatorio completamente caracterizado en el espacio de probabilidad $\boldsymbol{\Omega}$ por la distribución $f_{\mathbf{X}}(\mathbf{x})$ y $\mathbb{E}[\cdot]$ es el operador esperanza matemática. Por lo general, la resolución analítica o mediante integración 
numérica directa de dicha integral resulta imposible o de gran dificultad en problemas de alta dimensionalidad. Por ello, en los últimos años se han desarrollado métodos alternativos que abordan su resolución de forma eficiente y precisa. Uno de los retos del diseño óptimo robusto es encontrar un equilibrio entre la precisión y la eficiencia en la estimación de dichos momentos (Du y Chen, 1999).

El problema que se aborda en este capítulo consiste en la evaluación eficiente de las integrales multidimensionales de los momentos estadísticos de la respuesta estructural.

\subsubsection{Revisión del estado del arte y perspectivas}

Los métodos existentes en la literatura para la resolución del problema de propagación de incertidumbre se clasifican en cinco grupos: (1) métodos de simulación, (2) métodos de expansión, (3) métodos basados en el punto más probable, (4) métodos basados en meta-modelos y (5) métodos de integración aproximada. Una descripción en detalle de cada uno de ellos queda fuera del alcance de este trabajo, el lector interesado puede encontrarla en los trabajos de Ditlevsen y Madsen (1996), Melchers (1999) y Lemaire (2009). A continuación se describen las principales ventajas e desventajas de cada uno de los métodos.

Métodos de simulación. Conceptualmente son los más sencillos y su implementación es directa. Un ejemplo de estos métodos es la simulación MC (Niederreiter y Spanier, 2000). Entre sus principales ventajas destaca la independencia con la dimensionalidad del problema. Los principales inconvenientes son el alto coste computacional y el bajo orden de convergencia. A pesar de la existencia de métodos que intentan aliviar estos problemas como: el Quasi-MCS (Sobol, 1998), Latin hypercube sampling (McKay et al., 1979), Importance sampling, Directional sampling o Markov Chain Monte Carlo (Smith, 1984), su utilización es inviable en problemas que involucran modelos de elementos finitos de alto coste computacional. Por esta razón, se utilizan habitualmente como métodos de validación o cuando otros métodos no son aplicables.

Métodos de expansión. La idea de estos métodos es estimar los momentos estadísticos de la respuesta a partir de perturbaciones en las variables aleatorias del problema. Dentro de esta categoría destacan el método de perturbación o de expansión de Taylor (Jung y Lee, 2002; Liu et al., 1986), el método de expansión de Neumann (Spanos y Ghanem, 1989; Stefanou, 2009), el método basado en la solución estadística equivalente (Abdomian, 1980), los métodos de expansión estocástica espectral (Blatman y Sudret, 2011; Spanos y Ghanem, 1991) y los métodos de colocación estocástica (Eldred y Burkardt, 2009; Hu y Youn, 2011; Smolyak, 1963). Los métodos basados en expansión de Taylor pueden resultar computacionalmente muy costosos o poco precisos cuando 
el grado de incertidumbre en los parámetros de entrada es elevado o cuando la respuesta es altamente no lineal. Para reducir el error cometido requieren derivadas parciales de la respuesta estructural de alto orden, lo que puede ser problemático en problemas de gran escala. En comparación con los métodos de simulación, los métodos de expansión son más eficientes pero menos precisos. El método de expansión de Neumann se basa en la inversión de la matriz aleatoria del problema, lo que resulta problemático en el caso de un elevado número de variables aleatorias o en presencia de campos aleatorios. Los métodos basados en la solución estadística equivalente seleccionan los parámetros del modelo aproximado minimizando la diferencia entre la respuesta real y la aproximada. El principal problema es que la distribución de probabilidad de la respuesta es a priori desconocida, por lo que no es posible determinar esta diferencia analíticamente.

Dentro de la categoría de métodos de expansión estocástica espectral destaca la expansión en polinomios del caos (Stefanou, 2009; Wiener, 1938). La principal deficiencia de estos métodos reside en el incremento del coste computacional para problemas de alta dimensionalidad. Este problema a motivado el desarrollo de estrategias adaptativas como las propuestas por Blatman y Sudret (2011); Foo y Karniadakis (2010); Foo et al. (2008); Wan y Karniadakis (2006). El caracter intrusivo de los métodos basados en expansión estocástica espectral los convierte en poco útiles en la práctica. Para aliviar dicha limitación, han surgido métodos no intrusivos. Estos métodos utilizan el código de simulación como una caja negra para obtener evaluaciones a partir de las cuales calcular los coeficientes de la expansión. Destacan el Nonintrusive Polynomial Chaos method (NIPC) (Hosder et al., 2006), Stochastic Response Surface Method (SRSM) (Isukapalli et al., 1998), Deterministic Equivalent Modeling Method (DEMM) (Tatang, 1995), y principalmente Stochastic Collocation method (SC) (Eldred y Burkardt, 2009; Hu y Youn, 2011; Smolyak, 1963). Estos últimos son un tipo de superficie de respuesta estocástica que aproxima funciones aleatorias multidimensionales a partir de evaluaciones del simulador en un conjunto de puntos de colocación. Trabajos como los de Eldred y Burkardt (2009) reflejan la mayor eficiencia de los métodos de colocación estocástica en comparación con los métodos intrusivos basados en la expansión en polinomios del caos. Esta superioridad se debe a la utilización de métodos de colocación como las Sparse grids (Smolyak, 1963) que alcanzan una buena precisión en aplicaciones de integración (Gerstner y Griebel, 1998) y de interpolación (Barthelmann et al., 2000) con un menor número de puntos de colocación en comparación con métodos convencionales (Full grids). Además de reducir el número de puntos, permiten considerar la influencia de cada dimensión de forma independiente, lo que es un avance muy prometedor de cara al problema conocido como maldición de la dimensionalidad (curse of dimensionality (Bellman, 1961)). Muestra de ello son trabajos como los de Grestner y Griebel (2003) que abordan el problema de integración, Klimke (2006) que aborda el problema de interpolación, o más recientemente Hu y Youn (2011) que proponen un método adaptativo para análisis de fiabilidad. 
Métodos basados en el punto más probable. Estos métodos estiman la probabilidad de fallo mediante una aproximación lineal (FORM (Hasofer y Lind, 1974)) o cuadrática (SORM (Breitung, 1984)) de la respuesta. En primer lugar transforman el espacio de variables aleatorias de entrada en un espacio normal estándar utilizando transformaciones como la de Rosenblatt (Rosenblatt, 1952). Una vez obtenido dicho espacio buscan el punto más probable (Most Probable Point (MPP)), definido como el punto situado en la función de estado límite más cercano al origen del espacio estándar normalizado. Dicha distancia es conocida como Hasofer-Lind Reliability Index (HLRI) (Hasofer y Lind, 1974). Dicho índice es el punto de partida para la realización de análisis de fiabilidad. Estos métodos requieren sensibilidades de primer orden de la respuesta. Además, pueden dar lugar a errores significativos cuando la respuesta es altamente no lineal.

Métodos basados en meta-modelos. Estos métodos se basan en la utilización de un meta-modelo como sustituto del modelo de simulación (ej. superficie de respuesta polinómica, modelos Kriging, funciones de base radial o redes neuronales). El meta-modelo permite obtener evaluaciones de la respuesta con un menor coste computacional, lo que permite utilizarlo en combinación con métodos de simulación para la aproximación de la respuesta aleatoria. El principal inconveniente es que la precisión de los resultados obtenidos depende en gran medida de la precisión del meta-modelo. Además, no es aplicable a problemas de gran escala a causa del problema conocido como maldición de la dimensionalidad.

Métodos de integración aproximada. Utilizan técnicas de integración numérica para resolver la integral multidimensional (5.2). En esta categoría destacan los métodos de reducción dimensional (Rahman y Xu, 2004) y la representación de Modelos de Alta Dimensionalidad (High-Dimensional Model Representation (HDMR)) (Rabitz et al., 1999).

Gracias a su eficiencia y su carácter no intrusivo, los métodos de reducción dimensional han recibido una gran atención durante la última década en el contexto de la optimización bajo incertidumbre (Lee et al., 2008; Rahman y Xu, 2004; Won et al., 2008). Originalmente, el método de reducción unidimensional (Rahman y Xu, 2004) aproxima la función multidimensional y(x) (5.2) mediante una suma de funciones dependientes de una única variable, manteniendo el resto a su valor medio. El Método de Reducción Unidimensional (MRU) estima con buena precisión los momentos de bajo orden de $y(\mathbf{x}, \mathbf{Z})$. Sin embargo, puede derivar en grandes errores cuando los momentos son de mayor orden o cuando la respuesta tiene un alto grado de no linealidad. Estos errores se pueden disminuir incrementando el número de puntos de integración a costa de un incremento del coste computacional. Xu y Rahman (2004) y H. Xu (2005) generalizan la descomposición unidimensional y proponen los métodos de reducción bivariable y multivariable. Estos métodos permiten reducir el error de la aproximación cuando la contribución de los términos cruzados de alto orden es importante. 
El coste computacional de estos últimos es elevado, por lo que en la práctica el método más utilizado es la descomposición unidimensional. Recientemente, autores como Youn et al. (2008) y Huang et al. (2011) proponen mejoras del MRU utilizando aproximaciones de las funciones unidimensionales que permitan la utilización de un mayor número de puntos de integración con un bajo coste computacional. En esta línea Youn et al. (2008) utilizan stepwise moving least squares, Won et al. $(2009,2008)$ utilizan modelos Kriging y Huang et al. (2011) utilizan polinomios de Chebyshev.

Los métodos de reducción de dimensión presentan dos principales carencias. En primer lugar es difícil determinar a priori el nivel de descomposición para obtener una precisión suficiente. En segundo lugar, se muestran dimensiones con poca influencia en la función que se trata de aproximar, lo que sugiere el uso de técnicas adaptativas (Wang, 2011).

La representación de modelos de alta dimensionalidad (HDMR) (Alis y Rabitz, 2001; Li et al., 2001a,b; Rabitz y Alis, 1999; Rabitz et al., 1999) hace referencia al conjunto de técnicas utilizadas para tratar el problema de aproximación de modelos de alta dimensionalidad. La HDMR es una expansión de funciones correlacionadas jerárquicamente que emulan la relación entre las variables de entrada y la salida de un sistema multidimensional (Li et al., 2001a; Rabitz et al., 1999). En base a la idea de Owen (2000), la respuesta de un sistema en ingeniería depende fundamentalmente de interacciones de bajo orden entre las variables de entrada. Por esta razón, en la práctica dicha expansión se reduce a los componentes de bajo orden del sistema. La HDMR puede tener diferentes aplicaciones como: la creación de un meta-modelo en espacios de alta dimensión, la identificación de variables importantes del problema, la propagación eficiente de incertidumbre, etc.

Actualmente existen diferentes variantes de HDMR en función de la forma de determinar los componentes principales de la descomposición, destacando el ANOVA-HDMR (Sobol, 2003) y Cut-HDMR (Rabitz et al., 1999). El primero se basa en el análisis de la varianza de cada uno de los componentes, mientras que el método Cut-HDMR aproxima la función multidimensional de manera exacta en el hiperplano que pasa por un punto de referencia del dominio definido por las variables de entrada.

En los últimos años, dado el interés en la resolución del problema de cuantificación de la incertidumbre en problemas de alta dimensionalidad, se han desarrollado diferentes métodos teniendo como base el Cut-HDMR. Foo et al. (2008) utilizan Cut-HDMR para aproximar funciones de alta dimensionalidad con funciones de baja dimensionalidad, las cuales son integradas con técnicas de integración numérica. En el campo de la ingeniería financiera Griebel y Holtz (2010) combinan Cut-HDMR con sparse grids para la resolución adaptativa de problemas de integración en dominios de alta dimensión. Ma y Zabaras (2010) utilizan HDMR para representar la respuesta estocástica como una 
expansión en términos de las entradas estocásticas, desde las componentes de bajo orden hasta las de alto orden. Ma y Zabaras (2010) proponen una versión adaptativa de HDMR para detectar aquellas dimensiones que tienen una mayor influencia en la respuesta y construir los términos de alto orden que contienen dichas componentes. Así mismo, incorporan un método adaptativo de colocación estocástica para construir un modelo estocástico reducido de baja dimensionalidad del modelo original. Este modelo reducido se utiliza para realizar análisis estocásticos de la respuesta.

\subsubsection{Discusión}

La revisión de la literatura realizada pone de manifiesto que no existe un método de propagación de incertidumbre que sea óptimo para todas las aplicaciones. La selección de dicho método depende de la información disponible (derivadas o no), de la precisión que se desea obtener, del momento estadístico que se desea estimar y de los recursos computacionales.

Esta tesis aborda el problema de diseño óptimo robusto con un enfoque probabilista. El alcance del problema de propagación de la incertidumbre en este contexto se limita a la estimación de los momentos estadísticos de bajo orden de la respuesta. En este caso se requiere un método de PI no intrusivo, libre de derivadas que permita obtener momentos estadísticos de bajo orden de forma eficiente en dominios estocásticos de alta dimensionalidad. Así mismo, características como la posibilidad de paralelización del método son muy deseables.

Los gran mayoría de los métodos presentados anteriormente resultan poco eficientes (métodos de simulación) o poco precisos cuando el número de variables aleatorias es elevado (métodos basados en meta-modelos). Los métodos de reducción dimensional son los que mejor se adecuan al objetivo de este trabajo. Fruto de la revisión realizada se han identificado varias áreas donde los métodos de reducción dimensional pueden ser mejorados y/o extendidos:

- La utilización de meta-modelos permite aproximar funciones unidimensionales de alta no linealidad, permitiendo aumentar el número de puntos de integración con un reducido número de evaluaciones del modelo de simulación. Sin embargo, no está claro a priori el tipo de meta-modelo a utilizar ni donde evaluar el modelo de simulación para un mejor ajuste.

- Cuando la contribución de los términos cruzados es elevada se precisa una descomposición de orden dos o superior. En este caso el coste computacional del problema se puede incrementar drásticamente, lo que limita su aplicación práctica. Este problema se podría resolver mediante la utilización de técnicas de computación paralela o técnicas de aproximación. 


\subsection{Propagación de incertidumbre con métodos de reducción dimensional}

Los métodos de reducción dimensional permiten obtener tanto los momentos estadísticos de la respuesta estructural (Rahman y Xu, 2004; Xu y Rahman, 2004) como la probabilidad de fallo de la estructura (H. Xu, 2005) con un reducido número de evaluaciones. Además, son apropiados para la resolución del problema de propagación de incertidumbre de modelos de simulación no-intrusivos. En este sentido, una reciente comparación entre diversos métodos de propagación de incertidumbre realizada por Lee y Chen (2009), destaca su eficiencia en comparación con otros métodos de expansión o simulación, a la hora de caracterizar la respuesta aleatoria del sistema. Esta eficiencia ha propiciado su aplicación en diferentes formulaciones del problema de optimización bajo incertidumbre. Así, autores como Huang y Du (2007), Lee (2008) y Lee et al. (2009) han aplicado dichos métodos para la resolución del problema de diseño óptimo robusto. Otros autores como Lee (2008), Youn y Wang (2008), Choi et al. (2010), Won et al. (2008) y Youn y Xi (2009) resuelven el problema de diseño óptimo basado en fiabilidad. Lee et al. (2008) los aplican para la resolución del problema de diseño óptimo robusto y fiable.

El objetivo de los métodos de reducción dimensional es evaluar eficientemente los momentos estadísticos de la respuesta, a partir de lo cuales se construye la curva de densidad de probabilidad utilizando sistemas de Pearson. Durante el cálculo de los momentos estadísticos, la respuesta $\mathrm{N}$-dimensional se aproxima mediante una descomposición aditiva de $\mathrm{N}$ funciones de menor dimensión, donde $\mathrm{N}$ es el número de variables aleatorias. En el caso particular del método de reducción de dimensión univariable (Rahman y Xu, 2004; Xu y Rahman, 2004), las integrales resultantes son unidimenisonales. Como resultado, la integral $\mathrm{N}$-dimensional del momento estadístico de orden $m$ (ecuación (5.2)) se sustituye por un número finito de integrales unidimensionales que resultan menos costosas de evaluar. En esta sección se presentan las bases teóricas sobre las que se sustenta la aplicación de los métodos de reducción dimensional para el análisis estocásticos de sistemas estructurales.

\subsubsection{Aproximación univariable}

Sea $y(\mathbf{x}) \in \mathbb{R}$ una función continua y diferenciable que depende de un vector $\mathbf{x}=\left\{x_{1}, \ldots, x_{N}\right\}^{T} \in \mathbb{R}^{N}$. La expansión de Taylor de orden 2 de la función $y(\mathbf{x})$ centrada en el punto $\mathbf{x}=\mathbf{c}\left\{c_{1}, \ldots, c_{N}\right\}^{T}$ viene dada por la expresión:

$$
\begin{aligned}
y(\mathbf{x})= & y(\mathbf{c})+\sum_{j=1}^{\infty} \frac{1}{j !} \sum_{i=1}^{N} \frac{\partial^{j} y}{\partial x_{i}^{j}}(\mathbf{c})\left(x_{i}-c_{i}\right)^{j} \\
& +\sum_{j_{1}, j_{2}>0}^{\infty} \frac{1}{j_{1} ! j_{2} !} \sum_{i_{1}<i_{2}} \frac{\partial^{j_{1}+j_{2}} y}{\partial x_{i_{1}}^{j_{1}} \partial x_{i_{2}}^{j_{2}}}(\mathbf{c})\left(x_{i_{1}}-c_{i_{1}}\right)^{j_{1}}\left(x_{i_{2}}-c_{i_{2}}\right)^{j_{2}}+\mathscr{R}_{3}
\end{aligned}
$$


donde el término $\mathscr{R}_{3}$ representa todos los términos con dimensión mayor o igual a tres. De acuerdo con Rahman y Xu (2004), la función y(x) se puede aproximar mediante la descomposición univariable:

$$
\begin{aligned}
\hat{y}_{1}(\mathbf{x}) & \equiv \hat{y}_{1}\left(x_{1}, \ldots, x_{N}\right) \\
& =\sum_{i=1}^{N} y\left(c_{1}, \ldots, c_{i-1}, x_{i}, c_{i+1}, \ldots, c_{N}\right)-(N-1) y(\mathbf{c}),
\end{aligned}
$$

donde cada término $y\left(c_{1}, \ldots, c_{i-1}, x_{i}, c_{i+1}, \ldots, c_{N}\right)$ depende únicamente de la variable $i$-ésima. Tomando la expansión en serie de Taylor de orden 1 de cada una de las funciones univariable $\hat{y}_{i}$

$$
y\left(c_{1}, \ldots, c_{i-1}, x_{i}, c_{i+1}, \ldots, c_{N}\right)=y(\mathbf{c})+\sum_{j=1}^{\infty} \frac{1}{j !} \frac{\partial^{j} y}{\partial x_{i}^{j}}(\mathbf{c})\left(x_{i}-c_{i}\right)^{j}
$$

y sustituyendo (5.5) en (5.4) resulta:

$$
\hat{y}_{1}(\mathbf{x})=y(\mathbf{c})+\sum_{j=1}^{\infty} \frac{1}{j !} \sum_{i=1}^{N} \frac{\partial^{j} y}{\partial x_{i}^{j}}(\mathbf{c})\left(x_{i}-c_{i}\right)^{j}
$$

La comparación entre las ecuaciones (5.6) y (5.3) indica que el error cometido mediante la aproximación unidimensional definida en la ecuación (5.4) es $y(\mathbf{x})-$ $\hat{y}_{1}(\mathbf{x})=\mathscr{R}_{2}$, que incluye contribuciones de términos de orden mayor o igual a dos.

\subsubsection{Aproximación bivariable}

Del mismo modo, la función $y(\mathbf{x})$ se puede aproximar mediante una descomposición bivariable $\hat{y}_{2}$ definida como:

$$
\begin{aligned}
\hat{y}_{2}(\mathbf{x}) & \equiv \hat{y}_{2}\left(x_{1}, \ldots, x_{N}\right) \\
& =\sum_{i_{1}<i_{2}} y\left(c_{1}, \ldots, c_{i_{1}-1}, x_{i_{1}}, c_{i_{1}+1}, \ldots, c_{i_{2}-1}, x_{i_{2}}, c_{i_{2}}+1, \ldots, c_{N}\right) \\
& -(N-2) \sum_{i=1}^{N} y\left(c_{1}, \ldots, c_{i-1}, x_{i}, c_{i+1}, \ldots, c_{N}\right) \\
& +\frac{(N-1)(N-2)}{2} y(\mathbf{c}),
\end{aligned}
$$

donde cada término $y\left(c_{1}, \ldots, c_{i_{1}-1}, x_{i_{1}}, c_{i_{1}+1}, \ldots, c_{i_{2}-1}, x_{i_{2}}, c_{i_{2}+1}, \ldots, c_{N}\right)$ es función de dos variables $x_{i_{1}}, x_{i_{2}}$. Tomando la expansión en serie de Taylor de orden 2 de cada una de las funciones bivariable se tiene: 


$$
\begin{aligned}
& y\left(c_{1}, \ldots, c_{i_{1}-1}, x_{i_{1}}, c_{i_{1}+1}, \ldots, c_{i_{2}-1}, x_{i_{2}}, c_{i_{2}+1}, \ldots, c_{N}\right) \\
= & y(\mathbf{c})+\sum_{j=1}^{\infty} \frac{1}{j !} \frac{\partial^{j} y}{\partial x_{i_{1}}^{j}}(\mathbf{c})\left(x_{i_{1}}-c_{i_{1}}\right)^{j}+\sum_{j=1}^{\infty} \frac{1}{j !} \frac{\partial^{j} y}{\partial x_{i_{2}}^{j}}(\mathbf{c})\left(x_{i_{2}}-c_{i_{2}}\right)^{j} \\
& +\sum_{j_{1}, j_{2}>0}^{\infty} \frac{1}{j_{1} ! j_{2} !} \frac{\partial^{j_{1}+j_{2}} y}{\partial x_{i_{1}}^{j_{1}} \partial x_{i_{2}}^{j_{2}}}(\mathbf{c})\left(x_{i_{1}}-c_{i_{1}}\right)^{j_{1}}\left(x_{i_{2}}-c_{i_{2}}\right)^{j_{2}},
\end{aligned}
$$

y sustituyendo (5.8) y (5.5) en (5.7) resulta:

$$
\begin{aligned}
\hat{y}_{2}(\mathbf{x}) & =y(\mathbf{c})+\sum_{j=1}^{\infty} \frac{1}{j !} \sum_{i=1}^{N} \frac{\partial^{j} y}{\partial x_{i}^{j}}(\mathbf{c})\left(x_{i}-c_{i}\right)^{j} \\
& +\sum_{j_{1}, j_{2}>0}^{\infty} \frac{1}{j_{1} ! j_{2} !} \sum_{i_{1}<i_{2}} \frac{\partial^{j_{1}+j_{2}} y}{\partial x_{i_{1}}^{j_{1}} \partial x_{i_{2}}^{j_{2}}}(\mathbf{c})\left(x_{i_{1}}-c_{i_{1}}\right)^{j_{1}}\left(x_{i_{2}}-c_{i_{2}}\right)^{j_{2}} .
\end{aligned}
$$

La comparación entre las ecuaciones 5.9 y 5.3 indica que el error cometido mediante la aproximación bidimensional definida en la ecuación (5.7) es $y(\mathbf{x})-$ $\hat{y}_{2}(\mathbf{x})=\mathscr{R}_{3}$, que incluye contribuciones de términos de orden mayor o igual a tres.

\subsubsection{Aproximación multivariable}

Rahman y Xu (2004) generalizan el procedimiento anterior para el caso de un aproximación multidimensional de orden $S \in[1, N]$. La aproximación de orden $S$ de la función $y(\mathbf{x})$ viene dada por la suma finita:

$$
\hat{y}_{S}(\mathbf{x}) \equiv \underbrace{\underbrace{y_{0}+\sum_{i=1}^{N} y_{i}\left(x_{i}\right)}_{\hat{y}_{2}(\mathbf{x})}+\sum_{\substack{i_{1}(\mathbf{x}) \\ i_{1}<i_{2}}}^{N} y_{i_{1}, i_{2}}\left(x_{i_{1}}, x_{i_{2}}\right)+\ldots+\sum_{\substack{i_{1}, \ldots, i_{i}=1 \\ i_{1}<\cdots<i_{S}}}^{N} y_{i_{1}, \ldots, i_{S}}\left(x_{i_{1}}, \ldots, x_{i_{S}}\right)+\cdots+y_{1,2, \ldots, N}\left(x_{1}, \ldots, x_{N}\right), \quad(5.10)}_{\hat{y}_{S}(\mathbf{x})}
$$

donde $y\left(x_{i}\right)$ es la función univariable que representa la contribución a la respuesta total de cada uno de las variables $x_{i}$ de forma independiente; $y_{i 1, i 2}\left(x_{i_{1}}, x_{i_{2}}\right)$ es la contribución a la respuesta global de las variables $x_{i_{1}}$ y $x_{i_{2}}$ actuando conjuntamente; e $y_{i 1, \ldots, i s}\left(x_{i_{1}}, \ldots, x_{i_{S}}\right)$ representa el efecto de las variables $x_{i 1}, \ldots, x_{i_{S}}$ en la respuesta global. Rahman y Xu (2004) demuestran en su trabajo que la descomposición $\hat{y}_{S}(\mathbf{x})$ converge a la función real $y(\mathbf{x})$ cuando $S=N$. El error de la aproximación es del orden $\mathscr{R}_{S+1}$.

Teorema 2 (Aproximación S-variable generalizada (Rahman y Xu, 2004)) Para cualquier función $N$-dimensional $y(\mathbf{x})=y\left(x_{1}, \ldots, x_{N}\right)$, si

$$
\hat{y}(\mathbf{x}) \equiv \sum_{i=0}^{S}(-1)^{i}\left(\begin{array}{c}
N-S+i-1 \\
i
\end{array}\right) y_{S-i}
$$


representa una aproximación $S$-variable de $y\left(x_{1}, \ldots, x_{N}\right)$, donde $y_{0}=y(\mathbf{c}), S \leq N, y$

$$
y_{R}=\sum_{k_{1}<k_{2}<\cdots<k_{R}} y\left(c_{1}, \ldots, c_{k_{1}-1}, x_{k_{1}}, c_{k_{1}+1}, \ldots, c_{k_{R}-1}, x_{k_{R}}, c_{k_{R}+1}, \ldots, c_{N}\right), \quad 0 \leq R \leq S,
$$

entonces $\hat{y}$ consiste en todos los términos de la serie de Taylor de $y\left(x_{1}, \ldots, x_{N}\right)$ que tienen un número de variables menor o igual a $S$.

\subsubsection{Momentos estadísticos de la respuesta}

El momento estadístico de orden $m$ viene dado por la integral multidimensional:

$$
\mathbb{E}\left[y^{m}(\mathbf{x})\right]=\int_{\Omega} y^{m}(\mathbf{x}) \cdot p_{\mathbf{x}}(\mathbf{x}) \cdot d \mathbf{x}
$$

donde el vector $\mathbf{X}=\left\{x_{1}, \ldots, x_{N}\right\}^{T} \in \mathscr{R}^{N}$ representa la incertidumbre de los parámetros de entrada, $y(\mathbf{x})$ es la respuesta estructural de interés (ej. carga crítica, frecuencias principales, desplazamiento máximo o tensión), $p_{\mathbf{X}}(\mathbf{x})$ es la función de densidad de probabilidad conjunta de $\mathbf{X}$ y $\mathbb{E}[\cdot]$ es el operador esperanza matemática. Utilizando la descomposición multivariable (ecuación (5.11)) centrada en el valor medio de las variables $\mu_{i}=\mathbb{E}\left[x_{i}\right] \forall i=1, \ldots, N$, el momento estadístico de orden $m$ se puede aproximar por:

$$
\begin{aligned}
\mathbb{E}\left[\hat{y}^{m}(\mathbf{x})\right] & =\sum_{i=0}^{S}(-1)^{i}\left(\begin{array}{c}
N-S+i-1 \\
i
\end{array}\right) \\
& \times \sum_{k_{1}<k_{2}<\cdots<k_{S-i}} \underbrace{\mathbb{E}\left[\hat{y}^{m}\left(\mu_{1}, \ldots, \mu_{k_{1}-1}, x_{k_{1}}, \mu_{k_{1}+1}, \ldots, \mu_{k_{S-i}-1}, x_{k_{S-i}}, \mu_{k_{S-i}+1}, \ldots, \mu_{N}\right)\right]}_{\mathbb{E}\left[\hat{y}^{m}\left(x_{k_{1}}, x_{k_{2}}, \ldots, x_{k_{S-i}}\right)\right]} .
\end{aligned}
$$

Por simplicidad en la formulación, en adelante se considera la variable $g(\mathbf{x})=$ $y^{m}(\mathbf{x})$. Introduciendo un desarrollo en serie de Taylor para cada uno de los términos del sumatorio de la ecuación (5.13), Rahman y Xu (2004) demuestran que el error cometido por la aproximación de la integral (5.12) mediante la ecuación (5.13) es:

$$
\begin{aligned}
& \mathbb{E}[g(\mathbf{x}))]-\mathbb{E}[\hat{g}(\mathbf{x}))]= \\
& =\sum_{j_{S+1}=1}^{\infty} \sum_{j_{S}=1}^{\infty} \ldots \sum_{j_{1}=1}^{\infty} \frac{1}{j_{1} ! j_{2} ! \ldots j_{S+1} !} \sum_{i_{1}<i_{2}<\cdots<i_{S+1}} \frac{\partial^{j_{1}+j_{2}+\cdots+j_{S+1}} g(\mathbf{x})}{\partial j_{1} x_{i_{1}} \partial^{j_{2}} x_{i_{2}} \ldots \partial j_{S+1} x_{i_{S+1}}}\left(\mu_{x}\right) \\
& \left.\times \mathbb{E}\left[g\left(x_{1}, x_{2}, \ldots, x_{S+1}\right)\right)\right]+\ldots
\end{aligned}
$$

La ecuación (5.14) incluye la contribución de todos los términos con orden mayor o igual a $S+1$. Mediante la descomposición realizada, la integral múltiple de la ecuación (5.12) se sustituye por una suma de integrales de dimensiones $1,2, \ldots, S$. Estas integrales resultan más fáciles de calcular mediante métodos de 
cuadratura, seleccionados en función de la distribución de las variables aleatorias de entrada. La ecuación (5.13) presenta la forma generalizada para la resolución de la integral del momento estadístico mediante una descomposición de orden $S$, conocida como S-variate or multivariate dimension reduction.

\subsubsection{Integración de las integrales de dimensión reducida}

En el caso de distribuciones Gaussianas, los métodos de cuadratura más habituales son la cuadratura de Gauss-Legendre y la cuadratura de Gauss-Hermite (Davenport y Root, 1987). En el caso de variables aleatorias con distribuciones no Gaussianas, la integración se puede realizar por los siguientes cuatro procedimientos:

1. Obtención a partir de tablas. En aquellos casos en los que la función de densidad de probabilidad de una variable aleatoria pueda obtenerse mediante transformaciones a partir de la fórmula clásica de cuadratura Gaussiana.

2. Obtención a partir de los momentos de las variables de entrada. En el trabajo desarrollado inicialmente por Rahman y Xu (2004) se plantea la resolución numérica mediante una cuadratura basada en los momentos estadísticos de las variables aleatorias de entrada. Esta regla de cuadratura permite encontrar la posición y pesos de los puntos de integración para una variable aleatoria arbitraria mediante la resolución de un sistema de ecuaciones. Los correspondientes pesos y puntos de integración se obtienen de la resolución del sistema lineal de ecuaciones:

$$
\left[\begin{array}{ccccc}
\mu_{j, n-1} & -\mu_{j, n-2} & -\mu_{j, n-3} & \cdots & (-1)^{n-1} \mu_{j, 0} \\
\mu_{j, n} & -\mu_{j, n-1} & -\mu_{j, n-2} & \cdots & (-1)^{n-1} \mu_{j, 1} \\
\mu_{j, n+1} & -\mu_{j, n} & -\mu_{j, n-1} & \cdots & (-1)^{n-1} \mu_{j, 2} \\
\vdots & \vdots & \vdots & \vdots & \vdots \\
\mu_{j, 2 n-2} & -\mu_{j, 2 n-3} & -\mu_{j, 2 n-4} & \cdots & (-1)^{n-1} \mu_{j, n-1}
\end{array}\right]\left[\begin{array}{c}
r_{j, 1} \\
r_{j, 2} \\
r_{j, 3} \\
\vdots \\
r_{j, n}
\end{array}\right]=\left[\begin{array}{c}
\mu_{j, n} \\
\mu_{j, n+1} \\
\mu_{j, n+2} \\
\vdots \\
\mu_{j, 2 n-1}
\end{array}\right]
$$

donde $\mu_{j, i}$ es el momento estadístico de orden $i$ de la variable j-ésima y $n$ es el número de puntos de integración. La solución del sistema (5.15) proporciona los coeficientes de la ecuación (5.16)

$$
x_{j}^{n}+\sum_{i=1}^{n}(-1)^{i} \cdot r_{j, i} \cdot x_{j}^{n-i}=0,
$$

cuyas raíces representan los $n$ puntos de integración de la variable j-ésima $\left(x_{j, i}\right)$. Una vez obtenidos los puntos de integración, el peso del punto de integración k-ésimo para la variable aleatoria j-ésima se obtiene mediante la expresión: 


$$
w_{j, k}=\frac{\int_{-\infty}^{\infty} \prod_{l=1, l \neq k}^{n}\left(x_{j}-x_{j, l}\right) f_{X_{j}\left(x_{j}\right) d x_{j}}}{\prod_{l=1, l \neq k}^{n}\left(x_{j, k}-x_{j, l}\right)}=\frac{\prod_{l=0}^{n-1}(-1)^{l} \mu_{j, n-l-1} q_{j, k l}}{\prod_{j, k 0}^{n}\left(x_{j, k}-x_{j, l}\right)},
$$

La precisión de la cuadratura puede incrementarse mediante la utilización de un mayor número de puntos de integración. Sin embargo para problemas con un elevado número de puntos de integración $(n>5)$ la aplicación de este método está limitada por la dificultad asociada a la resolución del sistema de ecuaciones (5.15).

3. Obtención a partir de las raíces de polinomios ortogonales. Lee et al. (2009) proponen una metodología basada en fórmulas de cuadratura del tipo Gauss para la obtención de los momentos estadísticos de funciones que dependen de variables con distribuciones de densidad de probabilidad arbitrarias.

4. Transformación previa a una distribución normal estándar. Es posible realizar una transformación a una distribución normal estándar previamente a la integración numérica. En este sentido, son habituales la transformación de Rosenblatt (Rosenblatt, 1952) y la transformación de Nataf (Liu y Kiureghian, 1986). La utilización de una transformación sacrifica precisión a cambio de una mayor simplicidad a la hora de obtener pesos y nodos de integración. Estos últimos se pueden obtener de forma directa de tablas con las formulas de cuadratura de Gauss-Hermite. Utilizando una transformación a normal estándar, la integral (5.2) se puede resolver utilizando la cuadratura de Gauss-Hermite:

$$
\begin{aligned}
\mathbb{E}\left[y^{m}(\mathbf{x})\right] & =\int_{\Omega} y^{m}(\mathbf{x}) \cdot f_{\mathbf{X}}(\mathbf{x}) \cdot d \mathbf{x} \\
& =\int_{-\infty}^{\infty} \phi_{U}(\mathbf{u}) \cdot y^{m}\left(T^{-1}(\mathbf{u})\right) \cdot d \mathbf{u} \\
& \cong \sum_{i=1}^{n} \frac{w_{i}^{\mathrm{G}-\mathrm{H}}}{\sqrt{\pi}}\{a\} \cdot y^{m}\left(T^{-1}\left(\sqrt{2} \cdot l_{i}^{\mathrm{G}-\mathrm{H}}\right)\right)
\end{aligned}
$$

donde $\phi_{U}(\mathbf{u})$ representa la función de densidad de probabilidad de la distribución normal estándar. 


\subsection{Metodología propuesta}

\subsubsection{Motivación}

En esta tesis se propone una metodología basada en la utilización de métodos de reducción dimensional junto con modelos Kriging. Desde el punto de vista del diseño óptimo robusto, la utilización de métodos de reducción dimensional resulta apropiada porque permiten obtener de forma eficiente y precisa momentos estadísticos de bajo orden. Sin embargo, la revisión de la literatura realizada muestra como estos métodos pueden dar como resultado grandes errores en la estimación de momentos de mayor orden de respuestas altamente no lineales. Estos errores pueden ser disminuidos incrementando el número de puntos de integración a costa de un incremento del coste computacional. La metodología propuesta tiene por objetivo aproximar las funciones multivariable con modelos Kriging con los siguientes objetivos:

- Incrementar la precisión de la aproximación de funciones de alta no linealidad. Permitiendo aumentar el número de puntos de integración con un reducido número de evaluaciones del modelo de simulación.

- Reducir el problema de dimensionalidad asociado a la utilización directa de meta-modelos para aproximar modelos de simulación en dominios aleatorios de media-alta dimensionalidad.

- Reutilización del diseño de experimentos para la aproximación de la función de $(S-1)$ variables como parte del diseño de experimentos de la función de $S$ variables. Reduciendo el coste computacional de descomposiciones superiores a uno.

- El algoritmo propuesto en este trabajo es una extensión del propuesto por Won et al. $(2009,2008)$, que utilizan modelos de predicción del tipo Universal Kriging y el método de reducción unidimensional. Se resuelven algunos de los problemas detectados por Won et al. $(2009,2008)$ relacionados con la colocación de los puntos de la muestra y con la selección de las funciones de regresión y correlación del modelo. La metodología propuesta en esta tesis extiende la aproximación con modelos Kriging a métodos de descomposición de orden $S$. Así mismo, se propone un criterio de selección del mejor ajuste (función de regresión y de correlación) independiente de la localización del diseño de experimentos. Finalmente se establece un criterio de colocación de los diseños de experimentos a partir de las raíces de los polinomios de Hermite. 


\subsubsection{Implementación del algoritmo}

A continuación se describen las etapas del algoritmo utilizado para la propagación de la incertidumbre. Para hacer referencia al algoritmo propuesto se utilizarán las siglas EKDRM (Extended Kriging based Dimension Reduction Method). El diagrama de flujo se presenta en el Algoritmo 4.

Transformación a un espacio normalizado estándar. La primera etapa del algoritmo consiste en una transformación a un espacio normalizado estándar. El objetivo de esta transformación es generalizar el proceso de colocación de los puntos en el espacio aleatorio, con independencia del tipo de distribución de las variables aleatorias o de la correlación entre ellas. Formalmente dicha transformación se representa mediante la aplicación $\mathbf{T}: \mathbf{X}_{i} \rightarrow \mathbf{U}_{i}$, donde a cada vector de variables aleatorias del espacio original se le asigna un vector de variables aleatorias normalizado. El tipo de transformación realizada depende de la información disponible acerca de la distribución de probabilidad conjunta de las variables. Si la distribución está completamente caracterizada se emplea la transformación de Rosenblatt (Rosenblatt, 1952), mientras que si se dispone de las funciones de distribución marginales y correlaciones se utiliza la transformación de Nataff (Liu et al., 1986). Dado un conjunto de variables aleatorias $\mathbf{X}=\left(X_{1}, \ldots, X_{n}\right)$ la transformación de Rosenblatt del espacio $\mathbf{X}$ al espacio $\mathbf{U}$ se define como:

$$
\begin{aligned}
\phi\left(u_{1}\right) & =f_{X_{1}}\left(x_{1}\right), \\
\phi\left(u_{2}\right) & =f_{X_{2}}\left(x_{2} \mid x_{1}\right), \\
\phi\left(u_{3}\right) & =f_{X_{3}}\left(x_{3} \mid x_{1}, x_{2}\right), \\
& \vdots \\
\phi\left(u_{n}\right) & =f_{X_{n}}\left(x_{n} \mid x_{1}, x_{2}, \ldots, x_{n-1}\right),
\end{aligned}
$$

donde $n$ es el número de variables aleatorias, $f_{X_{i}}\left(x_{i} \mid x_{1}, x_{2}, \ldots, x_{i-1}\right)$ es la función de densidad de probabilidad condicionada de la variable $i$-ésima dadas las realizaciones de $x_{1}, x_{2}, \ldots, x_{n-1} \mathrm{y} \phi(\cdot)$ es la función de densidad de probabilidad estándar. Las variables aleatorias $\mathbf{X}$ se pueden expresar en términos de las variables normalizadas $\mathbf{U}$ utilizando la transformación inversa $\mathbf{T}^{-1}: \mathbf{U}_{i} \rightarrow \mathbf{X}_{i}$ :

$$
\begin{aligned}
x_{1} & =f_{X_{1}}^{-1}\left(\phi\left(u_{1}\right)\right), \\
x_{2} & =f_{X_{2}}^{-1}\left(\phi\left(u_{2} \mid x_{1}\right)\right), \\
& \vdots \\
x_{n} & =f_{X_{n}}^{-1}\left(\phi\left(u_{n} \mid x_{1}, x_{2}, \ldots, x_{n-1}\right)\right) .
\end{aligned}
$$

Colocación de la muestra. La transformación a un espacio normalizado estándar facilita el desarrollo de un procedimiento genérico de colocación de 


\section{Algoritmo 4 Algoritmo EKDRM}

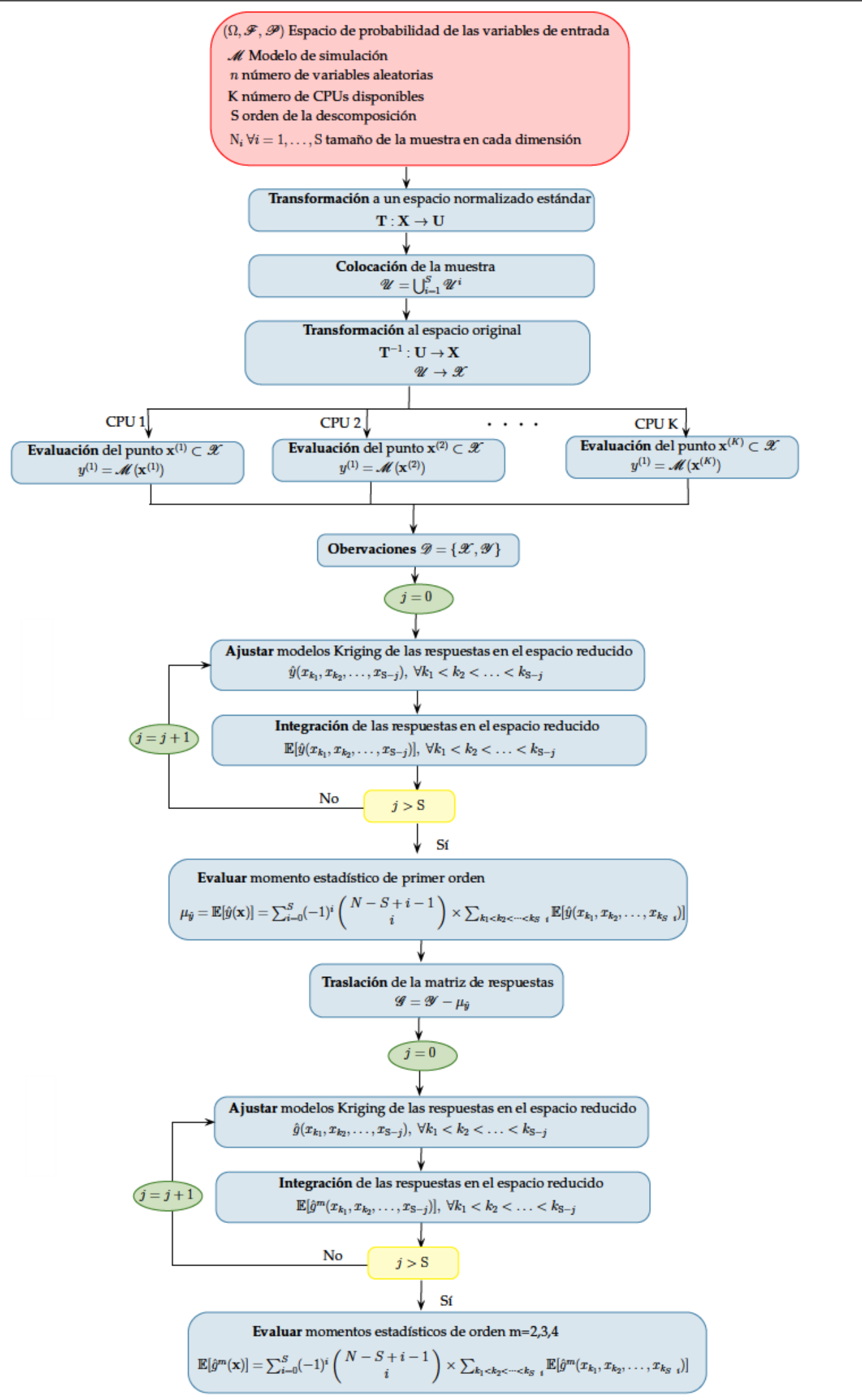




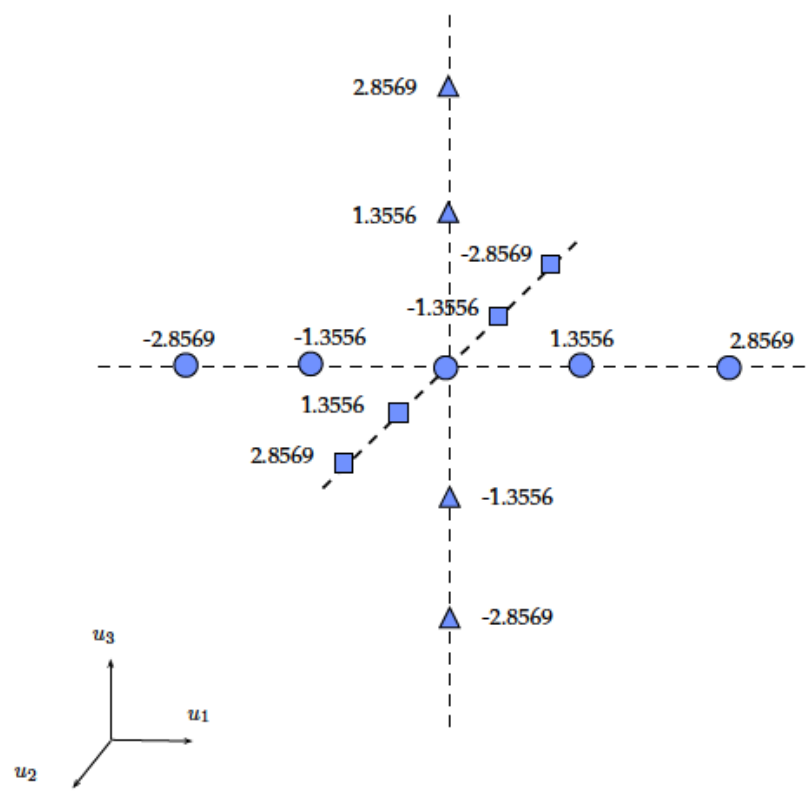

Figura 5.2: Colocación de puntos en un espacio normalizado estándar tridimensional $\left(S=1\right.$ y $\left.N_{1}=4\right)$.

puntos en un espacio multidimensional. En esta etapa se obtiene un conjunto de puntos $(\mathscr{U})$ que, una vez evaluados con el modelo de simulación $(\mathscr{Y}=\mathscr{M}(\mathscr{U}))$, aportan la información suficiente para ajustar los parámetros de los modelos de predicción de dimensión reducida $\hat{y}\left(x_{k_{1}}, x_{k_{2}}, \ldots, x_{k_{s-i}}\right), \forall i=0, \ldots, S$. Los modelos de predicción actúan como sustitutos de las respuestas en dimensión reducida durante el proceso de integración.

La posición de los puntos es función de: (1) el orden de la descomposición $(S)$ y (2) el número de puntos en cada dimensión distintos del origen $\left(N_{i}, \forall i=1, \ldots, S\right)$. Para $S=1$ (aproximación unidimensional) se sitúan $N_{1}$ puntos en las raíces de los polinomios de Hermite. Estos polinomios representan una selección óptima para distribuciones de tipo normal por sus propiedades de ortogonalidad respecto a la función de ponderación en la integración de Gauss-Hermite. El conjunto de puntos para una descomposición unidimensional viene dado por la expresión:

$$
\mathscr{U}^{S=1}=\bigcup_{i=1}^{n} \omega^{T} \cdot \hat{\mathbf{U}}_{i}
$$

donde $\omega \in \mathbb{R}^{1 x N_{1}}$ es un vector que contiene los $N_{1}$ ceros y $\hat{\mathbf{U}}_{i}$ es el autovector de la matriz de correlación, la cual coincide con la matriz identidad para un espacio normalizado estándar. La Figura 5.2 ilustra la colocación en un espacio normalizado tridimensional para una descomposición unidimensional $(S=1)$ y una muestra de cinco puntos en cada dimensión $\left(N_{1}=4\right)$. 
De forma genérica, para una descomposición de orden $S$ la muestra de puntos está formada por la unión:

$$
\begin{aligned}
\mathscr{U}^{S}= & \mathscr{U}^{S-1} \cup\left\{\left(x_{i}, \ldots, x_{j}, \ldots, x_{S}\right) \mid\right. \\
& x_{i}=x_{j}=\ldots=x_{S} \wedge \\
& \left(x_{i}, \ldots, 0, \ldots, 0\right) \subset \mathscr{U}^{1} \wedge \\
& \left(0, \ldots, x_{j}, \ldots, 0\right) \subset \mathscr{U}^{1} \wedge \\
& \left.\ldots \wedge\left(0, \ldots, 0, \ldots, x_{S}\right) \subset \mathscr{U}^{1}\right\}
\end{aligned}
$$

Cuando la descomposición incluye interacción de cuatro o más variables, el número de puntos necesarios se incrementa de forma considerable. Sin embargo, en la gran mayoría de problemas que se encuentran en ingeniería la contribución a la respuesta total de estos términos cruzados es baja. Por este motivo, una descomposición unidimensional o bidimensional suele ser suficiente para una integración precisa de los momentos estadísticos de la respuesta.

Obtención de observaciones. Esta etapa tiene por objeto obtener un conjunto de observaciones del modelo de simulación $(\mathscr{D}=\{\mathscr{X}, \mathscr{Y}\})$ que serán utilizadas para determinar los hiperparámetros del modelo de predicción. Estas observaciones se obtienen evaluando el modelo de simulación en los puntos generados en la etapa anterior. Para ello se transforma el diseño de experimentos $\mathscr{U}$ al espacio aleatorio original utilizando la inversa de la transformación $\mathbf{T}^{-1}$ (ecuación (5.20)). El diseño de experimentos resultante $(\mathscr{X})$ se evalúa utilizando el modelo de simulación $\mathscr{M}$. En función de los recursos computacionales, la evaluación se puede realizar en paralelo.

El coste computacional del proceso está dominado por el coste de las evaluaciones del modelo de simulación. El número de evaluaciones viene dado por la expresión:

$$
N_{\text {evals }}=\sum_{i=0}^{S}\left(\begin{array}{l}
n \\
i
\end{array}\right) \cdot N_{i}
$$

donde $n$ es el número de variables aleatorias, $S$ es el orden de la descomposición y $N_{i}$ es el número de puntos en la dimensión $i$ distintos del origen.

La introducción de los modelos de predicción reduce el número de evaluaciones necesarias respecto al método convencional. Esta reducción se aprecia gráficamente en la Figura 5.4. La Figura 5.4a muestra los puntos necesarios en una regla de cuadratura bidimensional. La Figura 5.4b representa los puntos evaluados con el modelo de simulación para la creación de los modelos de predicción. Este modelo de predicción actúa como soporte del proceso de integración. 

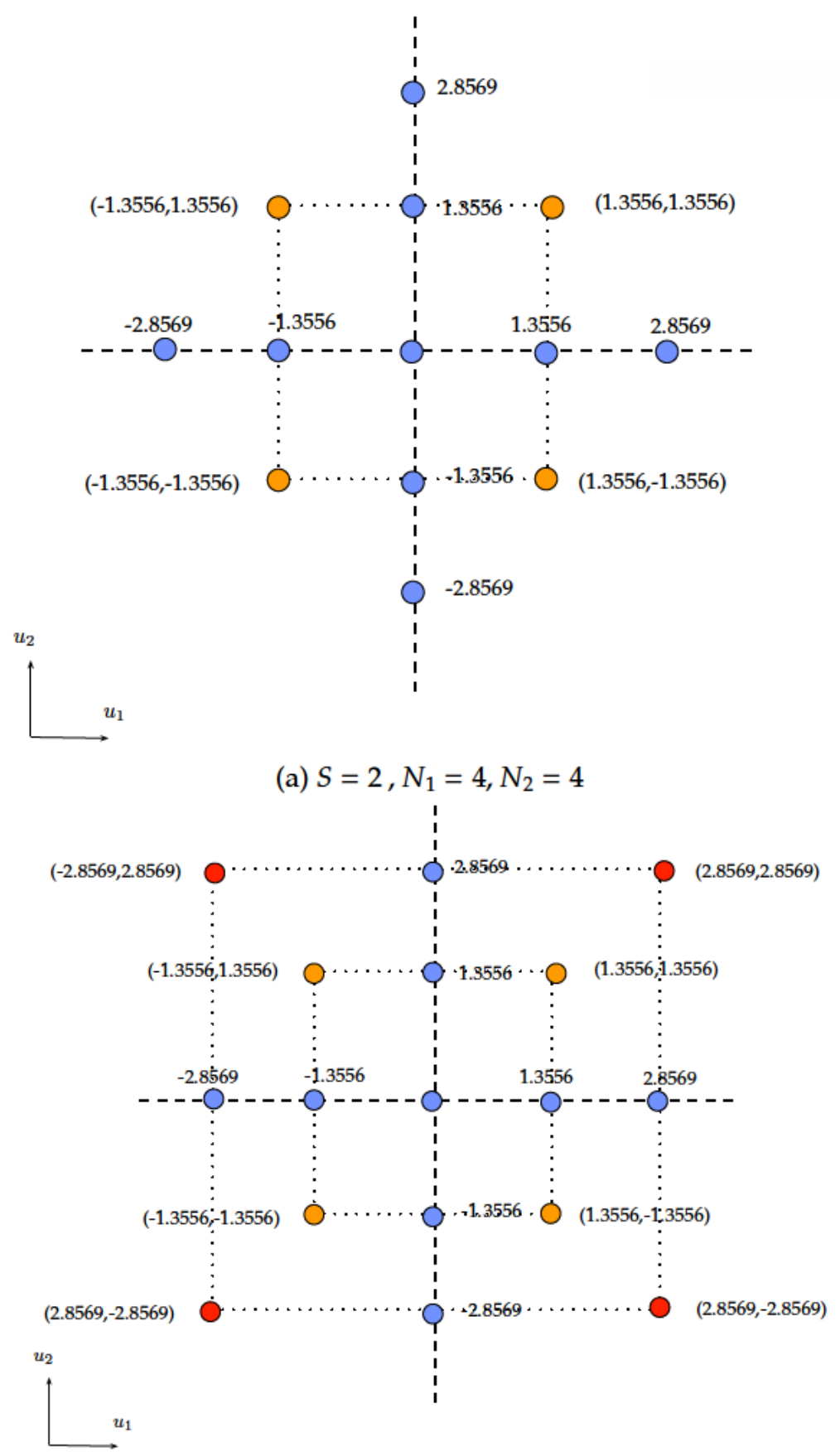

(b) $S=2, N_{1}=4, N_{2}=8$

Figura 5.3: Colocación de puntos en un espacio normalizado estándar bidimensional $\left(S=2, N_{1}=4, N_{2}=4,8\right)$. 


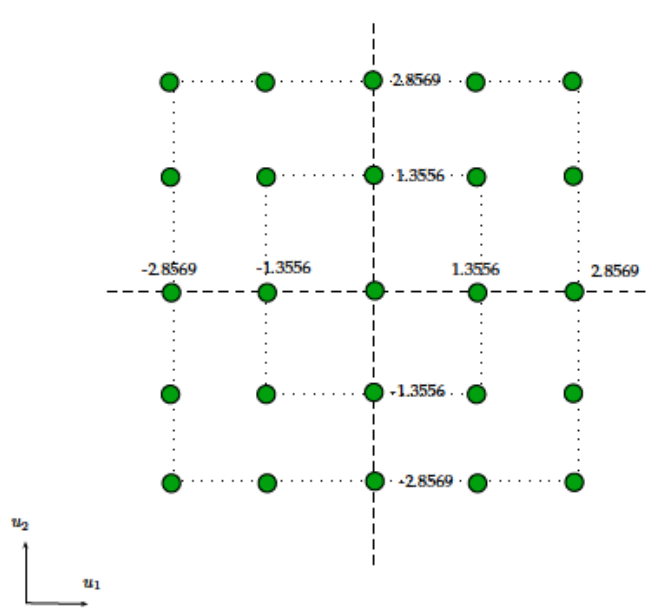

(a)

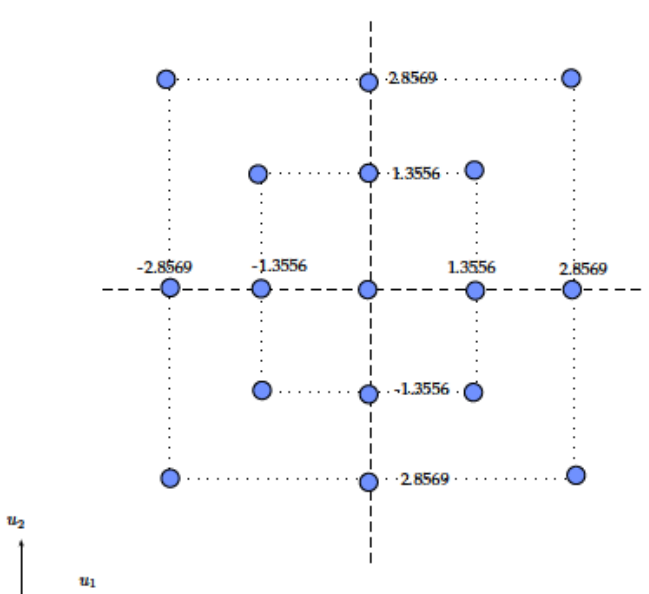

(b)

Figura 5.4: Colocación de puntos en un espacio normalizado estándar bidimensional. (a) puntos del método BIDRM . (b) $\left(S=2, N_{1}=4, N_{2}=8\right)$.

Modelos de predicción de la respuesta reducida centrada en el origen. Utilizando el conjunto de observaciones $\mathscr{D}$ se ajusta un modelo de predicción Kriging (Algoritmo 1) para cada una de las respuestas en el espacio reducido:

$$
\hat{y}\left(x_{k_{1}}, x_{k_{2}}, \ldots, x_{k_{S-i}}\right), \forall i=0, \ldots, S, k_{1}<k_{2}<\ldots<k_{S-i} \text {. }
$$

Estos modelos permiten obtener evaluaciones de la respuesta con un bajo coste computacional y posibilitan la utilización de métodos de simulación para la resolución de las integrales en el espacio reducido. El momento estadístico de primer orden de la respuesta $\left(\mu_{\hat{y}}\right)$ se obtiene con la ecuación (5.13).

Modelos de predicción de la respuesta reducida centrada en la media. Para obtener los momentos estadísticos centrados en la media se realiza una translación de la matriz de respuestas de la forma $\mathscr{G}=\mathscr{Y}-\mu_{\hat{y}}$. Utilizando el nuevo conjunto de observaciones $(\mathscr{D}=\{\mathscr{X}, \mathscr{G}\})$ se ajustan los modelos de predicción de las respuestas en el espacio reducido y centradas en la media. La ecuación (5.13), junto con los nuevos modelos de predicción, permiten obtener los momentos estadísticos de orden superior $\left.\mathbb{E}\left[\left(\hat{y}-\mu_{\hat{y}}\right)^{m}\right], \forall m=2,3,4\right)$. La desviación estándar, asimetría y curtosis se obtiene a partir de los momentos estadísticos centrados respecto de la media mediante las ecuaciones:

$$
\begin{aligned}
\sigma_{\hat{y}} & =\sqrt{\mathbb{E}\left[\hat{g}^{2}(\mathbf{x})\right]}=\sqrt{\mathbb{E}\left[\left(\hat{y}(\mathbf{x})-\mu_{\hat{y}}\right)^{2}\right]}, \\
\delta_{\hat{y}} & =\frac{\mathbb{E}\left[\hat{g}^{3}(\mathbf{x})\right]}{\sigma_{\hat{y}}^{3}}=\frac{\mathbb{E}\left[\left(\hat{y}(\mathbf{x})-\mu_{\left.\hat{y})^{3}\right]}\right.\right.}{\sigma_{\hat{y}}^{3}}, \\
\kappa_{\hat{y}} & =\frac{\mathbb{E}\left[\hat{g}^{4}(\mathbf{x})\right]}{\sigma_{\hat{y}}^{4}}=\frac{\mathbb{E}\left[\left(\hat{y}(\mathbf{x})-\mu_{\hat{y}}\right)^{4}\right]}{\sigma_{\hat{y}}^{4}} .
\end{aligned}
$$


Reconstrucción de la distribución de probabilidad. La función de distribución de probabilidad se reconstruye a partir de los cuatro primeros momentos estadísticos de la respuesta utilizando un sistema de Pearson (Pearson, 1895). De esta forma, la función de densidad de probabilidad es solución de la ecuación diferencial:

$$
\frac{1}{f} \frac{d f}{d x}=\frac{a x+b}{c+b x+d x^{2}}
$$

con:

$$
\begin{aligned}
a & =10 \kappa_{\hat{y}}-12 \delta_{\hat{y}}^{2}-18 \\
b & =\delta_{\hat{y}}\left(\kappa_{\hat{y}}+3\right) \\
c & =4 \kappa_{\hat{y}}-3 \delta_{\hat{y} \prime}^{2} \\
d & =2 \kappa_{\hat{y}}-3 \kappa_{\hat{y}}^{2}-6
\end{aligned}
$$

donde $x$ es una variable aleatoria normalizada, y $\delta_{\hat{y}}$ y $\kappa_{\hat{y}}$ son los momentos estadísticos centrados de tercer y cuarto orden (asimetría y curtosis respectivamente). La función de densidad de probabilidad aproximada permite estimar probabilidades de la respuesta.

\subsection{Ejemplos}

En esta sección se resuelve un conjunto de problemas de propagación de incertidumbre con el objetivo de analizar la precisión y eficiencia del algoritmo propuesto. Con los problemas seleccionados se busca cubrir un amplio abanico de situaciones que abarcan: problemas de baja y alta dimensionalidad, problemas con variables y campos aleatorios, problemas con un alto grado de no linealidad e interacción entre variables, diferentes tipos de distribuciones de probabilidad y problemas con modelos de elementos finitos de alto coste computacional. La Tabla 5.1 presenta un resumen de las principales características de cada uno de los problemas.

\subsubsection{Ejemplo TCPI-1}

Este ejemplo es una adaptación del propuesto en el trabajo de Huang et al. (2011). Se analiza la respuesta estocástica de una función analítica que presenta una alta no linealidad. La función es:

$$
Y(\mathbf{X})=\frac{1}{64} X_{3}^{3}-X_{2}-0,5\left(X_{1}-0,1\right)^{2}+5
$$




\begin{tabular}{|c|c|c|c|}
\hline Ejemplo & $n$ & $D_{t}$ & Características \\
\hline TCPI-1 Huang et al. (2011) & 3 & Normal & - Alta no linealidad \\
\hline TCPI-2 Creveling (2002); Huang y Du (2007) & 4 & $\begin{array}{l}\text { Beta } \\
\text { Normal } \\
\text { Rayleigh }\end{array}$ & $\begin{array}{l}\text { - Interacción entre } \\
\text { variables }\end{array}$ \\
\hline TCPI-3 Blatman y Sudret (2008) & 10 & $\begin{array}{l}\text { Lognormal } \\
\text { Gumble }\end{array}$ & $\begin{array}{l}\text { - Influencia de la } \\
\text { dimensionalidad } \\
\text { - 1er ejemplo FEM }\end{array}$ \\
\hline TCPI-4: Conexión T-Stub (Neves et al. (2001)) & 4 & Normal & $\begin{array}{l}\text { - Modelo FEM } \\
\text { - Material no lineal } \\
\text { - Grandes deformaciones } \\
\text { - Contacto }\end{array}$ \\
\hline TCPI-5: Lámina cilíndrica Scordelis-Lo Scordelis y Lo (1961) & $60^{1}$ & Lognormal & $\begin{array}{l}\text { - Modelo FEM } \\
\text { - } 60 \text { variables aliatorias } \\
\text { - Campos aleatorios }\end{array}$ \\
\hline
\end{tabular}

Tabla 5.1: Ejemplos numéricos.

donde $X_{1}, X_{2}$ y $X_{3}$ son variables independientes entre sí que siguen una distribución estándar Gaussiana con media 0 y desviación estándar 1 . Se utiliza una descomposición de orden $S=1$ y un número de puntos $N_{1}=4$. En total el número de evaluaciones de la función es:

$$
N_{\text {evals }}=N_{1} \cdot \mathrm{n}+1=4 \times 3+1=13 \text {. }
$$

Estos puntos son seleccionados a lo largo de los autovectores de la matriz de covarianza en los nodos [-2.857, $-1.356,1.356,2.857]$. El diseño de experimentos resultante se muestra en la Figura 5.5.

Las Figuras 5.6a a 5.6c muestran la predicción de las respuestas en el dominio unidimensional (línea azul), junto con las respuestas obtenidas con el modelo analítico (puntos negros). En cada modelo Kriging las funciones de regresión y correlación fueron seleccionadas utilizando los errores de validación cruzada. De acuerdo con estos errores se obtiene una regresión cuadrática y correlación Gaussiana para $Y\left(X_{1}\right)=-0,5\left(X_{1}-0,1\right)^{2}+5$, una regresión cuadrática y correlación general exponencial para $Y\left(X_{2}\right)=-X_{2}-0,005+5$, y una regresión cúbica junto con una correlación exponencial para $Y\left(X_{3}\right)=\frac{1}{64} X_{3}^{3}-0,005+5$. En los tres casos se consigue capturar el comportamiento lineal, cuadrático y cúbico, respectivamente. Para garantizar la independencia del procedimiento de selección de las funciones de correlación y regresión con respecto a la muestra inicial, el proceso de selección se repite 1000 veces partiendo de un diseño de experimentos aleatorio. La selección resultante se muestra en la Figura 5.7.

La Figura 5.6 muestra un excelente ajuste entre los modelos analíticos y los modelos de predicción. Estos modelos se utilizan para la resolución eficiente de las integrales unidimensionales utilizando una cuadratura de Gauss-Hermite (Burden y Faires, 1998) con 50 puntos de integración. 


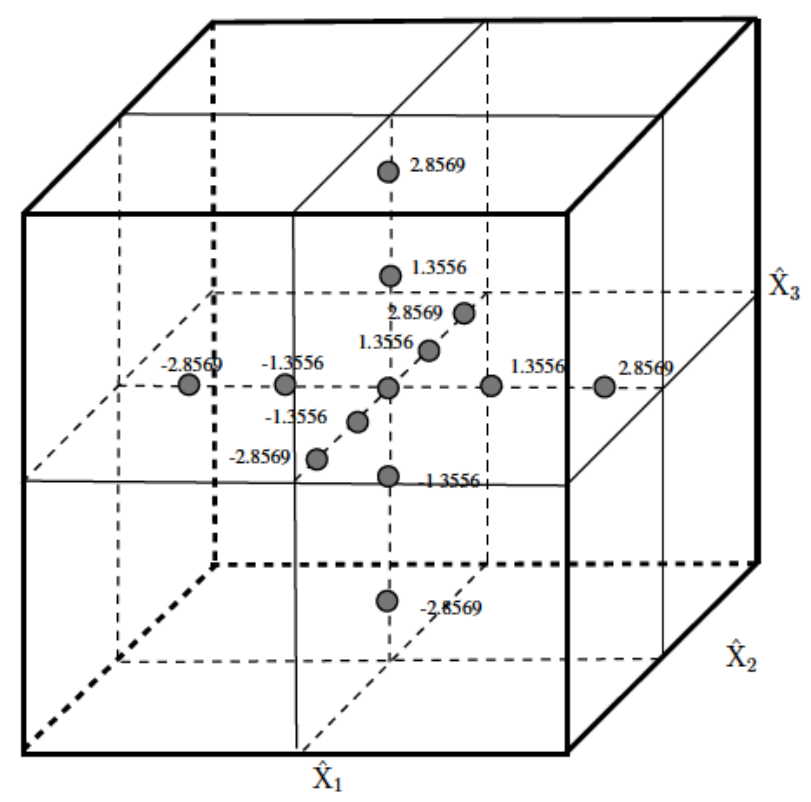

Figura 5.5: Problem TCPI-1. Localización de los experimentos $\left(S=1 ; N_{1}=4\right)$.

\begin{tabular}{cccc}
\hline & $\begin{array}{c}\text { EKDRM } \\
(S=1)\end{array}$ & $\begin{array}{c}\text { MC } \\
1 \mathrm{e}+06\end{array}$ & $\begin{array}{c}\text { Error relativo } \\
(\%)\end{array}$ \\
\hline \hline$\mu_{Y}$ & $4.4950 \mathrm{e}+00$ & $4.4953 \mathrm{e}+00$ & $-5.7170 \mathrm{e}-03$ \\
$\sigma_{Y}$ & $1.2303 \mathrm{e}+00$ & $1.2297 \mathrm{e}+00$ & $5.1018 \mathrm{e}-03$ \\
$\beta_{1, Y}$ & $-5.5309 \mathrm{e}-01$ & $-5.5308 \mathrm{e}-01$ & $5.9147 \mathrm{e}-04$ \\
$\beta_{2, Y}$ & $4.3620 \mathrm{e}+00$ & $4.3643 \mathrm{e}+00$ & $-5.1566 \mathrm{e}-02$ \\
\hline
\end{tabular}

Tabla 5.2: Problem TCPI-1. Momentos estadísticos obtenidos con EKDRM y simulación MonteCarlo. 


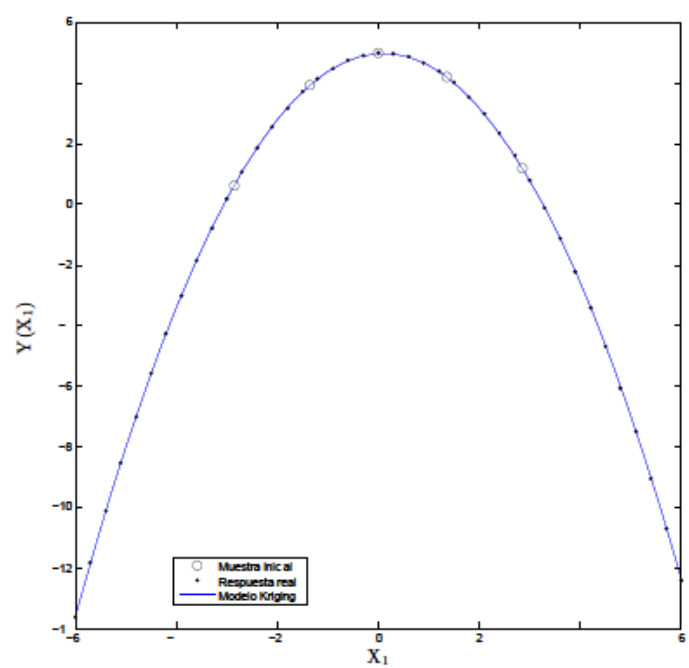

(a)

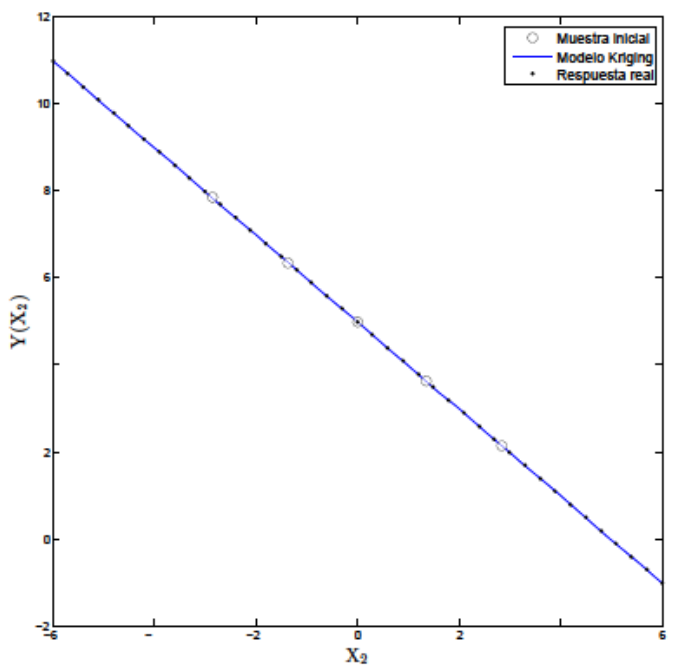

(b)

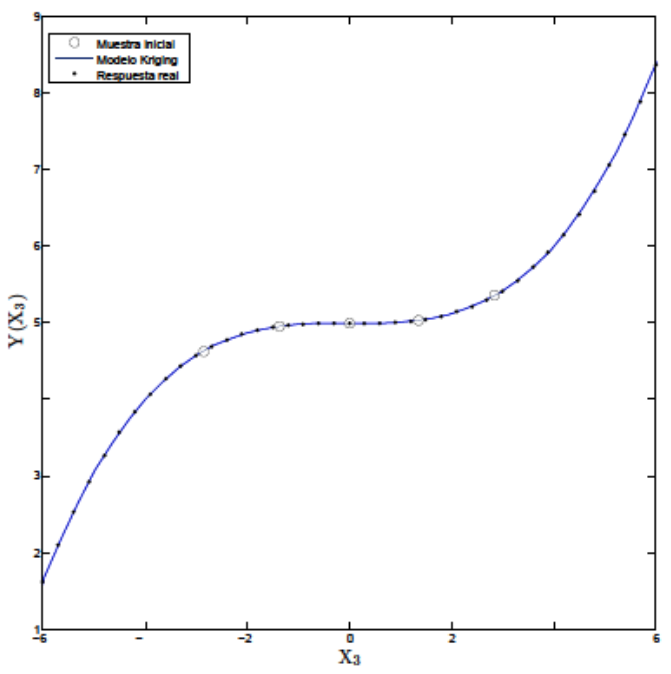

(c)

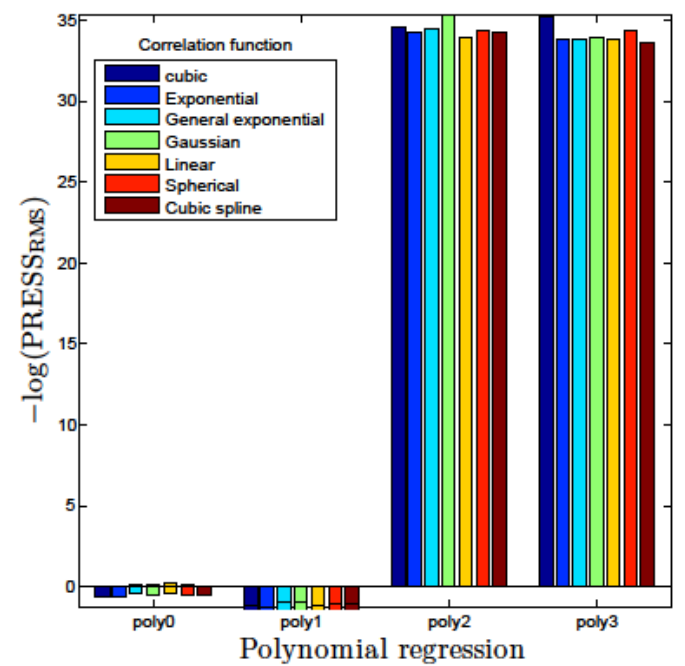

(d)

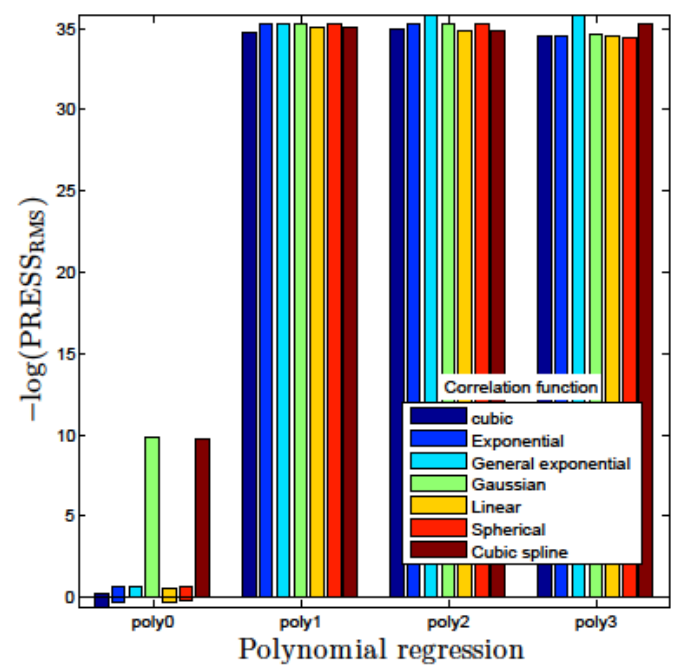

(e)

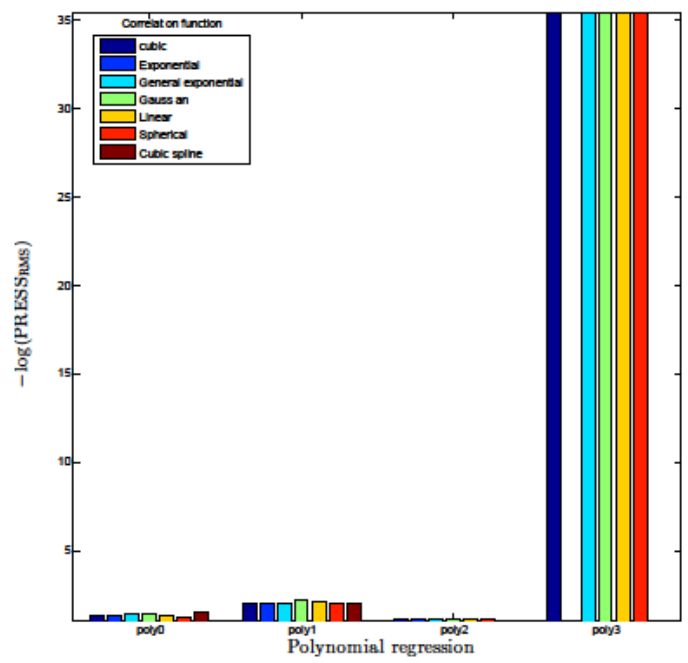

(f)

Figura 5.6: Problema TCPI-1. (a) $\hat{Y}\left(X_{1}\right)$, (b) $\hat{Y}\left(X_{2}\right)$, (c) $\hat{Y}\left(X_{3}\right)$, (d) $-\log \left(\operatorname{PRESS}_{\mathrm{RMS}}\right)$ de $\hat{Y}\left(\mathrm{X}_{1}\right)$, (e) $-\log \left(\mathrm{PRESS}_{\mathrm{RMS}}\right)$ de $\hat{\mathrm{Y}}\left(\mathrm{X}_{2}\right),(\mathrm{f})-\log \left(\mathrm{PRESS}_{\mathrm{RMS}}\right)$ de $\hat{\mathrm{Y}}\left(\mathrm{X}_{3}\right)$. 


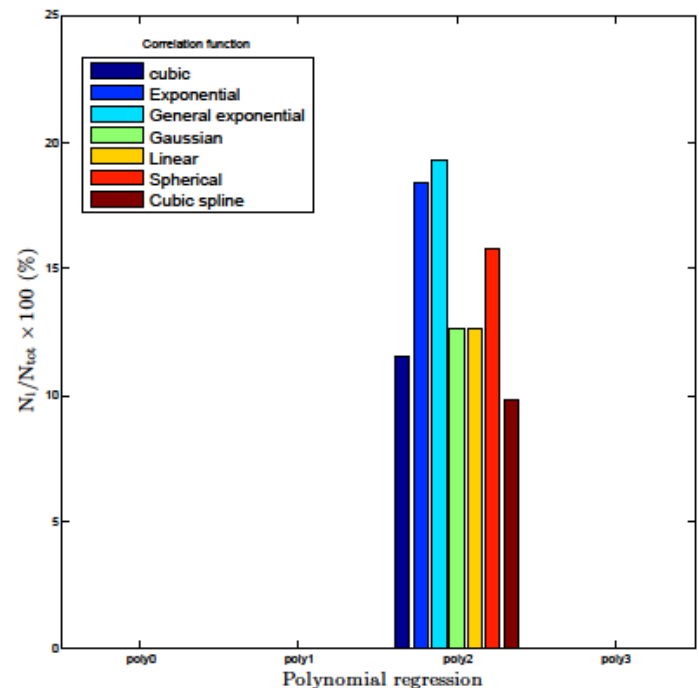

(a)

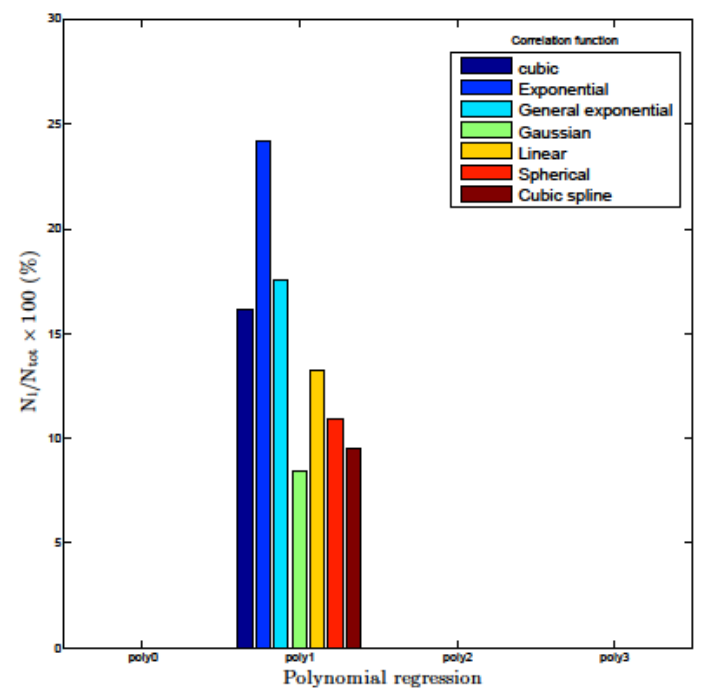

(b)

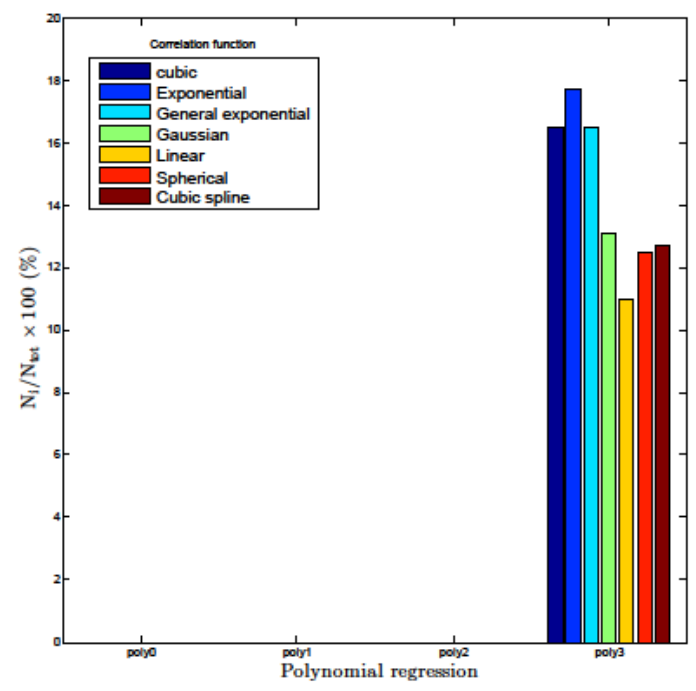

(c)

Figura 5.7: Problem TCPI-1. Funciones de regresión y correlación resultantes tras la realización de un total de 1000 experimentos. (a) $\mathrm{Y}\left(\mathrm{X}_{1}\right)$, (b) $\mathrm{Y}\left(\mathrm{X}_{2}\right)$, (c) $\mathrm{Y}\left(\mathrm{X}_{3}\right)$.

La Tabla 5.2 muestra los cuatro primeros momentos estadísticos de (5.34) obtenidos con el algoritmo EKDRM. Para analizar la precisión de los resultados se toma como referencia los momentos estadísticos obtenidos mediante una simulación MonteCarlo de 1e6 simulaciones. El error cometido se muestra en la cuarta columna de la Tabla 5.2. Este error se obtiene mediante la expresión:

$$
\text { Error }_{\mathrm{i}}=100\left|\frac{\mathbb{E}\left[\mathrm{Y}^{\mathrm{i}}(\mathbf{X})\right]_{\mathrm{EKDRM}}-\mathbb{E}\left[\mathrm{Y}^{\mathrm{i}}(\mathbf{X})\right]_{\mathrm{MC}}}{\mathbb{E}\left[\mathrm{Y}^{\mathrm{i}}(\mathbf{X})\right]_{\mathrm{MC}}}\right| i=1, \ldots, 4,
$$

donde $\mathbb{E}\left[Y^{i}(\mathbf{X})\right]_{\text {EKDRM y }} \mathbb{E}\left[Y^{i}(\mathbf{X})\right]_{\text {MC }}$ son el i-ésimo momento estadístico obtenido con el algoritmo EKDRM y la simulación MonteCarlo respectivamente. 


\begin{tabular}{|c|c|c|c|c|c|c|c|}
\hline & FORM $^{(1)}$ & $\operatorname{SORM}^{(1)}$ & BIDRM & TSPA $^{(1)}$ & $\begin{array}{l}\text { KDRM } \\
(S=1)\end{array}$ & $\begin{array}{c}\text { EKDRM } \\
(S=1)\end{array}$ & $\mathrm{MC}$ \\
\hline $\mathbb{P}[Y \leq 0.25]$ & 0.0024 & 0.0025 & 0.0033 & 0.0029 & 0.0033 & 0.0038 & 0.0039 \\
\hline $\mathbb{P}[Y \leq 0.50]$ & 0.0031 & 0.0033 & 0.0049 & 0.0044 & 0.0046 & 0.0052 & 0.0053 \\
\hline $\mathbb{P}[Y \leq 0.75]$ & 0.0041 & 0.0044 & 0.0072 & 0.0065 & 0.0065 & 0.0067 & 0.0069 \\
\hline $\mathbb{P}[Y \leq 8.25]$ & 0.9588 & 0.9997 & 0.9974 & 0.9980 & 0.9991 & 0.9994 & 0.9997 \\
\hline $\mathbb{P}[Y \leq 8.50]$ & 0.9689 & 0.9999 & 0.9984 & 0.9988 & 0.9995 & 0.9997 & 0.9998 \\
\hline $\mathbb{P}[Y \leq 8.75]$ & 0.9808 & 1.0000 & 0.9990 & 0.9993 & 0.9996 & 0.9998 & 0.9999 \\
\hline$N_{\text {eval }}$ & & & 61 & 13 & 13 & 13 & $1 \mathrm{e}+07$ \\
\hline
\end{tabular}

Tabla 5.3: Problem TCPI-1. Probabilidades de $Y$ para pequeños valores de la distribución de probabilidad acumulada.

Para completar este primer ejemplo se estudia la precisión de la metodología propuesta para la obtención de la probabilidad acumulada en puntos situados en los extremos del rango [-0.5,9.5]. Los resultados se comparan con los de otros métodos ampliamente utilizados en la literatura como el método FORM, el SORM, el BIvariate Dimension-Reduction Method (BIDRM), la simulación MonteCarlo, y otros de reciente aparición como el Tchebycheff Saddle Point Approximation (TSPA) (Huang et al., 2011). Un primer análisis de los resultados, presentados en la Tabla 5.3 y en la Figura 5.8, muestra que la precisión de los métodos FORM y SORM no es aceptable como consecuencia de la alta no linealidad del problema. Por otra parte los métodos BIDRM y TSPA aproximan las probabilidades con buena precisión. Sin embargo, en el caso de los primeros esta mejora es a costa de un aumento en el número de evaluaciones de la función analítica. Este problema se puede solventar utilizando un modelo Kriging para aproximar las repuestas unidimensionales (Kriging-based Dimension Reduction Method (KDRM)) (Won et al., 2009). La función de regresión y correlación de este modelo es desconocida a priori. El algoritmo KDRM utiliza un modelo de regresión constante y una función de correlación Gaussiana. A diferencia del KDRM, el algoritmo EKDRM propone la selección de las funciones de regresión y correlación que mejor se ajustan a la física del problema sin necesidad de nuevas simulaciones. Con esta metodología se alcanza, para este ejemplo, la mayor precisión con el menor número de simulaciones.

\subsubsection{Ejemplo TCPI-2}

En este ejemplo se realiza un análisis de fiabilidad del problema de ensamblado de un engranaje de rueda libre (Figura 5.9). Este problema, también conocido como Fortinis slutch, ha sido discutido por diversos autores como Creveling (2002) para el diseño de tolerancias, Huang y Du (2007) para comparar diferentes criterios de robustez, Huang et al. (2011) para validar la metodología propuesta TSPA, y Lee y Chen (2009) para comparar el funcionamiento de diferentes métodos de cuantificación de incertidumbre en problemas con funciones no intrusivas (black-box functions). Este ejemplo permite estudiar la precisión del 


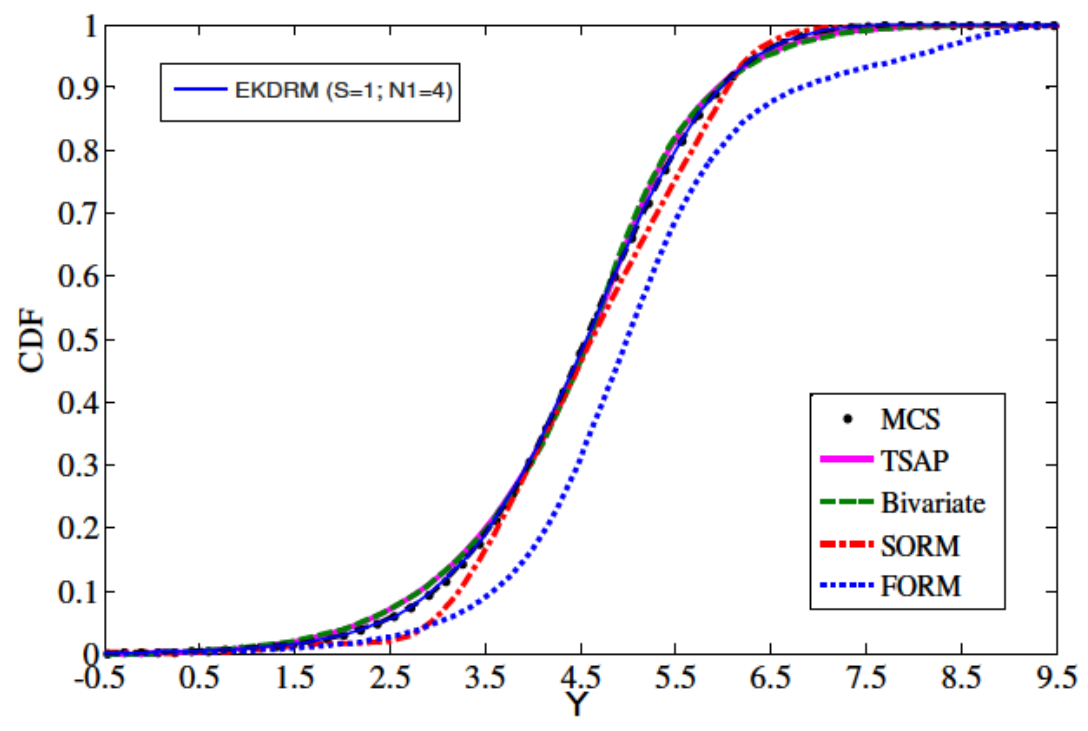

Figura 5.8: Problem TCPI-1. Función de densidad de probabilidad acumulada de 5.34.

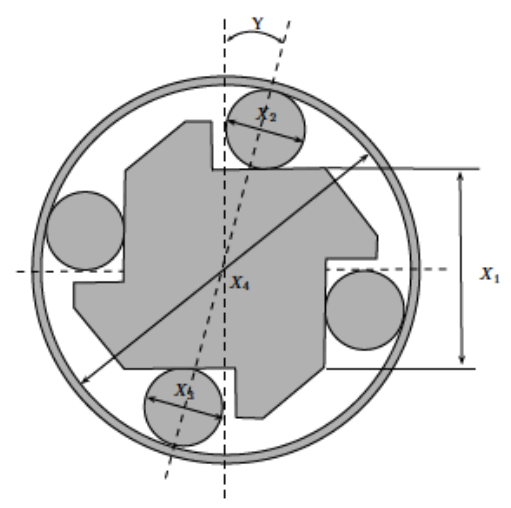

Figura 5.9: Fortinis clutch: esquema del ensamblado.

algoritmo propuesto frente a variables aleatorias no Gaussianas.

El ángulo de contacto $\mathrm{Y}$ es función de cuatro variables aleatorias independientes $\mathrm{X}_{1}, \mathrm{X}_{2}, \mathrm{X}_{3}, \mathrm{y} \mathrm{X}_{4} \mathrm{y}$ viene dado por la expresión:

$$
Y(\mathbf{X})=\arccos \left(\frac{X_{1}+0.5\left(X_{2}+X_{3}\right)}{X_{4}-0.5\left(X_{2}+X_{3}\right)}\right) .
$$

El criterio de diseño de este mecanismo es que el ángulo de contacto $(\mathrm{Y})$ esté situado entre los $8.726 e-02$ radianes $\left(5^{\circ}\right)$ y los $1.571 e-01$ radianes $\left(9^{\circ}\right)$. La media, desviación estándar y el tipo de distribución de las variables aleatorias se muestra en la Tabla 5.4.

En primer lugar, se analiza el problema utilizando una reducción unidimensional $(S=1)$ donde se varía el número de puntos de la muestra. 


\begin{tabular}{lllll}
\hline $\begin{array}{l}\text { Variable } \\
\text { aleatoria }\end{array}$ & Distribución & $\begin{array}{l}\text { Media } \\
(\mathrm{mm})\end{array}$ & $\begin{array}{l}\text { Desviación } \\
\text { estándar }(\mathrm{mm})\end{array}$ & parámetros \\
\hline \hline $\mathrm{X}_{1}$ & Beta & 55.29 & 0.0793 & $\mathrm{~A}=\mathrm{B}=5.0$ \\
& & & & $\left(55.0269 \geq \mathrm{X}_{1} \geq 55.5531\right)$ \\
$\mathrm{X}_{2}$ & Normal & 22.86 & 0.0043 & \\
$\mathrm{X}_{3}$ & Normal & 22.86 & 0.0043 & \\
$\mathrm{X}_{4}$ & Rayleigh & 101.60 & 0.0793 & $\begin{array}{l}\mathrm{A}=0.1211 \\
\left(\mathrm{X}_{4} \geq 101.45\right)\end{array}$ \\
\hline
\end{tabular}

Tabla 5.4: Problem TCPI-2. Distribución de las variables aleatorias.

\begin{tabular}{|c|c|c|c|c|}
\hline & $\begin{array}{l}\text { MC } \\
\text { (Ref.) }\end{array}$ & $\begin{array}{l}\text { EKDRM } \\
S=1 \\
N_{1}=2\end{array}$ & $\begin{array}{l}\text { EKDRM } \\
S=1 \\
N_{1}=4\end{array}$ & $\begin{array}{l}\text { EKDRM } \\
S=1 \\
N_{1}=6\end{array}$ \\
\hline Media $^{(1)}$ & 0.12210 & 0.12211 & 0.12212 & 0.12212 \\
\hline Des. Est. ${ }^{(2)}$ & 0.01166 & 0.01160 & 0.01162 & 0.01164 \\
\hline Asimetría ${ }^{(3)}$ & -0.05229 & 0.08905 & 0.09228 & 0.08564 \\
\hline Curtosis $^{(4)}$ & 2.88525 & 2.84799 & 2.87040 & 2.87774 \\
\hline (1) Error ( \%) & - & -0.01081 & -0.01482 & -0.01532 \\
\hline (2) Error (\%) & - & 0.50587 & 0.32123 & 0.17153 \\
\hline (3) Error ( \%) & - & 270.28071 & 276.46233 & 263.77893 \\
\hline (4) Error (\%) & - & 1.29134 & 0.51464 & 0.26029 \\
\hline$N_{\text {evals }}$ & $1 e+06$ & 9 & 17 & 25 \\
\hline
\end{tabular}

Tabla 5.5: Problem TCPI-2. Momentos estadísticos de la respuesta obtenidos mediante una descomposición unidimensional con $N_{1}=2, N_{1}=4$, y $N_{1}=6$ respectivamente.

Los resultados obtenidos para los cuatro primeros momentos estadísticos se presentan en la Tabla 5.5. Se muestran los errores cometidos en el cálculo de los momentos estadísticos con el algoritmo EKDRM tomando $N_{1}=2, N_{1}=4$, y $N_{1}=6$ puntos. Los resultados ponen de manifiesto que la aproximación unidimensional es suficiente para estimar con alta precisión los dos primeros momentos estadísticos de la respuesta. Sin embargo, para momentos estadísticos de orden superior se obtienen errores apreciables. Estos errores no pueden ser disminuidos con un aumento en el número de puntos de la muestra, lo que indica que están directamente relacionados con el error asociado al orden de la descomposición.

Para intentar reducir los errores cometidos en la estimación de los momentos estadísticos de tercer y cuarto orden, se resuelve el problema mediante una reducción bivariable. La localización de los puntos en el espacio aleatorio original se muestra en la Figura 5.10 para el caso de $S=2, N_{1}=6$ y $N_{2}=8$. El valor de los momentos estadísticos de la respuesta se muestran en la Tabla 5.6. Tomando como referencia la simulación MonteCarlo, se estudia la precisión y eficiencia del algoritmo EKDRM con $S=2$ y diferente número de evaluaciones. Los resultados se comparan con los obtenidos con polinomios del caos y con cuadratura. Se observa que los errores obtenidos en la estimación del tercer momento estadístico 

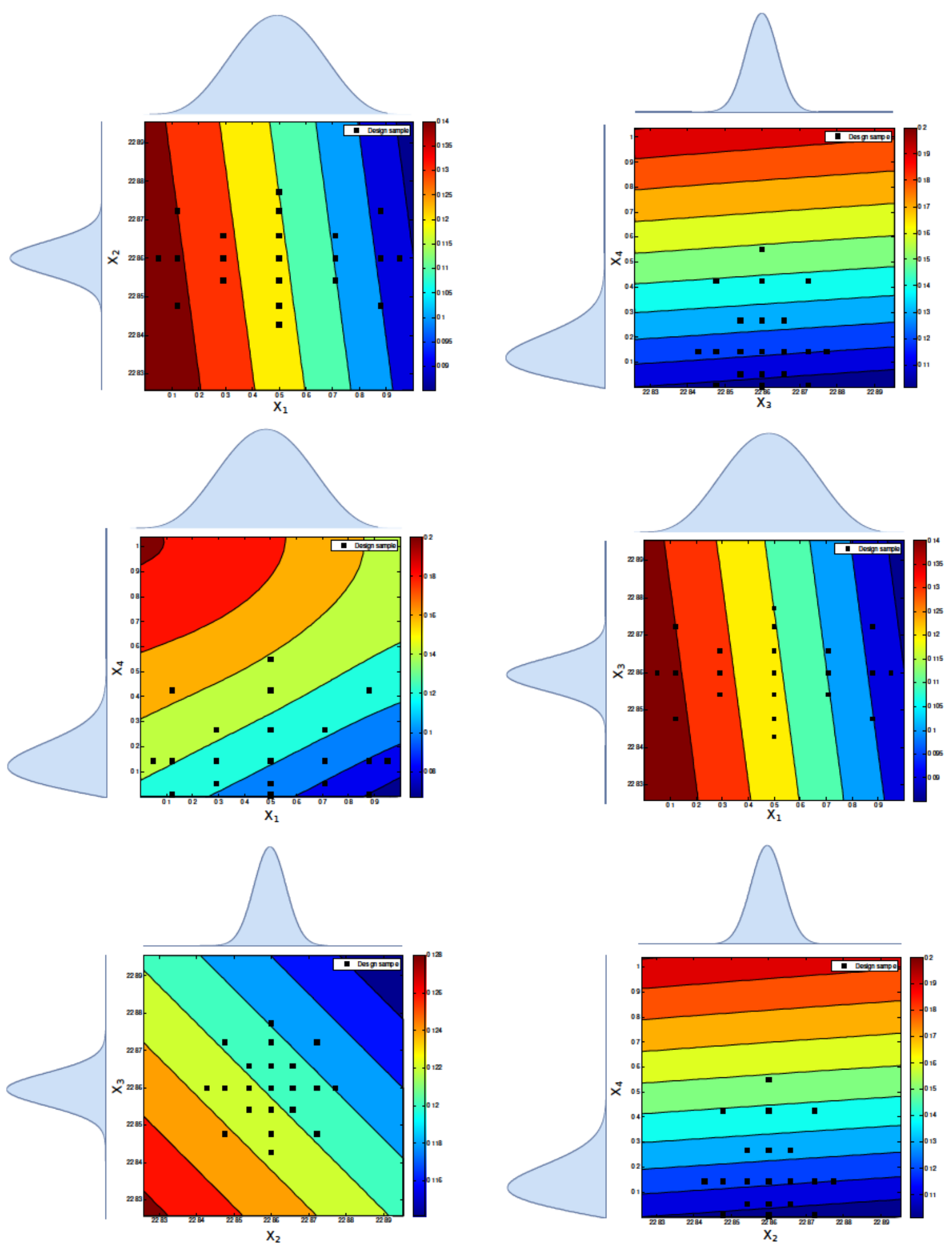

Figura 5.10: Problem TCPI-2. Localización de los experimentos para una descomposición bidimensional con $N_{1}=6$ y $N_{2}=8$. 


\begin{tabular}{|c|c|c|c|c|c|c|c|c|}
\hline & $\begin{array}{l}\text { MC } \\
\text { (Ref.) }\end{array}$ & $\begin{array}{l}\text { FFNI } \\
3^{n}\end{array}$ & $\begin{array}{l}\text { FFNI } \\
5^{n}\end{array}$ & $\begin{array}{l}\text { PCE } \\
\text { orden } 4\end{array}$ & $\begin{array}{l}\text { EKDRM } \\
S=2 \\
N_{1}=2 \\
N_{2}=4\end{array}$ & $\begin{array}{l}\text { EKDRM } \\
S=2 \\
N_{1}=4 \\
N_{2}=4\end{array}$ & $\begin{array}{l}\text { EKDRM } \\
S=2 \\
N_{1}=6 \\
N_{2}=4\end{array}$ & $\begin{array}{l}\text { EKDRM } \\
S=2 \\
N_{1}=6 \\
N_{2}=8\end{array}$ \\
\hline Media & $\overline{0.12210}$ & 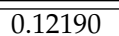 & $\overline{0.12190}$ & $\overline{0.12190}$ & 0.12212 & 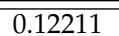 & $\overline{0.12211}$ & 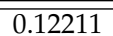 \\
\hline Des. Est. & 0.01166 & 0.01170 & 0.01170 & 0.01170 & 0.01153 & 0.01168 & 0.01167 & 0.01167 \\
\hline Asimetría & -0.05229 & -0.04970 & -0.05300 & -0.05770 & -0.04817 & -0.04939 & -0.05133 & -0.05222 \\
\hline Curtosis & 2.88525 & 2.84880 & 2.88270 & 2.89300 & 2.93261 & 2.93089 & 2.84277 & 2.92476 \\
\hline Error Media (\%) & - & 0.16476 & 0.16476 & 0.16476 & -0.01189 & -0.01124 & -0.00800 & -0.00889 \\
\hline Error Des. Est. (\%) & - & -0.32978 & -0.32978 & -0.32978 & 1.11695 & -0.15283 & -0.07022 & -0.06012 \\
\hline Error Asimetría (\%) & - & 4.95899 & -1.35158 & -10.33936 & 7.88068 & 5.54941 & 1.84689 & 0.14946 \\
\hline Error Media (\%) & - & 1.26319 & 0.08824 & -0.26875 & -1.64146 & -1.58213 & 1.47215 & -1.36962 \\
\hline Nevals. & $1.00 \mathrm{E}+06$ & 81 & 625 & 625 & 33 & 41 & 49 & 73 \\
\hline
\end{tabular}

Tabla 5.6: Problem TCPI-2. Momentos estadísticos de la respuesta obtenidos mediante una descomposición bidimensional, polinomios del caos y cuadratura.

se reducen drásticamente a costa de un mayor número de evaluaciones. En comparación con otros métodos de propagación de incertidumbre, como la expansión con polinomios del caos o la cuadratura, el algoritmo EKDRM iguala o mejora la precisión de los resultados con un menor número de evaluaciones del modelo de simulación.

\subsubsection{Ejemplo TCPI-3}

En este ejemplo se aborda un primer problema de análisis estocástico estructural. Se trata de la estructura de 23 barras (Blatman y Sudret, 2008) de la Figura 5.11. Se consideran diez variables aleatorias $\left(E_{1}, E_{2}, A_{1}, A_{2}, P_{1-6}\right)$ cuyas características probabilistas se muestran en la tabla 5.7. Se asume que todos los elementos del cordón inferior y del cordón superior tienen módulos de Young $\left(E_{1}\right)$ y áreas perfectamente correlacionadas $\left(A_{1}\right)$, al igual que ocurre con los elementos diagonales $\left(E_{2}\right.$ y $\left.A_{2}\right)$.

\begin{tabular}{llll}
\hline Variable & Distribución & Media & Des. estándar \\
\hline \hline$E_{1}\left(\mathrm{~N} / \mathrm{m}^{2}\right)$ & lognormal & $2.1 \mathrm{e} 11$ & $2.1 \mathrm{e} 10$ \\
$E_{2}\left(\mathrm{~N} / \mathrm{m}^{2}\right)$ & lognormal & $2.1 \mathrm{e} 11$ & $2.1 \mathrm{e} 10$ \\
$A_{1}\left(\mathrm{~m}^{2}\right)$ & lognormal & $2.0 \mathrm{e}-3$ & $2.0 \mathrm{e}-4$ \\
$A_{2}\left(m^{2}\right)$ & lognormal & $1.0 \mathrm{e}-3$ & $1.0 \mathrm{e}-4$ \\
$P_{1-6}(\mathrm{~N})$ & gumble & $5.0 \mathrm{e} 4$ & $7.5 \mathrm{e} 3$ \\
\hline
\end{tabular}

Tabla 5.7: Problem TCPI-3. Distribución de probabilidad de las variables aleatorias.

El requerimiento de diseño de la estructura es que el desplazamiento vertical del nudo central del cordón inferior $\left(V_{1}\right)$ sea menor de $0.11 \mathrm{~m}$. La función de estado límite viene dada por la expresión:

$$
Y(\mathbf{X})=0.11-V_{1} .
$$




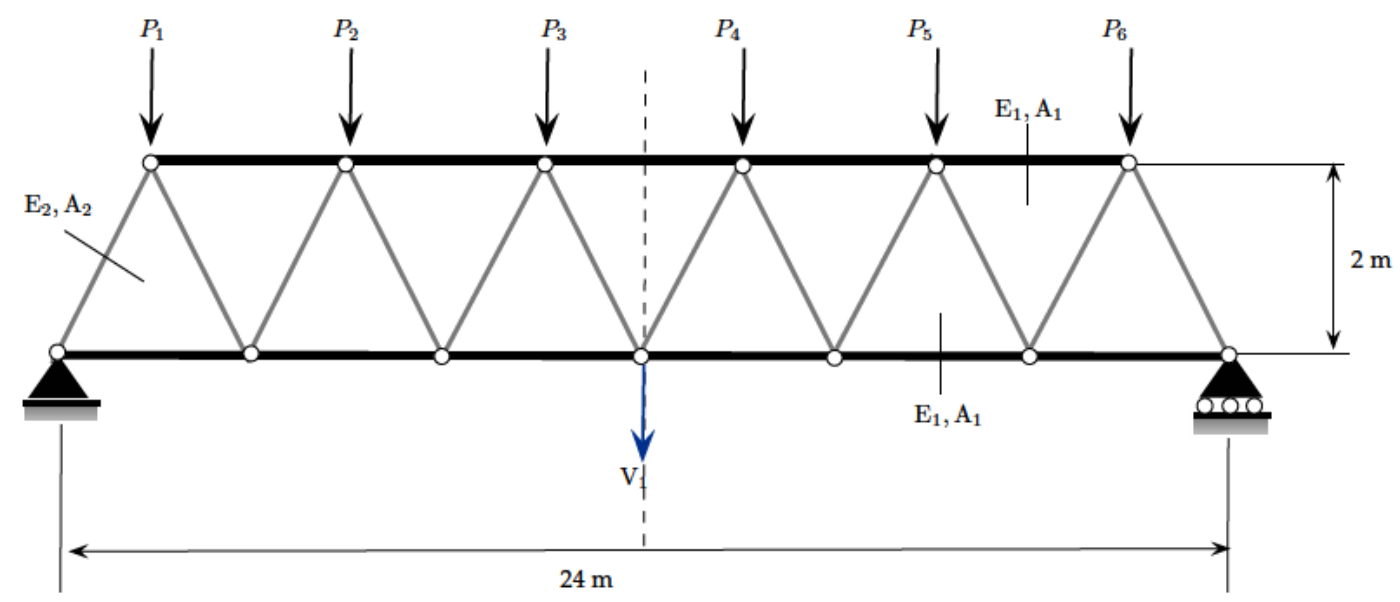

Figura 5.11: Problem TCPI-3. Estructura de 23 barras.

\begin{tabular}{llllll}
\hline & $\begin{array}{l}\text { MC } \\
\text { (Ref.) }\end{array}$ & $\begin{array}{l}\text { EKDRM } \\
S=1\end{array}$ & $\begin{array}{l}\text { EKDRM } \\
S=1\end{array}$ & $\begin{array}{l}\text { EKDRM } \\
S=1\end{array}$ & $\begin{array}{l}\text { EKDRM } \\
\text { N=2 }\end{array}$ \\
& & $N_{1}=2$ & $N_{1}=4$ & $N_{1}=6$ & $N_{1}=4, N_{2}=8$ \\
\hline \hline Media $^{(1)}$ & 0.03137 & 0.03130 & 0.03130 & 0.03132 & 0.03136 \\
Des. Est. $^{(2)}$ & 0.00994 & 0.01007 & 0.01006 & 0.00998 & 0.00994 \\
Asimetría $^{(3)}$ & -0.32691 & -0.03508 & -0.03988 & -0.04435 & -0.32559 \\
Curtosis $^{(4)}$ & 3.31030 & 3.03584 & 3.08753 & 3.08772 & 3.35235 \\
(1) Error (\%) $^{(1)}$ & - & 0.23323 & 0.20711 & 0.16459 & 0.02589 \\
${ }^{(2)}$ Error (\%) & - & -1.31346 & -1.27945 & -0.45998 & -0.03448 \\
${ }^{(3)}$ Error (\%) & - & 89.26841 & 87.80222 & 86.43266 & 0.40362 \\
${ }^{(4)}$ Error (\%) & - & 8.290979 & 6.72959 & 6.724006 & -1.27024 \\
$N_{\text {evals. }}$ & $1.00 \mathrm{E}+06$ & 21 & 41 & 61 & 401 \\
\hline
\end{tabular}

Tabla 5.8: Problem TCPI-3. momentos estadísticos de la función de estado límite obtenidos mediante descomposiciones unidimensionales y bidimensional.

La tabla 5.8 recoge los cuatro primeros momentos estadísticos de la función de estado límite obtenidos mediante una reducción unidimensional y bidimensional. Se toman como referencia los momentos estadísticos obtenidos mediante una simulación MonteCarlo de 1e6 evaluaciones. Mediante una descomposición unidimensional $(S=1)$ se aproxima con una buena precisión los dos primeros momentos estadísticos. Sin embargo, para los momentos de orden superior (asimetría y curtosis), se aprecia un error del orden del $90 \%$ y del 10\%, como consecuencia del efecto que tiene en la función de estado límite la interacción entre las variables aleatorias. Este error no se puede reducir incrementando el tamaño de la muestra $\left(N_{1}\right)$, y es preciso aumentar el orden de la descomposición para incluir términos cruzados. La quinta columna de la tabla 5.8 muestra los resultados obtenidos con una reducción de orden $2, N_{1}=4$ y $N_{2}=8$. En este caso el número total de evaluaciones del modelo de simulación es de 401 y los errores obtenidos están por debajo del $0.5 \%$ para los tres primeros momentos estadísticos y en torno al $1 \%$ para la curtosis.

La selección del orden y del número de puntos de la descomposición es un compromiso entre la eficiencia y la precisión deseada. Así, en el caso del 


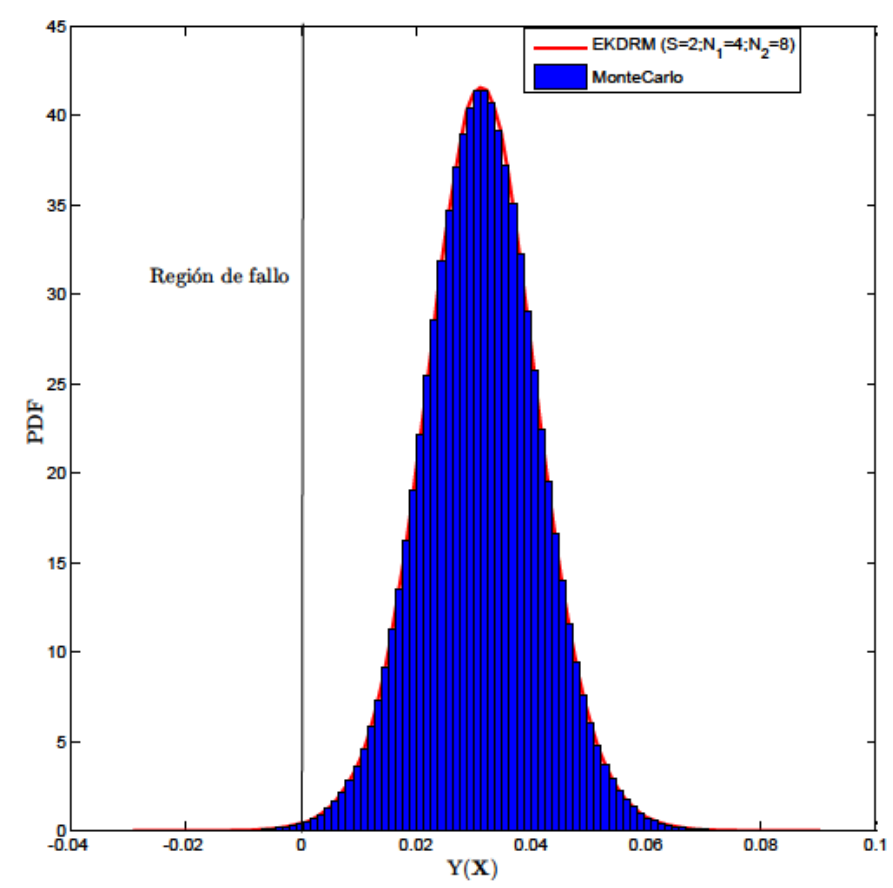

Figura 5.12: Problem TCPI-3. función de densidad de probabilidad de la función de estado límite.

DOR, una reducción unidimensional con $N_{1}=2$ permite estimar la media y la desviación de la respuesta con un bajo número de evaluaciones y con un error inferior al 1.5\%. En contraposición, si el objetivo es la resolución de un problema de DOF, se necesita la estimación de probabilidades de fallo. Para ello, se reconstruye la función de densidad de probabilidad utilizando los cuatro primeros momentos estadísticos. En este caso la descomposición de orden dos permite una reconstrucción precisa de la función de densidad de probabilidad. La Figura 5.12 muestra la función de densidad de probabilidad obtenida mediante un sistema de Pearson (línea roja continua) y la obtenida mediante simulación MonteCarlo (barras azules). Se observa el buen acuerdo entre ambas que se traduce en las siguientes probabilidades de fallo:

$$
\begin{aligned}
P_{F}^{M C} & =\mathbb{P}[Y(\mathbf{X})<0]=1.504 \cdot 10^{-03}, \\
P_{F}^{E K D R M} & =\mathbb{P}[Y(\mathbf{X})<0]=1.561 \cdot 10^{-03},
\end{aligned}
$$

y fiabilidades:

$$
\begin{aligned}
P_{S}^{M C} & =1-P_{F}^{M C}=0.998496 \\
P_{S}^{E K D R M} & =1-P_{F}^{E K D R M}=0.998439 .
\end{aligned}
$$

El error cometido en la probabilidad de fallo es del $0.0057 \%$. 


\subsubsection{Ejemplo TCPI-4}

En este ejemplo se aborda el problema de propagación de incertidumbre con un modelo de simulación de alto coste computacional. Se estudia la respuesta aleatoria de conexiones de acero frente a variaciones en los parámetros geométricos. Para ello se analiza, mediante un modelo de elementos finitos, la respuesta estocástica del principal componente de este tipo de conexión de acuerdo con el Eurocódigo 3 (EN1993-1-8, 1993), el T-Stub. Este componente es representativo del comportamiento global de la unión, puesto que en él intervienen varias partes de la misma: ala de la columna a flexión, chapa de testa a flexión y ala del perfil en $\mathrm{T}$ a flexión. A pesar de su simplicidad, el T-Stub resulta uno de los componentes principales de las uniones atornilladas de chapa de testa y determina la resistencia última, rigidez y capacidad rotacional de la unión. En este ejemplo, se busca determinar la función de densidad de probabilidad de la resistencia última de una conexión T-Stub. Para ello se reproduce numéricamente el ensayo experimental T1 realizado por Coelho (2004).

\subsubsection{Descripción del modelo}

Geometría. Las características geométricas del ensayo T1 se muestran en la Figura 5.13a. Se modela una octava parte de la conexión mediante las siguientes condiciones de contorno. Se aplican condiciones de simetría en los planos yz y $x y$. Los tornillos se modelan utilizando el concepto de tornillo equivalente (Girao Coelho et al., 2006), verificando las hipótesis de simetría en el plano $x z$. El tornillo equivalente se modela de tal forma que su rigidez es idéntica a la del tornillo real, es decir el alargamiento del tornillo equivalente es la mitad del alargamiento del tornillo real.

Discretización. El modelo de elementos finitos se desarrolla en el software comercial ANSYS (ANSYS, 2013). El modelo geométrico de la conexión T-Stub se discretiza utilizando elementos de elasticidad tridimensional. En particular, se realiza un malla estructurada con elementos hexaédricos (SOLID185) tal y como muestra la Figura 5.13b. El SOLID185 es un elemento de primer orden con tres grados de libertad por nodo. Se selecciona un orden de integración completa (2x2x2 puntos de Gauss) y una descripción cinemática basada en una formulación Lagrangiana actualizada que tiene en cuenta grandes desplazamientos y deformaciones moderadamente grandes.

El contacto entre la chapa superior e inferior, y entre la cabeza del tornillo y la chapa superior, se modela utilizando elementos de contacto superficial. El posible contacto entre la superficie interior del agujero y el vástago del tornillo no se considera. La interacción entre las superficies de contacto se modela utilizando un esquema maestro-esclavo con los elementos TARGET170 y CONTA174. 
Para determinar la densidad de la malla se realiza un análisis de sensibilidad de la misma respecto a dos parámetros: (1) la discretización de la chapa, para representar los fenómenos de flexión, y (2) el número de elementos en el espesor de la misma, para capturar de forma adecuada las líneas de plastificación. Los resultados obtenidos están en la línea de los de otros autores como Girao Coelho et al. (2006) y Bursi y Jaspart (1997), y sugiere un mínimo de tres elementos en el espesor de la chapa. La malla utilizada en este trabajo cumple con este criterio y es la mostrada en la Figura 5.13b.

Condiciones de contorno. Se restringe el desplazamiento en la dirección $z$ y en la dirección $x$ de los nodos contenidos en los planos $x y$ y zy respectivamente. Se consideran como superficies de contacto: (1) la interfaz entre el ala del perfil en T superior y el ala del perfil en T inferior (modelada como un soporte rígido) y (2) la interfaz entre la cabeza del tornillo y el ala del perfil en T superior. Se restringe el desplazamiento en dirección y de la base inferior del soporte rígido y del vástago. Con el fin de reducir el número de planos de contacto, se desprecia el contacto entre la cabeza del tornillo y la arandela, así como entre el vástago y la superficie interna del agujero. Esta simplificación lleva a un comportamiento ligeramente más rígido. Sin embargo no tiene una gran influencia en el comportamiento global de la conexión (Bursi y Jaspart, 1997; Zajdel, 1997).

Los contactos se modelan mediante una formulación del tipo maestro-esclavo. Esta formulación reconoce si el estado de la superficie de los elementos es contacto, deslizamiento o penetración, e impone las restricciones correspondientes a los nodos de la superficie esclavo. De esta forma, se impide la penetración de la superficie esclavo en la superficie maestro. El deslizamiento entre superficies se modela con la ley de fricción de Coulomb. El contacto entre el ala del perfil en $\mathrm{T}$ superior y la base rígida se considera libre de fricción. El contacto entre el ala del perfil en T superior y la cabeza del tornillo se considera con un coeficiente de fricción $\mu=0.25$ (Vasarhelyi y Chiang, 1967).

La carga se aplica de forma incremental, imponiendo desplazamientos en la dirección y a los nodos de la superficie superior del alma del perfil en $\mathrm{T}$, hasta alcanzar el colapso.

Propiedades mecánicas del acero. El modelo de elementos finitos tiene en cuenta la no linealidad del material a través de la curva tensión-deformación real en escala logarítmica del acero (Figura 5.14). El comportamiento plástico del acero se modela utilizando: (1) el criterio de plastificación de Von-Mises, (2) la regla de fluencia de Prandtl-Reuss y (3) un endurecimiento isotrópico. 


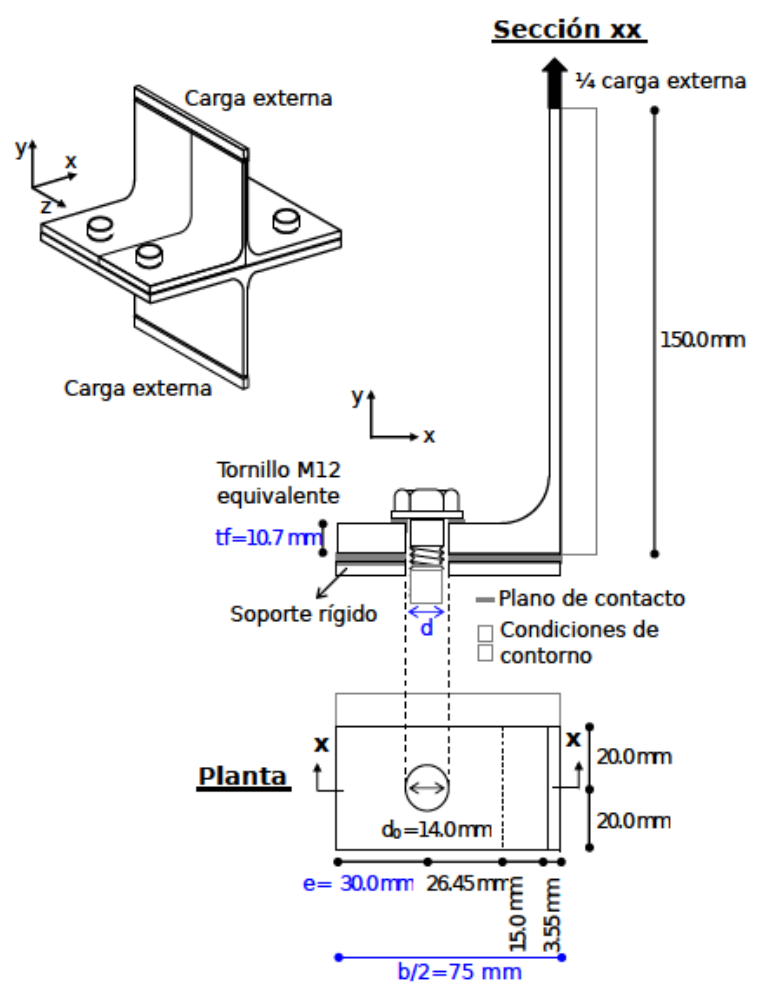

(a) Modelo geométrico y variables aleatorias (azul).

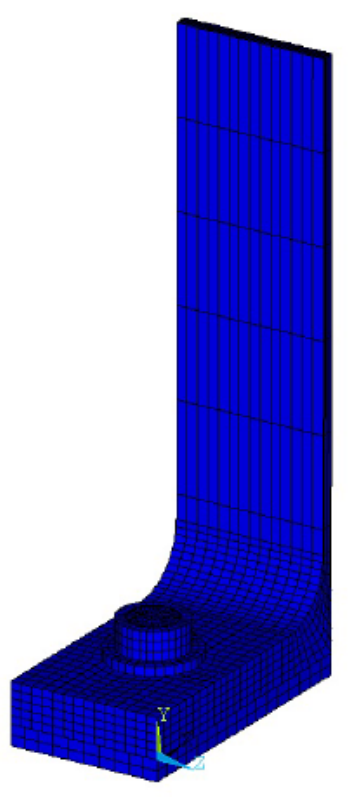

(b) Discretización.

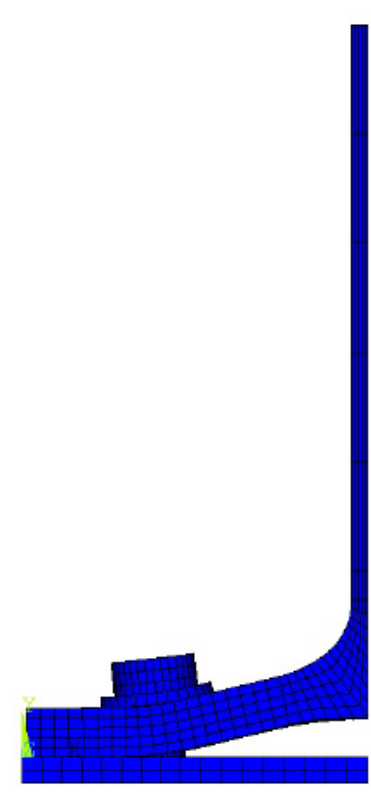

(c) Deformación.

Figura 5.13: Problem TCPI-4. Geometría, discretización y deformación del modelo de elementos finitos para el ensayo T1 (Coelho, 2004). 


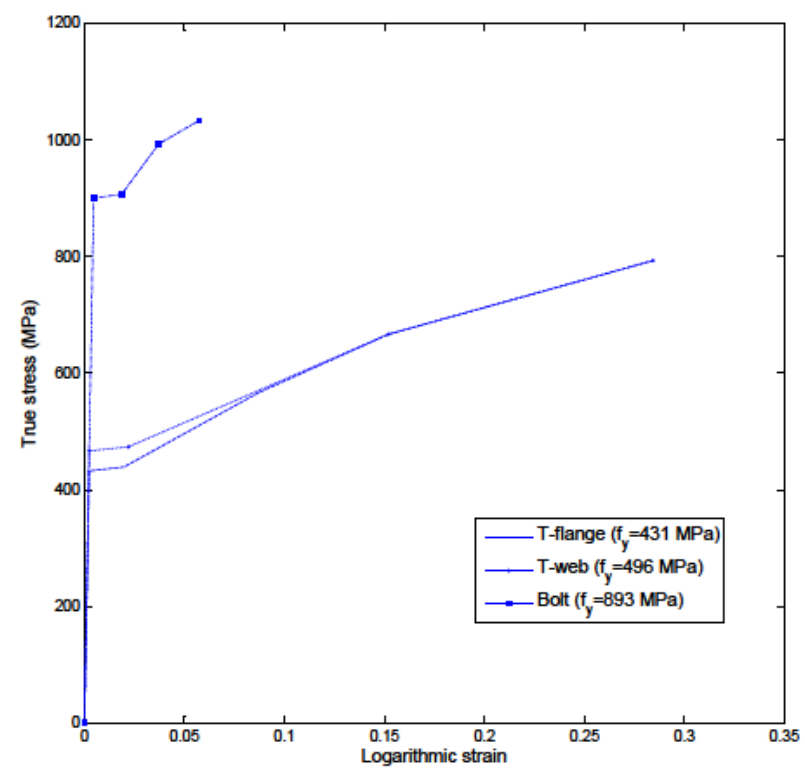

Figura 5.14: Problem TCPI-4. Curva tensión-deformación real en escala logarítmica del acero.

Criterio de fallo. La carga última de la conexión viene determinada por dos posibles modos de fallo: (1) el fallo del perfil como consecuencia de una excesiva deformación plástica y (2) el fallo del tornillo a tracción. En ambas situaciones se puede asumir (Girao Coelho et al., 2006) que la fractura se inicia cuando el valor medio de la deformación principal máxima en la sección crítica $\left(\varepsilon_{11, a v}\right)$ alcanza la deformación última $\left(\varepsilon_{u}\right)$. La capacidad de deformación de los tornillos $(5 \%$ $6 \%$ ) es considerablemente inferior a la del perfil (25\% - 30\%). Por este motivo, es probable que el fallo global de la conexión esté gobernado por la fractura del tornillo. Los tres mecanismos de fallo del tornillo son (EN1993-1-8, 1993): (1) fallo a tracción, (2) punzonamiento de la cabeza contra la chapa y (3) punzonamiento de la tuerca contra la chapa. En el caso de tornillos de alta resistencia, el fallo a tracción es anterior al fallo por punzonamiento por condiciones de diseño (Swanson, 1999), y como consecuencia es el modo de fallo predominante.

Resolución. La respuesta no lineal de la conexión se obtiene con una estrategia de resolución implícita. El problema se resuelve mediante control de desplazamientos. En cada incremento las ecuaciones de equilibrio se resuelve mediante el método de Newton-Raphson.

Validación. El modelo numérico se valida con los resultados obtenidos en el ensayo experimental T1 (Girao Coelho et al., 2006). Para comparar ambos resultados se toma como referencia la curva carga-desplazamiento de la conexión. Las curvas se muestran en la Figura 5.16a. Existe un buen acuerdo entre los resultados numéricos y los experimentales. La capacidad de deformación de la conexión corresponde con el último punto de la curva obtenido mediante la aplicación del criterio de fallo explicado anteriormente. Bajo este criterio, el fallo 
5. Propagación de incertidumbre con métodos de reducción dimensional ...

\begin{tabular}{lllllll}
\hline Variable & $x^{\text {inf }}$ & $x_{0}$ & $x^{\text {sup }}$ & Distribución & Media & Des. estándar \\
\hline \hline$d$ & 11.73 & 12.00 & 12.00 & normal & 12 & 0.0218 \\
$b$ & 148.00 & 150.00 & 154.00 & normal & 150 & 0.4854 \\
$t_{f}$ & 9.20 & 10.70 & 13.20 & normal & 10.7 & 0.3236 \\
$e$ & 29.55 & 30.00 & 30.46 & normal & 30 & 0.0504 \\
\hline
\end{tabular}

Tabla 5.9: Problem TCPI-4. Distribución de probabilidad de las variables aleatorias.

de la conexión está gobernado por la fractura del tornillo, que se produce para un desplazamiento de $8.997 \mathrm{~mm}$ y una carga de $204.8 \mathrm{kN}$ (Figura 5.16b a 5.16c). Los resultados experimentales muestran que el modo de fallo se debe a una plastificación del tornillo junto con niveles elevados de plastificación del ala del perfil. El fallo se alcanza para una carga de $205.6 \mathrm{kN}$ y un desplazamiento de 9.371 $\mathrm{mm}$.

\subsubsection{Análisis estocástico}

Las variables aleatorias se seleccionan a partir de estudios previos (Neves et al., 2001). Estos estudios concluyen que las variables geométricas que tienen una mayor influencia en la resistencia última de la T-Stub son: el diámetro de los tornillos $(d)$, el espesor del ala del perfil en $\mathrm{T}\left(\mathrm{t}_{\mathrm{f}}\right)$, la distancia de los agujeros al borde del perfil en dirección $\mathrm{x}(e)$ y el ancho del componente en dirección $x(b)$. Las variables aleatorias se muestran en la Figura 5.13a. La dispersión de las variables se establece en función de las dimensiones y las tolerancias dadas por las normas EN10034 y ISO4014 (Tabla 5.9). Las variables aleatorias están caracterizadas por una distribución normal truncada, donde la media y la desviación estándar vienen dadas por: $\mu=\left(x^{\text {sup }}+x^{\text {inf }}\right) / 2$ y $\sigma=\left(x^{\text {sup }}-x^{\text {inf }}\right) /(2 \times 3.09)$, siendo $x^{\text {sup }}$ y $x^{\text {inf }}$ los límites superior e inferior, respectivamente.

Se estiman los cuatro primeros momentos estadísticos de la resistencia última de la conexión utilizando reducciones de primer y segundo orden. Para la reducción de primer orden se utilizan $N_{1}=4$ evaluaciones, mientras que para la de segundo orden se utilizan $N_{1}=4$ y $N_{2}=8$. Los resultados se comparan con los obtenidos mediante una simulación MonteCarlo de 5000 puntos. La tabla 5.10 recoge los errores cometidos y el número de evaluaciones total con cada uno de los métodos. La descomposición unidimensional proporciona una estimación de los dos primeros momentos estadísticos con un error inferior al $4 \%$.

Los cuatro primeros momentos estadísticos se utilizan para reconstruir la distribución de probabilidad de la resistencia última de la conexión mediante un sistema de Pearson. Las distribuciones resultantes se muestran en la Figura 5.16. En función de la aplicación final (DOR o DOF), la utilización de una descomposición unidimensional puede resultar la solución más eficiente, ya que proporciona un error en torno al $4 \%$ para los dos primeros momentos estadísticos, con un número reducido de evaluaciones. 


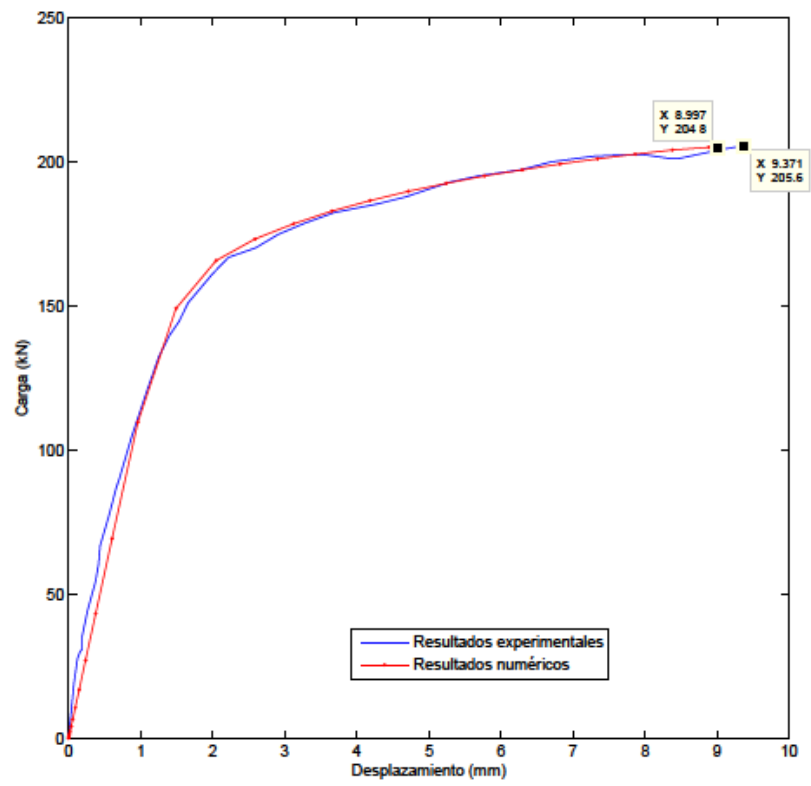

(a)

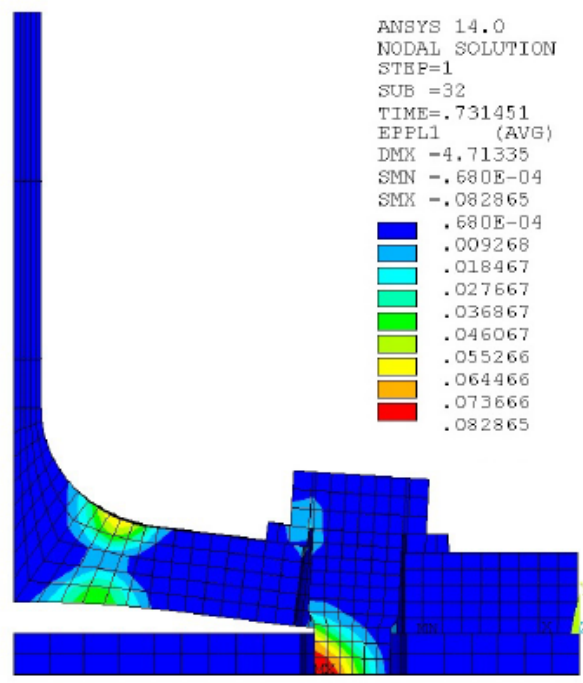

(b)

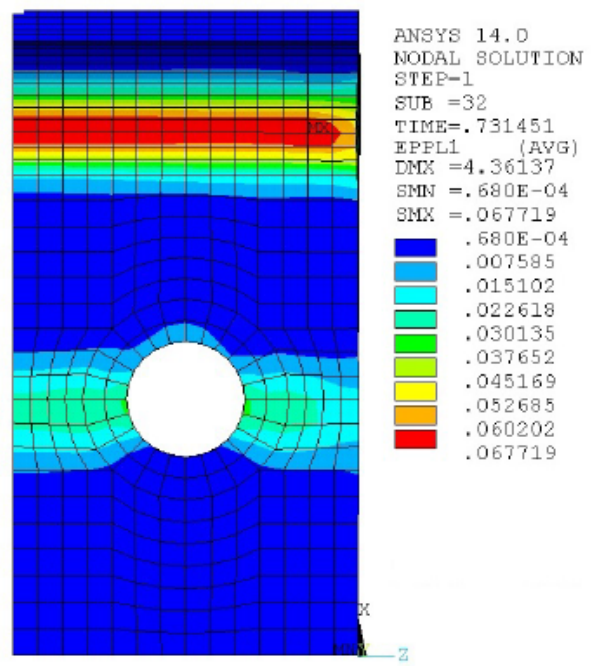

(c)

Figura 5.15: Problem TCPI-4. (a) Curva carga-desplazamiento; (b) deformación principal máxima; (c) detalle de la deformación principal máxima en el ala del perfil. 


\begin{tabular}{llll}
\hline & $\begin{array}{l}\text { MC } \\
\text { (Ref.) }\end{array}$ & $\begin{array}{l}\text { EKDRM } \\
(S=1) \\
N_{1}=4\end{array}$ & $\begin{array}{l}\text { EKDRM } \\
(S=2) \\
N_{1}=4 ; N_{1}=8\end{array}$ \\
\hline \hline Media $^{(1)}$ & 205.35567 & 205.86063 & 205.73079 \\
Des. Est. $^{(2)}$ & 4.13749 & 3.99472 & 4.19346 \\
Asimetria $^{(3)}$ & -1.73582 & -1.29296 & -1.69935 \\
Curtosis $^{(4)}$ & 9.84268 & 8.04817 & 8.66826 \\
(1) Error (\%) $^{(}-$ & -0.246 & -0.183 \\
(2) Error (\%) $^{2}-$ & 3.450 & -1.353 \\
${ }^{(3)}$ Error (\%) & - & 25.513 & 2.101 \\
${ }^{(4)}$ Error (\%) & - & 18.232 & 11.931 \\
$N_{\text {evals. }}$ & 5000 & 17 & 65 \\
\hline
\end{tabular}

Tabla 5.10: Problem TCPI-4. Momentos estadísticos de la función de la resistencia última obtenidos mediante descomposición unidimensionales y bidimensional.

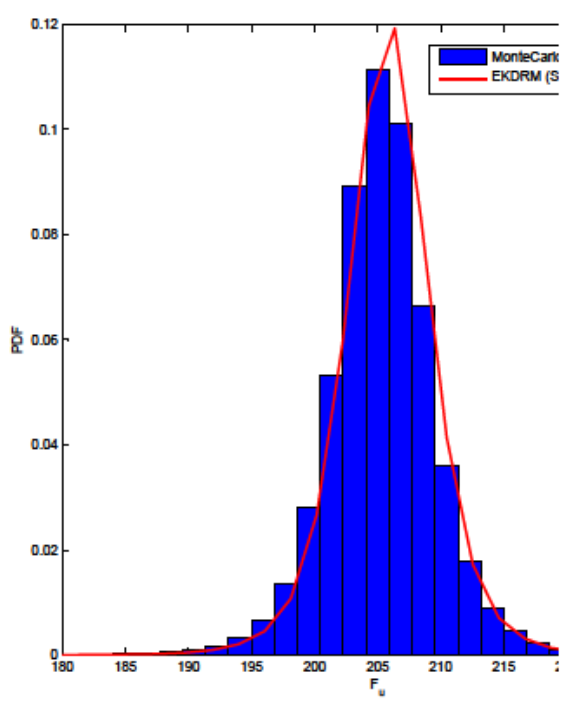

(a)

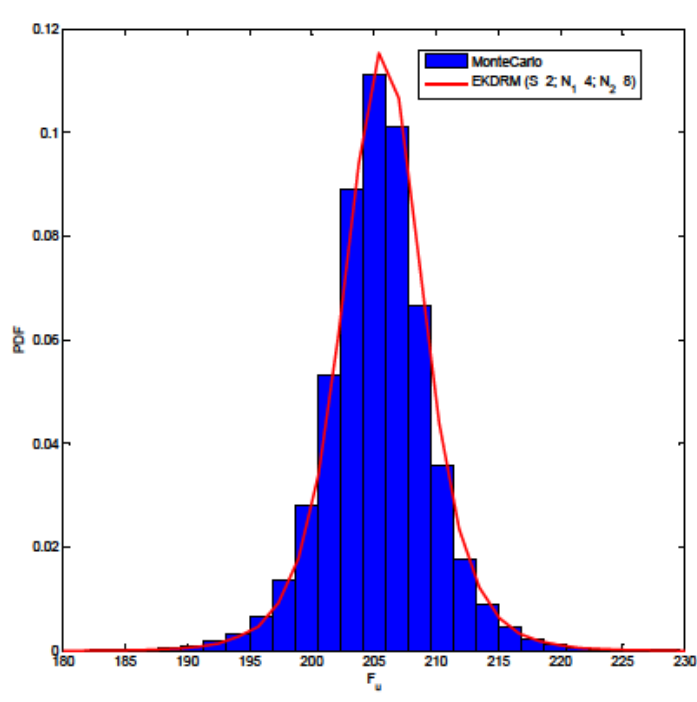

(b)

Figura 5.16: Problem TCPI-4. Función de densidad de probabilidad de la carga última del T-Stub. (a) Resultados para una reducción unidimensional, (b) resultados para una reducción bidimesional. 


\subsubsection{Ejemplo TCPI-5}

\subsubsection{Introducción}

Las láminas de pared delgada, metálicas o de material compuesto, son un tipo estructural ampliamente utilizado en aplicaciones de sectores como el aeronáutico o el espacial. Esta tipología estructural resulta muy sensible a imperfecciones geométricas, pudiendo dar lugar a cargas críticas mucho menores que las obtenidas para la configuración "perfecta" de la lámina (Budiansky y Hutchinson, 2006; Hutchinson y Amazigo, 1967). Además de imperfecciones geométricas, las láminas también pueden resultar muy sensibles a variaciones en otras propiedades como el material o el espesor. Por esta razón, incorporar información acerca de la imperfección real de la estructura resulta importante para obtener diseños óptimos más robustos y fiables.

Lo ideal es analizar el comportamiento de la lámina con la imperfección geométrica existente en la realidad. La forma y magnitud de la imperfección guarda una estrecha relación con el proceso de fabricación, el tipo de proceso y la calidad. Conociendo dicho proceso es posible estimar a priori la forma de la imperfección. Sin embargo, no siempre se dispone de información suficiente para realizar dicha estimación. Por esta razón, es práctica habitual considerar ciertas hipótesis para modelizar la imperfección, hipótesis que son verificadas a posteriori tras la fabricación. En este sentido, suele ser habitual utilizar los autovectores asociados con los primeros autovalores de la lámina. En estos casos, la imperfección se modeliza como una suma ponderada de dichos autovalores. Esta hipótesis resulta una solución de menor coste que utilizar datos reales a través de ensayos experimentales. Para un valor de amplitud definido, si las imperfecciones en la estructura real se asemejan a los autovectores o una combinaciones de estos, la reducción en la carga de pandeo será la mayor posible. Sin embargo, si las imperfecciones no se asemejan a los autovectores, la carga de pandeo calculada será menor que la real, produciendo un diseño conservador.

Autores como Faulkner y Das (1990),Groen y Kaminski (1996), Bourinet et al. (2000), Schenk y Schuëller (2003), Stefanou y Papadrakakis (2004), Fyllingen et al. (2007) y Lönn et al. (2010) proponen la utilización de una formulación probabilista para el diseño de láminas imperfectas. En este caso se genera un modelo probabilista de la imperfección geométrica en el espacio función del proceso de fabricación adoptado.

En este ejemplo, se aborda el problema de propagación de incertidumbre sobre un modelo continuo en el que existe una variación espacial de la incertidumbre. Se consideran variaciones de las propiedades del material (módulo de Young) y en el espesor de la lámina. Ambos parámetros aleatorios están correlacionados en el espacio y, siguiendo la línea de otros autores como Choi y Noh (2000); Dubourg et al. (2011); Graham y Siragy (2001); Stefanou (2009); Stefanou y Papadrakakis (2004), pueden ser descritos mediante dos campos aleatorios Gaussianos bidimensionales. 


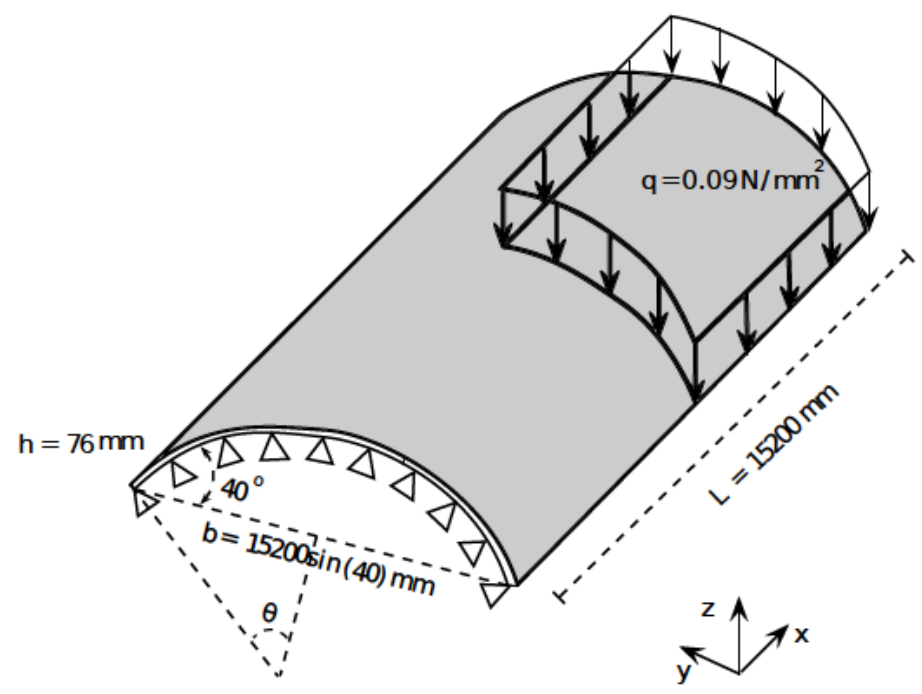

Figura 5.17: Problema Scordelis-Lo shell roof (I). Geometría de la lámina cilíndrica Scordelis-Lo Scordelis y Lo (1961).

Lámina cilíndrica Scordelis-Lo. El ejemplo seleccionado es la lámina cilíndrica mostrada en la Figura 5.17. Este ejemplo es una adaptación del problema original estudiado por Scordelis y Lo (1961), donde se han modificado el estado de cargas y las propiedades del material. El problema ha sido tratado desde un punto de vista probabilista por otros autores como (Dubourg et al., 2011; Stefanou y Papadrakakis, 2004).

Las dimensiones de la lámina se muestran en la Figura 5.17. La lámina está sometida a una carga distribuida de $0.09 \mathrm{MPa}$ actuando sobre la superficie superior de la misma. Los bordes longitudinales de la lámina están libres, mientras que el desplazamiento radial de los bordes circunferenciales está impedido. La lámina se analiza en régimen estático lineal con el programa de elementos finitos ANSYS. El modelo de elementos finitos consta de 28x40 elementos SHELL181.

Modelización de las variaciones aleatorias en el espacio. Las propiedades del material (módulo de Young $E$ ) y el espesor de la lámina $(h)$ se modelan mediante dos campos aleatorios de tipo lognormal no correlacionados. Las propiedades de las distribuciones de tipo lognormal de $E$ y $h$ se muestran en la Tabla 5.11.

Los campos aleatorios de tipo lognormal se obtienen a partir de la transformación:

$$
F(x, \theta)=\exp \left(\lambda_{F}+\xi_{F} U_{F}(x, \theta)\right), \quad F=E, h,
$$




\begin{tabular}{|c|c|c|c|c|c|}
\hline Variable & Distribución & Media & COV & $\begin{array}{l}\text { parámetro de localización } \\
\lambda\end{array}$ & $\begin{array}{l}\text { parámetro de escala } \\
\xi\end{array}$ \\
\hline$E(\mathrm{MPa})$ & Lognormal & 200,000 & $3 \%$ & 12.2056 & 0.03 \\
\hline$h(\mathrm{~mm})$ & Lognormal & 76 & $5 \%$ & 4.3295 & 0.05 \\
\hline
\end{tabular}

Tabla 5.11: Problema Scordelis-Lo shell roof (I). Parámetros de los campos aleatorios lognormales de $E$ y $h$.

donde $U_{E}$ y $U_{h}$ son dos campos Gaussianos estándar independientes entre sí, y $\lambda_{F}$ y $\xi_{F}$ son los parámetros de localización y escala definidos en la Tabla 5.11.

Los campos Gaussianos estándar tienen media cero y desviación unitaria y siguen una función de correlación isotrópica exponencial cuadrática:

$$
C\left(x-x^{\prime}, \theta-\theta^{\prime}\right)=\exp \left[-\frac{\left(x-x^{\prime}\right)^{2}}{l^{2}}-\frac{\left(R \theta-R \theta^{\prime}\right)^{2}}{l^{2}}\right]
$$

donde $l$ es la longitud de correlación, que es igual en ambas direcciones del espacio. Para analizar la influencia de la longitud de correlación en los resultados, se resuelve el problema estocástico para diferentes valores de la misma (350, 3500, y $5000 \mathrm{~mm}$ ). Los campos aleatorios se discretizan utilizando una expansión de Karhunen-Loève truncada en el término $\mathrm{M}=30$. La Figura 5.18 muestra realizaciones del campo aleatorio del módulo de Young y del espesor para diferentes longitudes de correlación.

Propagación de la incertidumbre. Se estiman los momentos estadísticos del desplazamiento en dirección $z$ del punto central de la lámina, y de la tensión máxima de Von Mises. Se utiliza una reducción unidimensional con $N_{1}=6$ puntos. Los resultados se comparan con los obtenidos mediante simulación MonteCarlo con un tamaño de 10,000 evaluaciones. Dado que el error de la aproximación depende en gran medida de la variabilidad de la incertidumbre en los parámetros de entrada, se han estudiado tres casos variando la longitud de correlación: $l=350 \mathrm{~mm}, l=3500 \mathrm{~mm}$, y $l=5000 \mathrm{~mm}$. El número total de evaluaciones del modelo de simulación es 181.

Los errores cometidos se presentan en la tabla 5.12 para el desplazamiento $U_{z}$ y en la tabla 5.13 para la tensión de Von Mises máxima. Tal y como era de esperar, los mayores errores se obtienen cuando la longitud de correlación es mayor, puesto que es el que incluye una mayor incertidumbre de entrada en el problema. Estos errores están por debajo del $0.5 \%$ en media y desviación estándar, tanto para desplazamiento como para tensión. Para momentos estadísticos de mayor orden se aprecian errores mayores.

Los resultados presentados en las tablas 5.12 y 5.13 se muestran en la Figura 5.19. En esta figura se representa la función de densidad de probabilidad del desplazamiento $U_{z}$ para longitudes de correlación de $350 \mathrm{~mm}$ y de $3500 \mathrm{~mm}$. En 


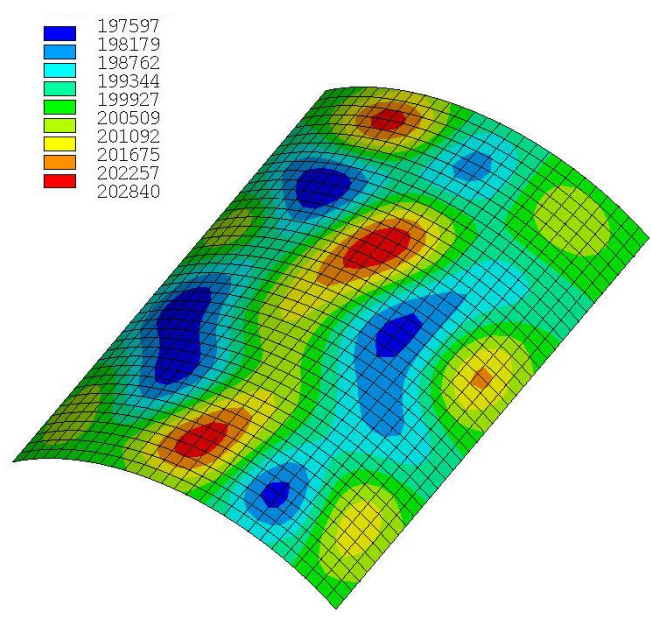

(a) Módulo de Young

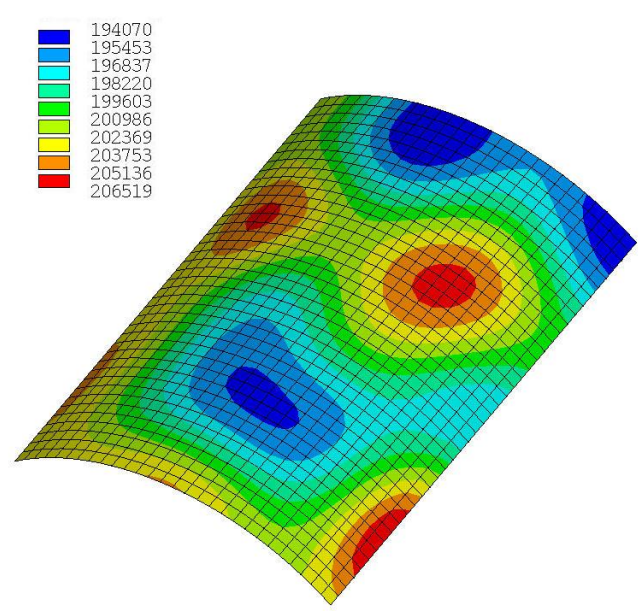

(c) Módulo de Young

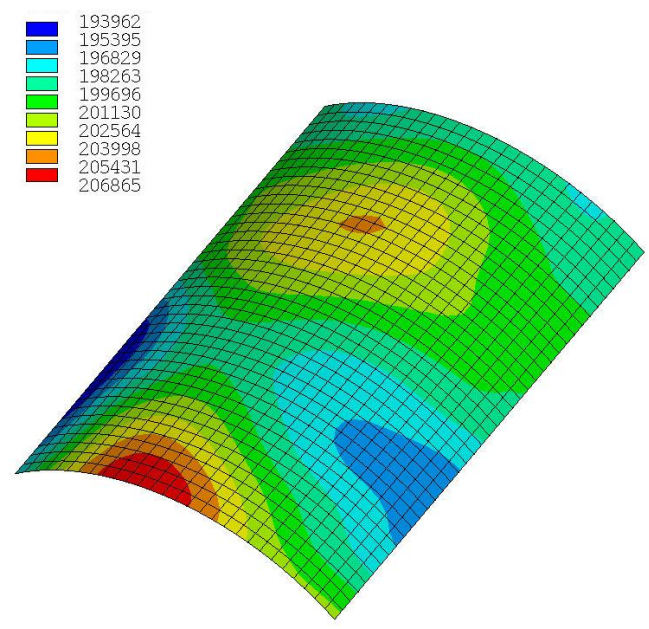

(e) Módulo de Young

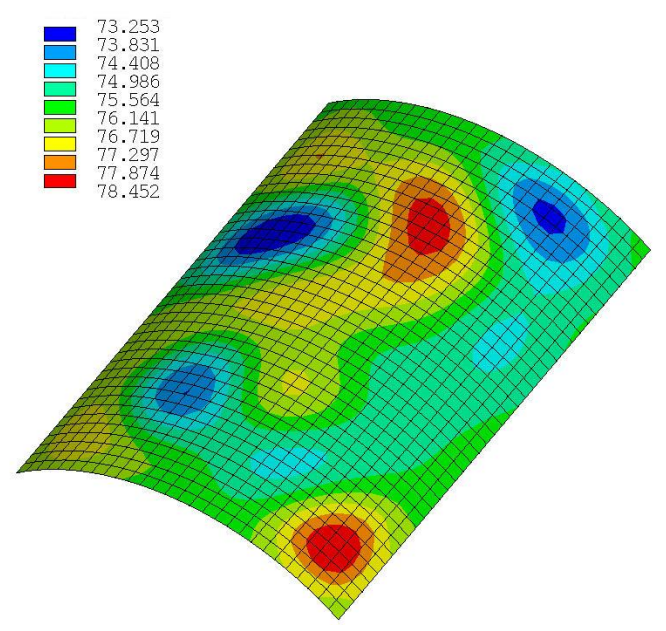

(b) Espesor

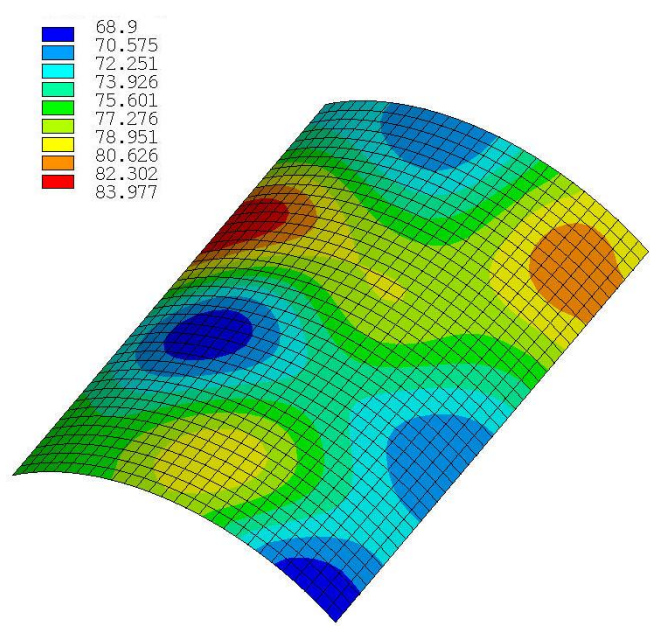

(d) Espesor

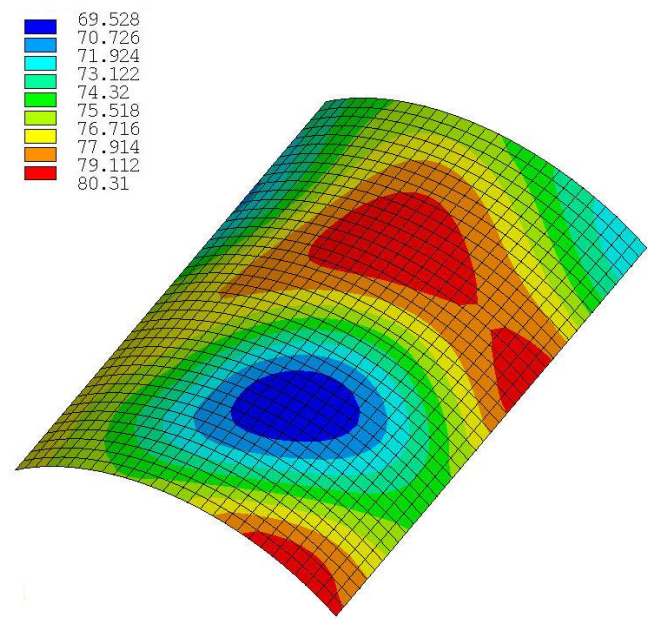

(f) Espesor

Figura 5.18: Problema Scordelis-Lo shell roof (I). Realizaciones de los campos aleatorios con diferentes factores de correlación (l): (a)-(b) $l=350 \mathrm{~mm}$; (b)-(c) $l=3500 \mathrm{~mm}$; (d)-(e) $l=5000 \mathrm{~mm}$. 


\begin{tabular}{|c|c|c|}
\hline & $\begin{array}{l}\text { MC } \\
\text { (Ref.) } \\
l=5000 / 3500 / 350 \mathrm{~mm}\end{array}$ & $\begin{array}{l}\text { EKDRM } \\
S=1 \\
n_{\text {doe } 1}=6 \\
l=5000 / 3500 / 350 \mathrm{~mm}\end{array}$ \\
\hline Media $^{(1)}$ & $24.255 / 24.294 / 24.310$ & $24.308 / 24.280 / 24.312$ \\
\hline Des. Est. ${ }^{(2)}$ & $3.236 / 2.629 / 0.324$ & $3.230 / 2.620 / 0.324$ \\
\hline Asimetría $^{(3)}$ & $0.155 / 0.108 / 0.004$ & $0.147 / 0.010 / 0.008$ \\
\hline Curtosis $^{(4)}$ & $3.096 / 3.112 / 3.067$ & $3.033 / 3.015 / 3.000$ \\
\hline (1) Error (\%) & - & $0.219 /-0.057 / 0.006$ \\
\hline (2) Error ( \%) & - & $-0.203 /-0.312 / 0.022$ \\
\hline (3) Error ( $\%)$ & - & $-5.079 /-7.399 / 93.708$ \\
\hline (4) Error ( $\%)$ & - & $-2.035 /-3.108 /-2.171$ \\
\hline$N_{\text {evals. }}$. & $1.00 \mathrm{E}+04$ & 181 \\
\hline
\end{tabular}

Tabla 5.12: Problema Scordelis-Lo shell roof (I). Momentos estadísticos del desplazamiento según el eje $z$ del punto central de la lámina.

\begin{tabular}{|c|c|c|}
\hline & $\begin{array}{l}\text { MC } \\
\text { (Ref.) } \\
l=5000 / 3500 / 350 \mathrm{~mm}\end{array}$ & $\begin{array}{l}\text { EKDRM } \\
S=1 \\
n_{\text {doe } 1}=6 \\
l=5000 / 3500 / 350 \mathrm{~mm}\end{array}$ \\
\hline Media $^{(1)}$ & $351.045 / 350.318 / 340.703$ & $350.742 / 349.509 / 340.770$ \\
\hline Des. Est. ${ }^{(2)}$ & $20.865 / 16.861 / 1.844$ & 20.906/16.893/1.857 \\
\hline Asimetría $^{(3)}$ & $0.202 / 0.172 / 0.027$ & $0.135 / 0.122 / 0.076$ \\
\hline Curtosis $^{(4)}$ & $3.149 / 3.101 / 2.989$ & $3.027 / 3.025 / 3.019$ \\
\hline (1) Error ( \%) & - & $-0.086 /-0.231 / 0.020$ \\
\hline (2) Error ( \%) & - & $0.197 / 0.188 / 0.690$ \\
\hline (3) Error ( $\%)$ & - & $-33.017 /-28.808 / 181.238$ \\
\hline (4) Error ( \%) & - & $-3.868 /-2.442 / 1.030$ \\
\hline$N_{\text {evals. }}$ & $1.00 \mathrm{E}+04$ & 181 \\
\hline
\end{tabular}

Tabla 5.13: Problema Scordelis-Lo shell roof (I). Momentos estadísticos de la tensión de Von Mises máxima de la lámina. 
ambos casos, la función de densidad de probabilidad reconstruida se aproxima con gran precisión a la obtenida mediante simulación MonteCarlo.

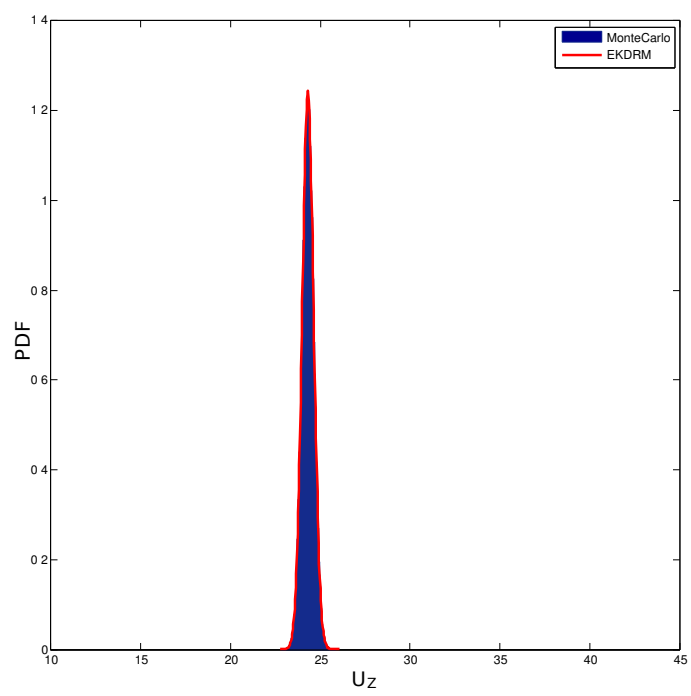

(a) $l=350 \mathrm{~mm}$

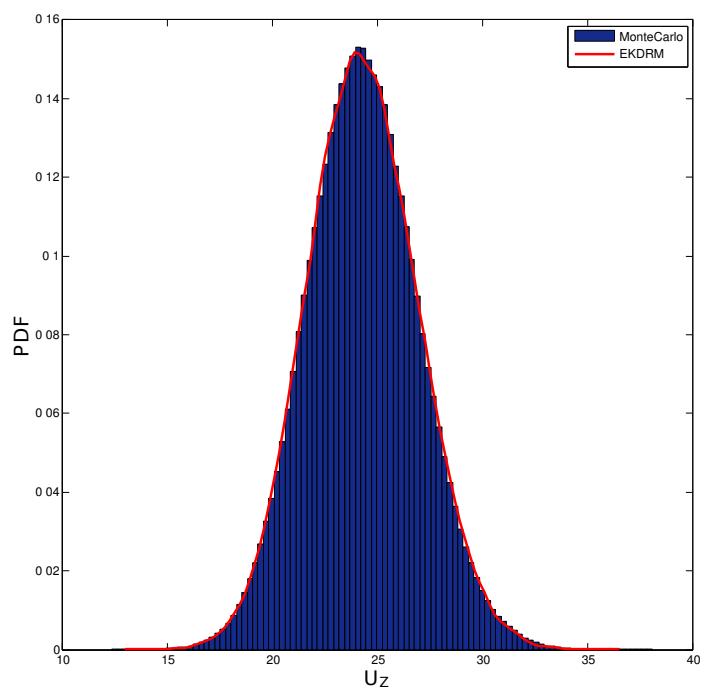

(b) $l=3500 \mathrm{~mm}$

Figura 5.19: Problema Scordelis-Lo shell roof (I). Función de densidad de probabilidad del desplazamiento vertical $U_{z}$.

Este ejemplo muestra la eficiencia y precisión del algoritmo EKDRM para la estimación de los momentos estadísticos en aplicaciones "reales" que incluyan modelos de elementos finitos y parámetros aleatorios con variabilidad espacial.

\subsection{Discusión y conclusiones}

En este capítulo se ha presentado el problema de propagación de incertidumbre en el contexto del diseño óptimo robusto. Se ha realizado una revisión de la literatura de los métodos existentes para la resolución de este problema. Esta revisión pone de manifiesto la necesidad de una mayor investigación en métodos de propagación de incertidumbre que traten con modelos de simulación de alto coste computacional, no intrusivos y/o de alta dimensionalidad. En este sentido, los métodos de reducción dimensional se muestran muy prometedores, ajustándose de forma adecuada a las características del problema que se aborda en esta tesis. En este capítulo se ha propuesto el algoritmo EKDRM para descomposiciones de orden superior a uno. El algoritmo propuesto incluye un criterio de selección del mejor ajuste (función de regresión y de correlación) independiente de la localización del diseño de experimentos. Además, se propone un criterio de colocación de los diseños de experimentos a partir de las raíces de los polinomios de Hermite. La precisión del algoritmo propuesto se ha validado mediante la resolución de problemas de diferentes características: baja y alta dimensionalidad, variables y campos aleatorios, alto grado de no linealidad e 
interacción entre variables, diferentes tipos de distribuciones de probabilidad y modelos de elementos finitos de alto coste computacional. Fruto del trabajo realizado se alcanzan las siguientes conclusiones:

1. La eficiencia y precisión del algoritmo está directamente relacionada con la aplicación final del proceso de propagación de incertidumbre. Si el objetivo final es un análisis de robustez de la respuesta, el algoritmo propuesto se muestra muy eficiente. Estima de forma precisa los dos primeros momentos estadísticos de la respuesta con un reducido número de evaluaciones del modelo de simulación. Si el objetivo final es un análisis de fiabilidad, a diferencia de otros métodos como la expansión en polinomios del caos, el algoritmo EKDRM no proporciona explícitamente una aproximación de la distribución de probabilidad. Sin embargo, es posible aproximar dicha distribución de forma empírica mediante un sistema de tipo Pearson, utilizando los cuatro primeros momentos estadísticos. En este caso la estimación de los momentos de tercer y cuarto orden requiere descomposiciones de orden superior a uno y un número de simulaciones mayor. En este contexto, el algoritmo EKDRM permite reducir el número de simulaciones en comparación con los métodos de reducción convencionales.

2. El algoritmo EKDRM es un método de integración aproximada enfocado a la estimación de momentos estadísticos de la respuesta aleatoria. Por este motivo, su precisión para la estimación de probabilidades depende de la capacidad de reconstruir la distribución de probabilidad original.

3. El algoritmo EKDRM no requiere el cálculo de derivadas parciales de la respuesta, como ocurre con métodos como la expansión de Taylor.

4. Desde un punto de vista computacional, el algoritmo EKDRM permite estimar los momentos estadísticos de la respuesta aleatoria con un menor número de evaluaciones que con otros métodos. Además, permite la explotación de arquitecturas de cálculo paralelo.

5. Las principales limitaciones del algoritmo propuesto vienen derivadas de las limitaciones del tipo de modelo de predicción utilizado y del sistema de reconstrucción de la distribución de probabilidad. La maldición de la dimensionalidad, asociada al modelo de predicción, limita el orden de la descomposición $(S)$ a valores $S \leq 10$. A pesar de esta limitación, en ingeniería no son muy frecuentes los problemas donde la interacción de diez o más variables tenga un peso considerable en la respuesta total del sistema. 

"It's no trick to get the answers when you have all the data. The trick is to get the answers when you only have half the data and half that is wrong and you don't know which half."

— William Thomson, Lord Kelvin (1824-1907)

\section{Diseño óptimo robusto utilizando meta-modelos estocásticos}

\section{Índice}

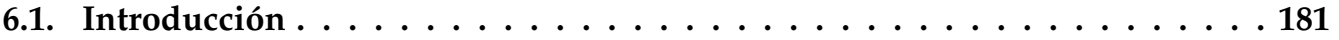

6.2. Meta-modelo estocástico utilizando modelos Kriging y métodos de reducción dimensional . . . . . . . . . . . . . . . . 190

6.3. Aplicación al problema multi-objetivo de diseño óptimo robusto . . . . . 193

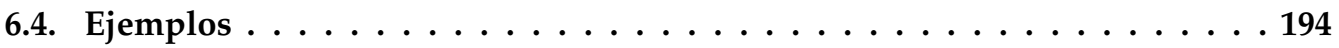

6.5. Discusión y conclusiones . . . . . . . . . . . . . . . . . 229 



\subsection{Introducción}

El problema de optimización bajo incertidumbre, y en particular el de diseño óptimo robusto, es un problema de una elevada exigencia computacional como consecuencia del acoplamiento entre el proceso de propagación de incertidumbre y el de optimización. Dicho acoplamiento puede transformar el problema en inabordable cuando se aplica a problemas estructurales de gran escala. En estos problemas, el coste computacional del algoritmo de optimización es muy inferior al coste de la simulación, por lo que es necesario reducir el número de evaluaciones. En este contexto resulta inevitable la utilización de técnicas de aproximación que sustituyan al modelo de simulación de alto coste computacional.

En los capítulos anteriores se abordaron problemas directamente relacionados con el proceso de diseño óptimo robusto como son: la creación de un modelo de predicción, la resolución eficiente del problema de propagación de incertidumbre, y la resolución eficiente del problema de optimización multi-objetivo utilizando modelos de predicción Gaussianos. En este capítulo se propone una estrategia para la resolución del problema de DOR basada en un meta-modelo estocástico no-intrusivo. Este meta-modelo utiliza modelos Kriging (Capítulo 3) junto con métodos de reducción dimensional (Capítulo 5) para aproximar la respuesta aleatoria del sistema estructural en el dominio de diseño. De esta forma se reduce considerablemente el coste computacional del proceso de optimización. La incertidumbre epistémica introducida por el meta-modelo se gestiona utilizando un algoritmo de optimización multi-objetivo basado en análisis Bayessiano (Capítulo 4). Como resultado se obtiene un conjunto de soluciones óptimas de Pareto del problema de DOR con un mínimo número de evaluaciones del modelo de simulación.

El resto de la sección presenta la formulación del problema de optimización bajo incertidumbre con meta-modelos, junto con una revisión de los diferentes enfoques existentes en la literatura. La sección 6.2 presenta las etapas de creación del meta-modelo estocástico. La sección 6.3 aplica el meta-modelo estocástico propuesto a la resolución del problema de diseño óptimo robusto con restricciones de probabilidad. La sección 6.4 recoge los resultados de la validación numérica de la metodología propuesta. Esta validación consta de la resolución tanto de ejemplos analíticos y como de modelos estructurales discretos y continuos. Finalmente la sección 6.5 presenta una discusión de la metodología propuesta así como las conclusiones del capítulo.

\subsubsection{Formulación del problema}

Uno de los principales problemas que se tratan cuando se aborda un problema de optimización bajo incertidumbre es el elevado coste computacional asociado al acoplamiento de los procesos de propagación de incertidumbre y de optimización 


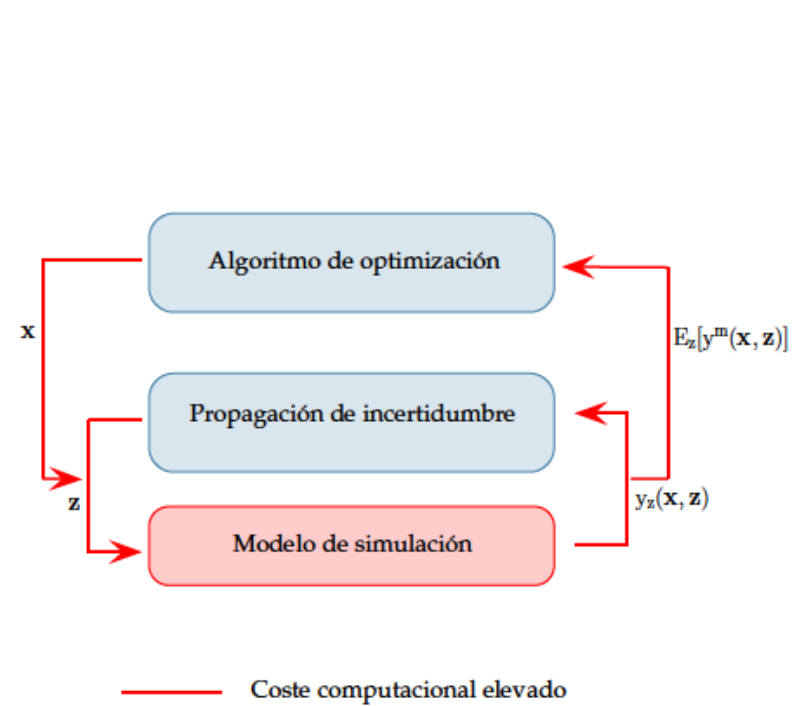

(a)

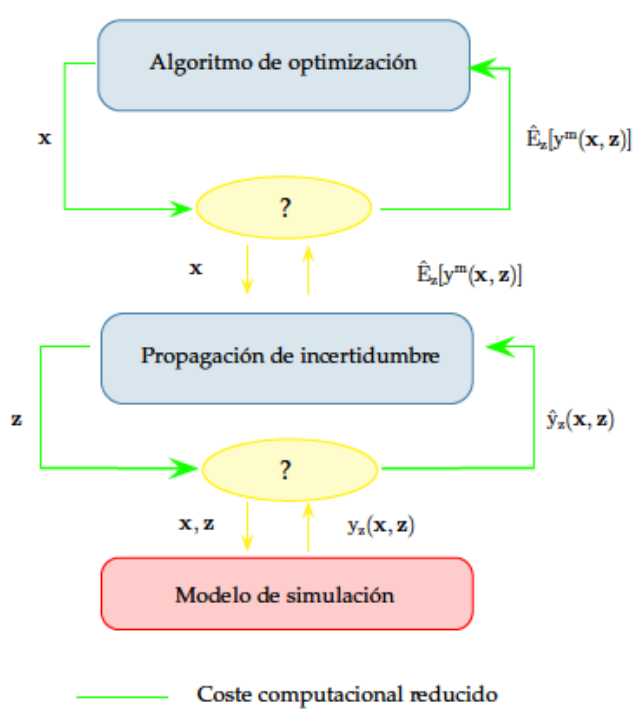

(b)

Figura 6.1: Diagrama de flujo del problema de optimización bajo incertidumbre. (a) Enfoque anidado convencional; (b) contexto del problema.

(Figura 6.1a). Este problema se ve amplificado cuando se trabaja con problemas estructurales de gran escala para los cuales el coste computacional del algoritmo de optimización es despreciable en comparación con el del modelo de simulación.

En el caso del diseño óptimo fiable el proceso de propagación de incertidumbre implica la estimación del punto más probable de fallo y la estimación de probabilidades de fallo de la función de estado límite en los extremos de la distribución. En el caso del diseño óptimo robusto, el problema de propagación de incertidumbre es menos exigente desde el punto de vista computacional. Requiere la estimación de los dos primeros momentos estadísticos de la respuesta. Sin embargo, precisa la resolución de un problema de optimización multi-objetivo para encontrar un conjunto de soluciones de compromiso entre el diseño con mejor performance y el diseño con mayor robustez.

En este contexto resulta inevitable la utilización de técnicas de aproximación que actúen como soporte de etapas de alto coste computacional durante el proceso de optimización. Dichas técnicas de aproximación, conocidas como meta-modelos, han sido ampliamente utilizadas en la literatura como sustitutos de modelos de simulación en procesos de alto coste computacional, tales como: optimización global, optimización multi-objetivo, análisis estocástico, o análisis de sensibilidad global (Wan y Karniadakis, 2006). A pesar de su creciente aplicación para aproximar respuestas deterministas, el desarrollo de meta-modelos estocásticos capaces de representar la respuesta aleatoria en el espacio de diseño es un campo de reciente explotación (Coelho et al., 2011), y son escasos los trabajos en esta línea. 
El problema que se aborda en este capítulo consiste en como aproximar la respuesta aleatoria del sistema en el dominio de diseño, gestionando de forma eficiente la incertidumbre epistémica de la aproximación durante el proceso de optimización multi-objetivo.

\subsubsection{Revisión del estado del arte y perspectivas}

En esta sección se realiza una revisión del problema de diseño óptimo robusto con meta-modelos. Para ello, se presentan en primer lugar las diferentes formulaciones del problema de optimización bajo incertidumbre con meta-modelos. En segundo lugar, se presentan los trabajos que resuelven el problema de diseño óptimo robusto con meta-modelos, analizando en profundidad el tipo de meta-modelo utilizado así como la estrategia utilizada para incorporar la aproximación en el proceso de optimización.

\subsubsection{Formulaciones del problema de optimización bajo incertidumbre con meta-modelos}

Cuando se aborda el problema de optimización bajo incertidumbre, el enfoque más directo y conceptualmente más simple es anidar los procesos de propagación de incertidumbre y de optimización tal y como muestra la Figura 6.2a. De este modo, para cada iteración del algoritmo de optimización se realiza una estimación completa de la incertidumbre en el modelo de simulación. Por lo general este enfoque resulta muy costoso desde el punto de vista computacional, especialmente si se utilizan modelos de simulación de alta fidelidad o métodos de simulación. Con el objetivo de aliviar este problema, la literatura presenta diferentes formulaciones del problema encaminadas a la separación de ambos procedimientos mediante meta-modelos (Eldred et al., 2002; Jin et al., 2001b). Atendiendo a la etapa en la que aparece el meta-modelo, las formulaciones existentes se pueden clasificar en tres categorías:

1. Formulación 1: anidada/meta-modelo (Figura 6.2b). La optimización se realiza directamente a partir de los resultados obtenidos del proceso de propagación de incertidumbre. Dicho proceso realiza evaluaciones sobre un meta-modelo que sustituye el modelo de simulación. Este Meta-modelo se puede ajustar en: (1) el dominio de diseño $\mathbf{x}$ y el dominio aleatorio $\mathbf{z}$, o (2) en el dominio aleatorio $\mathbf{z}$ para cada valor del vector de diseño x. Este último caso permite: (1) reducir el número de evaluaciones del modelo de simulación, ya que se reduce el espacio de ajuste y (2) una mejor calidad del ajuste al desacoplar el dominio de diseño y el dominio aleatorio.

2. Formulación 2: meta-modelo/anidada (Figura 6.2c). La optimización realiza evaluaciones sobre un meta-modelo que aproxima los resultados del proceso de propagación de incertidumbre en el dominio de diseño. La 
Figura 6.2c muestra el diagrama de flujo de esta formulación. En este caso se consigue desanidar los procesos de propagación de incertidumbre y de optimización mediante la utilización del meta-modelo. Sin embargo, el proceso de propagación de incertidumbre sigue anidado con el modelo de simulación. En las Figuras 6.2a a 6.2d las entidades obtenidas de forma aproximada y por lo tanto sometidas a incertidumbre epistémica son representadas con el símbolo $\hat{\bullet}$.

3. Formulación 3: meta-modelo/anidada/meta-modelo (Figura 6.2d). En este caso se utilizan dos meta-modelos. En primer lugar, la optimización realiza evaluaciones sobre un primer meta-modelo que aproxima las medidas estadísticas en el dominio de diseño $\mathbf{x}$. En segundo lugar, el proceso de propagación de incertidumbre realiza evaluaciones sobre un segundo meta-modelo que sustituye al modelo de simulación en el dominio de diseño $\mathbf{x}$ y aleatorio $\mathbf{z}$.

Las formulaciones presentadas en la Figura 6.2 hacen referencia al problema de optimización bajo incertidumbre con meta-modelos y por lo tanto se pueden utilizar en el contexto de diseño óptimo robusto o diseño óptimo fiable.

La mayor parte de los trabajos en el campo de la optimización bajo incertidumbre utilizando técnicas de meta-modelos pertenecen al campo del diseño óptimo fiable. En este campo, la búsqueda del punto de fallo más probable, junto con la estimación de la probabilidad de fallo, hacen imprescindible la utilización de meta-modelos para su aplicación a casos reales. En el caso del diseño óptimo robusto, el proceso de propagación de incertidumbre no resulta tan costoso desde el punto de vista computacional, puesto que requiere únicamente de la estimación de momentos estadísticos de bajo orden. Sin embargo en los últimos años, la utilización de modelos de simulación de alta fidelidad, la formulación multi-objetivo del problema, y el interés en formulación mixtas con restricciones de probabilidad, han motivado trabajos en el campo del diseño óptimo robusto que tienen como base la utilización de técnicas de aproximación.

\subsubsection{Diseño óptimo robusto con meta-modelos}

Como se discutió con anterioridad, la formulación 0 (Figura 6.2a) representa el enfoque más directo y conceptualmente más simple del problema de diseño óptimo robusto. Una revisión de los trabajos que abordan el problema de DOR en el campo de optimización estructural se presentó en la Sección 2.5. Esta forma de abordar el problema dificulta su aplicación a problemas con modelos de simulación de alta fidelidad. En este contexto, la utilización de métodos eficientes de propagación de incertidumbre es fundamental para reducir el coste computacional del problema. Autores como Huang y Du (2007) o Beyer y Sendhoff (2007) realizan una comparación de diferentes métodos para analizar la robustez en términos de precisión, eficiencia y fiabilidad. 


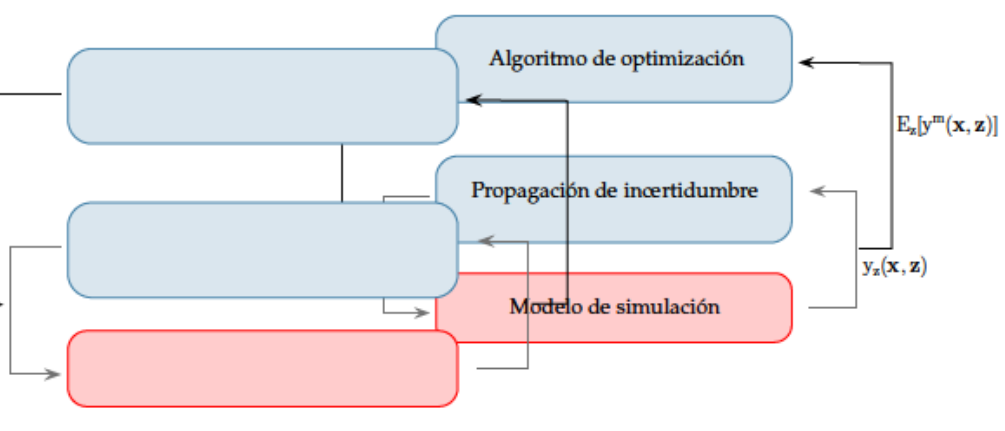

(a) Formulación 0 .

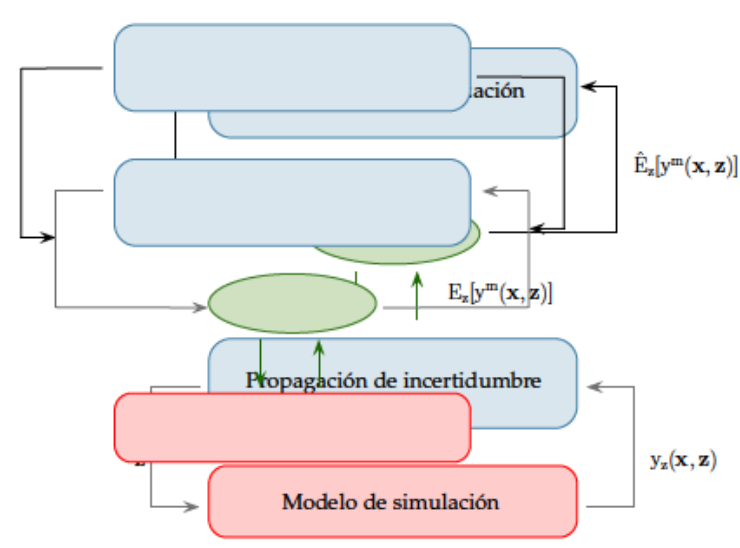

(c) Formulación 2.

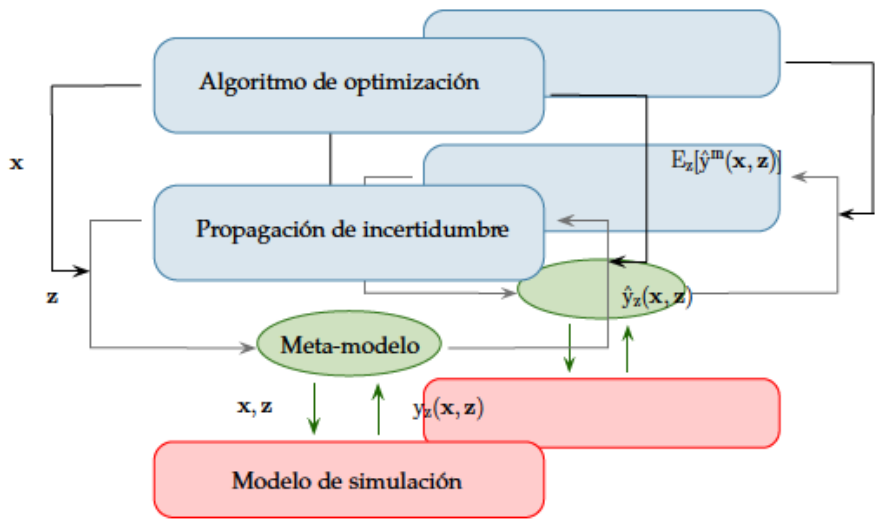

(b) Formulación 1.

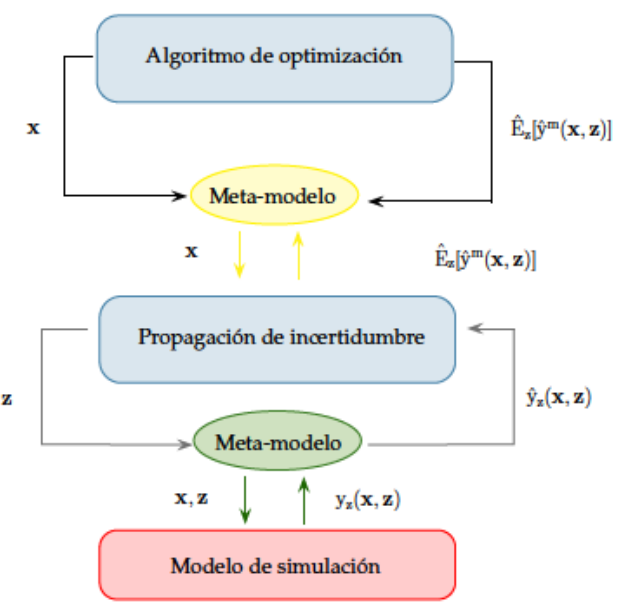

(d) Formulación 3.

Figura 6.2: Formulaciones del problema de optimización bajo incertidumbre con meta-modelos. (a) Anidada; (b) anidada/meta-modelo; (c) meta-modelo/anidada; (d) meta-modelo/anidada/meta-modelo.

En esta sección se presenta una revisión de la literatura cuyo alcance se limita a trabajos que abordan el problema de diseño óptimo robusto utilizando meta-modelos para reducir el coste computacional del proceso de propagación de incertidumbre, de la optimización o de ambos simultáneamente. Este criterio se utiliza para clasificar los trabajos en tres categorías.

En una primera categoría se encuentran aquellos trabajos que utilizan meta-modelos como sustitutos del modelo de simulación en el dominio aleatorio. Los meta-modelos actúan como soporte del proceso de propagación de incertidumbre, proporcionando evaluaciones con un reducido coste computacional. Guedri et al. (2009) utilizan una superficie de respuesta estocástica basada en una expansión en polinomios del caos. El problema de optimización 
multi-objetivo lo resuelven mediante el algoritmo NSGA-II. Xiang (2010) propone una regresión utilizando máquinas de soporte vectorial como sustituto del modelo de simulación. Estiman los dos primeros momentos estadísticos mediante una expansión en series de Taylor. Resuelve el problema multi-objetivo mediante una escalarización previa. Lagaros et al. (2007) y Papadrakakis y Plevris (2005) utilizan redes neuronales para aproximar la respuesta determinista del modelo de simulación. Estiman los dos primeros momentos estadísticos mediante una simulación MonteCarlo sobre el meta-modelo. Resuelven el problema multi-objetivo mediante una escalarización utilizando la función aumentada de Tchebycheff. den Boogaard et al. (2007) presentan una estrategia para el diseño óptimo robusto de procesos de conformado de metales mediante hidroforming. La estrategia consta de una modelización mediante FEM, una reducción del espacio de diseño mediante screening y una simulación MonteCarlo asistida por modelos Kriging para obtener los momentos estadísticos de la respuesta.

En una segunda categoría se encuentran las estrategias que utilizan un meta-modelo para aproximar el resultado del proceso de propagación de incertidumbre. En esta línea, Choi et al. (2008) utilizan superficies de respuesta polinómicas para aproximar la media y la desviación estándar obtenidas mediante métodos de reducción de dimensión. Lönn et al. (2010) estudian el problema de diseño óptimo robusto de perfiles tubulares frente a impacto. Evalúan los dos primeros momentos estadísticos utilizando métodos de simulación. Posteriormente, utilizan una red neuronal para aproximar los momentos estadísticos en el dominio aleatorio y de diseño. Resuelven el problema multi-objetivo mediante una suma ponderada. Utilizan el intervalo de confianza de la estimación de los momentos estadísticos como criterio de actualización. Coelho et al. (2011) proponen un meta-modelo jerárquico en el que utilizan técnicas de mínimos cuadrados móviles para aproximar los coeficientes de una expansión de polinomios del caos. Aplican el meta- modelo propuesto a la resolución del problema de diseño óptimo robusto multi-objetivo. Coelho y Bouillard (2011) extienden el trabajo anterior utilizando modelos Kriging.

En la tercera categoría se encuentran aquellos métodos que, de acuerdo a la formulación 3, introducen técnicas de meta-modelos en las etapas de propagación de incertidumbre y optimización. Tienen sus orígenes en el método de superficie de respuesta dual propuesto por (Vining y Myers, 1990), que propone la utilización de un meta-modelo individual para la media y la desviación estándar. Aunque este método fue propuesto para aproximar experimentos físicos con replicaciones, estudios realizados con posterioridad (del Castillo, 2007; Dellino et al., 2010; Lee y Park, 2006) muestran su utilidad para la aproximación de modelos de simulación deterministas en el contexto del diseño óptimo robusto. El método de superficie de respuesta dual presenta las siguientes ventajas: (1) proporciona una estimación de la media y la desviación estándar en cada punto del espacio de diseño, (2) aporta información acerca de la influencia de las variables de diseño en los momentos estadísticos de la respuesta y (3) permite 
la utilización de técnicas de optimización global para la obtención del diseño óptimo. Miró-Quesada y del Castillo (2004) extienden el método a casos con incertidumbres tanto en variables como en parámetros. Posteriormente Lee y Park (2006) y Lee (2010) proponen la utilización de modelos Kriging como modelos de aproximación. Construyen un primer modelo que aproxima la respuesta del sistema en el dominio de diseño y en el dominio aleatorio de forma conjunta. Este modelo se utiliza para estimar los momentos estadísticos mediante simulaciones MonteCarlo con un bajo coste computacional. A partir de los resultados del proceso de propagación de incertidumbre crean dos modelos Kriging que aproximan a la media y a la desviación estándar en el dominio de diseño. Estos nuevos meta-modelos asisten al proceso de optimización proporcionando estimaciones de los momentos estadísticos con un bajo coste computacional. Lee (2010); Lee y Park (2006) justifican la elección de modelos Kriging por su capacidad para aproximar respuestas con un alto comportamiento no lineal, lo cual es habitual en el caso de la varianza. Esta elección está apoyada por estudios previos como los de Jin et al. (2003) que realizan un estudio comparativo con diferentes técnicas de aproximación. La metodología propuesta por Lee y Park (2006) plantea tres claras limitaciones: (1) incremento de la dimensionalidad del problema debido a la unión del dominio de diseño y del dominio aleatorio, (2) es necesario un post-proceso para garantizar la precisión de los resultados y (3) por lo general es difícil garantizar una buena precisión en la totalidad del espacio de diseño.

Tomando como referencia los trabajos de Lee y Park (2006), autores como Dellino (2008), Dellino et al. (2009b) y Dellino et al. (2009a) desarrollan una metodología basada en la visión de Taguchi del problema de diseño óptimo robusto. Utilizan modelos Kriging para aproximar los momentos estadísticos de la respuesta. Más recientemente, Dellino et al. (2010) abordan el problema mediante un modelo de regresión. Utilizan una regresión de segundo orden para aproximar los factores de control, una regresión de primer orden para aproximar los factores de ruido y un término que incluye la interacción de ambos factores. A partir de la superficie de respuesta derivan una expresión analítica de la media y la varianza. Dellino et al. (2010) utilizan técnicas de programación matemática para minimizar la media manteniendo la desviación por debajo de un umbral admisible. El valor de dicho umbral debe ser especificado a priori, lo cual no resulta fácil. Finalmente, estiman un intervalo de confianza de la solución robusta alcanzada utilizando técnicas de bootstraping.

Los trabajos de Dellino et al. (2010), Lee y Park (2006) y Lee (2010) abordan el problema de creación del meta-modelo desde un punto de vista global. En este caso la precisión del resultado final depende de conseguir un meta-modelo de alta precisión en todo el dominio de ajuste. Esto no siempre es posible o requiere un elevado número de evaluaciones, especialmente cuando el dominio de ajuste combina variables de diseño y aleatorias conjuntamente. En estos casos es inevitable la presencia de incertidumbre epistémica en el proceso. El trabajo de Jurecka et al. (2007) es un primer intento de incorporar dicha 
incertidumbre en el proceso de diseño óptimo robusto. Jurecka et al. (2007) ajustan un modelo de predicción Kriging en el dominio conjunto de diseño y aleatorio. Realizan una estimación de la esperanza y la varianza de la respuesta a partir del valor esperado de la predicción y de la incertidumbre epistémica del modelo. El meta-modelo se actualiza de forma iterativa de acuerdo con el algoritmo de optimización global EGO (Algoritmo 2). Aproximan el error cuadrático medio en cada punto del espacio de diseño de forma empírica como el valor medio de dicho error en el dominio aleatorio. El principal inconveniente de esta formulación es: (1) no elimina el problema de la alta dimensionalidad, (2) no incorpora restricciones de alto coste computacional y (3) el criterio de actualización propuesto se obtuvo de forma empírica y solo ha demostrado ser válido para la resolución del problema $\mathrm{MO}$ escalarizado con una suma ponderada. Más recientemente, Janusevskis et al. (2012) proponen un método que combina de forma simultánea el proceso de propagación de incertidumbre con la minimización de la media. Utilizan modelos Kriging para aproximar de forma conjunta la respuesta determinista del modelo de simulación en el dominio aleatorio y de diseño. A diferencia de Jurecka et al. (2007), Janusevskis et al. (2012) obtienen una expresión analítica de la predicción del valor esperado y de la varianza de dicha predicción. Esta información estadística se utiliza junto con el algoritmo de análisis bayesiano EGO para actualizar el modelo de predicción en la búsqueda del óptimo global. La metodología propuesta destaca por su eficiencia puesto que el número de evaluaciones es reducido. Sin embargo, está limitada por: (1) las variables aleatorias son de tipo gaussiano, (2) solo se minimiza la media, y (3) la dimensionalidad del problema debe ser baja. La precisión depende de la capacidad del modelo de predicción de capturar el comportamiento del simulador por medio de la función de correlación.

\subsubsection{Discusión y perspectivas}

Discusión. En las formulaciones 0 y 1, la utilización de algoritmos de optimización basados en gradiente puede generar problemas de convergencia como consecuencia del error sistemático del proceso de propagación de incertidumbre. En contraposición, las formulaciones 2 y 3 suavizan el error sistemático producido por la propagación de incertidumbre, facilitando así la convergencia.

Desde el punto de vista de la propagación de incertidumbre las formulaciones 1 y 3 permiten la utilización de métodos de simulación con un elevado número de evaluaciones. Sin embargo, la utilización de un meta-modelo en el dominio conjunto de diseño y aleatorio está condicionado en gran medida por la dimensionalidad del problema.

Para el caso de las formulaciones 2 y 3 la verificación de los resultados óptimos conseguidos es crítica, siendo recomendable la utilización de procesos adaptativos que tengan en cuenta la incertidumbre epistémica de la aproximación durante la optimización. 
Perspectivas. A pesar de la gran eficiencia mostrada por los métodos basados en la formulación 3, los trabajos desarrollados en esta línea ponen de manifiesto grandes carencias y limitan su aplicación a casos "reales". La gran dimensionalidad del espacio de entrada puede dificultar su aplicación a casos reales que involucren un elevado número de variables de diseño o aleatorias, como es el caso de problemas con campos aleatorios. La unión del dominio de diseño y del dominio aleatorio dificulta la gestión de la incertidumbre epistémica del meta-modelo.

Los métodos basados en la formulación 2 son, desde el punto de vista del autor de esta tesis, más prometedores a la hora de incorporar el meta-modelo en el problema. Estos métodos representan una solución de compromiso entre la eficiencia y la precisión. Por este motivo, la línea a seguir en este trabajo es la propuesta por autores como Coelho et al. (2011) basada en la creación de un meta-modelo estocástico que aproxime la respuesta aleatoria del sistema. En esta línea resulta imprescindible la utilización de métodos de propagación de incertidumbre eficientes junto con procedimientos que incorporen de forma precisa y eficiente la incertidumbre epistémica durante la optimización (Eldred et al., 2002; Swiler et al., 2005).

La estrategia propuesta en esta tesis se diferencia de otros trabajos existentes en la literatura en los siguiente puntos:

- Aporta un criterio de actualización basado en el valor esperado del hipervolumen dominado, que permite incorporar la incertidumbre epistémica del modelo de predicción en la búsqueda de nuevos candidatos a formar parte del frente de soluciones no-dominadas. Así mismo, incorpora la incertidumbre de las restricciones.

- Resuelve el problema de optimización multi-objetivo subyacente en el problema de diseño óptimo robusto sin una escalarización previa.

- Propone un meta-modelo estocástico que aproxima de forma eficiente la respuesta aleatoria del sistema en el dominio de diseño. Cuando se aplica al problema de diseño óptimo robusto, el meta-modelo estocástico proporciona estimaciones de los dos primeros momentos estadísticos con un menor número de evaluaciones que otros meta-modelos de estas características, tales como los basados en PCE. Este meta-modelo posibilita la obtención de momentos estadísticos de la respuesta así como de probabilidades, por lo que su aplicación abarca un abanico más amplio de problemas que incluyan restricciones de probabilidad. 


\subsection{Meta-modelo estocástico utilizando modelos Kriging y métodos de reducción dimensional}

El meta-modelo estocástico propuesto está basado en: (1) métodos de reducción dimensional para la estimación eficiente de los momentos estadísticos de la respuesta estructural, (2) modelos de predicción Kriging para interpolar dichos momentos en el dominio de diseño, y (3) reconstrucción de la distribución de probabilidad mediante sistemas de Pearson.

Se considera que la respuesta estructural $y=\mathscr{M}(\mathbf{x}, \mathbf{z})$ depende de un vector de variables aleatorias $\mathbf{z}$ definido en un espacio de probabilidad $\Omega$ y de un conjunto de variables de diseño deterministas $\mathbf{x}$. La respuesta aleatoria está completamente caracterizada por su función de densidad de probabilidad $f_{Y}(\mathbf{x})(y(\mathbf{x}))$, que por extensión depende de un vector de variables deterministas $\mathbf{x}$.

Dado un vector de diseño $\mathbf{x}^{*}$, el momento estadístico de orden $m$ se puede estimar mediante el algoritmo de reducción dimensional propuesto en el Capítulo 5:

$$
\mathbb{E}\left[y^{m}\left(\mathbf{x}^{*}, \mathbf{z}\right)\right]=\sum_{i=0}^{S}(-1)^{i}\left(\begin{array}{c}
N_{S}-S+i-1 \\
i
\end{array}\right) \times \sum_{k_{1}<k_{2}<\cdots<k_{S-i}} \mathbb{E}\left[\hat{y}^{m}\left(\mathbf{x}^{*}, z_{k_{1}}, z_{k_{2}}, \ldots, z_{k_{S-i}}\right)\right],
$$

donde $S$ es el orden de la reducción y $N_{S}$ el número de puntos evaluados para aproximar las respuestas de orden $S$.

La función de densidad de probabilidad se puede expresar en función de los cuatro primeros momentos estadísticos de la respuesta mediante la resolución de la ecuación diferencial (Pearson, 1895):

$$
\frac{1}{f_{Y}} \frac{d f_{Y}}{d z}=\frac{a z+b}{c+b z+d z^{2}}
$$

con:

$$
\begin{aligned}
a & =10 \kappa_{\hat{y}}-12 \delta_{\hat{y}}^{2}-18 \\
b & =\delta_{\hat{y}}\left(\kappa_{\hat{y}}+3\right) \\
c & =4 \kappa_{\hat{y}}-3 \delta_{\hat{y}^{\prime}}^{2} \\
d & =2 \kappa_{\hat{y}}-3 \kappa_{\hat{y}}^{2}-6,
\end{aligned}
$$

donde $z$ es una variable aleatoria normalizada, y $\delta_{\hat{y}}$ y $\kappa_{\hat{y}}$ son los momentos estadísticos centrados de tercer y cuarto orden. 
La idea propuesta en esta tesis es la creación de un meta-modelo estocástico que aproxime los cuatro primeros momentos estadísticos de la respuesta en el espacio de diseño. Estos meta-modelos proporcionan predicciones de bajo coste computacional y permiten reconstruir la distribución de probabilidad de la respuesta en el dominio de diseño. El proceso de creación de los meta-modelos se ilustra en el Algoritmo 5 y consta de tres etapas: (1) la obtención de observaciones en el dominio de diseño, (2) la estimación de los hiperparámetros del modelo de interpolación y (3) la reconstrucción de la distribución de probabilidad a partir de la predicción en un punto de diseño.

Obtención de observaciones en el dominio de diseño. Esta etapa consiste en la generación de un diseño de experimentos (DoE) en el dominio de las variables de diseño $\left\{\mathbf{x}^{(1)}, \ldots, \mathbf{x}^{(n d o e)}\right\}$. Las muestras se colocan en el dominio de forma uniforme utilizando criterios de muestreo basados en el llenado del espacio de diseño. En este trabajo se utiliza un Hypercubo Latino (Latin Hypercube Sampling, Dam et al. (2007)).

Para cada punto de la muestra se obtienen los cuatro primeros momentos estadísticos utilizando el Algoritmo 4 propuesto en el capítulo 5. De esta forma se garantiza la estimación de dichos momentos con el mínimo número de evaluaciones del modelo de simulación:

$$
\left\{\mathbf{x}^{(1)}, \ldots, \mathbf{x}^{(\text {ndoe })}\right\} \Rightarrow \mathbb{E}\left[y^{m}\left(\mathbf{x}^{(1)}, \ldots, \mathbf{x}^{(n d o e)}\right)\right], \forall m=1, \ldots, 4
$$

Estimación de los hiperparámetros del meta-modelo. La información obtenida en la etapa anterior se utiliza para estimar los hiperparámtros del meta-modelo que aproxima los momentos estadísticos en el dominio de diseño. El tipo de meta-modelo seleccionado para realizar el ajuste es el modelo Kriging (Capítulo $3)$.

Ilustración 6.1. En este ejemplo se ilustra el proceso de creación del meta-modelo estocástico de la función no líneal:

$$
Y(X)=\frac{X^{2}(2+\sin (2 X))}{4}
$$

donde $X$ es una variable aleatoria que sigue una distribución normal con desviación estándar 0.3 y una media $\mu_{X}$. La variable de diseño del problema es el valor medio $\mu_{X}$ que puede tomar valores en el rango $[1,7]$.

El diseño de experimentos está formado por 20 puntos uniformemente repartidos en el espacio de diseño con un Hipercubo Latino (McKay et al., 1979; Tang, 1993). Para cada uno de estos puntos se evalúan los cuatro primeros 


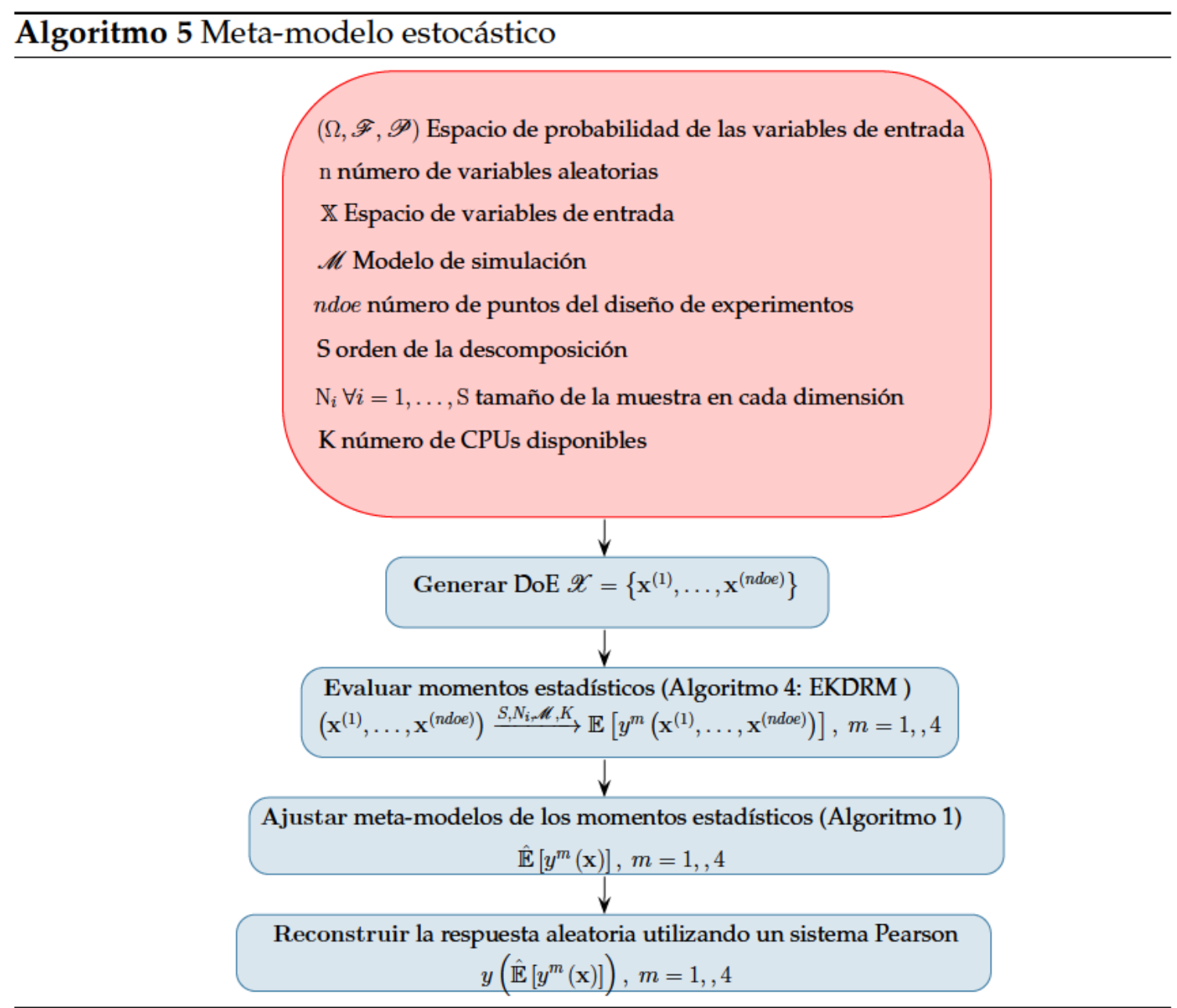

momentos estadísticos de la respuesta utilizando el algoritmo EKDRM con $S=1$ y $N_{1}=4$. Los resultados obtenidos se utilizan para ajustar los momentos estadísticos de la respuesta en el dominio de diseño. Las Figuras 6.3a y 6.3b muestran la interpolación en el espacio de diseño de los dos primeros momentos estadísticos.

La distribución de probabilidad se reconstruye utilizando un sistema de Pearson. Para ello se utiliza la función pearsrnd de MATLAB. Se obtienen los cuantiles 0.05 , 0.5, y 0.95 de la respuesta aleatoria. La Figura 6.3c muestra el valor de los cuantiles $0.05,0.5$, y 0.95 en el espacio de diseño obtenidos a partir de la función de densidad de probabilidad reconstruida. Los valores obtenidos se comparan con los resultantes de una simulación MonteCarlo de 1e5 puntos para 20 puntos en el dominio de diseño. 


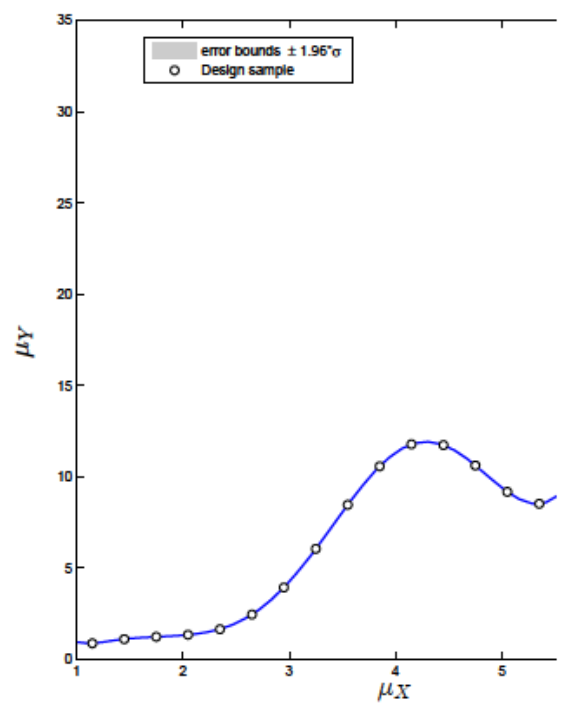

(a) $\hat{\mu}[Y(X)]$

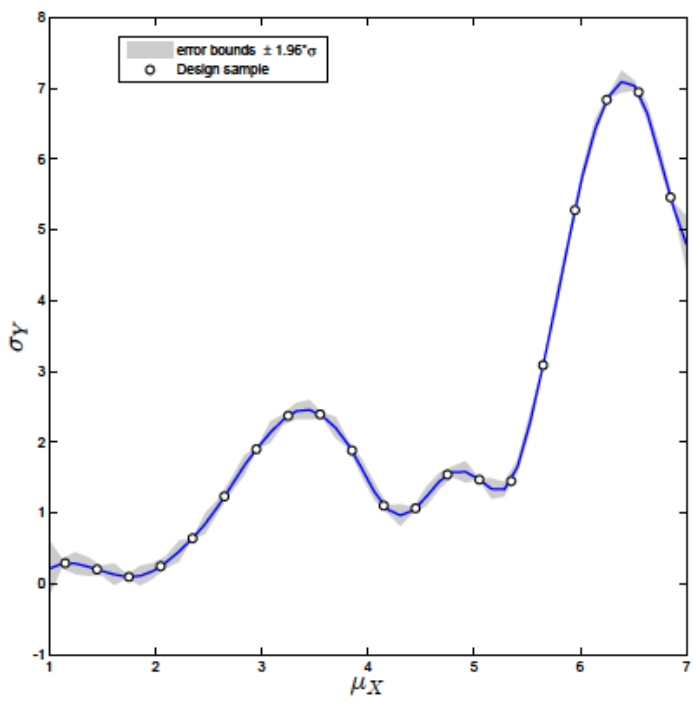

(b) $\hat{\sigma}[Y(X)]$

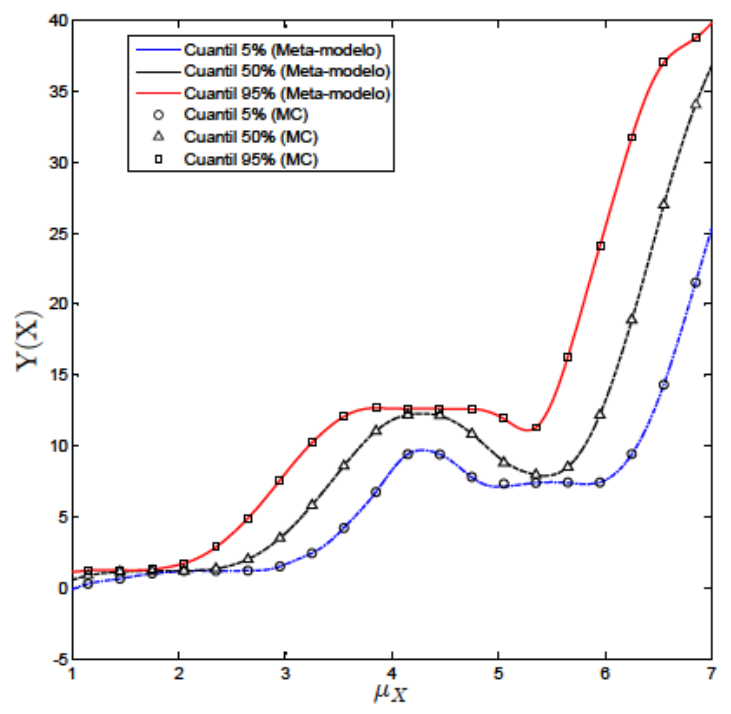

(c) $\hat{Q}_{Y}(0.95), \hat{Q}_{Y}(0.50), \hat{Q}_{Y}(0.05)$

Figura 6.3: Meta-modelos de los dos primeros momentos estadísticos y cuantiles de la función 6.8

\subsection{Aplicación al problema multi-objetivo de diseño óptimo robusto}

El meta-modelo estocástico obtenido en la sección 6.2 permite aproximar la respuesta aleatoria de la respuesta estructural en cada punto del dominio de diseño sin utilizar evaluaciones adicionales del modelo de simulación. Las predicciones del meta-modelo se pueden utilizar para la realización de: análisis de fiabilidad, análisis de sensibilidad, o junto con un algoritmo de optimización para la resolución de problemas de optimización bajo incertidumbre. 
En este trabajo se plantea la resolución del problema de diseño óptimo robusto. Tal y como se discute en el Capítulo 2, el problema DOR se formula como un problema multi-objetivo en el que se busca una solución de compromiso entre el diseño con mejor performance y el diseño menos sensible a incertidumbres. Este problema demanda un elevado coste computacional para su resolución, puesto que es un problema de carácter multi-objetivo que involucra momentos estadísticos de la respuesta estructural en su formulación. La idea propuesta en este trabajo es la de utilizar la información estadística (media de la predicción y varianza de la predicción) para encontrar de forma eficiente un conjunto de soluciones óptimas del problema MO. Para ello se utilizará el algoritmo EHV-EMO (Expected HyperVolume Efficient Multi-objective Optimization) propuesto en la Sección 4.4.

\subsection{Ejemplos}

La metodología propuesta se valida numéricamente mediante la resolución de problemas con funciones analíticas y con modelos de elementos finitos de estructuras discretas y continuas. Los problemas seleccionados para la validación se dividen en las siguientes tres categorías:

1. Categoría 1 (TCMO\#): aplicación al diseño óptimo robusto de problemas multi-objetivo con restricciones de fiabilidad.

2. Categoría 2 (TCSO\#): aplicación al diseño óptimo robusto de estructuras de nudos articulados.

3. Categoría 3: aplicación al diseño óptimo robusto de láminas con incertidumbre en geometría frente a pandeo.

Con la resolución de los problemas de validación propuestos se pretende: (1) estudiar la precisión y la eficiencia del algoritmo EHV-EMO en el contexto de diseño óptimo robusto, (2) estudiar la viabilidad de la metodología propuesta para la resolución de problemas de diseño óptimo robusto con restricciones de probabilidad y (3) comparar la precisión y la eficiencia de la metodología propuesta en comparación con formulaciones sin meta-modelos.

\subsubsection{Aplicación al diseño óptimo robusto de problemas multi-objetivo con restricciones de fiabilidad}

\subsubsection{Introducción}

En esta sección se aborda el problema de diseño óptimo robusto aplicado a problemas de optimización multi-objetivo (MORO; Multi-Objective Robust Optimization). Este problema ha sido tratado con anterioridad por otros autores como Deb y Gupta (2006), Gunawan y Azarm (2005), y Li et al. (2005). 


\begin{tabular}{cclll}
\hline TCMO\# & $\mathrm{n}$ & $\begin{array}{l}\text { Límites de } \\
\text { variables }\end{array}$ & $\begin{array}{l}\text { Funciones } \\
\text { objetivo }\end{array}$ & $\begin{array}{l}\text { Restricciones } \\
\text { de fiabilidad }\end{array}$ \\
\hline \hline 1 & 2 & $2 \leq x_{1} \leq 7$ & $f_{1}(\mathbf{x}, \xi)=-u_{1}-u_{2}^{2}$ & $\mathbb{P}\left[f_{3}(\mathbf{x}, \xi)=u_{1}+u_{2}-12 \leq 0\right] \geq P_{S}$ \\
& & $5 \leq x_{2} \leq 10$ & $f_{2}(\mathbf{x}, \xi)=-u_{1}^{2}-u_{2}$ & $\mathbb{P}\left[f_{4}(\mathbf{x}, \xi)=-u_{1}^{2}-10 u_{1}+u_{2}^{2}-16 u^{2}+80 \leq 0\right] \geq P_{S}$ \\
2 & 2 & $2 \leq x_{1} \leq 10$ & $f_{1}(\mathbf{x}, \xi)=-u_{1}^{2}-u_{2}$ & $\mathbb{P}\left[f_{3}(\mathbf{x}, \xi)=u_{1} / 6+u_{2}-13 / 2 \leq 0\right] \geq P_{S}$ \\
& & $0 \leq x_{2} \leq 10$ & $f_{2}(\mathbf{x}, \xi)=-0,5 u_{1}-u_{2}-1$ & $\mathbb{P}\left[f_{4}(\mathbf{x}, \xi)=u_{1} / 2+u_{2}-15 / 2 \leq 0\right] \geq P_{S}$ \\
& & & $\mathbb{P}\left[f_{5}(\mathbf{x}, \xi)=5 u_{1}+u_{2}-30 \leq 0\right] \geq P_{S}$ \\
& 2 & $-20 \leq x_{1} \leq 20$ & $f_{1}(\mathbf{x}, \xi)=2+\left(u_{1}-2\right)^{2}+\left(u_{2}-1\right)^{2}$ & $\mathbb{P}\left[f_{3}(\mathbf{x}, \xi)=u_{1}^{2}+u_{2}^{2}-225 \leq 0\right] \geq P_{S}$ \\
$4-5$ & 2 & $-20 \leq x_{2} \leq 20$ & $f_{2}(\mathbf{x}, \xi)=9 u_{1}-\left(u_{2}-1\right)^{2}$ & $\mathbb{P}\left[f_{4}(\mathbf{x}, \xi)=u_{1}-3 u_{2}+10 \leq 0\right] \geq P_{S}$ \\
& & $0 \leq x_{1} \leq \pi$ & $f_{1}(\mathbf{x}, \xi)=u_{1}$ & $\mathbb{P}\left[f_{3}(\mathbf{x}, \xi)=-u_{1}^{2}-u_{2}^{2}+1+0.1 \cos \left(16 \arctan \left(u_{1} / u_{2}\right)\right) \leq 0\right] \geq P_{S}$ \\
& $0 \leq x_{2} \leq \pi$ & $f_{2}(\mathbf{x}, \xi)=u_{2}$ & $\mathbb{P}\left[f_{4}(\mathbf{x}, \xi)=-1 / 2+\left(u_{1}-1 / 2\right)^{2}+\left(u_{2}-1 / 2\right)^{2} \leq 0\right] \geq P_{S}$ \\
\hline
\end{tabular}

Tabla 6.1: Formulación de los problemas TCMO\#.

Recientemente Shimoyama et al. (2009), Hu y Youn (2011) y Coelho et al. (2011) han propuesto la utilización de meta-modelos para la resolución del problema.

Entre los diferentes enfoques que tratan el problema MORO, se adopta la formulación propuesta por Mattson y Messac (2005):

$$
\begin{aligned}
& \min _{\mathbf{x}, \in \mathbb{R}^{\mathrm{n}}}\left\{\mu_{f_{1}(\mathbf{x}, \mathbf{z})}+k \sigma_{f_{1}(\mathbf{x}, \mathbf{z})}, \ldots, \mu_{f_{m}(\mathbf{x}, \mathbf{z})}+k \sigma_{f_{m}(\mathbf{x}, \mathbf{z})}\right\}, \\
& \text { s.a. } \mathbb{P}\left[g_{j}(\mathbf{x}, \mathbf{z}) \leq 0\right] \geq P_{S, j} j=1, \cdots, m_{i} \\
& \quad \mathbf{x}^{\text {mín }} \leq \mathbf{x} \leq \mathbf{x}^{\text {máx }},
\end{aligned}
$$

donde $\mathbf{z}$ es un vector de variables aleatorias, $\mu_{f_{i}}$ y $\sigma_{f_{i}}$ son la media y la desviación estándar de la función objetivo i-ésima.

Se estudiarán cinco benchmarks bajo la denominación TCMO\#. Los problemas están adaptados de estudios previos encontrados en la literatura. Todos ellos han sido utilizados como funciones test para la validación de algoritmos de optimización multi-objetivo deterministas. En particular los casos TCMO1-2 han sido tratados previamente por Veldhuizen y Lamont (1998. Air Force Inst. Technol.), mientras que los casos TCMO3-5 se recogen en Coello et al. (2002)). Todos los casos han sido transformados y adaptados para tratar las variables de diseño como variables aleatorias. Para ello las variables de diseño originales $x_{i}$ se transforman en variables de diseño aleatorias $u_{i}$ añadiendo un dispersión Gaussiana de media zero alrededor de cada variable:

$$
u_{i}=x_{i}+\xi_{i},
$$

donde $\xi$ sigue una distribución normal de media cero y desviación estándar $\sigma_{i}$.

\subsubsection{Configuración de los experimentos}

La información relacionada con cada uno de los casos: tamaño de población, número de generaciones, y tamaño del diseño de experimentos, se muestran en la Tabla 6.3. Los cinco problemas estudiados presentan las siguientes características en común: 


\begin{tabular}{cl}
\hline TCMO\# & $\begin{array}{l}\text { Distribución } \\
\text { de probabilidad }\end{array}$ \\
\hline \hline 1 & $\xi_{i} \sim \mathscr{N}\left(0,0.05\left(x_{i}^{\text {máx }}-x_{i}^{\text {mín }}\right)\right)$ \\
2 & $\xi_{i} \sim \mathscr{N}\left(0,0.01\left(x_{i}^{\text {máx }}-x_{i}^{\text {mín }}\right)\right)$ \\
3 & $\xi_{i} \sim \mathscr{N}\left(0,0.01\left(x_{i}^{\text {máx }}-x_{i}^{\text {mín }}\right)\right)$ \\
$4-5$ & $\xi_{i} \sim \mathscr{N}\left(0,0.01\left(x_{i}^{\text {máx }}-x_{i}^{\text {mín }}\right)\right)$ \\
\hline
\end{tabular}

Tabla 6.2: Distribución de probabilidad de los problemas TCMO\#.

1. Se adopta un valor $k=1$ en las funciones objetivo de la formulación (6.9). Así mismo todos los problemas se resuelven para niveles de fiabilidad de $P_{S}=0.75$ y $P_{S}=0.95$.

2. Cada simulación MonteCarlo está compuesta por diez mil evaluaciones del modelo de simulación, independientemente de si dicha simulación se realiza sobre el meta-modelo o sobre el modelo de simulación.

3. Se utiliza como algoritmo de optimización multi-objetivo el algoritmo NSGA-II (Deb et al., 2002). Los índices de distribución para el cruce y la mutación tienen un valor de 10 y 20 respectivamente, mientras que las tasas de cruce y mutación son de 0.9 y 0.2 (tal y como sugieren los diseñadores del algoritmo).

4. El diseño de experimentos inicial se genera mediante un Hipercubo Latino, utilizando el método doe.LHS de la toolbox de MATLAB ooSBOUU (Martinez-Frutos, 2013) junto con el criterio maximin (maximizar la distancia entre puntos) y un número de 1000 iteraciones.

5. Las funciones de regresión y correlación para cada uno de los modelos Kriging utilizados son las resultantes de la minimización del $P R E S S_{R M S}$ (Sección 3.4.5.2).

6. En todos los casos el problema de propagación de incertidumbre se resuelve con el algoritmo EKDRM tomando un orden de descomposición $\mathrm{S}=1$ y un número de puntos $N_{1}=4$.

7. Para los problema TCMO2-5 se utiliza el algoritmo EHV-EMO para la actualización del meta-modelo alrededor del frente de soluciones no dominadas del problema.

Todos los problemas se resuelven utilizando tres enfoques:

- Enfoque 1: resolución directa con MonteCarlo. Los momentos estadísticos y probabilidades se obtienen mediante una simulación MonteCarlo sobre el modelo de simulación. El problema de optimización se resuelve utilizando el algoritmo de optimización NSGA-II. 


\begin{tabular}{|c|c|c|c|c|c|}
\hline Problema & TCMO1 & TCMO2 & TCMO3 & TCMO4 & TCMO5 \\
\hline $\mathrm{N}^{\circ}$ de variables de diseño & 2 & 2 & 2 & 2 & 2 \\
\hline Tamaño de la población & 200 & 300 & 200 & 200 & 200 \\
\hline Número de generaciones & 100 & 200 & 40 & 200 & 200 \\
\hline Tamaño DoE inicial & 20 & 5 & 5 & 20 & 83 \\
\hline Tamaño actualizacion crit. $\mathscr{C}_{2}{ }^{(a)}$ & $(-)$ & 6 & 9 & 10 & $(-)$ \\
\hline Tamaño actualizacion crit. $\mathscr{C}_{1}(a)$ & $(-)$ & 44 & 61 & 53 & $(-)$ \\
\hline Tamaño total del DoE & 20 & 55 & 75 & 83 & 83 \\
\hline Número total de evaluaciones & 180 & 495 & 675 & 747 & 747 \\
\hline $\mathrm{It}_{\max }(a)$ & $(-)$ & 100 & 100 & 100 & $(-)$ \\
\hline Crit $_{\max }(a)$ & $(-)$ & $1 e-3$ & $1 e-3$ & $1 e-3$ & $(-)$ \\
\hline NumFeas $_{\min }{ }^{(a)}$ & $(-)$ & 5 & 5 & 10 & $(-)$ \\
\hline $\operatorname{tolg}(a)$ & $(-)$ & $1 e-3$ & $1 e-3$ & $1 \mathrm{e}-3$ & $(-)$ \\
\hline DIRECT maxiter $^{(a)}$ & $(-)$ & 1000 & 1000 & 1000 & $(-)$ \\
\hline DIRECT maxevals ${ }^{(a)}$ & $(-)$ & 1000 & 1000 & 1000 & $(-)$ \\
\hline$K^{(a)}$ & $(-)$ & 96 & 96 & 96 & $(-)$ \\
\hline
\end{tabular}

(a) Parámetros del algoritmo EHV-EMO.

Tabla 6.3: Parámetros utilizados para los casos TCMO\#.

- Enfoque 2: resolución directa con métodos de reducción dimensional. Los momentos estadísticos y las probabilidades se obtienen mediante la utilización de métodos de descomposición asistidos por modelos Kriging (Algoritmo 4). Este enfoque permite reducir considerablemente el número de evaluaciones necesarias durante el proceso de propagación de incertidumbre en comparación con el enfoque anterior. Sin embargo, los procesos de optimización y de propagación de incertidumbre están anidados por lo que el coste computacional sigue siendo elevado. El problema de optimización se resuelve utilizando el algoritmo de optimización NSGA-II.

- Enfoque 3: resolución con meta-modelos estocásticos. Los momentos estadísticos obtenidos mediante métodos de descomposición son aproximados en el dominio de diseño utilizando modelos Kriging. De esta forma se reduce drásticamente el coste computacional del proceso de optimización al realizarse las evaluaciones sobre dichos meta-modelos. Además estos meta-modelos proporcionan una información muy valiosa acerca de la incertidumbre epistémica de la predicción. El problema de optimización se resuelve utilizando el algoritmo de optimización EHV-EMO.

\subsubsection{Validación}

La validación de la precisión del meta-modelo se realiza atendiendo a dos criterios: (1) la precisión de la solución óptima multi-objetivo y (2) el grado de cumplimiento de las restricciones de probabilidad. Para evaluar como de cerca está la solución obtenida de la solución real, se utilizaron tres índices (Coello et al., 
2002; Zitzler et al., 2003) representativos de las diferencias entre los resultados de referencia (MonteCarlo) y los obtenidos mediante los meta-modelos estocásticos. Estos índices son: (1) la distancia entre generaciones (Generational distance (GD) (Veldhuizen, 1999)), (2) el máximo error en el frente de Pareto (Maximum Pareto front error (MPFE) (Veldhuizen, 1999)) y (3) El ratio de hipervolumen (Hypervolume ratio (HR)). Todos ellos se presentaron en la Sección 4.5 .

Para comprobar que las soluciones óptimas obtenidas satisfacen las restricciones de probabilidad, dichas restricciones son evaluadas mediante simulación MonteCarlo para el conjunto de soluciones óptimas de Pareto. Se toman como indicadores el valor medio de las restricciones violadas (VMRV) y el ratio de soluciones factibles (RFac) tras la simulación MonteCarlo. Dada la aleatoriedad de la simulación MonteCarlo, los diseños óptimos pertenecientes al frente de Pareto considerado de referencia pueden variar de una ejecución a otra. Por esta razón, para cada caso se han realizado 10 ejecuciones obteniendo las medias y desviaciones estándar de todos los indicadores.

\subsubsection{Problema TCMO1}

Los meta-modelos estocásticos de funciones objetivo y restricciones se ajustan utilizando las observaciones realizadas en 20 puntos en el espacio de diseño. En cada uno de estos puntos se estiman los 4 primeros momentos estadísticos utilizando el algoritmo EKDRM con un orden de descomposición $S=1$ y un número de puntos $N_{1}=4$. La Figura 6.4 muestra un mapa de contornos de los meta-modelos de los dos primeros momentos estadísticos de las funciones objetivo. La Figura 6.5 muestra un mapa de contornos de los meta-modelos de los cuantiles 0.75 y 0.95 de las restricciones. Dichos cuantiles se obtienen utilizando la distribución reconstruida a partir de los momentos estadísticos de las restricciones.

El coeficiente de determinación se estima utilizando una muestra de validación de 1e+05 puntos. El coeficiente de determinación obtenido es $\mathrm{R}^{2}=0.9999$ en todos los casos. Por lo tanto los meta-modelos obtenidos son capaces de proporcionar predicciones de los momentos estadísticos del problema con una elevada precisión en la totalidad del espacio de diseño. Por este motivo en este ejemplo se utilizan dichos meta-modelos para alimentar al algoritmo de optimización multi-objetivo NSGA-II.

Los resultados se muestran de forma gráfica en la Figura 6.6 y numéricamente en las Tablas 6.4 y 6.5. Tomando como referencia los resultados obtenidos con simulación MonteCarlo y el algoritmo NSGA-II se aprecia un gran acuerdo entre las soluciones obtenidas con los tres enfoques. Esta apreciación se corrobora numéricamente con valores cercanos a cero para la distancia entre generaciones y para el máximo error en el frente de Pareto, y valores muy cercanos a 1 para el ratio de hipervolumen. 


\begin{tabular}{lllllll}
\hline Caso & & TCMO1 & TCMO2 & TCMO3 & TCMO4 & TCMO5 \\
\hline \hline MonteCarlo versus EHV-EMO & $\mu_{G D}$ & $3.52 \mathrm{e}-04$ & $7.53 \mathrm{e}-04$ & $3.81 \mathrm{e}-03$ & $8.91 \mathrm{e}-04$ & $4.05 \mathrm{e}-03$ \\
& $\sigma_{G D}$ & $2.47 \mathrm{e}-05$ & $5.67 \mathrm{e}-05$ & $2.79 \mathrm{e}-04$ & $8.41 \mathrm{e}-05$ & $3.94 \mathrm{e}-04$ \\
& $\mu_{M P F E}$ & $2.77 \mathrm{e}-02$ & $7.34 \mathrm{e}-02$ & $7.58 \mathrm{e}-02$ & $1.25 \mathrm{e}-02$ & $1.16 \mathrm{e}-01$ \\
& $\sigma_{M P F E}$ & $3.65 \mathrm{e}-03$ & $9.42 \mathrm{e}-03$ & $9.75 \mathrm{e}-03$ & $1.06 \mathrm{e}-03$ & $1.39 \mathrm{e}-02$ \\
& $\mu_{H R}$ & $1.00 \mathrm{e}+00$ & $1.00 \mathrm{e}+00$ & $9.85 \mathrm{e}-01$ & $9.13 \mathrm{e}-01$ & $1.33 \mathrm{e}+00$ \\
& $\sigma_{H R}$ & $1.14 \mathrm{e}-01$ & $1.09 \mathrm{e}-01$ & $8.89 \mathrm{e}-02$ & $7.18 \mathrm{e}-02$ & $4.82 \mathrm{e}-01$ \\
MonteCarlo versus EKDRM $\left(S=1 ; N_{1}=4\right)$ & $\mu_{G D}$ & $3.15 \mathrm{e}-04$ & $9.47 \mathrm{e}-02$ & $2.13 \mathrm{e}-04$ & $7.13 \mathrm{e}-04$ & $7.13 \mathrm{e}-04$ \\
& $\sigma_{G D}$ & $2.63 \mathrm{e}-05$ & $6.60 \mathrm{e}-03$ & $1.62 \mathrm{e}-05$ & $5.13 \mathrm{e}-05$ & $5.13 \mathrm{e}-05$ \\
& $\mu_{M P F E}$ & $2.42 \mathrm{e}-02$ & $5.19 \mathrm{e}-02$ & $9.00 \mathrm{e}-03$ & $2.58 \mathrm{e}-02$ & $.58 \mathrm{e}-02$ \\
& $\sigma_{M P F E}$ & $3.52 \mathrm{e}-03$ & $4.97 \mathrm{e}-3$ & $9.61 \mathrm{e}-4$ & $3.16 \mathrm{e}-03$ & $3.16 \mathrm{e}-03$ \\
& $\mu_{H R}$ & $1.03 \mathrm{e}+00$ & $0.95 \mathrm{e}+00$ & $1.02 \mathrm{e}+00$ & $0.92 \mathrm{e}+00$ & $0.92 \mathrm{e}+00$ \\
Validación del Meta-modelo & $\sigma_{H R}$ & $3.36 \mathrm{e}-05$ & $1.74 \mathrm{e}-04$ & $9.35 \mathrm{e}-05$ & $7.49 \mathrm{e}-04$ & $7.49 \mathrm{e}-04$ \\
& $\mu_{V M R V}$ & $1.10 \mathrm{e}-03$ & $(-)$ & $(-)$ & $(-)$ & $1.46 \mathrm{e}-01$ \\
& $\sigma_{V M R V}$ & $2.81 \mathrm{e}-03$ & $(-)$ & $(-)$ & $(-)$ & $1.01 \mathrm{e}-01$ \\
& $\mu_{R F a c}$ & $0.99 \mathrm{e}+00$ & $1.00 \mathrm{e}+00$ & $1.00 \mathrm{e}+00$ & $1.00 \mathrm{e}+00$ & $0.25 \mathrm{e}+00$ \\
& $\sigma_{R F_{a c}}$ & $0.05 \mathrm{e}+00$ & $0.00 \mathrm{e}+00$ & $0.00 \mathrm{e}+00$ & $0.00 \mathrm{e}+00$ & $0.12 \mathrm{e}+00$
\end{tabular}

Tabla 6.4: Problemas TCMO\#. Resumen de los resultados numéricos para $P_{S}=0.75$. 


\begin{tabular}{lllllll}
\hline Caso & & TCMO1 & TCMO2 & TCMO3 & TCMO4 & TCMO5 \\
\hline \hline MonteCarlo versus EHV-EMO & $\mu_{G D}$ & $2.98 \mathrm{e}-04$ & $9.47 \mathrm{e}-04$ & $3.89 \mathrm{e}-03$ & $1.12 \mathrm{e}-03$ & $3.78 \mathrm{e}-03$ \\
& $\sigma_{G D}$ & $1.46 \mathrm{e}-05$ & $5.56 \mathrm{e}-05$ & $2.21 \mathrm{e}-04$ & $1.33 \mathrm{e}-04$ & $2.75 \mathrm{e}-04$ \\
& $\mu_{M P F E}$ & $1.38-02$ & $3.16 \mathrm{e}-02$ & $8.67 \mathrm{e}-02$ & $3.15 \mathrm{e}-02$ & $1.27 \mathrm{e}-01$ \\
& $\sigma_{M P F E}$ & $3.87 \mathrm{e}-03$ & $5.30 \mathrm{e}-03$ & $1.32 \mathrm{e}-02$ & $4.39 \mathrm{e}-03$ & $1.04 \mathrm{e}-02$ \\
& $\mu_{H R}$ & $1.03 \mathrm{e}+00$ & $0.95 \mathrm{e}+00$ & $9.51 \mathrm{e}-01$ & $9.22 \mathrm{e}-01$ & $1.28 \mathrm{e}+00$ \\
& $\sigma_{H R}$ & $1.02 \mathrm{e}-01$ & $9.37 \mathrm{e}-02$ & $7.95 \mathrm{e}-02$ & $6.54 \mathrm{e}-02$ & $2.91 \mathrm{e}-021$ \\
MonteCarlo versus EKDRM $\left(S=1 ; N_{1}=4\right)$ & $\mu_{G D}$ & $3.62 \mathrm{e}-04$ & $1.21 \mathrm{e}-03$ & $2.54 \mathrm{e}-04$ & $1.27 \mathrm{e}-03$ & $1.27 \mathrm{e}-03$ \\
& $\sigma_{G D}$ & $1.89 \mathrm{e}-05$ & $6.67 \mathrm{e}-05$ & $1.39 \mathrm{e}-05$ & $2.07 \mathrm{e}-04$ & $2.07 \mathrm{e}-04$ \\
& $\mu_{M P F E}$ & $1.99 \mathrm{e}-02$ & $1.59 \mathrm{e}-01$ & $1.88 \mathrm{e}-02$ & $4.19 \mathrm{e}-02$ & $4.19 \mathrm{e}-02$ \\
& $\sigma_{M P F E}$ & $2.75 \mathrm{e}-03$ & $1.43 \mathrm{e}-02$ & $1.41 \mathrm{e}-03$ & $3.56 \mathrm{e}-03$ & $3.56 \mathrm{e}-03$ \\
& $\mu_{H R}$ & $0.99 \mathrm{e}+00$ & $0.96 \mathrm{e}+00$ & $1.02 \mathrm{e}+00$ & 0.8984 & 0.8984 \\
Validación del Meta-modelo & $\sigma_{H R}$ & $2.33 \mathrm{e}-05$ & $1.84 \mathrm{e}-05$ & $8.93 \mathrm{e}-05$ & $7.21 \mathrm{e}-05$ & $7.21 \mathrm{e}-05$ \\
& $\mu_{V M R V}$ & $3.028 \mathrm{e}-3$ & $(-)$ & $(-)$ & $(-)$ & $7.83 \mathrm{e}-02$ \\
& $\sigma_{V M R V}$ & $1.32 \mathrm{e}-03$ & $(-)$ & $(-)$ & $(-)$ & $4.86 \mathrm{e}-02$ \\
& $\mu_{R F_{a c}}$ & $0.98 \mathrm{e}+00$ & $1.00 \mathrm{e}+00$ & $1.00 \mathrm{e}+00$ & $1.00 \mathrm{e}+00$ & $0.30 \mathrm{e}+00$ \\
& $\sigma_{R F_{a c}}$ & $0.07 \mathrm{e}+00$ & $0.00 \mathrm{e}+00$ & $0.00 \mathrm{e}+00$ & $0.00 \mathrm{e}+00$ & $0.09 \mathrm{e}+00$
\end{tabular}

Tabla 6.5: Problemas TCMO\#. Resumen de los resultados numéricos para $P_{S}=0.95$. 
En este primer problema se ha alcanzado un elevada precisión en los resultados aún sin utilizar técnicas adaptativas. Esto se debe a la alta linealidad de los momentos estadísticos en el dominio de diseño. Dicha linealidad permite una excelente aproximación mediante modelos Kriging de la respuesta aleatoria. Como consecuencia se consigue una gran precisión en la estimación de soluciones óptimas de Pareto así como un alto porcentaje de soluciones factibles en el conjunto final.

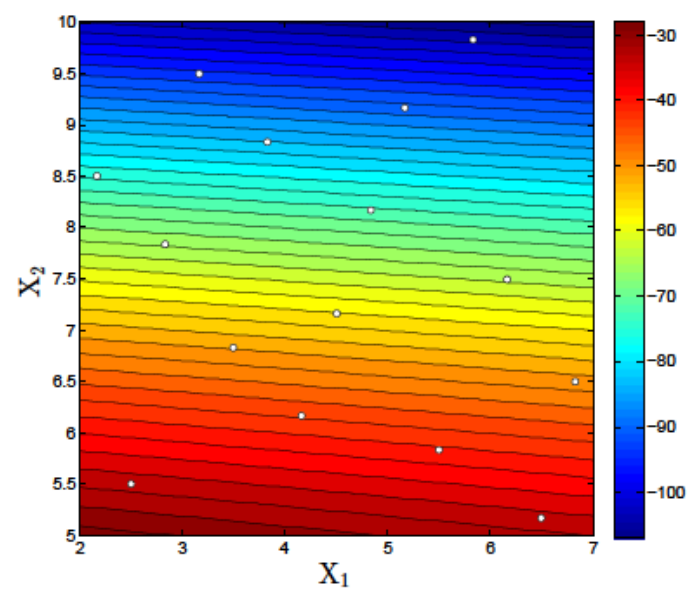

(a) $\hat{\mu}_{f_{1}}$

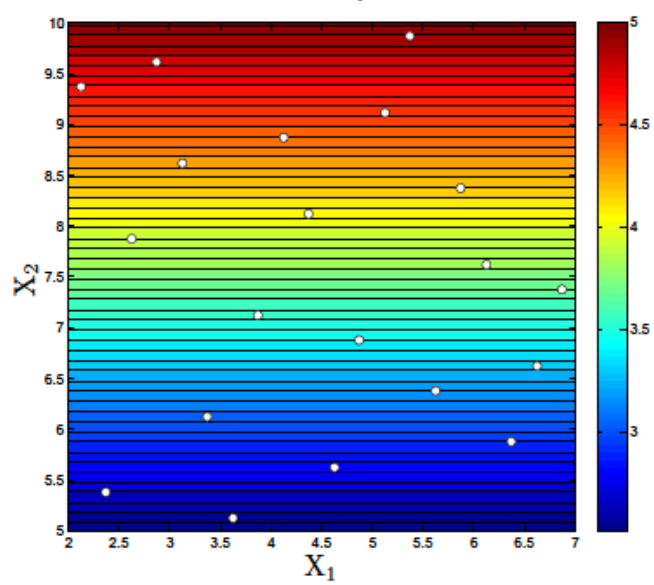

(c) $\hat{\sigma}_{f_{1}}$

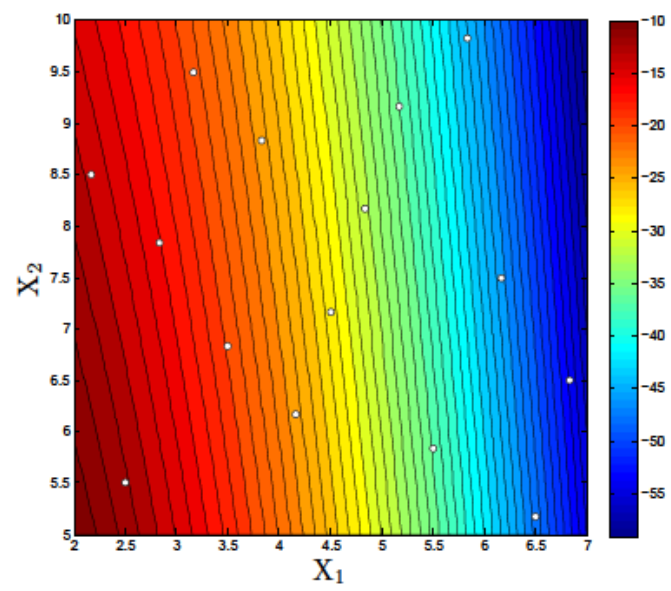

(b) $\hat{\mu}_{f_{2}}$

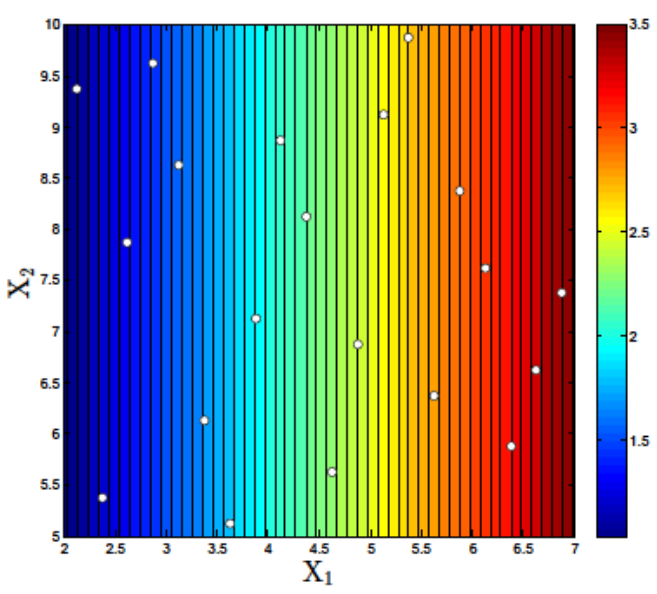

(d) $\hat{\sigma}_{f_{2}}$

Figura 6.4: Problema TCMO1. Meta-modelos de los momentos estadísticos.

La Tabla 6.6 recoge una comparación del coste computacional de los tres enfoques analizados, entendiendo dicho coste computacional como el número de evaluaciones del modelo de simulación. La utilización de meta-modelos supone una drástica disminución del número de evaluaciones requerido en comparación con los otros enfoques. 


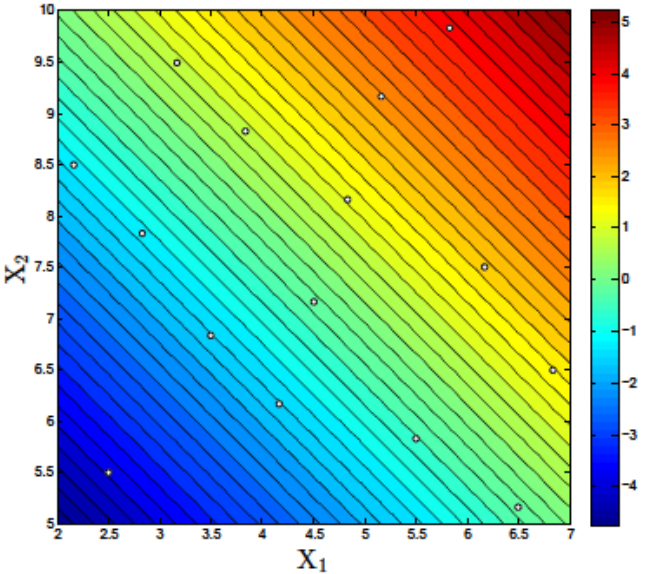

(e) $\hat{Q}_{f_{3}}(0.75)$

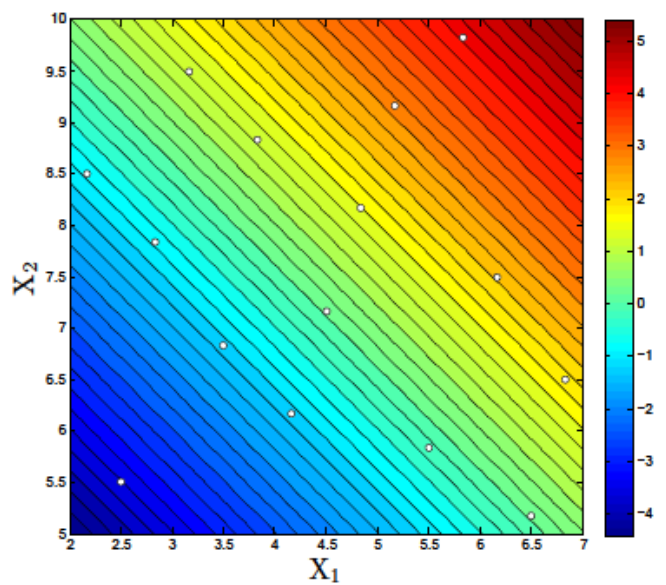

(g) $\hat{Q}_{f_{3}}(0.95)$

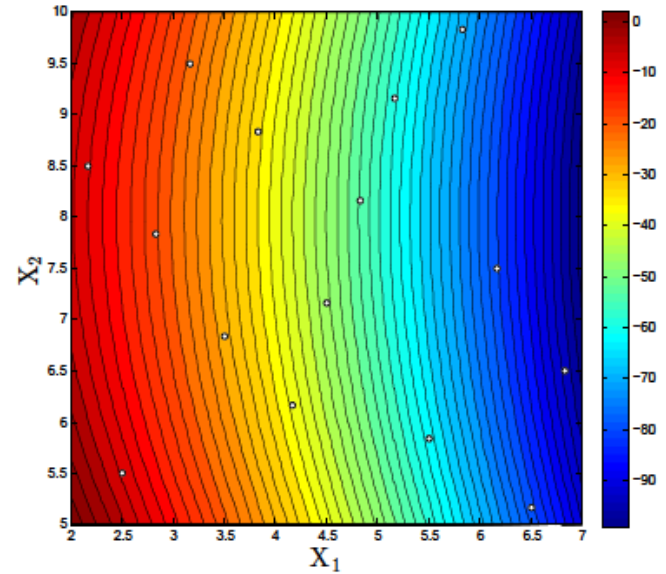

(f) $\hat{Q}_{f_{4}}(0.75)$

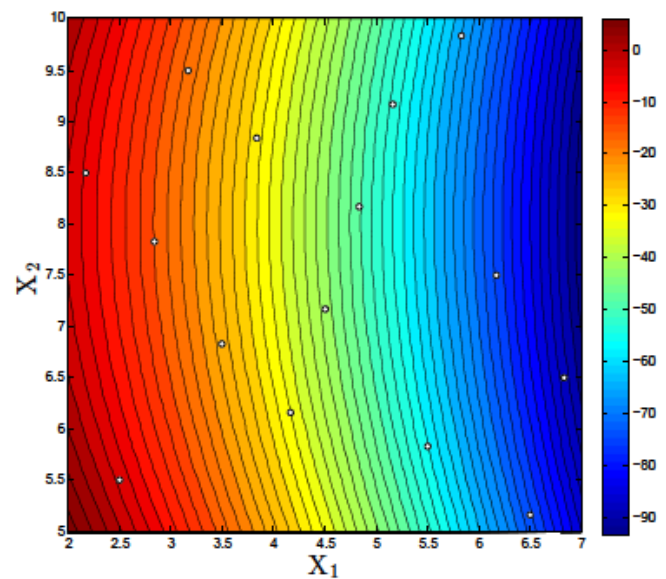

(h) $\hat{Q}_{f_{4}}(0.95)$

Figura 6.5: Problema TCMO1. Meta-modelos de los cuantiles 0.75 y 0.95 de las restricciones.

\begin{tabular}{lll}
\hline Enfoque & & Evaluaciones \\
\hline \hline Enfoque 1: NSGA-II+MC & pop $\times$ gen $\times M C_{\text {size }}$ & $2 \mathrm{e}+08$ \\
Enfoque 2: NSGA-II+EKDRM & pop $\times$ gen $\times K D R M_{\text {size }}$ & $1 \mathrm{e}+05$ \\
Enfoque 3: NSGA-II+Meta-modelo & $D O E_{\text {size }} \times K D R M_{\text {size }}$ & $1.8 \mathrm{e}+02$ \\
\hline
\end{tabular}

Tabla 6.6: Problema TCMO1. Número de evaluaciones total. 

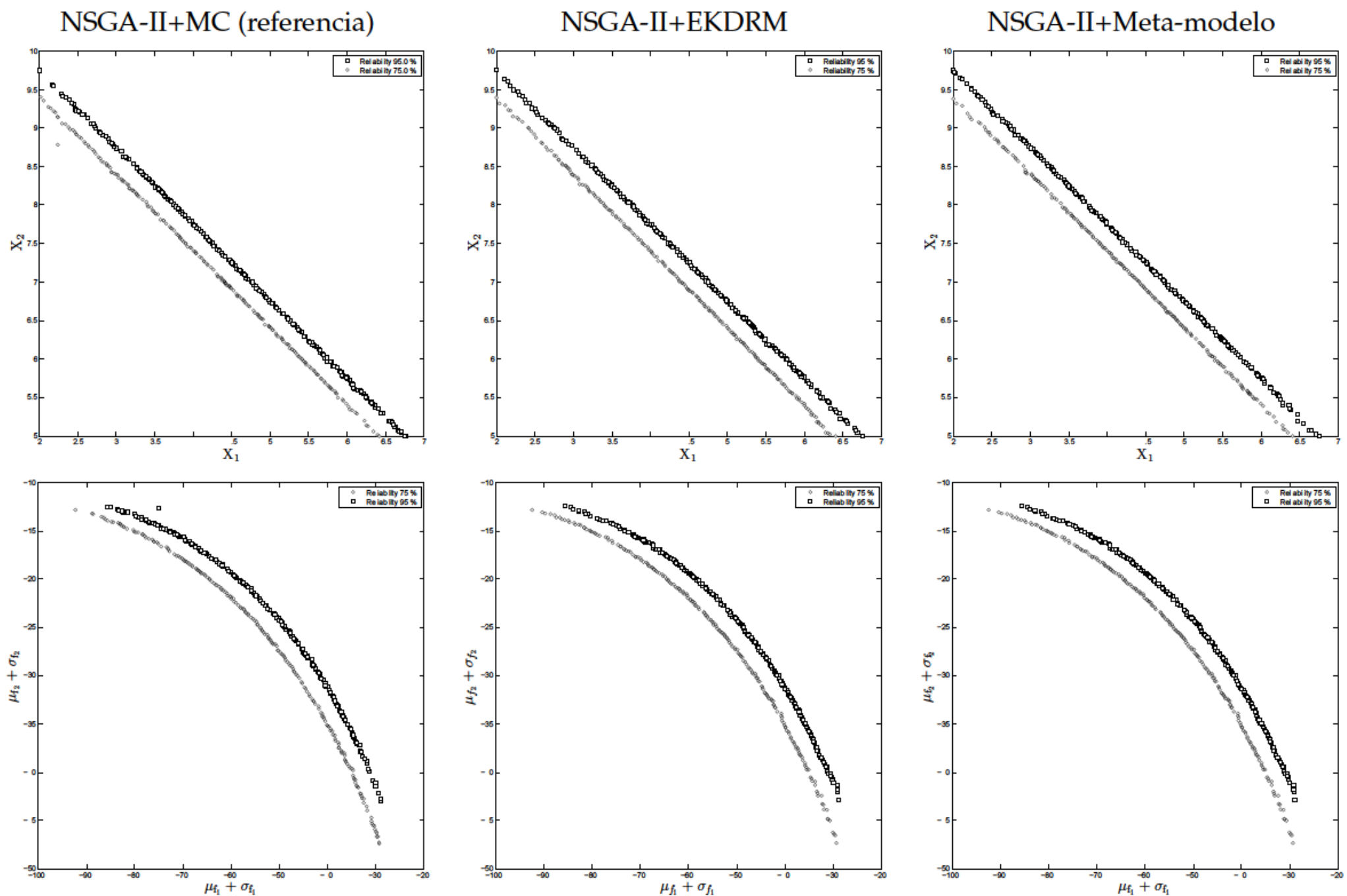

Figura 6.6: Problema TCMO1. Conjunto de soluciones óptimas de Pareto en el espacio de las variables de diseño (superior) y en el espacio de las funciones objetivo (inferior). 


\subsubsection{Problema TCMO2 a TCMO4}

Los problemas TCMO2 a TCMO4 se resuelven utilizando el algoritmo EHV-EMO. En cada caso los valores de los parámetros del algoritmo están recogidos en la Tabla 6.3. La convergencia se alcanza tras 44, 61 y 53 iteraciones para los problemas TCMO2, TCMO3, y TCMO4, respectivamente. La Figura 6.7 muestra la evolución del criterio de búsqueda con las iteraciones. Así mismo, las Figuras 6.10, 6.11, y 6.12, en su parte izquierda, representan los puntos añadidos en cada etapa sobre los contornos del criterio de búsqueda. En su parte derecha muestran la evolución del frente de Pareto aproximado junto con el frente de Pareto de referencia.

Los frentes de Pareto obtenidos mediante los tres enfoques propuestos (NSGAII+MC; NSGAII+EKDRM; EHV-EMO) se comparan gráficamente en las Figuras 6.8 y 6.9, y numéricamente en las Tablas 6.4 y 6.5. Los resultados muestran un buen acuerdo entre los frentes de Pareto correspondientes a los enfoques 1 y 2 (NSGA-II+ MC; NSGA-II+ EKDRM). Respecto a los frentes obtenidos con el algoritmo EHV-EMO se observa en primer lugar que el número de diseños encontrados es muy inferior al de los otros enfoques, aunque están muy próximos al frente de Pareto de referencia. Esto es debido a que, a diferencia del algoritmo NSGA-II, en el algoritmo EHV-EMO no se puede fijar a priori el número de diseños óptimos requeridos. Este es un aspecto a mejorar en futuras investigaciones. A pesar de ello, los meta-modelos obtenidos tras la actualización presentan una buena precisión en las regiones cercanas al frente, y pueden ser utilizados para incrementar el número de soluciones no-dominadas sin requerir evaluaciones adicionales del modelo de simulación.

Las conclusiones obtenidas mediante un primer análisis gráfico de los frentes de soluciones están en concordancia con los resultados numéricos para los indicadores seleccionados: la distancia entre generaciones (GD), el máximo error en el frente de Pareto (MPFE), y el ratio de hipervolumen (HR). Comparando el frente obtenido con los meta-modelos con el de referencia se observan valores cercanos a cero del GD y del MPFE y valores cercanos a uno para el HR. En cuanto a la validez de las soluciones, el algoritmo EHV-EMO minimiza la incertidumbre epistémica de los diseños óptimos conseguidos por lo que el $100 \%$ de ellos son factibles. 

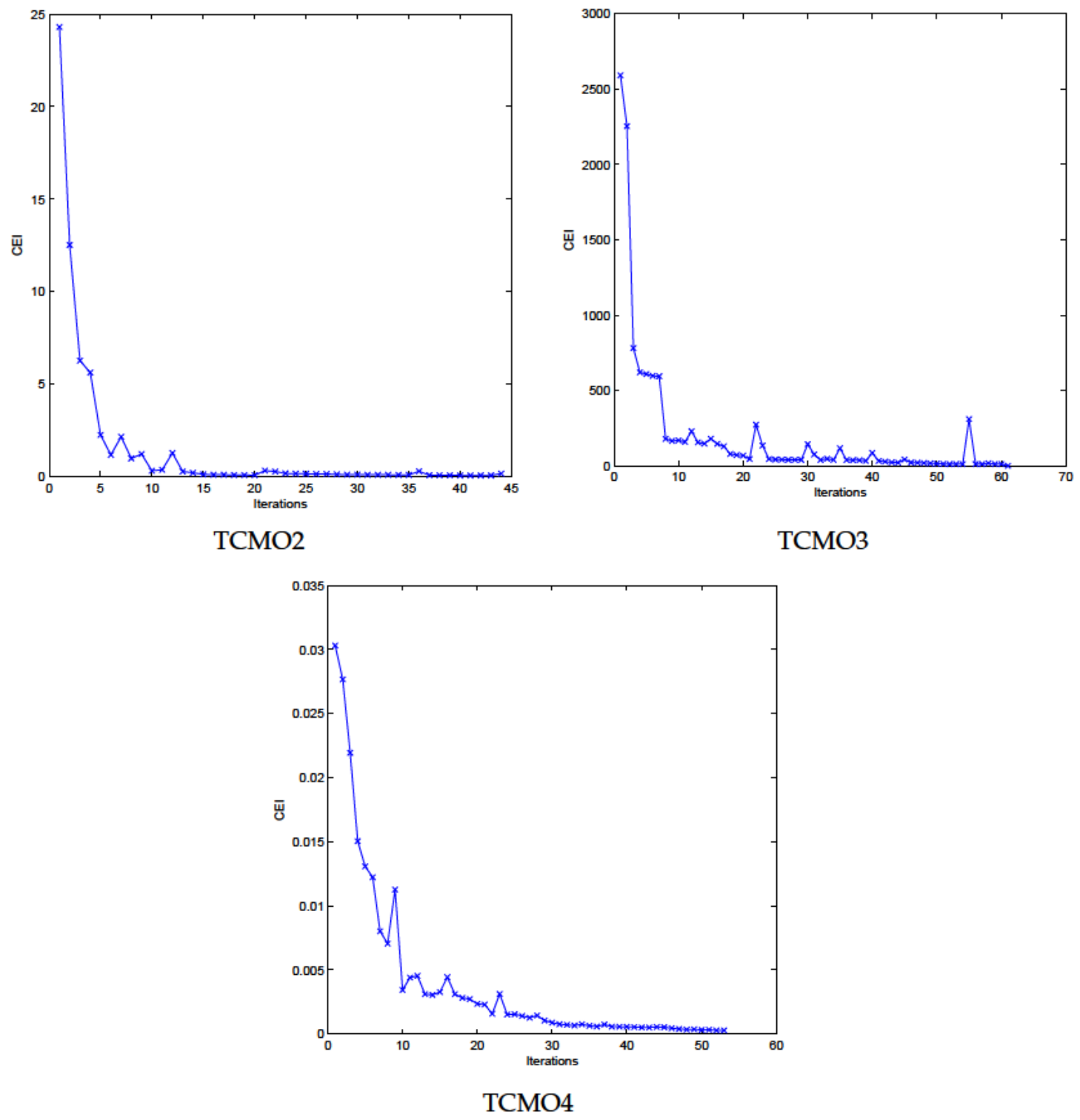

Figura 6.7: Problemas TCMO2 a TCMO4. Evolución del criterio de búsqueda. 


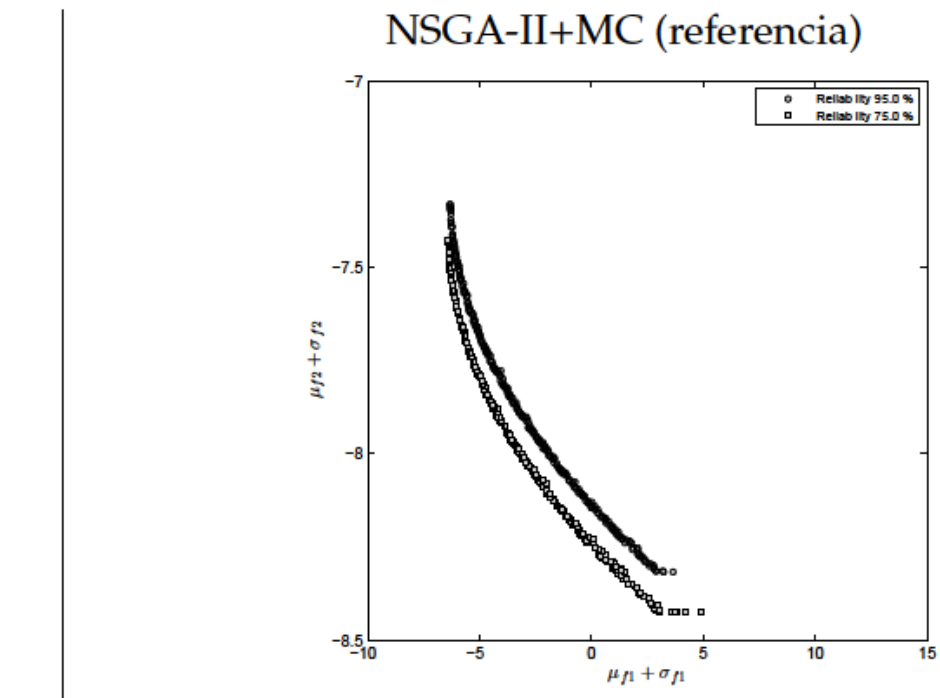

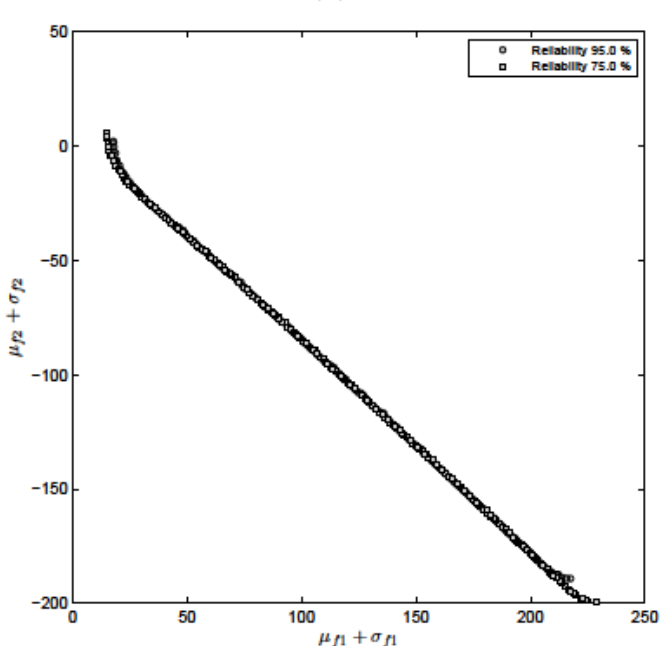

(d)

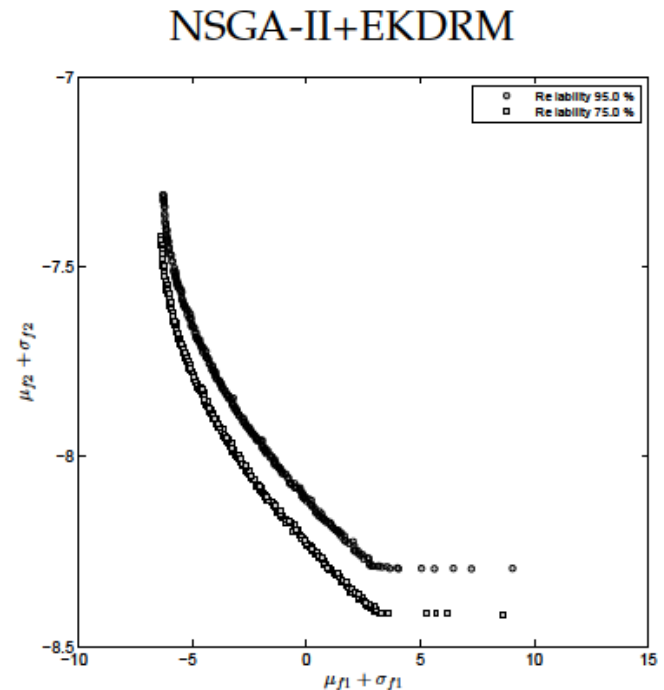

(b)

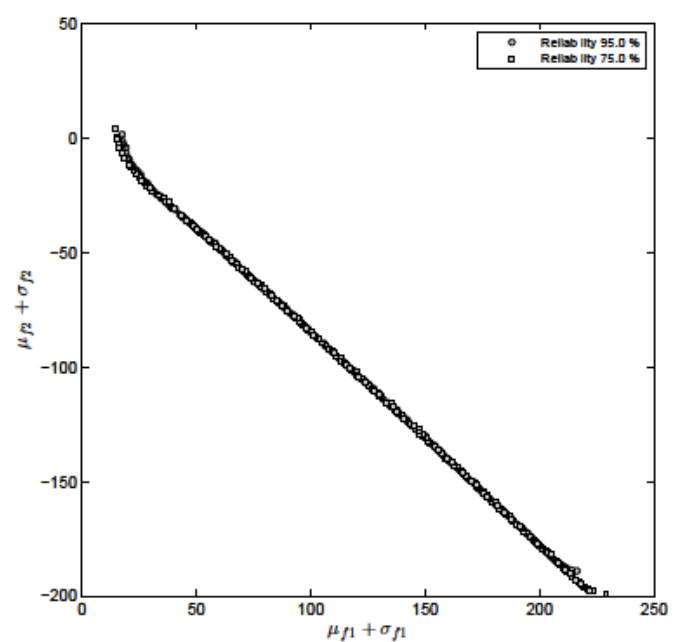

(e)

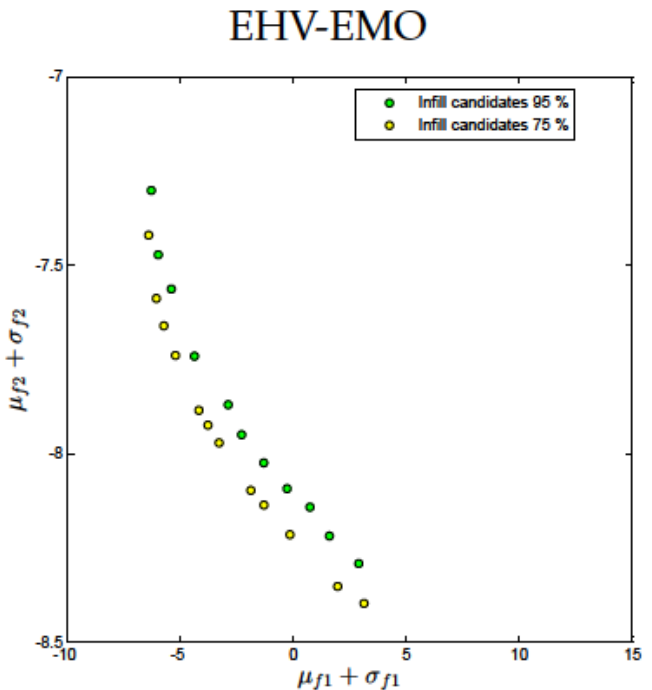

(c)

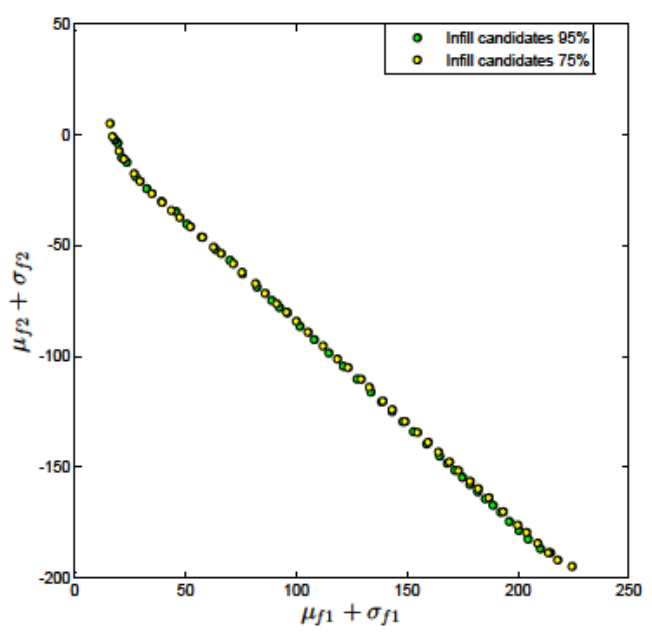

(f)

Figura 6.8: Problemas TCMO2 y TCMO3. Conjunto de soluciones óptimas de Pareto en el espacio de las variables de diseño (superior) y en el espacio de las funciones objetivo (inferior) obtenidas para el caso TCMO2 (a-c) y para el caso TCMO3 (d-f). 


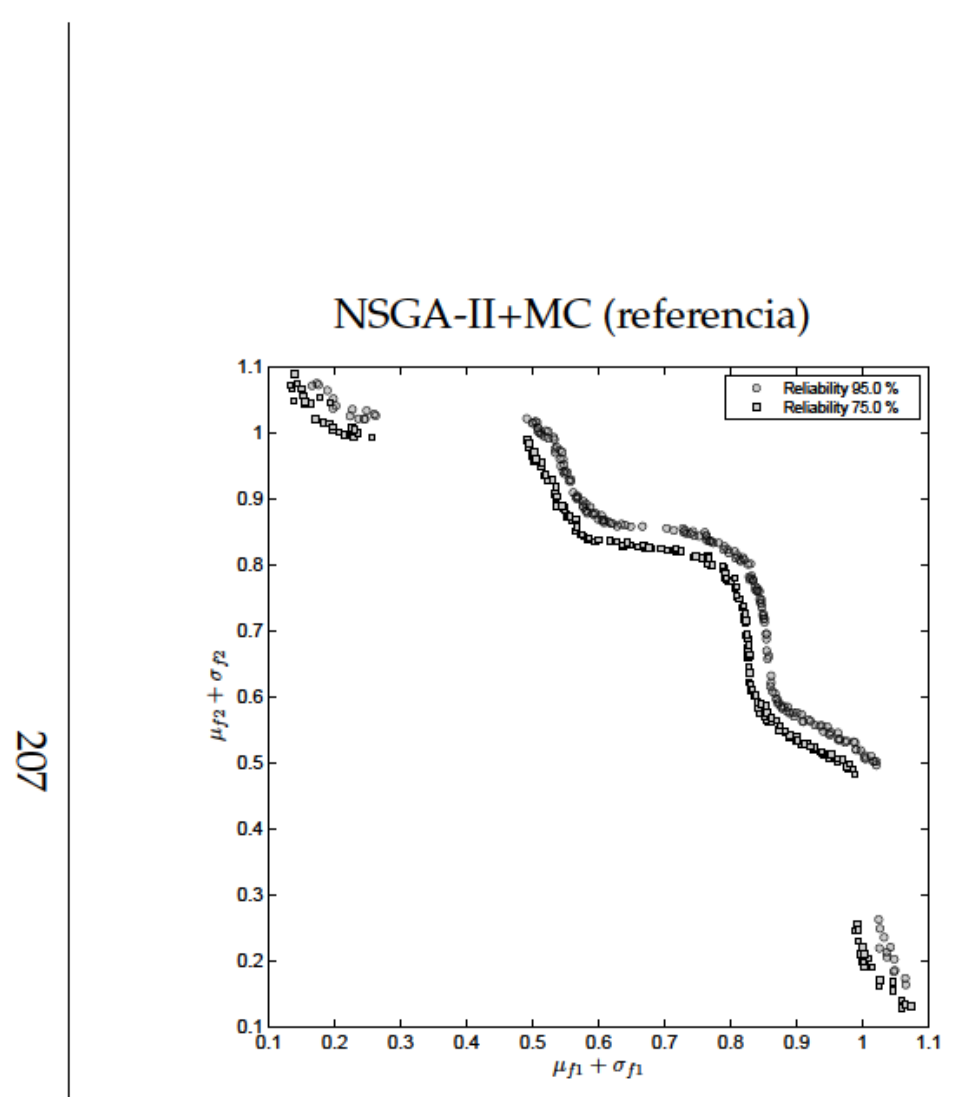

(a)

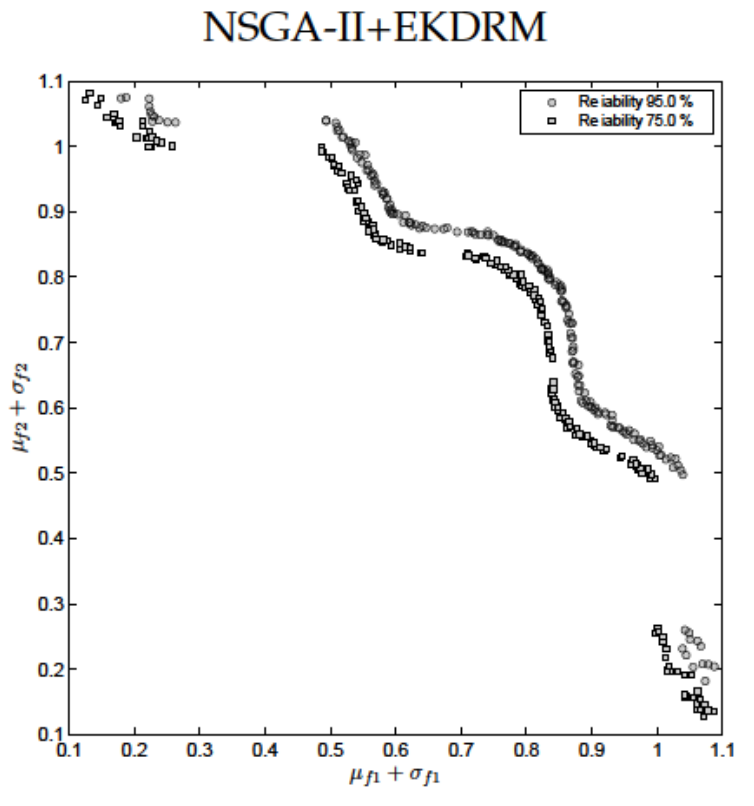

(b)

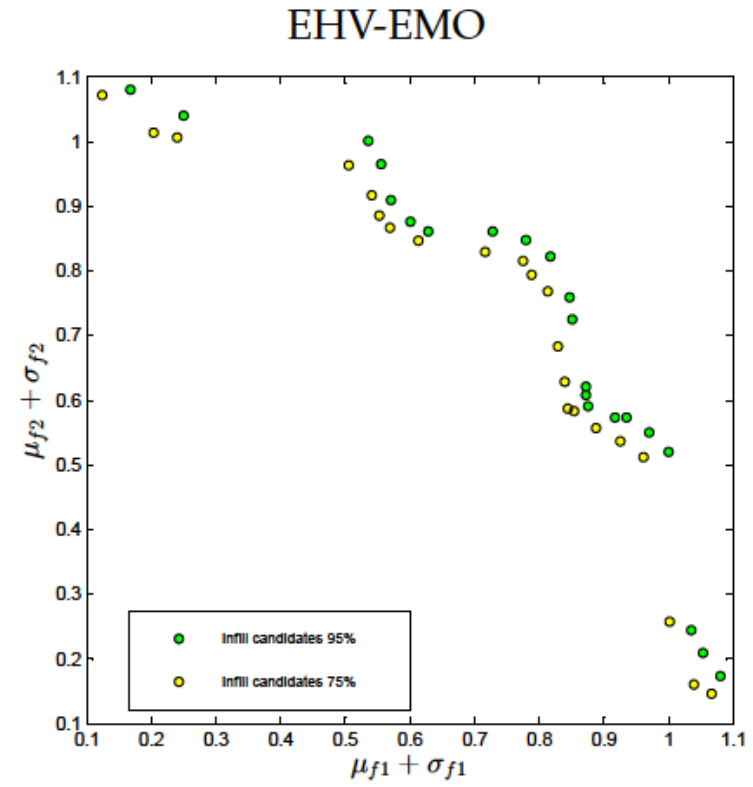

(c)

Figura 6.9: Problema TCMO4. Conjunto de soluciones óptimas de Pareto en el espacio de las funciones objetivo. 

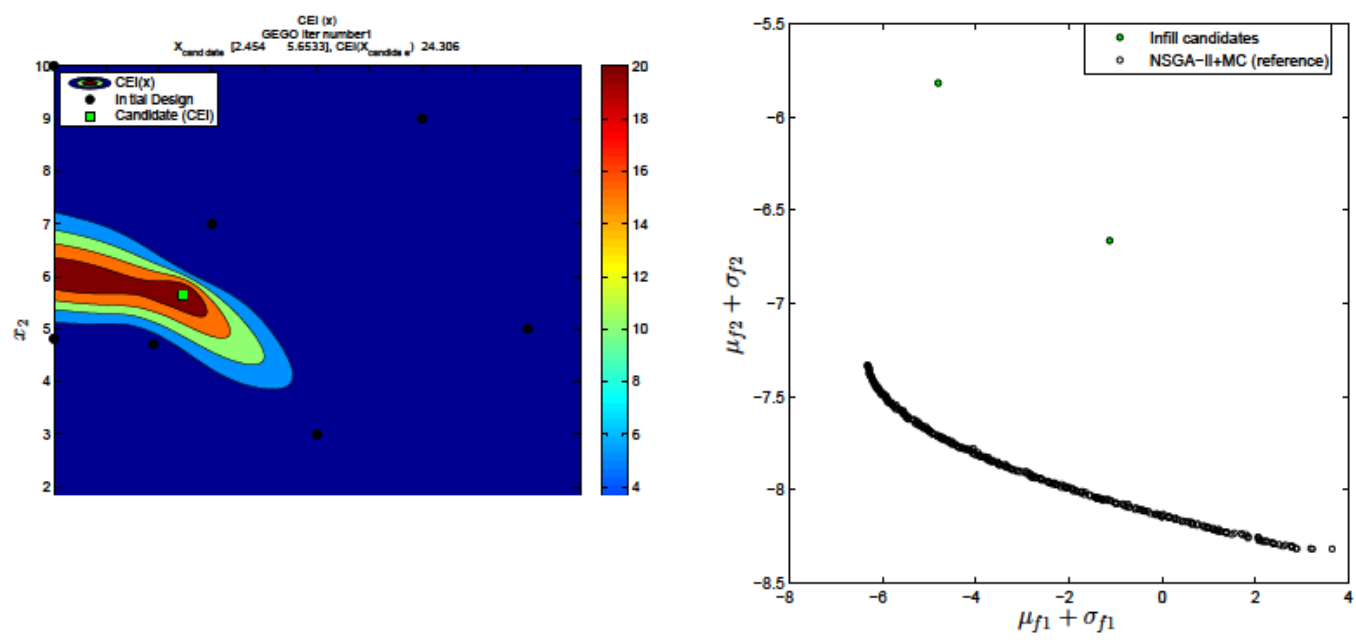

(b) Iter=1
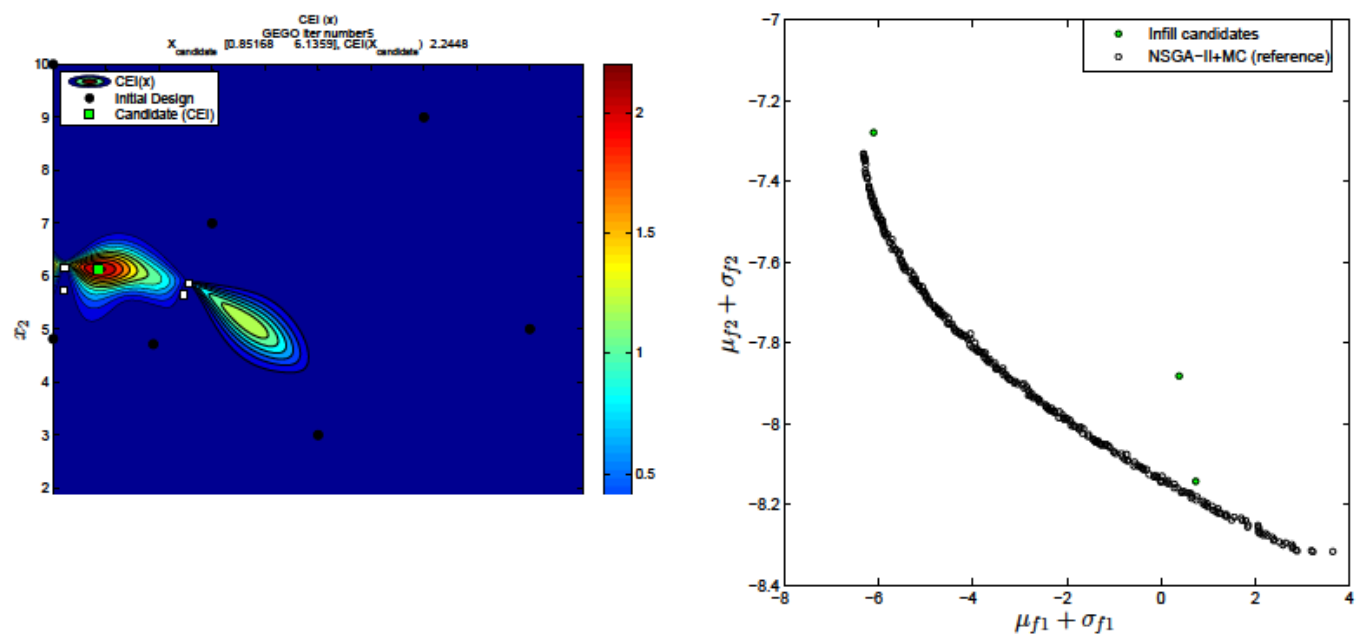

(d) Iter $=5$

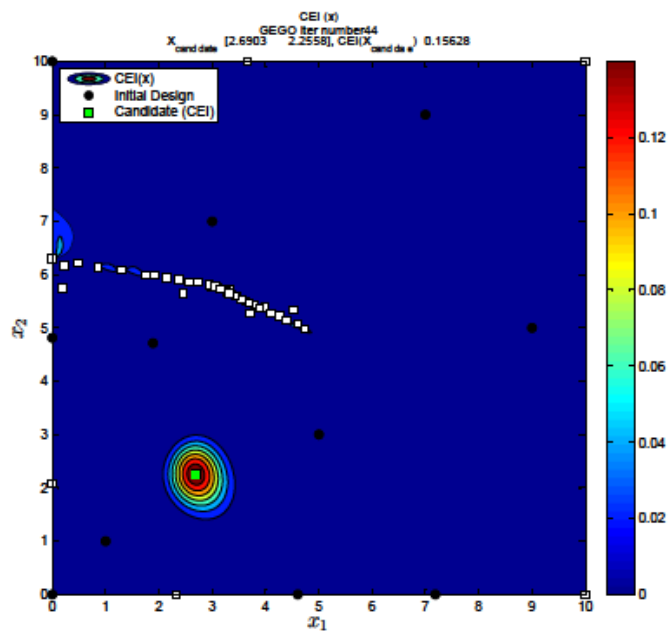

(g) Iter $=44$

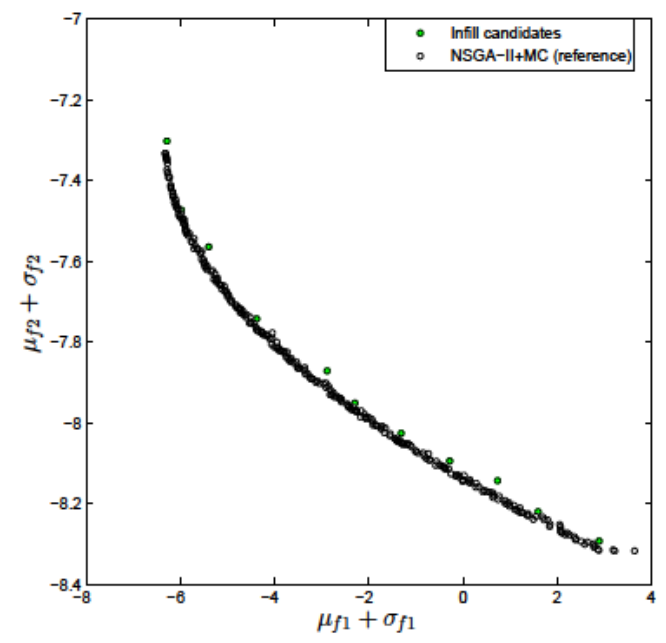

(h) Iter $=44$

Figura 6.10: Problema TCMO2. Evolución del criterio de búsqueda. La muestra inicial se presenta como círculos negros. Los cuadrados blancos representan los puntos añadidos hasta la iteración. Los cuadrados verdes representan el candidato que maximiza el criterio en cada iteración. 


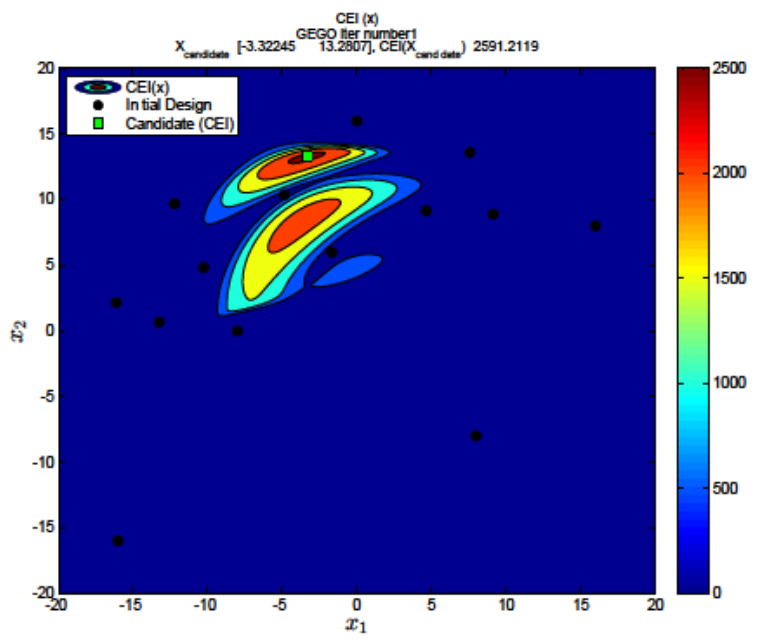

(a) Iter $=1$

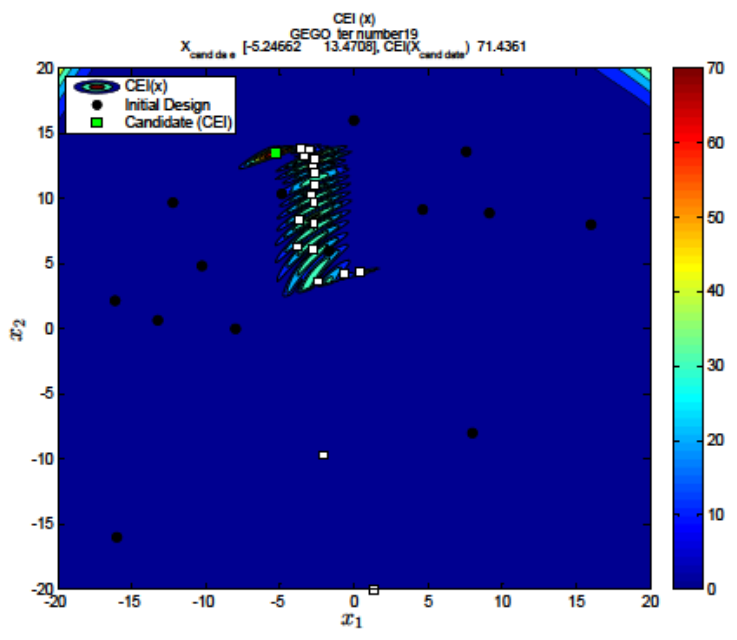

(c) Iter $=19$

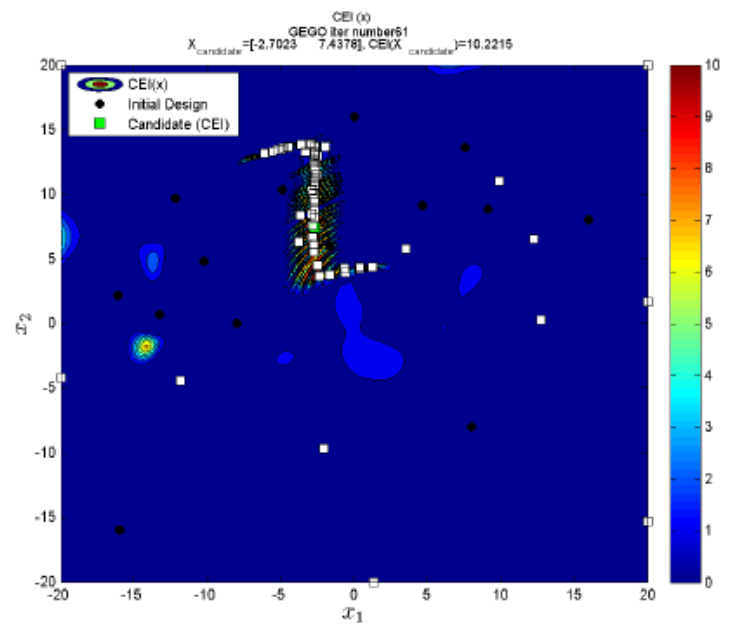

(g) Iter $=61$

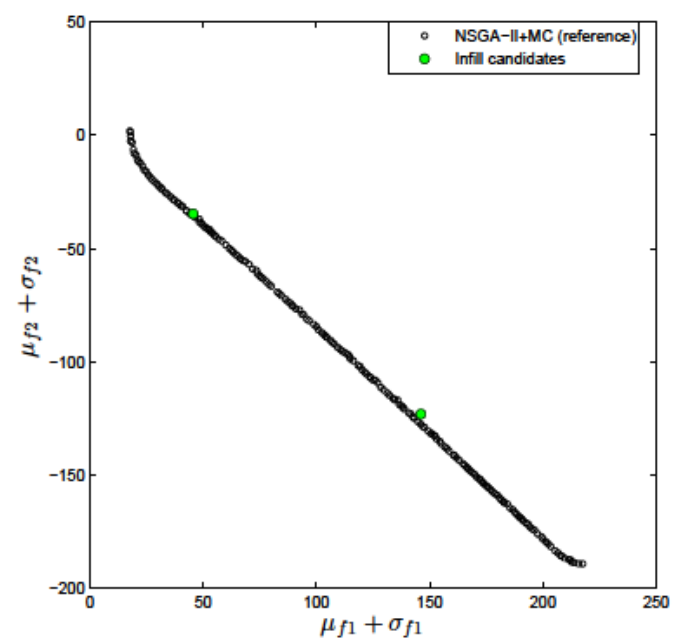

(b) Iter $=1$

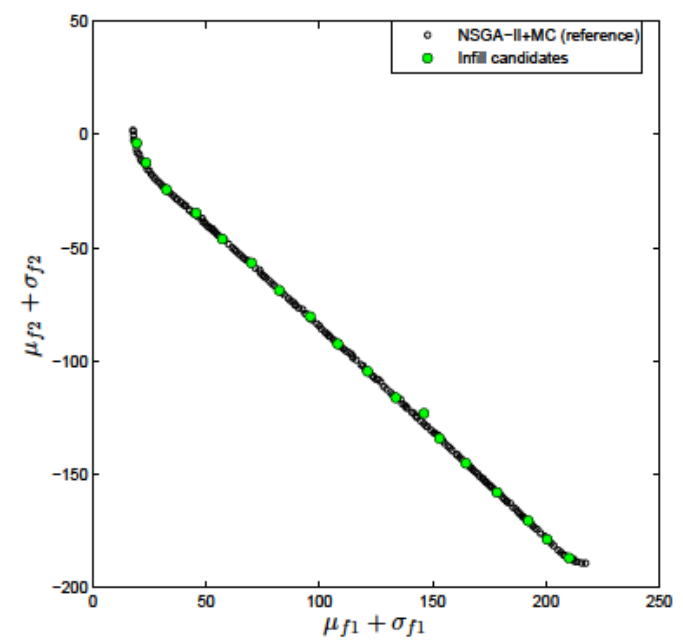

(d) Iter $=19$

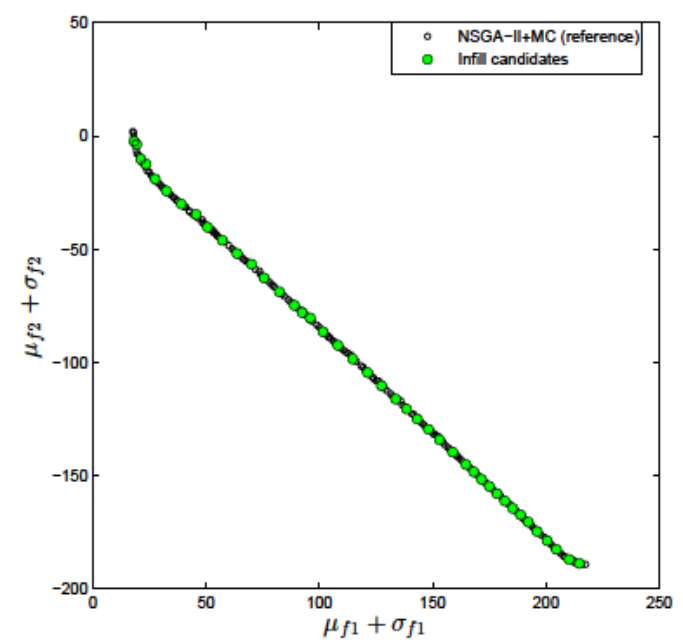

(h) Iter $=61$

Figura 6.11: Problema TCMO3. Evolución del criterio de búsqueda. La muestra inicial se presenta como círculos negros. Los cuadrados blancos representan los puntos añadidos hasta la iteración. Los cuadrados verdes representan el candidato que maximiza el criterio en cada iteración. 


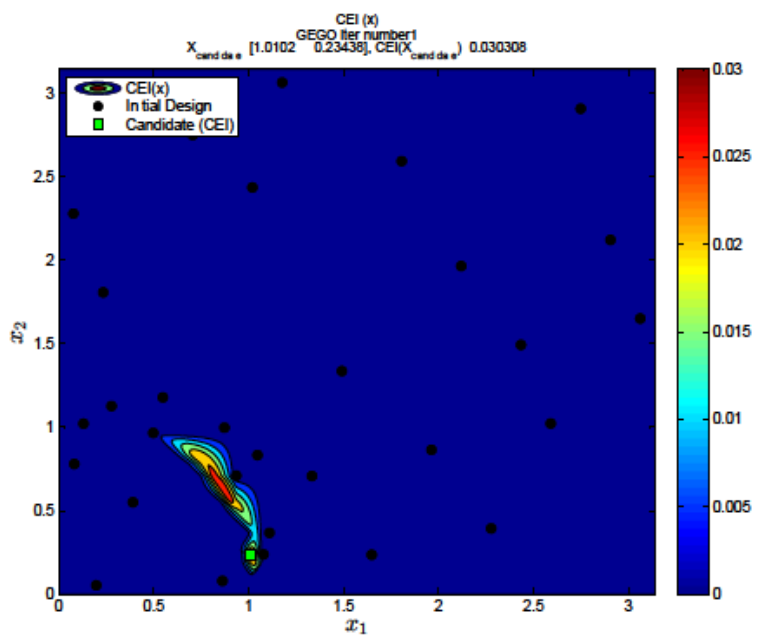

(a) Iter $=1$

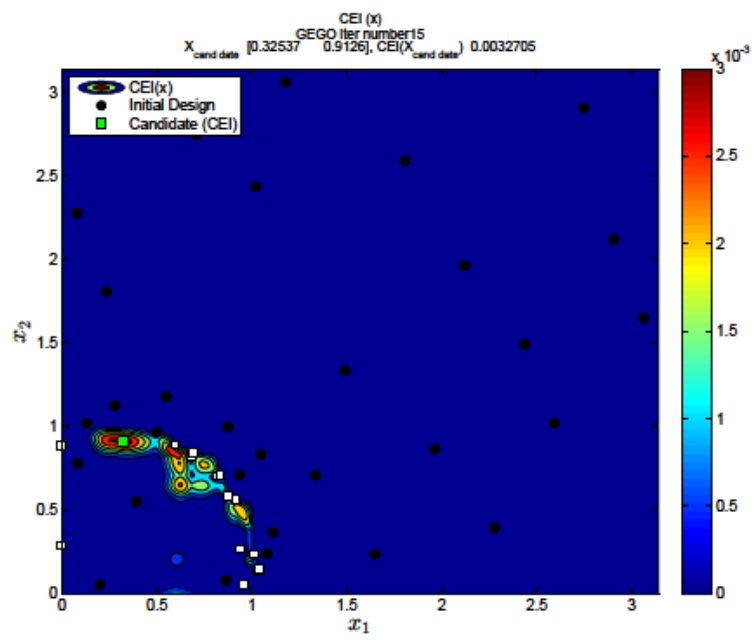

(c) Iter $=15$

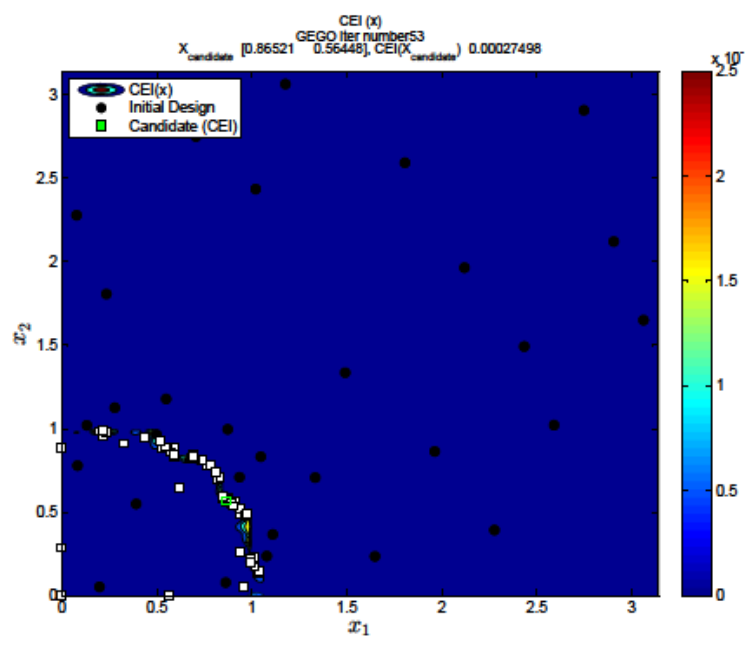

(g) Iter $=53$

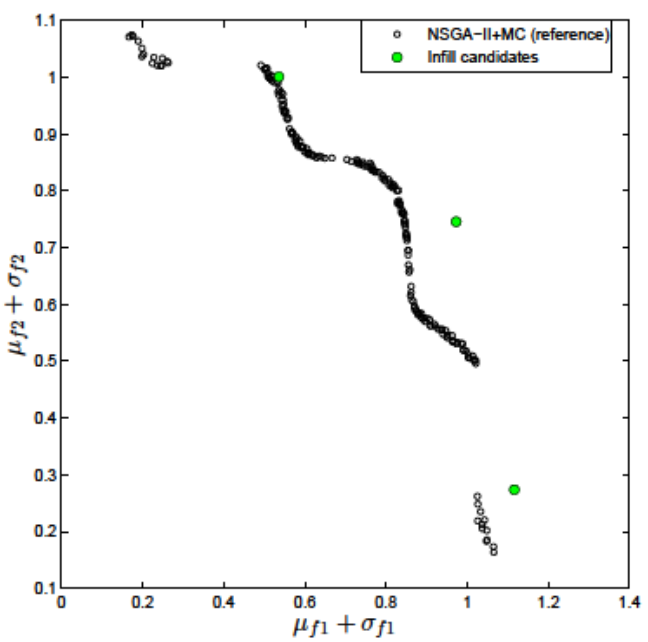

(b) Iter $=1$

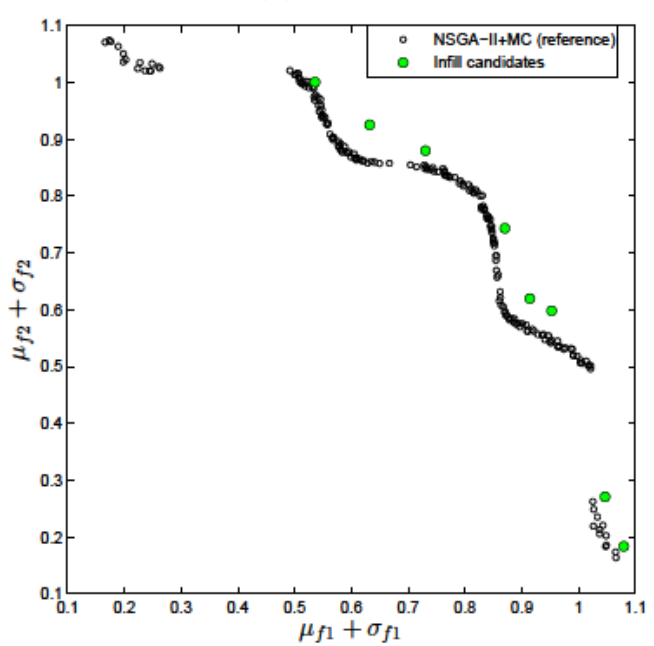

(d) Iter $=15$

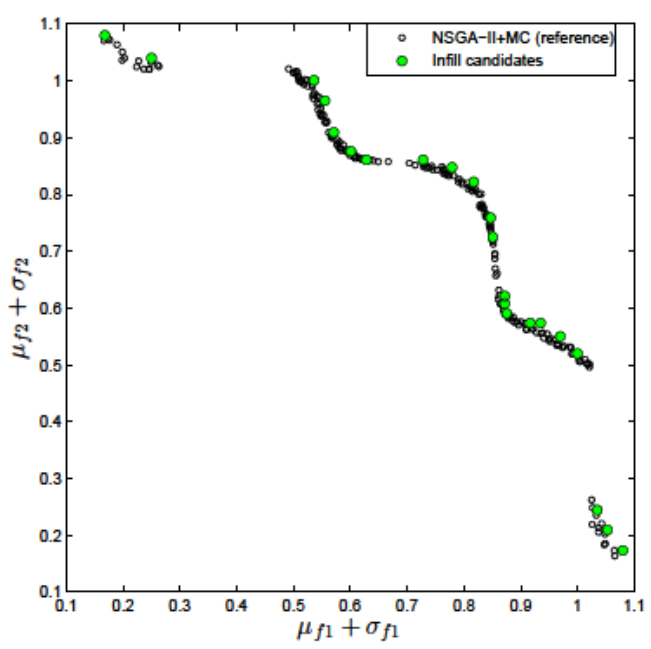

(h) Iter $=53$

Figura 6.12: Problema TCMO4. Evolución del criterio de búsqueda. La muestra inicial se presenta como círculos negros. Los cuadrados blancos representan los puntos añadidos hasta la iteración. Los cuadrados verdes representan el candidato que maximiza el criterio en cada iteración. 


\subsubsection{Problema TCMO5}

En este problema se resuelve el problema TCMO4, sin utilizar una estrategia adaptativa. En su lugar, se utiliza el algoritmo NSGA-II junto con meta-modelos estocásticos ajustados a partir de una muestra inicial.

Para comparar los resultados en igualdad de coste computacional, se considera una muestra inicial de tamaño equivalente al número total de evaluaciones realizadas por el algoritmo EHV-EMO en el problema TCMO4. El número de puntos utilizados en el ajuste es de 83 en los dos casos.

Los resultados se muestran gráficamente en la Figura 6.9. La explotación directa del meta-modelo con el algoritmo NSGA-II no consigue capturar de forma adecuada las regiones desconectadas del frente de Pareto del problema. Si lo comparamos con los resultados obtenidos en el caso TCMO4, en este caso se obtienen un mayor número de soluciones pero más alejadas del frente de referencia. Los meta-modelos actualizados obtenidos en el problema TCMO4 pueden ser explotados para la búsqueda de nuevas soluciones no dominadas (Figura 6.13).

Los indicadores utilizados para evaluar la precisión de la solución también muestran una clara diferencia entre el enfoque adaptativo y la explotación directa del meta-modelo. Para el problema TCMO5, los valores del GD y del MPFE son un orden de magnitud mayor que el caso TCMO4. Así mismo, el ratio de hipervolumen pasa de valores cercanos a uno a valores por encima de uno. A diferencia del enfoque adaptativo, la explotación directa del meta-modelo da lugar a un $25 \%$ y un $30 \%$ de soluciones no factibles para los casos con fiabilidad del $75 \%$ y del $95 \%$ respectivamente.

\subsubsection{Aplicación al diseño óptimo robusto de estructuras de nudos articulados}

En esta sección se estudia la eficiencia y precisión de la metodología propuesta para la resolución de problemas de diseño óptimo robusto de estructuras de nudos articulados. Se analizan tres problemas, adaptados de la literatura, en los que se consideran fuentes de incertidumbre relacionadas con el material, las cargas y la geometría de la estructura. La información relacionada con cada uno de los casos, tamaño de población, número de generaciones, tamaño del diseño de experimentos, se muestran en la Tabla 6.7.

\subsubsection{Problema TCSO1. Diseño óptimo robusto de una estructura de 4 barras con incertidumbre en material}

Este ejemplo fue tratado previamente por Doltsinis y Kang (2004). La estructura se muestra en la Figura 6.14. En el nudo 4 se aplica una fuerza horizontal 


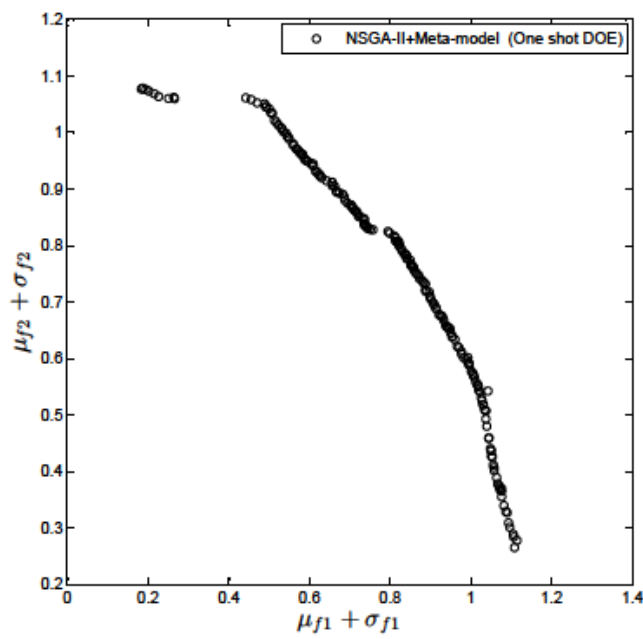

(a)

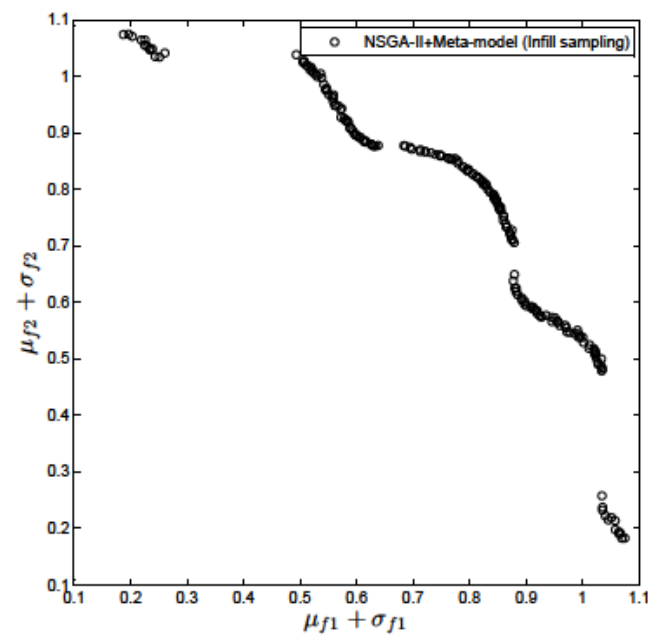

(b)

Figura 6.13: Problema TCMO5. Frentes de Pareto obtenidos tras aplicar el algoritmo NSGA-II sobre los meta-modelos actualizados (b) y sin actualizar (a).

\begin{tabular}{|c|c|c|c|}
\hline Problema & TCSO1 & TCSO2 & TCSO3 \\
\hline $\mathrm{N}^{\circ}$ de variables de diseño & 2 & 6 & 7 \\
\hline Tamaño de la población & 200 & 200 & 200 \\
\hline Número de generaciones & 100 & 270 & 200 \\
\hline Tamaño DoE inicial & 10 & 100 & 100 \\
\hline Tamaño actualizacion crit. $\mathscr{C}_{2}{ }^{(a)}$ & 6 & 43 & 33 \\
\hline Tamaño actualizacion crit. $\mathscr{C}_{1}^{(a)}$ & 50 & 151 & 102 \\
\hline Tamaño total del DoE & 66 & 294 & 235 \\
\hline Número total de evaluaciones & 330 & 13395 & 1175 \\
\hline $\mathrm{It}_{\max }(a)$ & 100 & 300 & 300 \\
\hline $\mathrm{Crit}_{\max }(a)$ & $1 \mathrm{E}-4$ & $1 \mathrm{E}-4$ & $1 \mathrm{E}-4$ \\
\hline NumFeas $_{\min }{ }^{(a)}$ & 11 & 20 & 20 \\
\hline $\operatorname{tolg}^{(a)}$ & $1 \mathrm{E}-6$ & $1 \mathrm{E}-6$ & $1 \mathrm{E}-6$ \\
\hline DIRECT maxiter $^{(a)}$ & 1000 & 1000 & 1000 \\
\hline DIRECT maxevals ${ }^{(a)}$ & 1000 & 1000 & 1000 \\
\hline $\mathrm{K}^{(a)}$ & 10 & 90 & 90 \\
\hline
\end{tabular}

(a) Parámetros del algoritmo EHV-EMO.

Tabla 6.7: Problema TCSO\#. Parámetros del algoritmo EHV-EMO . 


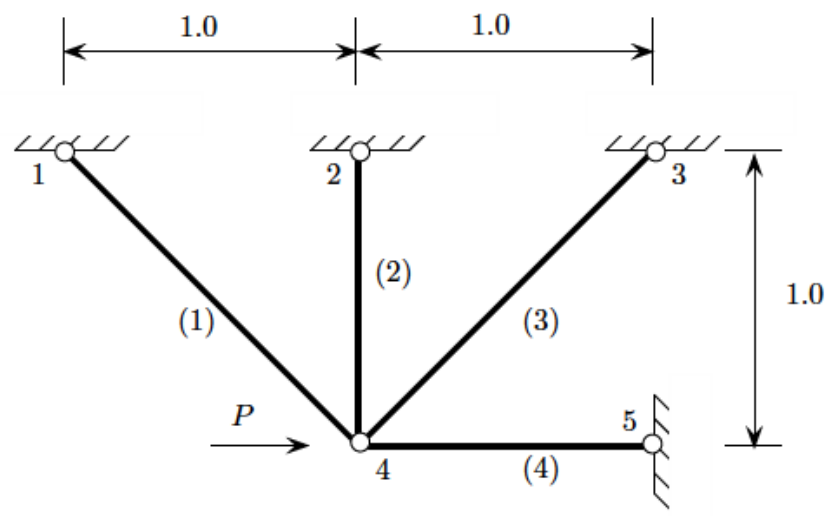

Figura 6.14: Problema TCSO1: geometría de la estructura.

$P$. Las áreas $A_{1}$ (barras 1 y 3 ) y $A_{2}$ (barras 2 y 4 ) se consideran variables de diseño deterministas. Los módulos de Young $E_{1}$ (barras 1 y 3) y $E_{2}$ (barras 2 y 4) se consideran variables aleatorias que siguen distribuciones normales con medias $\mu_{E_{1}}=210 \mathrm{y} \mu_{E_{2}}=100 \mathrm{y}$ desviaciones estándar $\sigma_{E_{1}}=21 \mathrm{y} \sigma_{E_{2}}=5$. La densidad del material es $\rho=1$. El problema consiste en la minimización del desplazamiento horizontal $(u)$ del nudo 4 , sujeto a una restricción de masa $w \leq 5$. La formulación del problema de diseño óptimo robusto es:

$$
\operatorname{TCS01}\left\{\begin{array}{c}
\operatorname{mín}_{\mu_{A_{1}} \mu_{A_{2}}}:\left\{\mu_{u_{4}}, \sigma_{u_{4}}\right\}, \\
\text { s.a. } \quad: \quad w \leq 5, \\
\quad 0 \leq \mu_{A_{1}} \leq 2,0 \leq \mu_{A_{2}} \leq 2 .
\end{array}\right.
$$

El problema se resuelve utilizando el algoritmo EHV-EMO junto con los meta-modelos de la media y la desviación estándar del desplazamiento. Los momentos estadísticos se obtienen utilizando una reducción unidimensional con $N_{1}=2$ puntos. El diseño de experimentos inicial está compuesto por 10 puntos, generados mediante un Hypercubo Latino con el criterio maximin (maximizar la distancia entre puntos) y 1000 iteraciones. Los parámetros del algoritmo están recogidos en la Tabla 6.7.

En la muestra inicial existen 5 diseños factibles. Este número se incrementa hasta 11 tras 6 iteraciones del algoritmo utilizando el criterio de búsqueda de diseños factibles. Tras verificar la condición NumFeas min $_{\text {min }} \geq 11$ comienza la $^{2}$ búsqueda de candidatos a formar parte del frente de soluciones no dominadas. La Figura 6.15, en su parte izquierda, muestra el mapa de contornos del criterio de búsqueda junto con los puntos pertenecientes a la muestra inicial (círculos negros), los añadidos en iteraciones previas (cuadrados blancos), y el candidato seleccionado en la iteración anterior (cuadrado verde). En la parte derecha de la Figura 6.15 se muestra la evolución del frente de Pareto estimado junto con el 
frente de Pareto de referencia. El algoritmo finaliza tras 50 iteraciones, en las que obtiene una aproximación del frente de Pareto formada por 16 puntos. El número total de evaluaciones del modelo de simulación es de 330.

La Figura 6.16 muestra las estructuras obtenidas para las soluciones extremas del frente. La estructura de la Figura 6.16a corresponde a la solución con mínima media o mejor performance, mientras que la estructura de la Figura $6.16 \mathrm{~b}$ la solución con mínima desviación o mayor robustez.

Para ilustrar la importancia de considerar la incertidumbre epistémica del meta-modelo durante la optimización, se resuelve el problema mediante la explotación directa de los meta-modelos de los momentos estadísticos (Figuras 6.17a y 6.17b) con el algoritmo NSGA-II. El tamaño de la población es de 200 y el número de generaciones es de 100. Para realizar una comparación justa desde el punto de vista computacional, los meta-modelos se ajustan a partir de un diseño de experimentos de 66 puntos. El frente de Pareto resultante se muestra en la Figura 6.17d. En comparación con el frente obtenido mediante la estrategia adaptativa, el número de soluciones es mayor pero están muy alejadas del frente de referencia. Esto es debido a que, a pesar de que el espacio de diseño es de baja dimensionalidad, los momentos estadísticos presentan una gran región horizontal, que dificulta tanto el ajuste global del meta-modelo como la optimización. Esta característica es común a los problemas de barras de nudos articulados, como consecuencia del crecimiento exponencial de la respuesta estructural cuando las áreas de los elementos tienden a cero.

La precisión de la solución del problema viene dada numéricamente por los siguientes indicadores: (1) distancia entre generaciones (GD=3.1366e-3), (2) máximo error en el frente de Pareto $(\mathrm{MPFE}=3.1044 \mathrm{e}-2)$ y (3) ratio de hipervolumen $(\mathrm{HR}=0.9375)$.

\subsubsection{Problema TCSO2. Diseño óptimo robusto de una torre de 25 barras}

El objetivo del problema es la minimización de la compliance de la estructura de 25 barras de la Figura 6.18 (Doltsinis y Kang, 2004). Las variables de diseño son las áreas de las barras, agrupadas en seis grupos. Las coordenadas de los elementos de la estructura y la agrupación se muestran en la Tabla 6.8 y en la Figura 6.18 respectivamente. La densidad del material es $\rho=1.0$. Los nudos 1 y 2 están sometidos a unas cargas $p_{1 y}=p_{2 y}=p_{1 z}=p_{2 z}=-1 e 4$, mientras que los nudos 3 y 6 están sometidos a cargas con magnitud aleatoria en la dirección $x$. Las fuerzas aplicadas a los nudos 3 y 6, el módulo de elasticidad longitudinal, y las áreas de los elementos son consideradas variables aleatorias cuyas propiedades se muestran en la Tabla 6.9. El valor medio del peso de la estructura está limitado a 750, y el valor máximo de las tensiones es de 5000 con una probabilidad del $99.99 \%$. 


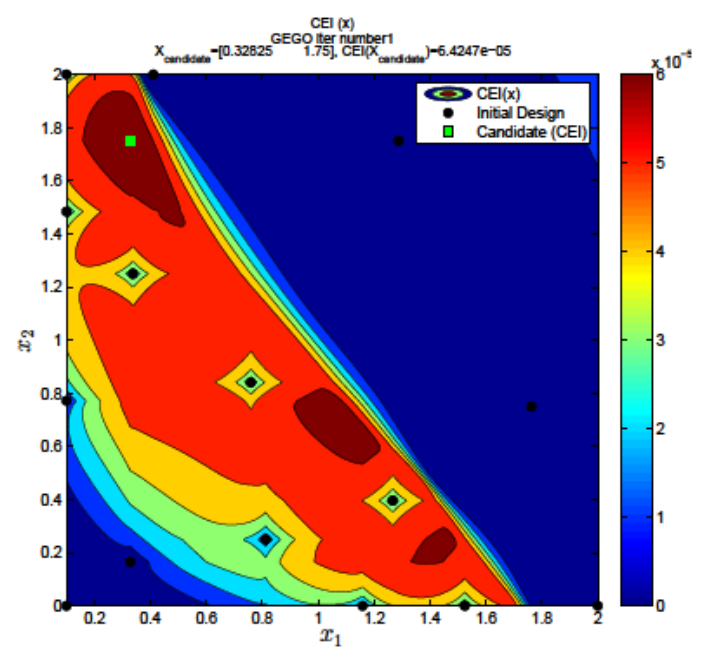

(a) Iter $=1$

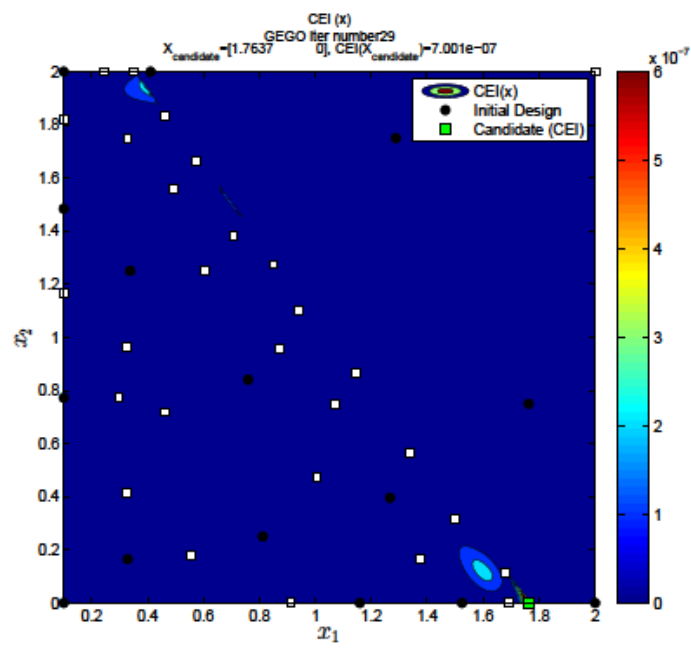

(c) Iter $=29$

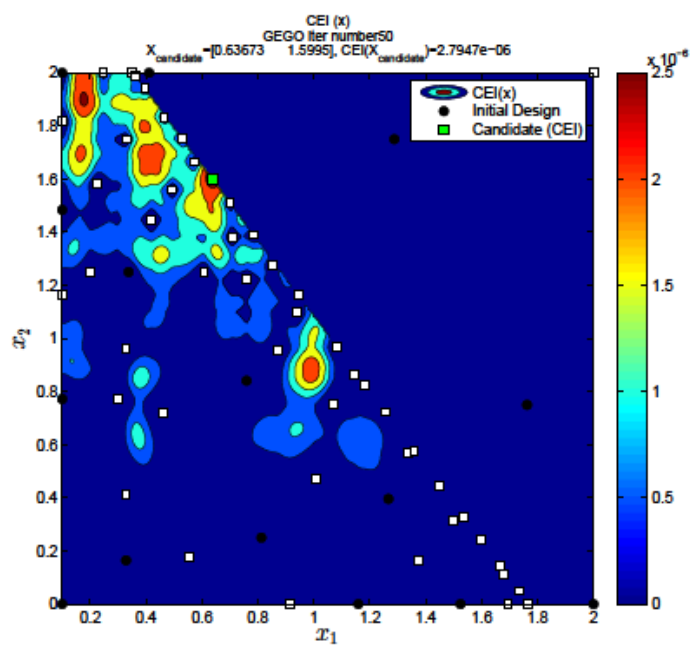

(e) Iter $=50$

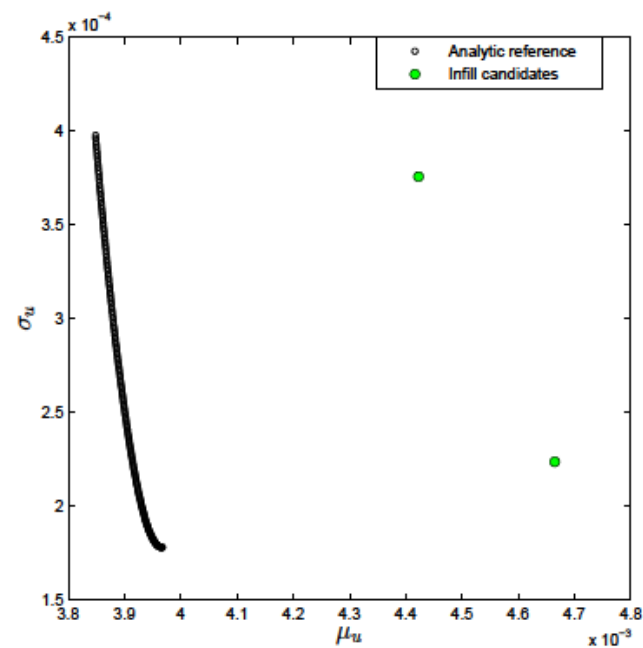

(b) Iter $=1$

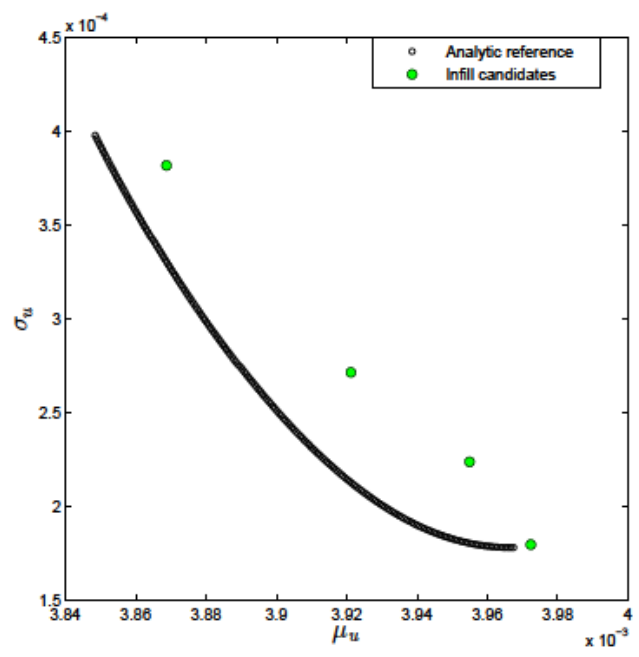

(d) Iter $=29$

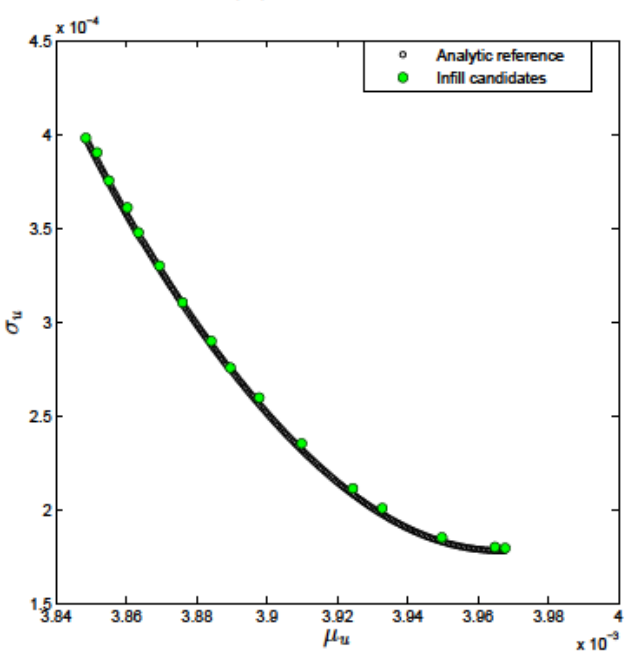

(f) Iter $=50$

Figura 6.15: Problema TCS01. Evolución del criterio de búsqueda. La muestra inicial se presenta como círculos negros. Los cuadrados blancos representan los puntos añadidos hasta la iteración. Los cuadrados verdes representan el candidato que maximiza el criterio en cada iteración. 


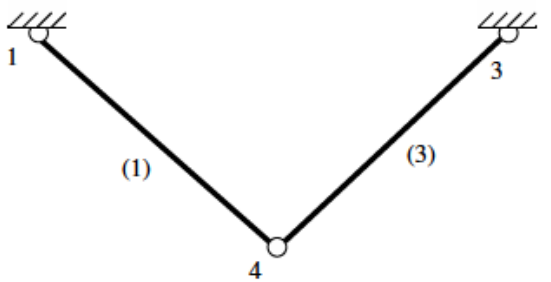

(a) Diseño A

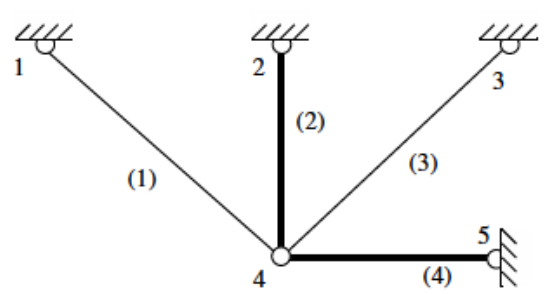

(b) Diseño B

Figura 6.16: Problema TCS01. Soluciones óptimas de máxima "performance" (Diseño A) y máxima robustez (Diseño B).

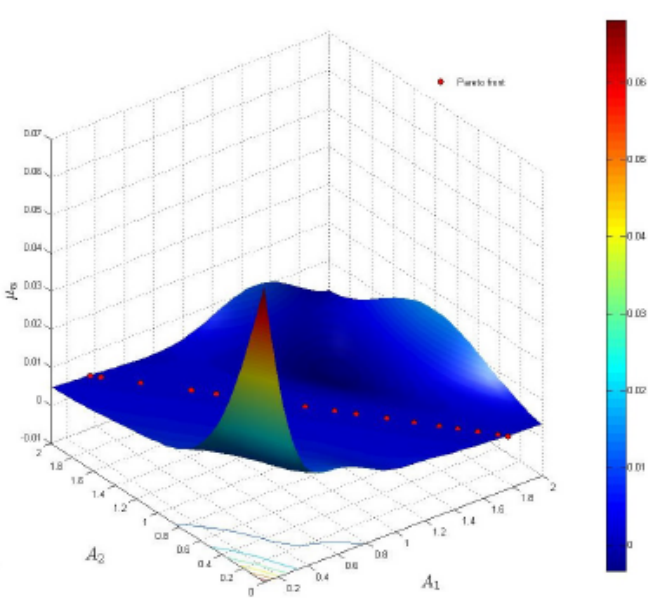

(a) $\mu_{u}$

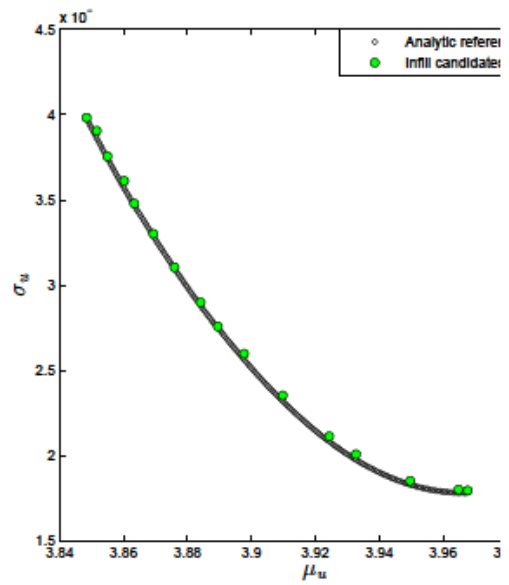

(c)

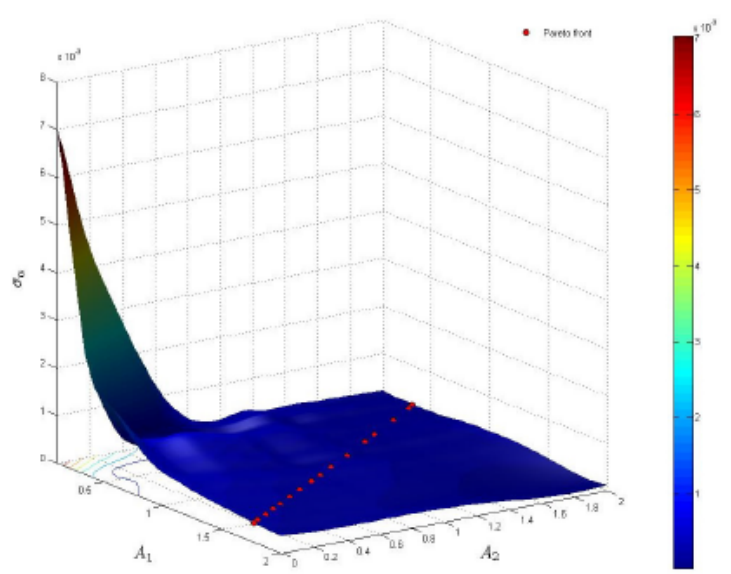

(b) $\sigma_{u}$

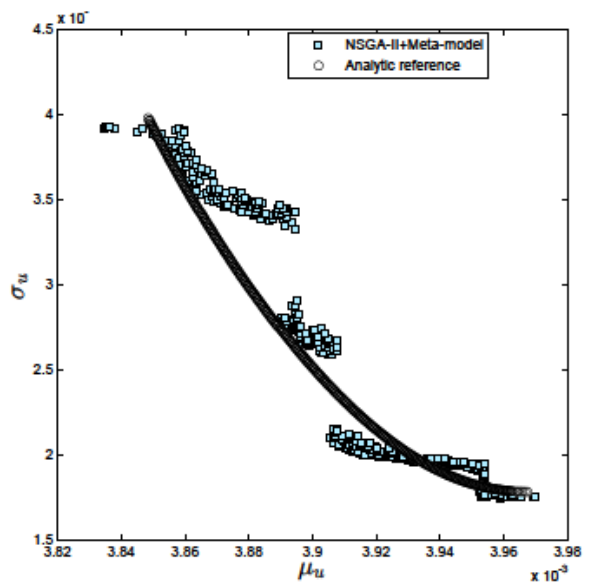

(d)

Figura 6.17: Problema TCS01. (a-b) conjunto de soluciones óptimas de Pareto sobre los modelos Kriging de las funciones objetivo tras 50 iteraciones del algoritmo de actualización. (c) frente de Pareto tras 50 iteración de la estrategia adaptativa (64 puntos en total); (d) frente de Pareto utilizando meta-modelos con una muestra inicial de 64 puntos. 


\begin{tabular}{cccc}
\hline Nudo & $\mathrm{X}$ & $\mathrm{Y}$ & $\mathrm{Z}$ \\
\hline \hline 1 & -37.5 & 0.0 & 200.0 \\
2 & 37.5 & 0.0 & 200.0 \\
3 & -37.5 & 37.5 & 100.0 \\
4 & 37.5 & 37.5 & 100.0 \\
5 & 37.5 & -37.5 & 100.0 \\
6 & -37.5 & -37.5 & 100.0 \\
7 & -100.0 & 100.0 & 0.0 \\
8 & 100.0 & 100.0 & 0.0 \\
9 & 100.0 & -100.0 & 0.0 \\
10 & -100.0 & -100.0 & 0.0 \\
\hline
\end{tabular}

Tabla 6.8: Problema TCSO2. Coordenadas de los nudos de la estructura de 25 barras.

\begin{tabular}{llll}
\hline Variable aleatoria & Distribución & Media & Desviación estandar \\
\hline \hline$E_{I}-E_{V}$ & Normal & $1.0 \mathrm{e} 7$ & $2.0 \mathrm{e} 5$ \\
$E_{V I}$ & Normal & $1.0 \mathrm{e} 7$ & $1.5 \mathrm{e} 6$ \\
$p_{3 x}$ & Normal & $5.0 \mathrm{e} 2$ & 50 \\
$p_{6 x}$ & Normal & $5.0 \mathrm{e} 2$ & 50 \\
$A_{I}-A_{V I}$ & Normal & $\mu_{\mathrm{A}_{I}-\mathrm{A}_{\mathrm{VI}}}$ & $0,05 \times \mu_{\mathrm{A}_{I}-\mathrm{A}_{\mathrm{VI}}}$ \\
\hline
\end{tabular}

Tabla 6.9: Problema TCSO2. Distribución de las variables aleatorias.

$$
\operatorname{TCS02~}\left\{\begin{aligned}
& \operatorname{mín}_{\mathrm{A}_{i}, \forall i=I, \ldots, V I}:\left\{\mu_{\text {compliance, }}, \sigma_{\text {compliance }}\right\}, \\
& \\
& \quad \mu_{w} \leq 750, \\
& \text { s.a. }: \quad \mathbb{P}\left[\left|S^{i}\right| \leq 5000\right] \geq 0.99, \forall i=\mathrm{I}, \ldots, \mathrm{VI}, \\
& 0.05 \leq A_{i} \leq 10, \forall i=\mathrm{I}, \ldots, \mathrm{VI},
\end{aligned}\right.
$$

El problema se resuelve utilizando tres enfoques. En los enfoques (1) y (2) se acopla el algoritmo de optimización NSGA-II con los resultados estadísticos obtenidos en el proceso de propagación de incertidumbre. En el enfoque (1) se utiliza una simulación MonteCarlo de 1e5 puntos, mientras que en el enfoque (2) se utiliza el algoritmo EKDRM con una reducción unidimensional con $N_{1}=4$ puntos. En este último caso se necesitan un total de 57 evaluaciones del modelo de simulación para una ejecución del algoritmo de propagación de incertidumbre. En el enfoque (3) se utiliza el algoritmo propuesto en este capítulo, basado en los meta-modelos estocásticos de la compliance, el volumen y las tensiones de los elementos. En este caso los meta-modelos de los momentos estadísticos obtenidos mediante reducción dimensional se ajustan a partir de un diseño de experimentos de 100 puntos, generado con un Hypercubo Latino en el espacio de diseño. A partir de la información estadística de la predicción, se ejecuta el algoritmo EHV-EMO con los parámetros definidos en la Tabla 6.7. 


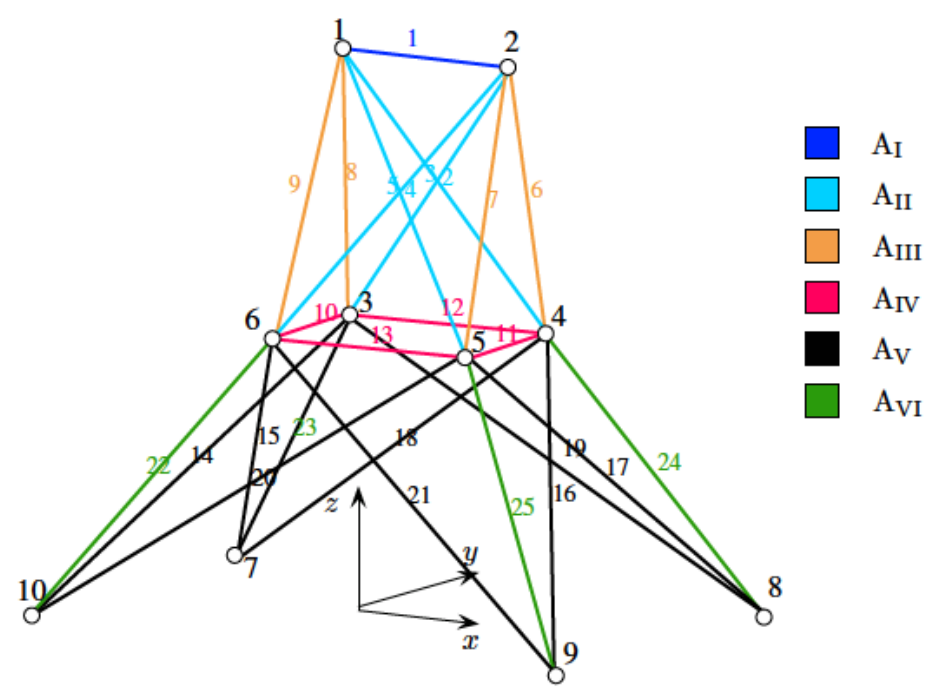

Figura 6.18: Problema TCSO2. Estructura de 25 barras.

\begin{tabular}{cccc}
\hline Comparación & HR & GD & MPFE \\
\hline \hline EHV-EMO vs EKDRM & 0.7781 & 0.0219 & 0.1469 \\
MonteCarlo vs EKDRM & 0.8642 & 0.0029 & 0.0643 \\
MonteCarlo vs EHV-EMO & 0.7140 & 0.0219 & 0.1531 \\
\hline
\end{tabular}

Tabla 6.10: Problema TCS02. Resumen de los resultados numéricos.

Tras 151 iteraciones con el algortimo EHV-EMO se alcanza una aproximación discreta del frente de Pareto formada por 12 diseños. Una primera comparación entre los frentes de Pareto obtenidos utilizando simulación MonteCarlo (Figura 6.19a) y reducción unidimensional (Figura 6.19b) muestra ciertas diferencias debidas al error sistemático originado por el método de reducción dimensional. Estas diferencias se traducen numéricamente en el valor de los indicadores recogidos en la Tabla 6.10 .

Respecto a los diseños obtenidos, la Figura 6.20 muestra los diseños extremos del frente. Las líneas de color rojo representan elementos a tracción y las líneas de color azul elementos a compresión. La Figura 6.20a presenta la solución con mínima masa. En este diseño, las áreas de los elementos del grupo I y II correspondientes a la parte superior de la torre, toman el valor mínimo permitido (línea discontinua). Para el diseño de mayor robustez (Figura 6.20b), el valor de dichas áreas se incrementa a costa de una disminución de las áreas de los elementos de los grupos III y IV.

En lo referente al coste computacional, el número total de evaluaciones del modelo de simulación es de $N_{\text {evals }}=13395$, que es dos órdenes de magnitud inferior al requerido por los enfoques acoplados, $N_{\text {evals }}=5.4 e 9$ para el basado en MonteCarlo y $N_{\text {evals }}=3.0 e 6$ para el basado en reducción dimensional. 


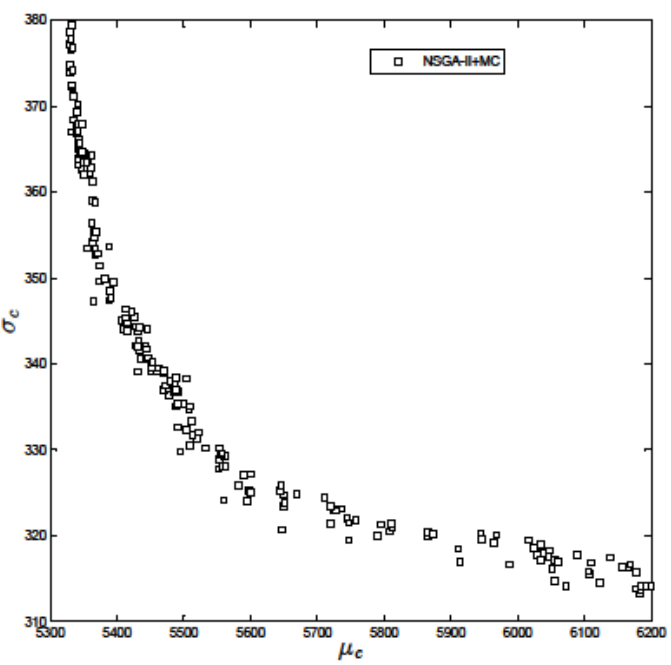

(a)

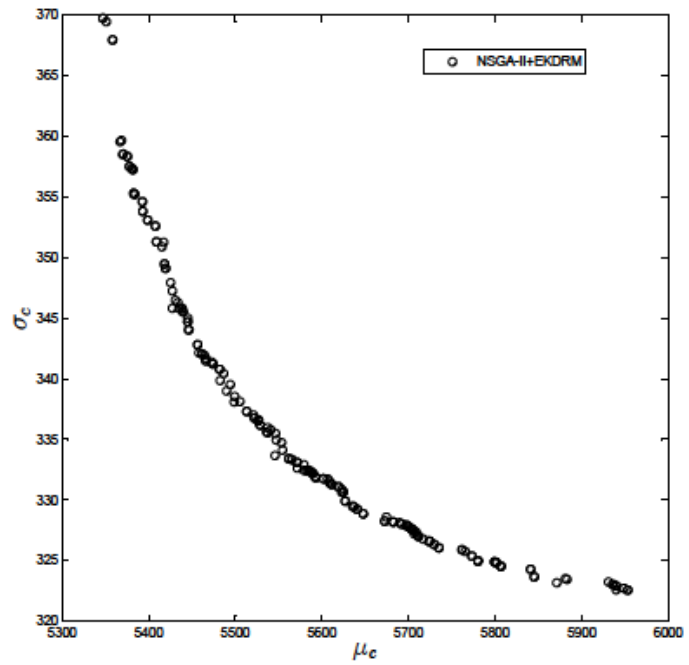

(b)

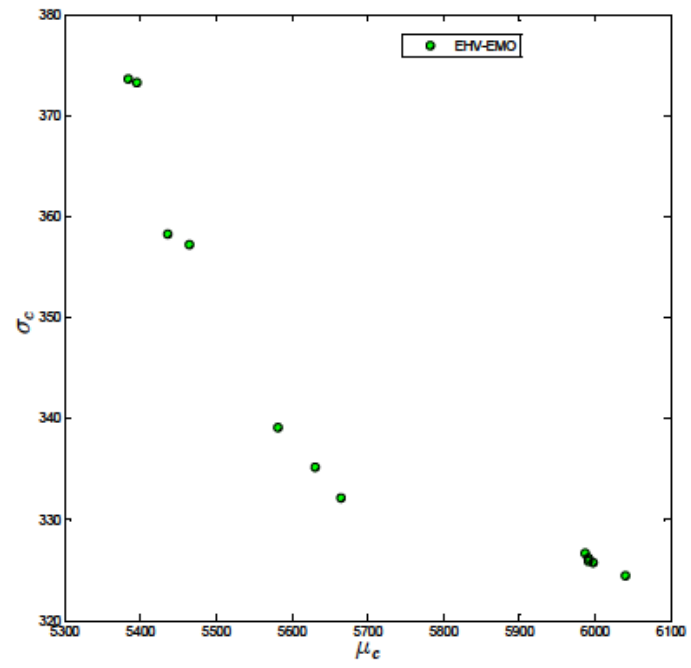

(c)

Figura 6.19: Problema TCS02. Frentes de Pareto: (a) Solución obtenida con NSGA-II y simulación MonteCarlo; (b) solución obtenida con NSGA-II y EKDRM; (c) Solución obtenida con el algoritmo EHV-EMO.

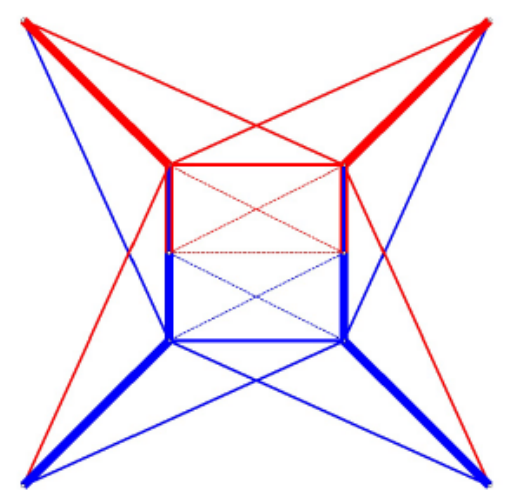

(a)

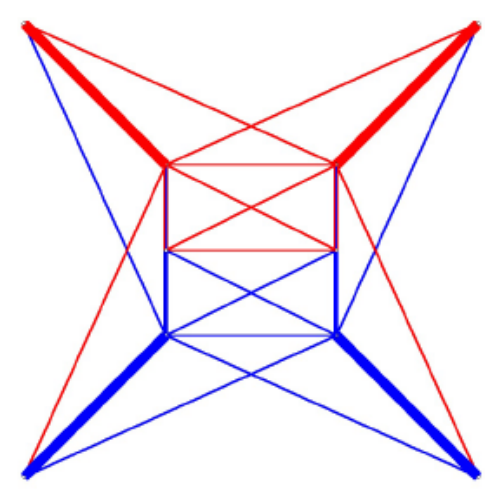

(b)

Figura 6.20: Problema TCS02. (a) diseño con mínima $\mu_{c}$; (b) diseño con mínima $\sigma_{c}$. 


\subsubsection{Problema TCSO3: Diseño óptimo robusto de un domo de 120 barras}

Se estudia el problema de diseño óptimo robusto de una estructura de 120 barras de nudos articulados, frente a incertidumbres en las condiciones de carga y propiedades del material. Las características geométricas de la estructura se muestran en la Figura 6.21.
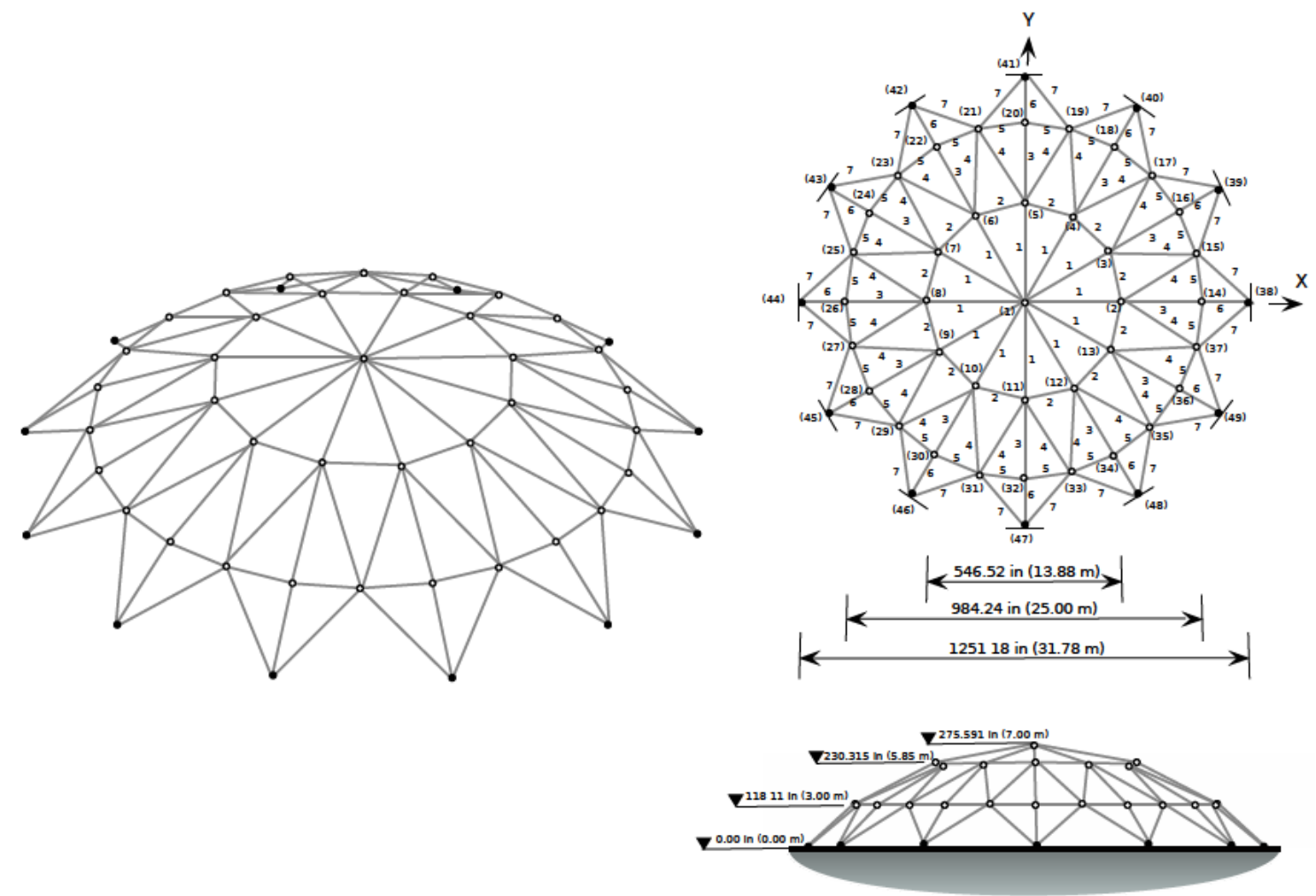

Figura 6.21: Problema TCS03. Domo de 120 barras de nudos articulados.

El problema está adaptado de trabajos previos como los de Soh y Yang (1996), Lee y Geem (2004) y Kaveh y Talatahari (2009), que abordan el problema de optimización determinista. Bajo este enfoque se busca dimensionar las áreas de las barras para obtener la estructura de mínimo peso. En el problema de optimización determinista se consideran las siguientes restricciones de tensión de acuerdo al código ASD (AISC, 1989):

$$
\begin{cases}\mathrm{S}_{i}^{+}=0.6 F_{y}, & \mathrm{~S}_{i} \geq 0 \\ \mathrm{~S}_{i}^{-}, & \mathrm{S}_{i}<0,\end{cases}
$$

donde $S_{i}^{-}$se calcula a partir del coeficiente de esbeltez $\left(\lambda_{i}\right)$ :

$$
\mathrm{S}_{i}^{-}= \begin{cases}{\left[\left(1-\frac{\lambda_{i}^{2}}{2 C_{c}^{2}}\right) F_{y}\right] /\left(\frac{5}{3}+\frac{3 \lambda_{i}}{8 C_{c}}-\frac{\lambda_{i}^{3}}{8 C_{c}^{3}}\right),} & \lambda_{i}<C_{c} \\ \frac{12 \pi^{2} E}{23 \lambda_{i}^{2}}, & \lambda_{i} \geq C_{c},\end{cases}
$$


siendo $E$ es el módulo de Young, $F_{y}$ el límite elástico del acero, $C_{c}=\sqrt{\frac{2 \pi^{2} E}{F_{y}}}$ divide las regiones de pandeo elástico e inelástico, $\lambda_{i}$ es el coeficiente de esbeltez $\left(\lambda_{i}=k L_{i} / r_{i}\right), k$ es el factor de longitud efectiva, $L_{i}$ es la longitud de la barra y $r_{i}$ es el radio de giro. El radio de giro $r_{i}$ se puede expresar en términos de la sección transversal de la barra, $r_{i}=a A_{i}^{b}$ (Saka, 1990). Las constantes $a$ y $b$ dependen del tipo de perfil seleccionado. En este ejemplo, se consideran perfiles tubulares para la totalidad de las barras que componen la estructura con $a=0.4993 \mathrm{y}$ $b=0.6777$. El módulo de elasticidad es $E=30,450 \mathrm{ksi}$ y la densidad del material $\rho=0.288 \mathrm{lb} / \mathrm{in}^{3}$. El límite elástico del acero es de $\sigma=58 \mathrm{ksi}$. La estructura está sometida a un estado de cargas vertical cuyo valor nominal es de -13.49 kips en el nudo 1, -6.744 kips en los nudos 2 a 14 y -2.248 kips en el resto de nudos. Las condiciones de contorno son restricciones en los tres grados de libertad de los nudos 38-49 (Figura 6.21). Las variables de diseño son las áreas de los elementos agrupadas en los 7 grupos identificados numéricamente en la Figura 6.21. El límite inferior de las variables de diseño es $x^{\mathrm{min}}=0.775 \mathrm{in}^{2}$.

La incertidumbre del problema se modeliza a través de dos variables aleatorias Gaussianas estándar $\xi_{1}$ y $\xi_{2}$. La variable $\xi_{1}$ está relacionada con la incertidumbre en las cargas externas mediante la expresión $F=F_{0} \cdot\left(1+\xi_{1}\right)$, mientras que la variable $\xi_{2}$ está relacionada con el módulo de Young mediante la expresión $E=E_{0} \cdot\left(1+\xi_{2}\right)$ y se asume que el valor es el mismo para todas las barras. Como indicador de la robustez de la estructura, frente a variaciones en las condiciones de carga y en las propiedades del material, se toma la desviación estándar del desplazamiento máximo $\left(\sigma_{\max \left(\mid u_{x, y, z}^{(i)}\right)}\right)$ en cualquiera de los tres grados de libertad. Como indicador de la calidad del diseño se mantiene la función objetivo determinista, la masa de la estructura $(w)$. Se consideran como restricciones de desigualdad el valor medio de las restricciones de tensión $\left(\mu_{S^{i}}\right)$ y el pandeo del problema determinista. El problema de optimización multi-objetivo se formula como sigue:

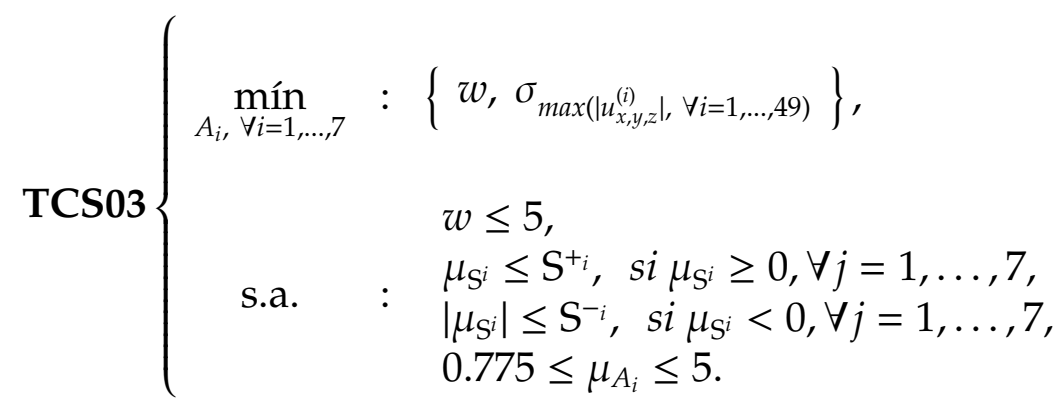

El problema se resuelve utilizando tres enfoques. En los dos primeros se acopla el algoritmo de optimización NSGA-II con los resultados estadísticos del proceso de propagación de incertidumbre. En el primer enfoque se propaga la incertidumbre con una simulación MonteCarlo de 1e3 puntos. En el segundo enfoque se propaga la incertidumbre con el algoritmo EKDRM propuesto en el capítulo anterior. En este último caso se utiliza una reducción unidimensional de $N_{1}=2$ puntos. El número total de evaluaciones del modelo de simulación para una ejecución del algoritmo de propagación de incertidumbre es de 5 . Como tercer enfoque se utiliza el algoritmo propuesto en este capítulo basado en los 
meta-modelos estocásticos del desplazamiento y de las tensiones en las barras de la estructura. En este caso, los meta-modelos de los momentos estadísticos obtenidos mediante reducción dimensional se ajustan a partir de un diseño de experimentos de 100 puntos, generados con un Hypercubo Latino en el espacio de diseño. A partir de la información estadística de la predicción proporcionada por los meta-modelos se ejecuta el algoritmo EHV-EMO con los parámetros definidos en la Tabla 6.7.

De acuerdo con Kaveh y Talatahari (2009), el diseño con mínimo peso determinista es 19707.77 lb. Este diseño presenta un desviación estándar del desplazamiento de 0.0485 in. Esta desviación se puede reducir a costa de un incremento en la masa de la estructura, tal y como muestran las Figuras 6.22a a 6.22c. En función del incremento de masa admitido por el diseñador, se puede alcanzar una reducción de la desviación estándar y por lo tanto un incremento de la robustez de la estructura de hasta un $56 \%$. La elección del mejor diseño forma parte de la etapa de toma de decisiones del problema multi-objetivo, y depende de los costes derivados de un incremento de masa así como de los coste generados por las consecuencias de una pérdida de robustez de la estructura.

Tras 102 iteraciones con el algortimo EHV-EMO se alcanza una aproximación discreta del frente de Pareto formada por 19 diseños. La evolución del criterio de búsqueda se muestra en la Figura 6.22d. Una primera comparación entre los frentes de Pareto obtenidos utilizando simulación MonteCarlo (Figura 6.22a) y reducción unidimensional (Figura 6.22b) muestra que existe una buena aproximación entre los dos frentes, exceptuando el valor de los diseños extremos. En ambos casos se utilizó el algoritmo NSGA-II con los mismos parámetros; sin embargo, se aprecia una mayor continuidad del frente generado con reducción dimensional. Esto se debe a que los resultados estadísticos proporcionados por el algoritmo EKDRM son "deterministas", es decir que para un mismo valor de los parámetros de entrada se obtiene un mismo valor de los momentos de salida. Esta situación no ocurre en el caso de la simulación MonteCarlo dado el carácter estocástico del método. El valor de los indicadores es: (1) distancia entre generaciones $(G D=0.0346),(2)$ máximo error en el frente de Pareto $(\mathrm{MPFE}=0.8525)$ y $(3)$ ratio de hipervolumen $(\mathrm{HR}=0.5510)$. El valor del hipervolumen es considerablemente inferior a 1 como consecuencia de las diferencias existentes en las regiones extremas de los frentes.

Comparando la aproximación discreta obtenida con el algoritmo EHV-EMO con los enfoques acoplados, se aprecia que dichos resultados tienen una mayor similitud con el enfoque basado en reducción unidimensional. Comparando estos dos frentes se obtienen los siguientes indicadores: (1) distancia entre generaciones $(\mathrm{GD}=0.0045)$, (2) máximo error en el frente de Pareto (MPFE=0.0366) y (3) ratio de hipervolumen $(\mathrm{HR}=0.9349)$. Tomando como referencia el frente obtenido con la simulación MonteCarlo, los valores de los indicadores son: (1) distancia entre generaciones (GD=0.1274), (2) máximo error en el frente de Pareto (MPFE=0.8503) y (3) ratio de hipervolumen $(\mathrm{HR}=0.5102)$. Se aprecia de nuevo una caída en el 

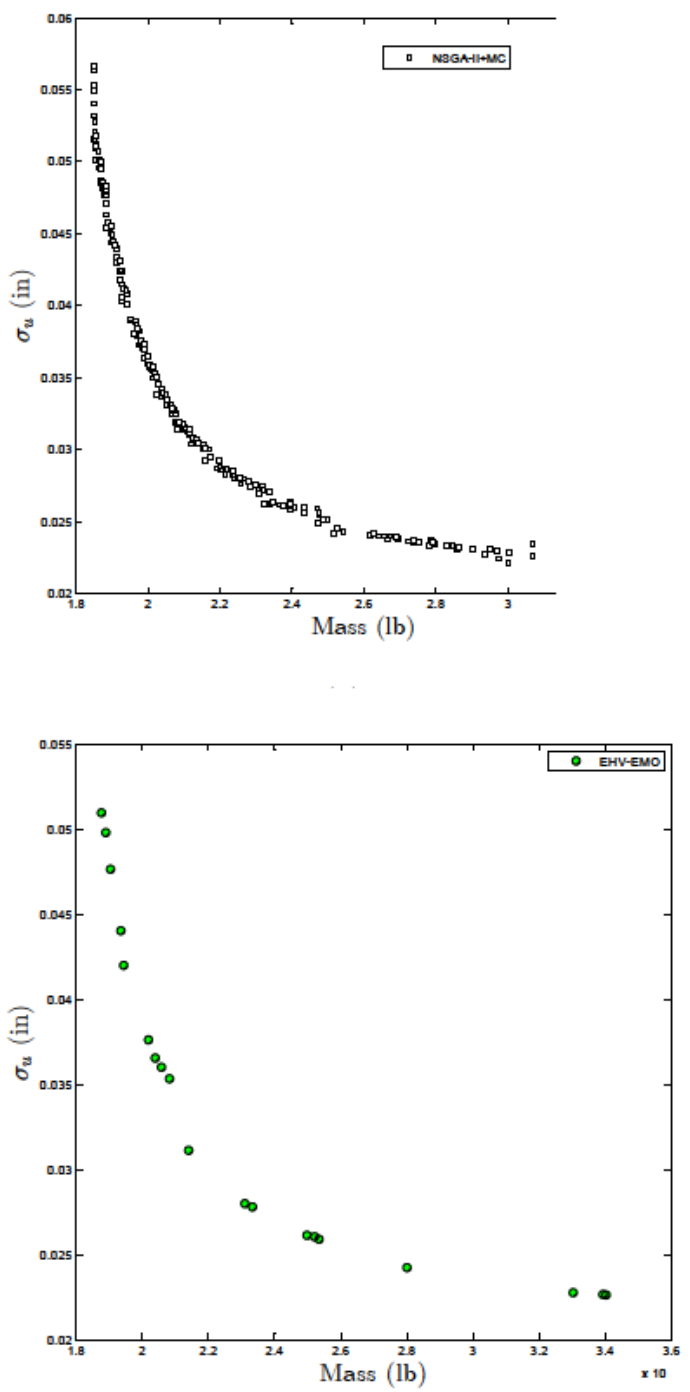

(c)

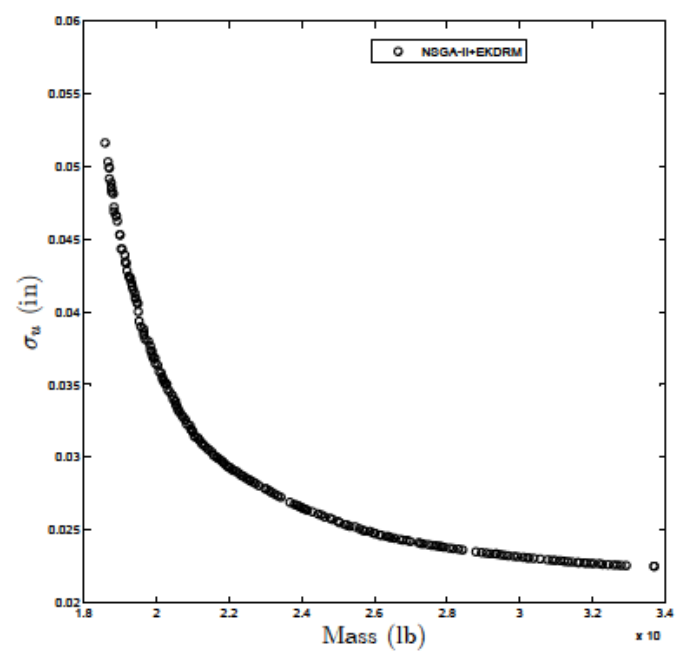

(b)

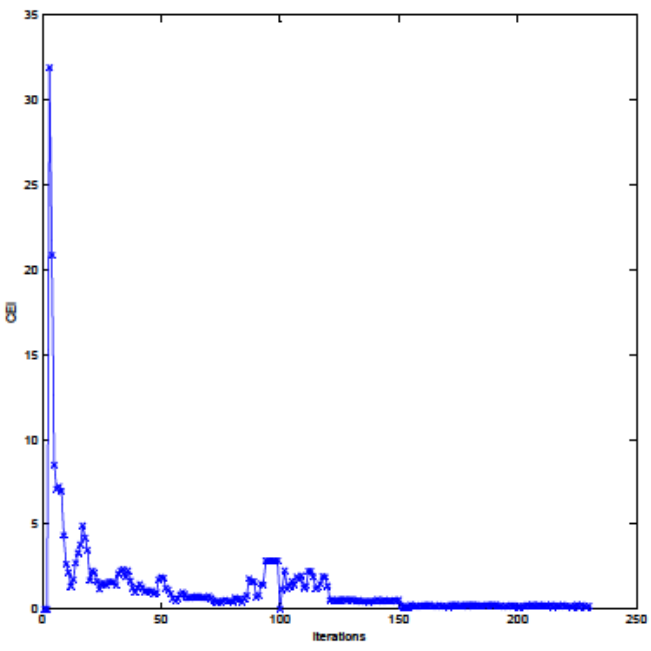

(d)

Figura 6.22: Problema TCS03. Frentes de Pareto: (a) solución obtenida con NSGA-II y simulación MonteCarlo; (b) solución obtenida con NSGA-II y EKDRM (S=1, N 1 = 1); (c) solución obtenida con EHV-EMO. (d) Evolución del criterio de búsqueda CEI.

valor del ratio de hipervolumen como consecuencia del error asociado con el procedimiento de propagación de incertidumbre.

Respecto al coste computacional, el número total de evaluaciones del modelo de simulación es de $N_{\text {evals }}=1150$, un número dos órdenes de magnitud inferior al requerido por los enfoques acoplados, $N_{\text {evals }}=8 e 7$ para el basado en MonteCarlo $\mathrm{y} N_{\text {evals }}=4 e 5$ para el basado en reducción dimensional. 


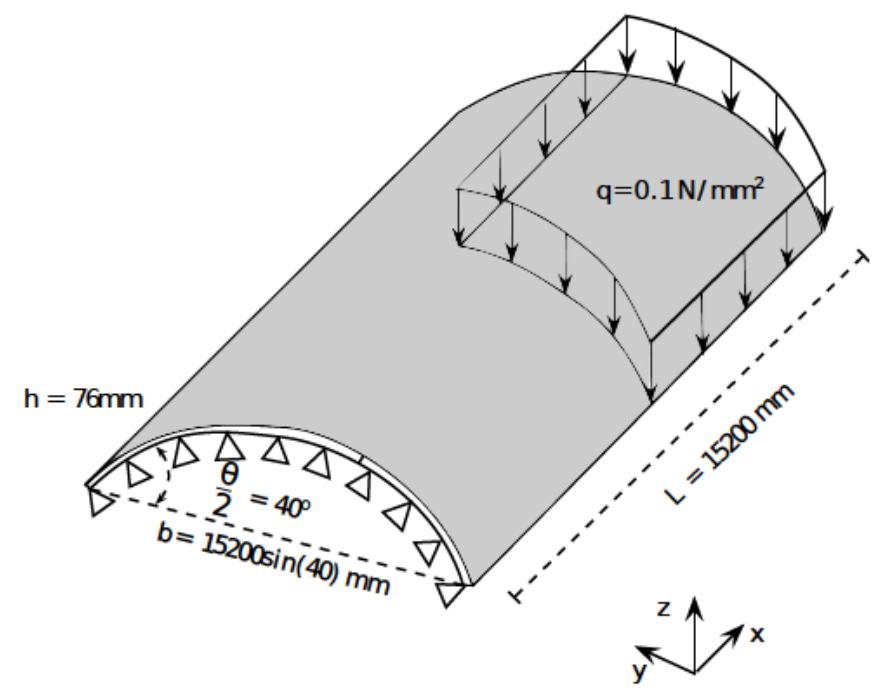

Figura 6.23: Geometría de la lámina cilíndrica Scordelis-Lo (Scordelis y Lo, 1961).

\subsubsection{Aplicación al diseño óptimo robusto de láminas imperfectas frente a pandeo}

El problema que se estudia en esta sección es una continuación del problema de propagación de incertidumbre abordado en la sección 5.4.5.

\subsubsection{Diseño óptimo robusto de una lámina cilíndrica sin rigidizadores. Scordelis-Lo shell roof}

Se estudia el problema de diseño óptimo robusto de la lámina cilíndrica de la Figura 6.23 abordado previamente por Dubourg et al. (2013). La lámina está sometida a fluctuaciones en el módulo de elasticidad, límite elástico, espesor e imperfección inicial. Las dimensiones de la lámina se muestran en la Figura 6.23. La lámina está sometida a una carga uniforme actuando sobre la superficie superior de la lámina. Los bordes longitudinales de la lámina están libres mientras que el desplazamiento radial de los bordes circunferenciales está impedido.

\subsubsection{Modelo de elementos finitos}

La lámina se analiza en régimen estático no lineal con el programa de elementos finitos ANSYS. Se considera una ley tensión deformación bi-lineal. El problema se discretiza con una malla de 28x40 elementos del tipo SHELL181 (elemento lámina de 4 nodos), obteniendo un total de 1189 nodos y 7134 grados de libertad. La carga crítica se obtiene mediante un algoritmo de longitud de arco. La Figura 6.24a ilustra la deformada amplificada de la lámina perfecta en el punto de carga crítica $P_{\text {crit }}=0.2449 \mathrm{MPa}$. La Figura $6.24 \mathrm{~b}$ representa la curva carga crítica frente a desplazamiento vertical del nodo localizado en el centro de la superficie exterior 


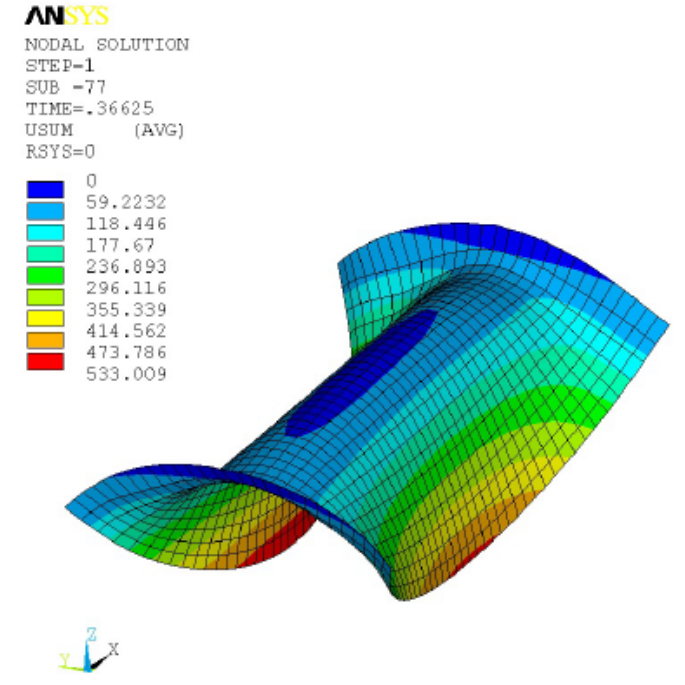

(a)

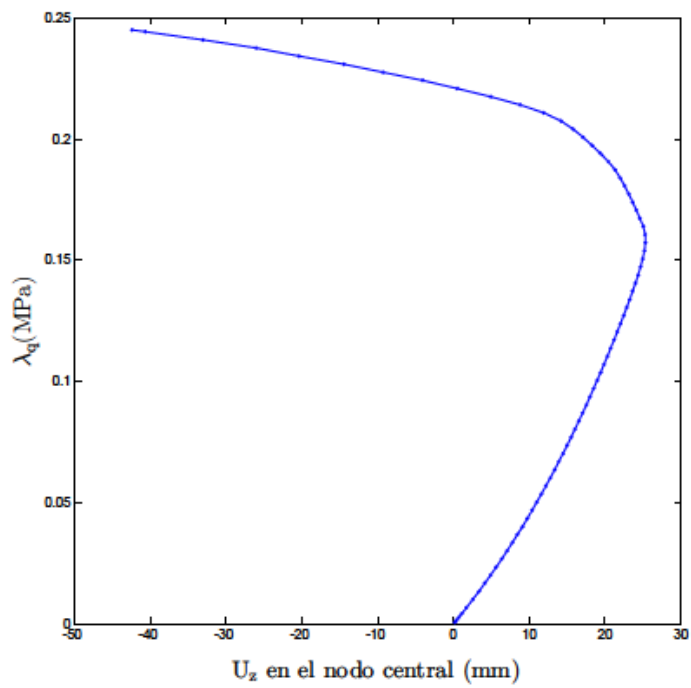

(b)

Figura 6.24: Análisis de no lineal de la lámina Scordelis-Lo shell roof con la configuración perfecta. (a) Deformada para la carga crítica (10x); (b) curva factor de carga crítica desplazamiento.

\begin{tabular}{llllll}
\hline Variable & Distribución & Media & COV & $\begin{array}{l}\text { parámetro de localización } \\
\lambda\end{array}$ & $\begin{array}{l}\text { parámetro de escala } \\
\xi\end{array}$ \\
\hline \hline$E(\mathrm{MPa})$ & Lognormal & 200,000 & $3 \%$ & 12.2056 & 0.03 \\
$F_{y}(\mathrm{MPa})$ & Lognormal & 390 & $7 \%$ & 5.9637 & 0.0699 \\
$h(\mathrm{~mm})$ & Lognormal & $\mu_{h}{ }^{(1)}$ & $5 \%$ & $\log \left(\frac{\mu_{h}}{\sqrt{1+0.05^{2}}}\right)$ & $\sqrt{\log \left(\frac{\left(0.05 \mu_{h}\right)^{2}}{1+\mu_{h}^{2}}\right)}$ \\
\hline
\end{tabular}

(1) variable de diseño.

Tabla 6.11: Problema Scordelis-Lo (II). Parámetros de los campos aleatorios de $E, F_{y}, \mathrm{y} h$.

de la lámina.

\subsubsection{Modelización de las variaciones aleatorias en el espacio}

Se consideran como fuentes de incertidumbre el módulo de Young $(E)$, el límite elástico $\left(F_{y}\right)$, el espesor de la lámina $(h)$, y la imperfección inicial $(\zeta(\mathbf{x}))$. La variabilidad espacial de las tres primeras se modela de forma probabilista mediante tres campos aleatorios de tipo lognormal. La Tabla 6.11 recoge las características de las distribuciones en cada caso. Los campos aleatorios se generan mediante la transformación presentada en la sección 5.4.5. Se utiliza una correlación isotrópica exponencial cuadrática con una longitud de correlación $l=3500(\mathrm{~mm})$. La Figura 6.25 muestra tres realizaciones de los campos aleatorios de $E, F_{y}, \mathrm{y} h$. 


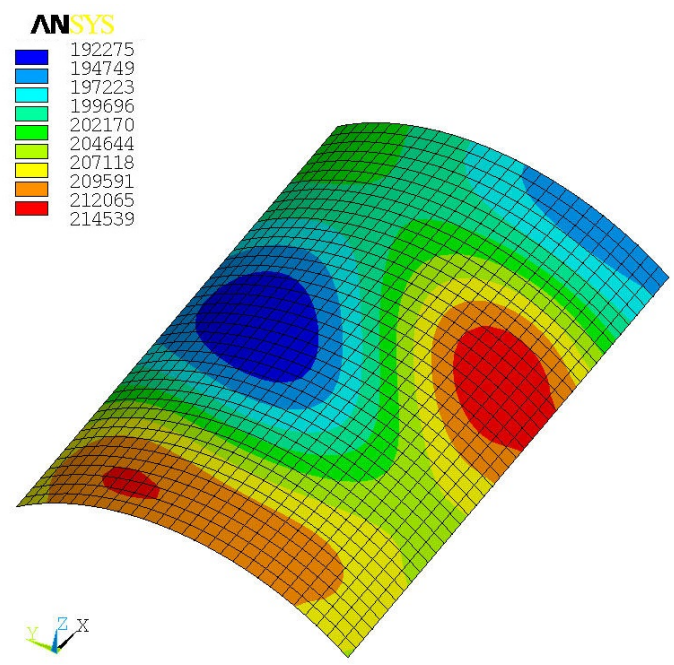

(a) $E(\mathrm{MPa})$

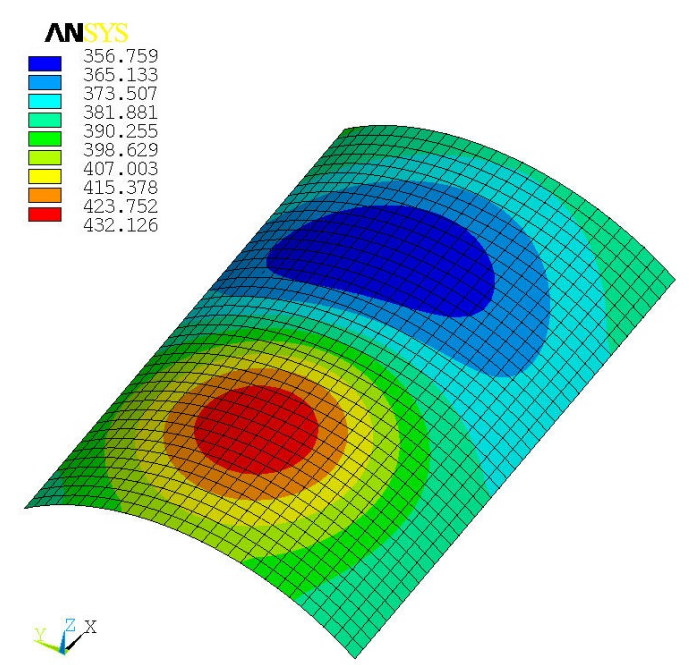

(b) $F_{y}(\mathrm{MPa})$

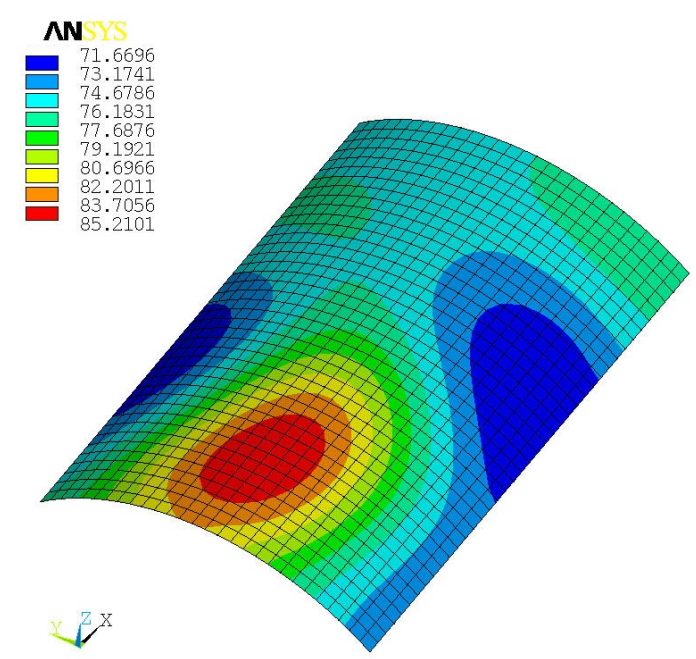

(c) $h(\mathrm{~mm})$

Figura 6.25: Problema Scordelis-Lo shell roof (II). Realizaciones de los campos aleatorios de E, $F_{y}, \mathrm{yh}$.

\subsubsection{Modelado de la imperfección}

La imperfección geométrica de la lámina se modela mediante una combinación aleatoria de los autovectores asociados a los primeros tres autovalores obtenidos mediante un análisis de pandeo lineal de la lámina perfecta. Las Figuras 6.26a a 6.26c muestran los tres primeros modos de pandeo de la lámina. La realización de la imperfección geométrica se obtiene a partir de la expresión:

$$
\zeta(\mathbf{x})=\sum_{i=1}^{3} \Xi_{\zeta, i} \mathbf{U}_{i}(\mathbf{x}),
$$


donde $\mathbf{U}_{i}(\mathbf{x})$ es el i-ésimo autovector y $\Xi_{\zeta, i}, \forall i=1,2,3$ son variables aleatorias Gaussianas independientes, con media cero y desviación estándar $9.5 \mathrm{~mm}$. Dichos valores se han seleccionado cumpliendo con las siguientes hipótesis:

- El valor medio de la imperfección debe coincidir con la geometría perfecta de la estructura.

- Para garantizar la validez de la formulación de la lámina utilizada, la máxima amplitud de la imperfección no debe superar una determinada fracción del espesor medio de la lámina. En este ejemplo se utiliza una desviación estándar de $9.5 \mathrm{~mm}$. Dicho valor de desviación se traduce en una amplitud de imperfección $\mu_{h} / 2=38 \mathrm{~mm}$ para \pm 2 desviaciones estándar.

\subsubsection{Formulación del problema de DOR}

El objetivo del problema de diseño óptimo robusto de la lámina es encontrar el diseño con mínimo volumen, máxima carga crítica y mínima desviación estándar de la carga crítica, cumpliendo las restricciones con un determinado nivel de fiabilidad. Para conseguir este objetivo se introduce como indicador de robustez el coeficiente de variación, puesto que tiene en cuenta simultáneamente el efecto de la media y de la desviación de la carga crítica $\left(P_{\text {crit }}\right)$. Las variables de diseño son el valor medio del espesor $\left(\mu_{h}\right)$ y el ángulo $(\theta)$. El problema multi-objetivo se formula como:

$$
\left\{\begin{aligned}
\min _{\mu_{h}, \theta}: & \left\{\mu_{\mathrm{vol}}, \mathrm{COV}_{\mathrm{P}_{\text {crit }}}\right\}, \\
& \\
& \mu_{P_{\text {crit }}}-3 \sigma_{P_{\text {crit }}} \geq 0.1, \\
\text { s.a. }: & \mu_{\sigma_{V M}}+3 \sigma_{\sigma_{V M}} \leq 390 \mathrm{MPa}, \\
& 40 \leq \mu_{h}(\mathrm{~mm}) \leq 90 ; 20 \leq \theta\left(^{\circ}\right) \leq 90,
\end{aligned}\right.
$$

donde las funciones objetivo son el valor medio del volumen de la estructura $\left(\mu_{\mathrm{vol}}\right)$ y el coeficiente de variación de la carga crítica $\left(\mathrm{COV}_{\mathrm{P}_{\text {crit }}}\right)$. El estado tensional de la estructura se comprueba utilizando el criterio de Von Mises para la comparación con el límite elástico del acero para un carga de 0.1 MPa. La carga crítica debe ser superior a $0.1 \mathrm{MPa}$ para un nivel de fiabilidad equivalente a $3 \sigma$.

\subsubsection{Metamodelos estocásticos}

El problema consta de 2 variables de diseño y 93 variables aleatorias. El problema de propagación de incertidumbre se abordó previamente en la Sección 5.4.5. Los resultados obtenidos ponen de manifiesto que una reducción unidimensional con $N_{1}=6$ es suficiente para aproximar los dos primeros momentos estadísticos de las respuestas con un error inferior al $0.5 \%$. En este ejemplo se utiliza el mismo procedimiento para estimar los dos primeros 


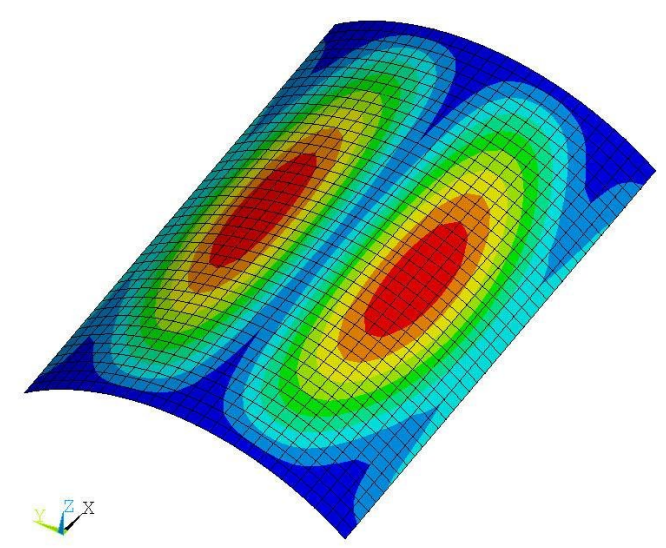

(a) Modo 1

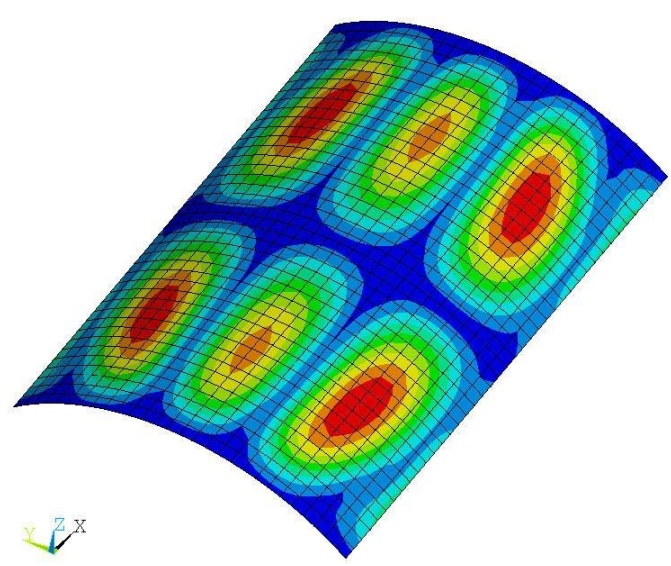

(c) Modo 3

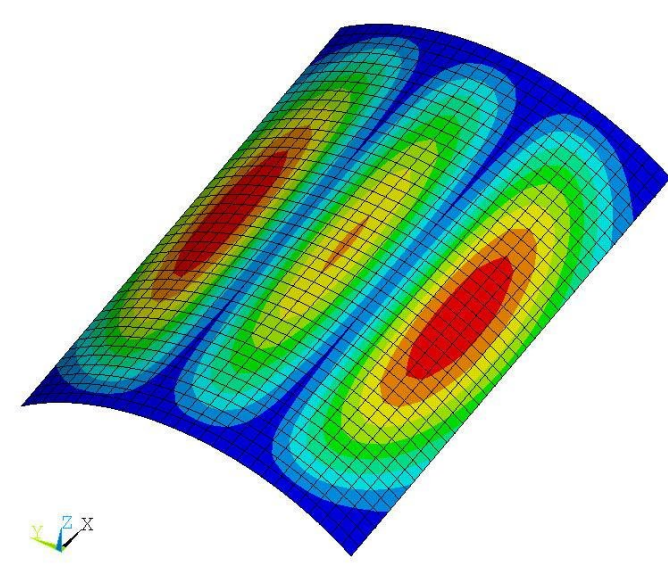

(b) Modo 2

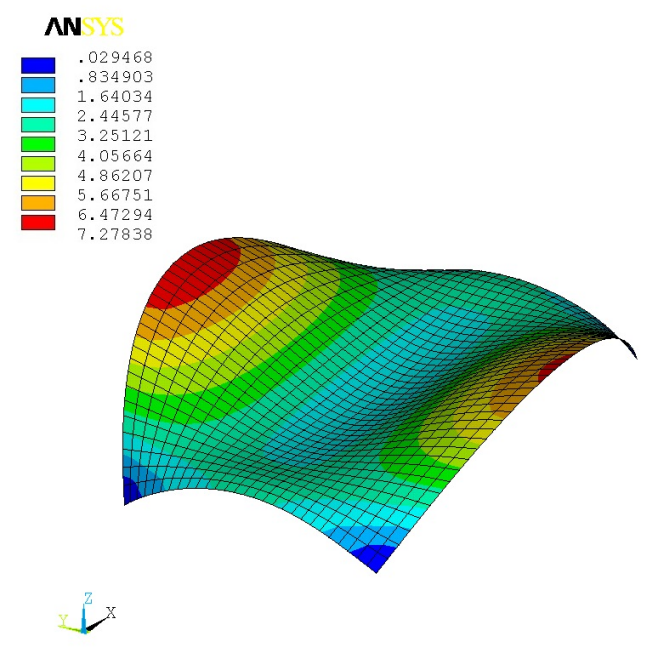

(d) Imperfección geometríca (mm) (1000x)

Figura 6.26: Problema Scordelis-Lo shell roof (II). Tres primeros modos de pandeo (a)-(c), y forma de la imperfección geométrica para una realización de las variables aleatorias que la definen $(\mathrm{d})$.

momentos estadísticos del volumen, tensión de Von Mises y carga crítica. El número total de evaluaciones del modelo de simulación para la estimación de los momentos estadísticos es de 199.

Los momentos estadísticos de las respuestas de la estructura se aproximan en el espacio de diseño mediante modelos Kriging ajustados a partir de un conjunto de 10 observaciones generadas mediante Hypercubo Latino. Esta muestra se incrementa en 2 puntos adicionales para conseguir un mínimo de 5 puntos en el espacio de diseño factible. El problema se resuelve utilizando dos enfoques: (1) un enfoque directo sin meta-modelos, en el que se utiliza el algoritmo NSGA-II junto con EKDRM y (2) un enfoque basado en meta-modelos con el algoritmo EHV-EMO. El primer enfoque se toma como referencia. La Figura 6.27 ilustra la 


\begin{tabular}{lll}
\hline Caso & & \\
\hline \hline NSGAII+EKDRM Vs EHV-EMO & GD & $1.63 \mathrm{e}-03$ \\
& MPFE & $7.34-02$ \\
& HR & $0.97 \mathrm{e}+00$ \\
Validación del Meta-modelo & VMRV & $(-)$ \\
& RF $_{\mathrm{ac}}$ & $1.00 \mathrm{e}+00$ \\
\hline
\end{tabular}

Tabla 6.12: Problema Scordelis-Lo shell roof (II). Resumen de los resultados numéricos.

evolución del algoritmo EHV-EMO para el problema propuesto. En ella la muestra inicial se presenta como círculos negros. Los cuadrados blancos representan los puntos añadidos hasta la iteración actual. Los cuadrados verdes representan el candidato que maximiza el criterio en cada iteración. El criterio de parada se cumple en la iteración número 21. El frente de Pareto aproximado está compuesto por 13 puntos y se muestra en la Figura 6.28a junto con la evolución del criterio de búsqueda (Figura 6.28b). La precisión de la aproximación se estima a través de los indicadores mostrados en la Tabla 6.12. La aproximación resulta de buena calidad con un valor del ratio de hipervolumen de 0.97 (cercano a 1) y un valor de la distancia generacional y del máximo error entre frentes cercano a 0.

Las soluciones no-dominadas obtenidas se muestran sobre los meta-modelos de las funciones objetivo en las Figuras 6.29a y 6.29c. En las Figuras 6.29b y 6.29d se muestra el error cuadrático medio de la predicción de las funciones objetivo. Se aprecia una clara reducción de dicho error en las regiones cercanas al frente de soluciones no-dominadas. Esto permite utilizar dichos meta-modelos para su explotación en una etapa posterior en la búsqueda de nuevas soluciones.

La Figura 6.30 muestra los diseños extremos del frente. El número total de evaluaciones del modelo de simulación es de 18447 con el enfoque 2, un valor muy inferior a las $3.98 \mathrm{e} 6$ requeridas por el enfoque 1.

\subsection{Discusión y conclusiones}

En este capítulo se ha presentado una estrategia basada en un meta-modelo estocástico para la resolución del problema multi-objetivo de diseño óptimo robusto. Fruto del trabajo realizado se obtienen las siguientes conclusiones:

\section{Revisión de la literatura}

- El problema de la alta dimensionalidad es una de las principales limitaciones de los enfoques estudiados para su aplicación a problemas "reales". Estos problemas involucran un elevado número de variables de diseño o aleatorias, como es el caso de problemas con campos aleatorios. 


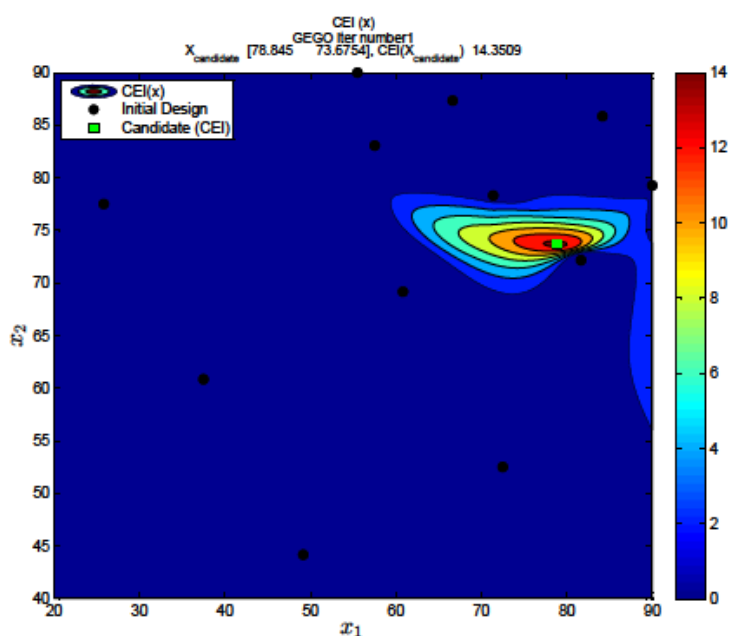

(a) Iter $=1$

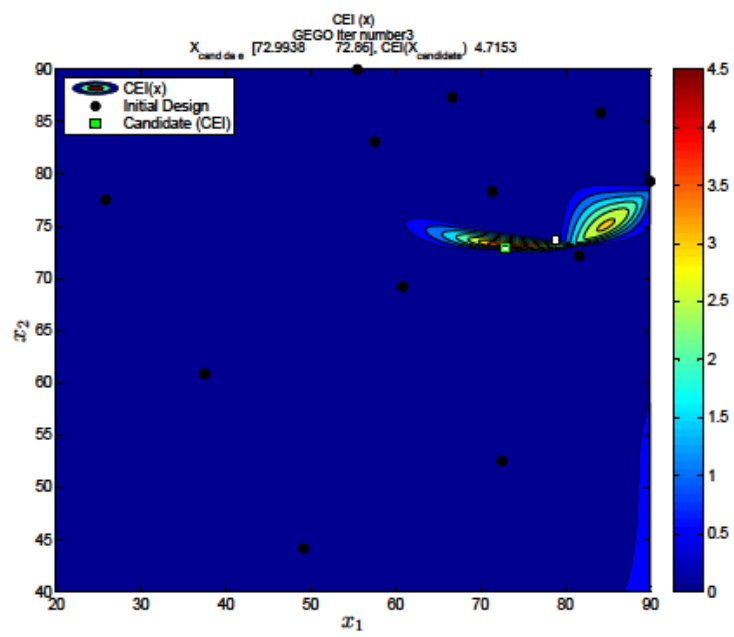

(c) Iter $=3$

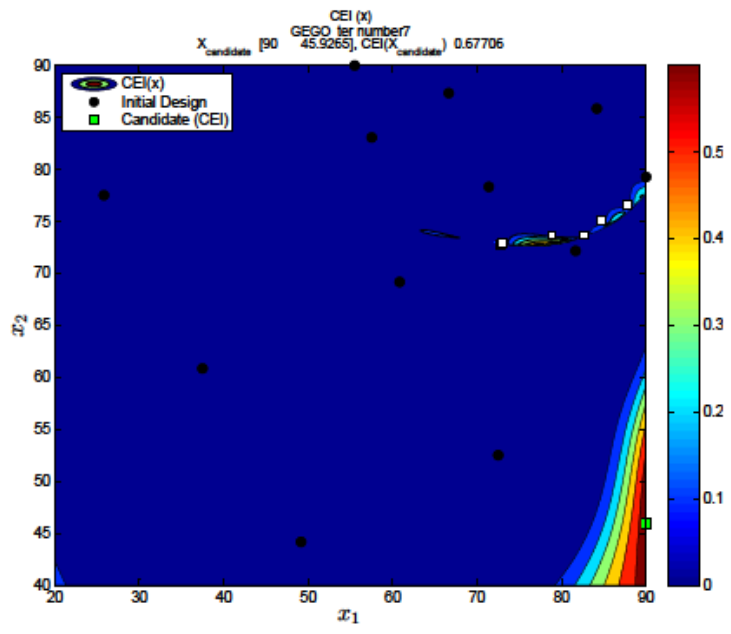

(e) Iter $=7$

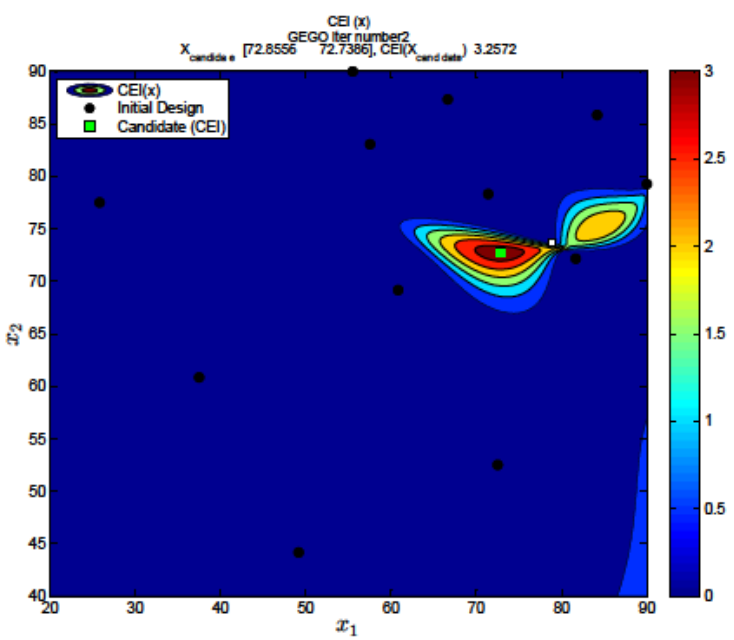

(b) Iter $=2$

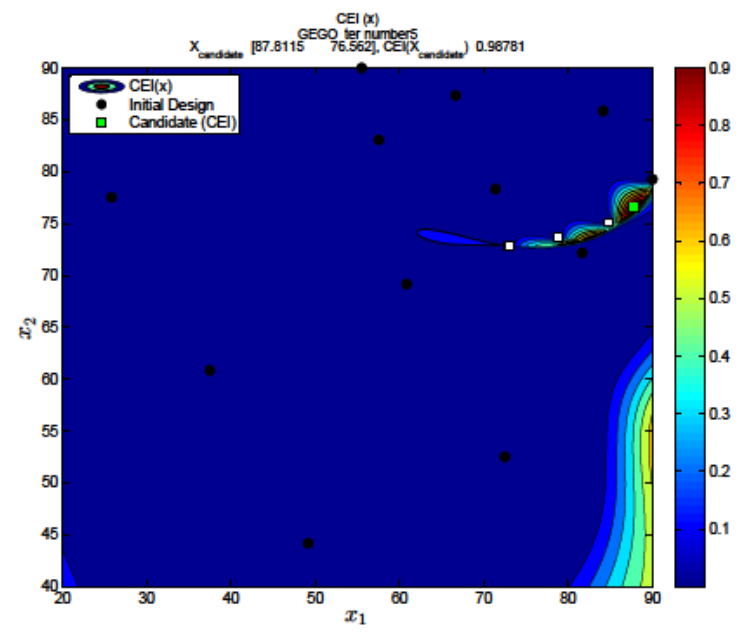

(d) Iter $=5$

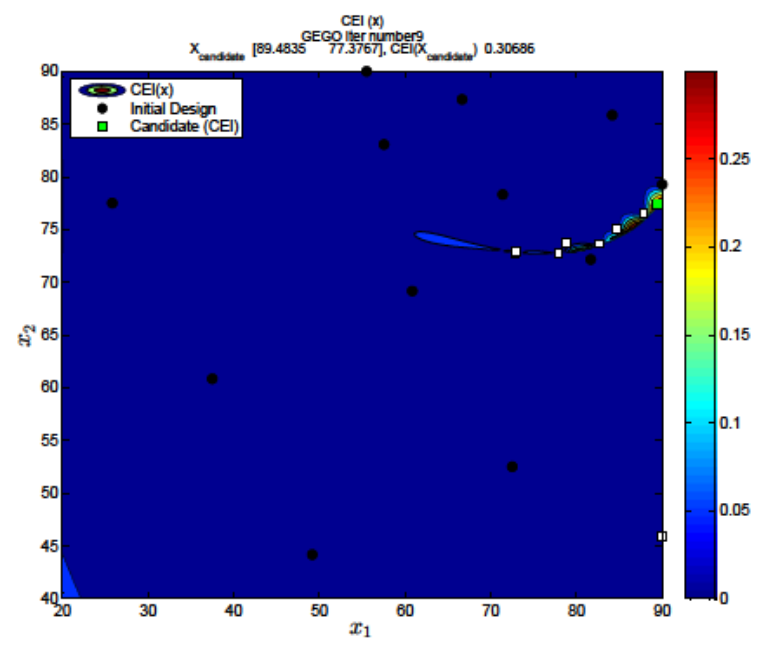

(f) Iter $=9$

Figura 6.27: Problema Scordelis-Lo shell roof (II). Evolución del criterio de búsqueda. La muestra inicial se presenta como círculos negros. Los cuadrados blancos representan los puntos añadidos hasta la iteración. Los cuadrados verdes representan el candidato que maximiza el criterio en cada iteración. 


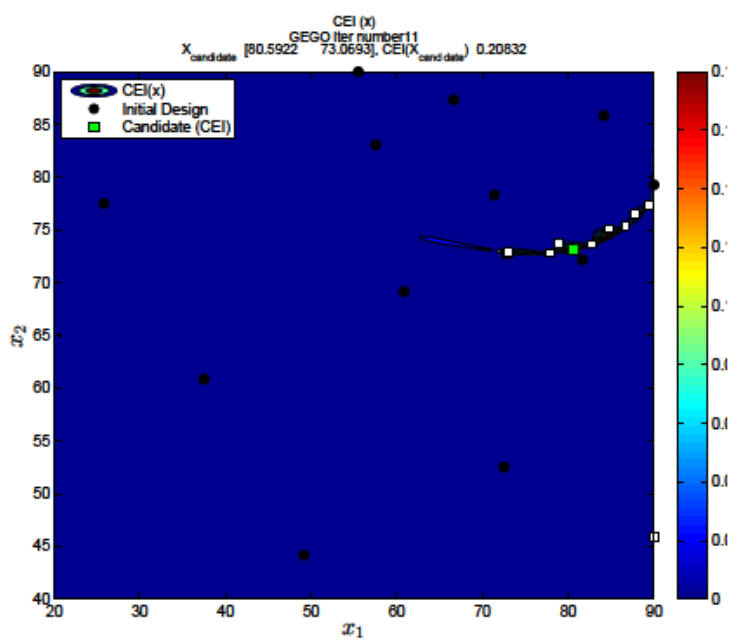

(g) Iter $=11$

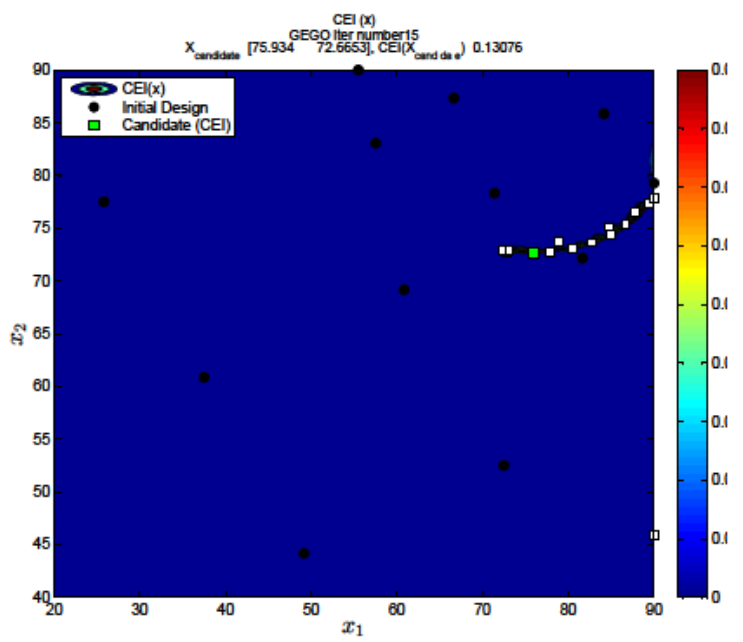

(i) Iter $=15$

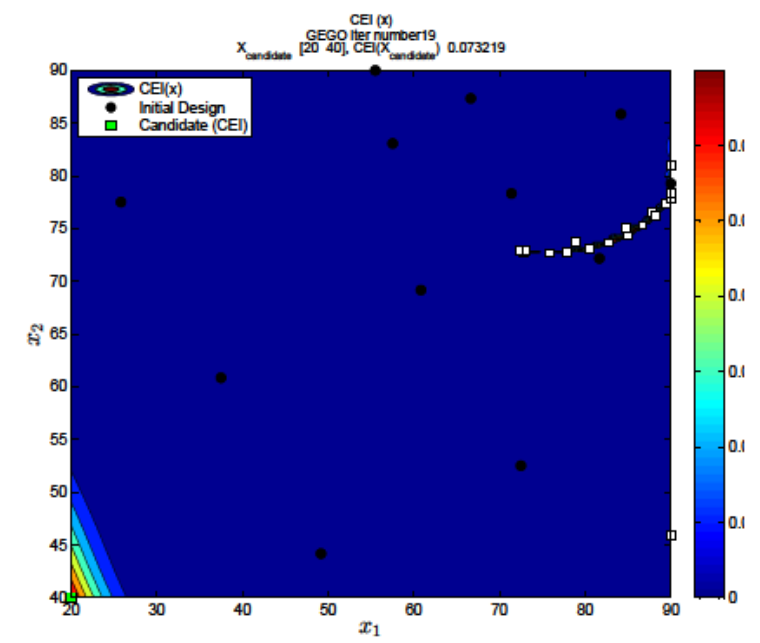

(k) Iter $=19$

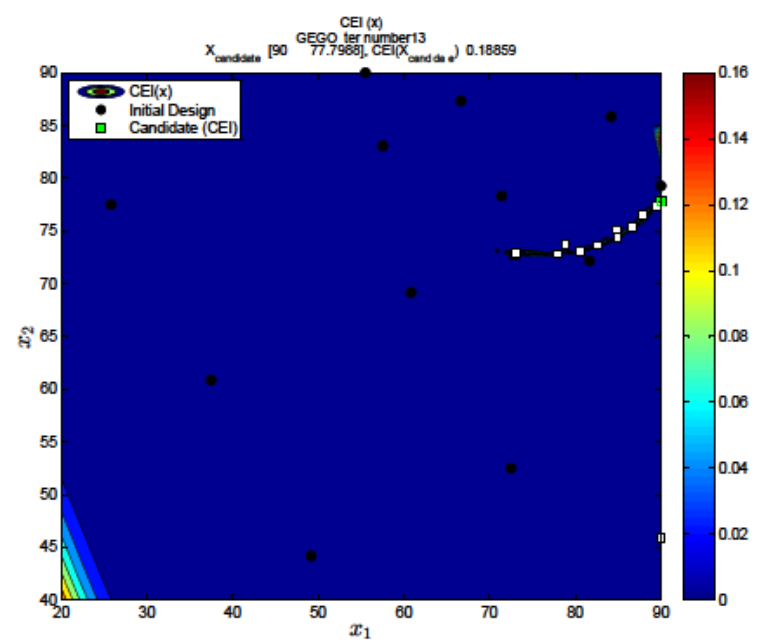

(h) Iter $=13$

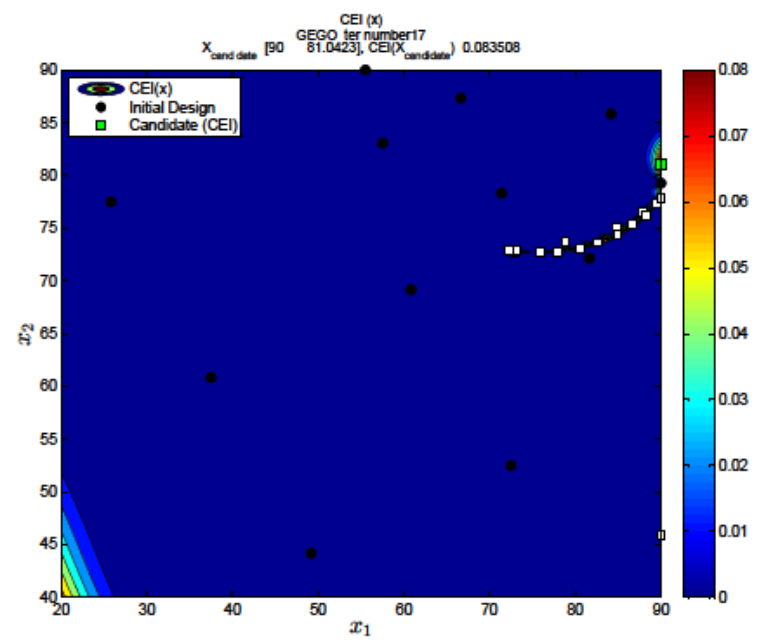

(j) Iter $=17$

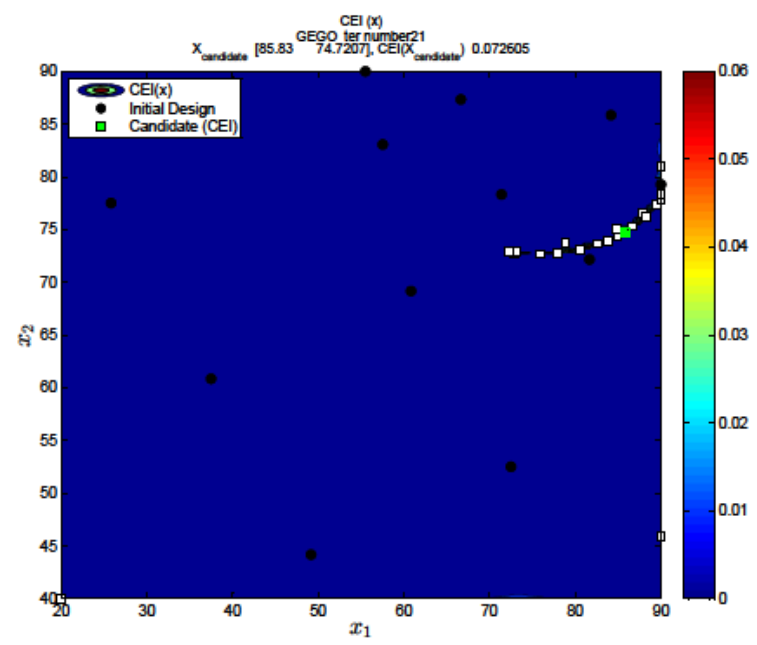

(1) Iter $=21$

Figura 6.27: (Continuación). Evolución del criterio de búsqueda. La muestra inicial se presenta como círculos negros. Los cuadrados blancos representan los puntos añadidos hasta la iteración. Los cuadrados verdes representan el candidato que maximiza el criterio en cada iteración. 


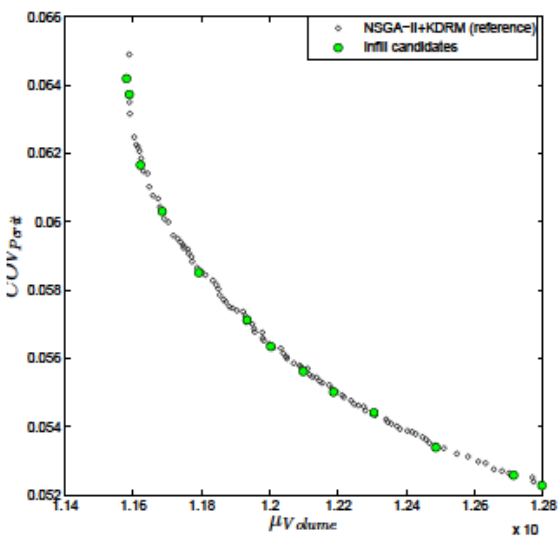

(a)

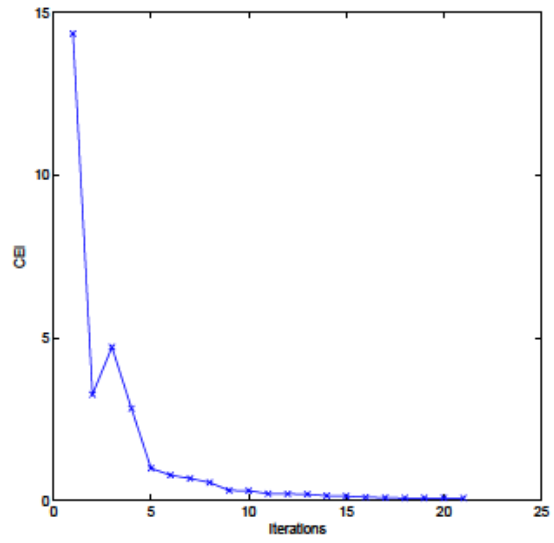

(b)

Figura 6.28: Problema Scordelis-Lo shell roof (II). (a) Frente de Pareto, (b) evolución del criterio de búsqueda CEI. $\hat{\mu}_{\text {vol }}$ en $\mathrm{m}^{3}$.

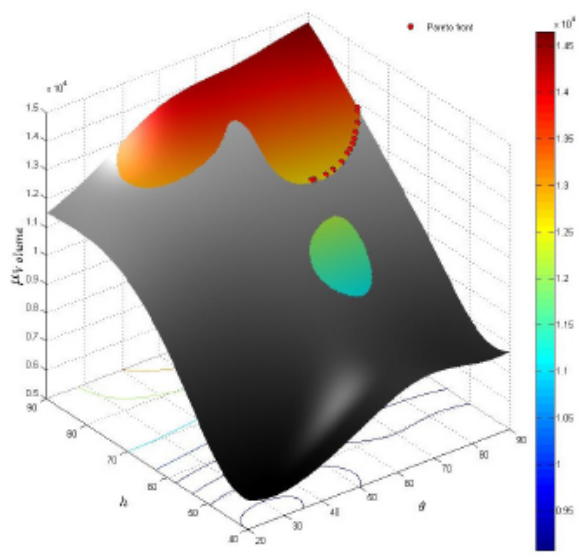

(a) $\hat{\mu}_{\text {vol }}$

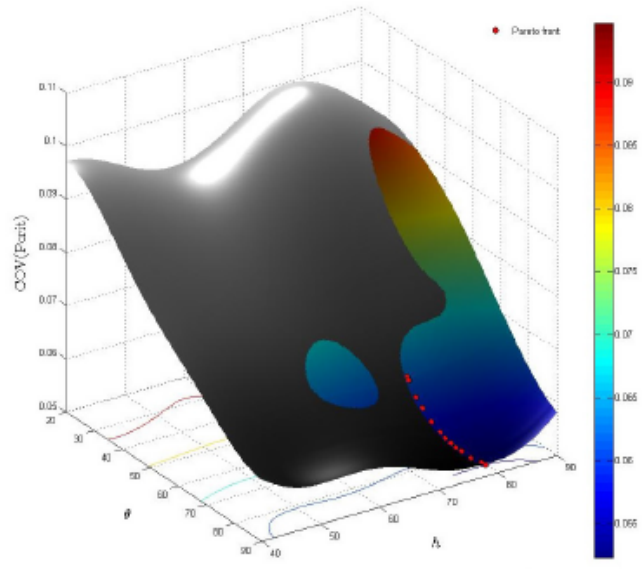

(c) $C O \hat{V} V_{\text {Prit }}$

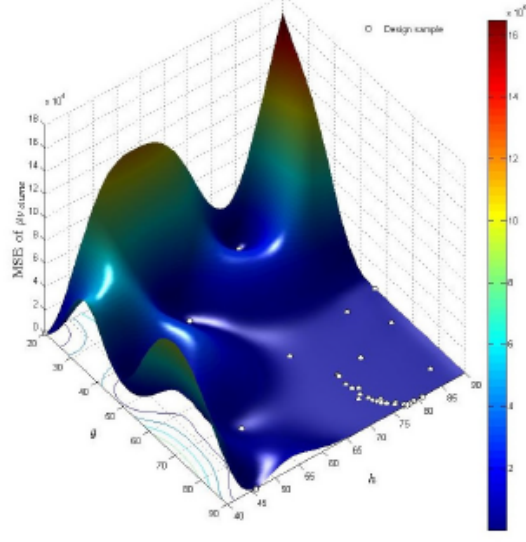

(b) Error cuadrático medio de $\hat{\mu}_{\text {vol }}$

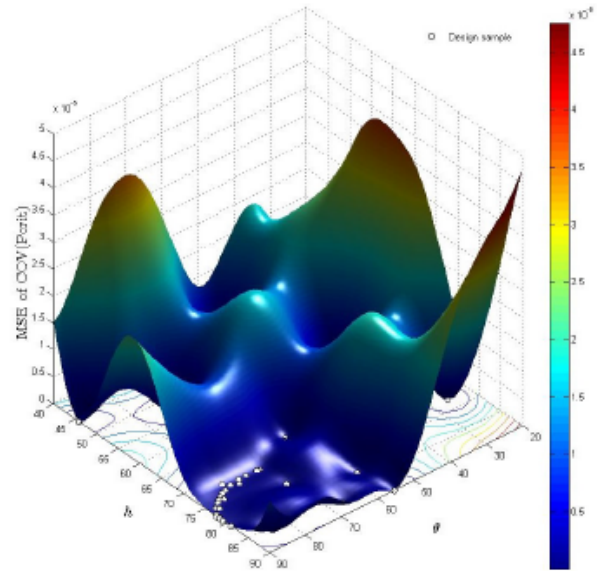

(d) Error cuadrático medio de $C O V_{\text {Pcrit }}$

Figura 6.29: Problema Scordelis-Lo shell roof (II). Soluciones óptimas de Pareto (puntos en color rojo). En color gris se representa el dominio de diseño no factible. 

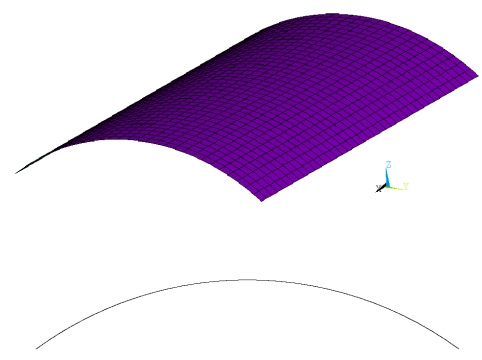

(a) $[\theta, h]=\left[72.3096^{\circ}, 72.9004 \mathrm{~mm}\right]$
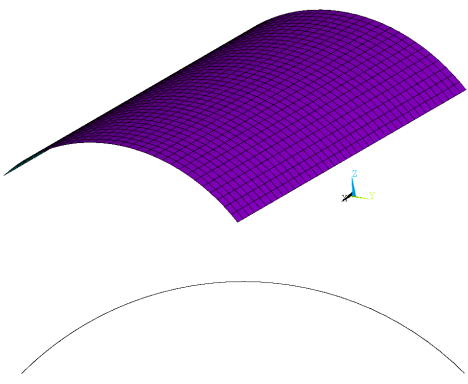

(b) $[\theta, h]=\left[90^{\circ}, 77.7988 \mathrm{~mm}\right]$

Figura 6.30: Problema Scordelis-Lo shell roof (II). (a) Diseño con minima $\mu_{\text {vol }}$. (b) Diseño con mínima $\operatorname{COV}_{P_{\text {crit }}}$.

- La unión del dominio de diseño y del dominio aleatorio dificulta la gestión de la incertidumbre epistémica del meta-modelo.

- Los métodos basados en la introducción de un meta-modelo tras el proceso de propagación de incertidumbre parecen ser la formulación más prometedora a la hora de incorporar el meta-modelo en el problema. Estos métodos representan una solución de compromiso entre la eficiencia y la precisión.

- Resulta imprescindible la utilización de métodos de propagación de incertidumbre eficientes junto con procedimientos que incorporen de forma precisa y eficiente la incertidumbre epistémica durante la optimización.

Metodología propuesta. El meta-modelo estocástico está basado en la utilización de forma conjunta de métodos de reducción dimensional para la estimación de los momentos estadísticos junto con modelos de predicción Kriging para la aproximación de dichos momentos en el espacio de diseño. La distribución de probabilidad de la respuesta se reconstruye a partir de los cuatro primeros momentos estadísticos utilizando una distribución de tipo Pearson. El meta-modelo estocástico permite estimar la respuesta aleatoria del sistema en cualquier punto del espacio de diseño sin necesidad de evaluaciones adicionales del modelo de simulación. Así mismo, la utilización de modelos Kriging proporciona información estadística acerca de la incertidumbre epistémica del meta-modelo que se utiliza para la resolución eficiente del problema de optimización multi-objetivo.

La metodología propuesta se ha validado numéricamente mediante la resolución de problemas con funciones analíticas y con modelos de elementos finitos de estructuras discretas y continuas. Los problemas seleccionados para 
la validación se dividen en las siguientes tres categorías: aplicación al diseño óptimo robusto de problemas multi-objetivo con restricciones de fiabilidad, (2) aplicación al diseño óptimo robusto de estructuras de nudos articulados y (3) aplicación al diseño óptimo robusto de láminas con incertidumbre en geometría frente a pandeo. A partir de los resultados obtenidos se concluye:

- La estrategia propuesta permite incorporar las restricciones de probabilidad durante la resolución del problema de optimización multi-objetivo, manteniendo un equilibrio entre la precisión de la solución y el coste computacional.

- La estrategia propuesta proporciona una aproximación discreta del frente de soluciones no-dominadas del problema multi-objetivo con un número de simulaciones considerablemente inferior a enfoques que no utilizan meta-modelos. En comparación con los enfoques basados en una explotación directa del meta-modelo, la incorporación de la incertidumbre epistémica durante la optimización multi-objetivo permite alcanzar soluciones con una mayor precisión.

- El número de diseños óptimos conseguidos mediante el algoritmo EHV-EMO es inferior al conseguido por enfoques basados en algoritmos evolutivos tales como el NSGA-II. Sin embargo, los meta-modelos finales actualizados presentan una alta precisión en las regiones cercanas al frente de soluciones, y pueden ser utilizados para su explotación directa en la búsqueda de un mayor número de soluciones óptimas sin necesidad de nuevas evaluaciones del modelo de simulación.

- El procedimiento propuesto es no intrusivo, no requiere derivadas y altamente paralelizable a nivel del problema de propagación de incertidumbre. Estas características favorecen la flexibilidad del mismo y su aplicación a un gran abanico de problemas y formulaciones. 


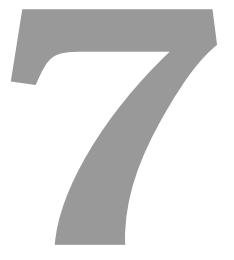

\section{Conclusiones}

Índice

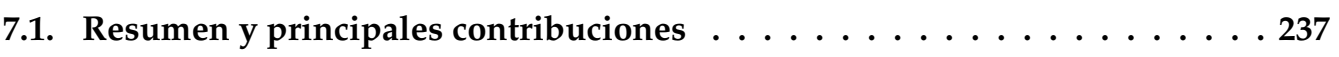

7.2. Trabajos futuros

239 



\subsection{Resumen y principales contribuciones}

El trabajo presentado en esta tesis tiene como objetivo la resolución eficiente del problema multi-objetivo de diseño óptimo robusto cuando se trabaja con modelos de simulación de alto coste computacional. Mediante el término eficiente se entiende la resolución del problema minimizando el número de evaluaciones del modelo de simulación. Para la resolución del problema planteado es necesario hacer frente a los siguientes retos: (1) resolución eficiente del problema de propagación de incertidumbre con modelos de simulación no intrusivos de alto coste computacional, (2) aproximación de la respuesta aleatoria de la estructura en el espacio de diseño y (3) resolución eficiente del problema de optimización multi-objetivo subyacente en el problema de diseño óptimo robusto. Para hacer frente a dichos retos, en esta tesis se han realizado las siguientes contribuciones:

\section{Algoritmo EKDRM. Resolución eficiente del problema de propagación de incertidumbre con modelos de simulación de alto coste computacional}

Esta contribución se ha desarrollado en el Capítulo 5. Se ha propuesto un algoritmo basado en métodos de reducción dimensional para evaluar las integrales multidimensionales de los momentos estadísticos de la respuesta estructural. La revisión de la literatura realizada pone de manifiesto que los métodos de reducción dimensional se ajustan al propósito de esta tesis por su carácter no intrusivo, por no requerir derivadas y por el reducido número de evaluaciones del modelo de simulación. En contraposición, el estado del arte de estos métodos muestra que pueden dar lugar a errores significativos cuando la respuesta es altamente no lineal o presenta un alto grado de interacción entre variables. Para solventar dichos problemas, se ha propuesto el algoritmo Extended Kriging based Dimension Reduction Method (EKDRM), que utiliza modelos de predicción Kriging para aproximar las funciones multivariable de la descomposición. El algoritmo propuesto permite:

- Incrementar la precisión de la aproximación de funciones de alta no linealidad, permitiendo aumentar el número de puntos de integración con un reducido número de evaluaciones del modelo de simulación.

- Reducir el problema de la maldición de la dimensionalidad, asociado a la utilización directa de meta-modelos para aproximar modelos de simulación en dominios aleatorios de media-alta dimensionalidad.

- Reutilizar el diseño de experimentos para la aproximación de la función de $(S-1)$ variables como parte del diseño de experimentos de la función de $S$ variables. De este modo se consigue reducir el coste computacional de las descomposiciones de orden superior a uno. 
- En comparación con trabajos similares que utilizan modelos Kriging junto con descomposición unidimensional (Won et al., 2009), la metodología propuesta: (1) extiende la aproximación con modelos Kriging a métodos de descomposición de orden $S$, (2) propone un criterio de selección del mejor ajuste (función de regresión y de correlación) independiente de la localización del diseño de experimentos y (3) establece un criterio de colocación de los diseños de experimentos para el ajuste a partir de las raíces de los polinomios de Hermite.

Esta contribución se ha validado numéricamente mediante la resolución de problemas de baja y alta dimensionalidad, variables y campos aleatorios, alto grado de no linealidad e interacción entre variables, diferentes tipos de distribuciones de probabilidad y modelos de elementos finitos de alto coste computacional.

\section{Algoritmo EHV-EMO. Resolución eficiente del problema de optimización MO con modelos de simulación de alto coste computacional}

Esta contribución se ha desarrollado en el Capítulo 4. Uno de los principales retos que plantea el problema de DOR cuando se aplica a problemas de ingeniería, es el alto coste computacional derivado del anidamiento entre los procesos de PI y de optimización MO. Por este motivo, es necesario minimizar el número de evaluaciones del proceso de PI. Se ha propuesto el algoritmo Expected HyperVolume Efficient Multi-objective Optimization (EHV-EMO) para la resolución eficiente del problema MO. El algoritmo Expected HyperVolume Efficient Multi-objective Optimization (EHV-EMO) utiliza modelos Kriging para aproximar las funciones objetivo y restricciones del problema. La información estadística proporcionada por dichos modelos permite estimar la esperanza de mejora del frente de soluciones no-dominadas en cada punto del espacio de diseño. Este criterio se utiliza para guiar el proceso de selección de nuevos puntos candidatos a formar parte de la muestra de observaciones inicial, mejorando la precisión de los meta-modelos en las regiones cercanas al conjunto de soluciones óptimas. El algoritmo EHV-EMO permite:

- Incorporar la incertidumbre epistémica de los meta-modelos de las restricciones en el criterio de búsqueda.

- Incrementar el número de puntos de diseño en el espacio de diseño factible de forma eficiente.

- Aproximar el conjunto de soluciones no-dominadas utilizando un número reducido de evaluaciones del modelo de simulación. 
Esta contribución se ha validado numéricamente mediante la resolución de un conjunto de problemas ampliamente utilizados como benchmark en el campo de la optimización multi-objetivo.

\section{Meta-modelo estocástico de la respuesta aleatoria basado en modelos Kriging y métodos de reducción dimensional}

Esta contribución se ha desarrollado en el Capítulo 6. Se ha propuesto una estrategia adaptativa basada en un meta-modelo estocástico de la respuesta aleatoria de la estructura. El meta-modelo estocástico emula la respuesta aleatoria en el espacio de diseño, proporcionando predicciones acerca de la estadística del problema con un bajo coste computacional. El meta-modelo estocástico propuesto se basa en: (1) la utilización de métodos de reducción dimensional para la estimación eficiente de los momentos estadísticos de la estructura, (2) modelos de predicción Kriging para la aproximación de los momentos estadísticos en el espacio de diseño y (3) la reconstrucción de la distribución de probabilidad de la respuesta, a partir de los momentos estadísticos utilizando una distribución Pearson. El meta-modelo estocástico propuesto permite:

- Aproximar la respuesta aleatoria de la estructura en el espacio de diseño.

- Incorporar la incertidumbre epistémica del meta-modelo en procesos de optimización bajo incertidumbre.

Esta contribución se ha validado numéricamente mediante la resolución de problemas de optimización robusta multi-objetivo con restricciones de probabilidad, problemas de diseño óptimo robusto de estructuras de nudos articulados y problemas de diseño óptimo robusto de láminas imperfectas frente a pandeo.

\section{Toolbox ooSBOUU}

Las diferentes contribuciones presentadas a lo largo de esta tesis se han integrado en una toolbox orientado a objetos desarrollada en el entorno MATLAB. Esta toolbox tiene por objetivo el diseño claro, flexible y encapsulado de algoritmos de optimización bajo incertidumbre asistida por meta-modelos. La toolbox está disponible bajo los términos de la licencia GNU (General Public License) desde el servidor web: http://gorgo.upct.es/public/ooSBOUU_web/.

\subsection{Trabajos futuros}

Cuando se abordan problemas que involucran modelos de simulación de alto coste computacional, resulta inevitable la utilización de técnicas de 
meta-modelos para la resolución de procesos de propagación de incertidumbre y de optimización. Aunque esta tesis presenta como principal contribución la resolución eficiente del problema multi-objetivo de DOR, gran parte de los algoritmos desarrollados pueden ser utilizados para abordar un rango más amplio de problemas. Como continuación al trabajo desarrollado en esta tesis se proponen las siguientes líneas de investigación como trabajos futuros.

\section{Propagación de incertidumbre con el algoritmo EKDRM.}

- Investigación en métodos adaptativos capaces de detectar las contribuciones de los términos cruzados con un mayor peso en la respuesta final.

- Propagación de la incertidumbre epistémica introducida por los modelos Kriging utilizados para aproximar las respuestas de dimensión reducida.

\section{Optimización multi-objetivo con el algoritmo EHV-EMO.}

- Estrategias adaptativas que permitan la actualización de los meta-modelos con múltiples puntos en cada iteración.

- La aplicación del algoritmo propuesto a problemas con una mayor dimensionalidad en el espacio objetivo.

- Investigación en modelos de predicción que permitan aproximar estadísticamente funciones de alta dimensionalidad y funciones con múltiples respuestas.

- Investigación en nuevos algoritmos para la evaluación en paralelo del valor esperado del hipervolumen.

DOR utilizando Meta-modelos estocásticos. La metodología propuesta se basa en la utilización de modelos de predicción Kriging. Por este motivo, dicha metodología no escapa de los problemas asociados a este tipo de modelos, tales como: la alta dimensionalidad o las discontinuidades en la respuesta. Se hace imprescindible la investigación de nuevas técnicas de predicción capaces de aproximar respuestas en espacios de alta dimensionalidad.

\section{Aplicaciones en ingeniería estructural.}

- Extensión del trabajo realizado a la resolución de nuevos problemas como: el problema de optimización de topología bajo incertidumbre y el problema de diseño óptimo fiable. 
- Aplicación a la resolución de un mayor número de problemas estructurales. Entre ellos se encuentra el problema de diseño óptimo fiable de uniones semirrígidas de acero con restricciones probabilistas de ductilidad y el diseño óptimo robusto de estructuras de pared delgada frente incertidumbres geométricas. 



\section{Referencias}

Abdomian, G. Applied Stochastic Processes. New York, NY: Academic Press, 1980. 134

Adeli, H. y Cheng, N. Concurrent genetic algorithms for optimization of large structures. Journal of Aerospace Engineering, ASCE, 7:276-296, 1994. 3

AISC. Manual of Steel Construction-Alloawable Stress Design, 1989. 220

Alis, O. F. y Rabitz, H. Efficient implementation of high dimensional model representations. Journal of Mathematical Chemistry, 29:127-142, 2001. 10.1023/A:1010979129659. 137

ANSYS. Version 14, (CANSYS, Inc. All Rights Reserved, 2013. 164

Aoues, Y. y Chateauneuf, A. Reliability-based optimization of structural systems by adaptive target safety - application to RC frames. Structural Safety, 30: 144-161, 2008. 34

Apley, D., J., L., y Chen, W. Understanding the effects of model uncertainty in robust design with computer experiments. ASME J Mech Des, 128(4):745-958, 2005. 17

Arora, J. S. Introduction to Optimum Design. McGraw-Hill Series in Mechanical Engineering, 1989. 93

Asadpoure, A., Tootkaboni, M., y Guest, J. K. Robust topology optimization of structures with uncertainties in stiffness - application to truss structures. Computers and Structures, 89:1131-1141, 2011. 34

Audet, C., Jr., J. D., Moore, D., Booker, A., y Frank, P. A surrogate-model-based method for constrained optimization. 2000. 104

Ayyub, B. M. y McCuen, R. H. Probability, Statistics, and Reliability for Engineers and Scientists. 2nd Ed., Chapman Hall/CRC, Boca Raton, Flo., 2003. 131

Baker, J. W., Schubert, M., y Faber, M. H. On the assessment of robustness. Structural Safety, 30:253-267, 2008. 38

Balling, R., Free, J., y Parkinson, A. Consideration of worst-case manufacturing tolerances in design optimization. ASME J Mech Des, 108:438-441, 1986. 17, 20, 33 
Barthelmann, V., Novak, E., y Ritter, K. High dimensional polynomial interpolation on sparse grids. Advances in Computational Mathematics, 12(4): 1118-39, 2000. 135

Bautista, D. C. A Sequential Design for Approximating the Pareto Front using the Expected Pareto Improvement Function. PhD thesis, Ohio State University, 2009. 91, 92

Bayes, T. An essay towards solving a problem in the doctrine of chances. Philosophical Transactions of the Royal Society of London, 53:370-418, 1763. 48

Belegundu, A. D. y Chandrupatla, T. Optimization Concepts and Applications in Engineering. Cambridge University Press, 2011. 63

Bellman, R. E. Adaptive Control Processes: A Guided Tour. Princeton University Press, 1961. 135

Ben-Haim, Y. y Elishakoff, I. Convex Models of Uncertainty in Applied Mechanics. Elsevier Science Publishers, Amsterdam, 1990. 23

Ben-Tal, A. y Nemirovski, A. Robust convex optimization. Mathematics of Operations Research, 23:769-805, 1998. 24

Bennett, J. y Lust, R. Conservative methods for structural optimization. AIAA Journal, 28(8):1491-6, 1990. 33

Beume, N., Naujoks, B., y Emmerich, M. SMS-EMOA: Multiobjective selection based on dominated hypervolume. European Journal of Operational Research, 181 (3):1653-1669, 2007. 89

Beyer, H.-G. y Sendhoff, B. Robust optimization - A comprehensive survey. Computer Methods in Applied Mechanics and Engineering, 196(33-34):3190-3218, 1 July 2007. 5, 32, 184

Blatman, G. y Sudret, B. Sparse polynomial chaos expansions and adaptive stochastic finite elements using a regression approach. Comptes Rendus Mecanique, 336(6):518 - 523, 2008. 153, 161

Blatman, G. y Sudret, B. An adaptive algorithm to build up sparse polynomial chaos expansions for stochastic finite element analysis. Prob. Eng. Mech., 25(2): 183-197, 2010. 59

Blatman, G. y Sudret, B. Adaptive sparse polynomial chaos expansion based on least angle regression. Journal of Computational Physics, 230(6):2345-2367, 2011. 134,135

Bourinet, J.-M., Gayton, N., Lemaire, M., y Combescure, A. Reliability analysis of the stability of shells based on combined finite element and response surface methods. In 4th Int. Coll. on Comput. of Shell \& Spat. Struct., IASS-IACM, 2000. 171 
Box, G. y Fung, C. Studies in Quality Improvement: Minimizing Transmitted Variation by Parameter Design. Technical Report 8, Center for Quality Improvement, University of Wisconsin, 1986. 33, 39

Box, G. E. P. y Wilson, K. B. On the Experimental Attainment of Optimum Conditions. Journal of the Royal Statistical Society, Series B, XIII(1):1-45, 1951. 41, 45,59

Boyd, S. y Vandenberghe, L. Convex Optimization. Cambridge University Press, 2004. 88

Branin, F. Widely convergent method for finding multiple solutions of simultaneous nonlinear equations. IBM journal of Research and Development, 16:504-522, 1972. 23

Breitung, K. Asymptotic approximations for multinormal integrals. Journal of Engineering Mechanics, 110(3):357-366, 1984. 21, 136

Budiansky, B. y Hutchinson, J. Dynamic buckling of imperfect sensitive structures. In 11th IUTAM Congress, páginas 636-651, 2006. 171

Burden, R. L. y Faires, J. D. 4.7: Cuadratura Gaussiana. Análisis Numérico. I.T.P. Latin America, 1998. 153

Bursi, O. y Jaspart, J. Calibration of a finite element model for isolated bolted end plate steel connections. Journal of Constructional Steel Research, 44(3):225-62, 1997. 165

Calafiore, G. y Dabbene, F. Optimization under uncertainty with applications to design of truss structures. Structural and Multidisciplinary Optimization, 35(3): 189-200, 2008. 17

Carter, R., Gablonsky, J., Patrick, A., Kelley, C., y Eslinger, O. Algorithms for noisy problems in gas transmission pipeline optimization. Optimization and Engineering, 2:139-157, 2002. 112

Chen, W., Garimella, R., y Michelena, N. Robust design for improved vehicle handling under a range of maneuver conditions. Eng Optim, 33(3):303-326, 2001. 17

Cherkassky, V. y Mulier, F. Learning from Data-Concepts, Theory, and Methods. John Wiley \& Sons, Ltd, Chichester, 1989. 46

Chi, H. y Bloebaum, C. Mixed variable optimization using taguchi's orthogonal arrays. Struct. Optim., 12:147-152, 1996. 33

Choi, C. y Noh, H. Stochastic analysis of shape imperfection in rc cooling tower shells. J. Struct. Eng., (3), 2000. 171 
Choi, J., Lee, W., Park, J., y Youn, B. A study on robust design optimization of layered plate bonding process considering uncertainties. Structural and Multidisciplinary Optimization, 35(6):531-540, 2008. 186

Choi, J., An, D., y Won, J. Bayesian Approach for Structural Reliability Analysis and Optimization Using the Kriging Dimension Reduction Method. Journal of Mechanical Design, 132(5), 2010. 139

Clarke, S. M., Griebsch, J. H., y Simpson, T. Analysis of support vector regression for approximation of complex engineering analyses. In Proceedings of the 29th Design Automation Conference (ASME Design Engineering Technical Conferences), (DAC/DETC'03), Sep 2003. 66

Coelho, A. Characterization of the ductility of bolted end plate beam-to-column steel connections. PhD thesis, University of Coimbra, 2004. xxvi, 164, 166

Coelho, R. F. y Bouillard, P. Multi-objective reliability-based optimization with stochastic metamodels. Evolutionary Computation, 19(4):525-560, 2011. 115, 186

Coelho, R. F., Lebon, J., y Bouillard, P. Hierarchical stochastic metamodels based on moving least squares and polynomial chaos expansion. Structural and Multidisciplinary Optimization, 43:707-729, 2011. 8, 9, 182, 186, 189, 195

Coello, C. A. C., Lamont, G. B., y Veldhuizen, D. A. V. Evolutionary Algorithms for Solving Multi-Objective Problems. Kluwer Academic Publishers, 2002. 89, 115, 195,197

Collette, Y. y Siarry, P. Multiobjective Optimization: Principles and Case Studies. Springer-Verlag, Berlin, 2003. 88

Couckuyt, I., Deschrijver, D., y Dhaene, T. Towards efficient multiobjective optimization: multiobjective statistical criterions. Brisbane, Australia, June, 10-15, 2012. 91, 92, 93, 113

Creveling, C. Tolerance Design: a Handbook for Developing Optimal Specification. Cambridge, MA: Addison-Wesley, 2002. 153, 157

Dam, E., van Husslage, B., den Hertog, D., y Melissen, J. Maximin latin hypercube designs in two dimensions. Operations Research, 55:158-169, 2007. 114, 191

Das, I. Robust optimization for constrained, nonlinear programming problems. Eng Optim, 32(5):585-618, 2000. 20

Davenport, W. B. y Root, W. L. An Introduction to the Theory of Random Signals and Noise. Wiley-IEEE Press, 1987. 143

Davis, P. K. y Bigelow, J. H. Motivated metamodels: Synthesis of cause-effect reasoning and statistical metamodeling. Technical report, Santa Monica, Calif.: RAND Corporation, MR-1570-AF, 2003, November 2003. 42 
Deb, K. y Gupta, H. Introducing robustness in multi-objective optimization. Evol. Comput., 14:463-494, 2006. 194

Deb, K., Pratap, A., Agarwal, S., y Meyarivan, T. A fast and elitist multiobjective genetic algorithm: NSGA-II. Evolutionary Computation, IEEE Transactions on, 6 (2):182-197, 2002. 31, 86, 89, 105, 113, 114, 196

del Castillo, E. Process Optimization: a Statistical Approach. Springer, New York, 2007. 186

Dellino, G. Robust Simulation-Optimization Methods using Kriging Metamodels. PhD thesis, Università degli studi di Bari. Dottorato di Ricerca in Matematica, 2008. 187

Dellino, G., Kleijnen, J. P., y Meloni, C. Robust Simulation-Optimization Methods using Metamodels. In Proceedings of the 2009 Winter Simulation Conference, páginas 540 - 550, Austin, TX, 2009a. 187

Dellino, G., Kleijnen, J. P. C., y Meloni, C. Robust optimization in simulation: Taguchi and krige combined. Technical Report 2009Â82, Tilburg University, October 2009b. 187

Dellino, G., P.C.Kleijnen, J., y Meloni, C. Robust optimization in simulation: Taguchi and Response Surface Methodology. International Journal of Production Economics., 125(1):52-59, 2010. 186, 187

den Boogaard, A. V., Bonte, M., y van Ravenswaaij, R. Computational optimisation of robust sheet forming processes. In Tisza, M., editor, IDDRG 2007 International Conference. 2007. 186

Desceliers, C., Soize, C., y Ghanem, R. Identification of chaos representations of elastic properties of random media using experimental vibration tests. Comput. Mech., 39:831-838, 2007. 54

Ditlevsen, O. y Madsen, H. Structural Reliability Methods. John Wiley \& Sons, 1996. 134

Doltsinis, I. y Kang, Z. Robust design of structures using optimization methods. Computer Methods in Applied Mechanics and Engineering, 193(23-26):2221-2237, 2004. 22, 30, 34, 211, 214

$\mathrm{Du}, \mathrm{X}$. y Chen, W. Towards a better understanding of modeling feasibility robustness in engineering design. ASME Journal of Mechanical Design, 122: 385-394, 1999. 17, 28, 134

Du, X. y Chen, W. Sequential optimization and reliability assessment method for efficient probabilistic design. Journal of Mechanical Design (Transactions of the ASME), 126(2):225-233, Mar. 2004. 28 
Dubourg, V., Bourinet, J.-M., Sudret, B., y Cazuguel, M. Reliability-based design optimization of an imperfect submarine pressure hull. In 11th Int. Conf. on Applications of Stat. and Prob. in Civil Engineering (ICASP11). 2011. 171, 172

Dubourg, V., Sudret, B., y Deheeger, F. Metamodel-based importance sampling for structural reliability analysis. Probabilistic Engineering Mechanics, 33:47-57, 2013. 224

Dyn, N., Levin, D., y Rippa, S. Numerical procedures for surface fitting of scattered data by radial basis functions. SIAM Journal of Scientific and Statistical Computing, 7:639-659, 1986. 45

Eggert, R. Quantifying design feasibility using probablistic feasibility analysis. Adv. Design Automation, 1:235-240, 1991. 19

Egorov, I. N., Kretinin, G. V., y Leshchenko, I. A. How to execute robust design optimization. In In 9th AIAA/ISSMO Symposium and Exhibit on Multidisciplinary Analysis and Optimization, 2002. 5, 15

Ehrgott, M. Multiple Criteria Optimization : Classification and Methodology. Shaker Verlag (Berichte aus der Mathematik), Aachen, 1997. 93

Eldred, M. y Burkardt, J. Comparison of non-intrusive polynomial chaos and stochastic collocation methods for uncertainty quantification. In Proceeding of the 47th AIAA Aerospace Sciences Meeting, Orlando, FL, 2009. 10, 134, 135

Eldred, M. S., Giunta, A. A., S. F. Wojtkiewicz, J., y Trucano, T. G. Formulations for Surrogate-Based Optimization Under Uncertainty. In Proceedings of the 9th AIAA/ISSMO Symposium on Multidisciplinary Analysis and Optimization, Atlanta, Sept. 4-6 2002. 183, 189

Elishakoff, I., Haftka, R., y Fang, J. Structural design under bounded uncertainty-optimization with anti-optimization. Computers and Structures, 53: 1401-1405, 1994. 24

Emmerich, M., Deutz, A. H., y Klinkenberg, J. W. The computation of the expected improvement in dominated hypervolume of Pareto front approximations. Technical report, Leiden University, Sept. 2008. 92, 99, 102, 104

Emmerich, M. T. M. Single- and Multi-objective Evolutionary Design Optimization Assisted by Gaussian Random Field Metamodels. PhD thesis, ELDORADO, TU-Dortmund, 2005. 81, 90

EN10034. Structural steel I and H sections - Tolerances on shape and dimensions. Technical report, European Committee for Standarisation (CEN), Brussells. 168

EN1993-1-8. Eurocode 3: Design of steel structures - part 1-8: Design of joints. Technical report, 1993. 164, 167

Enevoldsen, I. y Sorensen, J. Reliability-based optimization in structural engineering. Struct. Saf., 15(3):169-196, 1994. 21 
Fabian, T., Fisher, J. L., Sasieni, M. W., y Yardeni, A. Purchasing raw material on a fluctuating market. Operations Research, 7:107-122, 1959. 41

Fang, H. y Horstemeyer, M. F. Global response approximation with radial basis functions. Eng. Optimiz., 384:407-424, 2006. 45, 128

Fares, B., Noll, D., y Apkarian, P. Robust control via sequential semi definite programming. SIAM J Control Optim, 40(6), 2002. 19

Faulkner, D. y Das, P. Application of reliability theory to structural design and assessment of submarines and other externally pressurized cylindrical structures. In 4th Int. Symp. on Integrity of Offshore Structures., páginas 199-230, 1990. 171

Feynman, R. F. What Do You Care What Other People Think? Bantham Book, New York, NY, USA, 1988. 1

Finkel, D. E. DIRECT Optimization Algorithm User Guide. Center for Research in Scientific Computation. North Carolina State University., 2003. 112

Foo, J. y Karniadakis, G. Multi-element probabilistic collocation method in high dimensions. Journal of Computational Physics, 229:1536-1557, 2010. 135

Foo, J., Wan, X., y Karniadakis, G. The multi-element probabilistic collocation method (me-pcm): error analysis and applications. Journal of Computational Physics, 227:9572-9595, 2008. 135, 137

Forrester, A. I. y Keane, A. J. Recent advances in surrogate-based optimization. Progress in Aerospace Sciences, 45(1-3):50-79, 2009. 44, 90

Forrester, A. I., Sobester, A., y Keane, A. Engineering Design via Surrogate Modelling A Practical Guide. John Wiley and Sons, Ltd., 2008. xxIv, xxIv, xxIv, 46, 47, 65, $72,90,92,112$

Frangopol, D. M. y Maute, K. Reliability-Based Optimization of Civil and Aerospace Structural System. Engineering Design Handbook, CRC, Boca Raton, FL, Chap. 24, 2004. 5

Friedman, J. H. Multivariate adaptive regression splines. The Annals of Statistics, 19:1-67, 1991. 44

Fyllingen, Ø., Hopperstad, O., y Langseth, M. Stochastic simulations of square aluminium tubes subjected to axial loading. International Journal of Impact Engineering, 34:1619-36, 2007. 171

Gaspar-Cunha, A. y Vieira, A. A multi-objective evolutionary algorithm using neural networks to approximate fitness evaluations. International Journal of Computers, Systems, and Signals, 6(1):18-36, 2004. 89, 90

Gasser, M. y Schüller, G. Reliability-based optimization of structural systems. Math. Methods Oper. Res., 46(3):287-307, 1997. 21 
Gerstner, T. y Griebel, M. Numerical integration using sparse grids. Numerical Algorithms, 18:240-43, 1998. 135

Ghanem, R. y Doostan, A. On the construction and analysis of stochastic models: characterization and propagation of the errors associated with limited data. $J$. Comput. Phys., 217:63-81, 2006. 54

Ghanem, R. y Spanos, P. . Stochastic Finite Elements : A Spectral Approach. Courier Dover Publications, 2003. 53, 59

Girao Coelho, A., Simoes da Silva, L., y Bijlaard, F. Finite-element modeling of the nonlinear behavior of bolted t-stub connections. J. Struct. Eng., 132(6):918-928, 2006. $164,165,167$

Gomez, S. y Levy., A. The Tunnelling Method for Solving the Constrained Global Optimization Problem with Several Non-Connected Feasible Regions. Springer-Verlag, 1982. 107

Graham, L. y Siragy, E. Stochastic finite-element analysis for elastic buckling of stiffened panels. J. Eng. Mech., (1), 2001. 171

Grestner, T. y Griebel, M. Dimension-adaptive tensor-product quadrature. Computing, 71(1):65-87, 2003. 135

Griebel, M. y Holtz, M. Dimension-wise integration of high-dimensional functions with applications to finance. Journal of Complexity, 26(5):455 - 489, 2010. 137

Groen, H. y Kaminski, M. Optimisation of pressure vessels under reliability constraints. In 15th Int. Conf. Offshore Mech. \& Arctic Eng., OMAE96, páginas 177-185, 1996. 171

$\mathrm{Gu}, \mathrm{L}$. A comparison of polynomial based regression models in vehicle safety analysis. In 2001 ASME Design Engineering Technical Conferences - Design Automation Conference, Pittsburgh, PA, September 9-12 2001. ASME. 4

Gu, X., Renaud, J., Batill, S., Brach, R., y Budhiraja, A. Worst case propagated uncertainty of multidisciplinary systems in robust optimization. Struct Optim, 20(3):190-213, 2000. 17

Gu, Y. y Kang, Z. Sensitivity analysis of structural frequency responses and dynamic optimization of space structures. In 2nd World Congress of Structural and Multidisciplinary Optimization (WCSMO-2), páginas 211-216, 1997. 3

Guedri, M., Ghanmi, S., Majed, R., y Bouhaddi, N. Robust tools for prediction of variability and optimization in structural dynamics. Mechanical Systems and Signal Processing, 23(4):1123-1133, 2009. 185

Gunawan, S. y Azarm, S. Multi-objective robust optimization using a sensitivity region concept. Structural and Multidisciplinary Optimization, 29:50-60, 2005. 10.1007/s00158-004-0450-8. 17, 194 
H. Xu, S. R. Decomposition methods for structural reliability analysis. Probabilistic Engineering Mechanics, 20:239-250, 2005. 136, 139

Haftka, R. T., Scott, E. P., y Cruz, J. R. Optimization and experiments: A survey. Applied Mechanics Reviews, 51(7):435-448, 1998. 44

Hardy, R. L. Multiquadratic equations of topography and other irregular surfaces. Journal of Geophysical Research, 76:1905-1915, 1971. 45

Hasofer, A. M. y Lind, N. C. Exact and invariant second-moment code format. Journal of the Engineering Mechanics Division, 100(1):111-121, 1974. 21, 136

Hawe, G. y Sykulski, J. Scalarizing cost-effective multi-objective optimization algorithms made possible with kriging. COMPEL: The International Journal for Computation and Mathematics in Electrical and Electronic Engineering, 27:836-844, 2008. 91, 92

Hawke, G. I. y Sykulski, J. K. An enhanced probability of improvement utility function for locating pareto optimal solutions. 2008. 91

Henk, T. Understanding Probability. Cambridge Univ. Press., 2004. 47

Henkenjohann, N. y Kunert, J. An efficient sequential optimization approach based on the multivariate expected improvement criterion. Quality Engineering, 19(4):267-280, 2007. 92

Hosder, S., Walters, R., y Perez, R. A non-intrusive polynomial chaos method for uncertainty propagation in CFD simulations. In 44th AIAA Aerospace Sciences Meeting and Exhibit, Reno, NV, USA, 2006. 135

$\mathrm{Hu}, \mathrm{C}$. y Youn, B. An asymmetric dimension-adaptive tensor-product method for reliability analysis. Structural Safety, 33(218-231), 2011. 134, 135, 195

Huang, B. y Du, X. Analytical robustness assessment for robust design. Structural and Multidisciplinary Optimization, 34:123-137, 2007. 38, 139, 153, 157, 184

Huang, X., Zhang, Y., Jin, Y., y Lu, H. An improved decomposition method in probabilistic analysis using chebyshev approximations. Structural and Multidisciplinary Optimization, 43:785-797, 2011. 137, 152, 153, 157

Hurtado, J. Structural Reliability - Statistical Learning Perspectives, Volume 17 of Lecture notes in Applied and Computational Mechanics. Springer, 2004. 9

Hutchinson, J. y Amazigo, J. Imperfection sensitivity of eccentrically stiffened cylindrical shells. AIAA Journal, 5(3), 1967. 171

ISO4014. Hexagon head loads - product grades a and b. Technical report, European Committee for Standardisation (CEN). 168

Isukapalli, S., Roy, A., y Georgopoulos, P. Stochastic response surface methods (srsms) for uncertainty propagation: application to environmental and biological systems. Risk Anal, 18(3):351-363, 1998. 135 
Janusevskis, J., Riche, R., Ginsbourger, D., y Girdziusas, R. Expected improvements for the asynchronous parallel global optimization of expensive functions: Potentials and challenges. In Hamadi, Y. y Schoenauer, M., editors, Learning and Intelligent Optimization, Lecture Notes in Computer Science, páginas 413-418. Springer Berlin Heidelberg, 2012. 188

Jeong, S., Minemura, Y., y Obayashi, S. Optimization of combustion chamber for diesel engine using kriging model. J. Fluid Sci. Technol., 1(2):138-146, 2006. 91, 92

Jin, R., Chen, W., y Simpson, T. Comparative studies of metamodelling techniques under multiple modeling criteria. Struct. Multidisc. Optim., 23:1-13, 2001a. 68

Jin, R., Du, X., y Chen, W. The use of metamodeling techniques for optimization under uncertainty. In 2001 ASME Design Automation Conference, Paper No. DAC21039, páginas 99-116, 2001b. 183

Jin, R., Du, X., y Chen, W. The use of metamodeling techniques for optimization under uncertainty. Struct. Multidiscip. Optim., 25(2):99-116, 2003. 187

Jones, D. R. A taxonomy of global optimization methods based on response surfaces. Journal of Global Optimization, 21:345-383, 2001. 67, 91

Jones, D. R., Schonlau, M., y Welch, W. J. Efficient global optimization of expensive black-box functions. Journal of Global Optimization, 13(4):455-492, 1998. XIX, XXXI, $90,95,97,98,99,112$

Jung, D. H. y Lee, B. C. Development of a simple and efficient method for robust optimization. International Journal for Numerical Methods in Engineering, 53(9): 2201-2215, 2002. 134

Jurecka, F., Ganser, M., y Bletzinger, K.-U. Update scheme for sequential spatial correlation approximations in robust design optimisation. Computers Eamp; Structures, 85(10):606-614, 2007. 17, 187, 188

Kaveh, A. y Talatahari, S. Particle swarm optimizer, ant colony strategy and harmony search scheme hybridized for optimization of truss structures. Computers and Structures, 87(5-6):267-283, 2009. 220, 222

Keane, A. J. Statistical improvement criteria for use in multiobjective design optimization. AIAA Journal, 44:879-891, 2006. 91, 92

Kleijnen, J. P. A comment on blanningâs metamodel for sensitivity analysis: The regression metamodel in simulation. Interfaces, 5(5):21-23, 1975. 41

Kleijnen, J. P. C. Statistical Tools for Simulation Practitioners. Marcel Dekker, Inc., New York, NY, USA, 1986. 4

Klimke, A. Uncertainty Modeling Using Fuzzy Arithmetic and Sparse Grids. PhD thesis, Universität Stuttgart, Shaker Verlag, 2006. 135 
Knowles, J. Parego: a hybrid algorithm with on-line landscape approximation for expensive multiobjective optimization problems. IEEE Transactions on Evolutionary Computation, 10(1):50-66, 2006. 91, 92, 113

Knowles, J. y Nakayama, H. Meta-modeling in multiobjective optimization. Berlin, Heidelberg: Springer-Verlag, 2008. 90

Koehler, J. R. y Owen, A. B. Computer experiments. Handbook of Statistics. Elsevier Science, New York, 1996. 81

Kolmogorov, A. Foundations of the Theory of Probability. Chelsea Publishing Co., New York, 1950. 47

Krige, D. A statistical approach to some basic mine valuation problems on the witwatersrand. J. of the Chem., Metal. and Mining Soc. of South Africa, 52(6), 1951. 61

Lagaros, N. D., Plevris, V., y Papadrakakis, M. Reliability based robust design optimization of steel structures. International Journal for Simulation and Multidisciplinary Design Optimization, 1:19-29, 2007. 5, 34, 186

Lautenschlager, U. y Eschenauer, H. Design-of-experiments methods and their application to robust multi-criteria optimization problems. Z. Angew. Math. Mech., 79(51):71-74, 1999. 33

Lee, I. Reliabiblity-Based Design Optimization and Robust Design Optimization Using Univariate Dimension Reduction Method. PhD thesis, University of Iowa, 2008. 34,139

Lee, I., Choi, K. K., Du, L., y Gorsich, D. Dimension reduction method for reliability-based robust design optimization. Computers and Structures, 86: 1550-1562, 2008. 34, 136, 139

Lee, $K$. A robust structural design method using the kriging model to define the probability of design success. J. Mechanical Engineering Science, 224(379-388), 2010. 34, 187

Lee, K. y Park, G. Robust optimization considering tolerances of design variables. Computers and Structures, 79:77-86, 2001. 33, 34

Lee, K. y Park, G. A global robust optimization using kriging based approximation model. Journal of the Japan Society of Mechanical Engineering, 49(3):779-788, 2006. 186, 187

Lee, K., Eom, I., Park, G., y Lee, W. A study on the robust design for unconstrained optimization problems. Trans KSME, páginas 2825-36, 1994. 33

Lee, K. S. y Geem, Z. W. A new structural optimization method based on the harmony search algorithm. Computers and Structures, 82(9-10):781-798, 2004. 220 
Lee, S. H. y Chen, W. A comparative study of uncertainty propagation methods for black-box-type problems. Structural and Multidisciplinary Optimization, 37: 239-253, 2009. 10, 139, 157

Lee, S. H., Chen, W., y Kwak, B. M. Robust design with arbitrary distributions using gauss-type quadrature formula. Structural and Multidisciplinary Optimization, 39:227-243, 2009. 139, 144

Lemaire, M. Structural Reliability. Wiley, 2009. 134

Li, C. y Kiureghian, A. D. Optimal discretization of random fields. J. Eng. Mech., 119:1136-1154, 1993. 53

Li, G., Rosenthal, C., y Rabitz, H. High dimensional model representations. The Journal of Physical Chemistry A, 105(33):7765-7777, 2001a. 137

Li, G., Wang, S.-W., Rosenthal, C., y Rabitz, H. High dimensional model representations generated from low dimensional data samples. i. mp-cut-hdmr. Journal of Mathematical Chemistry, 30:1-30, 2001b. 10.1023/A:1013172329778. 137

Li, G., Li, M., Azarm, S., Al Hashimi, S., Al Ameri, T., y Al Qasas, N. Improving multi-objective genetic algorithms with adaptive design of experiments and online metamodeling. Structural and Multidisciplinary Optimization, 37(5): 447-461, 2009. 89

Li, M., Azarm, S., y Aute, V. A multi-objective genetic algorithm for robust design optimization. In Proceedings of the 2005 conference on Genetic and evolutionary computation, GECCO '05, páginas 771-778, New York, NY, USA, 2005. 194

Li, M., Li, G., y Azarm, S. A kriging metamodel assisted multi-objective genetic algorithm for design optimization. J. Mech. Des., 130(3), 2008. 89

Liu, H., Chen, W., Kokkolaras, M., Papalambros, P., y Kim, H. Probabilistic analytical target cascading: A moment matching formulation for multilevel optimization under uncertainty. ASME J Mech Des, 128:991-1000, 2006. 17

Liu, P.-L. y Kiureghian, A. D. Multivariate distribution models with prescribed marginals and covariances. Probabilistic Engineering Mechanics, 1(2):105 - 112, 1986. 144

Liu, W. K., Belytschko, T., y Mani, A. Random field finite elements. International Journal for Numerical Methods in Engineering, 23(10):1831-1845, 1986. 134, 146

Loeve, M. Probability Theory. Springer, 1977. 53, 54

Lombardi, M. y Haftka, R. Anti-optimization technique for structural design under load uncertainties. Computer Methods in Applied Mechanics and Engineering, 57:19-31, 1998. 24 
Lönn, D., Fyllingen, O., y Nilssona, L. An approach to robust optimization of impact problems using random samples and meta-modelling. International Journal of Impact Engineering, 37(6):723-734, 2010. 34, 171, 186

Ma, X. y Zabaras, N. An adaptive high-dimensional stochastic model representation technique for the solution of stochastic partial differential equations. Journal of Computational Physics, 229(10):3884 - 3915, 2010. 137, 138

Maitre, O. P. L. y Knio, O. M. Spectral Methods for Uncertainty Quantification. Springer-Verlag, New York, 2010. 59

Marano, G., Greco, R., y Sgobba, S. A comparison between different robust optimum design approaches: Application to tuned mass dampers. Probabilistic Engineering Mechanics, 25(1):108-118, 2010. 34

Marler, R. y Arora, J. survey of multi-objective optimization methods for engineering. Structural and Multidisciplinary Optimization, 26:369-395, 2004. 88

Martin, J. D. Computational improvements to estimating kriging metamodel parameters. J. Mech. Des., 131(8), 2009. 67

Martinez-Frutos, J. An object-oriented toolbox for surrogate-based optimization under uncertainty, 2013. 196

Martínez-Frutos, J. y Martí, P. Metamodel-based multi-objective robust design optimization of structures. Computer Aided Optimum Design in Engineering XII, 2011. 89,90

Martínez-Frutos, J. y Martí, P. Robust design optimization using kriging models: application to the robust design optimization of truss structures. Rev. int. métodos numér. cálc. diseño ing., 2013. 91

Matheron, G. Traité de Géostatistique Appliquée. Editions Technip, 1962. 61, 128

Mattson, C. A. y Messac, A. Pareto frontier based concept selection under uncertainty, with visualization. Optimization and Engineering, 6:85-115, 2005. 195

McAllister, C. y Simpson, T. Multidisciplinary robust design optimization of an internal combustion engine. ASME J Mech Des, 125(1):124-130, 2003. 17

McKay, M. D., Beckman, R. J., y Conover, W. J. A comparison of three methods for selecting values of input variables in the analysis of output from a computer code. Technometrics, 21(2):239-245, 1979. 134, 191

McWilliam, S. Anti-optimisation of uncertain structures using interval analysis. Computers and Structures, 79:421-430, 2000. 23, 24 
Meckesheimer, M., Booker, A. J., Barton, R. R., y Simpson, T. W. Computationally inexpensive metamodel assessment strategies. AIAA Journal, 40:2053-2060, 2002. 68,69

Melchers, R.-E. Structural Reliability Analysis and Prediction. John Wiley \& Sons, 1999. 134

Messac, A. y Ismail-Yahaya, A. Multiobjective robust design using physical programming. Struct Multidiscipl Optim, 23(5):357-371, 2002. 19, 22

Miró-Quesada, G. y del Castillo, E. Two approached for improving the dual response method in robust parameter design. Journal of Quality Technology, 36 (2):154-168, 2004. 187

Mockus, J., Tiesis, V., y Zilinskas, A. The Application of Bayesian Methods for Seeking the Extremum. North-Holland Publishing Company, New York, 1978. 44, 85, 90, 95

Montgomery, D. Design and analysis of experiments. 3rd ed. New York: John Wiley \& Sons, 1991. 33

Moore, G. Progress in digital integrated electronics. In Electron Devices Meeting, 1975 International, volume 21, páginas 11-13, 1975. 3

Mourelatos, Z. y Liang, J. A methodology for trading-off performance and robustness under uncertainty. ASME J Mech Des, 128:856-863, 2006. 17

Murphy, K. P. Machine Learning. Probabilistic Perspective. MIT press, 2012. 41

Nain, P. y Deb, K. A multi-objective optimization procedure with successive approximate models. Technical Report 2005002, Indian Institute of Technology, Kanpur, India, 2005. 89

Nair, V., Abraham, B., MacKay, J., Nelder, J., Box, G., Phadke, M., Kacker, R., Sacks, J., Welch, W., Lorenzen, T., Shoemaker, A., Tsui, K., Lucas, J., Taguchi, S., Myers, R., Vining, G., y Wu, C. Taguchiâs parameter design: a panel discussion. Technometrics, 34:127-161, 1992. 33

Neves, L., Cruz, P., y Henriques, A. Reliability analysis of steel connection components based on fem. Engineering Failure Analysis, 8(1):29 - 48, 2001. 153, 168

Niederreiter, H. y Spanier, J. Monte Carlo and quasi-Monte Carlo methods. Springer, Berlin, 2000. 134

Obayashi, S. Multiobjective Design Exploration Using EGO. TU Delft, The Netherlands, 2006. 92

Otto, K. y Antonsson, E. Extensions to the taguchi method of product design. In Third International Conference on Design Theory and Methodology, páginas 21-30, Miami, Florida, 1999. 20 
Owen, A. Assessing linearity in high dimensions. Ann Stat, 28(1):1-19, 2000. 137

Pantelides, C. y Ganzerli, S. Design of trusses under uncertain loads using convex models. Journal of Structural Engineering, 124:318-329, 1998. 23

Papadopoulos, V. y Lagaros, N. Vulnerability-based robust design optimization of imperfect shell structures. Structural Safety, 31:475-482, 2009. 34

Papadrakakis, M. y Plevris, N. L. V. Design optimization of steel structures considering uncertainties. Engrg. Struct., 27(9):408-1418, 2005. 21, 186

Papadrakakis, M., Lagaros, N. D., y Tsompanakis, Y. Structural optimization using evolution strategies and neural networks. Computer Methods in Applied Mechanics and Engineering, 156(1-4):309-333, 1998. 44

Park, G., Hwang, W., y Lee, W. Structural optimization post-process using Taguchi method. JSME International Journal, páginas 66-72, 1994. 33

Park, J. y Baek, J. Efficient computation of maximum likelihood estimators in a spatial linear model with power exponential covariogram. Computers $\mathcal{E}$ Geosciences, 27(1), 2001. 67

Parkinson, A., Sorensen, C., y Pourhassan, N. A general approach for robust optimal design. ASME J Mech Des, 115:74-80, 1993. 20, 22

Pearson, K. Contributions to the mathematical theory of evolution, II: Skew variation in homogeneous material. Philosophical Transactions of the Royal Society of London, 186:343-414, 1895. 152, 190

Perttunen, C., Jones, D., y Stuckman, B. Lipschitzian optimization without the lipschitz constant. Journal of Optimization Theory and Application, 79(1):157-181, 1993. 112

Ponweiser, W., Wagner, T., Biermann, D., y Vincze, M. Multiobjective optimization on a limited budget of evaluations using model-assisted s-metric selection. volume 5199, páginas 784-794. 2008. 92, 93

Purgachev, V. Theory of Random Functions and Its Application to Control Problems. Pergamon Press, 1967. 52

Putko, M., Taylor III, A., Newman, P., y Green, L. Approach for input uncertainty propagation and robust design in cfd using sensitivity derivatives. ASME J Fluids Eng, 124:60-69, 1991. 19

Queipo, N. V., Haftka, R. T., Shyy, W., Goel, T., Vaidyanathan, R., y Tucker, P. K. Surrogate-based analysis and optimization. Progress in Aerospace Sciences, 41(1): $1-28,2005.44$

Rabitz, H. y Alis, O. General foundations of high-dimensional model representations. Journal of Mathematical Chemistry, 25(2):197-233, 1999. 137 
Rabitz, H., Ömer F. Alis, Shorter, J., y Shim, K. Efficient input-output model representations. Computer Physics Communications, 117(1-2):11-20, 1999. 136, 137

Rahman, S. y Xu, H. A univariate dimension-reduction method for multi-dimensional integration in stochastic mechanics. Probabilistic Engineering Mechanics, 19:393-408, 2004. 136, 139, 140, 141, 142, 143

Ramakrishnan, B. y Rao, S. A robust optimization approach using Taguchi's loss function for solving nonlinear optimization problems. Adv. Design Automation, 32(1), 1991. 19, 33

Rangavajhala, S., Mullur, A., y Messac, A. The challenge of equality constraints in robust design optimization: examination and new approach. Structural and Multidisciplinary Optimization, 34(5):381-401, 2007. 20

Rasmussen, C. y Williams, C. Gaussian Processes for Machine Learning. Adaptive Computation and Machine Learning. MIT Press, Cambridge, Massachusetts, Internet edition., 2006. 41, 42, 59

Rosenblatt, M. Remarks on a multivariat transformation. The Annals of Mathematical Statistics, 23(3):470-472, 1952. 136, 144, 146

Roustant, O., Ginsbourger, D., y Deville, Y. Dicekriging: Kriging methods for computer experiments. Technical report, R package version 1.1., 2010. 67

Sacks, J., Welch, W., Mitchell, T., y Wynn, H. Design and analysis of computer experiments. Statistical science, 4(4):409-423, 1989. 67

Saka, M. Optimum design of pin-jointed steel structures with practical applications. J. Struct. Eng. ASCE, 116(10):2599-620, 1990. 221

Sakawa, M. Fuzzy Sets and Interactive Multi-objective Optimization. Plenum Press, 1993. 23

Sandgren, E. y Cameron, T. Robust design optimization of structures through consideration of variation. Comput. Struct., 80:1605-1613, 2002. 33, 34

Santner, B. W. T. y Notz, W. The Design and Analysis of Computer Experiments. Springer series in Statistics. Springer, 2003. 66

Sasena, M., Papalambros, P., y Goovaerts, P. The use of surrogate modeling algorithms to exploit disparities in function computation time within simulation-based optimization. In The Fourth World Congress of Structural and Multidisciplinary Optimization, 2001. 104

Sasena, M. J., Papalambros, P. Y., y Goovaerts, P. Metamodeling sampling criteria in a global optimization framework. In 8th AIAA/NASA/USAF/ISSMO Symposium on Multidisciplinary Analysis and Optimization, Long Beach, CA, Sept 2000. 90, 104 
Sasena, M. J., Papalambros, P. Y., y Goovaerts, P. Exploration of metamodeling sampling criteria for constrained global optimization. Eng. Optim., 34(3): 263-278, 2002. 90

Savage, L. J. The theory of statistical decision. Journal of the American Statistical Association, 46:55-67, 1951. 24

Schenk, C. y Schuëller, G. Buckling analysis of cylindrical shells with random geometric imperfections. Int. J. Nonlinear Mech., 38:1119-1132, 2003. 171

Schonlau, M. Computer Experiments and Global Optimization. PhD thesis, Waterloo, Ont., Canada, 1997. 99, 104

Schuëller, G. I. y Jensen, H. A. Computational Methods in Optimization Considering Uncertainties- An Overview. Computer Methods in Applied Mechanics and Engineering, 198:2-13, 2008. 5, 38

Scordelis, A. y Lo, K. Computer analysisi of cylindrical shells. ACI Journal, 61: 539-561, 1961. xxIV, xxIV, xxvI, xxvIII, 72, 73, 153, 172, 224

Sekhon, J. y Mebane, W. Genetic optimization using derivatives: the rgenoud package for r. J. Stat. Software, 42(11), 2011. 67

Shan, S. y Wang, G. Survey of modeling and optimization strategies to solve high-dimensional design problems with computationally-expensive black-box functions. Structural and Multidisciplinary Optimization, 41:219-241, 2010a. 3, 4, 7

Shan, S. y Wang, G. G. Metamodeling for high dimensional simulation-based design problems. Journal of Mechanical Design, 132(5):051009, 2010b. 3, 4

Shan, S. y Wang, G. G. Turning black-box into white functions. ASME Conference Proceedings, 2010(44090):599-609, 2010c. 3, 4

Shimoyama, K., Lim, J. N., Jeong, S., Obayashi, S., y Koishi, M. Practical implementation of robust design assisted by response surface approximation and visual data-mining. Journal of Mechanical Design, 131(6):061007, 2009. 195

Siddall, J. Probabilistic Engineering Design: Principles and Applications. Marcel Drekker New York, 1983. 19

Simpson, T. W., Toropov, V. V., Balabanov, V., y Viana, F. A. C. Design and analysis of computer experiments in multidisciplinary design optimization: a review of how far we have come - or not. In 12th AIAAISSMO Multidisciplinary Analysis and Optimization Conference. 2008. XXIII, 4, 44

Smith, R. L. Efficient monte carlo procedures for generating points uniformly distributed over bounded regions. Operations Research, 32(6):1296-1308, 1984. 134 
Smola, A. y Schölkopf, B. A tutorial on support vector regression. Statistics and Computing, 14:199-222, 2004. 44, 128

Smolyak, S. Quadrature and interpolation formulas for tensor product of certain classes of functions. Soviet Mathematics - Doklady, 4(3-4):209-32, 1963. 134, 135

Sóbester, A., Leary, S., y Keane, A. A parallel updating scheme for approximating and optimizing high fidelity computer simulations. Structural and Multidisciplinary Optimization, 27:371-383, 2004. 90

Sobieszczanski-Sobieski, J. y Haftka, R. T. Multidisciplinary aerospace design optimization: survey of recent developments. Structural Optimization, 14:1-23, 1997. 44

Sobol, I. On quasi-monte carlo integrations. Mathematics and Computers in Simulation, 47(2-5):103-112, 1998. 134

Sobol, I. Theorems and examples on high dimensional model representation. Reliability Engineering and System Safety, 79(2):187-193, 2003. 137

Soh, C. y Yang, J. Fuzzy controlled genetic algorithm search for shapeâoptimization. J. Comput. Civ. Eng., 10(2):143-150, 1996. 220

Spanos, P. y Ghanem, R. Stochastic finite element expansion for random media. Journal of Engineering Mechanics, 115(5):1035-1053, 1989. 134

Spanos, P. y Ghanem, R. Stochastic Finite Elements: A Spectral Approach. Springer-Verlag New Yotk Berlin Heidelberg, 1991. 134

Staum, J. Better simulation metamodeling: The why, what, and how of stochastic kriging. In Proceedings of the Winter Simulation Conference (WSC), páginas 119 133, Austin, TX, 13-16 Dec 2009. 67

Stefanou, G. The stochastic finite element method: Past, present and future. Computer Methods in Applied Mechanics and Engineering, 198(9-12):1031-1051, 2009. 134, 135, 171

Stefanou, G. y Papadrakakis, M. Stochastic finite element analysis of shells with combined random material and geometric properties. Comput. Methods Appl. Mech. Engrg., 193:139-160, 2004. 171, 172

Sudret, B. Meta-models for structural reliability and uncertainty quantification. In Asian-Pacific symposium on structural reliability and its applications, Singapore, 2012. 133

Sudret, B. y Kiureghian, A. D. Stochastic finite elements and reliability: a state-of-the-art report. Technical report, UCB/SEMM-2000/08. University of California, Berkeley., 2000. 53 
Sundaresan, S., Ishi, K., y Houser, D. A robust optimization procedure with variations on design variables and constraints. Adv. Design Automation, 65(1): 101-117, 1993. 19

Svanberg, K. The method of moving asymptotes - a new method for structural optimization. International Journal of Numerical Methods in Engineering, 24: 359-373, 1987. 35

Swanson, J. A. Characterization of the Strength, Stiffness and Ductility Behavior of T-stub Connections. PhD thesis, Georgia Institute of Technology, Atlanta, 1999. 167

Swiler, L. P., Wojtkiewicz, J. Steven F., Eldred, M. S., Giunta, A. A., y Trucano, T. G. Perspectives on optimization under uncertainty: Algorithms and applications. In Proceedings of the 10th AIAA/ISSMO Multidisciplinary Analysis and Optimization Conference, Albany, New York, 2005. 189

Taguchi, G. Introduction to Quality Engineering. Krauss International Publications, White Plains, NY, 1996. 20, 33

Tang, B. Orthogonal Array-Based Latin Hypercubes. Journal of the American Statistical Association, 88(424), 1993. 191

Tatang, M. Direct Incorporation of Uncertainty in Chemical and Environmental Engineering Systems. PhD thesis, Massachusetts Institute of Technology, 1995. 135

Toal, B. N. W. D. J. J. y Keane, A. J. Kriging hyperparameter tuning strategies. AIAA J., 46:1240-1252, 2008a. 67

Toal, B. N. W. D. J. J. y Keane, A. J. Geometric filtration using POD for aerodynamic design optimization. In Collection of Technical Papers-26th AIAA Applied Aerodynamics Conference, Honolulu, Hawai, 2008b. 67

Toal, D. J., Bressloff, N., Keane, A., y Holden, C. The development of a hybridized particle swarm for kriging hyperparameter tuning. Engineering Optimization, 43(6), 2011. 67

Törn, A. y Zilinskas, A. Global Optimization,. Springer-Verlag, Berlin, 1989. xxıv, 77,78

Trosset, M. Taguchi and robust optimization. Technical report, Department of Computational and Applied Mathematics, Rice University, 1996. 33

Ulmer, H., Streichert, F., y Zell, A. Evolution strategies assisted by gaussian processes with improved pre-selection criterion. páginas 692-699, Canberra, Australia, December 8-12, 2003. 90

Vasarhelyi, D. y Chiang, K. Coefficient of friction in joints of various steel. Journal of Structural Division ASCE, 93:227-243, 1967. 165 
Veldhuizen, D. A. V. Multiobjective Evolutionary Algorithms: Classifications, Analyses, and New Innovations. PhD thesis, Air Force Institute of Technology Wright Patterson AFB, OH, USA, 1999. 113, 114, 115, 198

Veldhuizen, D. A. V. y Lamont, G. B. Multiobjective Evolutionary Algorithm Research: A History and Analysis., 1998. Air Force Inst. Technol. 115, 195

Venkataraman, S. y Haftka, R. T. Structural optimization complexity: What has moore's law done for us? Structural and Multidisciplinary Optimization, 28(6): 375-387, 2004. 3

Viana, F. y Haftka, R. Surrogate-based optimization with parallel simulations using the probability of improvement. In 13th AIAA/ ISSMO Multidisciplinary Analysis and Optimization Conference, AIAA 2010-9392, Fort Worth, USA, September 13-15 2010. 90

Viana, F., Haftka, R., y Steffen, J. Valder. Multiple surrogates: how cross-validation errors can help us to obtain the best predictor. Structural and Multidisciplinary Optimization, 39(4):439-457, 2009. 68

Vining, G. G. y Myers, R. H. Combining Taguchi and response-surface philosophies - a dual response approach. Journal of Quality Technology, 22(1): 38-45, 1990. 186

Voutchkov, I. y Keane, A. Multiobjective optimization using surrogates. In Adaptive Computing in Design and Manufacture, páginas 18-36. 2006. 89, 90

Wald, A. Statistical Decision Functions. Wiley Publications in Statistics. John Wiley \& Sons, New York, NY, USA, 1950. 24

Wan, X. y Karniadakis, G. Multi-element generalized polynomial chaos for arbitrary probability measures. SIAM Journal of Scientific Computing, 28:901-928, 2006. 135,182

Wang, G. y Shan, S. An efficient pareto set identification approach for multiobjective optimization on black-box functions. Journal of Mechanical Design, 127:866-874, 2005. 89

Wang, G. G. y Shan, S. Review of Metamodeling Techniques in Support of Engineering Design Optimization. Journal of Mechanical Design, 129(4):370-380, 2007. 4,44

Wang, H. An efficient dimension-adaptive uncertainty propagation approach. Applied Mathematics and Computation, 218:3230-3237, 2011. 137

Wiener, N. The homogeneous chaos. American Journal of Mathematics, 60(4): 897-936, 1938. 135

Won, J., Choi, C., y Choi, J. Improved dimension reduction method (drm) in uncertainty analysis using kriging interpolation. Journal of Mechanical Science and Technology, 23:1249-1260, 2009. 137, 145, 157, 238 
Won, J. H., Choi, C. H., y Choi, J. H. Improving the Dimension Reduction Method (DRM) in the uncertainty analysis and application to the reliability based design optimization. In WCSMO-7, 2008. 136, 137, 139, 145

Xiang, G. A Metamodeling Method Based on Support Vector Regression for Robust Optimization. Chinese Journal of Mechanical Engineering, 23(02):242+, 2010. 186

$\mathrm{Xu}, \mathrm{H}$. y Rahman, S. A generalized dimension-reduction method for multidimensional integration in stochastic mechanics. International Journal for Numerical Methods in Engineering, 61:1992-2019, 2004. 136, 139

Yesilyurt, S., Ghaddar, C. K., Cruz, M. E., y Patera, A. T. Bayesian-validated surrogates for noisy computer simulations: application to random media. SIAM Journal on Scientific Computing, 17(4):973-992, 1996. 66

Youn, B. D. y Wang, P. Bayesian reliability-based design optimization using eigenvector dimension reduction (edr) method. Structural and Multidisciplinary Optimization, 36:107-123, 2008. 139

Youn, B. D. y Xi, Z. Reliability-based robust design optimization using the eigenvector dimension reduction (edr) method. Structural and Multidisciplinary Optimization, 37:475-492, 2009. 139

Youn, B. D., Xi, Z., y Wang, P. Eigenvector dimension reduction (EDR) method for sensitivity-free probability analysis. Structural and Multidisciplinary Optimization, 37:13-18, 2008. 137

Y.X. Gu, a. Z. K., Guan, Z., y Jia, Z. Dynamic sensitivity analysis and optimum design of aerospace structures. Int. J. Struct. Eng. Mech., 6:31-40, 1998. 3

Zajdel, M. Numerical analysis of bolted t-stub connections. tnoreport 97-con-r1123. Technical report, 1997. 165

Zhang, Q., Liu, W., Tsang, E., y Virginas, B. Expensive multiobjective optimization by MOEA/D with Gaussian process model. Trans. Evol. Comp, 14(3):456-474, June 2010. 89, 91, 92

Zhang, Y. y Leithead, W. Exploiting hessian matrix and trust-region algorithm in hyperparameters estimation of gaussian process. Applied Mathematics and Computation, 171(2):1264-1281, 2005. 67, 92

Zhou, A., Qu, B.-Y., Li, H., Zhao, S.-Z., Suganthan, P. N., y Zhang, Q. Multiobjective evolutionary algorithms: A survey of the state of the art. Swarm and Evolutionary Computation, 1:32-49, 2011. 90

Zilinskas, A. One-step bayesian method of the search for extremum of an one-dimensional function. Cybernetics, 1:139-144, 1975. 44, 85, 90

Zitzler, E. y Thiele, L. Multiobjective optimization using evolutionary algorithms-a comparative case study. páginas 292-301. 1998. 102 
Zitzler, E., Laumanns, M., , y Thiele, L. SPEA2: Improving the strength pareto evolutionary algorithm for multiobjective optimization. Evolutionary Methods for Design, Optimisation, and Control, páginas 95-100, 2002. 89

Zitzler, E., Thiele, L., Laumanns, M., Fonseca, C., y da Fonseca, V. Performance assessment of multiobjective optimizers: an analysis and review. Evolutionary Computation, IEEE Transactions on, 7(2):117 - 132, april 2003. 115, 198

Zitzler, E., Brockhoff, D., y Thiele, L. The hypervolume indicator revisited: on the design of pareto-compliant indicators via weighted integration. páginas 862-876. 2007. 92

Zuluaga, M., Krause, A., Sergent, G., y P., M. Active learning for multi-objective optimization. In Proceedings of the 30 International Conference on Machine Learning, Atlanta, Georgia, USA, 2013. 85, 113 
Este documento fue generado utilizando el sistema $\mathrm{LT}_{\mathrm{E}} \mathrm{X}{ }_{\varepsilon}(\mathrm{TeX}$ Live 2009/Debian) propuesto originalmente por Leslie Lamport, y basado en el lenguaje TeX desarrollado por Donald Knuth. Los ficheros fuentes fueron editados utilizando Kile 2.1beta4 en una distribución Ubuntu Linux 12.04.2 LTS. Las ilustaciones fueron generadas utilizando GIMP, Inkscape y Matlab ®.

Referencia (BibTEX):

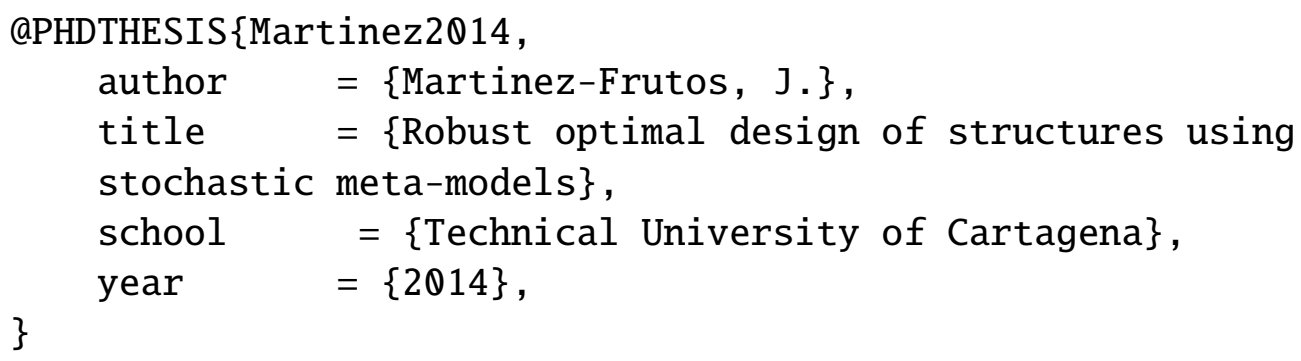

Todas las imagenes y algoritmos mostrados en este capítulo han sido obtenidas utilizando el software comercial MATLAB $®$ y la toolbox ooSBOUU (an object oriented toolbox for Surrogate-Based Optimization Under Uncertainty Toolbox) desarrollada durante esta tesis. 
REFERENCIAS 